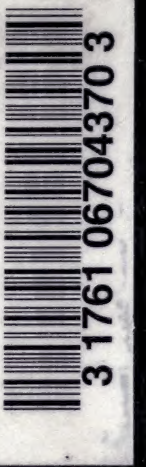




Digitized by the Internet Archive in 2007 with funding from Microsoft Corporation 


\section{COMPAR ATIVE}

\section{E LECT R O-PHYSIOL O G Y}




\section{WORKS BY THE SAME AUTHOR}

RESPONSE IN THE LIVING AND NON-

LIVING. With 117 Illustrations. 8vo. 10s. $6 d$. 1902.

PLANT RESPONSE: as a means of Physiological Investigations. With 278 Illustrations. 8 vo. $21 s$. 1906.

LONGMANS, GREEN \& CO., 39 Paternoster Row, London, New York, Bombay, and Calcutta 


\section{COMPARATIVE}

\section{ELECTRO-PHYSIOLOGY}

A PHYSICO-PHYSIOLOGICAL STUDY

BY

JAGADIS CHUNDER BOSE, M.A., D.Sc.

PROFESSOR, PRESIDENCY COLLEGE, CALCUTTA

WITH ILLUSTRATIONS

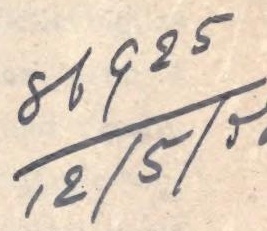

LONGMANS, GREEN, AND CO.

39 PATERNOSTER ROW, LONDON

NEW YORK, BOMBAY, AND CALCUTTA

1907 


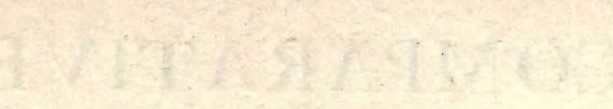

$10602=90$

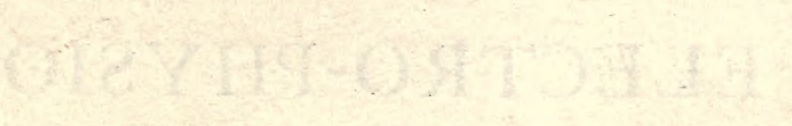




\section{PREFACE}

THIS volume concludes the line of investigation on responsive phenomena in general, which I commenced with the publication of a Memoir $^{1}$ at the International Congress of Science, Paris, I900. In this first of my publications on the subject I undertook to show the similarities of response in inorganic and living substances. The method which I at that time employed for obtaining my response-records was that of Conductivity Variation. With the object of showing that the similarity of response here demonstrated to exist was due to some fundamental molecular reaction, common to matter in general, and therefore to be detected by any method of recording response, I next undertook to record the Electro-motive Variation under stimulus. Believing, as I did, in the continuity of these responsive phenomena, I used the same experimental devices by which I had already succeeded in obtaining the electric response of inorganic substances, to test whether ordinary plants also, meaning those usually regarded as insensitive, would or would not exhibit excitatory electrical response to stimulus. The stimulation

I 'De la Généralité des Phénomènes Moléculaires produits par l'Electricité sur la Matière Inorganique et sur la Matière Vivante' (Travaux du Congrès International de Physique, Paris, 1900). See also 'On the Similarity of Effects of Electrical Stimulus on Inorganic and Living Substances,' Report Brit: Assoc., Bradford, September 1900 (Electrician). 
employed was mechanical and quantitative, thus obviating many sources of complication. By this method I was able to show that every plant, and every organ of every plant, gave true excitatory electrical response. As observations similar to these were subsequently made by another investigator, I quote here the following summary of my results. from the preliminary account which I communicated to the Royal Society, May 7, and afterwards read, with accompanying experimental demonstration, before the Society, on June 6, I90 I.

'An interesting link, between the response given by inorganic substances and the animal tissues, is that given by plant tissues. By methods somewhat resembling that described above, I have obtained from plants a strong electric response to mechanical stimulus. The response is not confined to sensitive plants like Mimosa, but is universally present. I have, for example, obtained such response from the roots, stems, and leaves of, among others, horse-chestnut, vine, white lily, rhubarb, and horse-radish. The "current of injury" is, generally speaking, from the injured to the uninjured part. A "negative variation" is also produced. I obtained both the single electric twitches and tetanus. Very interesting also are the effects of fatigue, of temperature, of stimulants, and of poison. Definite areas killed by poison exhibit no response, whereas neighbouring unaffected portions show the normal response.'

It may be well to point out here that at the time when this communication was made, the view that ordinary plants were excitable, and responded to mechanical stimulus by

1. A more complete account will be found in the report of my 'Friday Evening Discourse' before the Royal Institution, May 10, 1901, and in the Journal of the Linnean Society, vol. xxxv. p. 275. 
definite electro-motive changes, was regarded as highly controversial. Indeed, in the discussion which followed the reading of my Paper, on June 6, I90I, Sir John Burdon Sanderson went so far as to state that this excitatory response of ordinary plants to mechanical stimulation was an impossibility.

My next investigation was directed towards the question whether the responsive effects which I had shown to occur in ordinary plants might not be further exhibited by means of visible mechanical response, thus finally removing the distinction commonly assumed to exist between the 'sensitive' and supposed non-sensitive. These results were published in my work on Plant Response, ${ }^{1}$ where the effects of various environmental stimuli on the different plant organs were demonstrated by means of responsive movements. Many anomalous effects hitherto ascribed to specific sensibilities were here shown to be due to the differential excitability of anisotropic structures, and to the opposite effects of external and internal stimuli. Among other things, it was there shown that internal stimulus was in reality derived from external sources, and that the term 'autonomous response' was a misnomer, since all movements were due, either to the immediate effects of external stimulus, or to stimulus previously absorbed and held latent in the plant, to find subsequent expression. It was further shown that not gross mechanical movements alone, but also other invisible movements, were initiated by the action of stimulus; that external stimulus, so far from invariably causing a run-down of energy, more often brought about its accumulation by the plant; and that the various activities, such as the ascent of sap and growth,

' Plant Response as a Means of Physiological Investigation, 1906. 
were thus in reality different reactions to the stimulating action of energy supplied by the environment.

With regard to these points, my results have been in direct opposition to current views, according to which the effect induced by stimulus is always disproportionately greater than the stimulus. From the plausible analogy of the firing-off of a gun by the pulling of a trigger, or the action of a combustion-engine, it has been customary to suppose that all response to stimulus must be of the nature of an explosive chemical change, accompanied by an inevitable run-down of energy. This supposition, however, overlooks the obvious fact that the plant is not consumed by the incessant and multifarious stimuli of its environment. Rather, as we all know, it is the energy of the environment which is the agent that fashions the microscopic embryo into the gigantic banyan-tree. And it is clear that, for this to be possible, the energy contributed by the blow of external stimulus must have been largely conserved.

In the course of the present work, I have not only been able to corroborate, by means of electrical response, the various results which I had already established, with regard to the plant, by mechanical response, but I have also extended the electrical method in various directions, so as to include many more recondite problems in connection with the irritability of living tissues. It was my original intention to confine this investigation to the Electro-physiology of Plants. But, finding that in the results so obtained I possessed a key to that of the animal also, I proceeded to apply the same methods of inquiry, and to use the same experimental devices, in the one case as in the other. I have thus been able to trace out the gradual differentiation of various 
responsive peculiarities, characteristic of given tissues, from their simplest types in the plant to their most complex in the animal. The value of such a comparative method of study, for the elucidation of biological problems in general, is sufficiently obvious. Exception may be taken with regard to the unorthodox point of view from which various questions in animal physiology have been approached. It must be remembered, however, that in this work the attempt has been to explain responsive phenomena in general on the consideration of that fundamental molecular reaction which occurs even in inorganic matter. My mode of investigation has thus been determined by the necessary progression from simple to complex, and by my conviction as to the continuity which existed between them. And from this attempt it will be seen that various results, which, according to the so-called vitalistic assumption were anomalous, are, in fact, capable of an increasingly simple and satisfactory explanation. It must also be understood that my work deals mainly with the electrical response of plants, and that its extension into the field of Animal Electrophysiology was intended for the demonstration of the continuity between the two. It was therefore impossible, in the short space at my disposal, to make more than the brief necessary references to the different theories already in vogue concerning the response of various animal tissues. These will be found, in all their detail, in the excellent account given in the standard work of Biedermann. ${ }^{1}$

For the sake of clearness, however, I shall at this point enumerate a few only of the points of difference between current views and the results, obtained from actual experi-

' Biedermann, Electro-physiology (English translation), I896. 
ment, which I have set forth in the present volume. The reactions of different tissues have hitherto been regarded as specifically different. As against this, a continuity has here been shown to exist between them. Thus, nerve was universally regarded as typically non-motile; its responses were believed to be characteristically different from those of muscle. I have been able to show, however, that nerve is not only indisputably motile, but also that the investigation of its response by the mechanical method is capable of greater delicacy, and freedom from error, than that by the electrical. The characteristic variations in the response of nerve, moreover, are, generally speaking, similar to those of the muscle. It has been customary, again, to regard plants as devoid of the power to conduct true excitation. But I have shown that this view is incorrect. Experiments have been described, showing that the response of the isolated vegetal nerve is indistinguishable from that of animal nerve, throughout a long series of parallel variations of condition. So complete, indeed, has that similarity between the responses of plant and animal, of which this is an instance, been found, that the discovery of a given responsive characteristic in one case has proved a sure guide to its observation in the other, and the explanation of a phenomenon, under the simpler conditions of the plant, has been found fully sufficient for its elucidation under the more complex circumstances of the animal.

Many anomalous conclusions, with regard to the response of certain animal tissues, had arisen from the failure to take account of the differential excitability of anisotropic organs. Now this is a subject which, in the case of the simple plant organ, is capable of very exact investigation. I have been 
able to show that this differential excitability is widely present as a factor in determining the character of special responses, and that it finds its culminating expression in the electrical organs of certain well-known fishes.

Few conclusions in Electro-physiology have been supposed to rest on securer foundations than the generalisation known as Pfluiger's Law of the polar effects of currents. I have found, however, that this law is not by any means of such universal application as had been supposed, since, above and below a certain range of electromotive intensity, the polar effects of currents are precisely opposite to those enunciated by Pflüger.

Finally, that nervous impulse, which must necessarily form the basis of sensation, was supposed to lie beyond any conceivable power of visual scrutiny. But it has here been shown that this impulse is actually attended by change of form, and is therefore capable of direct observation. This wave of nerve-disturbance, moreover, instead of being single, has been shown to be of two different kinds, in which fact, as I have further explained, lies the significance of the two different qualities or tones of sensation.

In the concluding portion of the paper which I read before the Bradford meeting of the British Association in the year I900, I said :-

'In the phenomena described above there is little breach of continuity. It is difficult to draw a line and say: "Here the physical process ends, and the physiological process begins"; or "That is a phenomenon of inorganic matter, and this is a vital phenomenon, peculiar to living organisms"; or "These are the lines of demarcation that separate the physical, the physiological, and the beginning 
of psychical processes." Such arbitrary lines can hardly be drawn.

'We may explain each of the above classes of phenomena by making numerous and independent assumptions; or, finding some property of matter common and persistent in the living and non-living substances, attempt from this common underlying property to explain the many phenomena which at first appear so different. And for this it may be said that the tendency of science has always been to attempt to find, wherever facts justify it, an underlying unity in apparent diversity.'

It was for the demonstration of this underlying unity that I set out on these investigations seven years ago. And now, in bringing to its close another stage of their publication, I may, perhaps, be permitted to express the hope that by them not only may a deeper perception of this unity have been made attainable, but also that many regions of inquiry may prove to have been opened out, which had at one time been regarded as beyond the scope of experimental exploration.

I take this opportunity to thank my assistants for their efficient help in these researches.

J. C. BOSE.

Presidency College, Calcutta : August 1906. 


\title{
CONTENTS
}

\author{
CHAPTER I \\ THE MOLECUIAAR RESPONSIVENESS OF MATTER
}

Response to stimulus by change of form-Permeability variation-Variation of solubility-Method of resistivity variation : $(a)$ positive variation ; $(b)$ negative variation-Sign of response changed under different molecular modifications-Response of vegetable tissue by variation of electrical resistance-Response by electro-motive variation in inorganic substances - The method of block-Positive and negative responses-Similar responses in living tissues-Effects of fatigue, stimulants, and poisons on inorganic and organic responses-Method of relative depression, or negative variation, so called

\section{CHAPTER II}

THE ELECTRO-MOTIVE RESPONSE OF PLANTS TO DIFFERENT FORMS OF STIMULATION

Historical-Difficulties of investigation-Electrical response of pulvinus of Mimosa-Simultaneous mechanical and electrical records-Division of plants into 'ordinary' and 'sensitive' arbitrary-Mechanical and electrical response of 'ordinary' plants-Direct and transmitted stimulation-All forms of stimulus induce excitatory change of galvanometric negativity

\section{CHAPTER III}

THE APPLICATION OF QUANTITATIVE STIMULUS AND KELATION BETWEEN STIMULUS AND RESPONSE

Conditions of obtaining uniform response-Torsional vibration as a form of stimulus-Method of block-Effective intensity of stimulus dependent on period of vibration-Additive action of feeble stimuli-Response 
recorder-Uniform electric responses-List of suitable specimensEffect of season on excitability - Stimulation by thermal shocks - Thermal stimulator-Second method of confining excitation to one contact-Increasing response to increasing stimulus-Effect of fatigue-Tetanus .

\section{CHAPTER IV \\ OBSERVATION BY RHEOTOME ON ELECTRIC RESPONSE IN PLANTS}

Response-curve showing general time-relations-Instantaneous mechanical stimulation by electro-magnetic release-Arrangement of the rheotome - Tabular statement of results of rheotomic observations-Rhythmic multiple responses . . . . . . . . . .

\section{CHAPTER V}

THE ELECTRICAL INDICATIONS OF POSITIVE AND NEGATIVE TURGIDITY-VARIATIONS

Motile responses of opposite signs, characteristic of positive and negative turgidity-variations-Indirect hydrostatic effect of stimulus causes expansion and erection of leaf--Positive and negative work-Wave of increased hydrostatic tension transmitted with relatively greater velocity than wave of true excitation-Method of separating hydro-positive and excitatory effects-Indirect effect of stimulus, causing positive turgidityvariation induces galvanometric positivity-Antagonistic elements in the electrical response-Separation of hydro-positive from true excitatory effect by means of physiological block

\section{CHAPTER VI}

\section{EXTERNAL STIMULUS AND INTERNAL ENERGY}

Ifydraulic transmission of energy in plants--True meaning of tonic condition--Opposite expressions of internal energy and external stimulus seen in growth-response-Parallelism between responses of growing and motile organs-Increased internal energy caused by augmentation or temperature finds expression in enhanced rate of growth; erection of motile leaf ; curling movement of spiral tendril ; and galvanometric positivity-External stimulus induces opposite eftect in all these casesSudden variation of temperature, acting as a stimulus, induces transient retardation of growth; depression of motile leaf ; uncurling mevement of spiral tendril ; and galvanometric negativity-Laws of mechanical and electrical response 


\section{CHAPTER VII}

\section{ABSORPTION AND EMISSION OF ENERGY IN RESPONSE}

Sign of response determined by latent energy of tissue, and by intensity of external stimulus-Sub-tonic, normal and hyper-tonic conditions-The critical level-Outward manifestation of response possible only when critical level is exceeded-Three typical cases: response greater than stimulus; response equal to stimulus; and response less than stimulus -Investigation by growth-response-The sum of work, internal and external, performed by stimulus constant-Positive response of tissues characterised by feeble protoplasmic activity or sub-tonicity-Enhancement of normal excitability of sub-tonic tissue by absorption of stimulus

\section{CHAPTER VIII}

\section{VARIOUS TYPES OF RESPONSE}

Chemical theory of response-Insufficiency of the theory of assimilation and dissimilation-Similar responsive effects seen in inorganic matterModifying influence of molecular condition on response-Five molecular stages, A, B, C, D, E-Staircase effect, uniform response, fatigue-No sharp line of demarcation between physical and chemical phenomenaVolta-chemical effect and by-productions-Phasic alternation-Alternating fatigue-Rapid fatigue under continuous stimulation-In subtonic tissue summated effect of latent components raises tonicity and excitability-Response not always disproportionately greater than stimulus-Instances of stimulus partially held latent: staircase and additive effects, multiple response, renewed growth . . . .

\section{CHAPTER IX}

\section{DETECTION OF PHYSIOLCGICAL ANISOTROPY BY ELECTRIC RESPONSE}

Anomalies in mechanical and electrical response-Resultant response determined by differential excitability-Responsive current from the more to the less excitable-Laws of response in anisotropic organ-Demonstration by means of mechanical stimulation-Vibrational stimulus-Stimulation by pressure-Quantitative stimulation by thermal shocks . .

\section{CHAPTER $\mathrm{X}$}

\section{THE NATURAL CURRENT AND ITS VARIATIONS}

Natural current in anisotropic organ from the less to the more excitableExternal stimulus induces responsive current in opposite directionIncrease of internal energy induces positive, and decreàse negative, 
variation of natural current-Effect on natural current of variation of temperature-Effect of sudden variation-Variation of natural current by chemical agents, referred to physiological reaction-Agents which render tissue excitable, induce the positive, and those which cause excitation, the negative variation-Action of hydrochloric acid-Action of $\mathrm{Na}_{2} \mathrm{CO}_{3}$-Effect modified by strength of dose-Effect of $\mathrm{CO}_{2}$ and of alcohol vapour-Natural current and its variations-Extreme unreliability of negative variation so-called as a test of excitatory reactionReversal of natural current by excessive cold or by stimulation-Reversal of normal response under sub-tonicity or fatigue .

\section{CHAPTER XI}

VARIATIONS OF EXCITABIIITY UNDER CHEMICAL REAGENTS

Induced variation of excitability studied by two methods: (I) direct (2) transmitted stimulation-Fffect of chloroform-Effect of chloralEffect of formalin-Advantage of the Method of Block over that of negative variation-Effect of $\mathrm{KHO}$ - Response unaffected by variation of resistance-Stimulating action of solution of sugar-Of sodium carbonate-Effect of doses - Effect of hydrochloric acid-Diphasic response on application of potash-Conversion of normal negative into abnormal positive response by abolition of true excitability .

\section{CHAPTER XII}

VARIATIONS OF EXCITABILITY DETERMINED BY METHOD OF INTERFERENCE

Arrangement for interference of excitatory waves-Effect of increasing difference of phase-Interference effects causing change from positive to negative, through intermediate diphasic-Diametric balance--Effect of unilateral application of $\mathrm{KHO}-$ Effect of unilateral cooling .

\section{CHAPTER XIII}

\section{CURRENT OF INJURY AND NEGATIVE VARIATION}

Different theories of current of injury-Pre-existence theory of Du BoisReymond-Electrical distribution in a muscle-cylinder-Electro-molecular theory of Bernstein-Hermann's Alteration Theory-Experiments demonstrating that so-called current of injury is a persistent after-effect of over-stimulation-Residual galvanometric negativity of strongly excited tissue-Distribution of electrical potential in vegetable tissue with one end sectioned-Electrical distribution in plant-cylinder similar to that in muscle-cylinder-True significance of response by negative variation-. Apparent abnormalities in so-called current of injury-'Positive, current of injury 


\section{CHAPTER XIV}

\section{CURRENT OF DEATH-RESPONSE BY POSITIVE VARIATION}

Anomalous case of response by positive variation-Inquiry into the causeElectric exploration of dying and dead tissue : death being naturalDetermination of electric distribution in tissue with one end killedDying tissue shows maximum negativity, and dead tissue, positivity to living-Explanation of this peculiar distribution-Response by negative or positive variation, depending on degree of injury-Three typical cases - Explanation by theory of assimilation and dissimilation misleading All response finally traceable to simple fundamental reactions

\section{CHAPTER XV}

\section{EFFECT OF TEMPERATURE ON ELECTRICAL RESPONSE}

General observation of effect of temperature on plant-Effect of fall and rise of temperature on autonomous response of Desmodium-Effect of frost in abolition of electrical response-After-effects of application of cold, in Eucharis, Ivy and Holly-Effect of rise of temperature in diminishing height of response-This not probably due to diminution of excitability - Similar effect in autonomous motile response of Desmodium-Enhanced response as after-effect of cyclic variation of temperature-Abolition of response at a critical high temperature . . . . .

\section{CHAPTER XVI}

\section{THE ELECTRICAL SPASM OF DEATH}

Different post-mortem symptoms-Accurate methods for determination of death-point-Determination of death-point by abolition or reversal of normal electrical response-Determination of death-point by mechanical death-spasm-From thermo-mechanical inversion-By observation of electrical spasm : $(a)$ in anisotropic organs : $(b)$ in radial organs-Simultaneous record of electrical inversion and reversal of normal electrical response-Remarkable consistency of results obtained by different methods-Tabulation of observations

\section{CHAPTER XVII}

\section{MULTIPLE AND AUTONOMOUS ELECTRICAL RESPONSE}

Repeated responses under single strong stimulus-Multiple mechanical response in Biophytum-Multiple electrical responses in various animal and vegetable tissues - Continuity of multiple and autonomous response - Transition from multiple response to autonomous, and vice versa- 
Autonomous mechanical response of Desmodium gyrans and its timerelations-Simultaneous mechanical and electrical records of automatic pulsations in Desmodium-Double electrical pulsation, principal and subsidiary waves-Electrical pulsation of Desmodum leaflet under physical restraint - Growth-pulsation - So-called current of rest in growing plants .

\section{CHAPTER XVIII}

\section{RESPONSE OF LEAVES}

Observations of Burdon Sanderson on leaf-response in Dionca-Leaf and stalk currents-Their opposite variations under stimulus-Similar leafand-stalk currents shown to exist in ordinary leaf of Ficus religiosaOpposite-directioned currents in Citrus decumana-True explanation of these resting-currents and their variations-Electrical effect of section of petiolc on Dioncea and Ficus religiosa-Fundamental experiment of Burdon Sanderson on lamina of Dionaa-Subsequent results-Experimental arrangement with symmetrical contacts-Parallel experiments on sheathing leaf of Musa-Explanation of various results

\section{CHAPTER XIX}

\section{THE LEAF CONSIDERED AS AN ELECTRIC ORGAN}

Electrical organs in fishes-Typical instances, Torpedo and MalepterurusVegetal analogues, leaf of Pterospermum and carpel of Dillenia indica or pitcher of Nepenthe-Electrical response to transmitted excitation -Response to direct excitation-Uni-directioned response to homodromous and heterodromous shocks-Definite-directioned response shown to be due to differential excitability-Response to equi-alternating electrical shocks-Rheotomic observations-Multiple excitationsMultiplication of terminal electromotive effect, by pile-like arrangement, in bulb of Uriclis lily

\section{CHAPTER XX}

THE THEORY OF ELECTRICAL ORGANS

Existing theories-Their inadequacy--The 'blaze-current' so called-Response uni-directioned, to shocks homodromous or heterodromous, characteristic of electric organs-Similar results with inorganic specimens -Uni-directioned response due to differential excitability-Electrical response of pulvinus of Mimosa to equi-alternating electric shocks- $\mathrm{Re}$ sponse of petiole of $\mathrm{Musa}$ - Of plagiotropic stem of Cucurbita-Of EelThe organ-current of electric fishes-Multiple responses of electrical organ-Multiple responses of Biofhysum . . . . . . . 


\section{CHAPTER XXI}

\section{DETERMINATION OF DIFFERENTIAL EXCITABILITY UNDER}

\section{ELECTRICAL STIMULATION}

Advantage of electrical stimulation, in its flexibility-Drawbacks due to fluctuating factors of polar effects, and counter polarisation-currentDifficulties overcome by employment of equi-alternating electric shocks-Methods of the After-effect and Direct-effect-Experiment of Von Fleischl on response of nerve-Complications arising from use of make and break shocks-Rotating reverser-Motor transformer-Response of Musa to equi-alternating shocks-Abolition of this response by chloroform-Response records of plagiotropic Cucurbita and EelDifferential excitability of variegated leaves, demonstrated by electric response

\section{CHAPTER XXII}

\section{RESPONSE OF ANIMAL AND VEGETAL SKINS}

Currents of rest and action-Currents in animal skin-Theories regarding these-Response of vegetal skin-Stimulation by Rotary Mechanical Stimulator-Response of intact human skin--Isolated responses of upper and lower surfaces of specimens-Resultant response brought about by differential excitability of the two surfaces-Differences of excitability between two surfaces accounted for-Response of animal and vegetal skins not essentially different-General formula for all types of response of skin-Response of skin to different forms of stimulation gives similar results-Response to equi-alternating electric shocks : (I) Method of the After Effect; (2) Method of Direct Effect-Response of grape skin-Similar response of frog's skin-Phasic variation of current of rest induced as result of successive stimulation in $(a)$ grape skin ; $(b)$ frog's skin; (c) pulvinus of Mimosa-Phasic variation in autonomous mechanical response of Desmodium gyrans-Autonomous variation of current of rest-True current of rest in skin from outer to inner-This may be reversed as an excitatory after-effect of preparation-Electrical response of skin of neck of tortoise-Electrical response of skin of tomato-Normal response and positive after-effect-Response of skin of gecka-Explanation of abnormal response . . . . .

\section{CHAPTER XXIII}

\section{RESPONSE OF EPITHELIUM AND GLANDS}

Epidermal, epithelial, and secreting membranes in plant tissues-Natural resting-current from epidermal to epithelial or secretory surfaces-Current of response from epithelial or secretory to epidermal surfàces-Response 
of Dillenia-Response of water-melon-Response of foot of snail-The so-called current of rest from glandular surface really due to injuryMisinterpretation arising from response by so-called 'positive variation' -Natural current in intact foot of snail, and its variation on sectionResponse of intact human armpit-Response of intact human lipLingual response in man-Reversal of normal response under subminimal or super-maximal stimulation-Differential excitations of two surfaces under different intensities of stimulus, with consequent changes in direction of responsive currents, diagrammatically represented in characteristic curves-Records exhibiting responsive reversals

\section{CHAPTER XXIV}

\section{RESPONSE OF DIGESTIVE ORGANS}

Consideration of the functional peculiarities of the digestive organ-Alternating phases of secretion and absorption-Relation between secretory and contractile responses. Illustrated by $(a)$ preparation of Mimosa; (b) glandular tentacle of Drosera-General occurrence of contractile response-True current of rest in digestive organs-Experiments on the pitcher of Nepenthe-Three definite types of response under different conditions-Negative and positive electrical responses, concomitant with secretion and absorption-Multiple responses due to strong stimulation - Response in glandular leaf of Drosera-Normal negative response reversed to positive under continuous stimulation-Multiple response in Drosera-Response of frog's stomach to mechanical stimulation-Response of stomach of tortoise-Response of stomach of gecko-Multiple response of frog's stomach, showing three stages--negative, diphasic, and positive-Phasic variations . . . , . . .

\section{CHAPTER XXV}

\section{ABSORPTION OF FOOD BY PLANT AND ASCENT OF SAP}

Parallelism between responsive reactions of root and digestive organ-Alternating phases of secretion and absorption-Association of absorptive process with ascent of sap-Electrical response of young and old rootsDifferent phasic reactions, as in pitcher of Nepenthe-Response to chemical stimulation-Different theories of ascent of sap--Physical versus excitatory theories-Objections to excitatory theory-Assumption that wood dead unjustified-Demonstration of excitatory electrical response of sap-wood-Strasburger's experiments on effect of poisons on ascent of sap-Current inference unjustified 


\section{CHAPTER XXVI}

THE EXCITATORY CHARACTER OF SUCTIONAL RESPONSE

Propagation of excitatory wave in plant attended by progressive movement of water-Hydraulic response to stimulus-The Shoshungraph-Direct and photographic methods of record-Responsive variations of suction under physiological modifications induced by various agents-Effects of lowering and raising of temperature-Explanation of maintenance of suction, when root killed-Effect of poison influenced by tonic condition-Effect of anæsthetics on suctional response-Excitatory versus osmotic action - Stimulation by alternating induction-shocks-Terminal and sub-terminal modes of application-Three modes of obtaining response-records, namely (1) the unbalanced, (2) the balanced, (3) the over-balancedRenewal of suction previously at standstill, by action of stimulus-- Reponsive enhancement of suction by stimulus-After-effect of stimulusDiminution of latent period as after-effect of stimulus-Response under over-balance-Response under sub-terminal stimulation-Variation of response under seasonal changes .

\section{CHAPTER XXVII}

\section{RESPONSE TO STIMULUS OF LIGHT}

Heliotropic plant movements reducible to fundamental reaction of contraction or expansion-Various mechanical effects of light in pulvinated and growing organs-Electrical response induced by light not specific, but concomitant to excitatory effects-Electrical response of plant to light not determined by presence or absence of chloroplasts-Effect of unilateral application of stimulus on transwersely distal point-Positive response due to indirect effect and negative to transmission of true excitation-Mechanical response of leaf of Mimosa to light applied on upper half of pulvinus-Mechanical response consists of erection or positive movement, followed by fall or negative movement-Electrical response of leaf of Mimosa to light applied on upper half of pulvinus ; induction in lower half of pulvinus of positivity followed by negativityLongitudinal transmission of excitatory effect, with concomitant galvanometric negativity--Direct effect of light and positive after effectCircumstances which are effective in reversing normal response-Plants in slightly sub-tonic condition give positive followed by negative response -Exemplified by $(a)$ electrical and $(b)$ growth response-Examples of positive response to light-Periodic variation of excitability-Multiple mechanical response under light-Direct and after-effect-Multiple electrical response under light, with phasic alterations of $(-+-+)$ or $(+-+-)$--After-effects ; unmasking of antagonistic elements, either plus or minus-Three types of after-effects 


\section{CHAPTER XXVIII \\ RESPONSE OF RETINA TO STIMULUS OF LIGHT}

Response of retina-Determination of true current of rest-Determination of differential excitabilities of optic nerve and cornea, and optic nerve and retina-The so-called positive variation of previous observers indicates the true excitatory negative-Retino-motor effects-Motile responses in nerve-Varying responsive effects under different conditions -Reversal of the normal response of light due to (I) depression of excitability below par ; (2) fatigue-The sequence of responsive phases during and after application of light-Demonstration of multiple responses in retina under light, as analogous to those in vegetable tissues - Three types of after-effect-Multiple after-excitations in human retina-Binocular Alternation of Vision-Demonstration of pulsatory response in human retina during exposure to light

\section{CHAPTER XXIX}

\section{GEO-ELECTRIC RESPONSE}

Theory of Hydrostatic Pressure and Theory of Statoliths-Question regarding active factor of curvature in geotropic response, whether contraction or expansion-Crucial experiment by local application of cold-Reasons for delay in initiation of true geotropic response-Geo-electric response of shoot - Due to active contraction of upper side, with concomitant galvanometric negativity-Geo-electric response of an organ physically restrained .

\section{CHAPTER XXX}

\section{DETERMINATION OF VELOCITY OF TRANSMISSION OF}

\section{EXCITATION IN PLANT TISSUES}

Transmission of excitation in plants not due to hydromechanical disturbance, but instance of transmission of protoplasmic changes-Difficulties in accurate determination of velocity of transmission-A perfect methodDiminution of conductivity by fatigue-Increased velocity of transmission with increasing stimulus-Effect of cold in diminishing conductivity Effect of rise of temperature in enhancing conductivity-Excitatory concomitant of mechanical and electrical response-Electrical methods of determining velocity of transmission-Method of comparison of longitudinal and transverse conductivities-Tables of comparative velocities in animal and plant - Existence of two distinct nervous impulses, positive and negative 


\section{CHAPTER XXXI}

\section{ON A NEW METHOD FOR THE QUANTITATIVE STIMULATION} OF NERVE

Drawbacks to use of electrical stimulus in recording electrical response Response to equi-alternating electrical shocks-Modification of response by decline of injury-Positive after-effect-Stimulation of nerve by thermal shocks-Enhancement of normal response after tetanisation Untenability of theory of evolution of carbonic acid-Abnormal positive response converted into normal negative after tetanisation-Gradual transition from positive to negative, through intermediate diphasicEffect of depression of tonicity on excitability and conductivity-Conversion of abnormal into normal response by increase of stimulus-intensity - Cyclic variation of response under molecular modification . . .

\section{CHAPTER XXXII}

\section{ELECTRICAL RESPONSE OF ISOLATED VEGETAL NERVE}

Specialised conducting tissues-Isolated vegetal nerve-Method of obtaining electrical response in vegetal nerve-Similarity of responses of plant and animal nerve: $(a)$ action of ether $-(b)$ action of carbonic acid- $(c)$ action of vapour of alcohol- $(d)$ action of ammonia-(e) exhibition of three types of response, negative, diphasic and positive-$(f)$ effects of tetanisation of normal and modified specimens-Effect of increasing stimulus on response of modified tissue

\section{CHAPTER XXXIII}

\section{THE CONDUCTIVITY BALANCE}

Receptivity, conductivity, and responsivity-Necessity for distinguishing these-Advantages of the Method of Balance - Simultaneous comparison of variations of receptivity, conductivity and responsivity-The Conductivity Balance-Effect of $\mathrm{Na}_{2} \mathrm{CO}_{3}$ on frog's nerve-Effect of $\mathrm{CuSO}_{4}$-Effect of chemical reagents on plant nerve-Effect of $\mathrm{CaCl}_{2}$ on responsivityResponsivity variation under $\mathrm{KCl}$-Comparison of simultaneous effects of $\mathrm{NaCl}$ and $\mathrm{NaBr}$ on responsivity-Effects of $\mathrm{Na}_{2} \mathrm{CO}_{3}$ in different dilutions on conductivity-Demonstration of two different elements in conductivity, velocity and intensity-Conductivity versus responsivity(a) effect of $\mathrm{KI}-(b)$ Effect of $\mathrm{NaI}-\mathrm{Effect}$ of alcohol on receptivity, conductivity, and responsivity-Comparison of simultaneous effects of alcohol- $(a)$ on receptivity versus conductivity - (b) on receptivity versus responsivity 


\section{CHAPTER XXXIV}

\section{EFFECT OF TEMPERATURE AND AFTER-EFFECTS OF STIMULUS ON CONDUCTIVITY}

Effect of temperature in inducing variations of conductivity : $(a)$ by Method of Mechanical Response ; $(b)$ by Method of Electric Balance-Effect of cold-Effect of rising temperature-The Thermal Cell-After-effect of stimulation on conductivity - The Avalanche Theory-Determination of after-effect of stimulus on conductivity by the Electrical Balance-Aftereffects of moderate stimulation-After-effect of excessive stimulation .

\section{CHAPTER XXXV}

\section{MECHANICAL RESPONSE OF NERVE}

Current assumption of non-motility of nerve-Shortcomings of galvanometric modes of detecting excitation-Mechanical response to continuous electric shocks-Optical Kunchangraph-Effect of ammonia on the mechanical response of nerve-Effect of morphia-Action of alcoholOf chloroform-Abnormal positive or expansive response converted into normal contractile through diphasic, after tetanisation-Similar effects in mechanical response of vegetal nerve-Mechanical response due to transmitted effects of stimulation-Determination of velocity of transmission-Indeterminateness of velocity in isolated nerve-Kunchangraphic records on smoked glass-Oscillating recorder-Mechanical response of afferent nerve-Record of mechanical response of nerve due to transmitted stimulation, in gecko-Fatigue of conductivity-Conversion of normal contractile response into abnormal expansive, through diphasic, due to fatigue

\section{CHAPTER XXXVI}

\section{MULTIPLE RESPONSE OF NERVE}

Great sensitiveness of the high magnification Kunchangraph-Individual contractile twitches shown in tetanisation of nerve-Sudden enhancement of mechanical response of nerve on cessation of tetanisationSecondary excitation-Multiple mechanical excitation of nerve by single strong stimulation-Multiple mechanical excitation of nerve by drying .

\section{CHAPTER XXXVII}

\section{RESPONSE BY VARIATION OF ELECTRICAL RESISTIVITY}

Variation of resistance in Dioncea, by 'modification'-Excitatory change, its various independent expressions - Characteristic difficulties of investi- 
gation-Morographic record by variation of resistivity-Inversion of PAGE curves at death-point--Similarities between mechanical, electro-motive and resistivity curves of death-The true excitatory effect attended by diminution of resistance-Response of plant nerve by resistivity variation-Independence of resistivity and mechanical variations-Responsive resistivity variation in frog's nerve, and its modification under anæsthetics

\section{CHAPTER XXXVIII}

\section{FUNCTIONS OF VEGETAL NERVE}

Feeble conducting power of cortical tissues-Heliotropic and geotropic effects dependent on response of cortical tissues only-Phenomenon of correlation-Excitability of tissue maintained in normal condition only under action of stimulus-Physiological activities of growth, ascent of sap, and motile sensibility, maintained by action of stimulus-Critical importance of energy of light-Leaf-venation a catchment-basin-Transmission of energy to remotest parts of plants-Plant thus a connected and organised entity .

\section{CHAPTER XXXIX}

\section{ELECTROTONUS}

Extra-polar effects of electrotonic currents on vegetal nerve-Electrotonic variation of excitability-Bernstein's polarisation decrement-Hermann's polarisation increment-Investigation into the law of electrotonic variation of conductivity - Investigation on variation of excitability-Conductivity enhanced when excitation travels from places lower to higher electric potential, and depressed in opposite direction-When feeble, anode enhances and kathode depresses excitability-All electrotonic phenomena reducible to combined action of these factors-Explanation of apparent anomalies.

\section{CHAPTER XL}

\section{INADEQUACY OF PFLÜGER'S LAW}

Reversal of Pfliger's Law under high E.M.F.-Similar reversals under feeble E.M.F.-Investigation by responsive sensation-Experiments on living wounds-Under moderate E.M.F., intensity of sensation enhanced at kathode, and depressed at anode-Under feeble E.M.F., sensation intensified at anode and depressed at kathode-Application of electrical currents in medical practice 


\section{CHAPTER XLI \\ THE MOLECULAR THEORY OF EXCITATION AND ITS TRANSMISSION}

Two opposite responsive manifestations, negative and positive-Such opposite responses induced by polar effects of currents of different signs - Arbitrary nature of term ' excitatory'-Pro-excitatory and anti-excitatory agents-Molecular distortion under magnetisation in magnetic substances-Different forms of response under magnetic stimulationMechanical, magneto-metric, and electro-motive responses-Uniform magnetic responses-Response exhibiting periodic groupings-Ineffective stimulus made effective by repetition-Response by resistivityvariation-Molecular model-Response of inorganic substance to electric radiation-Effect of rise of temperature in hastening period of recovery and diminishing amplitude of response-Sign of response reversed under feeble stimulation-Conduction of magnetic excitation-The Magnetic Conductivity Balance-Effect of A-tonus and K-tonus, on excitability and conductivity - Conducting path fashioned by stimulus-Transmission of excitation temporarily blocked in iron wire, as in conducting nerveArtificial nerve-anḋ-muscle preparation

\section{CHAPTER XLII}

\section{MODIFICATION OF RESPONSE UNDER CYCLIC MOLECULAR VARIATION}

Anomalies of response-Explicable only from consideration of antecedent molecular changes-Continuous transformation from sub-tonic to hypertonic conditions-Two methods of inquiry, first by means of characteristic curves, second by progressive change of response--Abnormal response characteristic generally of A or sub-tonic state-Abnormal transformed into normal, after transitional $\mathbf{B}$ state-B state characterised by staircase response-Responses at C stage normal and uniform-At stages $\mathrm{D}$ and $\mathrm{E}$ responses undergo diminution and reversal-Responsive peculiarities seen during ascent of curve, repeated in reverse order during descent-All these peculiarities seen not only in living but also in inorganic substances, under different methods of observation-Elucidation of effect of drugs-Response modified by tonic condition and past history

\section{CHAPTER XLIII}

CERTAIN PSYCHO-PHYSIOLOGICAL PHENOMENA-THE PHYSICAL BASIS OF SENSATION

Indications of stimulatory changes in nerve : I, Electrical ; 2, MechanicalTransmission in both directions-Stimulatory changes in motor and 
sensory nerves similar-Responsive molecular changes and the correlated tones of sensation-Two kinds of nervous impulse, and their characteristics-Different manifestations of the same nervous impulse determined by nature of indicator--Electrical, motile, and sensory responses, and their mutual relations-The brain as a perceiving apparatus-WeberFechner's Law - Elimination of psychic assumption from explanation of particular relation between stimulus and resultant sensation-Explanation of the factor of quality in sensation-Explanation of conversion from positive to negative tone of sensation after tetanisation-Various effects of progressive molecular change in nerve-Effects of attention and inhibition-Polar variations of tonus, inducing acceleration and retardation .

\section{CHAPTER XLIV}

\section{DISSOCIATION OF COMPLEX SENSATION}

Conversion of pleasurable into painful sensation, and vice versa, by electrotonus-The Sensimeter-Mechanical stimulation-Stimulation by thermal shocks-Chemical stimulation-Opposite effects of anode and kathode-Normal effects reversed under feeble E.M.F. - Negative tone of sensation blocked by alcohol and anæsthetics-Separation of positive and negative sensations, by lag of one wave behind the other-Dissociation of sensation by depression of conductivity-Abolition of the negative or painful element by block of conduction . . . . . . 666

\section{CHAPTER XLV}

MEMORY • • • . . . 677

CHAPTER XLVI

REVIEW OF RESPONSE OF ISOTROPIC ORGANS . . 687

CHAPTER XLVII

REVIEW OF RESPONSE OF ANISOTROPIC ORGANS • 700

\section{CHAPTER XLVIII}

REVIEW OF RESPONSE OF NERVE AND RELATED PSYCHOLOGICAL

PHENOMENA . . . . . 718

CLASSIFIED LIST OF EXPERIMENTS . . . . . 735

INDEX 



\section{ILLUSTRATIONS}

FIG.

I. Series of Contractile Responses in Muscle . . . . . I

2. Response of Indiarubber . . . . . . . . . 2

3. Response of Selenium to the Stimulus of Light . • . . 3

4. Negative Response of Galena to Hertzian Radiation . . . . 3

5. Positive Response of $\mathrm{Ag}^{\prime}$ to Electric Radiation. . . . . . 4

6. Electric Response in Metals . . . . . . . . 5

7. Uniform Electric Response in Tin . . . . . . . 6

8. Fatigue in the Electric Response of Metals . $\quad$. . . . 7

9. Stimulating Action of $\mathrm{Na}_{2} \mathrm{CO}_{3}$ on Electric Response of Platinum - 8

IO. Abolition of Response in Metal by Oxalic Acid . . . . . 9

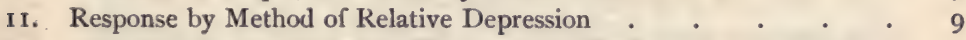

I2. Arrangement for observing Simultaneous Mechanical and Electrical Responses . . . . . . . . . . . I7

13. Simultaneous Mechanical and Electrical Responses in Biophytum . I9

14. Photographic Record of Electrical Response by Galvanometric Negativity of Pulvinus of Mimosa, when leaf is physically restrained from falling . . . . . . . . 20

15. Method of Transmitted Stimulation . . . . . . . 24

16. Excitation by Sudden Tension . . . . . . . . 25

I7. Excitatory Response to Tension and Compression . . . . 26

I8. The Mechanical Tapper . . . . . . . 26

19. The Torsional Vibrator . . . . . . . . 27

20. The Vibratory Stimulator . . . . . . . . 30

2I. Complete Apparatus for Method of Block and Vibratory Stimulation . 3 I

22. Influence of Suddenness on the Efficiency of Stimulus . . . . 32

23. Spring Attachment for obtaining Vibration of Uniform Rapidity . 33

24. Additive Effect . . . . . . . . . 34

25. Response Recorder . . . . . . . . . . 34

26. Photographic Record of Uniform Responses (Radish) . . . . 36

27. Stimulation by Thermal Shocks . . . . . . $\quad 38$

28. Photographic Record of Uniform Response in Petiole of Fern to transmitted excitation . . . . . . . . . . 39

29. Taps of increasing strength $I: 2: 3: 4$ producing increased response in leafstalk of turnip . . . . . . . 
FIG.

30. Increased Response with Increasing Vibrational Stimuli (Cauliflowerstalk)

3I. Responses to Increasing Stimulus obtained with Two Specimens of Stalk of Cauliflower

32. Genesis of Tetanus in Muscle Photographic Record of Genesis of Tetanus in Mechanical Response of Plants (Style of Datura alba) . . . . . . . .

34. Fusion of Effect of Rapidly Succeeding Stimuli . . - . .

35. Response of (a) quickly reacting Amaranth ; (b) of sluggish Colocasia 43

36. Arrangement for Instantaneous Stimulation . . . . . .

38. Enlarged View of Balanced Keys . • • • • . . . . 49

39. Curve showing Rise and Fall of Responsive E.M. Change, under moderate stimulation

40. Response Curve from Rheotomic Observation in Stem of Amaranth under strong stimulation .

41. Artificial Hydraulic Response of Mimosa .

42. Experimental Arrangement for obtaining Records on Smoked Drum of Responses given to Direct and Indirect Stimulation, by Leaf of Mimosa .

43. Mechanical Responses of Leaf of Mimosa.

• .57

44. Mechanical Response of Biophytum to Thermal Stimulation . . $\quad 5^{8}$

45. Record of Response of Mimosa Leaf, taken on a fast-moving drum - 60

46. The Abnormal Positive preceding the Normal Negative in Mechanical and Electrical Responses in Biophytum . . . . . . 6I

47. Photographic Record of Electrical Response of Petiole of Cauliflower. 62

48. Photographic Record of Electrical Responses of Potato-tuber . 64

49. Photographic Record of Electrical Response of Petiole of Fern . 66

50. Longitudinal Contraction and Retardation of Growth under Light in Hypocotyl of Sinapis nigra . . . . . . . . . . 73

51. Record of Growth in Crinum at Temperature of $34^{\circ} \mathrm{C}$. and $35^{\circ} \mathrm{C}$. .

52. Balanced Record of Variation of Growth in Flower bud of Crinum Lily under Diffuse Stimulation of Light

53. Diagrammatic Representation of the Tonic Level

54. Photographic Record of Abnormal Positive passing into Normal Negative Response in a Withered Specimen of Leaf-stalk of Cauliflower

55. Photographic Record of Staircase Response in Vegetable Nerve .

56. Staircase Increase in Electrical Response of Petiole of Bryophyllum rendered sluggish by cooling. . . . . . . . . 91

57. Photographic Record of Uniform Responses (Radish) . . 92

58. Photographic Record of Uniform Response in Petiole of Fern . 92

59. Record showing Diminution of Response, when sufficient Time is not allowed for Full Recovery . . . . . . . . . . 93

6o. Fatigue in Celery . . . . . . . . . . . 93

6i. Fatigue in Leaf-stalk of Cauliflower. $\quad . \quad \ldots \quad$ • $\quad . \quad 493$

62. Photographic Record showing Fatigue in Tin Wire which had been continuously stimulated for Several Days . 
63. Effect of Over-strain in producing Fatigue

64. Rapid Fatigue under Continuous Stimulation in $(a)$ Muscle ; $(b)$ Leafstalk of Celery (Electrical Response)

65. Photographic Records of Normal Mechanical Response of Mimosa to Single Stimulus (upper figure), and to Continuous Stimulation (lower figure)

66. Effect of Continuous Vibration (though $50^{\circ}$ ) in Carrot .

67. Oscillatory Response of Arsenic acted on Continuously by Hertzian Radiation

68. Alternate Fatigue $(a)$ in Electrical Responses of Petiole of Cauliflower; $(b)$ in Multiple Electric Responses of Peduncle of Biophytum; $(c)$ in Multiple Mechanical Responses of Leaflet of Biophytum; and $(d)$ in Autonomous Responses of Desmodium . .

69. Photographic Record of Periodic Fatigue in the Automatic Pulsation of Desmodium gyrans . . . . . . . . . .

70. Periodic Fatigue in Pulsation of Frog's Heart (Pembrey and Phillips) .

71. Photographic Record of Periodic Fatigue under Continuous Stimulation in.Contractile Response (Filament of Uriclis Lily) . . . . IO0

72. Fatigue in the Contractile Response of Indiarubber . . . . IOI

73. Reversed Response of Fatigued Nerve . . . . . . . . 102

74. Preliminary Staircase, followed by Fatigue, in the Responses of Muscle (Brodie)

75. Preliminary Staircase, Increase, followed by Fatigue, in the Response of Galena to Hertzian Radiation .

76. Photographic Record of Responses of Style of Datura alba in which Growth had come to a Temporary Stop

77. Differential Contractile Response of Artificial Strip . . .

78. Responses of Mimosa to Sunlight of not too long Duration . . . 109

79. Transverse Response of Pulvinus of Mimosa . . . . . I IO

8o. Diametric Method of Stimulation of an Anisotropic Organ . . . III

81. The Thermal Variator . . . . . . . . . II3

82. Responsive Current in. Petiole of Musa from Concave to Convex Side I 15

83. Parallelism of Natural Current in Pulvinus of Mimosa and Sheathing Petiole of Musa . . . . . . . . . . II8

84. Effect of Variation of Temperature on Natural Current, $\downarrow$, which in Petiole of Musa flows from Convex to Concave Side

85. Photographic Record showing effect of Sudden, followed by steady Rise of Temperature on Natural Current, $\downarrow$, in Musa

86. Action of 7 per cent. Solution of $\mathrm{Na}_{2} \mathrm{CO}_{3}$ on Natural Current of Musa

87. Effect of $\mathrm{CO}_{2}$ on Natural Current of Musa '. . . . .

88. Variation of the Transverse Natural and Responsive Currents in . Pulvinus of Mimosa.

89. Photographic Record of Effect of Chloroform on Responses of Carrot .

90. Photographic Record showing Action of Chloral Hydrate on the . Responses of Leaf-stalk of Cauliflower . . . . . . . I31

91. Photographic Record showing Action of Formalin (Radish) . . . I32

92. Abolition of Response at both $\mathrm{A}$ and $\mathrm{B}$ Ends by the Action of $\mathrm{NaOH}$ 
FIG.

93. Photographic Record showing the nearly complete Abolition of

94. Photographic Record showing the Stimulatory Action of Solution of Sugar

95. Photographic Record showing Continuous Action of 2 per cent. $\mathrm{Na}_{2} \mathrm{CO}_{3}$ Solution .

96. Photographic Record showing the Depressing Action of $\mathbf{5}$ per cent. $\mathrm{HCl}$ Acid .

97. Photographic Record showing Effect of I per cent. KHO

98. Photographic Record of Effect of 5 per cent. KHO.

99. Striking-rods for stimulation of two ends of specimen and inducing phase-difference . . . . . . . . . .

Ioo. Isolated and diphasic responses with increasing difference of phase .

IOI. Photographic Record showing Negative, Diphasic, and Positive Resultant Responses in Tin

102. Photographic Records of Response of Bryophyllum . . . .

103. Photographic Record of Response of Petiole of Cauliflower by the Diametric Method

I04. Distribution of Electrical Tension in Muscle-cylinder . . . .

105. Photographic Record showing Persistent Electrical After-Effect in Inorganic Substance under Strong Stimulation . . . .

106. Photographic Record exhibiting Persistent Galvanometric Negativity in Plant Tissue after Strong Stimulation

107. Experimental Arrangement for determining Electrical Effect due to Section

108. Records showing increasing Persistent Galvanometric Negativity, according as injury is caused nearer to proximal contact .

109. Curve showing the Electrical Distribution in Stem with one Sectioned End.

110. Electrical Distribution in Plant-cylinder with Opposite Ends

II I. Record of Responses in Plant (Leaf-stalk of Cauliflower) by Method of Negative Variation

112. Response by Positive Variation of Resting Current . . .

113. Distribution of Electric Potential in Lamina of Colocasia along a radial line from dead to living through intermediate stages . . . 169

1 I4. Straight Form Potentiometer . . . . • . . . I7 I

I15. Distribution of Electric Potential in Petiole of Nymphaa alba, one end of which has been killed

116. Photographic Records of Responses of Vegetable Nerve, one end of which has been injured .

I 17. Typical Cases of Variation of Current of Rest and Action-Current. Specimen originally isotropic

II8. Typical Cases of Variation of Current of Rest and Action-Current ; intermediate point naturally less or more excitable than either of terminal

I19. Typical Cases of Variation of Current of Rest and Action-Current. Anisotropic organ, B end originally more excitable than A . 
120. Photographic Record showing Effect of Rapid Cooling, by Ice-cold

Water, on Pulsations of Desmodium gyrans

I8I

121. Photographic Record of Pulsations of Desmodium during Continuous

Rise of Temperature from $30^{\circ} \mathrm{C}$. to $39^{\circ} \mathrm{C}$. . . . . 182

122. Diminution of Response in Eucharis by Lowering of Temperature . $\quad{ }_{183}$

123. After-effect of Cold on Ivy, Holly, and Eucharis Lily . . . 184

124. Photographic Record of Responses in Eucharis Lily during the Rise and Fall of Temperature . . . . . . . . . .

125. Diminished Amplitude of Response with Rising Temperature. (Stem . of Amaranth) . . . . . . . . . . . . . . . . .

126. Photographic Records of Autonomous Pulsations in Desmodium, showing Increase of Amplitude and Decrease of Frequency, with Lowering of Temperature

127. Photographic Record showing Effect of Steam in abolishing Response

128. Record of Electric Responses of Amaranth at various Temperatures .

129. Photographic Record of Thermo-mechanical Curve given by Coronal Filament of Passiflora . . . . . . . . . . .

130. Thermo-mechanical Curve of Two Different Specimens of Style of Datura alba, obtained from Flowers of the same Plant . . 198

131. The Thermal Chamber . . . . . . . 200

132. Photographic Record exhibiting Electric Spasm in the Petiole of Musa

133. Photographic Record showing Electric Inversion at Death-point, $59^{\circ} 5^{\circ}$, in the Petiole of Amaranth $\quad . \quad \ldots \quad . \quad . \quad . \quad$.

134. Record showing Inversion of Electric Curve and Simultaneous Reversal of Electric Response in Stem of Amaranth .

I35. Multiple Mechanical Response of Biophytum, due to a Single Strong Thermal Stimulus . . . . . . . .

136. Multiple Electro-tactile Response in Stem of Mimosa due to Single Strong Thermal Stimulus .

137. Photographic Record of Multiple Electrical Response in Leaf of Biophytum .

138. Multiple Electrical Responses under Different Forms of Stimulus in Different Organs

139. Photographic Record of Multiple Electrical Response to Single Thermal Shock in Frog's Stomach

140. Induction of Autonomous Response in Biophytum at Moderately High Temperature of $35^{\circ} \mathrm{C}$.

141. Initiation of Multiple Response in Lateral Leaflet of Desmodium originally at Standstill .

142. Photographic Record of Autonomous Mechanical Pulsation in Desmodium Leaflet .

143. Spark-record of Single Pulsation in Leaflet of Desmodium . .

144. Photographic Records of Simultaneous Mechanical and Electrical Pulsation of Desmodium Leaflet . . . . . .

145. Photographic Record of Simultaneous Mechanical and Electrical Pulsation in Leaflet of Desmodium, before and after Physical Restraint of Leaflet 
FIG.

146. Crescographic Record of Multiple Growth-responses in Peduncle of

Crocus... . . . . . . . . 221

147. Natural and Responsive Currents in Leaves . . . . . . 224

148. Burdon Sanderson's Fundamental Experiment on Dioncea Leaf . 229

149. Parallel Experiment in Sheathing Petiole of Musa - : . . 229

I50. Positive Response of certain Leaves of Dionaa . . . . . . 230

151. Diphasic Response of Leaf of Dionce ' in itsprime.' Positive followed by negative . . . . . . . $, \quad . \quad, \cdots, \ldots$

152. Positive Response of same Leaf when 'modified' by previous stimulation . . . .

153. Experimental Connections with Dionaa according to the second Experimental Method of Burdon Sanderson - $\because \cdots, \ldots$

i54. Response of Under-surface of Leaf of Dionca, with Electrical Connections as in Fig. 153 .

155. Photographic Records of Positive, Diphasic, and Negative Responses of Petiole of Musa depending on the Effective Intensity of Transmitted Stimulus . . . . . . . . . .

156. Electrical Response of Lamina of Nymphcea alba due to Transmitted Excitation from Petiole

157. Diagrammatic Representation by Du Bois-Reymond for Explanation. of Electrical Response in Organ of Torpedo . . . . . .

I 58. Photographic Records of Responses given by Leaf of Coleus aromaticus, when both Surfaces are Excited Simultaneously by Thermal Shock

159. Experimental Arrangement for Rheotomic Observations . . .

160. Records of Two Successive Responses in Leaf of Bryophyllum calycinum under Equi-alternating Electrical Shocks.

161. Response-curve from Rheotomic Observation on Leaf of $N y m p h a a$ alba

162. Series of Responses given by Leaf of Plerospermum suberifolium to Stimulus of Equi-alternating Electrical Shocks

163. Photographic Record of Responses of Carpel of Dillenia indica .

I64. Photographic Record of Normal Responses given by Pitcher of Nepenthe, under Equi-alternating Electric Shocks . . . 256

165. Responsive Currents in Lead Wire . . . . . . . . . . 264

166. Flat Strip of Lead, of which lower Surface is Brominated . . 265

167. Photographic Records of After-effect of Homodromous $\uparrow$ and Heterodromous $\downarrow$ Induction-shocks in prepared Strip of Lead . . . 266

168. Photographic Record of Responses to Equi-alternating Electric Shocks in Prepared Lead Strip . . . . . . .

169. Response of Pulvinus of Mimosa to Equi-alternating Electric Shocks . . . . . . . . . . . . .

170. Experimental Arrangement for Determination of Excitatory Aftereffect of Equi-alternating Electrical Shocks • . . . . 276

171. Method of Direct Effect of Excitation by Equi-alternating Shocks . 280

172. Excitation by Equi-alternating Shocks . . . . . . 281

173. Photographic Record of Response of Petiole of Musa to Equi-alternating Electric Shocks, before and after Application of Chloroform 
174. Thotographic Record of Responses of Plagiotropic Stem of Cucurbita to Equi-alternating Electric Shocks _... $\quad \ldots \quad \ldots 285$

175. Electrical Responses of Eel to Equi-alternating Electrical Shocks . $\quad 285$

176. Rotary Mechanical Stimulator . . . . . . . . . 29 I

177. Diagram Representing Different Levels of Excitability, Plus, Zero,

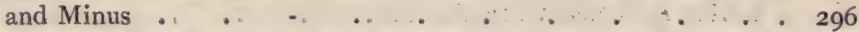

178. Electrical Response of Grape-skin to Rotary Mechanical Stimulation 299

179. Electrical Response of Frog's Skin to Rotary Mechanical Stimulation

180. Photographic Record of Electrical Responses of Upper Surface of Intact Human Forefinger to Rotary Mechanical Stimulation

300

301

18I. Photographic Record of Electrical Responses of Grape-skin to Thermal Shocks .. $\quad \ldots \quad \ldots \quad \ldots \quad \ldots \quad \ldots . \quad \ldots \quad \ldots$.

182. Photographic Record of Electrical Responses of Grape-skin to Stimulation by Equi-alternating Electrical Shocks . . .

I83. Photographic Record of Series of Electrical Responses of Frog's Skin to Equi-alternating Electrical Shocks .. . . . . . . .

184. Photographic Record of Transverse Response of Pulvinus of Mimosa to Equi-alternating Electrical Shocks.

185. Continuaus Photographic Record of Autonomous Pulsation of Desmodium gyrans from 6 P.M. to 6 A.M. . . . . . . .

I86. Photographic Record of Electrical Responses in Skin of Neck of Tortoise to Stimulus of Equi-alternating Electrical Shocks .. . .

187. Isolated Responses of Upper and Lower Surfaces of Skin of Tomato to Rotary Mechanical Stimulus _. . . . . . . .

I88. Photographic Record of Series of Responses in Skin of Tomato under Equi-alternatin Electrical Shocks . . . . . . .

189. A Single Response of Skin of Tomato to Equi-alternating Shock recorded on Faster Moving Drum . . . . . . .

190. Photographic Record of Series of Normal Responses in Skin of Gecko

191. Photographic Record of Abnormal Diphasic Responses in Skin of Gecko, converted to Normal, after Tetanisation _. $\quad . \quad 3$ II

192. Transverse Section of Tissue of Hollow Peduncle of Uriclis Lily . $\quad 313$

193. Photographic Record of Responses of Water-melon to Equi-alternating Electric Shocks .

194. Photographic Reer d of Electrical Responses of Intaet Human Armpit : . . . . . . . . 3

195. Experimental Arrangement for Response of Human Lip _ . . . 320

196. Photographic Record of Electrical Response of Intact Human Lip :

197. Possible Variations of Responsive Current, as between Two Surfaces $A$ and $\mathrm{B}$, shown by Means of Diagrammatic Representations of Characteristic Curves

198. Photographic Record showing Reversal of Normal Response in Pulvinus of Mimosa due to Fatigue

199. Photographic Record showing Reversal of -Response in Carpel of Dillenia indica, under Sub-minimal Stimulation . . . 328

200. Pitcher of Nepenthe, with lid removed $\ldots \quad \cdots 335$

201. Glandular Surface of a Portion of the Living Membrane of the Pitcher of Nepenthe. . 
FIG.

202. Transverse Section of Tissue of Pitcher of Nepenthe.

203. Photographic Record of Series of Normal Negative Responses of Glandular Surface of Nepenthe in Fresh Condition to Equi-alternating Electric Shocks

204. Photographic Record of Responses of Pitcher in Intermediate Stage, having Attracted a Few Insects .

205. Photographic Record of Responses of Pitcher in Third Stage, the whole Glandular Surface thickly Coated with Insects

206. Multiple Response of Pitcher of Nepenthe, in First or Fresh Stage, to Single Strong Thermal Shock

207. Multiple Response of Pitcher of Nepenthe, in Third Stage, to Single Strong Thermal Shock .

208. Photographic Record of Responses in Fresh Leaf of Drosera to Equialternating Electrical Shocks

209. Photographic Kecord of Multiple Response of Leaf of Drosera in Positive Phase

210. Photographic Record of Normal Negative Responses of Frog's Stomach to Mechanical Stimulation

21 I. Photographic Reçord of Normal Negative Responses of Stomach of Tortoise to Stimulus of Equi-alternating Electric Shocks .

212. Photographic Record of Normal Response in Stomach of Gecko to Equi-alternating Shocks, seen to be reversed after Tetanisation .

213. Photographic Record of Multiple Responses in Stomach of Frog to a Single Strong Thermal Shock

214. Photographic Record of Normal Negative Response of Young Root of Colocasia

215. Photographic Record of Positive Response in Older Root of Colocasia 216. Photographic Record of Electrical Response of Sap-wood . . . 217. Photographic Record showing Normal Responses of Living Wood to Vibrational Stimulus, and the Abolition of Response by a Toxic Dose of Copper Sulphate

218, The Shoshungraph - * .

219. Curve showing Normal Suction at $23^{\circ} \mathrm{C}$., Increased Suction at $35^{\circ}$ C., and the After-effect persisting on Return to Normal Temperature .

220. Action of Anæsthetics in Abolition of Suction . . . .

221. Effect of Strong $\mathrm{KNO}_{3}$ Solution . . . . . . .

222. Effect of Strong $\mathrm{NaCl}$ Solution . . . . . . . .

223. Record of Blank Experiment showing Absence of any Disturbance of Record from Induction-shocks as such . . . . $\quad 378$

224. Terminal Mode of Application of Stimulus . . . . . 380

225. Sub-terminal Mode of Application of Stimulus . . . . . 380

226. Renewal of Suction, Previously at Standstill, by Action of Stimulus .

227. Photographic Record of Effect of Stimulus in Enhancing Rate of Suction . . . . . . . . . . 383

228. Variation of Latent Period as After-effect of Stimulus . . - . 384

229. Photographic Record showing Variation of Latent Period as Aftereffect of Stimulus . . . . . . . . . . 385

230. Suctional Response under Over-balance . . . . . . $3^{86}$ 
231. Photographic Record of Effect of Stimulus on Over-balance .

232. Photographic Record of Response to Continuous Sub-terminal Stimulation

233. Experimental Arrangement for Detection of Electrical Change induced at the Point transversely Distal to Point stimulated .

234. Record of Response to Moderate Unilateral Stimulation under the Experimental Arrangement described .

235. Record of Different Specimen under same Experimental Arrangement when Stimulus is first Moderate and then Increased .

236. Mechanical Response of Pulvinus of Mimosa to Continuous Action of Light from Above $\quad . \quad \ldots \quad$. $\quad . \quad$. . .

237. Electrical Response in the Lower Half of the Pulvinus of Mimosa due to Stimulation of Distal Upper Half by Light . . .

238. Photographic Record of Series of Negative Responses of Petiole of

- Bryophyllum to Stimuli of Sunlight . . . . . .

239. Record of Responsive Growth-variation taken under condition of balance in slightly Sub-tonic Flower-bud of Crinum Lily under Diffuse Stimulation of Light.

240. Photographic Record of Positive Response of the Petiole of Cauliflower to Light . . . . . . . . . .

24I. Multiple Mechanical Response of Leaflet of Biophytum under the Continuous Action of Light

242. Photographic Record of Multiple Electrical Response in Leaf of Bryophyllum under Continuous Action of Light . . . . .

243. Diagrammatic Representation of Phasic Alternations, and After-effect in Type $I$.

244. Photographic Record of Phasic Alternations, showing Direct and After Effects of Light in Type I., represented by Bryophyllum .

245. Diagrammatic Representation of Phasic Alternations, and After Effect in Type III.

246. Photographic Record of Phasic Alternation, showing Direct and After Effects in Type III., represented by Petiole of Cauliflower .

247. Photographic Record of Pair of Responses obtained with a Second Specimen of Caulitlower, representative of Type III. . . .

248. Experimental Arrangement for Determination of Differential Excitability of Optic Nerve and Cornea.

249. Series of Photographic Records of Excitatory Responses in Frog's Eye to Equi-alternating Electric Shocks

250. Experimental Arrangement for Demonstration of Differential Excitability as between Retina and Optic Nerve.

25I. Series of Photographic Records of Excitatory Responses in Frog's Retina to Equi-alternating Electric Shocks . . . . .

252. Photographic Record of Multiple Response of Retina of Frog under Continuous Action of Light

253. Response of petiole of Bryophyllum. Light was cut off on attainment of maximum positivity in the second of the multiple responses 
FIG.

254. Similar effect in response of retina of Ophiocephalus fish :

255. The same with another specimen. Light was here cut off after the first oscillation

256. Response of retina of Ophiocephatus when slightly fatigued

257. Response of frog's eye (Kühne and Steiner) _ _ . . . . . 428

258. After-effect of Light on Silver Bromide . $+. \quad+\quad . \quad 429$

259. Response of petiole of cauliflower. Light was here cut off on attainment of maximum negativity $\quad \ldots$

260. Response of retina of Ophiocephalus fish when depressed . . .

26r. Response of isolated retina of fish as observed by Küne and Steiner

262. Inclined Slits for Stereoscope and Composite Image formed in the Two Eyes

263. Composite Indecipherable Word, of which Components are Seen Clearly on Shutting the Eyes.

264. Diagrammatic Representation of a Multicellular Organ laid Horizontally and Exposed to Geotropic Stimulus : . : .

265. Effect on Apogeotropic Movement of Temporary Application of Cold on Upper and Lower Surfaces respectively

266. Diagrammatic Representation of Experiment showing Curvature Induced by Unilateral Pressure Exerted by Particles . . . .

267. Record of Responsive Curvature Induced in Bud of Crinum Lily by Unilateral Pressure of Particles

268. Record of Apogeotropic Response in Scape of Uriclis Lily . .

269. Photographic Record of Geo-electric Response in the Scape of Uriclis Lily laid horizontally. $\quad \ldots \quad \ldots \ldots$

270. Experimental Arrangement. for Subjecting Organ to Geotropic Stimulus, Mechanical Response being Restrained . . .

27.I. Geo-electric Response of the Physically Restrained Scape of Uriclis Lily.

272. Diagrammatic Representation of.Electrical Connections for Determination of Velocities of Centrifugal and Centripetal Transmissions :

273. Experimental Arrangement for Comparing the Relative Conductivities in Transverse and Longitudinal Directions

274. Response of Frog's Nerve under Simultaneous Excitation of both Contacts, by Equi-alternating Electrical Shocks, one Contact being Injured

275. Enhancement of Amplitude of Response, as After-effect of Thermal Tetanisation, in Frog's Nerve.

276. Conversion of Abnormal Positive into Normal Negative Response after Thermal Tetanisation :

277. Gradual Transition from Abnormal Positive, through Diphasic, to Normal Negative Responses in Frog's Nerve ... . . . . .

278. Abnormal Positive Response converted through Diphasic to Normal Negative under the increasingly Effective Intensity of Stimulus, brought about by Lessening the Distance between the Responding and Stimulated Points. .

279. Frond of Fern with Conducting Nerves exposed. 
280. Photographic Record of Effect of Ether on the Electrical Response of Plant-nerve .

281. Photographic Record of Effect of $\mathrm{CO}_{2}$ on Electrical Response of

282. Photographic Record of Abolition of Response by Strong Application

283. Photographic Record of Effect of Ammonia on Ordinary Tissue of Petiole of Walnut

284. Photographic Record of Effect of Similar Application of Ammonia on Plant-nerve. . . . . .

285. Photographic Record of Exhibition of Three Types of Response, Normal Negative, Diphasic, and Abnormal Positive, in Nerve of Fern under Different Conditions

286. Photographic Record of Effect of Tetanisation in Inducing Enhance. ment of Normal Negative Response in Nerve of Fern . . .

287. Photographic Record of Conversion of the Abnormal Di-phasic into Normal Negative, after Tetanisation,.T, in. Nerve of Fern

288. Photographic Record showing how the Abnormal Positive Response is converted through Diphasic into Normal Negative by the Increasing Effective Intensity of Stimulus, due to Lessening the Distance between the Responding and Stimulated Points .

289. Diagrammatic Representation of the Conductivity Balance . .

290. Photographic Record made during Preliminary Adjustment for

Balance of Nerve of Fern . . . . . . . . . 482

291. Complete Apparatus of Conductivity Balance . . . . . 484

292. Effect of $\mathrm{Na}_{2} \mathrm{CO}_{3}$ Solution on Responsive Excitability of Frog's Nerve 485

293. Effect of $\mathrm{CuSO}_{4}$ on Frog's Nerve + . . . . . . 485

294. Photographic Record showing Enhancement of Responsivity by Application of $\mathrm{CaCl}_{2}$. . . . . . . . . 486

295. Photographic Record showing Depression of Responsive Excitability by Application of $\mathrm{KCl}$.

296. Photographic Record exhibiting Comparative Effects of $\mathrm{NaCl}$ and $\mathrm{NaBr}$ on Responsivity . . . . . . . .

297. Photographic Record of Effect of Dilute ( 5 per cent.) Solution of $\mathrm{Na}_{2} \mathrm{CO}_{3}$ on Variation of Conductivity . . . . .

298. Photographic Record of Effect of Stronger Dose (2 per cent.) of $\mathrm{Na}_{2} \mathrm{CO}_{8}$ Solution on Conductivity _. . . . . . 489

299. . Responsivity versus Conductivity under KI . . . . 49I

300. Responsivity versus Conductivity under NaI . . . . 492

301. Effect of Alcohol on the Responsivity of Frog's Nerve . . . 492

302. Photographic Record of Effect of Alcohol Vapour on Receptivity - 493

303. Photographic Record of Effect of Alcohof on Conductivity . . . 494

304. Photographic Record showing Effect of Alcohol on Responsivity . 494

305. Diagrammatic Representation of Experimental Arrangement for

Demonstration of Receptivity versus Conductivity, or of

ReCEPTIVity versus Responsivity . . . . . . . 495

306. Receptivity versus Responsivity under Alcohol : . . . 495

307. Photographic Record showing Effect of Cooling on Conductivity of Plant-nerve . 
FIG:

308. The Cork Chamber for Gradual Raising of the Temperature of one Arm of the Balance

309. Photographic Record Showing Effect of Rising Temperature on Conductivity

310. Experimental Arrangement for Studying After-effect of Stimulus on Conductivity and Excitability

311. Photographic Record Showing Effect of Moderate Stimulation in Enhancing Conductivity and Excitability

312. Photographic Record showing Effect of Excessive Stimulation in Depressing Excitability and Conductivity

313. Record of Contractile Response in Frog's Nerve under Continuous Electric Tetanisation.

314. Optical Kunchangraph for Mechanical Response of Nerve .

315. Diagrammatic Representation of Arrangement for Obtaining Trans. mitted Effect of Stimulus

316. Photographic Record of Effect of Ammonia on Mechanical Response -of Frog's Nerve

317. Photographic Record showing Abolition of Mechanical Response of Frog's Nerve by Action of Solution of Morphia

318. Photographic Record showing Preliminary Exaltation in Mechanical Response of Frog's Nerve after Application of Alcohol . . .

319. Photographic Record showing Effect of Chloroform on Mechanical Response of Frog's Nerve . . • • . • • .

320. Photographic Record showing Abnormal Positive converted into Negative Response after Tetanisation . . . . . .

321. Photographic Records showing Gradual Disappearance of Positive Element in diphasic Mechanical Responses of Frog's Nerve and Plant-nerve

322. Photographic Record showing Staircase Effect in Mechanical Response of Frog's Nerve .

323. Photographic Reproduction of Record of Mechanical Responses of Frog's Nerve and Plant-nerve obtained on Smoked Glass Surface of Oscillating Recorder

324. Record of Mechanical Responses to Electrical Stimulus obtained on Smoked Glass, and given by the Optic Nerve of Fish Ophiocephalus

325. Record, obtained on Smoked Glass, of Transmitted Effect of Stimulation on Nerve of Gecko

326. Initiation of Multiple Response by Drying of Nerve

PAGE 500 501

510

5 I I

327. Diagrammatic Representation of Experimental Arrangement for Recording Response by Resistivity Variation

328. Photographic Record of the Morographic Curve taken by Method of Resistivity Variation in Pistil of Hibiscus. Critical point of inversion at $60 \cdot 8^{\circ} \mathrm{C}$.

329. Photographic Record of the Morographic Curve taken by Method of Electromotive Variation in Petiole of Musa. Critical point of inversion at 59:6 C. . . . . . . . . .

330. Photographic Record of the Morographic Curve taken by Method of Mechanical Response in Filament of Passiflora. Critical point of inversion at $59^{\circ} 6$ C. . 
332. Effect of Chloroform seen in Modification of Resistivity Variation in Frog's Nerve

333. Photographic Record of Effect of Tetanisation in Enhancing Mechanical Response of Plant-nerve . . . . . . .

334. Photographic Record showing Enhancement of Excitability under Action of Light in Nerve of Fern

335. Distribution of Fibro-vascular Elements in Single Layer of Stem of

\section{Papaya}

336. Extra-polar Kat-electrotonic Effect .

337. Extra-polar An-electrotonic Effect

338. Extra-polar Electrotonic Effects under an Acting E.M.F. which rises from $\cdot 6$ to $\mathrm{I} \cdot 4$ Volts .

560

339. Diagram illustrating Bernstein's Decrement of Kat-electrotonic Current

340. Diagram illustrating Bernstein's Decrement of An-electrotonic Current

34I. Diagram representing Hermann's Polarisation-increment under Tetanising Shocks, with reversed polarising Current . . .

342. Diagram representing Hermann's Polarisation-increment under

Tetanising Shocks, with reversed polarising Current
343. Experiment with Petiole of Fern demonstrating Variation of Conductivity by Polarising Current, Excitation travelling electrically Downhill

344. Experiment with Petiole of Fern demonstrating Variation of Conductivity by Polarising Current, Excitation travelling electrically Uphill

345. Photographic Records of Responses taken in last Experiment, when Excitation was transmitted with and against the Polarising Current.

346. Photographic Record of Modification of Conduction during Passage of Excitation from Anodic to Kathodic Region, under Increasing Intensity of Polarising E.M.F.

347. Photographic Record showing Enhanced Conduction from Kathodic to Anodic Region .

348. Experimental Arrangement to Exhibit the Enhancement of Excitability at Anode, when the Acting E.M.F. is feeble

349. Experimental Arrangement to Exhibit Depression of Excitability at Kathode, when the Acting E.M.F. is feeble . . . . .

350. Photographic Records of Response, illustrating the Enhancement of Excitability at Anode, and Depression at Kathode, under Feeble Acting E.M.F. in two Specimens of Nerve of Fern $a$ and $b$.

351. Experimental Arrangement demonstrating the Joint Effects of
Variation of Conductivity and Excitability by Polarising Current
352. Experimental Arrangement demonstrating the Joint Effects of

351. Experimental Arrangement demonstrating the Joint Effects of
Variation of Conductivity and Excitability by Polarising Current
352. Experimental Arrangement demonstrating the Joint Effects of Variation of Conductivity and Excitability by Polarising Current, when Current is Reversed .

353. Photographic Record of Response under the Arrangements given in Figs. 351, 352 in Nerve of Fern . . . . . .

354. Experimental Arrangements for Showing so-called Polarisationincrement by the Joint Effect of Increased Excitability at Anode and Enhanced Conduction of Excitation electrically Uphill . 
FIG.

355. Experimental Arrangements for Showing so-called Polarisationincrement by the Joint Effect of Increased Excitability at Anode and Enhanced Conduction of Excitation electrically Uphill. Direction of Current in this is Reversed

356. Photographic Record of Responses in Nerve of Fern, under Anodic and Kathodic Action as described in Figs. 354 and 355.

357. Photographic Record of Similar Effects in Nerve of Frog . .

358. Make-kathode and Break-anode Effects in Biophytum . . .

359. Effect of Anode and Kathode on Responsive Sensation in Human Hand

360. Polar Effects of Currents due to Localised Application on Upper Half of Pulvinus of Erythrina indica

36r. Experimental Arrangement for Magnetometric Method of Record

362. Photographic Record of Uniform Magnetic Responses of Iron . .

363. Photographic Record of Periodic Groupings in Magnetic Responses

364. Photographic Record of Response and Recovery of Steel under Moderate and strong Magnetic Stimulus

365. Photographic Record showing Ineffective Stimulus made Effective by Repetition

366. Molecular Model

367. Method of Resistivity Variation .

595

598

600

368. Photographic Record of Response of Aluminium Powder in Sluggish Condition to Stimulus of Electric Radiation

601

369. Photographic Record Showing Uniform Response of Aluminium Powder to Uniform Stimulus of Electric Radiation . . . . . 602

370. Photographic Record of Response of Tungsten . . . . . . 603

371. Experimental Arrangement for obtaining Response in Iron by Induction Current

372. Magnetic Conductivity Balance

373. Process of Balancing illustrated by Photographic Record of Responses.

374. Effect of $\mathrm{K}$ - and A-Tonus on Magnetic Conduction . . . .

375. Opposite Effects of $\mathrm{K}$-Tonus when moderate and strong . . .

37ó. Effects of $\mathrm{K}$ - and A-Tonus on Magnetic Excitability . . .

377. Gradual Enhancement of Conductivity by the Action of Stimulus

379. Characteristic Conductivity Curve of Sensitive Metallic Particles belonging to Negative Class, under increasing Electro-motive Force.

380. Cyclic Curves of Magnetisation and of Conductivity

381. Photographic Record of Magnetic Tetanisation of Steel, exhibiting Transient Enhancement of Response on Cessation .

382. Mechanical Response of Frog's Nerve to successive equal Stimuli, applied at Intervals of One Minute

383. Mechanical Response of Frog's Nerve, showing Conversion of Abnormal Positive into Normal Negative Response after Tetanisation

384. Photographic Record showing Conversion of Abnormal 'Down' Response in Tin to Normal 'Up,' after Tetanisation. 
385. Gradual Transformation from Abnormal to Normal Response in Platinum :

PAGE

386. Normal Electro-motive Response in Tin, enhanced after Tetanisation

387. Photographic Record of Abnormal Response of Selenium Cell converted into Normal after Tetanisation

388. Photographic Record showing Moderate Normal Response of Selenium enhanced after Tetanisation

389. Photugraphic Record of Abnormal Response of Tungsten to Electric Radiation, converted after Tetanisation into Diphasic and Normal .

390. Moderate Normal Response of Aluminium, enhanced after Tetanisation

39I. Photographic Record of Enhancement of Magnetic Response after Tetanisation

632

632

392. Vertical Series of Records showing Transformation of Abnormal into Normal Response after Tetanisation in Living and Inorganic alike in the $A$ phase

393. Series showing how Tetanisation enhances Normal Response in the B Phase

394. Photographic Record showing Responses corresponding with different parts of characteristic curve in frog's nerve . . . . .

395. Photographic Record of Response of Tungsten showing Enhancement of Response after moderate Tetanisation, and Reversal of Response, due to Fatigue under stronger Tetanisation . . .

396. Series showing reversal of Normal Response by fatigue due to strong Tetanisation inducing the $\mathrm{E}$ phase .

397. Fatigue in Indiarubber giving rise to Diphasic and Reversed Responses . . . . . . . . . .

398. Fatigue inducing Diphasic Variation and Reversal of Normal Response in Frog's Nerve

399. Abnormal Response of Muscle by Relaxation, followed by Normal Response of Contraction

400. Record of Response in Nerve of Gecko showing the Effect of Arithmetically increasing Stimulus . . . . . . .

401. Response of Nerve of Bull-frog to Stimuli 1, 2, 3, . . . I 2, increasing in Arithmetical Progression. . . . . . .

402. Response of Optic Nerve of Ophiocephalus to Arithmetically increasing Stimuli $1,2,3,4,5,6,7$. . . . . .

403. Mechanical Response of Nerve of Fern to Arithmetically increasing Stimulus

404. Photographic Record of Magnetic Responses in Steel to Arithmetically increasing Stimulus . . . . . . 660

405. The Sensimeter . . . . . . . . . . 670

406. Revival of latent Image in Metal . . . . . . . $\quad$. 683 



\section{COMPARATIVE EI.ECTRO-PHYSIOLOGY}

\section{CHAPTER I}

THE MOLECULAR RESPONSIVENESS OF MATTER

Response to stimulus by change of form-Permeability variation-Variation of solubility - Method of resistivity variation: $(a)$ positive variation; (b) negative variation-Sign of response changed under different molecular modifications-Response of vegetable tissue by variation of electrical resistance-Response by electro-motive variation in inorganic substancesThe method of block-l'ositive and negative responses--Similar responses in living tissues-Effects of fatigue, stimulants, and poisons on inorganic and organic responses-Method of relative depression, or negative variation, so called.

In studying the properties of living tissues, we find one of their most important characteristics is found in the fact that they exhibit the state of excitation under the impact of stimulus. On the cessation of stimulus, again, the excited tissue returns to its original condition. The excitatory change thus undergone is fundamentally due to the derangement, or upset, of the molecules of the living tissue from their normal equilibrium, recovery being brought about by their restoration to that state. The excitatory condition is sometimes shown by change of form, as in the case of the shortened length of excited muscle (fig. I). This might be compared with the shortening of stretched india-rubber under thermal stimulus (fig. 2).

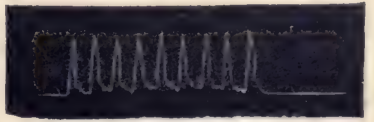

FIG. I. Series of Contractile Responses in Muscle

Now it is clear that the molecular change consequent on excitation must occasion various concomitant physical 
changes, and it should be theoretically possible to detect and measure this induced molecular change by recording such concomitant variations. Thus the stimulus of light, for

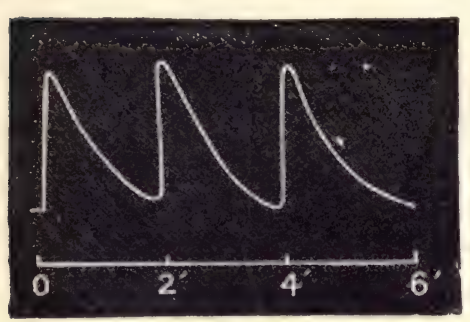

FIG. 2. Response of India-rubber

Thermal stimulus for I second at intervals of two minutes. example, may induce a molecular change which may in its turn induce, say, a variation in the permeability of the substance to liquid. Bichromated gelatine becomes less permeable under the action of light. The solubility of a substance may again undergo variation under external stimulus - sulphur, for example, usually soluble in carbon disulphide, is rendered insoluble under the action of light.

In order, then, to study the effect of a given stimulus with accuracy, we should be able to detect and measure the extent of the changes induced. The two effects which have just been referred to are not, as will be seen, highly susceptible of accurate measurement. But in the detection of molecular changes by electrical means, we have at our disposal methods for the measurement of such changes, the ease and delicacy of which leave nothing to be desired. Two such methods may be used-that of Resistivity and that of Electro-motive Variation. According to the method of resistivity variation, the substance to be experimented on is placed in an electrical circuit, including a delicate galvanometer and a suitable electro-motive force, such as to cause a small deflection of the galvanometer. The impact of the stimulus on the substance under examination now induces in it a molecular change by which its resistance is made to undergo a variation, which in the case of certain substances may be an increase, or in that of others a diminution. On the cessation of external stimulus, the substance shows recovery, with a corresponding return to its original conductivity. Thus in the case of selenium, for instance, the conductivity is increased, or the 
resistance decreased, under the action of light. In fig. 3 is shown a number of responses to light, given on a series of separate exposures, each of one second's duration, the intervening periods allowed for recovery being of one minute each.

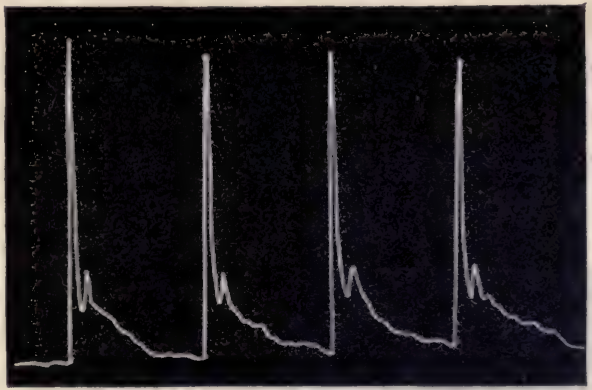

FIG. 3. Response of Selenium to the Stimulus of Light (Resistivity variation method)

These responses were obtained by recording the increased deflection due to decreased resistance under the impact of light, and the subsequent recovery. Such responses, by means of decreased resistance, we may arbitrarily distinguish as negative. Similar responses are also given by a mass of metallic particles when acted upon by electric radiation. In fig. 4 are seen several of these negative responses given by galena under the action of this stimulus.

There are, on the other hand, some substances which give positive responses; that is to say, their resistance is increased, or conductivity decreased, under

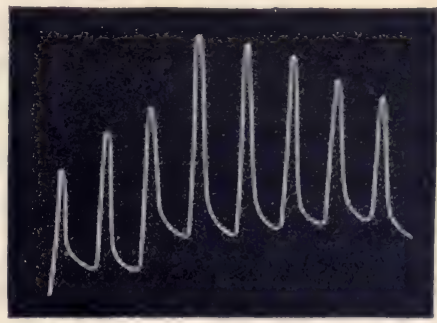

FIG. 4. Negative Response of Galena to Hertzian Radiation

(Resistivity variation method) the action of stimulus. The deflection of the galvanometer under a constant electro-motive force now undergoes diminution during the impact of stimulus. Such positive responses are obtained with potassium and sodium. 'That the sign 
of response does not depend on the electro-positivity or negativity of the substance is seen in the fact that while highly electro-positive potassium gives positive response, the equally electro-negative dioxide of lead gives a response of the same sign. Substances like magnesium, aluminium, and iron give negative response.

It is found, again, that the same substance, under different molecular conditions, will give responses of opposite signs. For example, a particular molecular variety of silver, Ag', gives positive (fig. 5), whereas ordinary silver gives nega-

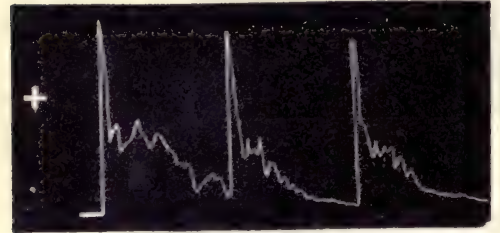

FIG. 5. Positive Response of $\mathrm{Ag}^{\prime}$ to Electric Radiation tive response. Again, while Ag' normally gives positive, yet the sign of this response is gradually reversed to negative, under the long-continued action of very strong stimulus. By the employment of the same method of resistivity variation, I have been able to obtain excitatory response records from living tissues also. Details of these will be given in a subsequent chapter.

The electric response, however, employed to obtain the excitatory reaction of living tissues, depends upon the electro-motive variation of the substance under stimulation. This electric reaction has been regarded as vitalistic in contradistinction to physical. But I have shown that similar responses are given by inorganic substances also. That is to say, the molecular excitability on which the phenomenon of response depends is not distinctive of animal tissues alone, but is common to all matter, both organic and inorganic. If, then, we desire to understand those fundamental reactions which underlie the response of living tissues, it will be well to observe its occurrence in the much simpler case of the inorganic body. ${ }^{1}$

If we take an inorganic substance, say a piece of metal

1 For a detailed account cf. Bose, Kesponse in the Living and the Non. Living. 
wire, and if its molecular condition be the same throughout, it is obvious that its physical properties will likewise be uniform. Hence its electrical condition will also be the same at every point; in other words, it will be iso-electric. But if a portion of this wire should now be made to undergo a molecular change, as, say, by hammering, the physical condition of this portion will be made different from that of the rest. There will, therefore, be an electrical difference, and the wire will no longer be iso-electric. This fact can be verified by making suitable connections between the molecularly strained and unstrained portions of the wire, and a galvanometer, when a current will be found to flow through the galvanometer, showing that a difference of electrical potential has been brought about by the induced

(a)

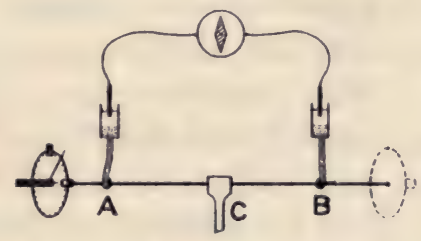

(b)

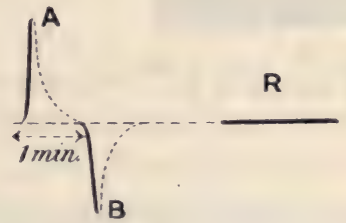

FIG. 6. Electric Response in Metals

(a) Method of block; (b) Equal and opposite responses when the ends A and $\mathrm{B}$ are stimulated; the dotted portions of the curves show recovery; (c) Balancing effect, $\mathrm{R}$, when both the ends are stimulated simultaneously.

inequality of molecular conditions in different parts of the same wire.

I shall now describe the method by which electrical responses to molecular disturbance may be obtained from inorganic substances. For this purpose, two different methods may be employed-first, the method of block, and, secondly, the method of relative depression. According to the first of these, the wire to be experimented on is held clamped at the middle, electrical connections being made with a galvanometer at two points, $A$ and $B$, by means of two non-polarisable electrodes (fig. 6). We may now produce 
excitation of the A end of the wire, by imparting a torsional vibration, the molecular disturbance being prevented from reaching the $B$ end by the intervening block. Using this method of experiment, I have obtained with different substances two different types of response-namely, positive and negative. In the positive, the responsive current flows through the wire from the unexcited to the excited, or towards the excited-that is to say, the excited point becomes galvanometrically positive. Responses of this kind are given by tin, zinc, platinum, and other metals. In fig. 7

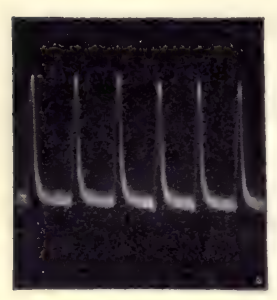

FIG. 7. Uniform Electric Response in Tin is seen a uniform series of such responses to uniform stimulus. The intensity of the response, moreover, does not appear to depend on the chemical activity of the substance. For the response of the chemically inactive tin is much stronger than that of the active zinc. The very inactive platinum is also found to give a fairly strong response, although the electrolytic contacts are made with pure water.

The electro-motive response may also be obtained by other modes of molecular excitation. Thus, instead of torsional, we may use longitudinal vibration. A metallic rod of brass, A C, is clamped in the middle. A thin copper wire is led sideways from the clamp and connected with a piece of brass, B. A and B are connected with a galvanometer by means of non-polarisable electrodes. If now the $\mathrm{C}$ end of the rod be rubbed with resined cloth, A may be thrown into longitudinal vibration, B being little affected by this. It is here interesting to observe the concomitance of the electrical response with the sonorous iesponse of the rod, and the dying of the electrical response with the waning of the musical note. The stronger the molecular vibration, the stronger the sound, and also the stronger the response. The direction of the responsive current in the metal is from the less excited $\mathrm{B}$ to the more excited $\mathrm{A}$.

We found under the method of conductivity variation 
that when a substance is molecularly modified, the sign of its response tends to be reversed. Thus, as already said, ordinary silver gives positive, and modified silver, $\mathrm{Ag}^{\prime}$, negative response. But the latter, under strong and longcontinued stimulation, has its response re-converted, as it were, to the normal positive. In the same way, under the method of electro-motive variation also, we find the normal positive response of, say, tin, or platinum, becoming converted by molecular modification into negative, to be again reconverted under continuous stimulation to the normal positive.

There are, again, certain other substances, of which the normal response is negative. Thus a wire of brominated lead, for instance, when suitably prepared, is found to give an electro-motive response in which the current flows from the excited to the unexcited or away from the excited, the excited point becoming galvanometrically negative.

These electro-motive responses of the inorganic have thus the same characteristics as those which have been observed in the case of animal tissues. Certain tissues, such as highly excitable muscle and nerve, give negative response-that is to say, the excited point becomes galvanometrically negative. Other tissues, again, the skin for example, give positive response. The normal responses, moreover, are sometimes found to be reversed under molecular modification, and to be rereversed to normal response under long-continued stimulation.

The electrical responses of metals, again, are subject to an increase or decrease

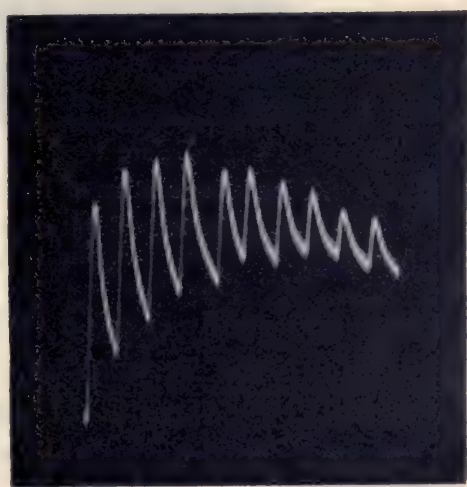

FIG. 8. Fatigue in the Electric Response of Metals which is paralleled by the same phenomenon in the response of animal tissues under similar circumstances. That is to 
say, fatigue is found to depress the response of the inorganic as of the organic (fig. 8). As in the case of animal tissues, again, so also in that of metals, certain chemical substances act as excitants, enhancing the response (fig. 9), others as depressors and still a third class-such as oxalic acid-as poisons, abolishing response altogether (fig. IO).

By taking advantage of the last of these facts, we arrive at a second means of obtaining response-that is to say, the method of relative depression. If both the contact points

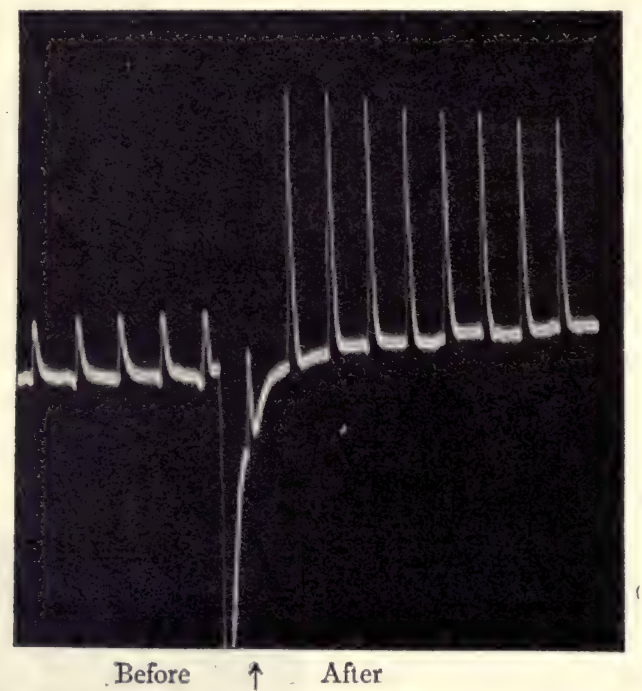

Fig. 9. Stimulating Action of $\mathrm{Na}_{2} \mathrm{CO}_{3}$ on Electric Response of Platinum

Records to the left exhibit response before, to the right after the application of reagent.

$\mathrm{A}$ and $\mathrm{B}$ be equally excited--that is to say, subjected to diffuse stimulation-the responsive currents will be opposed, and there will be no resultant galvanometric effect. This was overcome, according to the method of block, by localising excitation at one point, say, A ; we might, however, neutralise altogether the counteracting excitatory effect at $\mathrm{B}$ by abolishing the excitability of that point, as, say, by the application of oxalic acid, A being left in its normal condition. If now the wire be subjected to diffuse stimulation 
by vibrating it as a whole, a resultant response will occur. But by the application of oxalic acid to one contact, a resting or permanent current has been induced in the circuit. The responsive or action current originated under stimulation is now found to flow in a direction opposite to that of this resting current - that is to say, it causes a negative variation of it (fig. II). The method of response by the so-called negative variation, which is generally employed in studying responsive phenomena in animal tissues, is in reality, as will be seen later, a form of this method of relative depression.

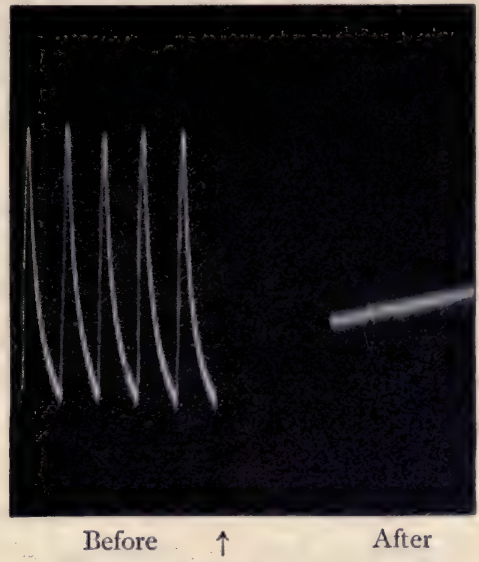

FIG. 10. Abolition of Response in metal by Oxalic Acid

Various means have been described, in the course of this chapter, for the detection and record of that excitatory change which is brought about by the upsetting of molecular equilibrium under stimulus, and the subsequent recovery. The responsive change may find expression in different ways. This expression may, for instance, in the case of living tissues, take the form of mechanical contraction, or of the electrical variation of galvanometric negativity.
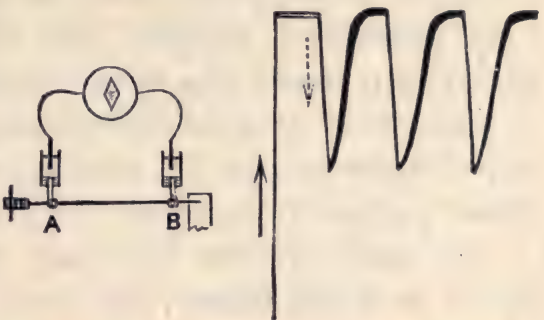

FIG. I I. Response by Method of Relative Depression

$\uparrow$ represents current of rest ; $\downarrow$ represents the action current.

Or the opposite change, expressed mechanically as expansion, will be evidenced by galvanometric positivity. But it must be borne in mind that neither of these expressions 
is consequent on the other It would be as incorrect to suppose that the electrical effect depended on the mechanical, as to assume that the mechanical was brought about by the electrical. The two are independent expressions of the same fundamental molecular change, brought about by the shock of stimulus.

Again, those various responsive phenomena and their modifications which are the subject of our inquiry, are such as are induced by different external agencies. Under the influence of certain conditions, the responses of living matter undergo an abolition - the change which we associate with death. In inorganic matter also, we find a similar change of responsiveness into irresponsiveness, to take place. The death-change in the case of living matter is thus not due to a change from the organic to the inorganic condition, but to some molecular transformation. And the nature of this very obscure transformation may one day be elucidated by a careful study of the changes which take place in inorganic matter, when it passes from responsivity to irresponsivity.

The word 'physiological' is generally used to distinguish phenomena which are believed to be exclusively characteristic of the properties of living matter. Such phenomena, however, are found, as our power of investigation grows, to be increasingly capable of analysis into physico-chemical processes. In my own use of the term 'physiological,' therefore, it will be understood as a convenient expression for describing the response-phenomena of plant or animal tissues, but as in no sense opposed to the word 'physical.'

We shall, in the following chapters, study excitatory effects in living tissues, and their variations under different conditions, using the methods of electrical response. These phenomena will be studied with special detail in the case of vegetable tissues, and it will be found that there is no responsive reaction exhibited by any one amongst the various types of animal tissues, which has not its exact correspondence in the vegetable. Those anomalies, further, which have been observed in the response of the animal, will be seen 
to be fully elucidated by the study of similar phenomena under the simpler conditions of the plant. And finally an attempt will be made to arrive at some generalisation which will show the continuity between the simplest form of response in the inorganic and the most complex which occur in the highest type of animal tissue. 


\section{CHAPTER II}

THE ELECTRO-MOTIVE RESPONSE OF PLANTS TO DIFFERENT FORMS OF STIMULATION

Historical-Difficulties of investigation-Electrical response of pulvinus of Mimosa-Simultaneous mechanical and electrical records-Division of plants into 'ordinary' and 'sensitive' arbitrary - Mechanical and electrical response of 'ordinary' plants-Direct and transmitted stimulation-All forms of stimulus induce excitatory change of galvanometric negativity.

IT has been customary to divide plants, as regards their responsiveness, into two distinct classes: 'ordinary' and 'sensitive.' Of these only the latter class, represented by such plants as Mimosa and Dioncea, was regarded as cxcitable. Hence the attention of observers desirous of investigating excitatory electro-motive phenomena in vegetable tissues was mainly attracted towards the reactions exhibited by these plants. The experiments undertaken in this field by Kunkel, Burdon Sanderson, and Munk are well known. Burdon Sanderson and Munk worked on the sensitive leaves of Dioncea and Kunkel on those of Mimosa. ${ }^{1}$

Electro-motive variations were observed to take place in all these plants on stimulation, but the conclusions arrived at by the investigators were not concordant. Burdon Sander. son and Munk found out that the resting-current between two selected points of Dioncea exhibited variation on application of stimulus to the leaf. They thus obtained di-phasic and sometimes even tri-phasic responses, consisting of positive and negative variations. More definite results were obtained by Burdon Sanderson, in the responsive variations of the normal leaf-current, flowing between the proximal and distal

' For a more detailed account cf. Biedermann, Electro-physiology, English translation, I898, vol. ii. pp. I-3I. 
points of the midrib of the leaf. On stimulation of the lamina, this current was found to undergo a diminution, or negative variation. But the current in the stalk, or petiole was found to undergo the opposite change-that is to say, a positive variation. Kunkel, in working with the pulvinus of Mimosa, found that on excitation a series of opposite or oscillatory electro-motive variations was induced. $\mathrm{He}$ also found electro-motive differences to be induced as the result of the flexure or injury of ordinary stcms He believed all these electrical phenomena to be consequent on hydrostatic disturbance or water-movement.

By means of this ' migration of water' Kunkel attempted to explain all the electrical phenomena in vegetable organs. The electro-motive difference between different parts of an organ was due, according to him, to their different powers of absorption of water. The greater absorptiveness of one point, with its consequent greater movement of water, would render that point relatively positive. As against this, however, Haake pointed out that electrical differences between different points were also to be found, even in submerged plants, like Valisnaria and Nitella, in which there could not possibly be any differences of absorptiveness. This difference, therefore, he suggested, must be ascribed to some vital process, inasmuch as the P.D. is seen to undergo a change whenever the respiratory process is interfered with, as, say, by the substitution of hydrogen for oxygen.

We shall study later in greater detail the conditions on which this 'current of rest,' so called, actually depends (Chapter X.). But more important is the excitatory variation induced by stimulus. It has already been stated that Kunkel found oscillatory variations of the current to occur on stimulation of the leaf of Mimosa. These alternating variations were difficult to reconcile with his theory of the active, single-phased displacement of water, as he did not fail to see, and he suggested that the first negative swing observed might be due to the disturbance of the diffusionprocess through alterations of the protoplasm. 
Munk attempted to explain the complicated electrical effects which he observed in Dioncea by assuming the existence of two different kinds of electro-motive elements, affected in opposite ways, the maximum changes being initiated in onc set earlier than in the other. In this way, he thought, it might be possible to explain the occurrence of positive and negative variations, holding that the upper parenchymatous layer of the leaf, and the upper midrib, went through the negative, and the under layer and the under midrib through the positive change.

Burdon Sanderson, in his 'fundamental experiment ' on the lamina of Dionar, had his led-off circuit connected with the upper and lower surfaces of one lobe, stimulus being applied at the other. In the experiments described in the 'Phil. Trans.' of 1882 , he found that the upper or inner surface of the leaf become positive on excitation. This he regarded as the true excitatory change. The upper contact now, however, after a certain interval became negative, a change which Burdon Sanderson designated as the after-effect. This aftereffect he ascribed to the electrical variations caused by that movement of water which had been observed by Kunkel. But with regard to the preceding positive variation he says :

'The excitatory disturbance which immediately follows excitation is an explosive molecular change, which by the mode of its origin, the suddenness of its incidence, and the rapidity of its propagation is distinguished from every other phenomena except the one with which I have identified it, namely, the corresponding process in the excitable tissues of animals. . . The direction of the excitatory effect in the fundamental experiment is such as to indicate that in excitation excited cells become positive to unexcited, whereas in animal tissues excited parts always become negative to unexcited.' ${ }^{1}$

In a subsequent series of experiments, however, given in 'Phil. Trans,' for I 888 Burdon Sanderson finds the reaction

I Phil. Trans. vol, clxxiii. 
of leaves 'in their prime' to be somewhat different. A strong negative phase was now observed on stimulation, but preceded by a short-lived positive reaction. These results will be discussed in detail in a subsequent chapter, but it may be said here that from the records given by Burdon Sanderson it is difficult to know which of the various responsive phases are to be regarded as those of true excitation, and which as the results of some other cause.

It will thus be seen that the results arrived at and the theories advanced by different observers in this field are somewhat at variance with each other. This must have been due to the difficulties met with in disentangling the fundamental reaction from those various subsidiary effects which are apt to be found in combination with it. Chief among these difficulties was (I) the fact that positive and negative variations are generally measured in terms of an existing current of rest. But, as a matter of fact, these two apparently opposite responsive variations, positive and negative, are not always indicative of opposite reactions. For an identical excitatory reaction, added algebraically to the resting-current, might appear, according to circumstances, as either of the two. There was also (2) the difficulty of discriminating two opposed electrical effects, one of which was due, as I shall show, to true excitation, and the other to increase of internal energy brought about by mechanical movement of water. Under different conditions, it may be either the one or the other of these which becomes prominent. I shall hope to show that each of them is definite and distinguishable from the other.

With regard to the response of plants in general, then, it may be well to state here that the first fact to be demonstrated in the course of the present work is that such responsive phenomena as may be observed in the case of sensitive plants like Dioncea, are not unique, but occur under similar circumstances even in ordinary plants, and are characteristic of all plant organs. I shall be able to show, moreover, that an explanation of these phenomena, much 
simpler than the theory of electro-motive molecules, is available. It will also be proved that the electrical response due to true excitation is quite distinct from that which is brought about by the hydrostatic disturbance, its sign being in fact opposite. This true excitatory electrical response, again, will be shown to be modified by all those conditions which affect the physiological state of the tissue. And, lastly, it will be proved that there is no breach of continuity as between the electrical responses in plant and animal, for not only is the sign of response in both cases the same, but it is also true that every type of response and modification of response, which occurs in the animal tissue, is to be found under parallel circumstances in that of the plant also.

In order to determine what is the electrical response characteristic of excitation, we first select for experiment a sensitive plant, say Mimosa, because here, in the responsive fall of the leaf, we have a visible indication of the excitatory reaction. It is desirable at this point to say a few words regarding the use of the terms 'excitatory ' and 'true excitation.' We are all familiar with the fact that, when muscle is excited by stimulus, it responds in a conspicuous manner by contraction. This is universally accepted as the phenomenon of excitation, its electrical concomitant being galvanometric negativity. Having once applied the term 'excitatory' to this particular aspect of molecular response in living tissues, it is of course important that we should henceforth distinguish carefully between it and its possible opposite, namely, expansion, with concomitant galvanometric positivity. Under stimulation there is a contraction of, and expulsion of water from, the excited pulvinus, which brings about the depression of the leaf. It is generally. supposed that only the lower half of the pulvinus is excitable. This, however, is an error, for both upper and lower halves are excitable, and contract under stimulation. If localised stimulus be applied on the upper side, that side contracts, and, by the concavity thus induced, the leaf is erected. But, though both halves are 
sensitive, yet the excitability of the lower is, generally speaking, greater, and diffuse stimulation therefore causes greater contraction of that half. Hence the resultant fall is due to the differential contraction of the two sides of the organ. The excitatory reaction of the organ, then, consists of a contraction; expulsion of water with consequent diminu-

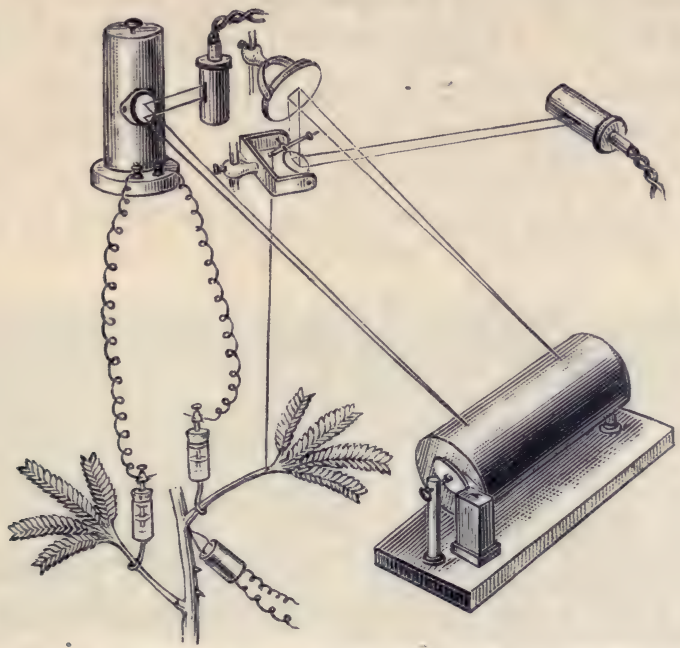

FIG. 12. Arrangement for ubserving Simultaneous Mechanical and Electrical Responses

Leaf stimulated by electro-thermic stimulator. Mechanical record obtained by excursion of spot of light reflected from Optic Lever, which falls on the right of drum. Electric record obtained by excursion of galvanometer spot of light adjusted to fall on the lett side of the drum.

tion of turgidity, or negative turgidity variation; and fall of the leaf.

In order next to determine the electrical concomitant of this reaction, we make suitable electrical connections by non-polarisable electrodes, with a galvanometer. One of these is made with the pulvinus, whose excitation is to be observed, and the other with a distant indifferent point. In this way we can obtain the excitatory effect at the pulvinus, uncomplicated by that at the distal point. A spot of light, reflected from the galvanometer mirror, is thrown on the 
recording drum. This spot of light, hitherto quiescent, shows, by its sudden deflection, the occurrence of the excitatory change.

To show the concomitance of the mechanical and electrical responses, and in order to detect with certainty the exact moment of the initiation of the former, a magnifying arrangement is obtained by attaching the end of the leaf to an Optical Lever. The pull exerted by the falling leaf rotates the fulcrum-rod, carrying a light mirror. The spot of light from this mirror moves in a vertical direction, and that of the galvanometer horizontally or laterally. For the purpose of simultaneous record, it is necessary to have the two in one direction. The up and down movement of the spot from the Optic Mirror is therefore converted into lateral, by means of reflection from a second mirror, suitably inclined.

Stimulation may be effected in the neighbourhood of the pulvinus by means of the electro-thermic stimulator, which has the advantage of producing no mechanical disturbance. This consists of a V-shaped platinum wire, suddenly heated by the momentary passage of an electric current. On applying stimulus in this manner it is found that the two responses-mechanical and electrical-take place at the same moment, the mechanical fall of the leaf being practically coincident with the induced electrical variation. As regards the sign of this electrical change, the excited point is found to become galvanometrically negative : that is to say, the electro-motive variation induced in the excited vegetable, is the same as that observed in animal tissues. I give below a series of these simultaneous records of mechanical and electrical response (fig. I3), obtained from Biophytum, whose lateral leaflets give motile indications. It will be seen that the responsive fall of the leaflet, and the subsequent recovery, are synchronous with the electrical variation of galvanometric negativity, and its subsequent recovery. For convenience of inspection. I shall always, unless specially stated to the contrary, represent the normal 
responses of mechanical depression and galvanometric negativity by up-curves. The erectile movement and galvanometric positivity will, conversely, be represented as down.

A few words may be said here as regards the synchronism between the two forms of response. The excitatory molecular change takes place instantaneously, and the electro-motive variation is, as far as can be judged, strictly concomitant with it. This is shown by the rheotomic method of observation, described in Chapter IV. It will there be seen that a very considerable electro-motive change has already been induced, in a period so short as 'OI of a second after the reception by the tissue of the stimulating shock.

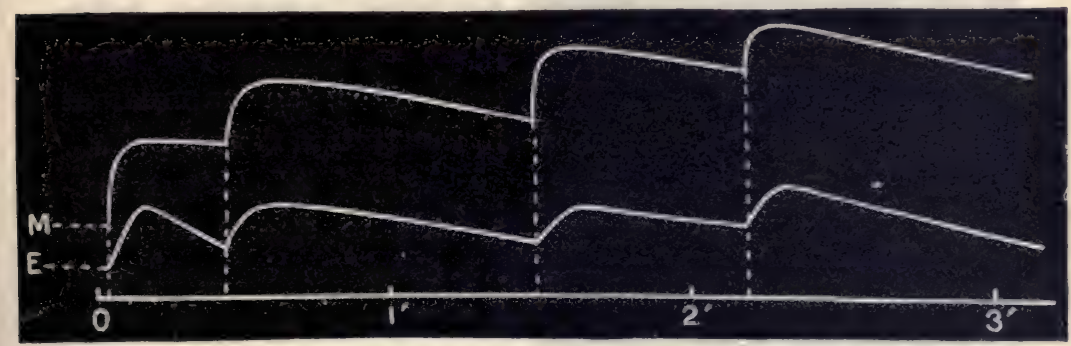

FIG. I3. Simultaneous Mechanical (M) and Electrical (E) Responses in Biophylum These responses are seen to take place at the same moment.

In the electrical response, then, of highly excitable tissues there is practically no latent period. But if the same electrical variation be recorded by the galvanometer, there will be a lag in the response, owing to the inertia of the galvanometer needle. Similarly, in the mechanical response, though the excitatory reaction is immediate, yet the motile response is delayed, by the antagonistic actions of the upper and lower halves of the pulvinus, the sluggishness of the tissue, and the mechanical inertia of the indicating leaf. The latent period of the mechanical response of a vigorous Mimosa, owing to all these causes, I find to be about twenty-four-hundredths of a second. But this may be still further prolonged when the tissue is in a state of depressed 
excitability. It will thus be seen that even when the fundamental excitatory reaction is instantaneous, its outward expression, whether mechanical or electrical, may nevertheless appear to be subject to delay in consequence of the inertia of the particular indicator concerned.

As regards these two forms of response, it should further be remembered that the mechanical and electrical responses are independent indications of the fundamental excitatory

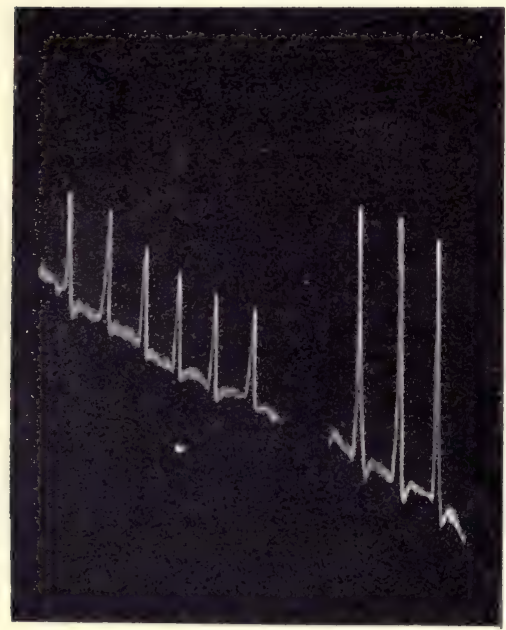

FIG. I4. Photographic record of Electrical Response by Galvanometric Negativity of Pulvinus of Mimosa, when leaf is physically restrained from falling. The first series in response to given uniform stimuli; the second series to stimuli twice as strong. reaction, and that neither is dependent for its occurrence on the other. Thus, when the mechanical response is physically restrained, the electrical response takes place unimpeded. I shall here relate an experiment in illustration of this point.

A leaf of Mimosa responds to strong stimulus by complete collapse, and the recovery from this state is somewhat prolonged, taking from five to eighteen minutes, according to the season. In order to obtain a series of responses with these recoveries, within a reasonable time, I find that it is necessary to apply moderate stimulus. There is then a moderate fall, without complete collapse, and recovery under such circumstances is found to take place within a minute or so. In order to show that electrical response takes place, even when the leaf is prevented from giving mechanical expression, I held the petiole in a clamp, and obtained the set of electrical responses seen in fig. I4. The first series of this record were taken in answer to uniform stimuli of a given intensity, and the 
second to stimuli twice as strong. We may here see how response is increased by increased intensity of stimulus. One peculiarity to be noticed in this figure is the trend of the base-line downwards, showing the increasing positivity of the pulvinus. In order to obtain a photographic record the experiment had to be carried out in a dark room, and under these circumstances the pulvinus undergoes an increase, or positive variation, of turgidity. And we shall see later that a positive turgidity variation is associated with galvanometric positivity in the same way as the negative turgidity variation is found to be accompanied by galvanometric negativity.

In consequence of the impression produced by the conspicuous movements of the leaf of Mimosa, it was assumed that only plants showing such movements were to be regarded as excitable. I have already shown elsewhere, however, that this test of lateral motile responses, as a sign of sensitiveness, is fallacious in the extreme. Such mechanical display is possible only when the two halves of an organ are unequally contractile, and there is consequently a greater expulsion of water from one, in response to stimulus, than from the other. If these conditions are not fulfilled, even the so-called 'sensitive' Mimosa would appear to be insensitive. Thus, when we place a cut branch of Mimosa in water, the pulvini of the leaves, on account of vigorous suction at the cut end, are rendered over-turgid, and the leaves become highly erected. On now applying stimulation, no responsive fall is found to take place; this is due to the difficulty encountered in the expulsion of water from the gorged tissue. An intact plant, again, which in the light has been found highly sensitive, will often be found insensitive after a short time spent in a dark room. It will then be difficult to believe that the plant is of the sensitive class, for the hardest blow will fail to evoke any mechanical response. And not only does the Mimosa cease to show responsive movement under these circumstances, which may perhaps be regarded as exceptional ; but under perfectly normal conditions also, its sensitiveness 
varies so much that its motile response would seem at times almost to have disappeared. I have already pointed out that it is by the unequal excitabilities of the upper and lower halves of the pulvinus that that differential contraction is induced which brings about the lateral response of the Mimosa leaf. Now it is clear from this that if the differential excitability should be reduced or abolished, by any means whatsoever, there will then be a corresponding diminution or abolition of response. We shall see later that the excitability of a tissue depends upon its state of turgor, and in Mimosa, from internal causes, a periodic variation is induced in the relative turgescence of the two halves of the pulvinus. We might then expect, in consequence of this, to find a periodic variation of motile sensibility. And certainly, whatever may be the cause, a long course of observation will convince the inquirer of the occurrence of great variations in the sensibility of Mimosa at different times of the day. Thus, I had six specimens of this plant growing in pots in the open, and I found, watching them in the month of August, that at eight o'clock in the morning the pulvini of the leaves of all these plants were sensitive in the highest degree. Half an hour later, however, this sensitiveness had so far waned that they would give hardly any motile indication. It is, perhaps, worth while to remark, in connection with this, that a constant observer is able to judge, by a peculiar, though indescribable, attitude of the leaves, whether or not this condition of insensitiveness has supervened. Thus the mechanical movements of the belauded sensitive plants, such as Mimosa, on which depended the arbitrary assumption that 'ordinary' plants were insensitive, rest on a basis which is itself extremely unreliable. For by this standard one identical plant ought to be classed as belonging to both the sensitive and insensitive groups, according to the time of day at which any particular observation is made.

The fact that when the mechanical response of the leaf of Mimosa is physically restrained, excitatory electrical response takes place unimpeded, shows that we have a criterion by 
which to test the excitability of a plant, independently of any motile indication. On applying this test, I have found that not the so-called sensitive plants alone, but all plants and all organs of all plants, respond to stimulation. And from this I was led to the discovery that ordinary plants also, in spite of current misconceptions, exhibit motile response by mechanical contraction. The common error of regarding these plants as insensitive has arisen from the fact that in a radial organ, diffuse stimulation induces equal contractions on all sides. Hence those lateral movements, dependent on differential contraction, which are seen so conspicuously displayed in Mimosa, cannot take place here. But that the organ as a whole undergoes a responsive contraction has been demonstrated by recording the consequent induced shortening of its length. Such longitudinal contraction is sometimes very considerable; for instance, in the filamentous corona of Passiflora it may sometimes be as much as 20 per cent. of the original length.

Having thus shown that all plants are excitable, I shall proceed to demonstrate the fact by means of electrical response. In studying the excitatory effect on ordinary plants, we must bear in mind that there are two different ways of stimulating a given point: that is to say, locally or directly, and by transmission of excitation from a distance. Conducting tissues are capable of stimulation in either of these ways, but the feebly conducting must be subjected to local excitation, since the effect of stimulus applied at a distance cannot, in their case, reach the responding point. Organs containing fibro-vascular elements are fairly good conductors, and stimulus applied on them at one or two centimetres from the point to be stimulated will thus easily reach it. It must, however, be remembered that stimulus becomes enfeebled by transmission through a long tract, its effect at a great distance being negligible. Parenchymatous tissues are bad conductors of excitation, and in order to excite them, stimulus must therefore be applied directly.

Turning first to the transmitted mode of stimulation, 
we take a petiole or stem, and making suitable electrical connections (fig. I 5), apply stimulus, say by contact of hot wire at the point marked $x$. After a short interval, necessary for the excitation to traverse the intervening distance, an electrical response is obtained, of galvanometric negativity. It is thus seen that the electrical response of 'ordinary' is of the same sign as that of 'sensitive' plants, and that in both, again, it is like that of animal tissues.

I shall next proceed to demonstrate a very important proposition: namely, that all effective forms of stimulation induce an identical excitatory response of galvanometric negativity. Any sudden change of environmental conditions may constitute an efficient stimulus. Such are : sudden rise

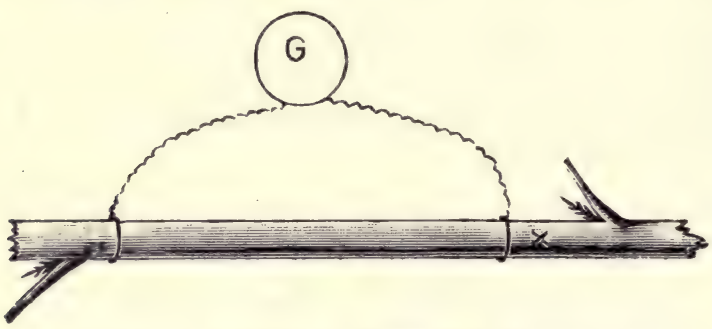

FIG. 15. Method of Transmitted Stimulation

Stimulus applied to the right at $\times$. Excitation reaches right contact first, causing galvanometric negativity of the point.

of temperature; any variation of pressure, whether of tension or compression ; mechanical blows or torsional vibration; any prick or cut; the application of a chemical agent, such as acid; the application of light ; the incidence or variation of electrical currents; and, lastly, the action of gravity. The stimulatory action of all these agents has already been demonstrated in my work on 'Plant Response,' " the excitation induced being there shown to find expression in appropriate mechanical movements. In the present volume I shall deal more particularly with the electrical reactions which they induce. The effects of the stimulating action of

1 Bose, Plant Response as a Means of Physiological Investigation, 1906. 
electrical currents, of light and of gravity, will be taken up in special chapters devoted to their consideration, while here I shall demonstrate the excitatory effects of the other forms of stimulus enumerated.

We have already observed the responsive effect which results from the sudden application of heat, by means of a hot wire. The effects of various forms of mechanical stimulation may now be subjected to demonstration, and first we have to observe the effect of the stimulus resulting from sudden tension. The specimen is clamped securely in the middle (fig. I6), so that when a vertical pull is given to the upper half, that half alone is subjected to a suddenly increased tension, the lower being left entirely unaffected.

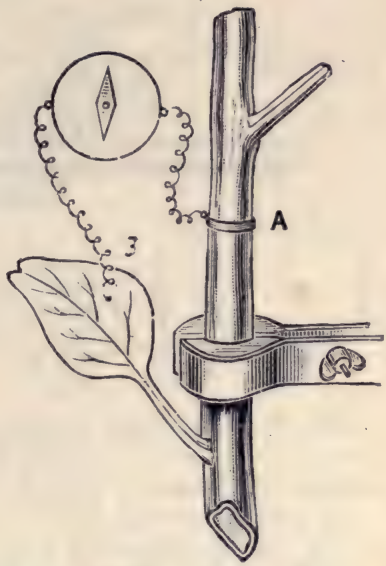

F1G. 16. Excitation by Sudden Tension

Plant securely clamped. When suddenly pulled, tension induces galvanometric nega. tivity of A.

Under these circumstances, there is an electrical response, A becoming galvanometrically negative. A is next subjected to mechanical compression, and for this purpose the piece of moistened cloth surrounding the specimen, and making the electrical connection at $\mathrm{A}$, is placed between the two grooved halves of a cork. The enclosed plant tissue at A may now be made to undergo sudden compression, by squeezing the pieces of cork together. This gives rise to the same electrical response as before.

This fact, that both tension and compression will give rise to similar excitatory responses of galvanometric negativity, may receive independent demonstration by first making an electric connection at $\mathrm{A}$ with the upper side of the specimen (fig. I 7). When the tissue at $\mathrm{A}$ is now suddenly bent down, this upper side becomes convex : that is to say, it is subjected to tension. This gives rise to the excitatory 
response of negativity. The electrical connection at A is next removed to the lower side of the specimen, at a point $\mathrm{A}^{\prime}$. On now repeating the sudden flexure, $\mathrm{A}^{\prime}$ undergoes com-

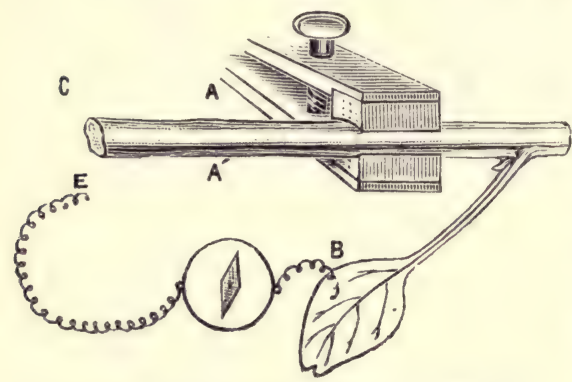

FIG. 17. Excitatory Response to Tension and Compression

When $\mathbf{E}$ is connected with the upper point A a sudden bending down causes tension of A. When connection is made with $\mathbf{A}^{\prime}$ the same flexure causes compression of $\mathbf{A}^{\prime}$. Both induce galvanometric negativity. pression instead of tension. The result is a similar negative electrical response.

Excitation may, again, be produced by means of a sudden blow at a point. This blow may be delivered by means of a spring-tapper (fig. I8), in which $\mathrm{S}$ is the spring proper and the attached rod $\mathrm{R}$ carries at its end the tapping head T. A projecting rod-the lifter L-passes through $\mathrm{S} R$. It is provided with a screw-thread, by means of which its length, projecting downwards, is regulated. By means of this the height or intensity of the stroke may

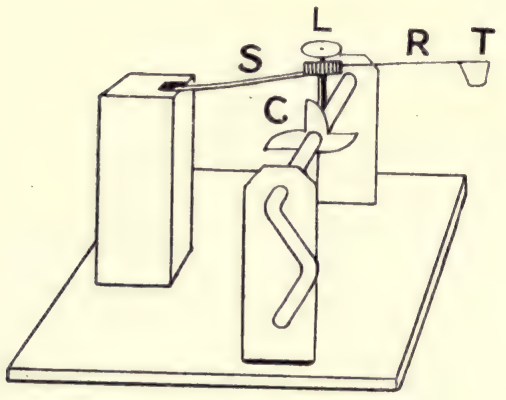

FIG. 18. The Mechanical Tapper be varied. As one of the spokes of the cog-wheel $\mathrm{C}$ is rotated past $\mathrm{L}$, the spring is lifted and released, and $\mathrm{T}$ delivers a sharp tap. The height of the lift, and therefore the intensity of the stroke, is measured by a graduated scale not shown in the figure. We can increase the intensity of this stroke through a wide range, first, by augmenting the projecting length of the spring by a sliding catch. We may give isolated single taps, or superpose a series in rapid succession according as the wheel is rotated slowly or quickly. 
Stimulation, again, may be effected by the prick of a needle or pin in the neighbourhood of A. Response to this also occurs by the normal galvanometric negativity. Successive pricks may thus give rise to successive responses.

Or the specimen may be subjected to torsional vibration. It is here held in the middle by a clamp, and stimulus of torsional vibration is applied at one end. - The stimulation of $\mathrm{A}$ makes that end galvanometrically negative, the direction of the current outside the circuit being towards, and in the tissue away from, A. Vibration of $B$ induces responsive negativity of B (fig. I9), the current of response being now reversed. In the cases just described, it will be noticed that stimulus is applied directly. This method

(a)

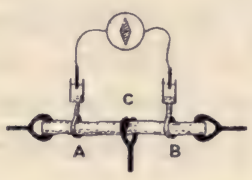

Current of response when $A$ is stimulated $\rightarrow$ Current of responce when $B$ is stimulated $\rightarrow$ (b)

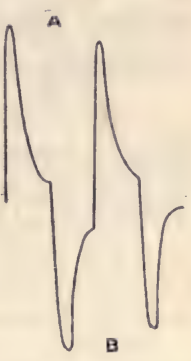

FIG. 19. The Torsional Vibrator

(a) The plant is clamped at $\mathrm{C}$, between $A$ and $B$.

(b) Responses obtained by alternately stimulating the two ends. Stimulation of A produces upward response; of $\mathrm{B}$ gives downward response.

is, therefore, specially applicable when we wish to study the excitability of such tissues as are not good conductors of excitation, the method of transmitted stimulation being here, therefore, inapplicable.

In order to observe the effect of chemical stimulation, the given agent-sulphuric or hydrochloric acid-is applied at $x$ at a short distance from the proximal contact. The transmitted excitation is now again demonstrated by the induced galvanometric negativity of that contact. It will thus be seen that, whatever be the effective form of stimulus employed, it gives rise to a definite and invariable electrical response whose sign is always one of galvanometric negativity.

It was shown, then, in the course of this chapter that the excitatory change in 'sensitive' plants is characterised by contraction, negative turgidity variation, mechanical depression of the leaf, and by the electrical response of 
galvanometric negativity, all these effects being concomitant. It was further shown that electrical response is independent of the mechanical, being unimpeded in its occurrence when the leaf is physically restrained.

The same electrical response of galvanometric negativity is also obtained from the tissues of the so called 'ordinary' plants. And these electrical responses of plant tissues, it was further noted, are identical in sign with the corresponding responses given by animal tissues.

All forms of stimulus, moreover-mechanical, thermal, photic, chemical, and electrical-induce the same excitatory response of galvanometric negativity. 


\section{CHAPTER III}

\section{THE APPLICATION OF QUANTITATIVE STIMULUS AND}

RELATION BETWEEN STIMULUS AND RESPONSE

Conditions of obtaining uniform response-Torsional vibration as a form of stimulus-Method of block-Effective intensity of stimulus dependent on period of vibration-Additive action of feeble stimuli-Response recorderUniform electric responses-List of suitable specimens-Effect of season on excitability-Stimulation by thermal shocks-Thermal stimulator-Second method of confining excitation to one contact-- Increasing response to increasing stimulus-Effect of fatigue-Tetanus.

A QUALITATIVE demonstration has been given in the last chapter of the induction of galvanometric negativity in plant tissues, in response to the excitation caused by various forms of stimulus. This galvanometric response is thus a sign or indication of the state of excitation; and under normal conditions it will be of uniform extent, provided only that the stimuli are also uniform. Assuming this ideal condition to be secured, it is clear that the physiological modifications induced by various agents will be manifested by a corresponding modification of response. The conditions essential to such application of stimulus are, then, (I) that it should be capable of uniform repetition; (2) that it should be capable of increase or decrease by definite amounts; and (3) that it should be of such a nature as to cause no injury, by which the excitability of the tissue might be changed in some unknown degree. These conditions, on which the success of the electro-physiological investigation depends, are very difficult to meet. Chemical stimulation, for example, cannot be uniformly repeated. Electrical stimulation, again, which has the advantage of being easy to render quantitative, is open to the objection that by escape of current it may induce 
galvanometric disturbance. Indeed, as the response is electrical, it is obvious that if we are to obtain unimpugnable results, a non-electrical form of stimulus is almost a necessity. But it is only after providing against various sources of error that the electrical form of stimulation can be used with confidence. The stimulation caused by mechanical blows can be repeated, it is true, with uniform intensity. But the point struck is subjected to increasing injury, and its excitability thus undergoes an unknown variation.

I have, however, been able to devise two different modes of stimulation, in which all these difficulties have been
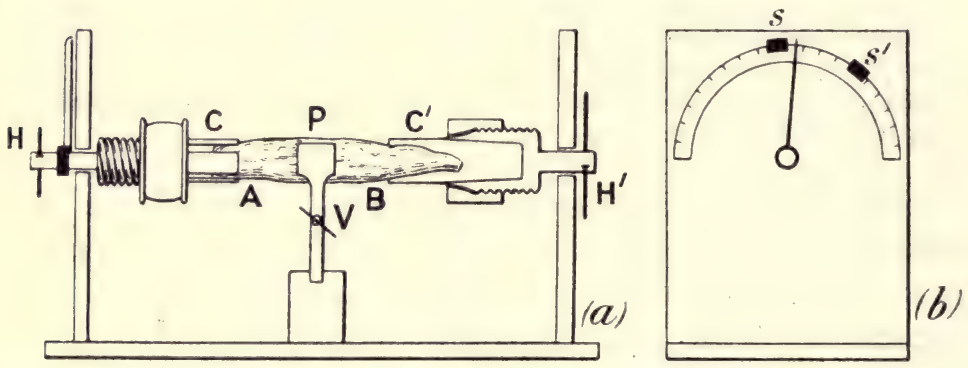

FIG. 20. The Vibratory Stimulator

Plant $\mathrm{P}$ is securely held by a vice $\mathrm{V}$. The two ends are clamped by holders $\mathrm{C} \mathrm{C}^{\prime}$. By means of handles $\mathrm{H} \mathrm{H}^{\prime}$, torsional vibration may be imparted to either the end A or end B of the plant. The end view $(b)$ shows how the amplitude of vibration is predetermined by means of movable stops, s s'.

successfully overcome. rendering the results as perfect as possible. These are (I) torsional vibration, and (2) the application of thermal shocks. For the obtaining of perfect responses, it must be said here that there is still another condition to be fulfilled. If we wish to obtain the pure effect of stimulus at one contact, say A, special care must be taken that excitation does not reach the second contact, B; for otherwise, unknown effects of interference will occur. This may, it is true, be obviated by means of the method of relative depression or method of negative variation, so called, to be described in a subsequent chapter. But the experimental mode which I am about to describe, in which a block 
is interposed between $A$ and $B$, is much more perfect. According to this arrangement, the specimen is tightly clamped in the middle, by which device the excitation of either end is practically precluded from affecting the other.

Stimulation is brought about by means of torsional vibration. The stem or petiole is fixed, at its middle, in a vice, $\mathrm{V}$, the free ends being held in tubes, $\mathrm{C} \mathrm{C}^{\prime}$, each provided

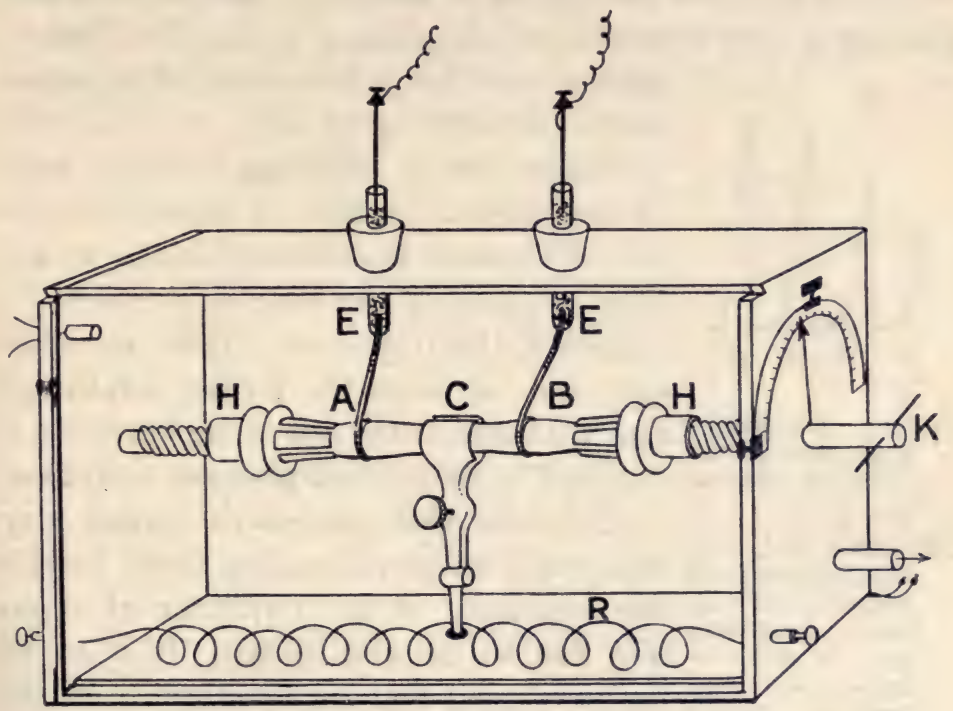

FIG. 2I. Complete Apparatus for Method of Block and Vibratory Stimulation

Amplitude of vibration which determines the intensity of stimulus is measured by the graduated circle seen to the right. Temperature is regulated by the electric heating coil $\mathrm{R}$. For experiments on action of anæsthetics, vapour of chloroform is blown in through the side tube.

with three clamping jaws. A torsional vibration may now be imparted to the specimen at either end by means of the handles $\mathrm{H}$ and $\mathrm{H}^{\prime}$ (fig. 20). The amplitude of vibration which determines the intensity of stimulus can be accurately measured by the graduated circle, and may be predetermined by means of the sliding stops S S.' The complete vibrational apparatus, by means of which various experimental investigations may be carried out, is given in fig. $2 \mathrm{I}$. 
Moistened cotton threads in connection with the non-polarisable electrodes, E E, make secure electrical contacts with A and B. For experimenting on the effects of temperature, there is an electrical heating coil, $\mathrm{R}$, inside the chamber. For the study of the effects of different gases, there are inlet and outlet tubes, which enable a stream of the required gas or vapour to be circulated through the chamber.

If the A end of the specimen be now suddenly torsioned through a given number of degrees, a responsive electromotive variation takes place, which after-

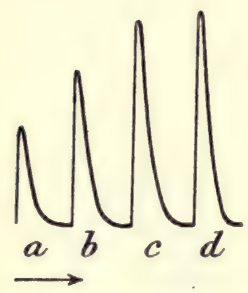

FIG. 22. Influence of Suddenness on the Efficiency of Stimulus

The curves $a, b, c, d$, are responses to vibrations of the same amplitude, $30^{\circ}$. In $a$ the vibration was very slow; in $b$ it was less slow; it was rapid in $c$, and very rapid in $d$. wards subsides gradually. If next the torsioned end be suddenly brought back to the original position, a second electromotive response is obtained, similar to the first. Hence, in the case of a to-and-fro vibration, the responsive effects are additive, and we have the further advantage that the tissue at the end of the operation is returned to its original physical condition.

In order that successive stimuli may be equally effective, another factor besides the constancy of the amplitude of vibration has to be considered. It is to be borne in mind that the effectiveness of the stimulus in evoking response depends also on the rapidity of the onset of the disturbance. In the application of vibratory stimulation to plants, I find the extent of response to depend to some degree on the quickness with which the vibration is effected. I give below records of responscs to successive stimuli, induced by vibration through the same amplitude, which were delivered with increasing rapidity (fig. 22). It will be noticed that an increasing quickness of vibration increased the response, but that this reached a limit. If we wish, then, to maintain the effective intensity of stimulus constant, we must meet two conditions. First, the amplitude of vibration must be kept the same. This is done by means 
of the graduated circle and movable stops: and, second, the vibration period must be uniform. This last condition is effected by an arrangement shown in fig. 23. The torsionhead is kept tense by means of a stretched spiral spring, s, made of steel. From this torsion-head there projects an elastic brass piece, $B . \quad R$ is a striker which can be made to give a quick stroke to $\mathrm{B}$, by the rotation of the handle. A quick to-and-fro vibration is thus produced, by the blow given to $\mathrm{B}$, acting against the tension of the antagonistic spring S. The amplitude of the angular vibration is at the

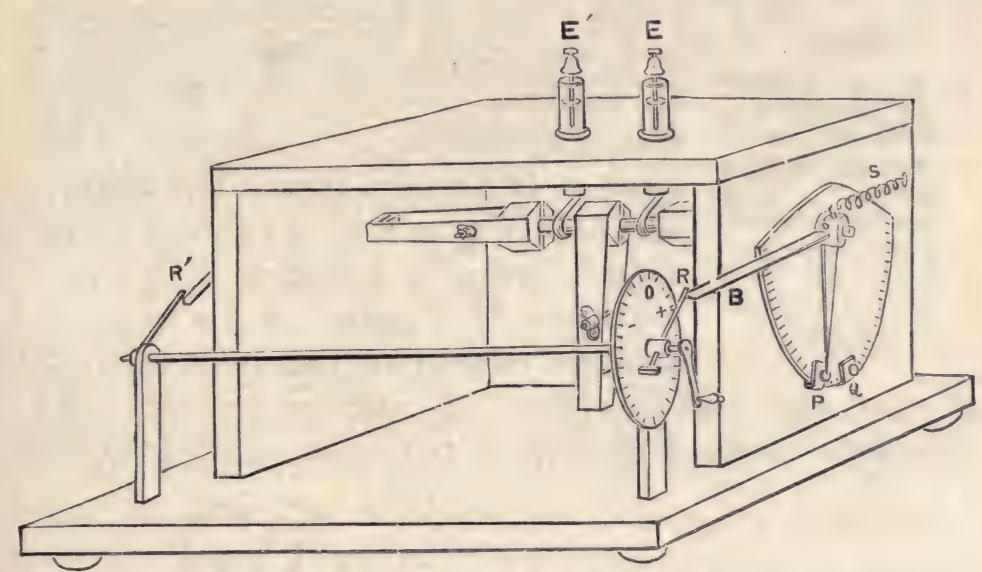

FIG. 23. Spring Attachment for obtaining Vibration of Uniform Rapidity

same time predetermined by means of the stops $P$ and $Q$. The arrangements described are as used in ordinary work. But for certain experiments on differential excitability, a second striker, $R^{\prime}$, may be attached to the other end of the apparatus, and by this means the opposite contacts in connection with $\mathrm{E}$ and $\mathrm{E}^{\prime}$ may be excited simultaneously.

In order to obtain responses of great amplitude, it is now necessary to increase the amplitude of vibration. But this may give rise to fatigue. By way of avoiding this, therefore, it is still possible to obtain enlarged response by the additive effect of repeated feeble stimuli. In the electrical response of plants a sub-minimal stimulus, singly ineffective, is found 
to become effective by the summation of several. This is seen in fig. 24, where a single vibrational stimulus of $3^{\circ}$,

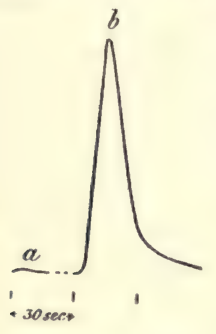

F1G. 24. Additive Effect

(a) A single stimulus of $3^{\circ}$ vibration produced little or no effect, but the same stimulus when rapidly superposed thirty times produced the large effect $(b)$. (Leaf stalk of turnip.) alone ineffective, was found to evoke a large response when repeated with rapidity thirty times in succession.

For the delivering of such equal and rapidly succeeding stimuli, I substitute for the single striker $\mathrm{R}$ an eight-spoked wheel, a complete rotation of which, by means of the handle, gives rise to a definite summated effect: and a series of responses to such summated stimulations is found to be uniform. The galvanometer used for these experiments is a dead-beat instrument of D'A rsonval type. The sensitiveness of this is such that a current of $\mathrm{IO}^{-9}$ ampere causes a deflection of $\mathrm{I} \mathrm{mm}$. at a distance of I metre. For a quick and accurate method of obtaining records, I devised the following form of response-recorder. The curves are obtained directly, by tracing the excursion of the

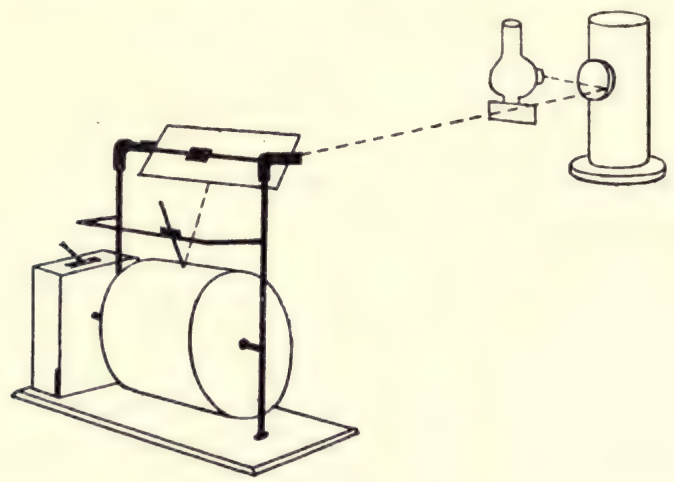

FIG. 25. Response Recorder

galvanometer spot of light on a revolving drum (fig. 25). This drum, on which is wrapped the paper for receiving the record, is driven by clockwork. Different speeds of 
revolution can be given to it by adjustment of the clockgovernor, or by changing the size of the driving-wheel. The galvanometer spot is thrown down on the drum by the inclined mirror M. The galvanometer deflection takes place at right angles to the motion of the paper; a stylographic pen attached to a carrier rests on the writing surface. The carrier slides over a rod parallel to the drum. As has been said before, the galvanometer deflection takes place parallel to the axis of the drum, and as long as the plant rests unstimulated, the pen, remaining coincident with the stationary galvanometer spot on the revolving paper, describes a straight line. If, on stimulation, we trace the resulting excursion of the spot of light, by moving the carrier which holds the pen, the rising portion of the response curve will be obtained. The galvanometer spot will then return more or less gradually to its original position, and that part of the curve which is traced during this process constitutes the recovery. The ordinate in these curves represents the electro-motive variation, and the abscissa the time.

We can calibrate the value of the deflection by applying a small known E.M.F., say of ' $I$ volt, to the circuit, and noting the deflection which results. This gives us the value of the ordinate. The value of the abscissa which represents time is determined by the distance through which the recording surface moves, in unit time. In this simple manner accurate records are obtained. It has the additional advantage of enabling the observer to see at once whether the specimen is suitable for the purpose of investigation. A large number of records might be taken by this means, in a comparatively short time.

It is also easy to take the records photographically by wrapping a photographic film round the recording drum.

I give in fig. 26 a series of responses taken from the root of radish (Raphanus sativus), in which the stimuli were applied at intervals of one minute. This shows how extremely uniform the responses may be rendered, if proper precautions are taken. It may here be once more pointed 
out, that for convenience of inspection, the records in this book have been so taken that the normal electrical responses of galvanometric negativity, unless specially stated to the contrary, are seen as up-curves, galvanometric positivity being represented by down-curves. These excitatory responses of

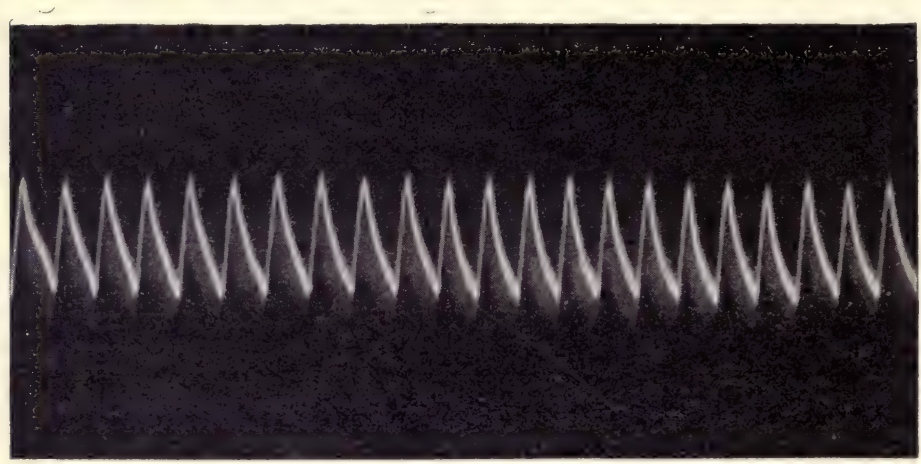

FIG. 26. Photographic Record of Uniform Responses (Radish)

galvanometric negativity are obtained with all plants, and with every organ of the plant. I give here a table containing a list of specimens which will be found on stimulation to give fairly large electro-motive effects, occasionally as high as - I volt.

\begin{tabular}{|c|c|c|c|c|}
\hline \multicolumn{4}{|c|}{ Organ } & Specimen \\
\hline Root & · & · & . & $\begin{array}{l}\text { Carrot (Daucus carota) } \\
\text { Radish (Raphanus satious) }\end{array}$ \\
\hline Stem & - & - & - & $\begin{array}{l}\text { Geranium (Pelargonium) } \\
\text { Vine (Vitis vinifera) } \\
\text { Amaranth (Amaranthus) }\end{array}$ \\
\hline Petiole & - & - & - & $\begin{array}{l}\text { Horse Chestnut ( Esculus hippocastanum) } \\
\text { Turnip (Brassica napus) } \\
\text { Cauliflower (Brassica oleracea) } \\
\text { Celery (Apium graveolens) } \\
\text { Eucharis lily (Eucharis amazonica) }\end{array}$ \\
\hline Peduncle & & - & - & Arum lily (Ricardia africana) \\
\hline Fruit & . & . & . & Egg-plant (Solanum melongena) \\
\hline
\end{tabular}


These responses, being physiological, vary in intensity with the condition of the specimen. The same plant which gives strong electrical response in spring or summer, may exhibit but feeble responsiveness in autumn or winter. Again, we shall see in a subsequent chapter that any agent which depresses physiological activity will also depress the electrical response; and, lastly, when the specimen is killed, the normal response is abolished.

I shall next describe a second and equally perfect method of stimulation, by means, namely, of thermal shocks. We have seen that a sudden thermal variation acts as an efficient stimulus. I have also shown in my 'Plant Response' that thermal radiation acts as a stimulating agent, in inducing excitatory contraction. Hence, if a tissue be surrounded by a platinum wire, through which an electrical heating-current can be sent, the enclosed tissue will be subjected to a sudden variation of temperature, and also to the thermal radiation proceeding from the heated wire. Now if in successive experiments the duration and intensity of the current flowing through the wire be maintained constant, the stimuli also will thereby be rendered constant. The thermal stimulator, as already said, surrounds the specimen, but is not in actual contact with it. This is to prevent any injury to the tissue, by scorching. The current is so adjusted as to make the platinum wire red-hot and this heating-current is closed for about half a second at a time. Should larger response be desired, it can be obtained by the summated effect of a number of such shocks, or the thermal stimulator may be put in direct contact with the tissue, if care be taken that the rise of temperature is not so great as to injure it.

The difficulty of ensuring similarity of duration to each individual shock is overcome by the use of a balanced key actuated by a metronome (fig. 27). A second rod is attached at right angles to the vibrating rod of the metronome, and carries a bent piece of brass in the form of two prongs. During the course of each vibration these pròngs dip into 
two cups of mercury, thus closing the electrical circuit for a brief and definite time. When a second press-key, not shown in the figure, is open, the circuit is incomplete, and there is no thermal stimulation. The observer then presses this

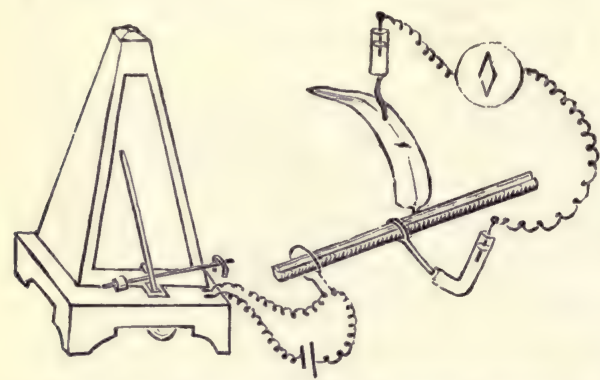

FIG. 27. Stimulation by Thermal Shocks key, and counts, say, five strokes of the metronome, after which the press-key is again opened. In this way, the summated effect is obtained, of five equal thermal shocks. This process is repeated as often as desired, at intervals of, say, one minute, by which time the tissue is generally found to have completely recovered from its excitatory electrical variation.

In the case of the experimental arrangements of which the diagram is given in fig. 27 , stimulation is confined to one contact of the responding circuit. The method by which excitation was here prevented from reaching the distal contact is important. I shall, in the course of the present work, show that the parenchymatous tissue of the lamina of a leaf or leaflet is a bad conductor of excitation. Hence if the second contact of the circuit be made with this tissue, the stimulus does not reach the distal point. It is true that a certain small proportion might conceivably be conducted through the attenuated fibro-vascular channel of the midrib. But even so remote a contingency is provided against by a transverse cut across the midrib on the hither side of the contact.

The arrangements, then, being made in the manner described, the tissue may be subjected to the action of successive uniform stimuli. How regular the resulting responses may be rendered will be seen from fig. 28 , in which is given a series of responses obtained from the petiole of a fern (fig. 28) 
under successive thermal shocks, imparted at intervals of one minute. We have hitherto studied the responses caused by uniform stimuli. We shall next observe the increase of responsive effects brought about by increase of stimulus. In

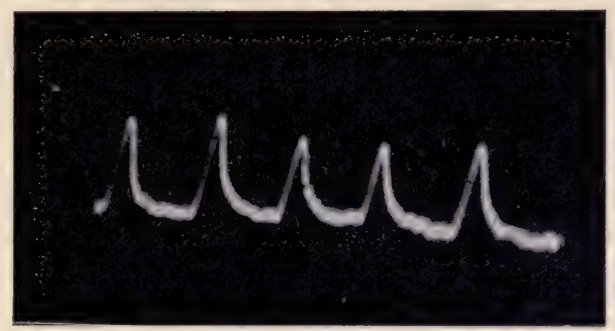

FIG. 28. Photographic Record of Uniform Response in Petiole of Fern to transmitted excitation

animal tissues it is found, speaking generally, that increasing stimuli induce increasing effects, but that this process has a limit; and in plant tissues the same is found to be the case. In order to obtain effects of the simplest type, not complicated by any secondary phenomena, it is necessary to choose specimens which exhibit little fatigue. In the first of these the stimulus was applied by means of the spring-tapper. The first stimulus was given by a fall of the striking-lever from the height $\mathrm{h}$; the second from $2 \mathrm{~h}$; and the third from $3 \mathrm{~h}$. The responsecurves (fig. 29) clearly show the increase of effect due to this increasing stimulus.

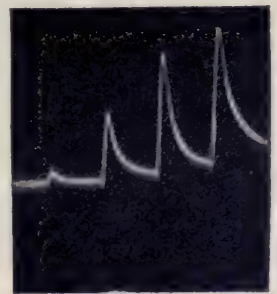

FIG. 29

Taps of increasing strength I : $2: 3: 4$ producing increased response in leafstalk of turnip.

In the second series, the stimulus applied was vibrational, and increased from $2.5^{\circ}$ to $12.5^{\circ}$ by steps of $2.5^{\circ}$ at a time. Fig. 30 shows how the intensity of response tends under these conditions to approach a limit. The following table gives the absolute values of the responsive electro-motive. variations. 


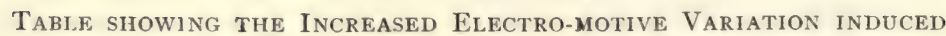
by Increasing Stimulus.

\begin{tabular}{|c|c|}
\hline Angle of vibration & Induced E.M.F. \\
\hline $2.5^{\circ}$ & 044 volt. \\
$55^{\circ}$ & $075,$, \\
$7: 5^{\circ}$ & $090,$, \\
$10^{\circ}$ & 100 ,, \\
$12.5^{\circ}$ & 106 ,", \\
\hline
\end{tabular}

In such normal cases an increase of response is always induced with increasing stimulation. A diminution of response may, however, sometimes appear, with increasing

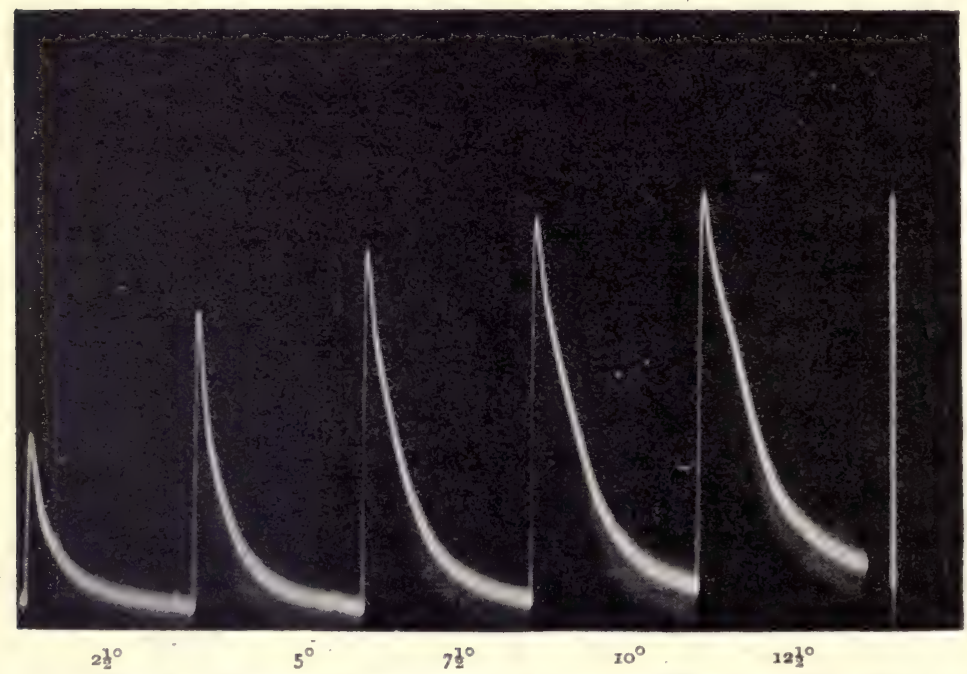

FIG. 30. Increased Response with Increasing Vibrational Stimuli (Cauliflower-stalk)

Vertical line $=\cdot$ I volt. Stimuli applied at intervals of three minutes.

stimulus. But this is merely a secondary effect, due to fatigue. The following records (fig. 3I) will show in what manner this may be brought about. They were taken with specimens of the petiole of cauliflower, in one of which (A) fatigue was absent, while in the other (B) it was present. In the first specimen the recovery from each stimulus was 
complete. Every response in this series starts, therefore, from a position of equilibrium, and the height of each single response increases with increasing stimulation. In the second case, however, the molecular derangement consequent on stimulation is not completely removed after any single
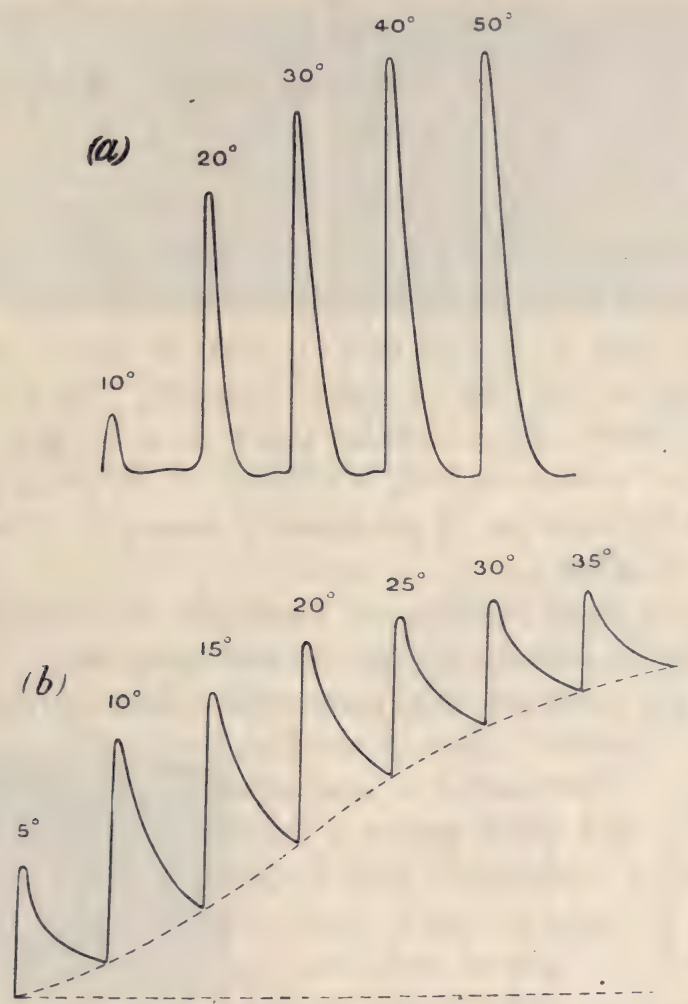

FIG. 31. Responses to Increasing Stimulus obtained with Two Specimens of Stalk of Cauliflower

In $(a)$ recovery is complete, in $(b)$ it is incomplete.

stimulus of the series. That the recovery is only partial is seen in the gradual shifting of the base-line upwards. In the former case the base-linè had been horizontal, representing a condition of complete equilibrium. Now, however, the base-line, or line of modified equilibrium, is tilted upwards. Thus, even here, if we measure the heights of successive 
responses from the line of absolute equilibrium, they will be found to increase with increasing stimulus. Ordinarily, however, no allowance is made for the shifting of the base-line, the responses being measured instead from the place of its previous recovery, or point of modified equilibrium. In this way these responses undergo an apparent diminution.

I have occasionally observed another curious phenomenon in connection with the subject of response under increasing stimulus. During the gradual increase of the stimulus from a low value, there would at first be no response. But on reaching a certain critical value, a response would suddenly be evoked which was maximum - that is to say, would not be exceeded, even when the stimulus was further increased. We have here a parallel case to what is known in animal physiology as the 'all or none' principle. In the case of cardiac muscle, for example, there is a certain minimal intensity of stimulus which is effective in inducing response. But further increase of stimulation causes no concomitant increase of effect.

When a tissue is subjected to rapidly succeeding stimuli, the excitatory effects are superposed upon each other. In muscle, for example, the contractile effect of the second stimulus is added to that of the first, before that has time to disappear. The result is a summation of effects more or less complete; and these attain a maximum. With moderate frequency of stimulation, such a tetanic effect is incomplete, tending to become more and more complete, with the progressive increase of frequency (fig. 52). I have obtained results in every way similar to these, with the mechanical response of ordinary plants. In fig. 33 is given a photographic record of tetanus, taken from the longitudinal motile responses of the style of Datura alba. Similar tetanic effects are also obtained in the electric response of plants, of which the records seen in fig. 34 form an example.

The difficulties in the quantitative observation of electrical response have thus been overcome by the employment of two different methods of stimulation-namely, torsional vibration, and stimulation by thermal shocks. In the case of the 
former, the intensity of stimulation was seen to depend on the amplitude of vibration. In the latter, stimulus intensity was determined by that of the thermal variation, which again was regulated by the intensity and duration of the electrical heating-current. It was also seen to be important that the
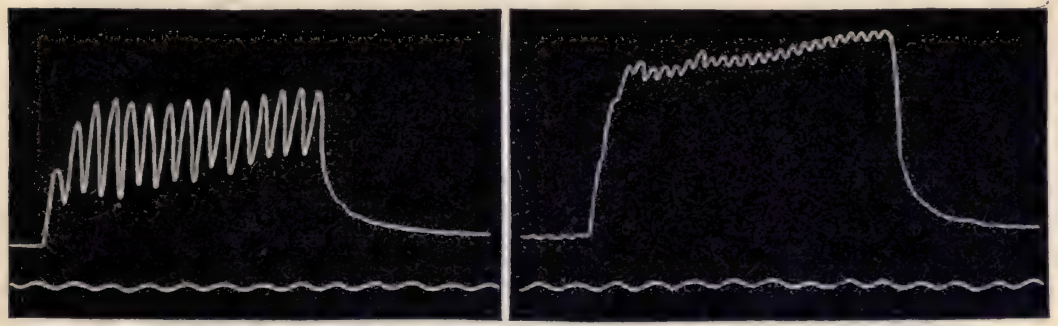

FIG. 32. Genesis of Tetanus in Muscle

Record to left shows incomplete tetanus, with moderate frequency of stimulation. Record to right shows tetanus more complete, with greater frequency of stimulation (Brodie).

excitation of one contact should be prevented from reaching the other, and this was provided against in two different ways. In the first of these, a physical block was interposed between the two contacts. In the second, the distal contact was made with the non-conducting tissue of a lateral leaf.

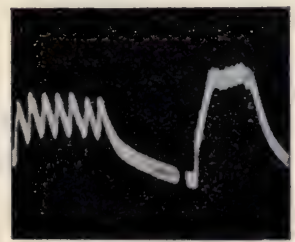

FIG. 33. Photographic Record of Genesis of Tetanus in Mechanical Response of Plants (Style of Datura alba)
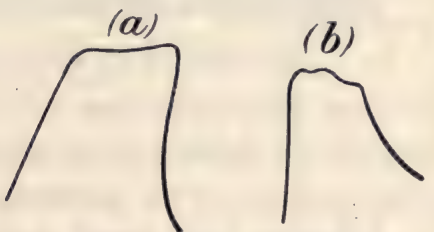

FIG. 34. Fusion of Effect or Rapidly Succeeding Stimuli

$(a)$ in muscle ; $(b)$ in carrot.

When these precautions were observed, it was found that uniform stimuli induced uniform response. Stimuli which were singly ineffective, were found on repetition to become effective. A tetanic effect was obtained by the rapid superposition of stimuli. 


\section{CHAPTER IV}

\section{OBSERVATION BY RHEOTOME ON ELECTRIC RESPONSE}

\section{IN PLANTS}

Response-curve showing general time-relations-Instantancous mechanical stimulation by electro-magnetic release--Arrangement of the rheotomeTabular statement of results of rheotomic observations-Rhythmic multiple responses.

IN taking records of the electric response of plants, a galvanometer of fairly high sensitiveness is required. One which gives a deflection of $\mathrm{I} \mathrm{mm}$. at a scale-distance of I metre, under a current of $1 \mathrm{O}^{-9}$ ampere is found, as already said, to be suitable for practical purposes. I used for most of the experiments in this work a dead-beat galvanometer of the D'Arsonval type. The natural period of swing in these galvanometers is somewhat long, however, and the responserecord thus lags behind the electro-motive changes induced by stimulus.

In order, therefore, to investigate the time-relations of a growing electro-motive reaction in a plant, after the reception of the stimulating shock, it is necessary to employ a rheotomic mode of investigation. An account of this, and of the results obtained, will be given in the course of the present chapter. The after-effect of stimulus is found to be somewhat persistent and to vary in duration in different specimens. In some cases, recovery is complete in a very short time; in others it takes very much longer. For the purpose of forming a general idea of this difference two response-records are given here, one of which was taken from a stem of the quickly-reacting Amaranth (fig. 35), and the other from the more sluggish Colocasia. It will be seen that 
while in the first of these the recovery was completed in fifteen seconds, in the latter, even after the lapse of forty seconds, it was still far from complete. Indeed, in this case it was not altogether accomplished till after several minutes. The character of the tissue again is an important factor in determining the time required for recovery. Thus it will be shown that in a vegetable structure functioning as nerve, recovery is much more rapid than in ordinary tissue. The physiological modification induced by season, moreover, is seen in the fact that response and recovery are quicker in

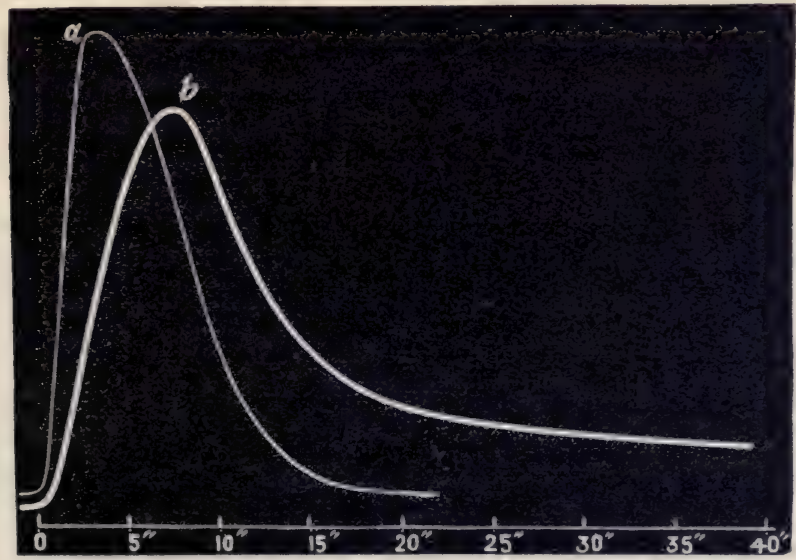

FIG. 35. Response of $(a)$ quickly reacting Amaranth ;

(b) of sluggish Colocasia

summer than in winter. This difference is demonstrated by mechanical response also, for in that of the leaf of Mimosa, as already stated, it is found that, whereas in summer the period is six minutes, in winter it is as long as eighteen, or three times as much.

In the study of the time-relations of response, we may overcome the difficulty of the galvanometer-inertia by using, as already said, some modification of the rheotome, originally devised by Bernstein. The relative values of electrical variation induced at various periods after the impact of the excitatory shock may here be found by making brief 
galvanometric contacts of equal period at the required intervals.

The difficulty in this investigation lies in the instantaneous application of a stimulus at a definite moment, and in the successful adjustment of the subsequent interval at which the resulting responsive current is to be led to the galvanometer and recorded. Instantaneous stimulation can, it is true, be effected by electrical shock. But polarisation, and other disturbances caused by it, might give rise to unknown variations in the responsive effect. Hence, it is advisable when recording the electrical response to employ, if possible, a non-electrical form of stimulus. And it is only after the successful employment of such an unimpeachable method, that we can feel any confidence in the use, after due precautions have been taken, of the electrical stimulus itself, as will be described in a later chapter. Another obstacle to be overcome is the elimination of the unknown element of time required for transmission when stimulus is applied at a distance from the responding point. This uncertainty can only be removed by applying the stimulus directly on the responding point itself. All these difficulties I have successfully met by employing the mechanical form of stimulation, which I am now about to describe. We have seen that a stimulus of definite intensity may be imparted by a quick torsional vibration of either twist or un-twist, or of the one followed by the other. The intensity of this stimulus, as we saw, depends on the angle of torsion, and remains constant as long as that angle is maintained the same. For this purpose I use the vibrational apparatus already described, successive excitations being produced on one side only, say the right. The torsion-head is set by pulling a vertical thread by which the index is made to rest against the stop $P$. This pull of the vertical thread is against the antagonistic action of the spiral spring $\mathrm{S}$ (fig. 36). During the process of the setting, which is carried out slowly, there is a slight excitatory disturbance. But this is allowed to subside. The vertical thread by which the torsion-head 
is 'set,' is kept pulled up by an electro-magnetic arrangement shown in fig. 37, where the electro-magnet is seen to hold a soft iron armature at the end of the thread. At the moment when stimulation is to be effected the current which energises the electromagnet is interrupted by an automatic arrangement which will be described later. By the break of the current the armature is released and a semi-vibration of the torsion-

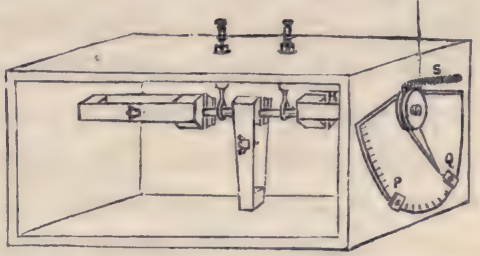

FIG. 36. Arrangement for Instantaneous Stimulation

Torsion-head set by string against stop Q is suddenly let go by electro-magnetic release seen in fig. 37 . head is suddenly produced, the amplitude of which has been predetermined by suitable adjustment of the stop P. Suc-

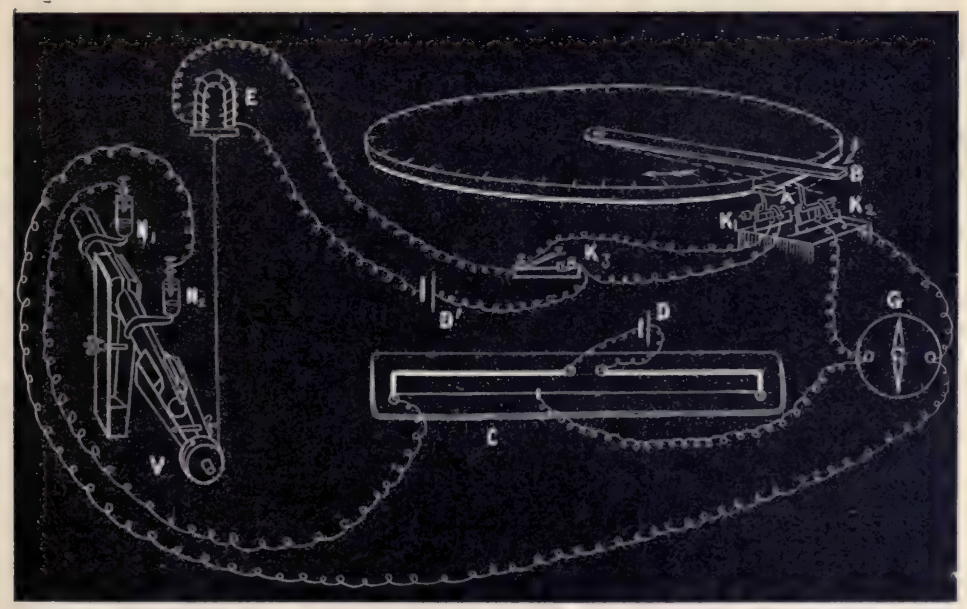

FIG. 37. General arrangement for Rheotomic Observation

$\mathrm{A}$, B, striking rods attached to revolving rheotomic disc; $\mathrm{K}_{1}$, key for electro-magnetic release of torsional stimulator ; $\mathrm{K}_{2}$, for unshunting the galvanometer, G; E, electro-magnet with its armature by which the vibration-head, $\mathrm{v}$, is set at a definite torsion-angle; $\mathrm{N}_{1}, \mathrm{~N}_{2}$, non. polarisable electrodes making electric contacts with specimen;? $\mathrm{C}$, compensator.

cessive stimuli of equal intensity may thus be applied on the experimental tissue at whatever time may be desired. 
The next point is to secure an automatic arrangement by which galvanometric connections can be made with the experimental tissue, for a short period of time, say, or of a second. In order to study the growing electro-motive changes, these short-lived contacts are to be effected in successive experiments at gradually increasing intervals of ${ }^{\circ} \mathrm{OI},{ }^{\circ} \mathrm{O} 2,{ }^{\circ} \mathrm{O} 3$ seconds, and so on after stimulation. It should be remembered in connection with this subject that the reactions in plant tissues are much more sluggish than those in the animal. The time-intervals here provided for, therefore, are even smaller than would have been strictly necessary;

The general plan of the apparatus for carrying out this investigation is seen in fig. 37. The revolving rheotome-disc carries two striking-bars, A and B, of which $\mathrm{A}$ is fixed, and $\mathrm{B}$ capable of an increasing angular adjustment behind $\mathrm{A}$. The bar A, striking against the key $\mathrm{K}_{1}$, interrupts the electromagnetic circuit E, thus causing stimulation. All this time, the galvanometer $\mathrm{G}$ is short-circuited by key $\mathrm{K}_{2}$, and it is only when the striker B unshunts the galvanometer, by striking against $\mathrm{K}_{2}$, that the responsive current can act on the galvanometer. $\mathrm{C}$ is the compensating potentiometer, the object of which will be described presently. The rheotomedisc is rotated by means of a motor, provided with a perfect governor, the period of a single rotation being adjusted to one second. The circumference of the disc is $100 \mathrm{~cm}$. One centimetre of this circumference therefore represents an interval of time of $O I$ second. The breadth of the striker $B$ is also I $\mathrm{cm}$. and it will therefore pass over a given point in the course of oI second. These striking-rods attached to the disc, impinge, as already said, against two electrical keys $K_{1}$, and $\mathrm{K}_{2}$ which are adjusted along the same radius of the disc. $\mathrm{K}_{1}$ is a balanced key, one end of which carries a two-pronged brass fork, both prongs of which are normally dipped in cups of mercury, thus completing the particular electric circuit. By the blow given by the striker A on a projecting rod attached to $\mathrm{K}_{1}$, this fork is tilted upwards, and the circuit is broken. The striker $\mathrm{B}$ then impinges on the second key, $\mathrm{K}_{2}$. 
Here, the prong is kept down by a spring $\mathrm{S}$, the circuit being re-made, as soon as the breadth of the striker $\mathrm{B}$ has passed over the projecting rod-that is to say, in oI second (fig. 38 ). The interval of time between the actions on the two keys, by which the two different electrical circuits are broken in succession, can be gradually increased, by increasing the angle between $\mathrm{A}$ and $\mathrm{B}$.

The key $\mathrm{K}_{1}$, as already said, controls the electromagnet, which, on its release, instantaneously effects. the mechanical stimulation of the tissue. The rotation of the rheo-

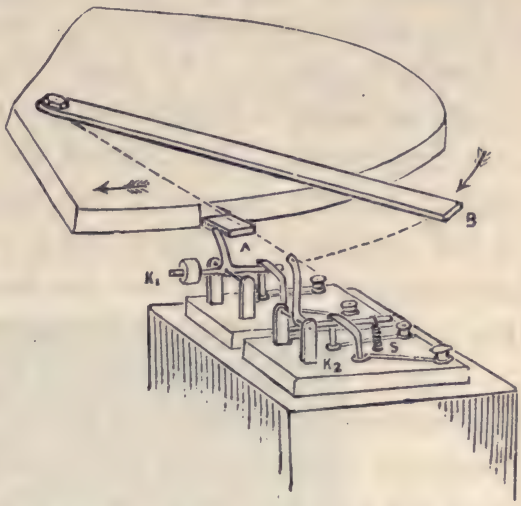

Fig. 38. Enlarged View of Balanced Keys

$\mathrm{K}_{1}$, actuated by $\operatorname{rod} \mathrm{A}, \mathrm{K}_{2}$ by $\mathrm{B}$. tome-disc does not at once become uniform, on the starting of the motor, but attains this when one revolution has been completed. Therefore the experimental observations are not made till the speed has become steady. By pressing the key $\mathrm{K}_{3}$ during the first revolution (fig. 37), the break-action of $\mathrm{A}$ on $\mathrm{K}_{1}$ is postponed. $\mathrm{K}_{3}$ is then opened, and during the next revolution, stimulation is effected.

In order to obtain the galvanometric effect of excitation at definite short intervals of time after the stimulus has been applied, the galvanometer short circuit, as stated before, is removed at those definite intervals. The adjustment of the striking-rod $\mathrm{B}$, in relation to $\mathrm{A}$, enables us to open the short circuit, for 'OI of a second, at increasing intervals. When the $\operatorname{rod} \mathrm{B}$ is at a distance of $\mathrm{I} \mathrm{cm}$. from $\mathrm{A}$, the short circuit is removed after OI second, when at $2 \mathrm{~cm}$. after ' $\mathrm{O} 2$ second, and so on. Thus, in the arrangement just described, the galvanometer is short-circuited, except at those definite intervals required for observation. In a second 
arrangement, the galvanometer is kept open, and closed only during $O I$ of a second at the required increasing intervals of time.

There may be a pre-existing difference of potential in the plant, as between the two points of galvanometric contact, $\mathrm{N}_{1}$ and $\mathrm{N}_{2}$. In order that this may not be a source of disturbance, a compensating potentiometer arrangement, $\mathrm{C}$, is employed. The slider of the compensator is so adjusted that it exactly balances the resting difference of potential in the specimen. Under these circumstances, neither make nor break of the galvanometer occasions any deflection. And this balance is readjusted for each experiment of the series.

I give below two tables which summarise rheotomic observations on specimens of the petiole of cauliflower. By successive intervals of a given length should always be understood the mean interval : that is to say, the period between the application of stimulus and the mid-point in the removal of the short circuit. Thus, for the mean interval of $\mathrm{O} 2$ of a second, the middle of the striking-rod $B$, whose breadth is I cm., placed at a distance of $2 \mathrm{~cm}$. from A. The galvanometer is therefore acted on for 'OI of a second, throughout the period from $\mathrm{O} I 5$ to $\mathrm{O} 25$ of a second, after stimulation. In the first two sets of results here given, the observations will be seen to have been taken at the somewhat long intervals of 'I of a second. The stimulus applied in these cases was moderate. In the case of a third specimen, the observations on which will be given subsequently, the results were recorded at intervals of $\mathrm{O}$ I of a second.

Specimen I

Specimen II

\begin{tabular}{|c|c|c|c|c|c|c|c|}
\hline \multicolumn{2}{|c|}{$\begin{array}{l}\text { Mean interval } \\
\text { after excitaton }\end{array}$} & \multicolumn{2}{|c|}{$\begin{array}{l}\text { Galvanometric } \\
\text { deflection }\end{array}$} & \multicolumn{2}{|c|}{$\begin{array}{l}\text { Mean interval } \\
\text { after excitation }\end{array}$} & \multicolumn{2}{|c|}{$\begin{array}{c}\text { Galvanometric } \\
\text { deflection }\end{array}$} \\
\hline \multicolumn{2}{|c|}{ •I second } & \multicolumn{2}{|c|}{70 divisions } & \multicolumn{2}{|c|}{ 'I second } & 26 & risions \\
\hline$\cdot 2$ & , : & 100 & , & $\cdot 2$ & , & IOO & , \\
\hline 3 & , & 310 & , & 3 & , & 70 & , \\
\hline 4 & , & 220 & , & 4 & , & 64 & , \\
\hline$\cdot 5$ & , & 104 & ;, & $\cdot 5$ & , & 56 & , \\
\hline I $\cdot O$ & , & 70 &, & I'O &, & 42 & , \\
\hline $2 \cdot 0$ & , & I5 & , & $\begin{array}{l}2 \cdot 0 \\
6 \cdot 0\end{array}$ & , & $\begin{array}{r}24 \\
4\end{array}$ & , \\
\hline
\end{tabular}


It will be seen from the observations made on the first of these two specimens that the maximum electro-motive effect was attained in three-tenths of a second after excitation. In the second case, the maximum was reached in two-tenths of a second. The curve given in fig. 39 shows how quickly the electro-motive variation attains a maximum, and how rapid is its decline after reaching this point. There apppears to be practically no latent period, the induction of the electromotive effect being apparently immediate. This will be made evident by the results given in the next series.

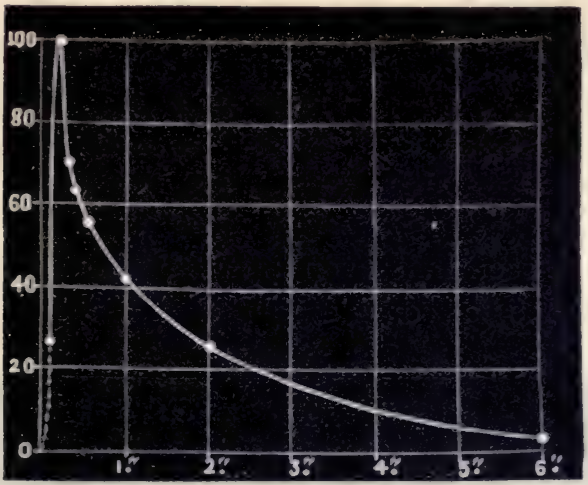

FIG. 39. Curve showing Rise and Fall of Responsive E.M. Change, under moderate stimulation

Ordinate represents galvanometric deflection; abscissa, time. Large division $=1$ second. $\quad$ (Petiole of cauliflower.)

For the next experiment, I took the stem of Amaranth, which I find to be more excitable and more quickly reacting than the petiole of cauliflower. The intensity of stimulus was here greater than in the last case. I may state now what will be demonstrated in full later, that a strong stimulus often gives rise, not to a single, but to multiple responses. I had previously detected these multiple responses by means of both mechanical and galvanometric indications, and found them to have periodicities varying from some 15 seconds to several minutes. Indeed, had they been much quicker than they were, they could not have been detected, owing to the 
inertia of the motile leaflets or of the galvanometer needle. By the employment of the rheotomic method, however, I was able to detect multiple responses having periodicities of the order of one-tenth or so of a second. All the experiments carried out on Amaranth gave two or three waves of electromotive variation, the character of which will be understood from the following table and curve (fig. 40).

\begin{tabular}{|c|c|c|c|}
\hline \multicolumn{2}{|c|}{ Mean interval after excitation } & \multicolumn{2}{|c|}{ Galvanometric deflectior } \\
\hline$\circ \mathrm{OI}$ & second & 63 & visions \\
\hline .05 & ,, & 77 &,,$\ldots$ \\
\hline$\cdot{ }^{\prime} 5$ & ," & 82 & ,, \\
\hline 20 &,, & 68 &, \\
\hline 30 & , & 76 & ", \\
\hline 40 &, & 75 & ", \\
\hline 60 & ,", & I04 & ", \\
\hline 70 & , & 86 & , \\
\hline •80 & , & 67 & ," \\
\hline
\end{tabular}

It will here be seen that even after so short an interval as OI of a second, a considerable electromotive change had

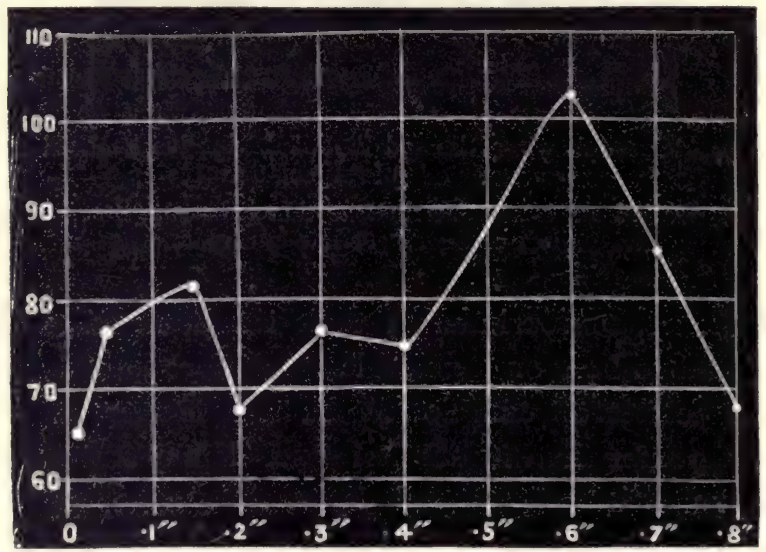

FIG. 40. Response Curve from Rheotomic Observation in Stem of Amaranth under strong stimulation

Note occurrence of multiple response. Large division of abscissa $=\cdot \mathbf{I}$ second.

already been induced. The first maximum occurred after ' 15 , the second after 3 , and the third after 6 of a second. 
We thus see that there is a rhythm in these multiple responses, the successive maxima being here found to occur at periods which constitute multiples of 15 second. The third of these rhythms may be presumed to be missing, owing to the fact that no observation was taken at $\cdot 45$ second, as, at the time when these experiments were carried out, I was unaware of the existence of such rhythmicities. It was by the curves plotted from these data that my attention was first drawn to their occurrence.

It will thus be seen that the electro-motive variation is initiated practically simultaneously with the impact of stimulus on the organ. With moderate stimulus, the maximum variation is reached within two-tenths of a second, or this period may be made still shorter by the employment either of a more quickly reacting tissue, or of a greater intensity of stimulus. Strong stimulation is apt to give rise to rhythmic multiple responses. 


\section{CHAPTER V}

\section{THE ELECTRICAL INDICATIONS OF POSITIVE AND NEGATIVE TURGIDITY VARIATIONS}

Motile responses of opposite signs, characteristic of positive and negative turgidity-variations-Indirect hydrostatic effect of stimulus causes expansion and erection of leaf-Positive and negative work-Wave of increased hydrostatic tension transmitted with relatively greater velocity than wave of true excitation-Method of separating hydro-positive and excitatory effects-Indirect effect of stimulus, causing positive turgidity-variation induces galvanometric positivity-Antagonistic elements in the electrical response-Separation of hydro-positive from true excitatory effect by means of physiological block.

HAVING now described that fundamental electrical response of galvanometric negativity which is characteristic of excitation, I shall next proceed to deal with an opposite type of response-namely, that of galvanometric positivity. The combination of these two factors, in varying degrees of each, in the electrical response of plants, has been a source in the past of the greatest perplexity, leading investigators to contradictory results. And it can only be by disentangling them, and by ascertaining the conditions under which each invariably occurs, that precision will be arrived at in the field of electrical response.

We have seen that excitation of the pulvinus of Mimosa induces negative turgidity-variation, with fall of the leaf, and galvanometric negativity. What, then, would be the outward expression of an increase of turgidity - that is to say, of the positive turgidity-variation?

With regard, first, to the mechanical expression, we may subject the question to an experimental test. The cut end of a branch of Mimosa, bearing leaves, is fixed watertight in one end of a U-tube, filled with water, and the other 
end is connected alternately with a vacuum and a forcopump, by means of which a diminution or increase of hydrostatic pressure may be induced at will. In this way it is possible to suck water away from, or force it into, the plant and its organs, thus producing negative and positive turgidityvariations at will. When turgidity is thus diminished the indicating leaf is seen to fall. This is what happens also under the ordinary negative turgidity-variation induced by excitation. When turgidity is increased, on the other hand, the leaf is erected (fig. 4I). In the case of the negative variation of turgidity, the pulvinus as a whole loses water,

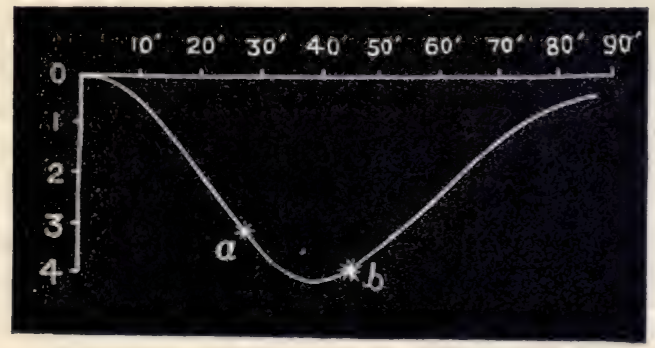

FIG. 4I. Artificial Hydraulic Response of Mimosa

The plant was subjected to diminished pressure up to $a$, and to nornial pressure to $b$, after which the pressure was increased. The effect of diminished pressure, in the depression of the leaf, continues for a while. The ordinate represents movement of tip of leaf in $\mathrm{cm}$., abscissa represents time.

but more from the lower and more excitable half than from the upper. In the case of the positive turgidityvariation, also, it is again the more excitable lower half which absorbs the greater quantity of water. Thus, in Mimosa, and in Biophytum, the mechanical indication of increased turgidity is an erection of the leaf or leaflet. The characteristic electrical indication of this will be observed presently.

It has already been mentioned that the direct application of stimulus at a point causes a negative turgidity-variation of that point. We shall now see whether, under any 
circumstances, stimulus will induce a positive turgidityvariation. If we now apply moderate stimulus on the stem of Mimosa, at a certain distance below the pulvinus, an excitatory expulsion of water will occur at the point directly stimulated. Such an expulsion of water will then cause, it is clear, a hydrostatic disturbance of increased pressure. And this hydro-mechanical disturbance will be transmitted with relatively great velocity. Now such an increase of pressure, as we have seen, causes an increase of turgidity at

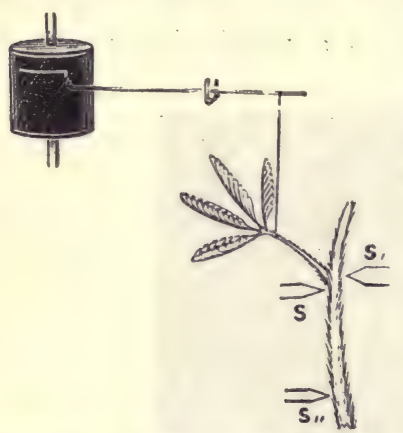

FIG. 42. Experimental Arrangement, for obtaining Records on Smoked Drum of Responses given to Direct and Indirect Stimulation, by Leaf of Mimosa

Thermal stimulator at $s$ produces direct stimulation, and consequent fall of leaf. Moderate stimulation, at a distant point, $\mathrm{s}_{1,}$, gives rise to indirect effect of erection. the puivinus, in consequence of which the leaf ought to be erected. And although this hydrostatic disturbance is transmitted very quickly, yet a certain time is consumed in the process of forcing water into the pulvinus, by which to bring about the erection of the leaf. After the passage of the hydrostatic wave, there follows the wave of true excitation, passing from cell to cell, and inducing the characteristic reaction, of negative turgidity-variation. And when this excitatory wave reaches the pulvinus, the previous erectile movement should give place to excitatory depression, or fall of the leaf. In fig. 42 are seen the arrangements for an experiment on Mimosa by which these inductions may be verified. Moderate thermal stimulus is applied at $\mathrm{S}$, at a certain distance below the indicating leaf. This latter is attached by a thread to a writing-lever, which traces the response-record on a smoked revolving drum. When the stimulus is applied at a point $\mathrm{S}$ very near the pulvinus, the response takes place by a negative turgidityvariation, with a concomitant fall of the leaf, seen in fig. 43 (a) as an up-curve. When a moderate stimulus is applied 
at a greater distance $\mathrm{S}_{\iota,}$, the hydrostatic wave causing the positive turgidity-variation brings about an erectile twitch. This is followed by a responsive fall, when the true excitation reaches the organ (fig. $43, b$ ).

It has thus been shown that by separating the responding point from the point stimulated, or the receptive point, it is possible to discriminate two different effects which are both brought about by stimulus. It is most important, moreover, to distinguish between these two factors : namely, the direct effect of stimulus causing contraction, and its indirect effect, causing expansion. We have seen that direct excitation and transmitted excitation both induce contraction, negative turgidity-variation and fall of the leaf. Unfortunately, in animal physiology, it has been customary to apply the term indirect to that form of stimulation which is applied at a distance. And it has not been noticed that such stimulus is capable of inducing diametrically opposite results, according as the true excitatory effect reaches, or does not reach, the responding organ. When the intervening tissue is highly

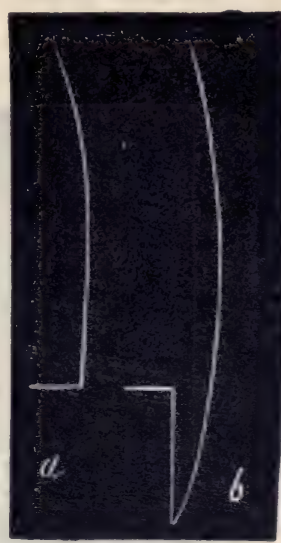

FIG. 43. Mechanical Responses of Leaf of Mimosa

(a) record of responsive fall when stimulus applied near the responding organ; (b) response when stimulus is applied on same side, but at greater distance, $s_{1,}$. A preliminary erectile response is here followed by the true excitatory depression. This is due to the indirect effect first transmitted being succeeded by the direct. Had the stimulus applied been feebler, or more distant, there would have been only the first, or indirect erectile effect. conducting, the transmitted effect induces exactly the same result as if stimulus were applied directly. But we shall see that when the intervening tissue is non-conducting or feebly conducting, true excitation is not transmitted, and the effect which makes its appearance at the responding point is then due to increase of hydrostatic tension, causing positive 
turgidity-variation, with the concomitant effect of expansion, and, in the case of Mimosa, of erection of the leaf. This latter effect of positive turgidity-variation and expansion, I shall therefore distinguish as the INDIRECT EFFECT of stimulus, in contradistinction to the term INDIRECT STIMULATION, as it is generally used. The last-named, however, I shall myself always refer to under the title of TRANSmitted Stimulation. If the intervening tissue be of

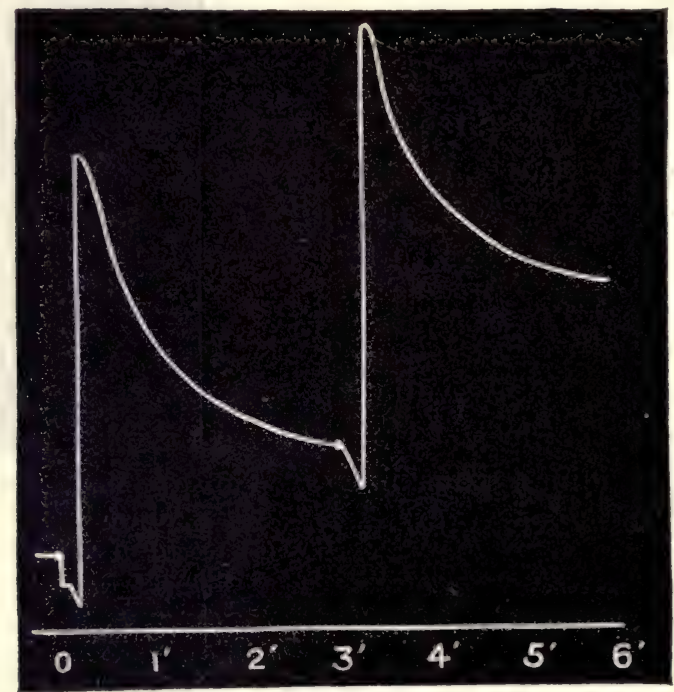

FIG. 44. Mechanical Response of Biophytum to Thermal Stimulation

Stimulus was applied at some distance from the responding leaflet. And the preliminary erectile twitch is due to the prior arrival of the hydrostatic disturbance. Thick dot represents moment of application of stimulus.

moderate conducting power, we shall, as in the case of the experiments on Mimosa, obtain a preliminary erectile twitch, due to the indirect effect of stimulus, followed by a fall, in consequence of the transmission of the true excitatory effect. In fig. 44 is seen this twofold expression of the indirect and transmitted effects of stimulus, given by the leaflet of Biophytum.

These two waves, then, of increased hydrostatic tension 
and of true excitation, induce, as we have now seen, opposite responsive reactions. But of these, that due to true excitation is, generally speaking, greatly predominant. Hence, when these two waves reach the responding organ in close succession, as is the case when the point of stimulation is very near, the excitatory effect masks the hydrostatic. In order, then; to separate them, we may employ various methods. First, in the case of highly conducting tissues, the stimulus must be applied at a sufficient distance to make the slow excitatory wave lag adequately behind the quickly travelling hydrostatic wave. Or we may choose a direction of transmission of excitation which will be relatively slow. Thus I have found that transmission across a stem, for example, is very much slower than along its length. Hence, on applying moderate stimulus at $\mathrm{S},($ fig. 42$)$ at a point on the stem diametrically opposite the pulvinus, of the given leaf, it is found that the excitation reaches the pulvinus only after a measurable interval, the hydrostatic effect inducing erectile response much earlier. Thus in a given experiment, whose record was taken on a fast-moving drum (fig. 45), the erectile response took place 6 second after the application of stimu. lus, whereas the true excitatory fall did not occur till 3.45 seconds had elapsed - that is to say, 2.85 seconds later. It is to be borne in mind that a certain interval of time passes, even after the arrival of the respective waves, before the turgidity-variation is able to give rise to the motile indication.

Let us next examine the results at the responding tissue of the indirect effect of stimulus. The distant receptive point contracts on stimulation, and sends to the responding organ a wave of increased hydrostatic tension. This, as we have seen, forces water in, and expands the tissue. Work is thus done on the tissue which increases its store of energy. In this the indirect is unlike the typical direct effect of stimulus. For the latter causes the impulsive fall of the leaf, which represents work done by the tissue, and an expenditure of energy. We must, therefore, recognise two distinct responsive effects, according as the work done is positive-done on 
the tissue-or negative: that is to say, done by the tissue. The outward manifestations of these two processes are respectively expansion and contraction. The positive, as we shall see, is not the result of hydrostatic disturbance as such, but is the effect of energy transmitted hydraulically.

The indirect effect of stimulus, then, gives rise to positive turgidity-variation, and increases the internal or potential

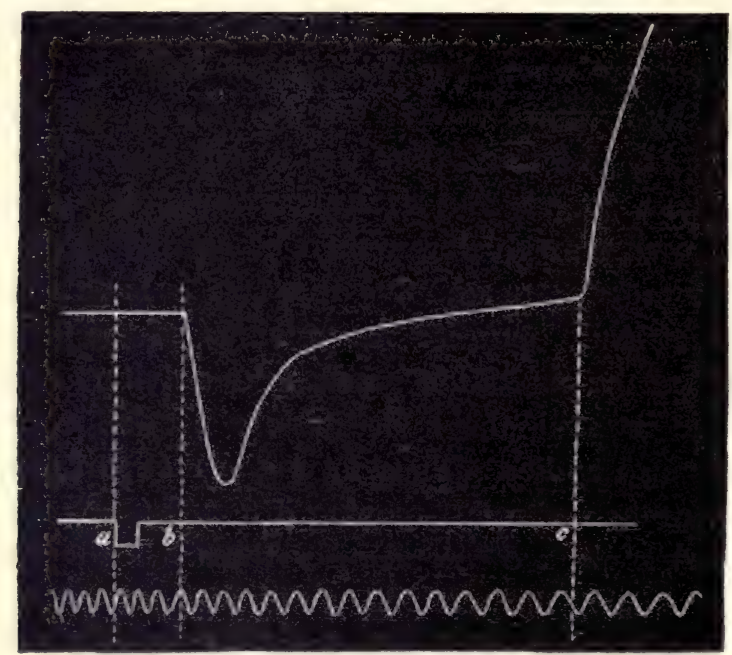

FIG. 45. Record of Response of Mimosa Leaf, taken on a fastmoving drum

Stimulus applied at moment $a$, on point of stem diametrically opposite to responding leaf. Hydro-positive erectile response occurs at $b, \cdot 6$ second after application of stimulus. True excitatory response of fall takes place at $c, 3.45$ seconds after application of stimulus. Time-marks represent fifths of a second.

energy of the organ. A positive turgidity-variation is thus concomitant with an increase of internal energy and a negative turgidity-variation with the reverse. In observing the mechanical response, we saw that the expression of positive turgidity-variation, due to the indirect effect of stimulus, consisted of an erection, and was therefore of opposite sign to that of the negative turgidity-variation, caused by true excitation, and expressing itself in a fall, of 
the leaf. We shall now see whether a similar difference exists between the electrical expressions of the positive and negative turgidity-variations.

In carrying out this experiment, I took a specimen of Biophytum and applied stimulus at a distance from the particular leaflet whose responses were to be observed, arranging, at the same time, for a simultaneous record of the mechanical and electrical responses. It will be seen from fig. 46 that the preliminary erectile twitch, due to the positive turgidityvariation, has, as its concomitant, galvanometric positivity. And this is followed in both records by its opposite: namely, the contractile fall and the galvanometric negativity of true excitation.

It will thus be seen that the increase of internal energy, with its positiye turgidity-variation, has, as its electrical expression, galvanometric positivity. Besides this, the mere physical movement of water in the tissue gives rise to a certain electrical variation of positivity, and this can still be detected, even after the tissue is killed. The question of how to discriminate what proportion of the electropositivity was due to this mere water-movement, and what to the increase of turgidity, associated with the increase of internal energy, I at first found it very difficult to decide. But I ultimately succeeded in doing this by bringing a plant to

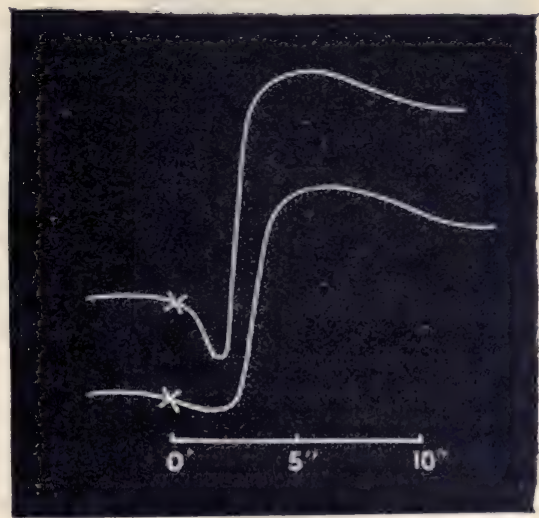

F1G. 46. The Abnormal Positive preceding the Normal Negative in Mechanical and Electrical Responses in Biophytum

$x$ represents the moment of application of stimulus. The upper is the mechanical and the lower the electrical record. The records downward indicate erection of the leaf or galvanometric positivity.

a condition just short of death, and thus abolishing its true excitatory reaction. In this condition, the responsive 
indication was found to be one of considerable electropositivity. On finally killing the plant, however, the positive change due to water-movement was found to represent so insignificant a proportion of the whole as to be negligible,

In order to exhibit the electrical expression of the excitatory and hydro-positive effects of stimulation, in ordinary plants, I took a petiole of cauliflower and made one connection, the proximal,

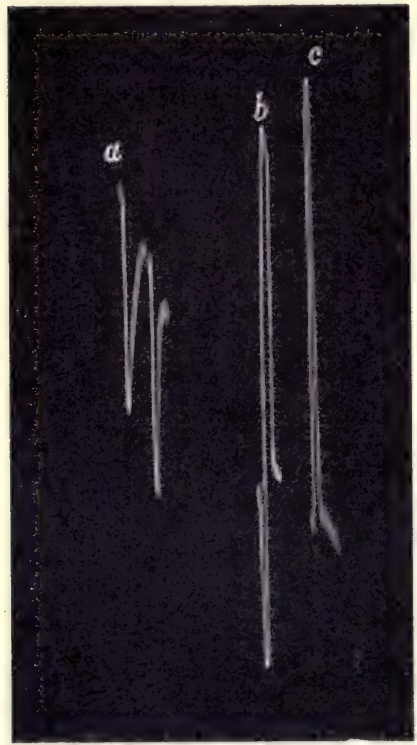

FIG. 47. Photographic Record of Electrical Response of Petiole of Cauliflower

$a$, hydro-positive ; $b$, di-phasic ; $c$, excitatory negative responses. with a point on it, and the other with an indifferent point on the surface of the lamina. In order to obtain the unmistakable hydrostatic effect, the petiole was suddenly squeezed, at a distance of $6 \mathrm{~cm}$. from the proximal contact, and this gave rise, as will be seen (fig. $47, a$ ), to a positive response, represented downwards. This was repeated once more, and the same effect observed. I next applied thermal stimulus at a distance of $4 \mathrm{~cm}$. from the responding point. In this case hydrostatic and excitatory disturbances reached the contact, the hydrostatic shortly followed by the excitatory, as in the case of the experiment on Mimosa. This is seen in the record, as a diphasic response, the hydrostatic positive being followed by the excitatory negative (fig. $47, b$ ).

Reference has already been made to the observation of Burdon Sanderson, that in the lamina of the Dioncea leaf the immediate response was one of galvanometric positivity. Mistaking this for the true excitatory effect, he concluded that the response of the plant was of opposite sign to that of the 
animal. From the experiment just described, however, it will be seen that the effect observed by him was in reality due, not to true excitation, but to the hydrostatic disturbance, or indirect effect of stimulus.

In the next record (fig. $47, c$ ) we see the effect of stimulus applied nearer : that is to say, at a distance of $2 \mathrm{~cm}$. from the proximal contact. Owing to the propinquity of the point of stimulation, the two disturbances are not now sufficiently separated, and the excitatory negative reaction completely masks the hydrostatic positive effect.

It is thus seen that, as has been said, one method of exhibiting these two effects separately is to apply stimulus at a point so distant from the proximal contact that there is an interval between the arrival of the two waves of hydrostatic and excitatory disturbance respectively. It is obvious, then, that if the tissue under experiment be a good conductor of excitation, we must place the point of stimulation at a long distance from the first electrode, in order that the effect of excitation may lag sufficiently behind the hydrostatic wave. Similarly, in a bad conductor of excitation, it will be the indirect effect alone which will reach the proximal contact; unless the stimulus applied be very near, and very strong.

In order to distinguish these two opposite effects from each other, I shall in future refer to that hydrostatic effect which causes expansion and galvanometric positivity as 'the hydro-positive effect,' by way of differentiating it from 'the true excitatory effect,' of negative turgidityvariation and galvanometric negativity.

It has already been said that tissues which exhibit a high degree of conduction are characterised by more or less of protoplasmic continuity. Hence, fibro-vascular elements are relatively good, and parenchymatous tissues bad, conductors of excitation. The cells of the potato tuber for this reason exhibit very little power of transmitting excitation. When, therefore, in experimenting with this 
tissue, stimulation was caused by application of a hot wire so near as I $\mathrm{cm}$. to the proximal contact, it was the hydropositive effect alone which reached it, giving rise to positive response. It was only, indeed, by applying the stimulus very near, at a distance of $3 \mathrm{~mm}$., that the true excitatory

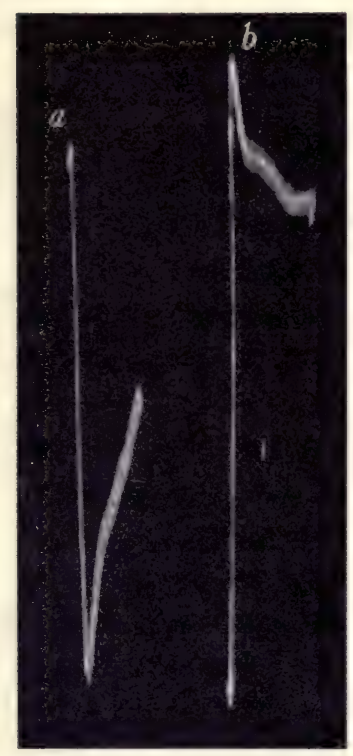

FIG. 48. Photographic Record of Electrical Responses of Potato-tuber

$a$, Positive response to stimulus applied at distance $; b$, Negative response to stimulus applied near. response of galvanometric negativity was in this case obtained (fig. 48).

From what has been said, it will be seen that when a given point is excited by transmitted stimulation, two antagonistic electrical effects are induced-one of positivity, due to hydro-positive action, and the other of negativity, due to true excitation. When the stimulator is near to, or coincides with, the responding point, the tissue is subjected to rapidly succeeding positive and negative turgidity-variations, and the electrical indication of the latter being the more intense, it masks the former, and the resulting response is determined by an algebraical summation of the two. In a vigorous specimen, whose excitability is great, the excitatory galvanometric negativity masks the positivity. The resultant electrical response in general is expressed by the formula $\mathrm{N}_{E}-\mathrm{P}_{H}$, where $\mathrm{N}_{\mathrm{R}}$ is the galvanometric negativity due to the true excitatory effect, and $P_{H}$ the positivity due to the hydro-positive effect, which immediately precedes it. From this, it is clear that, as regards the resultant galvanometric response of vegetable tissues under stimulation, there may occur the two typical cases displayed in the following table: 
Typical Cases of Resultant Response

\begin{tabular}{|c|c|c|}
\hline Conditions & Constituent Factors & Resultant Response \\
\hline $\begin{array}{l}\text { Excitability great } \\
\text { Excitability diminished } \\
\text { or nearly abolished }\end{array}$ & $\begin{array}{l}\mathrm{N}_{\mathrm{E}}>\mathrm{P}_{\mathrm{H}} \\
\mathrm{N}_{\mathrm{E}}<\mathrm{P}_{\mathrm{H}}\end{array}$ & $\begin{array}{l}\text { Galvanometric negativity } \\
\text { Galvanometric positivity }\end{array}$ \\
\hline
\end{tabular}

We thus see how, under the physiological modifications induced by various agents, the normal negative response of a tissue may undergo diminution, or even reversal. We have, for the sake of simplicity, assumed that the two antagonistic effects act on the specimen simultaneously. But, as a matter of fact, under certain circumstances, their time-relations may be subjected to change. Hence various effects of interference may be seen, giving rise to diphasic responses, such as positivity followed by negativity, or the reverse. Or, instead of diphasic, there may even be multiphasic responses, since it will be shown that very strong stimulus may cause not a single but repeated responses. Some of these effects will be described in detail in a subsequent chapter.

I shall here meanwhile describe another method, in which, by means of a selective physiological block, we can unmask the contained hydrostatic effect of positivity, from the ordinary response of galvanometric negativity. The occurrence of the excitatory contraction, in Mimosa for example, as shown by the fall of the leaf, depends on a favourable excitatory condition of the tissue. If this motile excitability should be in any way depressed or abolished as, say, by application of ice-cold water on the pulvinus, the true excitatory response could not take place. But if, under these circumstances, we applied stimulus at the diametrically

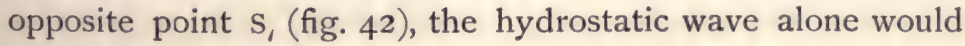
be found to reach the organ. and, vi et armis, to produce expansion there, with a consequent erection of the leaf. It will thus be seen that the hydrostatic transmission of the indirect effect of stimulus is not to any great extent affected 
by the loss of excitability of the tissue. Similarly, the transmission of true excitation may be selectively blocked, in a tissue, by the application of various depressing agents, such as anæsthetics, without appreciably affecting the passage of the wave of increased hydrostatic tension.

For the present experiment I took a leaf of fern, and made the proximal electrical connection with the petiole, and the distal with an indifferent point on a leaflet. By means of the thermal stimulator (p.38), I now applied successive uniform stimuli at intervals of one minute, on a point I.5 $\mathrm{cm}$. below the proximal contact. The first three

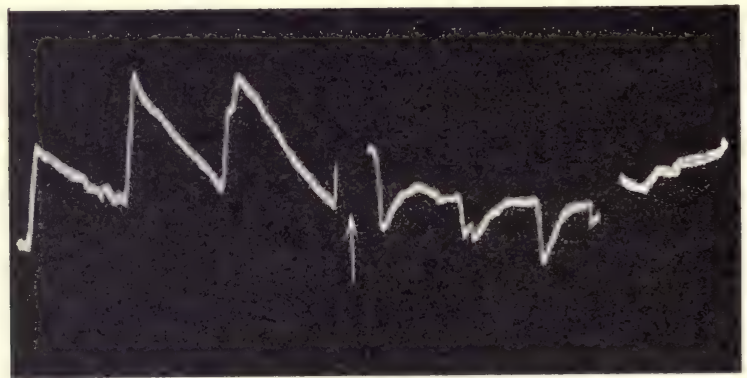

FIG. 49. Photographic Record of Electrical Response of Petiole of Fern First part of record shows normal negative responses; second part shows positive response unmasked by selective physiological block of chloro. form ; in the third part is seen the abolition of response when stimulus is applied on anæsthetised area itself.

responses of the series are seen (fig. 49) to be more or less uniform, and negative. Such resultant response is, as has been pointed out, due to the summation of two antagonistic effects, of which the excitatory is predominant. In order therefore to eliminate from this resultant response its excitatory component, I applied chloroform on a narrow belt intermediate between the point of stimulation and the proximal contact. From the next three responses it will be seen that the hydrostatic effect of positivity, represented by downresponses, was thus successfully unmasked. The application of chloroform is thus seen to act here as a selective block, 
the wave of increased tension being transmitted across its area, whereas the wave of true excitation is arrested.

But the hydrostatic disturbance itself was caused by the excitatory contraction of the distant point. The abolition of the excitability of an intermediate point did not, as we have seen, block the hydrostatic wave. If now the stimulator be brought nearer, and placed over the strongly anæsthetised area, the expulsion of water dependent on true excitation can no longer take place. On doing this, therefore, we find that neither the true excitatory negative, nor its consequent hydrostatic positive effect, is exhibited at the responding point. Response is thus totally abolished.

We thus see that incident stimulus gives rise in the plant to two distinct responsive expressions. In that which we shall consider first-namely, the direct, or true excitatory effect-there is an expenditure of energy, which is indicated by a contraction, negative turgidity-variation, mechanical fall, and galvanometric negativity. In the second, or indirect, of these two effects, we have the opposite of all these. That is to say, we have here an increase of internal energy, expansion, positive turgidity-variation, erection of leaf, and galvanometric positivity. The galvanometric negativity, due to negative turgidity-variation, is generally speaking of much greater intensity than the galvanometric positivity, due to positive turgidity-variation. Hence, when the two effects act on a tissue in rapid succession, that of electro-negativity masks the positive. The wave of increased hydrostatic tension, by which the indirect effect of stimulus is transmitted, has a greater speed of transmission than that of true excitation. It may be transmitted even across tissues of which the excitability has been depressed or abolished, whereas the transmission of true excitation is arrested by the intervention of such a physiological block.

The occurrence of these opposed effects of galvanometric negativity and positivity has long been regarded as a phenomenon of a highly perplexing nature. It wàs too hastily assumed that wherever these opposed electrical changes took 
place, they were necessarily to be ascribed to opposite or antagonistic chemical processes - namely, of assimilation and dissimilation. That this, however, cannot be universally true, has here been proved by tracing the two effects to their definite respective origins, in positive and negative turgidityvariations. I shall enter into this subject in greater detail in the subsequent chapters. 


\section{CHAPTER VI}

\section{EXTERNAL STIMULUS AND INTERNAL ENERGY}

Hydraulic transmission of energy in living tissue - True meaning of tonic condition-Opposite expressions of internal energy and external stimulus seen in growth-response-Parallelism between responses of growing and motile organs - Increased internal energy caused by augmentation of temperature finds expression in enhanced rate of growth ; erection of motile leaf ; curling movement of spiral tendril ; and galvanometric positivity-External stimulus induces opposite effect in all these cases-Sudden variation of temperature, acting as a stimulus, induces transient retardation of growth ; depression of motile leaf ; uncurling movement of spiral tendril ; and galvanometric negativity-Laws of mechanical and electrical response.

We have seen in the last chapter that external stimulus, directly applied, induces in an excitable vegetable tissue a contractile response, with concomitant galvanometric negativity. An increase of internal energy, on the other hand, was there seen to give rise to expansion, and galvanometric positivity. These two factors of external stimulus and internal energy are thus seen to be antagonistic in their general expressions.

But while stimulus from outside, impinging on an excitable area, thus caused an expenditure of energy at that area, by inducing excitatory response there, yet it was also found that, by its indirect effect, it brought about an increase of energy in neighbouring tissues. By the sudden contraction, and expulsion of water from the excited area, energy was transmitted hydraulically, and the consequent positive turgidity-variation caused an erectile response of a neighbouring motile organ. In the simple case in which the point of receptivity was at a distance from that of response, it was seen that these two effects of stimulus, direct and indirect, were easily discriminated from one another. But as the 
stimulator was brought nearer and nearer, the two effects became superposed, and one was masked by the other. Even in this case, however, on careful examination, it is possible to infer the results due to the action of the internal factor.

Thus, we may suppose stimulus to be applied directly on the pulvinus of Mimosa, bringing about a responsive fall of the leaf. The expelled water from the excited pulvinus will now be forced into the neighbouring tissues, making them over-turgid, and raising their energy above par. On the cessation of stimulus, the water that has been forced away will flow back from the region of heightened, to that of lowered tension, and re-establish the normal turgidity of the pulvinus, which had undergone a negative variation. The erectile recovery of the leaf is thus seen to be, not a merely passive process, but an effect dependent on the internal energy of the plant. This is also shown by the fact that in autumn and winter, when the internal energy is low, the period of recovery is very long, being sometimes as much as eighteen minutes; whereas in summer, on the other hand, with the increased internal energy of the plant, it takes place nearly three times as quickly. I have elsewhere shown that it is this internal energy which is vaguely referred to as the tonic condition of the tissue, and that it consists of the sum total of energy derived from external stimuli previously absorbed and held latent by the plant. The different forms of stimulus may be very various. We have for instance tonicity, as imparted by light, or phototonus; by favourable temperature, thermotonus; by electrical current, electrotonus; by internal hydrostatic pressure, hydrotonus; or by the presence of favourable chemical substances, chemotonus.

We have seen that, as a general rule, external stimulus and internal energy find responsive expressions of opposite sign, the former inducing a negative, and the latter, a positive turgidity-variation. It is, nevertheless, important to demonstrate the extensive applicability of this law, by many different results and different modes of manifestation. And such a 
demonstration would undoubtedly become still more convincing, if we should succeed in discovering some mode of response in which the antagonistic effects of internal energy and external stimulus found opposite expressions.

Such an example, of a very striking character, I have in my work on 'Plant Response' shown to be found in growthresponse. But the same opposition, between the effects of external stimulus and internal energy, I shall now proceed to demonstrate not only by means of growth-response, but also through mechanical and electrical responses. In the case of growth, the responsive expression of the growing organ, under increased turgidity, consists of an expansive elongation. If the organ be growing at a uniform rate, an increase of internal energy will enhance that rate. But when external stimulus acts directly on the growing organ, the normal rate of growth is retarded during the action of stimulus. Thus it will be seen that though the mechanical response of a motile organ, and the movement of a growing organ, appear so different, yet these two expressions are not fundamentally distinct. For while in one, the application of direct stimulus, causing negative turgidity-variation and contraction, induces depression of the leaf, in the other, the same negative turgidity-variation and contraction under external stimulus causes a depression of the rate of growth. And, on the other hand, indirect effect of stimulus, or increase of internal energy, inducing positive turgidity-variation, brings about in the one case the erection of the leaf, in the other an enhancement of the normal rate of growth. This parallelism is displayed in detail in the table given below.

It is thus understood that the indication of response to external stimulus is the depression of the motile leaf, or depression of the rate of growth, while the effect of increased internal energy is the erection of the motile leaf, or enhancement of the rate of growth.

We shall first deal with the responsive expression to that positive turgidity-variation which is due to the increase of internal energy. One mode of increasing the internal 
Tabular Statement showing Comparative Effects of External Stimulus and Internal Energy on Pulvinated and Growing Organs

\begin{tabular}{|c|c|}
\hline Mechanical response & Growth response \\
\hline $\begin{array}{l}\text { Effect of normal turgidity: } \\
\text { Normal horizontal position of leaf. } \\
\text { Local action of external stimulus : } \\
\text { Contraction ; } \\
\text { Diminution of turgidity; } \\
\text { and concomitant depression of leaf. } \\
\text { Action of internal energy exhibited by } \\
\text { (a) Recovery; } \\
\text { Re-establishment of turgidity and } \\
\text { gradual return of leaf to normal } \\
\text { horizontal position. } \\
\text { (b) Increased hydrostatic pressure : } \\
\text { Erection of leaf. }\end{array}$ & $\begin{array}{l}\text { Effect of normal turgidity : } \\
\text { Uniform rate of growth. } \\
\text { Local action of external stimulus : } \\
\text { Contraction ; } \\
\text { Diminution of turgidity ; } \\
\text { and concomitant depression of rate } \\
\text { of growth. } \\
\text { Action of internal energy exhibited by } \\
\text { (a) Recovery: } \\
\text { Re-establishment of turgidity and } \\
\text { gradual return of organ to normal } \\
\text { rate of growth. } \\
\text { (b) Increased hydrostatic pressure : } \\
\text { Increased rate of growth. }\end{array}$ \\
\hline
\end{tabular}

energy of a plant is by a moderate rise of temperature. And this finds expression in the case of a motile organ, by the erection of the leaf. Thus, when Mimosa is raised in temperature, all its leaves become highly erect. A diminution of energy, on the other hand, by cooling, brings about a depression of the leaves.

In the same way, the rate of growth is exalted by rise of temperature. Thus in a growing flower of Crinum lily the normal rate of growth at $30^{\circ} \mathrm{C}$. was $0040 \mathrm{~mm}$. per minute, and this was exalted to OII $3 \mathrm{~mm}$. per minute, or nearly three times, when the temperature was raised to $35^{\circ} 5^{\circ} \mathrm{C}$. Lowering of temperature, on the other hand, greatly depresses the rate, and may even, if it proceed far enough, cause arrest of growth.

As regards external stimulus, on the contrary, we have seen that its effect on the motile organ is one of depression. The following record (fig. 50) shows that it has a similar influence on the rate of growth. The first part of the curve shows the normal rate of elongation. But after the application of stimulus of light, growth is not only retarded, but there is an actual shortening of the organ. On the cessation of stimulus, the normal rate of growth is gradually reestablished. 
I shall next give examples in which the opposite effects of external stimulus and internal energy are exhibited in growth response, motile response, and electrical response. To take first the response of growth: we have seen that a steady rise of temperature brings about an increase of internal energy, while a sudden variation of temperature acts as a stimulus. Thus, if we effect a sudden augmentation of temperature, this will act on the organ, during the period of variation, as a stimulus; but afterwards, when the temperature itself, or its rate of rise, has become

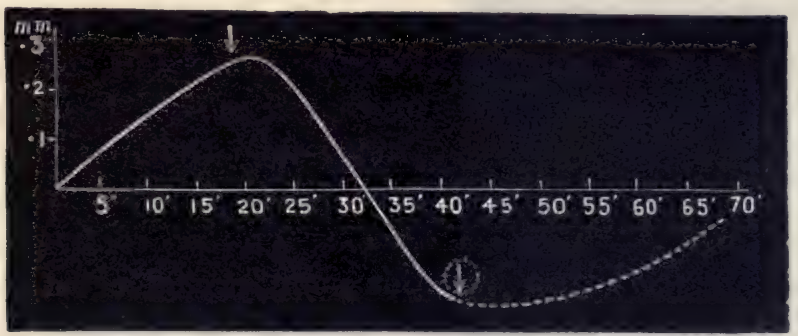

FiG. 50. Longitudinal Contraction and Retardation of Growth under Light in Hypocotyl of Sinapis nigra

The first part of the curve shows the normal rate of growth. Arrow ( $\downarrow$ ) indicates moment of application of diffuse light, which is seen not only to retard growth, but also to induce a marked contraction. The second arrow indicates moment of withdrawal of light, and dotted portion of the curve shows recovery.

steady, the condition will act by increasing the internal energy of the organ. These opposite results are seen to be strikingly illustrated by growth response in the case of the following record (fig. $5 \mathrm{I}$ ),

The normal rate of growth at $34^{\circ} \mathrm{C}$. was here $015 \mathrm{~mm}$. per two minutes. By a sudden application of heat, raising the temperature of the chamber ultimately by $\mathrm{I}^{\circ} \mathrm{C}$., a responsive contraction was caused, as is seen in the record. But, on the attainment of a steady augmented temperature of $35^{\circ} \mathrm{C}$., an increased rate of growth, which now amounted to 'O24 $\mathrm{mm}$. per two minutes, was observed, owing to the increase of internal energy. 
Or the same difference may be demonstrated by means of mechanical response. A Mimosa is placed in a small chamber and subjected to a sudden rise of temperature. In consequence of this there is a preliminary excitatory depression, followed, on the attainment of a steady rise, by

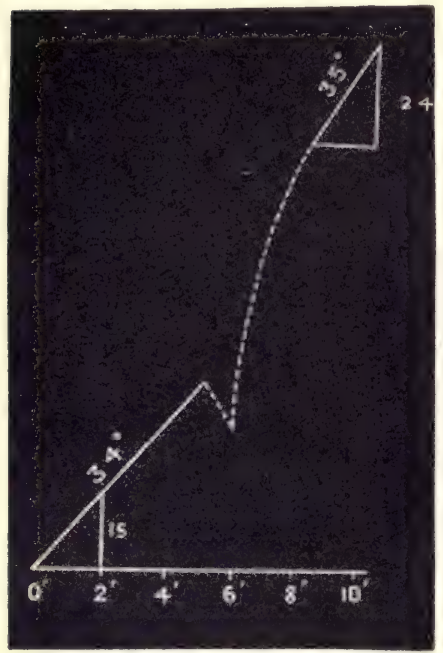

FIG. 5I. Record of Growth in Crinum at Temperature of $34^{\circ} \mathrm{C}$. and $35^{\circ} \mathrm{C}$.

The dotted line represents the variable period of temperature change. Note the contractile twitch and transient highly accelerated growth which follows. The rate of growth became constant when the temperature became permanent at $35^{\circ} \mathrm{C}$. gradually increasing erectile response, which carries the leaf above its original level.

These opposed motile effects can be shown, moreover, even in the case of ordinary plants. We take a spiral tendril of Passiflora. In this, the outer or convex surface is more excitable than the inner or concave, and external stimulus, causing greater contraction of this more excitable outer side, induces a movement of uncurling. This movement corresponds to the excitatory fall of Mimosa. The response by increase of internal energy is, however, the opposite of this, and consists of a movement of curling. When the tendril is placed in a vessel of water, of which the temperature can be varied at will, a sudden rise of temperature causes a preliminary excitatory movement of uncurling, followed by a movement of curling, when the higher temperature has become steady.

The electrical expressions of external stimulus and internal energy are similarly opposed. In fig. 85 (Chapter X.) will be seen a record showing that sudden variation of temperature, acting as an external stimulus, induced a responsive galvanometric negativity, whereas steady rise of 
temperature had the opposite effect - that, namely, of inducing galvanometric positivity.

It is thus seen that while the characteristic effect of external stimulus on an excitable tissue is to cause a negative turgidity-variation, that of increased internal energy is to induce a positive turgidity-variation. The former of these, or negative turgidity-variation, finds electrical expression in galvanometric negativity; in a motile organ by the fall of the leaf, and in a growing organ by retardation of the rate of growth. The latter, or increase of internal energy, on the other hand, is expressed electrically by galvanometric positivity; mechanically, by erection of the leaf; and in a growing organ by an acceleration of the normal rate of growth.

We thus arrive at the following laws of response of isotropic organs :

1. Effective stimulation induces contraction and galvanometric negativity.

2. Increase of internal energy induces expansion and galvanometric positivity. 


\section{CHAPTER VII}

\section{ABSORPTION AND EMISSION OF ENERGY IN RESPONSE}

Sign of response determined by latent energy of tissue, and by intensity of external stimulus - Sub-tonic, normal and hyper-tonic conditions-The critical level-Outward manifestation of response possible only when critical level is exceeded-Three typical cases: response greater than stimulus; response equal to stimulus; and response less than stimulus-Investigation by growth-response-Instance of sum of work, internal and external, performed by stimulus constant-Positive response of tissues characterised by feeble protoplasmic activity or sub-tonicity-Enhancement of normal excitability of sub-tonic tissue by absorption of stimulus.

WE shall find, in this and succeeding chapters, that the nature and intensity of response are determined not merely by the intensity of stimulus, but also by the molecular condition of the responding substance. The excitatory manifestation is dependent upon the occurrence of a particular directioned molecular distortion. Hence, if by the action of the stimuli of the environment, an incipient distortion in this direction has already been induced in the tissue, the incidence of even moderate stimulus will then prove sufficient to precipitate visible excitatory manifestation. A tissue in this condition is said to be highly excitable or fully tonic. If, on the other hand, the tonic condition be less favourable, longcontinued stimulation will be necessary to evoke the excitatory effect. Here, during the first part of application, stimulus will appear to be ineffective. As a matter of fact, however, it is at work to give such a predisposition to the molecules that the action of subsequent stimulus shall be rendered effective.

In the simple case in which the tonic condition is favourable, a given stimulus will induce a given responsive expres- 
sion. If further, the tissue, on recovery, return to its original condition, then a second similar stimulus will induce the same responsive expression as the first. These responses will thus be uniform. But if, owing to the after-effect of stimulus, the condition of the tissue itself be changed, the responses will be found to exhibit either staircase increase or fatigue decline, according to the particular molecular considerations involved, which will be described in detail in the following chapter.

It will be interesting here, however, to take an extreme case of a tissue in the opposite condition-namely, of great sub-tonicity. It is clear that since the excitability of the tissue is here feeble, there will be little or no outward manifestation of excitatory response. The incident stimulus will thus be absorbed, and entirely held latent. And the increase of internal energy thus brought about may find expression mechanically by expansion, or electrically by galvanometric positivity.

In the case which we have selected, the excitability of the tissue is too low for the ordinary excitatory expression to occur. Hence the incident stimulus will be entirely absorbed, and there will be a gain of energy without any loss. The whole responsive expression will in this case consist, not of contraction but of expansion. In other words, it will be exactly opposite to that which is usually consequent upon external stimulus. In order to demonstrate this, I took a seedling of Tamarindus indicus which had been cut off from its supply of external energy, and was consequently sub-tonic. Owing to the insufficiency of internal energy, its growth had, in fact, come to a standstill. On now subjecting this seedling to thermal stimulation, the absorbed stimulus raised the internal energy and found expression in growth expansion. We have seen that the effect of external stimulus on a growing organ in normal tonic condition was the retardation or arrest of growth. But here, in a tissue which is sub-tonic, we find that the effect is exactly opposite.

Without, however, taking so extreme a case, as that in 
which sub-tonicity is manifested by arrest of growth, we may select a specimen in which, while the tissue is not fully tonic, there is still, nevertheless, a feeble rate of growth. In such a case we may expect the income from the absorption of stimulus to prove greater than the expenditure in the form of true excitatory response. Hence, if we subject such a tissue to the constant action of an external stimulus, we shall in the first stage obtain the predominant effect of the internal factor, with its positive turgidity-variation and enhanced rate of growth.

But by the continued action of this accumulating income, the tonic condition of the tissue will be raised to the normal, with a concomitant increase of excitability. It will now therefore be the excitatory component which becomes predominant, resulting in the negative turgidity-variation, contraction, and retardation of growth.

In order to detect these variations of the normal rate of growth, under the action of stimulus, it is necessary to have at our disposal some very delicate means of record. This need I have, however, been able to meet, by devising the Balanced Crescograph, more fully described in my book on 'Plant Response.' Here, the uniform rate of elongation of a growing organ causes a rotation of the recording Optic Lever. The spot of light from this lever falls upon a second mirror, which is subject to a compensating movement. When the balance is exact, the spot of light, reflected from the two mirrors, remains quiescent. When, however, the normal rate of growth, under the action of any agent, undergoes variation, the balance is upset. Thus, when growth is accelerated, there is a movement of the recording spot of light in one direction, say up, and when retarded, a movement in the opposite, say down.

In order to study the effect of external stimulus on a tissue in a slightly sub-tonic condition, I took a flower-bud of Crinum lily, and first obtained a balanced record, seen as a horizontal line (fig. 52). Stimulus of light was now applied, and it will be seen that after a short latent period the 
absorbed stimulus induced a positive turgidity-variation, with enhanced rate of growth, as seen by the up-curve. But by this absorption of the stimulus itself, the tonic condition of the specimen was raised, with consequent increase of excitability, and the response became normal. That is to say, it now consisted of contraction and retardation of growth as seen by the downward curve. The external stimulus was now cut off and the dotted portion of the curve shows the aftereffect. The after-effect is thus not a mere recovery, but an enhanced rate of growth, due to the increased energy which remains latent. It was only when this was exhausted that the normal rate of growth was reestablished, as seen in the horizontal part of the curve. And as the tissue was now in full tonic condition the renewed application of stimulus of light did not again induce a preliminary enhancement of the rate of growth, but the normal contraction and retarda-

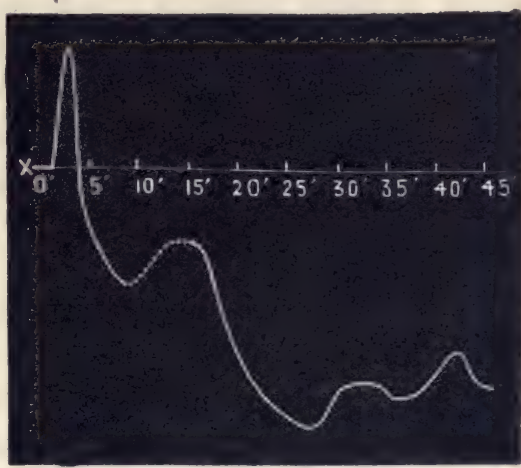

FIG. 52. Balanced Record of Variation of Growth in Flower-bud of Crinum Lily under Diffuse Stimulation of Light

Continuous lines represent the effect during application of light, the dotted line on withdrawal of light. The plant was originally in a sub-tonic condition, and application of light at $x$, after short latent period, induces preliminary acceleration of growth. After this follows the normal retardation. On withdrawal of light, in the dotted portion of the curve is seen the after-effect, followed by return to the normal rate of growth. A second and long-continued application of light induces retardation, followed by oscillatory response.

tion. Its long-continued application gave rise to the further phenomenon of multiple response, a subject which will be fully dealt with in a future chapter.

Now owing to this fact that the response of growth gives us by means of the enhancement or depression of its rate, effects which correspond to the positive and negative, we are able clearly to perceive : 
(I) That when the tonic condition or the excitability of the tissue is low, the predominant effect will be the positive. This has been shown during the course of the present chapter in the case of a very sub-tonic tissue of Tamarindus indicus, where the positive or growth-expansion effect was initiated by the action of stimulus.

(2) That when the sub-tonicity of a tissue is not very great, incident stimulus will at first give the positive effect of an enhanced rate of growth. But with the absorption of the stimulus itself, the tonic condition of the tissue will be raised, and we shall then obtain the true excitatory reaction of contraction and retardation of growth. Thus, in this intermediate case, the positive response will be seen to pass into normal negative.

(3) And, lastly, that when the tonic condition is already high, the excitatory negative response will predominate and we shall obtain normal contractile response. Both this and the previous intermediate cases are illustrated by the experiment described on Crinum lily. It was there seen that the first effect of incident light was positive, the tissue being sub-tonic; subsequently, the tonic condition being raised, this response was converted into the excitatory negative. And on renewed application of stimulus thereafter the immediate response continued to be negative.

The fact that by means of growth-response, it is possible to obtain indications of the external and internal work performed by absorbed stimulus, enables us to demonstrate a proposition of great importance, that, namely, under certain conditions, the sum of the work done, internally and externally, by a given stimulus, is constant. This will be the case where there is little or no dissipation of energy in the course of transformation. In considering the question of the relative proportions of the incident stimulus utilised for internal and external work respectively, we find it clear, from considerations already adduced, that the lower the tonic condition the greater will be the proportion of stimulus held latent for the performance of internal work. The nearer is 
the tonic condition, on the other hand, to the critical level, the greater will be the excitatory overflow, and the smaller the latent component. The internal and external factors will thus be complementary to each other.

On subjecting this inference to experimental demonstration by means of growth-response, I fully succeeded in verifying it. According to this method of growth-response, it will be remembered, the true excitatory effect is measured by retardation of the normal rate of growth, the internal factor of increased latent energy being represented, on the other hand, by a corresponding enhancement of the rate of growth. This being understood, it was found that in a particular specimen of growing tissue, whose tonic condition was somewhat low, the external and internal effects caused by a given stimulus were in the proportion of 32 to 13.5 . When the tonic condition of the specimen was raised, however, and the same stimulus was applied, the external effect was found to be enhanced to 38 at the expense of the internal, which was now found to be lowered to 8.5 . The sum of the work done, both internally and externally, is seen to be in both these cases approximately the same, being in the former experiment $45^{\circ} 5$, and in the latter 46.5 .

We have seen that, of the two antagonistic factors of response, the positive will predominate if the excitability of the tissue be in any way diminished. Such a loss of excitability may occur in either of two ways: (I) by the sub-tonicity of the tissue itself; (2) by the depression consequent on fatigue. Under either of these conditions then we may expect to obtain the exhibition of the positive effect.

The exhibition of the positive effect under fatigue will be described in the course of the next chapter. We shall here consider instances in addition to those already given, of the occurrence of the positive effect in a tissue which is sub-tonic. We have to bear in mind that the work which incident stimulus is called upon to perform is two-fold, both internal and external, and that there is a certain critical excitatory level, above which only is the normal responsive expression 
possible. The actual potential or excitatory level of a tissue depends on its tonic condition and the intensity of the incident stimulus. Now this existing potential of the tissue may be anything within a wide range, $\mathrm{S} T$, when sub-tonic, N when normal, or $\mathbf{H} \mathbf{T}$ when hyper-tonic or above the

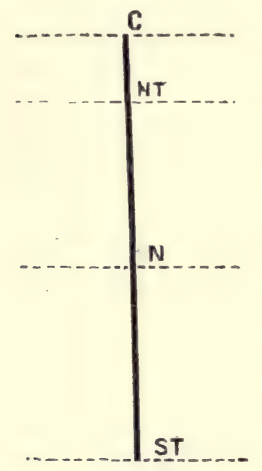

FIG. 53. Diagrammatic Representation of the Tonic Level

N, normal ; S T, sub-tonic ; $\mathrm{H} \mathrm{T}$, hyper-tonic ; and c, the critical level. ordinary normal degree (fig. 53). Since it is necessary that the incident stimulus should cause the critical level $\mathrm{C}$ to be slightly exceeded, if there is to be an excitatory overflow, we can see that the intensity of the stimulus requisite to evoke response will be greater in proportion as the tonicity of the tissue itself is low. Thus when the tissue is extremely sub-tonic, a stimulus of ordinary intensity could never avail to raise the energy of the system above the critical point, and the response must then therefore be positive. Under these circumstances it will only be by the impact of excessively strong stimulus, or by the cumulative action of a series of moderate stimuli, that the critical point can be reached and passed, and the normal negative response evoked.

Thus the intensity of the minimally-effective stimulus in evoking normal response will afford us a measure of the tonicity of the tissue. If the latter be high, then the feeblest stimulus will precipitate outward response, and indeed, if excessive, response will occur on little or no provocation, and such movements we call 'autonomous.' It must be remembered, however, that it was by the previous absorption of stimuli that the tissue was brought to this point of unstable equilibrium at which the added impact of an infinitesimal stimulus causes it to bubble over, as it were, into apparently spontaneous activity.

The predominant expression of the highly tonic tissue 
being thus negative, we must go to the other extreme of great sub-tonicity if we are to be successful in demonstrating the occurrence of the unmixed positive response. This consideration leads us to expect that positive response will be evoked on moderate stimulation from tissues that are either not highly tonic or protoplasmically defective. I shall show in Chapter XXII. that in cells of epidermis, where the protoplasmic contents have been reduced to a minimum, response to moderate stimulus tends in general to be positive, Even highly excitable tissues like nerve, as will be shown later, when cut off from their supply of energy, often become so sub-tonic as to give positive response. I shall here show how ordinary tissues exhibit this effect, when the tonic condition is allowed to fall to such an extent as to render the tissue extremely sub-tonic. For this purpose I took a cut specimen of petiole of cauliflower, and kept it without water for a couple of days. By this process the specimen became somewhat withered. I next proceeded to take records of its electrical responses under increasing stimuli. The intensity of these stimuli rose from I to IO units. It will be seen from the record (fig. 54) that each stimulus up to $g$ evoked positive response, and that it was the strong stimulus of Io which gave rise to the normal response of negativity.

This constitutes the first instance of a phenomenon which I shall show later to be of very extended occurrence-the induction, namely, of one effect under moderate, and its opposite under very feeble stimulation. It is not so easy to demonstrate this fact with a highly excitable, as with a somewhat sub-tonic tissue, where the critical intensity of stimulus for the evoking of normal response need not be impracticably low. A point to be taken into account here is the after-effect of sub-minimal stimulus in enhancing subsequent normal excitability. Thus it is found in taking the record of responses to a succession of feeble stimuli, that though they are at first abnormal positive, they are afterwards converted into normal negative. That it is the after-effect of the previous stimulation which thus enhances previous excitability. 
may also be demonstrated by subjecting the tissue to continuous stimulation or tetanisation, when the abnormal positive is found to pass into normal negative.

From the experiments that have been described, it would appear that the several kinds of response characteristic of various tissues are relatively rather than absolutely different. The true excitatory reaction of an excitable tissue, is one of galvanometric negativity. Any diminution of the excitability - whether by lowering of tonic condition or other

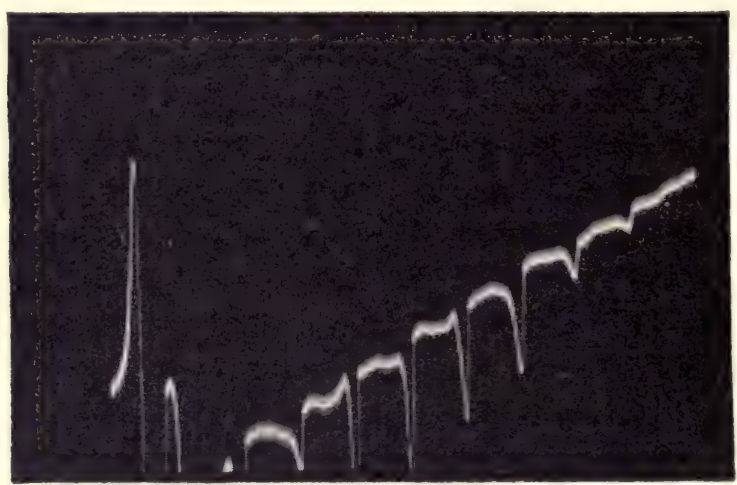

FIG. 54. Photographic Record of Abnormal Positive passing into Normal Negative Response in a Withered Specimen of Leaf-stalk of Cauliflower

Stimulus was gradually increased from I to Io, by means of spring-tapper. When the stimulus intensity was IO, the response became reversed into normal negative. (Parts of 8 and 9 are out of the plate.) This record is to be read from right to left. Down-records stand for positive, and up-curves for negative responses.

causes-will bring about a decrease of this negativity, which may culminate in actual positivity. Thus negative is not separated from positive response by any break of continuity ; but we are able, on the contrary, to trace a gradual transition from one to the other. Moreover, in every response we have the two antagonistic elements, positive and negative, either actually or potentially present. The form taken by the resultant response is entirely determined by the question of what proportion of the stimulus impinging upon the tissue becomes latent; and this in its turn depends upon the tonic 
condition of the tissue. When the absorbed stimulus is wholly retained, response is positive, but by this absorption the tonicity of the tissue and its excitability are both raised. When the tonic condition of the tissue, on the other hand, is already high, and its excitability great, a large proportion of the energy finds outward expression, and we obtain the normal negative response. Between these two extremes, we may observe many effects of interference, due to the play of the two antagonistic elements. If, then, the time-relations be not coincident, variations will be induced which will find expression in different types, diphasic response, positive followed by negative, and vice versa.

The question considered in the course of the present chapter has been that of the energy received and given out by the tissue, and the molecular work, positive and negative, performed during these processes. Such work, however, is itself the result of molecular distortions brought about by stimulus, and the question of the amplitude of response, as related to the degree of distortion, will be discussed in the following chapter. 


\section{CHAPTER VIII}

\section{VARIOUS TYPES OF RESPONSE}

Chemical theory of response-Insufficiency of the theory of assimilation and dissimilation-Similar responsive effects seen in inorganic matter-Modifying influence of molecular condition on response-Five molecular stages, A, B, C, D, E - Staircase effect, uniform response, fatigue-No sharp line of demarcation between physical and chemical phenomena-Volta-chemical effect and byproducts-Phasic alternation-Alternating fatigue-Rapid fatigue under continuous stimulation-In sub-tonic tissue summated effect of latent components raises tonicity and excitability-Response not always disproportionately greater than stimulus-Instances of stimulus partially held latent: staircase and additive effects, multiple response, renewed growth-Bifurcated responsive expression.

ACCORDING to current theories, living matter is maintained in a state of equilibrium by the two opposed chemical processes of assimilation and dissimilation. It is supposed that stimulus causes a down or dissimilatory change, which is again compensated during recovery by the building-up or assimilative change. In the case of uniform responses, again, these two processes are regarded as balancing each other. On this theory, when the down change is the greater of the two, the potential energy of the system falls below par; for the building-up process cannot then sufficiently repair the chemical depreciation caused by it. Hence occurs diminution of response, or fatigue, which is supposed to be further accentuated by the accumulation of deleterious fatigue-stuffs. The disappearance of fatigue after a period of rest is explained by the renovating action of the blood-supply, which is also regarded as the means of carrying away the fatiguestuffs.

A serious objection to these explanations lies, however, in the fact, that even excised and bloodless muscles exhibit recovery from fatigue after a period of rest. In isolated 
vegetable tissues, again, where there is no active circulation of renovating material, the same effect, and its removal after a period of rest, are observed. Thus the difficulties encountered in explaining fatigue, on purely chemical considerations, are great enough; but still greater are those difficulties which arise when we come to deal with the staircase effect-typically shown in cardiac muscle-in which successive responses to uniform stimuli exhibit a gradual enhancement of amplitude. The results obtained here are in direct opposition to the theory described; for in this particular case we have to assume that the same stimulus which is usually supposed to cause a chemical breakdown, has become efficient to induce an effect exactly the reverse.

Of the two antagonistic elements in the electrical response, moreover, it is the positive which is supposed to be associated with the assimilative, and the negative with the dissimilative change. If this supposition were correct, however, it would be natural to expect that the positive response would be manifested predominantly in vigorously growing tissues, in which assimilation must be at its greatest. Fatigued tissues on the other hand, in which dissimilatory changes are supposed to be predominant, should manifest negativity as their characteristic response ; moribund tissues, in contrast with the actively growing, might also be expected to exhibit responsive negativity. In actual fact, however, the very reverse is the case. For in vigorous tissues, normal response is by galvanometric negativity; and it is the over-fatigued or moribund which characteristically exhibit the positive response.

It would be difficult again to conceive of assimilation and dissimilation in the case of inorganic matter. Yet even in inorganic matter we find reproduced all the various types met with in the response of living tissues : namely, uniform response, the staircase effect, and fatigue. Response being really due to molecular upset from a condition of equilibrium, we can see how different forms of responsive expression will occur, according to the various molecular conditions of the 
substance at the time being. One of the most important factors, then, in determining the character of response is the molecular condition of the substance itself. The numerous anomalies hitherto encountered in our interpretation of responsive phenomena are all traceable to our failure to take this factor of molecular condition into account. For a full exposition of the modifying influence which it exercises on response, however, though I shall here state some of the principal conclusions which I have arrived at, the reader is referred to Chapter XLII.

From the fact, that every type of response is to be obtained from inorganic matter, where chemical assimilation and dissimilation are obviously out of the question, it is clear that the fundamental phenomenon must be dependent on physical or molecular, and not on such hypothetical chemical changes. It must, however, be remembered that though response phenomena and their modifications are undoubtedly in the first place physical or molecular, yet in the borderland between physics and chemistry there is no sharp line of demarcation. For example, yellow phosphorus becomes converted, under the stimulus of light, into the red, or allotropic, variety. This molecular change, however, is also attended by a concomitant change in the chemical activity, phosphorus in its allotropic condition being less active than in the yellow. Under certain circumstances, further, it is possible to have a secondary series of chemical events following upon a condition of unequal molecular strain. A homogeneous living tissue, when unstimulated, is iso-electric. When stimulated, however, an electro-motive difference is induced, as between the stimulated and unstimulated parts of the tissue. The result is an electrical current attended by electro-chemical changes. As a consequence of such volta-chemical action, when prolonged, by-products (fatigue stuffs?) may be accumulated, and these may have a depressing effect on the activity of the tissue. Hence, just as, after very prolonged activity of a voltaic combination, it is necessary to renew the active element and change the 
electrolyte, surcharged with by-products, so after sustained activity of a living tissue, the process of renewal, or renovation, will be necessary. It is thus seen how upon the fundamental molecular derangement, a chain of very various chemical events may follow, as its after-effect. And it is only by going in this way to the very root of the phenomenon that we can avoid the many contradictions with which we are confronted by the chemical theory.

In studying various response phenomena, our conclusions are necessarily based upon the observation of the amplitude of responses. It is therefore important at this point to draw attention to the danger of hasty inferences. On finding, for instance, that the amplitude of response in a given case is diminished, we are apt to infer that the responding tissue has undergone depreciation. But this is not invariably the case. In the entire process of response, while stimulus induces molecular upset, we must remember that there is also an internal factor, which brings about molecular restitution. Now, if this force of restitution be in any way enhanced, it is easy to see that the responsive distortion of the molecules will find itself opposed, with consequent diminution of amplitude. We shall thus often find that a rise of temperature, by enhancing the force of recovery, actually causes a diminution of response. That this is not due, however, to any depreciation of the tissue is seen from the fact that the same rise of temperature enhances another excitatory property of the tissue-namely, the speed of its conduction.

I shall now give a brief account of the modifying influence exercised on response by the molecular condition. It will be shown, in the Chapter (XLII) on the Modification of Response under Cyclic Molecular Variation, that a given response is not determined merely by the nature of the responding substance, but also by the amount of the energy which it possesses. Starting from the lowest condition of sub-tonicity, a substance undergoes progressive molecular transformation by the action of the impinging stimulus itself. 'Five stages may be roughly distinguished in this transformation. In the 
first, or $\mathbf{A}$, stage of extreme sub-tonicity, we have absorption without excitatory response. By this absorption the substance passes into the next, or $\mathbf{B}$, stage, which is the stage of transition, where response is converted from the abnormal to normal. Above this stage the rate of molecular transformation is very rapid. From the residual after-effect of stimulus, the substance now passes from the stage $\mathbf{B}$ to the stage $\mathbf{C}$, which is a condition of more or less stability. Further stimulation carries the substance to stages $\mathbf{D}$ and $\mathbf{E}$. Here the molecular distortion from the normal equilibrium is very great. Stimulation applied in this condition has little further effect in inducing response. That is to say, excitability is here reduced to a minimum. In this extremely distorted position, moreover, the substance has a strong tendency to revert to the position of normal equilibrium.

In the $\mathbf{A}$ condition of extreme sub-tonicity, since there is absorption without excitation, the response which we obtain is abnormal positive. Intense or long-continued stimulation carries the substance into the $\mathbf{B}$ stage, with its normal negative response often preceded by diphasic. An example of this has already been given in fig. 54, obtained from the sub-tonic petiole of cauliflower. We shall meet, however, with numerous other examples in a great variety of tissues. Arriving at the B stage, the substance is still somewhat sub-tonic, and the rate of molecular transformation here is rapid. From the after-effect of stimulus the molecules of the somewhat inert substance become incipiently distorted in the same direction as that of normal response. A proportion of the incident stimulus is thus utilised in inducing a favourable molecular disposition. A repetition of the original stimulus will now give rise to a greater excitatory reaction than before. Thus at the $\mathbf{B}$ stage we obtain a staircase increase of response. This fact - that by the after-effect of previous stimulation the molecules may be incipiently distorted in a direction favourable to excitatory response-finds illustration in stimuli individually ineffective being made effective by repetition. The result here is evidently made 
conspicuous by the summation of the after-effects of all the preceding stimuli with the direct effects of their successors. The staircase effect is seen in the two accompanying records. In fig. 55 is given a photographic record of the staircase increase in the electrical response of a vegetable nerve ${ }^{1}$ in somewhat sub-tonic condition. In fig. 56 we have a second example of this effect, seen in the electrical response of the petiole of Bryophyllum, rendered artificially sub-tonic by cooling.

We next arrive at the $\mathbf{C}$ stage, which is, as has been said, one of more or less stability. Expenditure is here, for a

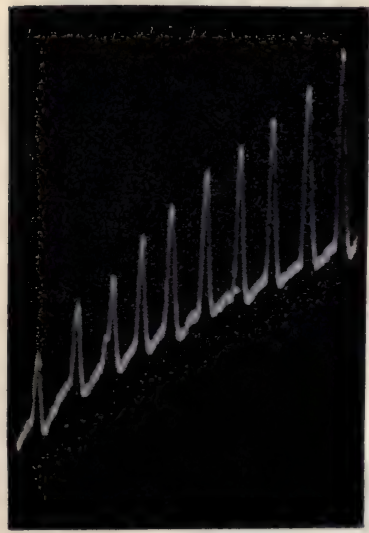

FIG. 55. Photographic Record of Staircase Response in Vegetable Nerve certain length of time, balanced by income. The molecular condition of the tissue being thus constant, the responses are uniform. I give below records of such uniform responses to uniform stimuli, ex-

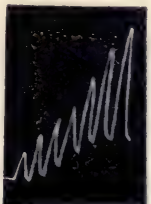

Fig. 56. Staircase Increase in Electrical Response of Petiole of Bryophyllum, rendered sluggish by cooling

hibited by different tissues. In fig. 57 are seen uniform electrical responses to uniform mechanical stimuli, given by the root of radish. Fig. 58 shows uniform electrical responses to uniform thermal stimuli, given by the petiole of fern.

The $\mathbf{C}$ is succeeded by stages $\mathbf{D}$ and $\mathbf{E}$, representing a condition of over-strain. In fig. 59, $a$, are shown uniform responses to uniform stimuli, applied at intervals

1 An account of the discovery of certain vegetable tissues, wịth the function of ṇervẹs, will be found in Chapter XXXII, 
of one minute. An inspection of the record shows that there is in such cases a complete recovery, at the end of which the molecular condition is the same as before stimulation. Hence, successive responses are exactly similar to each other. The stimulation-rhythm was now changed, to intervals

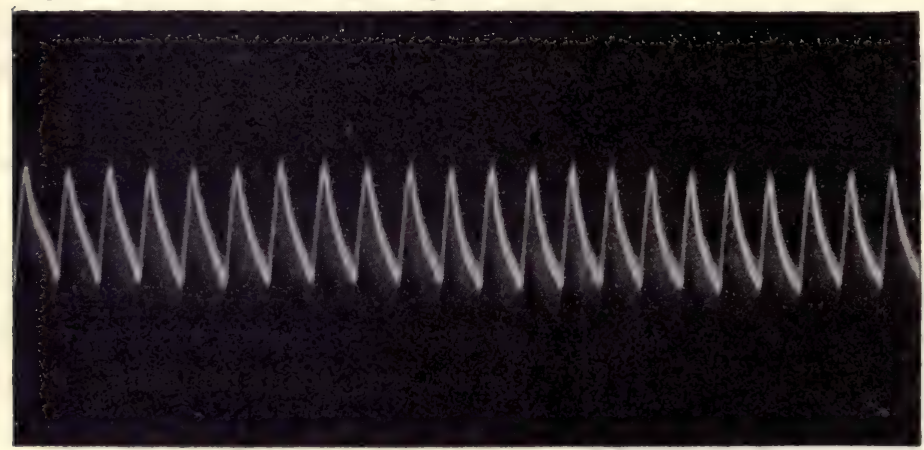

FIG. 57. Photographic Record of Uniform Responses (Radish)

of half a minute instead of one, while the stimuli were maintained at the same intensity as before. It will be noticed (fig. 59, b) that these responses are now of much smaller

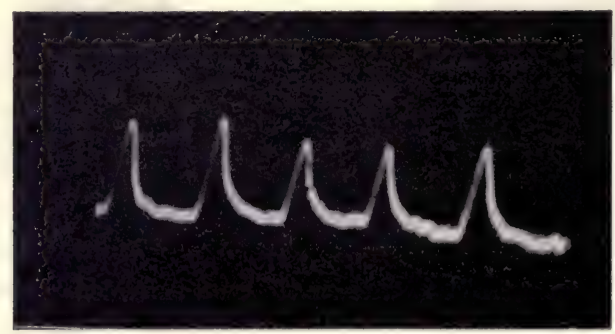

FIG. 58. Photographic Record of Uniform Response in Petiole of Fern

amplitude, in spite of the equality of stimulus. An inspection of the figure also shows that, when greater frequency of stimulation was introduced, the tissue had not had time to effect complete recovery from previous strain. The molecular swing towards equilibrium had not yet abated, when 
the new stimulus with its opposing impulse was received. There is thus a diminution of height in the resultant

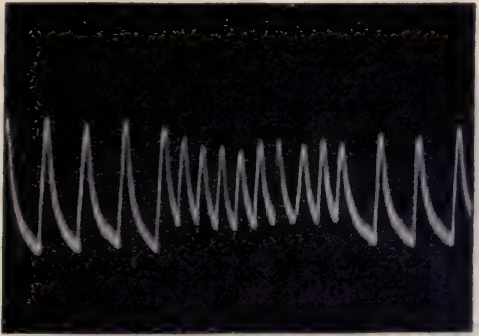

(a) (b)

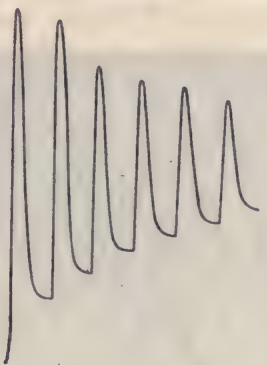

FIG. 6o. Fatigue in Celery

Vibration of $30^{\circ}$ at in tervals of half a minute.

response. The original rhythm of one minute was now restored, and the succeeding records (fig. 59, c) at once show increased response.

Residual strain is thus seen to be one of the principal reasons of reduced response or fatigue. This is also shown in a record which I have obtained with a petiole of celery (fig. 60). It will be noticed there that, owing to imperfect molecular recovery, during the time allowed for rest, the heights of succeeding responses undergo a continuous diminution. Fig. 6r

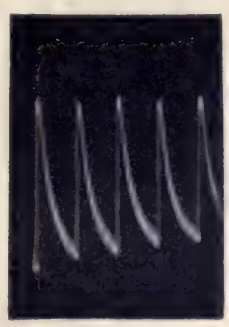

FIG. 61. Fatigue in Leaf-stalk of Cauliflower

Stimulus : $30^{\circ}$ vibration at interval of one minute. gives a photographic record of fatigue in the petiole of cauliflower, and fig. 62 of fatigue in inorganic response.

It is evident that residual strain, other things being equal, will be greater if the stimuli have been excessive. This is 
seen in fig. 63, where the first set of these responses, $\mathrm{A}$, is for an intensity of mechanical stimulation of $45^{\circ}$ vibration, and the second set, B, of augmented amplitude, for an intensity of $90^{\circ}$ vibration. On reverting, in $\mathrm{C}$, to the first stimulus-

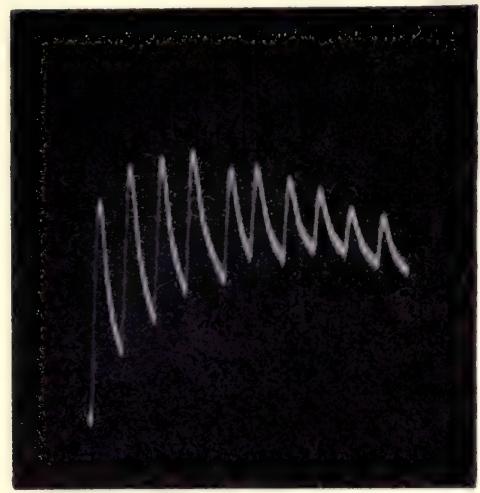

FIG. 62. Photographic Record showing Fatigue in Tin Wire which had been continuously stimulated for several Days intensity of $45^{\circ}$, the responses are seen to undergo a great diminution, as compared with the first set, A. This change is due to the over-strain of the previous excessive stimulation. But we should expect that the effect of such over-strain would disappear with time, and the responses regain their former height, after a period of rest. In order to verify this, therefore, I renewed stimulation (at the intensity of $45^{\circ}$ ) fifteen minutes after $\mathrm{C}$. 'It will be seen from the record $\mathrm{D}$ how far fatigue had been removed in this interval.

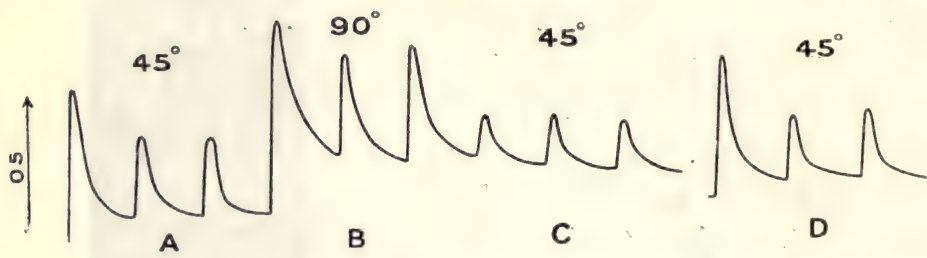

FIG. 63. Effect of Over-strain in producing Fatigue

Successive stimuli applied at intervals of one minute. The intensity of stimulus in $\mathrm{C}$ is the same as that of $\mathbf{A}$, but response is feebler owing to previous over-stimulation. Fatigue is to a great extent removed after fifteen minutes' rest, and the responses in D are stronger than those in c. The vertical line between arrows represents 05 volt. (Turnip leaf-stalk.)

One peculiarity that will be noticed in these curves is that, owing to the presence of comparatively little strain, the first response of each set is relatively large. The succeeding 
responses are approximately equal, where the residual strains are similar. The first response in fig. 63, $\mathrm{A}$, shows this, because there had been long previous rest. The first of B shows it, because we are there passing for the first time to an increased intensity of stimulus. The first of $\mathrm{C}$ does not show it, because of the strong residual strain from the preceding excessive stimulation. And the first of D, again, does show it, because the strain has now been removed, by the interval of fifteen minutes' rest.

Of the antagonistic elements of positivity and negativity which are present in response, we have seen that the positive becomes predominant when the excitability of the tissue is in any way depressed. And since a tissue under fatigue has its excitability lowered, it follows that in this condition it may be expected to exhibit a tendency towards positive response : that is to say, expansion in the case of mechanical. and galvanometric positivity in the case of electrical, response. Thus, when a tissue is subjected to continuous stimulation, the first effect will be the maximum negative response, contraction and galvanometric negativity. But on the settingin of fatigue, the positive effect will predominate, inducing a fatigue-reversal of the response. In cases where such fatigue is very great, as, for instance, in certain muscles, the top of the tetanic curve undergoes rapid decline (fig. 64, $a$ ). The normal contraction now exhibits a reversal, or relaxation. In the sensitive plant, Mimosa, similarly, continuous stimulation by electrical shocks gives rise to results which are essentially the same. It will be noticed that after the responsive fall of the leaf it returns to its former erect position, in spite of the fact that stimulus is still being continued. Here also, as in the corresponding case of muscle, we have the usual sequence, of ( $\mathrm{I}$ ) normal contraction and (2) fatigue relaxation (fig. 65).

In electrical response, also, under continuous stimulation, the normal galvanometric negativity, owing to the increasing positive effect, undergoes decline or abolition. 'This is seen in fig. $64, b$, which exhibits the decline of electrical response 
under continuous stimulation in the petiole of celery. The fatigue in the mechanical response of muscle under similar conditions is given in $\boldsymbol{a}$ for the purpose of comparison. The effect of rest in inducing molecular recovery, and hence in the removal of fatigue, is illustrated in the following set of photographic records (fig. 66). The first of these shows the curve of electrical response, obtained with a fresh plant. It will be seen that under a continuous stimulation of two minutes the response first attains a large amplitude, after which it declines, in a fatigue-reversal. Another two minutes were now
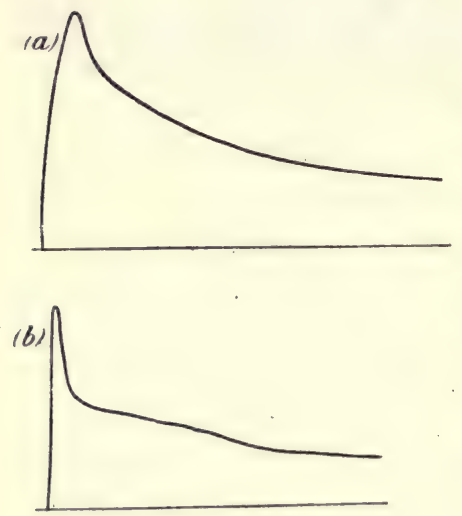

FIG. 64. Rapid Fatigue under Continuous Stimulation in (a) Muscle ; (b) Leaf-stalk of Celery (Electrical Response)

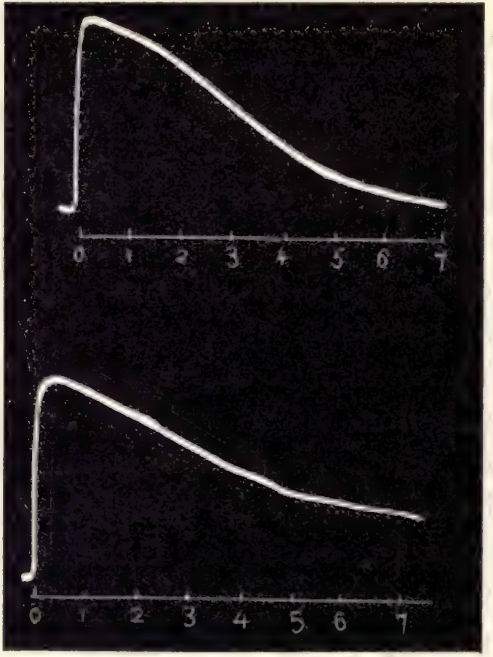

FIG. 65. Photographic Records of Normal Mechanical Response of Mimosa to Single Stimulus (upper figure), and to Continuous Stimulation (lower figure)

In the latter case the leaf is erected in spite of continuous stimulation.

allowed for recovery, and we observe that a partial recovery takes place. Stimulation was now repeated throughout the succeeding two minutes, to be followed once more by two minutes' rest. The response in this case is seen to be decidedly smaller than at first. The same effects are seen in the third response. A period of rest of five minutes was next given, and the curve subsequently obtained under the 
same two minutes' stimulation as before shows greater response than the preceding, owing to the partial removal of residual strain.

There is one aspect of the subject of fatigue-reversal which now demands our attention. We have seen that under continuous stimulation, a maximum contraction is induced, which is attended by the depression of the leaf of Mimosa.

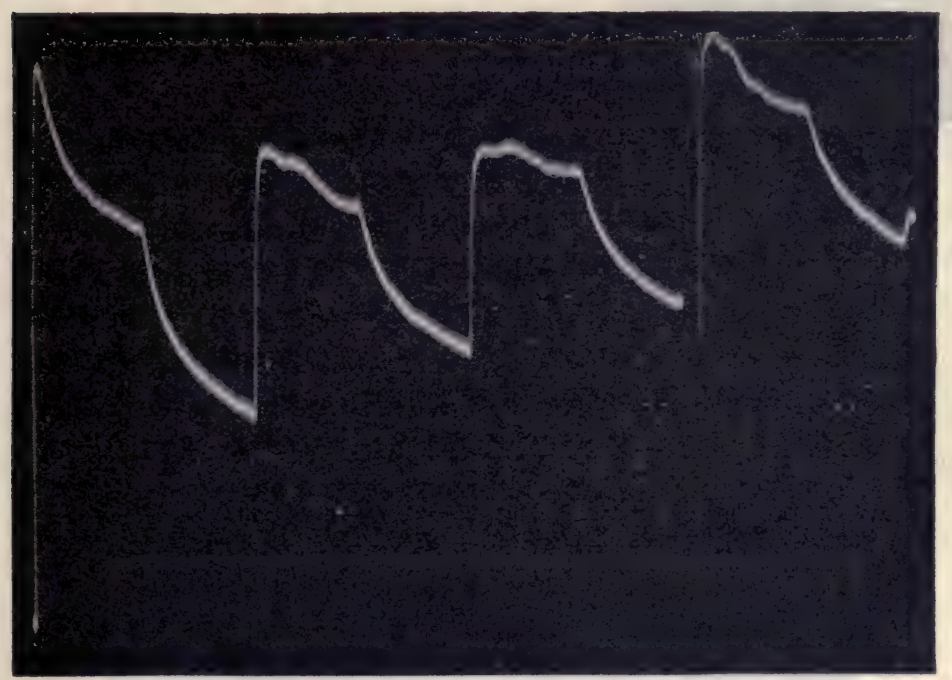

FIG. 66. Effect of Continuous Vibration (through $50^{\circ}$ ) in Carrot

In the first three records, two minutes' stimulation is followed by two minutes' recovery. The last record was taken after the specimen had a rest of five minutes. The response, owing to removal of fatigue by rest, is stronger.

This is followed, however, by a reversal-namely, expansion, with re-erection of the leaf. According to the chemical theory of assimilation and dissimilation, the fatigue-effect is assumed to be due to an explosive dissimilatory change, with consequent run-down of energy. In the case of Mimosa, however, it is difficult to understand how, by a mere rundown of energy and consequent passivity of the tissue, an active movement of erection-involving the performance of work in lifting the weight of the leaf-could be brought 
about. Now we have seen that the diminution of normal response may be brought about by the augmentation of the internal factor, tending to enhance the force of restitution,

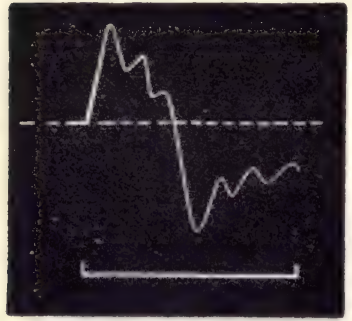

Fig. 67. Oscillatory Response of Arsenic acted on Continuously by Hertzian Radiation

Taken by method of conductivity variation. and the necessary augmentation of the internal factor may be the result of an increase of internal energy. Thus while the plant is the recipient of a continuous income, its responsive expression is alternately one of emission and absorption of energy. Thus negative and positive succeed each other or vice versa. Such a phasic alternation is widely present, as we shall see, in the response, not only of various living tissues, but also of inorganic substances. The following record (fig. 67) exhibits this oscillatory response in arsenic under the continuous stimulation of electric radiation. In the case of Mimosa, under continuous stimulation, we obtain

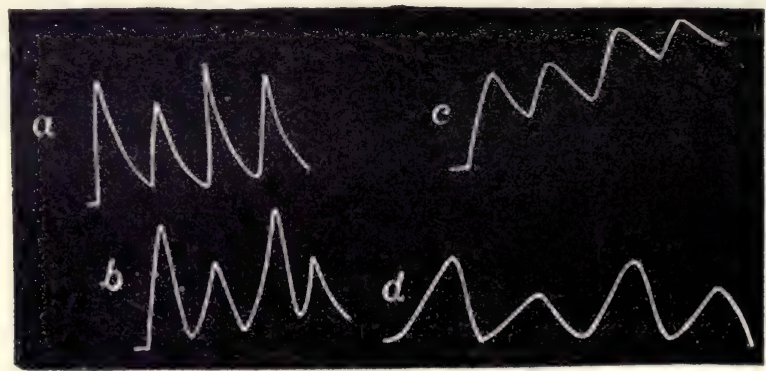

Fig. 68. Alternate Fatigue $(a)$ in Electrical Responses of Petiole of Cauliflower; $(b)$ in Multiple Electric Responses of Peduncle of Biophytum; (c) in Multiple Mechanical Responses of Leaflet of Biophytum ; and $(d)$ in Autonomous Responses of Desmodium

a single alternation, and a certain period must then elapse, before the response can be repeated. In other cases, however, continuous stimulation may give rise to two, or three, or a large number of similar alternations. 
In connection with this subject of phasic alternation I may describe a certain curious phenomenon, which I have often noticed; I refer to the periodic waxing and waning of both mechanical and electrical responses. The simplest example of this will be a case in which the responses are alternately large and small. But others are to be found in which the groupings are more complex. In fig. $68 a$ is seen such a simple alternation, in the electrical response of the petiole of cauliflower, under successive uniform stimuli. In

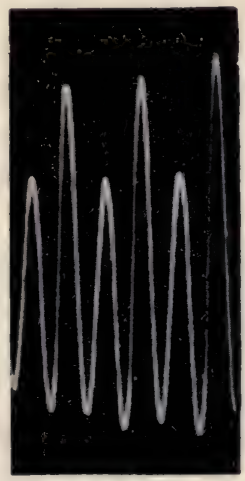

F1G. 69. Photographic Record of Periodic Fatigue in the Automatic Pulsation of Des. modium gyrans $b, c$, and $d$ are shown similar alternations in multiple and autonomous responses. I give also a photographic record (fig. 69) of a similar alternation in the automatic pulsations of the leaflet of Desmodium gyrans.

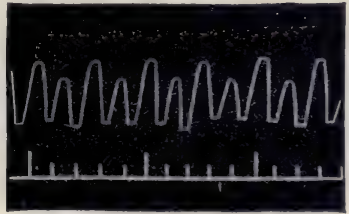

Fig. 7o. Periodic Fatigue in Pulsation of Frog's Heart (Pembrey and Phillips)

Similar alternations are sometimes observed in the beating of frog's heart (fig. 70).

In the following record of mechanical response (fig. $7 \mathrm{r}$ ), taken from the style of Datura alba, we find that fatigue, as already understood, would not explain the phenomenon observed. For here, under the continuous action of stimulus, without any intervening period of rest for the so-called 'assimilatory' recuperation, we see that a second response occurs. I shall later give other instances in which pulsating responses, with their alternating negative and positive phases, are given, under the action of continuous stimulation. We pass here imperceptibly from the ordinary phenomenon of 
individual response to individual stimulus, into that of multiple response, either to continuous, or to a single strong stimulation. The excess of energy derived from impinging stimulus is in the latter case held latent in the tissue to find subsequent expression in phasic alternations of negative and positive variations in series (cf. Chapter XVII).

There can be no doubt that these effects of periodic alternation of phase are due to two antagonistic reactions, becoming effectively predominant by turns. Thus the continuous impact of stimulus on a tissue may first give rise to the negative phase of response. But by the continuous absorption of incident stimulus, the internal energy, is in-

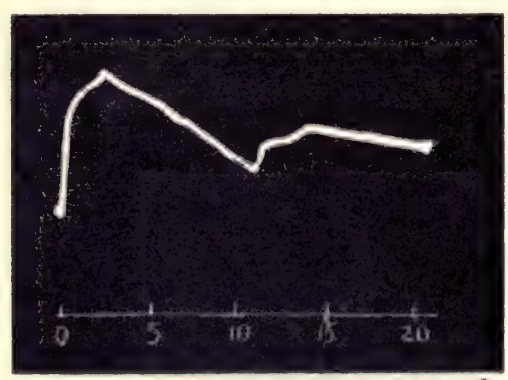

FIG. 71. Photographic Record of Periodic Fatigue under Continuous Stimulation in Contractile Response (Filament of Uriclis Lily) creased, with its opposite reaction of positivity. Hence, the negativity will be gradually diminished, and the positive phase become predominant. The existence of these two antagonistic factors will be understood, from an inspection of the top of a tetanic curve. Here, the more or less horizontal line represents a state of balance between the two opposite forces of excitatory response by contraction, with galvanometric negativity and recovery or expansion, with galvanometric positivity. When this state of balance is disturbed, by a sudden cessation of the hitherto continuously acting stimulus, a brief overshooting of the response in the negative direction is sometimes seen, followed by recovery. We shall meet with examples of this in, among others, the responses of retina and certain vegetable tissues under light. Such facts it has been suggested afford a demonstration of the two antagonistic processes of assimilation and dissimilation, characteristic of living tissues. But that they are really to be accounted for 
from molecular considerations will be seen from the fact that effects exactly similar are met with in the response of inorganic matter (cf. figs. 258 and 383.)

In the case of responses exhibiting fatigue from overstrain, we have a diminution of normal response, which may ultimately culminate in reversal. We may imagine a spiral spring, undergoing increasing compression from a gradually augmenting force. The responsive compression will at first be considerable. But this wili soon reach a limit, beyond which added force will seem to have but little power to induce further responsive distortion. In a somewhat similar way, we may visualise the condition of the responding molecule at the stage $\mathbf{D}$ or $\mathbf{E}$. Here, molecular distortion has almost reached its limit. It follows that added stimulus can induce

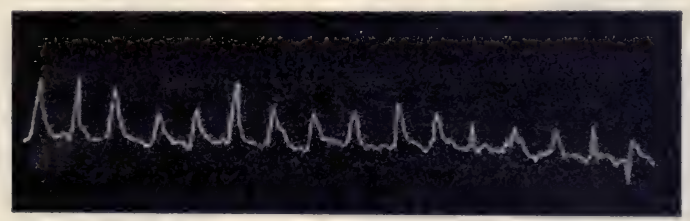

FIG. 72. Fatigue in the Contractile Response of India-rubber Note the periodic alternation and the reversal at the end.

little further distortion. But the maximally distorted molecule has now a great tendency to revert to the position of equilibrium, and the shock of stimulus, instead of inducing excitatory action, induces the reverse. That this is to be explained by molecular rather than chemical considerations, is seen in the following record (fig. 72) of the contractile response of india-rubber to thermal stimulation. This represents the last part of a long series of responses, whose amplitude was already undergoing a progressive decline. Further symptoms of growing fatigue are seen in the periodic alternations of amplitude, and in the final reversal of response to one of expansion. I shall later give another record in which the normal negative response is seen reversed to positive through an intermediate diphasic. 
The fact that the normal response of living tissues may be reversed under fatigue, I am here able to show by an experiment of an unexpected character. It is usually supposed that fatigue is typical of such tissues as muscle, and absent from nerve. But I shall show with regard to all the various types of response, that there is none of these which is distinctive of any one tissue. The difference is one of degree and not of kind. The same intensity and duration of stimulus which is efficient to cause

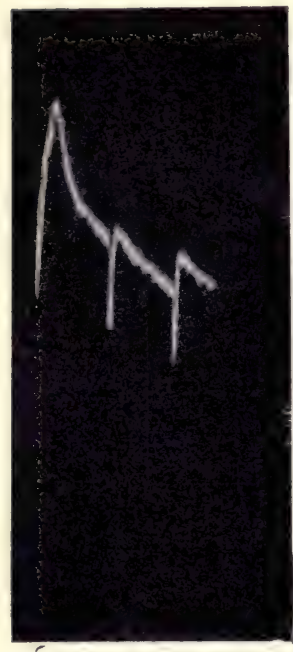

Fig. 73. Reversed Response of Fatigued Nerve fatigue in muscle will not be enough to do so in the case of nerve. But even nerve will display fatigue when excessively stimulated. In the record given in fig. 73, a particular nerve of frog had been previously fatigued, by overstimulation, and on now taking individual responses to individual stimuli, it was found that they had become reversed to positive.

Thus a particular type of response is the result of a particular condition of the responding substance, and there is none which is exclusively characteristic of any one tissue. Were it otherwise, ordinary muscle, in which the explosive molecular change is supposed to be so predominant, should typically show only the fatigue, and never the staircase effect. But the following record (fig. 74) shows that this is not the case. For at first it exhibits a characteristic staircase effect; the responses are then for a time uniform; and lastly, we see fatigue, in a manner exactly corresponding to the theoretical considerations which we have anticipated in stages $\mathbf{B}, \mathbf{C}$, and $\mathbf{D}$. The staircase response is thus not peculiar to cardiac muscle, but is to be seen, under appropriate conditions, in skeletal muscle, in nerve, and even in inorganic substances. In fig. 75 is given a series of responses of Galena to Hertzian 
radiation, which in its various phases of staircase, uniform and fatigue-decline, is parallel to that just seen in muscle. The phasic change, due to molecular transformation, which I have already pointed out under continuous stimulation, is seen in both these records in the shifting of the base-line. I $r_{n}$ fig. $64 a$ under continuous stimulation, we see the mechanical response of muscle passing from a condition of growing contraction into one of relaxation. In the record of individual responses given in fig. 74 , the same is seen to take place:

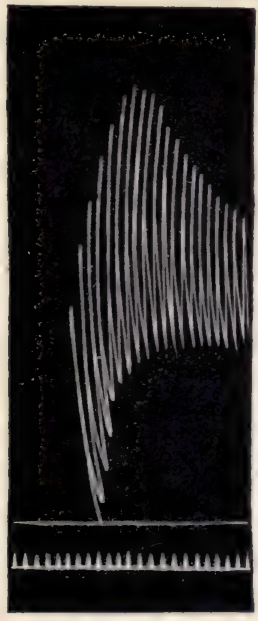

FIG. 74. Preliminary Staircase, followed by Fatigue, in the Responses of Muscle (Brodie) A similar phenomenon is observed in the mechanical response of Mimosa (fig. 65). When the mode of record, however, is electro-motive, in-

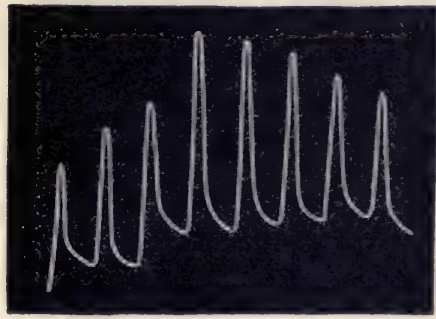

FIG. 75. Preliminary Staircase, Increase, followed by Fatigue, in the Response of Galena to Hertzian Radiation

(Resistivity variation method)

stead of mechanical, the increasing galvanometric negativity which corresponds to increasing contraction, is found gradually to give place to positivity (fig. $64 b$ ). And finally, when the mode of record is by resistivity-variation, we find, by the shifting of the-base line in fig. 75 , that the residual negative variation of resistance at first waxes and then wanes.

Instances have been given, in which a portion of the incident stimulus has been seen to be held latent to do internal work. And from this it is clear that the current assumption that response must always be larger than stimulus is quite un- 
tenable. There are cases, again, in which a large portion of the incident stimulus is held latent for a time, to find subsequent manifestation externally. This I have been able to demonstrate by the discovery of multiple response in plants. Thus while a single moderate stimulus in such cases evokes a single response, a single strong stimulus is found to give repeated or multiple responses. This I have shown, not

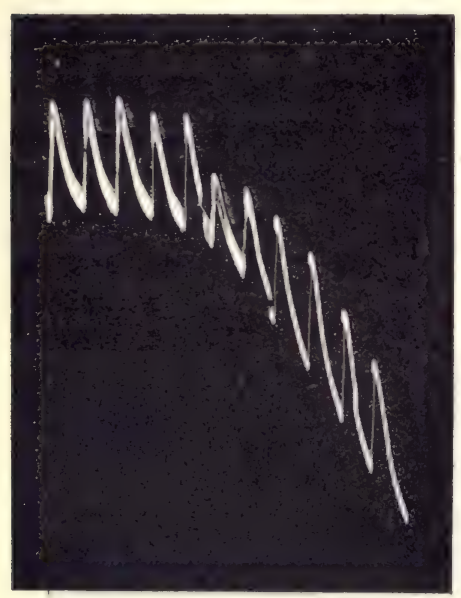

FIG. 76. - Photographic Record of Responses of Style of Datura alba in which Growth had come to a Temporary Stop

The up curve shows contraction. As long as the base-line is horizontal, growth is seen to be at standstill. Renewal of growth at sixth response, after which growth-elongation is shown by the trend of the base-line downwards. only in mechanical, but also in electrical response, and the latter subject will be taken up in detail in a subsequent chapter.

And, lastly, it follows from what has been said, that incident stimulus need not always cause depreciation of the energy of the tissue, but that, on the contrary, it may actually raise it above par. I shall now describe an example in which incident stimulus was seen to find bifurcated expression. In fig. 76 is given a photographic record of contractile responses in the style of Datura alba, in which growth had previously been in a state of standstill. The first five responses of this series are seen to be uniform. A portion of the stimulus applied must, however, from the first have been absorbed and held latent in the organ, thus increasing that internal energy, or tonic condition, on which growth depends. For at the sixth response we find that growth recommences, and the stimulus now finds bifurcated expression, in maintaining response and in renewing growth, as seen in the trend downwards of the 
hitherto horizontal base-line. This bifurcation causes the first contractile response of the now growing organ-sixth of the series-to be smaller than usual. But, as a favourable tonic condition is gradually established by the absorption of energy and the molecular mobility of the responding organ is increased, the contractile response becomes larger, and growth goes on at a certain steady rate. This constitutes an instance in which stimulus, so far from lowering the energy of the responding system, has actually raised it above par.

It would thus appear that while the theory of assimilation and dissimilation is insufficient for the explanation of the various characteristics of response, the difficulties there encountered are, on the contrary, satisfactorily explained, on taking full account of the influence on response of the molecular condition of the responding substance. From the chemical hypothesis of an explosive molecular change, with its attendant dissimilation and run-down of energy, it would follow that previous stimulation should always induce a depression of the subsequent responses. Instead of this, however, it is found that previous stimulation sometimes exalts, and at other times depresses, the subsequent responses. This apparent anomaly we have seen to be explained by the consideration of molecular transformation. From the sluggish condition $\mathbf{A}$, we have seen tissues transformed, by the impact of moderate stimulus, to condition $\mathbf{B}$, with its greater excitability. It is only when the molecular condition has been brought to $\mathbf{D}$ or $\mathbf{E}$, that the responses undergo a diminution or reversal.

The molecular condition, then, undergoes a continuous transformation, in consequence of the action of stimulus, from the extreme of sub-tonicity $\mathbf{A}$ to the overstrained molecular conditions $\mathbf{D}$ and $\mathbf{E}$. In the $\mathbf{A}$ stage, there is no true excitatory expression, response to stimulus being here by the abnormal positive variation. The substance is next transformed into stage $\mathbf{B}$, where response exhibits a staircase character. In the next stage $\mathbf{C}$, the responses are uniform. 
Under over-stimulation, the stages $\mathbf{D}$ and $\mathbf{E}$ are reached, characterised by diminished amplitude of response, or actual reversal into positive. There are thus two conditions under which we obtain abnormal positive responses. One of these is that of sub-tonicity, and the other, the reversal due to fatigue.

There is, again, no tissue which is exclusively characterised by any specific type of response. All these-staircase, uniform, and fatigue-will occur in muscle, nerve, plant, and even inorganic matter, under certain definite and appropriate conditions. In a future chapter, we shall study in detail the characteristic molecular curve, from which light will be thrown on the internal molecular condition of the tissue, and the influence of that condition on response. 


\section{CHAPTER IX}

DETECTION OF PHYSIOLOGICAL ANISOTROPY BY ELECTRIC RESPONSE

Anomalies in mechanical and electrical response-Resultant response determined by differential excitability-Responsive current from the more to the less excitable-Laws of response in anisotropic organ-Demonstration by means of mechanical stimulation-Vibrational stimulus-Stimulation by pressureQuantitative stimulation by thermal shocks.

IT has been customary, as we know, to ascribe the varied movements of plant-organs under external stimulus, to the presence of different specific sensibilities; and, indeed, it would seem at first sight impossible to reduce such highly complex and apparently unrelated phenomena, to the terms of a single fundamental reaction, common to all alike. There is no denying, for instance, that certain plant-organs, when acted on by light, bend towards it, and others away. I have elsewhere shown, ${ }^{1}$ however, that all these diverse movements are clearly traceable to one fundamental excitatory reaction, and that the different effects observed are due merely to the differential excitabilities of various parts of the structure; and that the resultant movement is in all cases brought about by the greater contraction of the more excited side.

Passing next to the electrical response of living tissues, animal and vegetable, we encounter many anomalies. Not only will one tissue give positive, and another negative response, but we find also that the same tissue will give sometimes one and sometimes the other. These apparent inconsistencies are often due, as we shall find, to the differential. excitability of anisotropic structures-a factor in the problem which has not hitherto been recognised. An investigation on

- Bose, Plant Response. 
this subject, then, demands that we first discover some means of determining the relative excitabilities of different parts of a tissue.

As the simplest example of an anisotropic structure, we may take a compound strip of ebonite and stretched indiarubber, glued firmly together throughout their length. Of these, the india-rubber is the more contractile, and when the strip as a whole is subjected to periodic thermal stimulation, response takes place by the greater contraction induced in the india-rubber. If the strip be held, with the india-rubber below, response will be by the induced concavity of the lower side. In fig. 77 is shown a series of these responses of

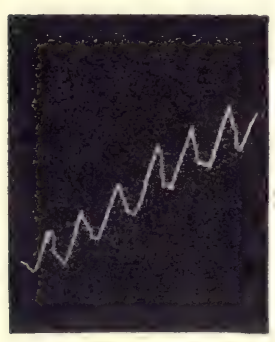

FIG. 77. Differential

Contractile Response of Artificial Strip the compound strip, taken on a smoked surface by means of a recording lever.

In anisotropic motile organs, such as the pulvinus of Mimosa, response takes place by differential contraction, the more excitable side being that which under diffuse stimulation becomes concave. If we apply very moderate stimulus locally on the upper half of the pulvinus, we shall find that, by the excitatory contraction of this half, the leaf is raised. A similar contractile effect, though of greater intensity, is induced when the lower half of the pulvinus is stimulated locally, the leaf in this case undergoing a depression. When both upper and lower halves, then, are excited simultaneously, the resulting fall of the leaf shows that the contraction of the lower half must in this case be the greater, or, in other words, that this half is the more excitable of the two. This experiment may be carried out very easily by using the stimulus of light. Fig. 78 gives the results observed (a), showing the up movement consequent on stimulation of the upper half; $(b)$ that caused by equal stimulation of the lower half; and $(c)$ the resultant fall when the two are excited simultancously. In the case of mechanical response, then, we find it true that response is by the greater contraction of the more excitable. 
We shall next observe what is the electrical mode of response for a tissue which is anisotropic, or unequally excitable on two sides. For this purpose we may again take the pulvinus of Mimosa, and make electrical connections at two diametrically opposite points on the upper and lower halves of the pulvinus respectively. It is to be remembered that electrical response takes place on excitation, whether the leaf be free to move, or physically restrained. We may, therefore, hold it in a fixed position; and indeed this is advisable, in order to avoid that shifting of the electrical contacts which might possibly take place if it were allowed to fall.

The two contacts are made with two fine straws filled with kaolin paste, moistened in normal saline. On now applying a series of thermal stimuli, on the petiole, near the pulvinus, I obtained the responses given in fig. 79. It will be seen that the responsive current flows in the tissue from the rela-

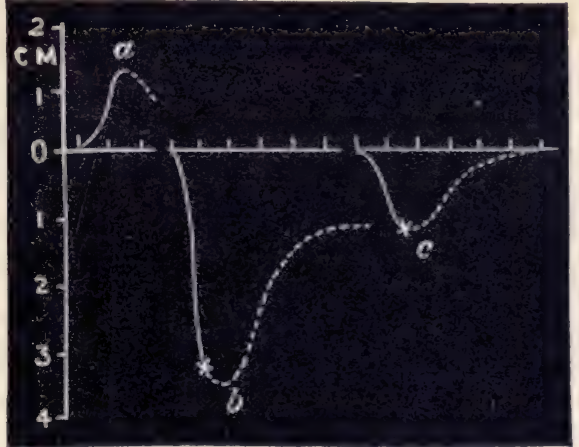

FIG. 78. Responses of Mimosa to Sunlight of not too long Duration

(a) Light acting on pulvinus from above; (b) light acting on pulvinus from below ; $(c)$ light acting simultaneously from above and below. Dotted line represents recovery on cessation of light.

tively more excited lower, to the less excited upper; half of the organ.

We thus arrive at a comprehensive law of the mechanical and electrical response of anisotropic organs:

Diffuse stimulation induces greater contraction and galvanometric negativity of the more excitable side.

The laws of electric response in the anisotropic organ may then be detailed as follows :-

1. On simultaneous excitation of two points, $\mathbf{A}$ and $\mathbf{B}$, the responsive current flows in the tissue from the more to the less excited. 
2. Conversely, if under simultaneous excitation the responsive current be from $\mathbf{B}$ to $\mathbf{A}$, then $\mathbf{B}$ is the more excitable of these two points.

These form only an instance of the general law that the responsive current always flows from the more to the less excited. For when a point, B, is excited locally-this point, that is to say, being the more excited-the responsive current is found to flow away from it to a neutral or indifferent point, A, for which any distant point will serve, provided the tissue be non-conducting. Should it be conducting, the neutrality of $\mathrm{A}$ is maintained by interposing a
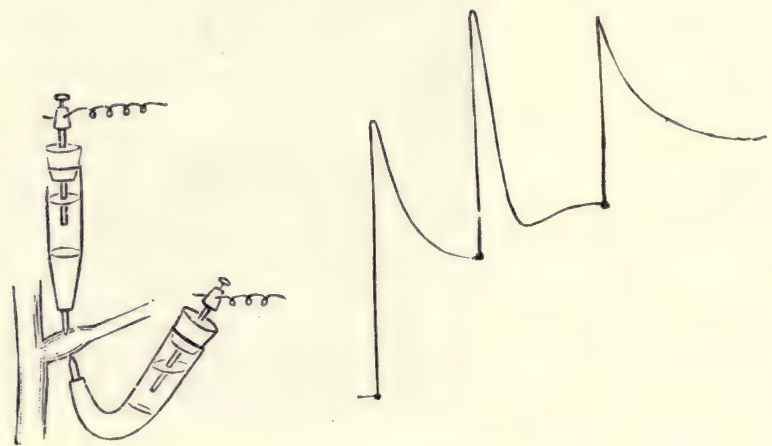

FIG. 79. Transverse Response of Pulvinus of Mimosa

The petiole is securely held to prevent movement, and diametric electric contacts made in the upper and lower surfaces of pulvinus. Responsive current is from lower to upper surface.

block. Should the stimulus, however, not be local, but diffuse, a resultant response may still be obtained by injuring or killing the point A, and thus diminishing or abolishing its excitability. On stimulation, the point $\mathrm{B}$ is now necessarily the more excited, and the responsive current is still away from B, towards A. And finally, owing to physiological anisotropy, B may be naturally more excitable than A, and on stimulation the responsive current will then be found to flow from the more excited $\mathrm{B}$ to the less excited A.

The comparison of the excitabilities of the two points $A$ and $B$, therefore, reduces itself to the application of similar 
stimuli to the two points simultaneously, and then ascertaining the direction of the responsive current.

For this purpose we might employ any form of stimulus, and it is extremely interesting to find that, however diverse the stimuli, the results obtained by them are always identical. And here we have not merely a means of qualitative demonstration, but in some cases one of quantitative also.

If we take an erect stem of Cucurbita, it being radial and isotropic, all its flanks will be found equally excitable. Hence, if two diametrically opposite contacts are made, there

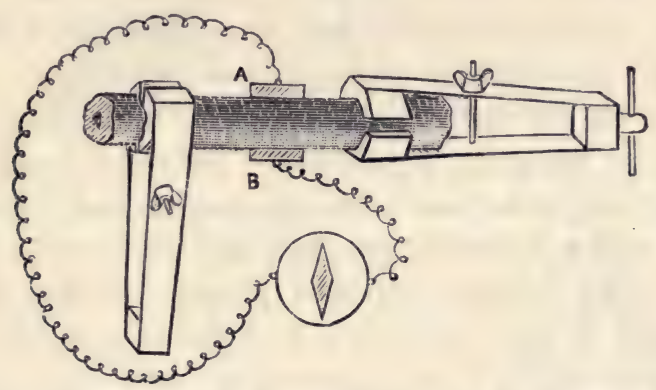

FIG. 8o. Diametric Method of Stimulation of an Anisotropic Organ

Diametrically opposite contacts are made at $\mathrm{A}$ and $\mathrm{B}$, and tissue subjected to vibrational stimulus.

will, on diffuse stimulation, be no resultant response. But when such a stem becomes recumbent, the upper side, being now constantly exposed to light, becomes fatigued by overstimulation, with consequent diminution of its excitability. This is true only when the stimulus has been excessive and long continued; for we have seen moderate stimulus may sometimes enhance the excitability. By the unilateral action of light, then, the organ has been converted from radial into anisotropic, the lower side being that which we shall expect to find the more excitable.

On mounting such a stem in the vibratory apparatus (fig. 80), and making diametrically opposite contacts on the two anisotropic surfaces, we find that on applying vibration 
both sides are subjected to similar stimulus simultaneously; and the responsive current is now found to flow across the tissue, from the lower to the upper side. The lower is thus, as we expected, the more excitable. Since we can by means of vibration apply measured stimuli, it will be seen that we have here a quantitative method of investigation. Moreover, as the stimulus is applied directly, it is applicable not only to conducting but also to non-conducting tissues.

If we next take a radial stem or petiole of Cucurbita, and slit it longitudinally, we obtain, in either of the halves, a specimen having an inner and an outer surface. As one of these has been exposed to light and the other protected from it, we should expect to find, on examination, that there has been an induction of physiological anisotropy. As such a specimen is not very well adapted for vibrational stimulation, we may use that of pressure. Two moistened rags, in connection with non-polarisable electrodes, pass through two pieces of cork, adjusted on the two surfaces-outer and inner-at diametrically opposite points. When the interposed tissue is now subjected to sudden pressure its two surfaces are excited simultaneously, and the responsive current is found to flow from the inner concave to the outer convex surface, proving that the former was the more excitable.

We might again use the chemical form of stimulation, and the results obtained by this method will be described in the course of the next chapter. But these forms of stimulus -by pressure, or by chemical means-are not capable of exact measurement. For quantitative observations, then, it is necessary to employ some other form of stimulus, and the electrical offers us in this respect many advantages. There are, however, in this case many possible disturbing influences to be considered, all of which must be carefully eliminated before the method can be used without misgiving. How this may be done will be shown in a future chapter. For the present I shall describe another method of stimulation which I have been able to bring to great perfection, by which 
two points of an anisotropic organ may be simultaneously excited, under a series of stimuli of uniform or increasing intensity.

This mode of excitation, by thermal shocks, will be found in every way satisfactory and convenient. The Thermal Variator, by which stimulation is effected, consists of a spiral of german-silver wire, the diameter of the spiral being about $3 \mathrm{~cm}$. The electrical circuit, through which the heatingcurrent is sent, is closed periodically for a definite length of

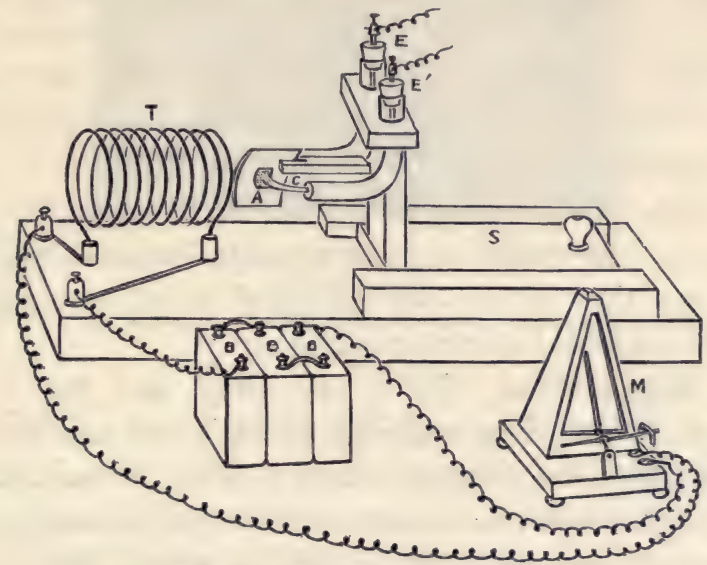

Fig. 81. The Thermal Variator

The anisotropic tissue-petiole of Musa is held in ebonite clip, c. E, E', electrodes connected with opposite sides. Specimen after adjustment pushed inside heating-spiral, $T$, by slide, S. Spiral heated periodically by closure of electric circuit by metronome, $\mathrm{M}$.

time, by means of a metronome (fig. 8I). The thermal variation within the coil can be controlled by a suitable adjustment of the battery-power, or by the duration of closure, or both. The experimental tissue is held in an ebonite clip, C, fixed on a slide, S, on the same stand as the heating-spiral. This slide is pulled out for the purpose of adjustment. Square or circular pieces of wetted muslin make contacts with equal areas on two opposite sides of the experimental tissue, these pieces of cloth being connected with non-polarisable electrodes, $\mathrm{E}_{1}$ and $\mathrm{E}^{\prime}$ After the adjust- 
ment is made, the slide is pushed in, till the tissue is well in the centre of the coil. When the circuit is completed, for a brief period, both the sides $\mathrm{A}$ and $\mathrm{B}$ are subjected to the same sudden variation of temperature, which, as we know, acts as a stimulus. As the two contacts are thus in practice raised to the same temperature, there will be no thermo-electrical disturbance. The responsive current, therefore, will be determined by any difference of excitability which may exist as between $\mathrm{A}$ and $\mathrm{B}$. The spiral also gives out heat-radiation, which acts as a contributory stimulus. That it is the thermal variation, and not the temperature, which acts as the efficient external stimulus, is seen from the fact that when the tissue is subjected to the higher temperature continuously, the galvanometric deflection obtained is opposite in direction to that induced by the thermal shock. This is because the absorption of heat, as such, increases the internal energy, and thus induces an electrical effect opposite to that caused by external stimulation.

As experimental tissue, we may use the sheathing petiole of Musa. The required piece is cut and mounted in the apparatus, the concave surface being taken, say, as B, and the convex as A. I have mentioned Musa as suitable for this purpose, because I find it, when fresh, to show practically no sign of fatigue in its responses. There are many other sheathing petioles, which would doubtless answer the same purpose more or less perfectly.

In obtaining records with this specimen, it is found that the responsive current flows across the petiole, from the inner concave surface B to the outer convex surface A, showing that it is the inside which is more excitable. Uniform stimuli of short duration were applied at intervals of one minute, and the responses obtained are seen to be fairly uniform (fig. 82). The specimen was next subjected to the anæsthetic action of chloroform. This, it will be seen, induced a very great depression of the response.

It has thus been shown that just as the greater contraction and concavity of a motile organ enables us to discrimi- 
nate which side of two is the more excitable, so here also the more excitable side is that which, on diffuse stimulation, exhibits galvanometric negativity relatively to the other. From this it becomes possible to determine the relative excitabilities of any anisotropic organ, even though it be non-motile, and therefore incapable of exhibiting any conspicuous mechanical response. The difficulty of applying equal and quantitative stimulus on two sides simultaneously

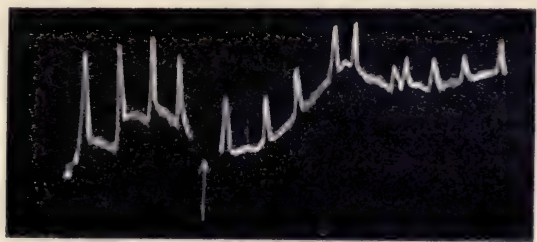

FIG. 82. Responsive Current in Petiole of Musa from Concave to Convex Side

First series, normal ; after application of chloroform subsequent depression.

has now been overcome by vibrational stimulation, and by the perfection of the method of thermal shocks. Thus a definite resultant response has been shown to be determined by the differential excitabilities of two parts of an experimental tissue. And that from this consideration it becomes further possible to resolve many of the remaining anomalies of electrical response will be fully demonstrated in a subsequent chapter. 


\section{CHAPTER $\mathrm{X}$}

THE NATURAL CURRENT AND ITS VARIATIONS

Natural current in anisotropic organ from the less to the more excitable-External stimulus induces responsive current in opposite direction-Increase of internal energy induces positive, and decrease negative, variation of natural currentEffect on natural current of variation of temperature-Effect of sudden variation-Variation of natural current by chemical agents, referred to physiological reaction-Agents which render tissue excitable, induce the positive, and those which cause excitation, the negative variation-Action of hydrochloric acid-Action of $\mathrm{Na}_{2} \mathrm{CO}_{3}-$ Effect modified by strength of doseEffect of $\mathrm{CO}_{2}$ and of alcohol vapour-Natural current and its variationsExtreme unreliability of negative variation so-called as test of excitatory reaction-Reversal of natural current by excessive cold or by stimulationReversal of normal response under sub-tonicity or fatigue.

WE have seen that when the pulvinus of Mimosa is excited by an external stimulus, there is a relatively greater expulsion of water from the more excitable lower half, with a concomitant greater contraction. Conversely, the lower half of the pulvinus is capable of absorbing more water, and of expanding to a greater extent, than the upper. Increased internal energy, in contrast to the action of external stimulus, has the effect of causing a greater expansion of the lower half of the pulvinus, and thus raising the leaf. This we saw exemplified when the plant was subjected to a gradually rising temperature, so as to increase its internal energy, its leaves being thereby made to show increased erection (p. 72). Hence the more excitable tissue in the pulvinus of Mimosa is characterised, both by greater power of absorption and by greater emission of energy, according to circumstances. In this we see a close analogy to the action of inorganic bodies, in which also we find the greatest power of emission to be associated with a correspondingly great power of absorption of energy, 
We have thus seen that in order to maintain a high state of excitability, absorption of energy is necessary. On excitation, emission of energy occurs. In this latter case, of emission, we observe a concomitant galvanometric negativity of the more excited lower side. Since to have maintained its excitability the opposite process of absorption would have been necessary, it follows that the more excitable lower side must under normal conditions be galvanometrically positive. This is found to be the case. For when the leaf is in an excitable condition, there is an electro-motive difference between the upper and lower halves of the pulvinus, in consequence of which a current flows across the tissue, from the less excitable upper to the more excitable lower half, which is thus galvanometrically positive, in relation to the upper. We have here, then, an additional instance of the opposite effects of internal energy and external stimulus. Internal energy, maintaining a greater excitability of the lower half of the pulvinus, induces in it a relative galvanometric positivity. External stimulus, on the other hand, gives rise to precisely the opposite effect-namely, the relative galvanometric negativity of the lower half. Under typical conditions, then, we may expect the more excitable point to be galvanometrically positive; and the more excited to be galvanometrically negative.

Turning next to non-motile tissues, we find the same conclusions to hold good. We saw that in the case of the sheathing petiole of Musa, the concave was more excitable than the convex side. The concave is thus normally positive to the convex side, and the natural current flows across the tissue from the convex to the concave. While the natural current flows from the more excitable to the less excitable, external stimulus gives rise to a responsive current in the opposite direction, from the more excited to the less excited, constituting a negative variation of the current of rest.

Let us next consider what would be the effect of an increase of internal energy on the natural current. Since the action of internal energy is opposite to that of external stimulus, we should expect it to induce a positive variation of 
the natural current. Diminution of internal energy on the other hand might be expected to cause a negative variation. These effects are diagrammatically represented in fig. 83, which also exhibits the parallelism between the electric responses of motile pulvinus and non-motile anisotropic organ.

We next proceed to subject the question of the effect of increased or diminished internal energy on the natural current to experimental verification. As regards the increase of internal energy, we have already seen that this can
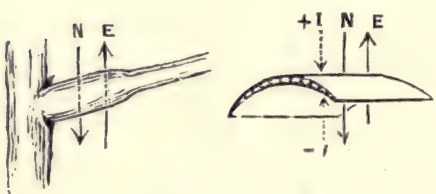

Fig. 83. Parallelism of Natural Current in Pulvinus of Mimosa and Sheathing Petiole of Musa

Upper and less excitable surface of former corresponds with outer or convex surface of latter. The natural current, N, is in both from the less to the more excitable. In both excitatory current, E, is in opposite direction, i.e. from the more to the less excitable. In Musa increase of internal energy $(+\downarrow)$ induces a positive, and diminution of internal energy $(-\uparrow)$ a negative, variation of the natural current. be secured by a gradually rising temperature, its diminution being, contrariwise, secured by a falling temperature. In the case of Mimosa, we saw that the former induced an erection of the leaves, and the latter a gradual depression. In order, then, to observe the effect of increase or decrease of internal energy on the existing current of rest, we have only to subject the specimen togradual thermal ascent or descent, and record the consequent variation of current.

The specimen of Musa is placed in a chamber and two diametrically opposite contacts are made, with the internal and external surfaces, and led off to the galvanometer. To take first the effect of cooling : a stream of ice-cold water is sent through a hollow tube in the chamber: this gradually lowers the temperature, say from $30^{\circ} \mathrm{C}$. to $27^{\circ} \mathrm{C}$. It will be seen from left-hand curve of fig. 84 that this has the effect of diminishing the natural current of rest in the tissue, as represented by the dotted arrow $\downarrow$. When the chamber is 
allowed to return to the temperature of the room, this diminution of current is annulled.

To study the effect, on the other hand, of a rising temperature, the chamber is gradually heated, by means of the electric heating-coil already described. In thus raising the temperature, it is found (fig. 84 , right hand curve) that the natural resting-current undergoes an increase. On cooling again to the original temperature, this increase is annulled. That these effects are due to induced electro-motive variations, and not to any changes of resistance, is demonstrated from the fact that the effect described takes place even when the original E. M. F. is exactly balanced by a potentiometer.

It was specially stated that these observations with regard to the effect of varying temperatures apply only to steady variations. In the case of

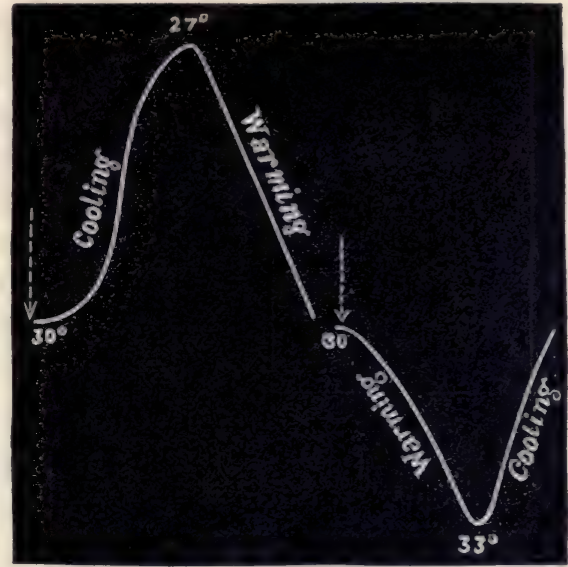

FIG. 84. Effect of Variation of Temperature on Natural Current, $\downarrow$, which in Petiole of Musa flows from Convex to Concave Side

Effect of cooling from $30^{\circ} \mathrm{C}$. to $27^{\circ} \mathrm{C}$, , seen on left, induces negative variation of natural current. Restoration to original value on return to surrounding temperature. Warming induces positive variation (see record to right). In this and subsequent figures in the present chapter $\downarrow$ indicates the direction of the natural current of rest.

\section{thermal ascent, we}

have seen that a steady rise brings about an increase of the existing current. But since sudden variation of temperature acts as a stimulus, we shall, in the preliminary stage, obtain an excitatory reaction, which will cause a transient diminution of the current of rest. This will be followed, when the rise of temperature is steady, by an increase of the current of rest. I give here (fig. 85) a photographic 
record of these contrasted effects. In the first part of the curve we observe a sudden movement of the record upwards corresponding to the sudden rise of temperature. This is so great as to carry the curve out of the photographic field. We have here, then, a sudden excitatory diminution of the natural

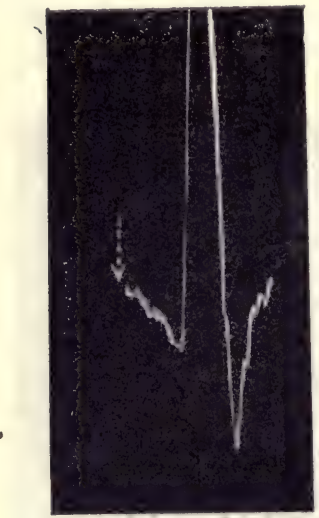

FIG. 85. Photographic Record showing effect of Sudden, followed by steady Rise of Temperature on Natural Current, $\underset{v}{ }$, in Musa

During sudden variation of temperature an excitatory negative variation of natural current takes place, as shown by first up curve; when rise of temperature becomes steady there is a positive variation, as shown by the down curve; on return to surrounding temperature, the normal current is restored to its original value.

current. In the next stage, while the temperature is steadily ascending, we find a reversal of the curve, and the natural current is enhanced above the normal. On now allowing the chamber to cool down to the original temperature of the room, the natural current was found to return more or less to its normal value.

We shall next study the effect of chemical agents on the natural current. The mode of procedure is to apply the given agent on both the contacts at the same time. If the substance be liquid, it can be applied by a pipette. If it be gaseous, the specimen is placed in a chamber through which the gas or vapour is allowed to stream. In observing the effects of various agents we obtain results which are at first sight very perplexing. For example, certain substances will be found to induce a diminution of the natural current, and others an increase. The effect, moreover, is found to be modified by the strength of the dose. Thus an agent which, in a given strength, will cause a diminution of the natural current, may often be found to cause an increase, when sufficiently diluted. This inquiry is of great importance, since it is directly connected with many equally obscure problems in medical practice, where the effect of a drug 
is well known to be modified by the amount of the dose.

Much light appears to be thrown on this subject, when we consider the electrical reactions of the chemical agents as due to their physiological action. If a drop of hydrochloric acid be applied to the pulvinus of Mimosa, the leaf falls, showing that the more excitable side has undergone a greater excitatory contraction. We have also seen that when a drop of this acid is applied on any tissue in the neighbourhood of, but not directly touching, an electrical contact, it induces an excitatory galvanometric negativity. If now we apply it in solution, say, of Io per cent. on the two diametrically opposite contacts of Musa, we shall expect that the greater excitatory reaction induced on the concave side will give rise on that side to a relative galvanometric negativity, resulting in a negative variation of current of rest. On the application of the reagent this is found to be the case, the responsive current flowing in a direction opposite to that of rest: that is to say, it flows from the more excited concave to the less excited convex.

It is by considering chemical agents from the point of view of their physiological reaction, that we are able to explain their diversity of effects, according to the strength of the dose and the duration of application. We have seen that while a strong stimulus induces the excitatory effect of negativity, a feeble stimulus will bring about the opposite, or positivity. This abnormal positive response, however, by the continued action of moderately feeble stimulus becomes converted into normal negative. Now a chemical substance which in a certain strength acts as an efficient excitatory agent, may, when sufficiently diluted, act as a feeble stimulus, inducing a positive response. If the same agent again were applied in a slightly greater concentration, its immediate effect might be positive, to be succeeded under continued application by the normal negative.

We might thus expect, using a strong solution of a given chemical reagent, to obtain a negative variation of the current 
of rest; using a dilute solution, to obtain a positive variation; and, lastly, applying a dose of intermediate strength, to discover the very interesting case in which the reagent would give rise immediately to a positive variation, and after a longer or shorter continuance of its action, to a reversed, or negative variation, of the current of rest. These inductions are found fully verified in the experiment which I am now about to describe.

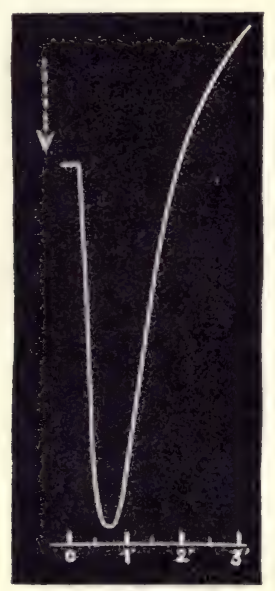

FIG. 86. Action of 7 per cent. Solution of $\mathrm{Na}_{2} \mathrm{CO}_{3}$ on Natural Current of Musa

Preliminary positive variation represented by down curve followed by reversal, 50 seconds after application.

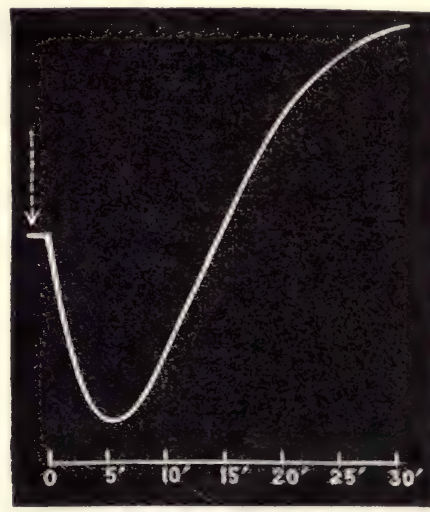

Fig. 87. Effect of $\mathrm{CO}_{2}$ on Natural Current of Musa

Preliminary positive seen to be ${ }^{\prime}$ succeeded by negative variation 5 minutes after application.

Applying a strong solution of sodium carbonate- IO per cent. or above-at the electrical contacts on Musa, the result is a negative variation of the natural current. If now a dilute solution of I per cent. be applied on a similar specimen, we obtain a response by positive variation. And if, lastly, we use a 7 per cent. solution, we obtain, as will be seen from the record (fig. 86) the preliminary positive, succeeded, under the continued action of the agent, by reversal to the negative, variation.

We pass next to the question of the effect of gases. In fig. 87 is given a record of the action of carbonic acid on 
the natural current in Musa. It will be seen here that in the first stage there was an enhancement, or positive variation, of the existing current. In a later stage, however, this is followed by a reversal, the resting-current now undergoing a diminution. We have here an effect parallel to that of the intermediate dose of sodium carbonate. Vapour of alcohol also exerts an effect very similar; that is to say, it induces a preliminary exaltation, followed by a depression of the natural current.

In connection with this subject, of the changes induced in the natural electro-motive difference between the two surfaces, by the action of a chemical reagent, it is well to distinguish between the effects of two different factors: namely, the electrical variation caused by the chemical substance as such, and that brought about by the excitatory reaction. Let us suppose both the electrical contacts to be made on iso-electrical surfaces, with normal saline solution; there will then be no difference of potential, as between the two. But this state of things will be disturbed, by the application of another chemical solution, say acid, on either one of the two contacts. The resulting disturbance may be distinguished as due to heterogeneity of chemical application. But if the same chemical agent be applied at both the contacts, no such chemical heterogeneity will ensue. If, then, any electro-motive difference be induced, it must be due primarily to some induced physiological change. The contact which has been rendered more excitable will become increas. ingly positive; that which is more excited, on the other hand, will become increasingly negative. That the induced electromotive variation under such circumstances is indicative of a variation of excitability or excitation, was seen in the fact that the same chemical agent-for example, $\mathrm{Na}_{2} \mathrm{CO}_{3}$ - caused a positive variation when dilute, a negative when strong, and positive followed by negative under the continued action of an intermediate dose. This conclusion-that the variation of the existing current, by the simultaneous application at the two contacts of the same chemical reagent, is due 
to a physiological reaction, the positive variation being a sign of relatively increased, and the negative of decreased, excitability - will be verified by an independent mode of inquiry, to be described in the following chapter.

It follows from the experimental demonstration which has just been given that the phenomena of the natural current and its variations may be summarised in general as follows :

I. Under normal conditions, the current of rest flows in the tissue from the less to the more excitable. In other words, the more excitable is galvanometrically positive to the less excitable.

2. Increase of internal energy induces an increase or positive variation of the existing current; and diminution of internal energy induces a negative variation.

3. External stimulus induces a negative variation of the true or natural current of rest.

The natural current and its variations under normal conditions have now been studied. We shall next proceed to trace out those conditions under which abnormal results may occur. Excessive cooling, by diminishing internal energy, may thus reverse the normal current, which reversal may become more or less persistent. It has been shown, moreover, that in an anisotropic organ, external stimulus gives rise to a current opposite in direction to the natural current. By this excitatory reaction the more excitable side, hitherto positive, is rendered negative, and if the excitatory reaction be great, it may remain for a considerable period in this reversed condition of galvanometric negativity.

We have seen that under normal conditions, the direction of the natural or true current of rest is from the less to the more excitable, and that external stimulus causes a responsive current in the opposite direction, which thus constitutes a negative variation of the current of rest. This state of things we shall distinguish as the primary condition. It frequently happens, however, in consequence of previous stimulation, with its after-effect, that extremely varied effects, appearing at first very anomalous, occur, with regard to the 
direction, not only of the current of rest, but also of the current of response. We shall be able to obtain a clear understanding of these effects, on subjecting the underlying phenomena to close analysis. As a concrete example, we may take for our investigation the various effects to be observed in the pulvinus of Mimosa.

In this anisotropic organ, the directions of the resultant current of rest and the current of response are determined, as we have seen, by the differential excitability and differential excitation of the two sides of the organ. We shall fix our attention, however, for the sake of simplicity, on the changes which occur in the more effective lower half. I shall here succinctly describe the various post-primary phases in the changing effect, culminating in the onset of fatigue from overstimulation. In the primary condition, as we have seen, the lower half of the pulvinus is positive to the upper, the direction of the resting-current being down $\downarrow$. On stimulation, the lower half becomes negative and the response current is up $\uparrow$. Response thus takes place by a negative variation of the resting-current.

We may now suppose the pulvinus to be in a state of slight excitation, its molecular condition at or about the $\mathbf{B}$ stage. This existing state of moderate excitation will annul the previous positivity, and the current of rest will now be zero. At this stage, however, as we have seen, the excitability of a tissue is enhanced. Hence, on stimulation, the lower half of the pulvinus will exhibit responsive negativity, and the direction of the response current will be up $\uparrow$. We are, however, unable to describe this variation in terms of the current of rest, since that, as we have already seen, is zero.

A condition of still stronger excitation, bringing the tissue to the $\mathbf{C}$ condition, will induce galvanometric negativity of the lower half. The so-called current of rest is thus upwards $\uparrow$. But as the excitability of the lower half is still relatively greater than that of the upper, it follows that external stimulus will bring about a responsive current whose direction is upwards $\uparrow$. This normal excitatory response, 
then, in this particular case, appears as a positive variation of the resting-current.

And, finally, we may imagine the pulvinus to have been strongly excited, so as to be in the condition $\mathbf{D}$ or $\mathbf{E}$. The resting-current will in this case be upwards $\uparrow$. But the excitability of the lower half, owing to fatigue, has now become depressed, a condition which, as we have seen, tends to give rise to the abnormal positive response, the responsive current being thus downwards $\downarrow$. As the modified current of rest is upwards, this abnormal current of response will appear as a negative variation of it.

All these cases are conveniently tabulated as follows :

Tabular Statement of the Relative Directions of the Current of Rest and the Responsive Curkent under Various Conditions.

\begin{tabular}{|c|c|c|c|}
\hline Condition. & Current of rest. & $\begin{array}{c}\text { Current of re- } \\
\text { sponse. }\end{array}$ & Variation of current of rest. \\
\cline { 1 - 2 } \begin{tabular}{c|c} 
Primary condition \\
Feebly excited
\end{tabular} & $\downarrow$ & $\uparrow$ & Negative variation \\
$\begin{array}{c}\text { Moderately excited } \\
\text { Strongly excited and } \\
\text { fatigued. }\end{array}$ & $\uparrow$ & $\uparrow$ & ? \\
\hline
\end{tabular}

The various conditions mentioned may be induced accidentally in the responding organ, or may be brought about by the excitatory effect of experimental preparation. I give here the records of certain experiments performed on Mimosa, in which some of these changes were seen to occur as the result of stimulation (fig. 88). The normal natural current is seen to be from above to below, as represented by the dotted arrow. The first strong stimulus, applied at the moment represented by the thick dot, gives rise to a responsive current whose direction is from below to above. Owing to the strong intensity of the stimulation, there is here a slight indication of multiple response. As an after-effect of stimulus, we observe that the normal restingcurrent has undergone a reversal, the lower surface, which was formerly positive, having now become relatively negative, A second stimulus now gave rise to a response 
similar to the first. But after this, owing to the greater fatigue with loss of excitability induced in the lower half of the pulvinus, the succeeding responses are seen to be reversed, the responsive current being henceforth from above to below.

From the table given on the previous page, it will be seen that hardly could any standard have been devised for the study of excitatory reaction, so likely to be prolific of confusion as this, of the so called variation of the restingcurrent. For in the first three cases displayed, we see one identical excitatory effect, appearing now as a negative, again as doubtful, and a third time as a positive variation of the current of rest. In the fourth case, again, it is actually the abnormal response which appears as the normal negative variation! But while these responses appear to be so various, the underlying reaction is nevertheless constant. The direction of the responsive current is always from the more to the less excited.

It has thus been shown

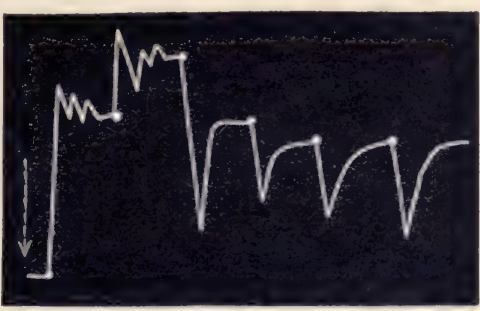

Fig. 88. Variation of the Transverse Natural and Responsive Currents in Pulvinus of Mimosa

Natural current i which is normally down, reversed in consequence of strong external stimulus. The first two responses are normal, i.e. current being from below to above. Strong stimulus is here seen to induce mul. tiple responses. After the second response on account of the greater fatigue induced in the lower half of the pulvinus, the direction of the responsive current is seen to be reversed. Thick dots represent moment of application of stimulus.

in the course of the present chapter that under normal conditions the current of rest flows in the tissue from the less to the more excitable; that increase of internal energy causes a positive variation of the current of rest ; while its diminution gives rise to a negative variation; that reagents which increase excitability induce a positive, and those which cause excitation a negative, variation of the resting-current ; and, finally, that external stimulus induces a negative variation of the resting-current. While these are 
the normal reactions, however, under abnormal conditions, they may be reversed. Thus, excessive cooling or strong external stimulation may reverse the normal current of rest. There are, moreover, two different conditions, those, namely, of sub-tonicity ( $c f$. p. I06) and fatigue, which may be effective in bringing about a reversal of the normal direction of the responsive current. In this way, by means of induced variations of the resting-current and of the responsive current, many very varied effects become possible. 


\title{
CHAPTER XI
}

\section{VARIATIONS OF EXCITABILITY UNDER CHEMICAI.}

\author{
REAGENTS
}

Induced variation of excitability studied by two methods : (I) direct (2) transmitted stimulation-Effect of chloroform-Effect of chloral-Effect of formalin -Advantage of the Method of Block over that of negative variation-Effect of $\mathrm{KHO}-$ Response unaffected by variation of resistance-Stimulating action of solution of sugar-Of sodium carbonate-Effect of doses-Effect of hydrochloric acid-Di-phasic response on application of potash-Conversion of normal negative into abnormal positive response by abolition of true excitability.

IT has been said in a previous chapter that the electrical response is a true physiological response. This is demonstrated by the fact that, while a vigorous specimen gives strong electrical response of galvanometric negativity, the same specimen, when killed, whether by heat or by poison, ceases to respond. This particular electrical response is thus seen to be a concomitant of physiological efficiency. It follows that, whatever diminishes physiological activity will, pari passu, modify the amplitude of the response. But in cases in which the death of the tissue is brought about by steam or by poison, it is the last stage only, namely the abolition of response, that can be observed. It is also important, however, to be able to trace the growth of physiological changes through the concomitant modification of response. In this way it is possible not only to study the gradual onset of death, as induced by a poison, but also the action of other chemical agents, some of which might be of such a nature as to induce exaltation, others depression, and still others, like the narcotics, a temporary abolition of the electrical response. 
An essential condition of this investigation is first to obtain a uniform series of responses. Having once done this, those subsequent changes in the response which are due to the application of a given reagent can be demonstrated in an unmistakable manner. I have already explained in Chapter III. that this may be done by either of two different methods: namely, those of direct and of transmitted stimulation. In the first of these we employ vibrational stimulus, using the Method

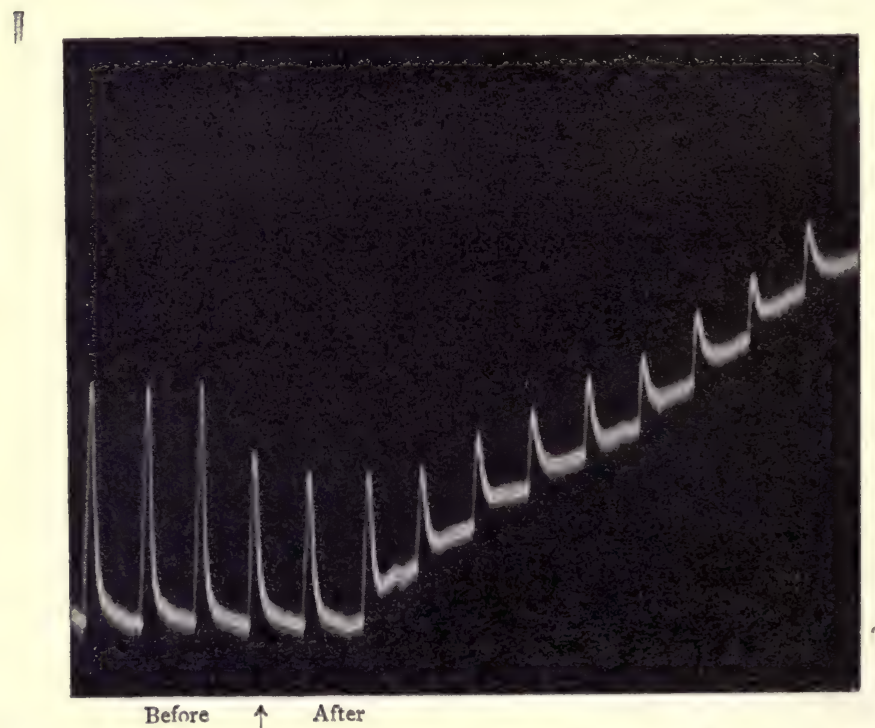

FIG. 89. Photographic Record of Effect of Chloroform on Responses of Carrot Stimuli of $25^{\circ}$ torsional vibration at intervals of one minute.

of Block. In the second, the stimulus of thermal shocks is used, the excitation of the proximal contact being due to transmitted stimulation.

We shall investigate the effect of chemical reagents by both these methods. And first I shall give results obtained by the employment of the Method of Block, the tissue being subject to direct stimulation. In cases where the effect of gaseous reagents, like chloroform, is to be studied, the vapour is blown into the plant-chamber (see fig. 21). In 
cases of liquid reagents, they are applied on the points of contact $\mathrm{A}$ and $\mathrm{B}$, and in their close neighbourhood. The experiment is carried out by first obtaining a series of normal responses to uniform stimuli, applied at regular intervals of time, say one minute, the record being taken the while on a photographic plate. Then, without interrupting this procedure, the given agent-say, vapour of chloroformis applied, by being blown into the chamber. It will be seen from fig. 84 how rapidly chloroform induces depression of response, and how the effect grows with time. If the specimen be subjected for a short time only to the anæsthetic, the depressing action proves transient, passing off on the reintroduction of fresh air. But too strong or too prolonged an application induces a permanent abolition of response.

I give below (figs. 90, 91), two sets of records, one of which shows the effect of chloral and the other formalin.

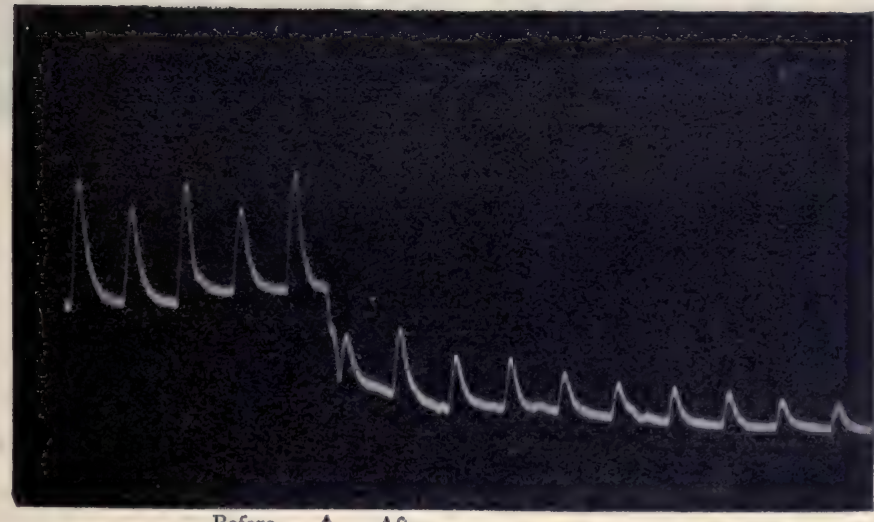

Before $\uparrow \quad$ After

Fig. 90. Photographic Record showing Action of Chloral Hydrate on the Responses of Leaf-stalk of Cauliflower

Torsional vibration of $25^{\circ}$ at intervals of one minute.

These reagents were applied as solutions on the tissue at the two leading contacts and adjacent surfaces. Both are seen to induce a rapid decline of the response. In the 
normal responses, shown in fig. 9I, is seen a very interesting instance of alternating fatigue.

In order to bring out clearly the main phenomena, I have postponed till now the consideration of a point of some difficulty. To determine the influence of a reagent in modifying the excitability of a tissue, we rely upon its effect in exalting or depressing the responsive E.M. Variation, and we read this effect by means of changes induced in the galvanometric deflection. Now as long as the resistance of the circuit remains constant, an increase or decrease of galvanometric deflection will accurately indicate a heightened or depressed E.M. Variation, due to augmented or lowered

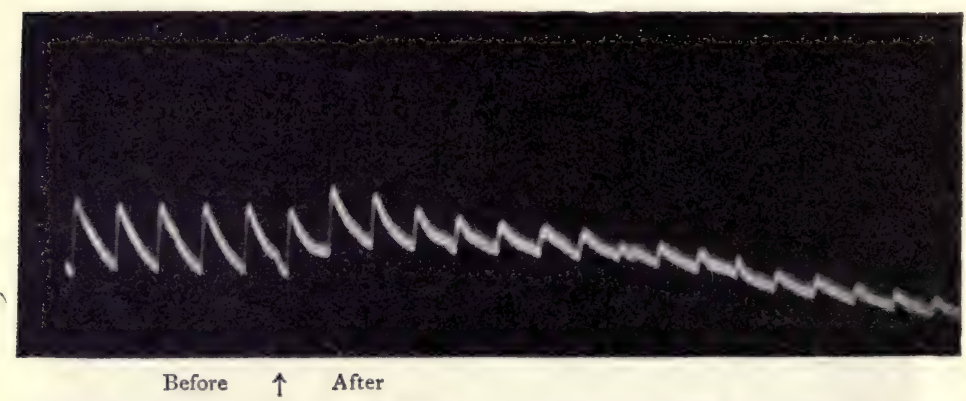

FIG. 9r. Photographic Record showing Action of Formalin (Radish)

excitability, induced by the reagent in the tissue. But by the introduction of the chemical reagent the resistance of the tissue may undergo a change, and, owing to this cause, modification of response, as read by the galvanometer, may be induced without any E.M. Variation: The observed variation of response may thus be partly owing to some unknown change of resistance, as well as to that of the E.M. Variation.

This difficulty may, however, be obviated by interposing a very large and constant resistance in the external circuit. The variation in the tissue then becomes negligible, the galvanometric deflections being now proportional to the electro-motive variation. An actual experiment will make 
this point clear. Taking a carrot as a specimen, I found its resistance plus the resistance of the non-polarisable electrodes to be 20,000 ohms. The application of a chemical reagent reduced this to $19,000 \mathrm{ohms}$. The resistance of the galvanometer used was I,O0O ohms, and the high constant external resistance interposed was I million ohms. The variation of resistance induced in the circuit by the application of the reagent was thus $\mathrm{I}, 000$ in $\mathrm{I}, 020,000$, or less than one part in a thousand.

In studying the variation of excitability in animal tissues, the method of negative variation is employed. But I may here draw attention to the advantage which is afforded by the employment of the Method of Block instead. For, in the method of negative variation, one contact being injured, the chemical reagents act on injured and uninjured unequally. It thus happens that by this unequal action the resting difference of potential is indefinitely altered. But the intensity of response in this method of injury may to a certain extent be dependent on the resting difference. It is thus seen that, when this method is employed, a factor is introduced which may give rise to complications.

According to the Block Method, however, the two contacts are made with uninjured surfaces, and the effect of the reagents on both is similar. Thus no advantage is given to either contact over the other. The changes now detected in the response are therefore due to no adventitious circumstance, but to the reagent itself. If further proof be desired of the effect ascribed to the action of the reagent, we can now obtain it by the alternate stimulation of the two ends $A$ and B. I give below (fig. 92) a record of responses obtained in this way from the petiole of turnip. This petiole was somewhat conical in form, and owing to this difference between the $\mathrm{A}$ and $\mathrm{B}$ ends, the responses given by one were slightly smaller than those given by the other, though the stimuli were equal in the two cases. A few drops of a IO per cent. solution of $\mathrm{NaOH}$ were applied at both ends. The record shows how quickly this reagent abolished the 
response of both. In the next figure (fig. 93) is given a photographic record, showing the marked depression of response induced by a strong solution of $\mathrm{KOH}$, and in order to show that under the given experimental conditions, the variation of resistance does not in any way affect the responses, the deflection produced in the galvanometer by the application of an E.M.F. of 'I volt to the circuit is shown at the beginning and end of the record. The equality of these two deflections shows that the resistance in the circuit has remained practically the same throughout the experiment.

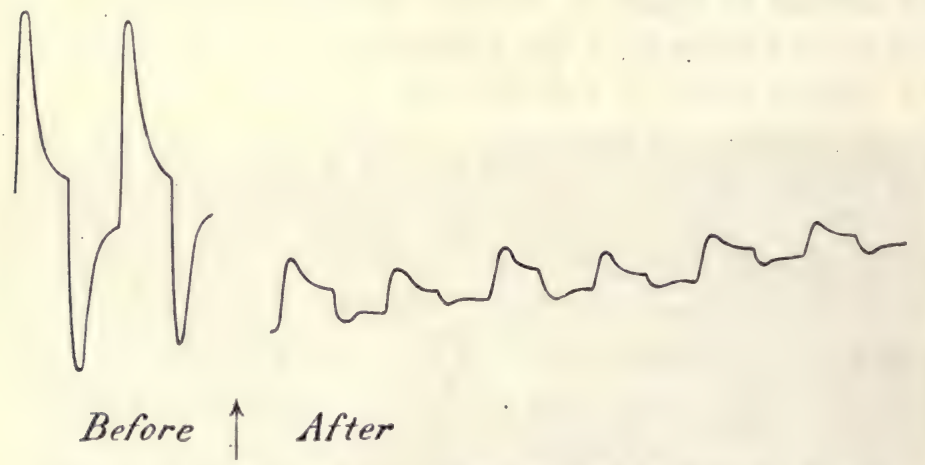

Fig. 92. Abolition of Response at both $\mathrm{A}$ and в Ends by the Action of $\mathrm{NaOH}$

Stimuli of $30^{\circ}$ vibration were applied at intervals of one minute to A and $B$ alternately. Response was completely abolished twenty-four minutes after application of $\mathrm{NaOH}$.

Therefore, the change in the amplitude of the E. M. responses recorded may be taken as due entirely to the variation in the excitability of the tissue.

In the experiments just described, the stimulus was applied directly at the responding point. By the application of a chemical reagent, not only was the responsive excitability of the tissue modified, but its receptivity, or power of receiving stimulus, also underwent a change. It will be shown later that the receptive excitability and the responsive excitability are not necessarily the same. The records which have just been given show what is, strictly speaking, the 
effect of the reagent on both receptivity and responsivity jointly.

If, however, we wish to study the effect of the reagent on responsive excitability alone, it will be necessary to separate the receptive from the responding point, and apply the reagent on the latter. This may be done by the method of transmitted stimulation described previously. Successive uniform

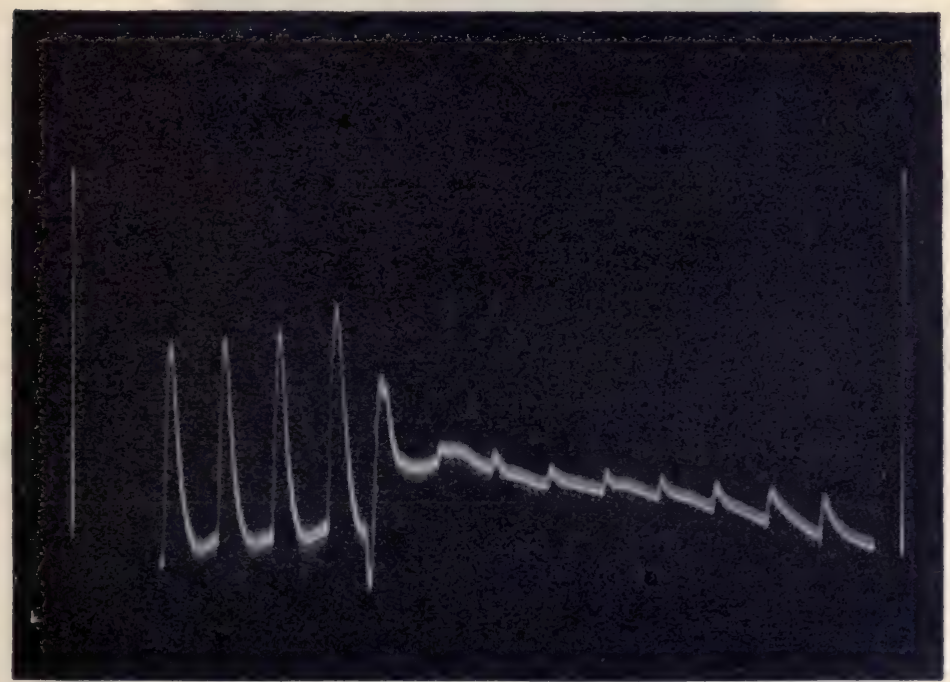

Before $\uparrow$ Afier

FIG. 93. Photographic Record showing the nearly complete Abolition of Response by strong $\mathrm{KOH}$

The two vertical lines are galvanometer deflections due to 1 I volt, before and after the application of reagent. It will be noticed that the total resistance remains unchanged.

stimuli applied at a given point cause excitatory response at the separate responding point, the record of which is taken ; after this, the chemical reagent is applied locally at the responding point. It will be seen that the receptive excitability and the conductivity of the intervening tissue remain unaffected, changes being induced at the responding area alone.

I shall now describe effects obtained by this method. 
The specimen employed was the petiole of fern. The thermal stimulator was at a distance of $\mathrm{I}^{\cdot} 5 \mathrm{~cm}$. from the proximal electrode. In fig. 94 is shown the stimulating action of a

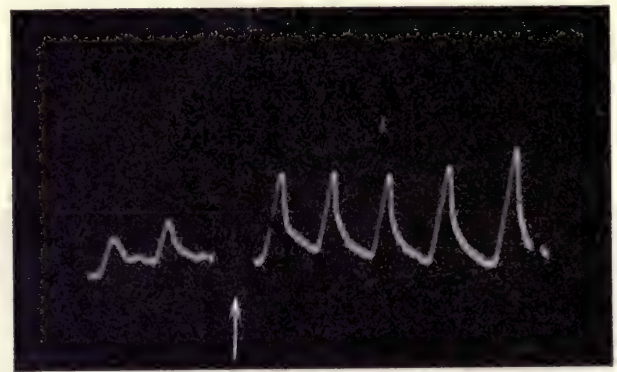

FIG. 94. Photographic Record showing the Stimulatory Action of Solution of Sugar

2 per cent. solution of sugar, inducing a continuous enhancement of response for some time.

Another stimulating agent is a dilute solution of $\mathrm{Na}_{2} \mathrm{Co}_{3}$. This when applied in I per cent. solution induces an

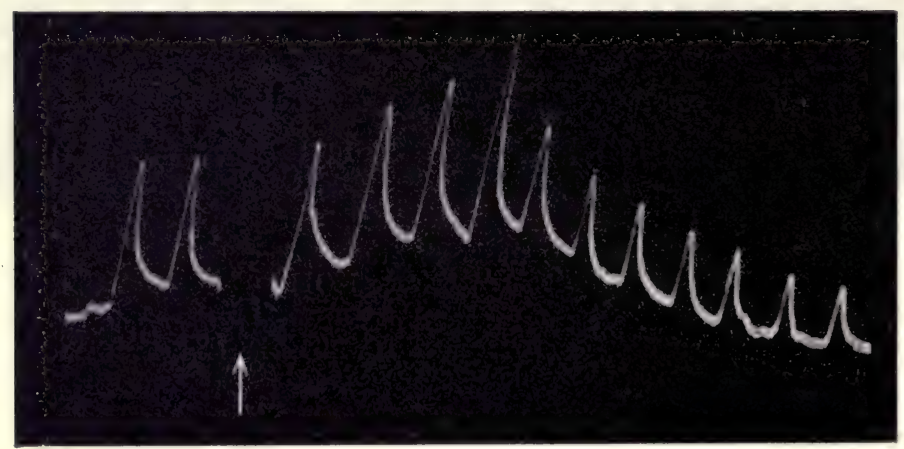

FIG. 95. Photographic Record showing Continuous Action of 2 per cent. $\mathrm{Na}_{2} \mathrm{CO}_{3}$ Solution

Preliminary exaltation followed by depression.

enhancement of amplitude of response, but when given in strong solution, induces depression. An intermediate strength of solution shows preliminary enhancement followed by depression (fig. 95). 
While pursuing another line of inquiry on the effect of various strengths of solution of $\mathrm{Na}_{2} \mathrm{Co}_{3}$ on the natural current, I obtained results which were parallel (p. I22). It was there shown that dilute solution of $\mathrm{Na}_{2} \mathrm{Co}_{3}$ induced a positive variation of the natural current; a strong solution, a negative variation, and that a solution of intermediate strength induced a preliminary positive followed by a negative variation. Thus the positive variation in the last-named experiments, already shown to be indicative of increased excitability, was here seen to correspond with heightened amplitude of response, while the negative variation on the other hand is seen to coincide with depression of excitability. The application of a strong solution inducing excitation, carries the molecular condition of the tissue to the stage $\mathbf{E}$, where, as we know, the excitability is depressed.

Another fact elucidated by this and similar inquiries, which I have pursued elsewhere, ${ }^{1}$ lies in the fact that the difference between stimulants and poisons, so called, is often one merely of degree. Thus a stimulatory reagent, if given in large quantities, will be found to induce a profound depression, whereas a poisonous reagent in minute quantities may be found to act as a stimulant. In carrying out a similar investigation with regard to growth response, I found that sugar, for instance, which is stimulating in solutions of, say, I to 5 per cent., becomes depressing when the solution is very strong. Copper sulphate again, which is regarded as a poison, is only so at I per cent. and upwards, a solution of - 2 per cent. being actually a stimulant. The difference between sugar and copper sulphate is here seen to lie in the fact that in the latter case the range of safety is very narrow. Another fact, which must be borne in mind in this connection, is that a substance like sugar is used by the plant for general metabolic processes, and thus removed from the sphere of action. Thus continuous absorption of sugar could not for a long time bring about sufficient accumulation to cause depression. With copper sulphate, however, the case

1 Bose, Plant Response, p. 488. 
is different. Here, the constant absorption of the minimal stimulatory dose would cause accumulation in the system, and thus ultimately bring about the death of the plant.

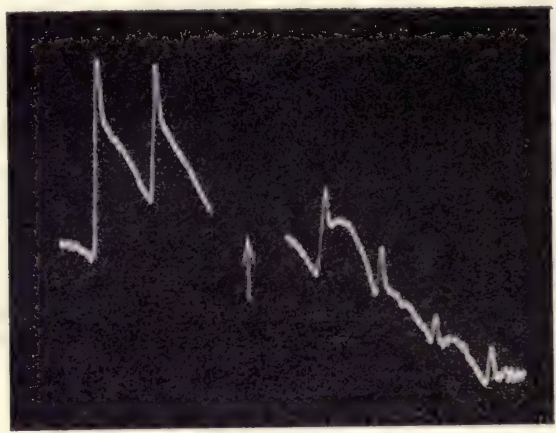

FiG. 96. Photographic Record showing the Depressing Action of 5 per $\mathrm{c} \in \mathrm{nt}$. $\mathrm{HCl}$ Acid

The effect of very dilute acids is often to induce an enhancement of excitability, while strong solutions induce depression and abolition. In fig. 96 is shown the depression

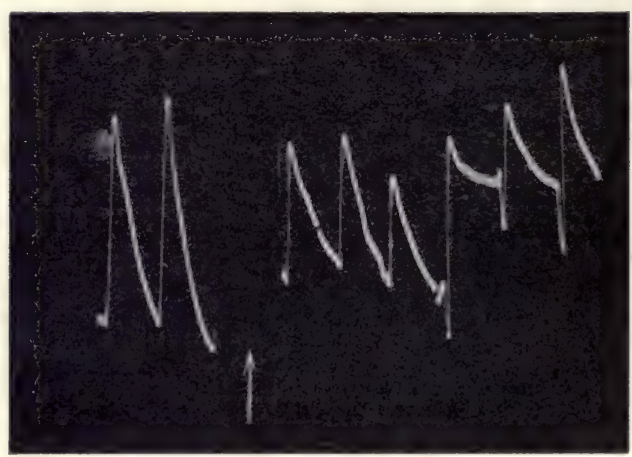

FIG. 97. Photographic Record showing Effect of I per cent. KHO

Note the preliminary positive twitch at the fourth response after application.

and abolition induced by the application of a 5 per cent. solution of hydrochloric acid.

In dealing with the question of electrical response, we have seen that two opposed electrical effects occur in the 
tissue subjected to stimulation. One of these is the positive effect, and the other, the true excitatory change of galvanometric negativity. As the latter is, under normal conditions, predominant, the simultaneous effect of both is a resultant negativity. The positive effect may, however, be unmasked, as we have seen, by abolishing the true excitatory effect of negativity (p. 66). This positivity may also be unmasked, if, by the action of a chemical reagent, the time-

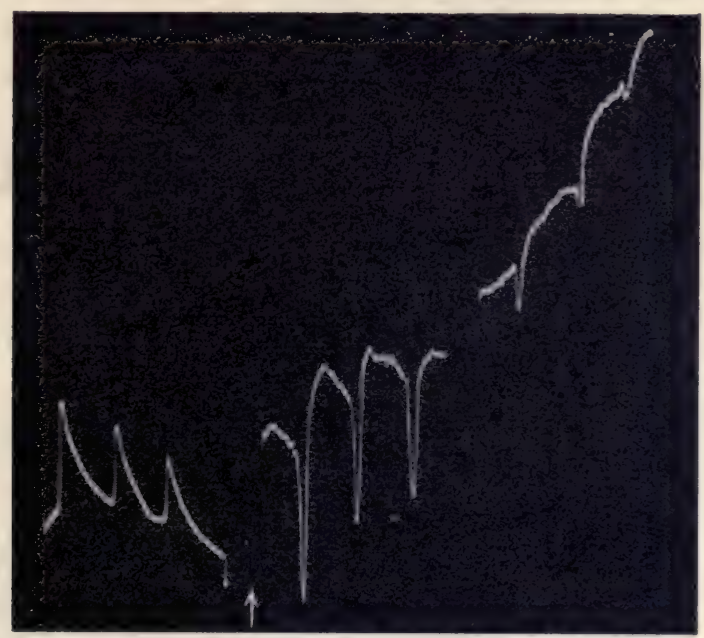

FIG. 98. Photographic Record of Effect of 5 per cent. KHO

Note the complete reversal of response to positive at the beginning, and its subsequent abolition.

relations of the two responses are changed, so that instead of occurring simultaneously, the one is made to lag behind the other. This case will be seen very strikingly illustrated in fig. 97, which exhibits the effect of a I per cent. solution of $\mathrm{KHO}$, on response to transmitted stimulation in the petiole of fern. In the normal responses here given, we observe the resultant response of galvanometric negativity. The application of KHO is first seen to reduce the excitability, as indicated by the reduced height of the responses. Later, we observe that the true excitatory effect is delayed. 
Hence the positive effect is no longer completely masked. Its existence is now seen as a preliminary downward twitch in a di-phasic response, in the case of the fourth and succeeding records, after the application of KHO. In fig. 98, a stronger, namely a 5 per cent. solution of $\mathrm{KOH}$, was used. And here, by the almost complete abolition of the excitatory factor, the response has undergone an apparent conversion to positive; this positive response is, however, subsequently abolished by the death of the plant. 


\section{CHAPTER XII}

VARIATIONS OF EXCITABILITY DETERMINED BY METHOD OF INTERFERENCE

Arrangement for interference of excitatory waves-Effect of increasing difference of phase-Interference effects causing change from positive to negative, through intermediate di-phasic-Diametric balance-Effect of unilateral application of $\mathrm{KHO}-$ Effect of unilateral cooling.

I HAVE explained how the variations of excitability brought about by various agencies may be determined, by recording the corresponding amplitudes of response. I shall now proceed to describe a new and interesting method of making such determinations, by means of which it will be found possible to elucidate certain questions which without it must remain obscure. This method is, moreover, of extreme delicacy, enabling the investigator to detect the slightest variation of excitability, induced by any agent.

Let two points in the experimental tissue, say A on the right, and $\mathrm{B}$ on the left, be suitably connected with the galvanometer, and let the occurrence of excitation at $\mathbf{A}$ on the right be represented by an ' $u$ p' response record, the excitatory effect at $B$, on the left, being represented as 'down.' If now the two points, $A$ and $B$, be excited simultaneously, the resultant electrical response will be due to the algebraical summation of the two excitatory electro-motive effects $E_{A}$ and $E_{B}$, these standing for the individual electrical effects at the two points A and B. Now if the intensities of the two effects be the same, and if their time-relations be also the same, it is evident that these two excitatory electrical waves, being of equal amplitude and having the same phase but of opposite signs, will, by their mutual interference, neutralise 
each other. Under such balanced conditions, therefore, on simultaneous excitation of $\mathrm{A}$ and $\mathrm{B}$, the resultant response will be zero. If now, under the modifying action of any external agency, the excitability of $\mathrm{A}$ be enhanced, it is clear that the resultant response will be 'up,' showing the greater excitability of the right-hand point. A similar effect will also be produced if the excitability of $B$ be depressed. Similarly the depression of the excitability of A, or enhancement of $B$, would cause a resultant response which would be 'down.' If, again, the two waves of excitation be not of the same phase, we shall obtain various di-phasic effects resulting from the algebraical summation of the constituent responsecurves. The resultant zero-response may thus be converted into di-phasic, by the action of any agency which is capable of changing the time-relations of either of the constituent responses.

I shall now proceed to describe the experimental arrangements by which two points in connection with $\mathrm{E}$ and $\mathrm{E}^{\prime}$ may be excited, and the resulting electrical disturbances made to interfere with each other. For this purpose we may use the vibrational stimulation which has already been described, with certain necessary additions (fig. 99). The angle of torsional vibration which regulates the intensity of excitation is determined by two stops, $P$ and $Q$. An elastic piece of brass, $\mathrm{B}$, projects from the torsion-head. When a single stroke is given to this, a quick to-and-fro vibration is induced, the backward pull being supplied by the attached spring, $\mathrm{s}$. The amplitude of this vibration remains always the same, as determined beforehand by the setting of the stops $\mathrm{P}$ and $\mathrm{Q}$. The stroke is given by the striking-rod $\mathrm{R}$, set in motion by the turning of a handle. What has already been said about the excitation of the right-hand side of the specimen applies equally to the left-hand, arrangements for the purpose being a duplicate of those just described. After deciding on a suitable angle of torsional vibration for the right, and taking the response at that point, we proceed to adjust the torsional angle on the left, so that the response there may be exactly 
the same as that on the right. If the excitability of the two points had been exactly the same, equal amplitudes of vibration would have resulted in the equal stimulation of both. But in practice the excitabilities are found to be slightly different and the angle of vibration of the one must, therefore, be so adjusted as to induce an excitatory effect exactly equal to that of the other.

The two striking-rods, one on the right, $\mathrm{R}$, and the other on the left, $R^{\prime}$, can be adjusted so that both are in the same

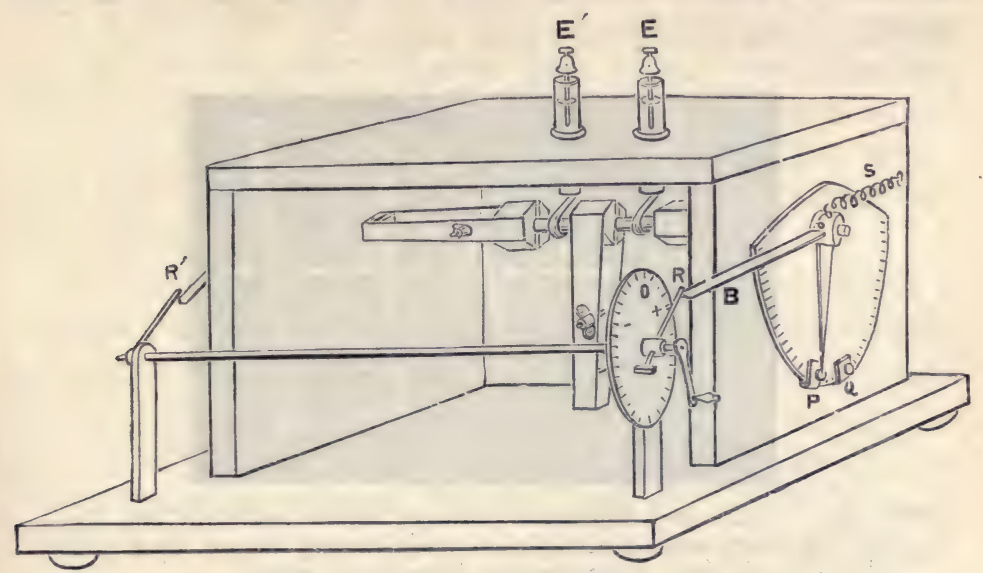

FiG. 99. R, R', striking-rods for stimulation of two ends of specimen; B, elastic brass tongue projecting from torsion-head. For producing phase-difference $R$ is adjustable in azimuth.

vertical plane, or so that one is in advance of the other. The left rod is permanently fixed to the rotating axis, but the right can be set at any angle that is desired, with the other. When the right striking-rod is set, pointing to zero of the scale, the two rods are in the same vertical plane, and the rotation of the handle causes equal vibrational stimulus by the two at the same moment. The excitatory reactions on right and left are now, therefore, of the same phase and of equal intensity, but opposed to each other. In fig. I00, $a$, are reproduced the two separate and equal constituent responses given by a specimen of stem of Amaranth. The 
'down' curve was given by the individual excitation of the left, and the 'up' by the right. On the simultaneous excitation of the two points, the resultant response was zero (b). But if the excitation of one-say, the right-be increased by increasing the angle of vibration, the resultant differential response is found to be 'up.' It is obvious that a similar effect would have been observed had the stimulation of the right been kept the same, while its excitability was increased by any external agent. In these cases we have two opposed excitatory waves of similar phase, and of the same or unequal intensities, interfering with each other.

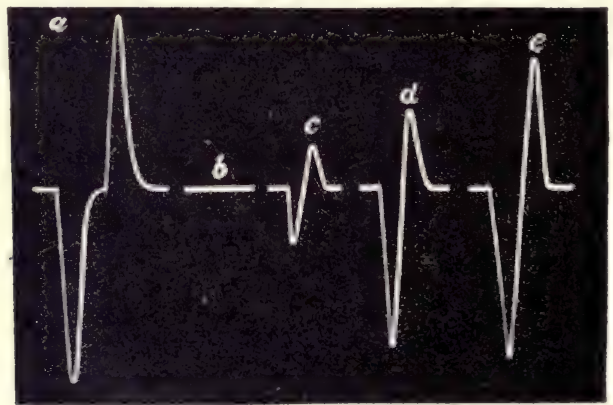

FIG. Ioo. (a) Isolated response of left side (down) and right side (up); $(b)$ null-effect when excitations are simultaneous; $(c),(d),(e)$ di-phasic responses obtained with increasing difference of phase.

We shall next take some simple instances in which, while the stimulation is maintained constant, there is an increasing difference of phase. If the right-hand striking-rod $\mathrm{R}$, instead of being set at zero, be set to the right, or at a plus angle, the rotation of the handle will cause a slightly earlier excitation of the right than of the left. If, on the other hand, the rod be set at a minus angle, the excitation of the right will be later than that of the left. Under these circumstances, instead of the null-effect due to continuous balance, we shall have a di-phasic response. It is also clear that as the phase difference is increased, the neutralisation of effects will become. less and less perfect, the separate constituent responses being thus rendered increasingly apparent. In fig. $100, c$, 
is seen the di-phasic effect which was induced when the excitation of the right was made to lag slightly behind that of the left, by the adjustment of the striking-rod at a small minus angle. The first of the two twitches, which is downwards, indicates the relatively earlier excitation of the left-hand contact. As the phase-difference was increased progressively as in $(d)$ and $(e)$, it is seen that the constituent elements of the di-phasic response are increased correspondingly. It is also clear from this that, having obtained the null-effect, if any agents were afterwards applied locally which would make the excitation of the one point earlier than that of the other, we must then expect the null-effect to be modified to di-phasic. An earlier ' up' twitch would now indicate that the right-hand contact, having had its reaction quickened, was the first to respond ; an earlier 'down' twitch the opposite.

We thus see how the conversion of the null-effect into a resultant 'down' negative or 'up' positive, could be utilised as a test of the excitatory or depressing nature of a given reagent. We also see how the conversion of this null into a di-phasic effect would give us indications as to the change of time-relations induced by the reagent. I shall here, before going on to describe the results obtained with plants, give a photographic record (fig. IOI) of certain positive, negative, and di-phasic effects obtained in the electrical response of the inorganic substance, tin, under appropriate modification of the excitability of its two contacts by various chemical reayents. ${ }^{1}$

Turning now to the question of the determination of the effects of the various reagents by the Method of Interference, we may, as we have seen, cause simultaneous excitation of right and left, by means of the apparatus which has just been described, and which I shall distinguish as the Longitudinal Balance. There is, again, another and simple method of accomplishing the same object, by means, namely, of the Diametric Balance, the diagram of which has already

${ }^{1}$ Bose, Response in the Living and Non-Living, p. I 5 . 
bee $\mathrm{n}$ given in fig. 80 . Using this arrangement, the specimen is clamped at one end, the vibration-head being at the other. Electrical connections are now made with the two diametrically opposite points, A and B, of which one, say A, is the upper, and $\mathrm{B}$ the lower. In a tissue which is isotropic, vibrational stimulus will induce equal and simultaneous excitation at the two points $\mathrm{A}$ and $\mathrm{B}$. The effect of any given agency is tested by applying it locally, say at A, and observing the resultant variation of the response. I shall

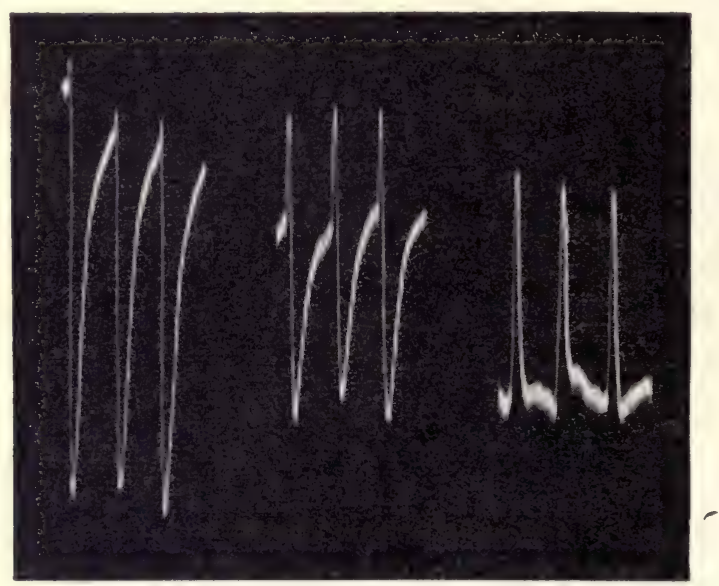

(a)

(b)

(c)

Fig. IOI. Photographic Record showing Negative, Di-phasic, and

Positive Resultant Responses in Tin under appropriate modifications of excitation of the two contacts

here give examples of results obtained by both these methods, thus affording an indication of the extent of their applicability in various investigations.

We have seen in the previous chapter that the application of strong solution of potash will abolish the excitability of a tissue. Using the Longitudinal Balance, I took a petiole of Bryophyllum and first made such adjustments that the right 'up' and left 'down' responses were almost equal. On now producing simultaneous excitation of the two ends, a di-phasic response was obtained, due to the fact that the left-hand point was the quicker to respond. Strong solution 
of potash was next applied on the right-hand point, and from the record it is seen that the 'up' part of the di-phasic response, due to the excitation of the right-hand side, was thus completely abolished, the 'down' response being at the

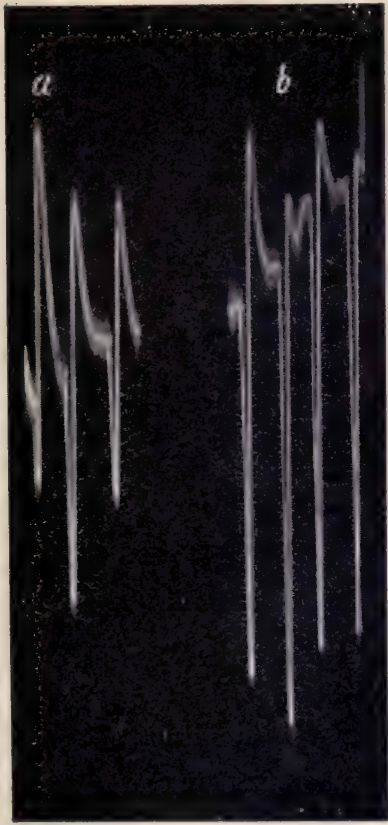

FIf. 102. Photographic Records.

(a) Di-phasic response of petiole of Bryophyllum, the up component being due to the excitation of right side. Strong application of $\mathrm{KHO}$ on the right abolished this responsive component, giving rise in $(b)$ to enhanced down response same time increased by the suppression of this opposing response (fig. $\mathrm{IO} 2$ ).

In order to demonstrate the use of the Diametric Balance Method, I undertook to investigate by its means the influence of the lowering of temperature on excitability. For this

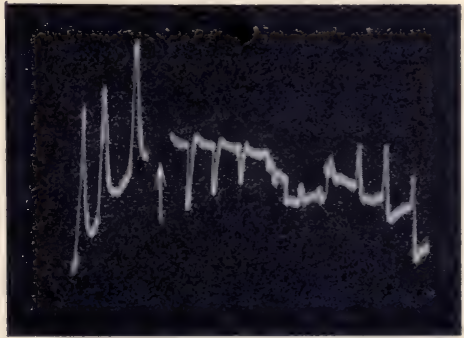

FIG. I03. Photographic Record of Response of Petiole of Cauliflower by the Diametric Method

A contact was naturally more excitable, hence resultant ' up'-response. Excitability of A being depressed by local application of ice, the resultant response became converted to 'down'; normal 'up'-response was restored on allowing the tissue to return to surrounding temperature.

purpose, I took a petiole of cauliflower. In this instance, the natural excitability of the upper contact, A, was greater than that of the lower, B. Hence the resultant response was not zero, but 'up.' The point A was now cooled locally by ice. This process so lowered its excitability that that of $\mathrm{B}$ was now relatively the greater, hence the resultant response was found to be reversed or 'down.' The point A was next 
allowed to return to the surrounding temperature of the room, records of the response being taken meanwhile, at intervals of one minute. It will be seen how, by means of the gradual restoration of the original excitability of $\mathrm{A}$, the resultant response changes gradually from negative to zero, and then again from zero back to positive, indicating the restoration of the naturally greater excitability of A (fig. IO3).

We have thus studied two different methods, both of which depend on interference, for the determination of the variations of excitability induced by different external agents. In a subsequent chapter we shall study a modification of this method, by means of which it is possible to demonstrate the variations not only of excitability but also of conductivity under various reagents. 


\section{C.HAPTER XIII}

\section{CURRENT OF INJURY AND NEGATIVE VARIATION}

Different theories of current of injury-Pre-existence theory of Du BoisReymond-Electrical distribution in a muscle-cylinder-Electro-molecular theory of Bernstein-Hermann's Alteration Theory-Experiments demonstrating that so-called current of injury is a persistent after-effect of overstimulation-Residual galvanometric negativity of strongly excited tissueDistribution of electrical potential in vegetable tissue with one end sectioned-Electrical distribution in plant-cylinder similar to that in musclecylinder-True significance of response by negative variation-Apparent abnormalities in so-called current of injury- 'Positive' current of injury.

IF a section be made of an uninjured nerve or muscle, the transverse contact will be found to be galvanometrically negative, as compared with an uninjured longitudinal contact. I shall have occasion in the present chapter to give a simple explanation of this phenomenon and of the excitatory negative variation of the current of injury. It is, therefore, only necessary to recapitulate briefly the three theories which have hitherto been proposed on this subject.

The Pre-existence Theory of Du Bois-Reymond supposed that the smallest particle had the same electro-motive characteristics as the entire tissue, each such electro-motive molecule consisting of two bi-polar portions, the positive poles of any two molecules being always face to face with each other. This theory was based upon the fact that a muscle-cylinder, for example, exhibited a peculiar distribution of electrical tension. There are in such a cylinder, one longitudinal and two transverse surfaces. Midway in the cylinder is the equatorial zone of the longitudinal surface, and this zone is positive to all the rest. Thus the electromotive difference between one electrode placed on the 
equator, and the other, is increased as the latter is moved further and further away, say towards the right transverse section. The distribution

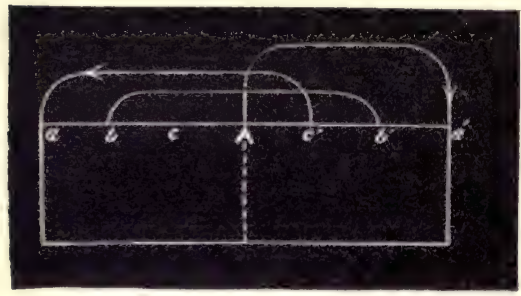

FIG. 104. Distribution of Electrical Tension in Muscle-cylinder. of electrical tension on the left side of the equator is symmetrical with this (fig. I04). On these facts was based the theory of $\mathrm{Du}$ BoisReymond; but this has since been found to be inadequate. I shall later return to the explanation of the particular distribution of electrical tension involved.

According to the theory of Bernstein, known as the Electro-chemical Molecular Theory, the fundamental attribute of the molecule is chemical. Its poles are supposed to attach to themselves electro-negative groups of atoms, while its sides attach oxygen, and stimulation is supposed to be attended by explosive chemical changes.

According to Hermann's Alteration Theory, finally, all the electro-motive activities of living tissues are supposed to be due to chemical rather than molecular changes of the substance. In amplification of this theory, Hering attributes all electromotive phenomena to the disturbance of equilibrium by up and down chemical changes.

It is my intention to show in the course of the present chapter that the current of injury is an after-effect of over-stimulation. And since excitation is fundamentally due to molecular upset, we shall best understand the electro-motive changes concomitant with it, if we first study it and its after-effect under the simplest conditions, namely those of inorganic substances. For here the action of such complicating factors as assimilation and dissimilation is clearly out of the question.

We have found for example that a piece of well-annealed wire was iso-electric throughout its length. In the first 
place, when a portion of it was subjected to any molecular disturbance, an electro-motive difference was induced, as between the molecularly disturbed or excited and the undisturbed areas. The intensity of this electro-motive change, in the second place, was seen to increase with intensity of excitation. And, thirdly, the recovery from excitation was seen to be delayed, where the intensity of stimulus was strong (fig. I05). This is shown in the electrical

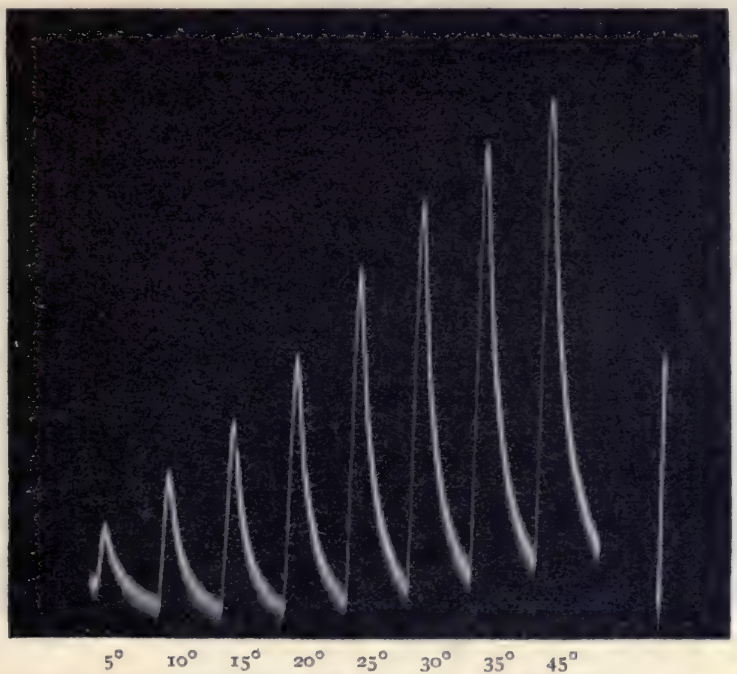

FIG. 105. Photographic Record showing Persistent Electrical After-Effect in Inorganic Substance under Strong Stimulation. Note the tilt of base-line upwards

The vertical line to the right represents $\cdot I$ volt.

response of tin as a persistent after-effect, the sign of which is the same as that of the excitatory electro-motive change.

A similar state of things is exhibited mechanically in a torsioned wire. When the torsion is moderate, and the molecular distortion slight, the released wire quickly recovers its original position of equilibrium. But when the torsion is excessive and the wire strained beyond a certain limit, it remains for a long time in a torsioned condition, 
even after it has been set free. Recovery is thus, in such a case, indefinitely delayed. In other words, a molecularly over-strained substance exhibits a persistent after-effect.

Turning next to plant response, we find a similar persistence of the after-effect to occur in consequence of overstimulation. And first we shall take the simplest casethat in which the tissue is directly stimulated. Here the specimen was petiole of cauliflower, and increasing stimuli

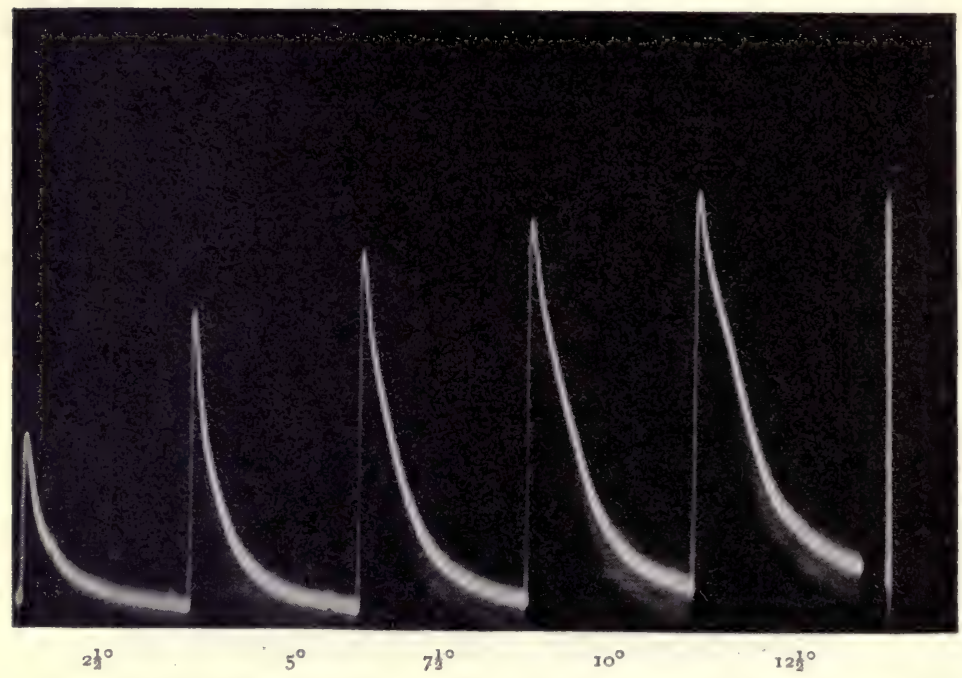

FIG. 106. Photographic Record exhibiting Persistent Galvanometric

Negativity in Plant Tissue after Strong Stimulation

Stimuli applied at intervals of three minutes. Vertical line $=\cdot I$ volt.

were applied, at intervals of three minutes, by means of a gradually increasing angle of torsional vibration. It will be noticed that whereas the electrical recovery from moderate stimulation - as seen in the first of the series-is complete, it becomes, with increasing stimulus, more and more incomplete (fig. I06). In other words, the tissue, after strong stimulation, is seen to exhibit an after-effect of residual galvanometric negativity, which is really due to incomplete molecular recovery, in consequence of over-strain.

In the cases just given, stimulation was applied directly. 
We shall now, however, take an instance in which excitation is transmitted and observe the persistent negative after-effect, due to strong stimulation. We know that a cut (mechanical section) or the application of a hot wire (thermal section) acts as a strong stimulus, and the effective intensity of such stimulation will obviously decrease with increasing distance from the point of stimulation. Hence, if we observe the persistent excitatory change of galvanometric negativity, which is induced as between an indifferent point-say, the surface of a leaf-and points increasingly near to the zone of section, we shall find that the electro-motive change is greatest at the point of section, and is progressively lessened as we recede from it. This induction may be verified experimentally by taking readings of the persistent negativity, as between an indifferent point, B, and points such as the contacts $a, b, c, d$, A (fig. I07), which are further and further removed from the point of section. For this purpose we may employ a capillary electrometer, whose indications are independent of the varying resistance of the interposed tissue. The magnifying power of the observing microscope was so adjusted that ' I volt gave a reading of Ioo divisions of the micrometer. In carrying out an experiment on the leaf of Colocasia I found the electrical distribution, as between an indifferent point on the lamina and points on the sectioned petiole, at increasing distances from the section, to be as shown in the following table :

Table showing Electrical Distribution in Specimen of Colocasia.

[The sectioned end was negative. 100 divisions $=\cdot \mathbf{I}$ volt.]

\begin{tabular}{|c|c|}
\hline Distance from section & $\begin{array}{c}\text { E. M. difference between indifferent } \\
\text { and given points }\end{array}$ \\
\hline $.5 \mathrm{~cm}$ & 50 divisions \\
\hline I $\quad$, & $40 \quad$, \\
\hline $2 \quad 3$ & 33 \\
\hline 3, & 29 \\
\hline $4 \quad 9$ & 27 \\
\hline
\end{tabular}

It will thus be seen that points near the sectioned end are more negative than others further away. 
I shall next describe the more sensitive galvanometric method of investigation. The resistance is here maintained constant by having the permanent contacts at $\mathrm{A}$ and the indifferent point B (fig. IO7). The specimen is a stem of Calotropis gigantea. A thermal section is made at first, say, at a distance of $3 \mathrm{~cm}$. from $\mathrm{A}$. The persistent galvanometric negativity of A will now be due to the after-effect of stimulation by section. The thermal injury is now repeatcd at

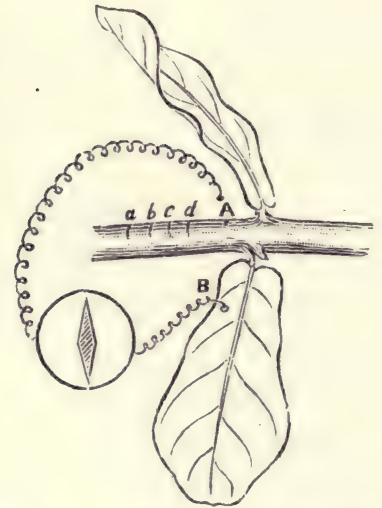

Fig. 107. Experimental Arrangement for determining Electrical Effect due to Section

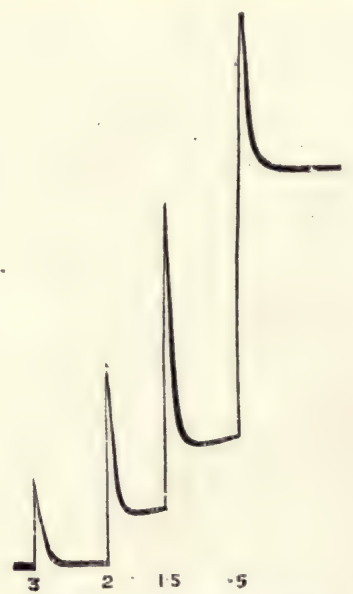

FIG. 108. Records showing increasing Persistent Galvanometric Negativity, according as injury is caused nearer to proximal contact A, i.e. moved from 3 to $5 \mathrm{~cm}$. distance

decreasing distances from A. I give a series of records (fig. I08), from which it will be seen that when the stimulus of thermal section occurs at some distance, there is no persistent after-effect, recovery being complete. But as the injury is made nearer and nearer $\mathrm{A}$, the permanent aftereffect becomes greater and greater. From observations made in the course of a similar experiment, I obtained the following results, given in tabular form, which show the increasing value, with lessening distance, of this persistent galvanometric negativity. 
Table showing Permanent Galvanometric Negativity at DifFERENT Distances From POINT OF INJURY.

\begin{tabular}{|c|c|}
\hline Distance from section & Galvanometric negativity \\
\hline $.25 \mathrm{~cm}$. & 220 divisions \\
\hline 5, & $180 \quad$, \\
\hline 1.0, & I 20 \\
\hline $1 \cdot 5$, & 75 \\
\hline 20, & 40 \\
\hline $3 \circ$, & ," \\
\hline
\end{tabular}

In fig. Iog we have a curve which illustrates these results, and explains why the maximum negativity is at the zone of section, diminishing rapidly as we recede from it. It is obvious that if these sections had been made to the right as

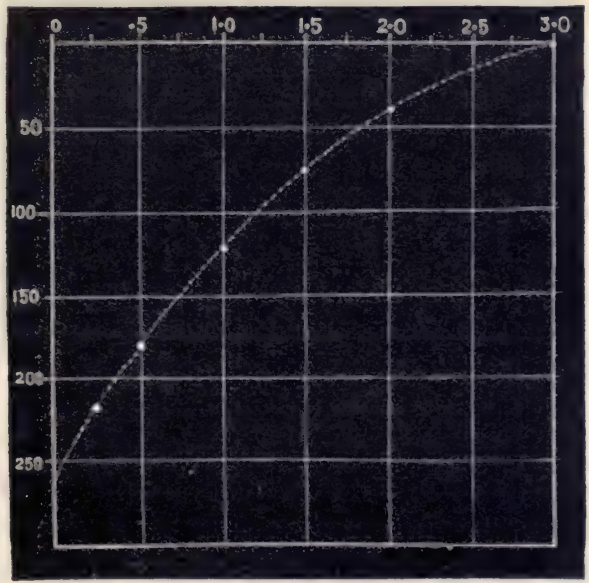

FIG. 109. Curve showing the Electrical Distribution in Stem with one Sectioned End

Ordinate represents galvanometric negativity; abscissa, the distance from sectioned end.

well as to the left of $\mathrm{A}$, the result would have been a duplicate series of changes of galvanometric negativity in reference to $\mathrm{A}$, on that side also. Such a series is represented in fig. I IO, by means of dotted lines. It will also be seen from this figure that the greatest electro-motive difference exists 
as between the equatorial point $\mathrm{A}$ and the two terminal sections $a$ and $a^{\prime}$; that symmetrical points $c c^{\prime}, b b^{\prime}, a a^{\prime}$, are equipotential ; and that a point relatively nearer the terminal section is galvanometrically negative, in reference to one further away from it. It will also be seen that this electrical distribution is exactly the same as that seen in a musclecylinder, with terminal sections, as given in fig. IO4.

Thus, without the postulation of any electro-motive molecules so-called, these experimental results afford a simple and direct explanation of the so-called current of injury, as the excitatory after-effect of strong stimulation.

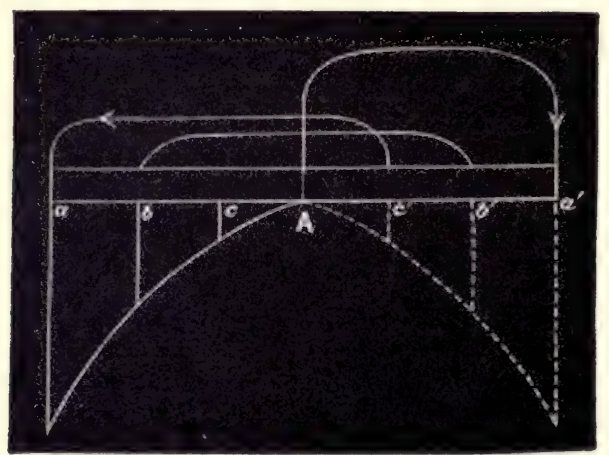

FIG. I IO. Electrical Distribution in Plant-cylinder with Opposite Ends Sectioned

The Current of Injury is simply therefore an excitatory after-effect, due to incomplete recovery from over-strain. But even after strong stimulation a slow recovery may occur, and the Current of Injury will thus undergo a progressive diminution. This will probably account for Engelmann's observation that in medullated nerves the E.M.F. of the artificial cross-section fell, by as much as from 25 to 60 per cent., in the first two hours after section, and disappeared altogether within twenty-four. The renewal of the crosssection he found to renew the original difference. This is an obvious case of renewal of the effect by re-stimulation.

The disappearance of negativity, owing to recovery, only 
takes place when the injury has not been excessive. If, however this has been too great, the injured tissue will then pass gradually into a condition of permanent death. But the electrical change concomitant with death is one of positivity, as I shall show in the next chapter. Thus the subsidence of the galvanometric negativity of an injured point may be brought about by either of two processes, which are exactly opposite-namely, recovery or death.

Turning next to the subject of the Negative Variation of an existing current of rest, as a reliable index to the state of excitation, two different questions arise. First: why, in order to obtain response to diffuse stimulation, is it necessary previously to subject one of the contacts to injury? And secondly: why is the responsive action-current opposite in direction to the resting-current, thus constituting a negative variation of it?

With reference to the first of these questions, we have already seen that when two points, $\mathrm{A}$ and $\mathrm{B}$, are simultaneously excited, the resultant electro-motive response is equal to $E_{A}-E_{B}$. If, then, the excitabilities of these two points are the same, it is clear that the resultant response will be zero. From this we can see that, in order to obtain a resultant response, we must depress or abolish the excitability of one of the two contacts.

This inference may be verified by the employment of the Method of Block and of longitudinal balance. Two equal and opposite responses are first obtained at $\mathrm{A}$ and $\mathrm{B}$. Then one end, say B, is injured by thermal section. The specimen being now replaced in the vibratory apparatus, it is found that, whereas the A half gives strong response, the end $\mathrm{B}$ gives none. Or the $\mathrm{B}$ end of the specimen may be injured by a few drops of strong potash, the other end remaining uninjured. The end $\mathrm{A}$ is then stimulated, and a strong response is obtained. The end $B$ is next stimulated, and there is little or no response. The block between A and B is now removed, and the specimen stimulated throughout its length. Though the stimulus now acts on both contacts, 
yet, owing to the irresponsive condition of $\mathrm{B}$, there is a resultant response, and the direction of this action-current is found to be from A to B. We have thus experimentally verified the assumption that in the same tissue an uninjured portion will be thrown into a greater excitatory state than an injured, by the action of the same stimulus.

When the point $\mathrm{B}$ is injured, there is generally speaking a more or less persistent current set up which flows from $B$ to A. But we saw that the direction of the action-current was opposite-that is to say, from A to B. This will explain the reason why the action-current causes a diminution or negative variation of the current of injury, so called. One method of doing this is to cause injury to one of the two points. If this be such as to kill the tissue, then its excitability is permanently abolished. Or by causing the excessive stimulation of injury, we may simply depress the excitability of the tissue for a longer or shorter time.

I shall now give a few instances of response in plants by negative variation. Taking the petiole of turnip, we injure an area on its surface, say B. A current is now observed to flow in the petiole from the injured $B$ to the uninjured $A$. The induced difference of potential depends on the condition of the plant, and the season. In the experiment here described, its value was I 3 volt. A sharp mechanical tap was now given to the petiole, between $A$ and $B$, and a sudden diminution, or negative variation, of current occurred, the resting potential difference being decreased by ' 026 volt. A second and stronger tap induced a second response, causing a greater diminution of potential difference by 047 volt.

In another experiment, the specimen employed was a petiole of cauliflower (Brassica oleracea). The first up-line to the right indicates the current of injury. The three responses which succeed are induced by a given intensity of stimulus, the next series of six, being in response to stimulus nearly twice as strong, exhibit signs of fatigue (fig. I I I).

The current of injury generally undergoes a diminution with time. This is often, as has been explained, on account 
of slow recovery from the excessive stimulation of injury. Response by negative variation is then found to undergo a decline. It is in general vaguely accepted that, in order to obtain a response by negative variation an antecedent current of injury is necessary, by whose induced variation we may be able to record responsive effects. In cases of the disappearance of the current of injury, it is supposed that response must necessarily vanish, since its antecedent condition no longer existș. But I have already shown, and shall

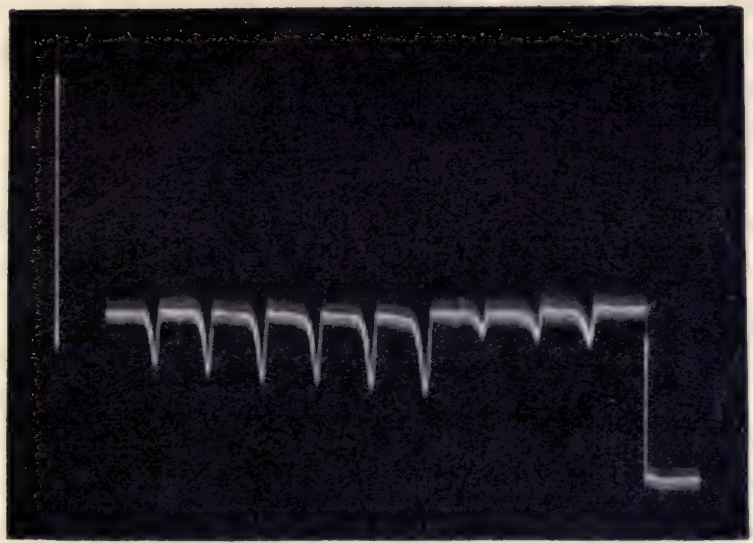

Fig. II I. Record of Responses in Plant (Leaf-stalk of Cauliflower) by Method of Negative Variation

The first three records are for stimulus intensity $\mathbf{I}$; the next six are for intensity twice as strong; the successive responses exhibit fatigue. The vertical line to the left represents I volt. The record is to be read from right to left.

have occasion again to show in the next chapter, that these suppositions are altogether erroneous. For we may obtain the usual response when the current of injury is zero, or even positive. In fact, the only essential condition for the obtaining of resultant response is that at one contact the excitability should be in a state of relative depression.

In that case in which response becomes enfeebled, with the gradual decline and vanishing of the current of injury, a simple explanation is often applicable. Whèn the tissue is injured, it does not necessarily die. In fact, I have often 
found that, in order to ensure death-in the case for instance of thermal section-a prolonged application of the fatal temperature is necessary. In ordinary cases of injury caused by the application of heat, I find that we have merely excessive stimulation of the point, with depression of excitability. But after a long interval, excitability is more or less restored, with the gradual passing away of the effect of injury. The subsidence of the current of injury thus also denotes the restoration of excitability to a greater or less extent. Hence that differential action between the uninjured and injured contacts, which determines the amplitude of the resultant response, will become correspondingly diminished. And when response has undergone diminution from this cause, a fresh injury is found to renew its amplitude. This is due to the reduction of excitability now freshly brought about at one of the contacts.

There are, however, two other additional factors which may further contribute to the enhancement of response after a recent injury. We have seen that, as a general rule, the resultant response will be $\mathrm{E}_{\mathrm{A}}-\mathrm{E}_{\mathrm{B}}$, where $\mathrm{E}_{\mathrm{A}}$ means the excitatory electrical change induced at $\mathrm{A}$, and $\mathrm{E}_{\mathrm{B}}$ that induced at B. It would therefore appear that this value will be at its maximum when the excitability of $\mathrm{B}$ is totally abolished by reason of injury; the resultant effect being due to the unopposed electrical excitation at A. But we have seen that when the true excitatory negative variation of a point is abolished, it may nevertheless exhibit a positive electrical variation, due to hydro-positive action. When this happens to be the case, this positive effect at $\mathrm{B}$, conspiring with the true excitatory effect at A, may bring about a response larger than we should have supposed to be maximum.

Again, though over-stimulation of a point diminishes its excitability, yet moderate stimulation often enhances it. The effect of this, in enhancing resultant response, is well seen in the case of conducting nerves. Thus, when the point $B$ is injured, the excitation caused by injury reaches $\mathrm{A}$, and causes moderate stimulation of that point. As an after-effect of this 
moderate stimulation, A often becomes more than normally excitable. ${ }^{1}$ It is thus seen how, after a recent injury, these two factors-of a hydro-positive effect at the injured, and of increased excitability at the uninjured contact, in consequence of moderate transmitted stimulation-may act to enhance the response.

We have seen that the common effect of injury is to induce a galvanometric negativity of the point injured. We have further seen that in such a case the response to external stimulus is by a negative variation of the current of injury. We have next, then, to take up various instances which appear highly anomalous, cases, that is to say, in which the injured point, relatively to the uninjured, is, for some hitherto unknown reason, galvanometrically positive. As a result of this and other causes, there are, in addition to the cases already described in a previous chapter, instances in which response is found to take place, not by a negative, but by a positive, variation of the current of rest or of injury as the case may be.

The first point to be considered in connection with such abnormal responses is whether the experimental tissue is physiologically isotropic, that is to say, of equal excitability throughout, or anisotropic, possessed of unequal excitabilities at different points. The discussion of the first of these cases, the isotropic, I propose to defer to the following chapter. The anisotropic will be touched upon here, though its detailed consideration will be entered upon in the next.

As an example of the anisotropic organ, we may take the pulvinus of Mimosa, in which the lower side is more excitable than the upper. In animal tissues also, such anisotropy is not uncommon. For example, we may have a muscular tissue terminating in a glandular. Owing to this anisotropy, the muscular and glandular surfaces are unequally excitable, and it will be shown in a later chapter that, generally speaking, it is the glandular which exhibits more intense excitatory galvanometric negativity. When such a

I For further details, see Chapter XLII. 
preparation is made, by cutting across the muscle, it is found that an electrical current flows from the uninjured gland to the injured muscle. From this it has been supposed that such a current was not the current of injury at all, but something of an unknown nature, essentially different. The consequent perplexity is the result of a failure to understand on the one hand that there is no such thing as a current of injury per se, except as the after-effect of strong stimulation, and on the other, that the current induced in the tissue is always from the more excited to the less excited. In the present case of muscle-and-gland preparation, the excessive stimulation due to section becomes diffused all over the tissue, and since the glandular surface is the more excitable, its excitatory galvanometric negativity is greater than that of the sectioned muscle, which thus becomes relatively positive. We have here a striking demonstration of the necessity for regarding the electrical reaction as the sign, not of injury, but of the excitation caused by injury. In the case described, for instance, the physical injury is obviously incapable of transmission, and it is the consequent excitation which is conducted to the gland.

The account of an experiment on a sensitive leaf of Mimosa will serve to elucidate the foregoing argument. If one contact, A, be made with the upper half of the pulvinus, and the other, $\mathrm{C}$, with a distant and indifferent point, then, on giving a prick near $\mathrm{A}$, we shall find that that contact, owing to excitation by injury, becomes galvanometrically negative. If, next, we make two contacts at diametrically opposite points of the pulvinus, A on the upper, and B on the lower, suríaces, it will then be found, on causing injury at the upper point $\mathrm{A}$, that that point, relatively to $\mathrm{B}$, becomes galvanometrically positive. This is because the stimulus caused bv the injury has become diffused throughout the pulvinus, witn the effect of causing greater excitation and consequent greater galvanometric negativity at the more excitable $\mathrm{B}$.

It has been seen that a mechanical or thermal section acts as a strong stimulus. It has also been shown that 
recovery from a strong stimulus is very protracted. Hence, after such stimulation, there is persistent galvanometric negativity as an after-effect. As the intensity of this aftereffect depends upon the intensity of stimulation, it will be seen that the galvanometric negativity near the section will be greater than at a distant point, where the transmitted effect of stimulation is feeble. From this it follows, that the so-called current of injury will flow in the tissue from the neighbourhood of the cut, to the distant and relatively unexcited end. The current of injury is thus an after-effect of strong stimulus. The peculiar electrical distribution which occurs in a muscle-cylinder is also found in a plant-cylinder, and both are equally explicable from the fact that the greatest excitatory after-effect occurs at the two sectioned ends, and that this decreases progressively towards the equator. The over-stimulated area of injury has its excitability depressed or abolished; diffuse stimulation, causing greater excitation of the uninjured contact, induces in it a greater excitatory effect of negativity, and this gives rise to a diminution of the existing difference of potential, as between the injured and uninjured. This is the explanation of response by the so-called negative variation.

In an anisotropic tissue the excitation caused by injury, when diffused, induces greater galvanometric negativity of the more excitable part. If this be the distal end, the resultant persistent current will be from the distal uninjured to the proximal injured. An apparently anomalous case will thus arise of a 'positive' current of injury, so-called. 


\section{CHAPTER XIV}

CURRENT OF DEATH-RESPONSE BY POSITIVE VARIATION

Anomalous case of response by positive variation-Inquiry into the causeElectric exploration of dying and dead tissue : death being natural-Determination of electric distribution in tissue with one end killed-Dying tissue shows maximum negativity, and dead tissue, positivity to living-Explanation of this peculiar distribution-Response by negative or positive variation, depending on degree of injury-Three typical cases-Explanation by theory of assimilation and dissimilation misleading-All response finally traceable to simple fundamental reactions.

WE have seen in the last chapter that, in order to obtain response by negative variation, it is customary among investigators on animal physiology to kill one end of the experimental tissue, say by scalding. It is generally supposed also that dead tissue is negative to living. On stimulation, the induced negativity of the living contact, now superposed on the existing P.D. of the unilaterally killed tissue, causes a negative variation of it. This mode of investigation, by means of the negative variation, is one which has hitherto, as we have seen, been universally regarded as reliable.

In the course of my investigations on the response of vegetable tissues, by this mode of negative variation, however, I have sometimes found response to take place by the positive variation. Taking, for example, a stem of Balsam, I killed one end by immersion in boiling water. On now subjecting this to diffuse vibrational stimulus, the responsive action was found to induce a positive variation of the existing current. On further investigation, I found that the excitatory electrical variation at the living contact had remained normal; that is to say, the direction of the responsive current was away from the excited living, and towards the killed end. I next 
found that the so-called 'current of injury' had in this case, owing to some hitherto unknown cause, undergone a reversal, and was now from the living to the dead, the latter being galvanometrically positive to the former, to the extent of .08 volt. The abnormality of the response lay, then, in this fact, that the current of reference had become reversed, and that the responsive current, due to excitation, was now concordant with it, instead of antagonistic, thus constituting a positive variation (fig. I 12). Later on, I discovered many instances in which the killed end was positive to the unkilled. Since, then, it is possible for the current of reference itself to undergo such obscure and spontaneous reversals, from unknown causes, it is easy to see how uncertain the study of responsive phenomena must become, if we are to depend upon the negative variation as our only reliable means for their investigation. I next, therefore, turned my attention towards an inquiry into the causes of these anomalous reversals.

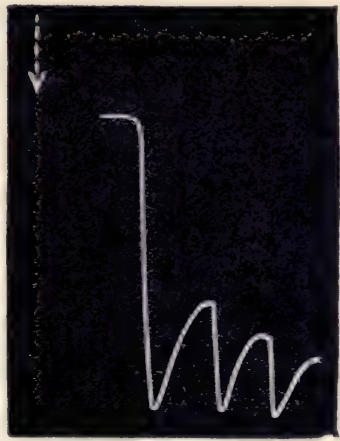

FIG. I12. Response by Positive Variation of Resting Current

The current of injury, shown by $\checkmark$, had in this case become reversed, the killed end having become galvanometrically positive.

The subject therefore resolved itself into an investigation as to what conditions determined the negativity or positivity of a tissue at the onset of death. My first attempt, then, was to study a case in which the approach of death was natural, and not the result of any sudden or violent change, such as might conceivably give rise to abnormal reactions. And in my search for suitable specimens, I noticed that often, owing to local mal-nutrition or other causes, the leaves of plants exhibited spots or areas, from which, as centres, death proceeded in constantly widening circles. Thus, in the leaves of Colocasia, for example, we find such dead and dying areas in otherwise fairly healthy leaves. The innermost of these 
patches may be quite dark and discoloured, while, as the living tissue is approached, this dark passes imperceptibly into yellow colour. And beyond this, again, we find the discolouration of yellow passing into the vivid green of living tissue. Proceeding thus in a radial direction inwards, towards the centre of such a patch from the living green, we shall find all possible stages of death, from its initiation, somewhere on the border-line between green and yellow, to its phase of completion, in the dark central area. On testing the electrical conditions of these different parts, I found that the border between green and yellow was negative to the living green surface. But the same point was also negative to the dead central area, and more negative to this than to the living tissue. Hence the dead was relatively positive to the living.

Or if we make one fixed contact on the living tissue, and if the second exploring contact be made with various points successively on a radial line passing from this to the centre of the dead area, these contacts will pass in succession through the living, the dying, and the dead. The variation of electrical potential will be found to be at its greatest along this line. The electro-motive difference between the point which has been fixed on the living tissue, and the exploring second contact, will at first be found to increase. The maximum difference is attained on reaching the border-line between green and yellow, or very little beyond this, this point being galvanometrically the most negative. On now passing further inward from this point, the maximum difference is found to decrease, till we come to a point in the dead tissue which is iso-electric with the living. On now again passing inwards, to the still more completely dead tissue of the central area, we find that we are approaching points which are more and more galvanometrically positive, as compared with the living tissue. The dying point on the border-line between green and yellow is thus the most negative, and points to the right or left of this are positive in comparison with it, the dead, however, being more positive than the living. It has been said that the electro-motive variation is most rapid along the 
radial line. On the other hand, we obtain series of equipotential surfaces whose outlines closely follow those of the boundaries of the different degrees of discolouration.

I shall next proceed to give quantitative measurements. The first point to be considered is that of the choice of a definite electrical level, which is to be used as a standard. If this point be selected in the living tissue, we shall find that our standard of comparison is extremely variable, since the tonic condition, on which its electrical level depends, is itself subject to change. The only condition which cannot be modified in any way is that of complete death. This may be taken, then, as the standard level. The method of experiment will thus consist in selecting a series of equidistant points, $a b c d$, and so on, $5 \mathrm{~mm}$. apart, along a radial line, passing outwards from the central area, which is completely dead, to the green tissue. The non-polarisable contacts $\mathrm{E}$ and $\mathrm{E}^{\prime}$ are first placed on $a$ and $b$, then on $b$ and $c, c$ and $d$, and so forth. The external circuit contains a high resistance, compared with which any difference of resistance, as between any $5 \mathrm{~mm}$. of interposed tissue, becomes negligible. Hence, the successive deflections of the galvanometer indicate the electro-motive difference that exists between $a$ and $b, b$ and $c$, and so on.

One difficulty which is experienced, in these measurements of small electro-motive differences, lies in securing the iso-electric condition of the non-polarisable electrodes themselves. Whatever precautions are taken in the construction of these, a small electro-motive difference will sometimes be found to exist between them. The existence of such a difference is easily tested by bringing the kaolin ends of the two electrodes in contact, or by dipping both of them close together in a vessel of normal saline solution. Any electromotive difference of the electrodes, however small, will now give rise to a large galvanometric deflection.

This difficulty may be overcome by first taking special precautions as to the purity of zinc rods and the chemicals employed, and secondly, by keeping the electrodes for a long time short-circuited, with their ends dipped in normal 
saline. In very obstinate cases, however, I succeeded in eliminating all differences by subjecting the electrodes to cyclic variations of alternating electro-motive force. By means of a Pohl's commutator, without cross-bars, the electrodes were put in connection with an alternating source of E.M.F., and with the galvanometer intended to test the resulting variation in the E.M. difference, by turns. A small hand-driven alternating-current generator was used for this purpose. The speed of rotation of this machine was gradually raised to a maximum, and afterwards as gradually slowed down. Thus at each cycle the electrodes were subjected to ascending and descending intensities of alternating electro-motive variations. The effect of such cyclic changes, in diminishing the existing electro-motive difference between a pair of electrodes, specially selected for carelessness of preparation, will be clearly seen from the following tabular results :

\begin{tabular}{|c|c|c|c|}
\hline Condition at starting & & Galvanometric deflection & E.M. difference \\
\hline Original difference. & • & 360 divisions & 'oog volt \\
\hline After first cycle . & . & $40 \quad$, & . \\
\hline After second cycle. & . & o & o , , \\
\hline After third cycle . & . & ", & o ,", \\
\hline
\end{tabular}

It will thus be seen that, after a very short time of this treatment, the two electrodes were rendered iso-electric.

I next proceeded to determine the distribution of electrical potential in the various portions, living and dead, of the leaf. In order to remove any accidental strain, the leaf was placed in tepid water, and kept there for about half an hour, till the water was cooled to the surrounding temperature. The experiment was then carried out, in the manner already described, and the following tabular statement shows the results obtained. The electrodes, it will be remembered, were placed successively at points $5 \mathrm{~mm}$. apart from each other, along a radial line proceeding from the dead tissue to the living, the first point being taken as zero: 


\begin{tabular}{|c|c|}
\hline Position of Electrodes & Galvanometric Deflection \\
\hline $0-5 \mathrm{~mm}$. & o division \\
$5-10, "$ & $-10,$, \\
$10-15, "$ & -20, \\
$15-20, "$ & -45, \\
$20-25$, & -140, \\
$25-30, "$ & -140, \\
$30-40, "$ & $+110,$, \\
$40-45, "$ & $+20,$, \\
\hline
\end{tabular}

It will be observed that as we proceed from the dead to the dying, the negativity of the latter rapidly increases, the maximum being at $30 \mathrm{~mm}$. from the zero-point taken on the

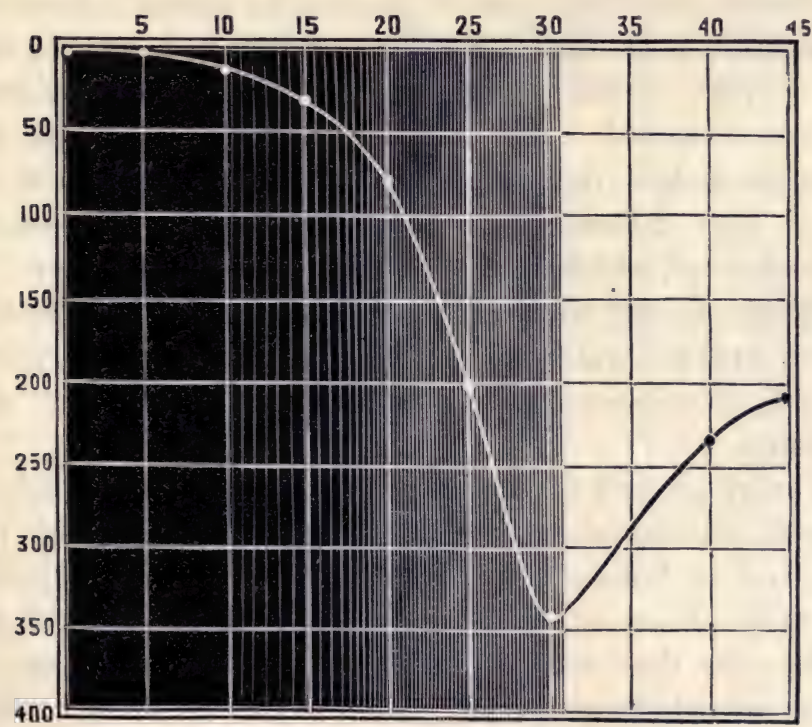

FIG. II3. Distribution of Electric Potential in Lamina of Colocasia along a radial line from dead to living through intermediate stages. $\mathrm{Ab}$ scissa gives distance in $\mathrm{mm}$. from chosen centre in dead tissue, ordinate represents galvanometric negativity in divisions. Dead tissue represented dark, dying shaded, and living white.

dead tissue. This point of maximum-negativity almost coincides with the visible border-line between the yellow and the green. Beyond this, however, there is an electrical 
reversal, the living becoming increasingly positive, as compared with the dying. An inspection of the curve (fig. I I 3) shows that while there is a point in the tissue between the dying and the dead, which is equipotential with the living, the completely dead tissue is positive to the living.

I next carried out an experiment in which death was artificially induced, by immersing a portion of the tissue in boiling water. In connection with this, I may say that it is extremely difficult to ensure the complete death of a thick tissue. It is only the outside layers which undergo death easily, but the interior tissues, from their protected position, are extremely resistant, and it is only after prolonged immersion in boiling water that death can really be ensured throughout. In the present experiment, however, where only a part of the tissue is to be killed, such prolonged immersion would cause death to encroach upon those portions of the tissue which were intended to be kept alive. This difficulty was met by choosing a specimen, the inside of which was accessible to boiling water. The peduncle of the water-lily (Nymphcea alba) in transverse section appears extremely reticulated, and there is thus no difficulty in exposing all its parts to the direct action of the hot water.

The upper end of the peduncle was kept surrounded by a cloth moistened in ice-cold water, the lower end being immersed in boiling water for ten minutes. The specimen was then placed in tepid water, and allowed to cool down slowly. In this way a length of the peduncle was obtained, in which one end was completely killed, whereas the other remained fully alive, the intermediate portions showing all stages of the transition from the living to the dead condition. In order to determine the electrical distribution in its different parts, I now employed the potentiometer method of balance. One electrode was permanently connected with that dying point which by a previous test had been found to exhibit maximum negativity. The second electrode was placed at successive points, 
each of which was nearer than the last by $5 \mathrm{~mm}$. to the dead end, which was to the left. The same process was now repeated, the successive readings however being taken towards the right or living end. At each point, the electro-motive difference was balanced by the potentiometer. This straight form of potentiometer had a scale divided into one thousand parts (fig. I I 4), and when its terminal electro-motive force was adjusted to I volt, each

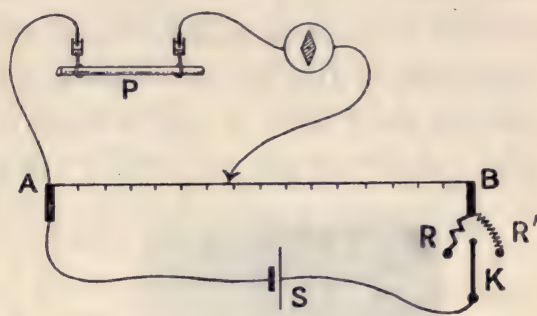

FIG. II4. Straight Form Potentiometer A $B$ is a stretched wire with added resistances, $\mathbf{R}$ and $\mathbf{R}^{\prime}$. $\mathbf{S}$ is a storage cell. When the key, $\mathrm{K}$, is turned to the right, one scale division $=\cdot 00 \mathrm{I}$ volt, when turned to the left one scale division $=$ OI volt. $P$ is the plant.

division of the potentiometer was equal to OOI volt. The following table gives the results obtained:

\begin{tabular}{|c|c|c|c|}
\hline $\begin{array}{l}\text { Towards left or dead end, } \\
\text { E.M. difference in } \frac{t}{\Sigma 000} \text { volt }\end{array}$ & $\begin{array}{l}\text { Distance from } \\
\text { negative point to } \\
\text { to the rig }\end{array}$ & $\begin{array}{l}\text { maximum } \\
\text { the left }(-) \text { or } \\
\text { ght }(+)\end{array}$ & $\begin{array}{l}\text { Towards right or living end, } \\
\text { E.M. difference in } \frac{1}{\square 00} \text { volt }\end{array}$ \\
\hline$I \cdot 2$ & & $\mathrm{~cm}$. & $\circ 9$ \\
\hline $5^{\circ} \mathrm{O}$ & Iי & , & 2.5 \\
\hline $18 \cdot 2$ & I.5 &, & 4.7 \\
\hline $22{ }^{\circ} 0$ & $2 \cdot 0$ & ,, & 6.8 \\
\hline $23^{\circ} 0$ & $2 \cdot 5$ & , & $10^{\circ} 0$ \\
\hline $23 \cdot 3$ & $3^{\circ} \circ$ & ," & $12 \cdot 8$ \\
\hline - & 3.5 & , & 15.6 \\
\hline - & $4^{\circ} \mathrm{O}$ & , & $16 \cdot 8$ \\
\hline- & $4 \div 5$ & , & $17 \cdot 5$ \\
\hline - & $5^{\circ} \mathrm{O}$ & ," & $18^{\circ} \mathrm{O}$ \\
\hline
\end{tabular}

Here, also, as in the case of natural death, we find a point in the dying tissue which is most negative. From the curve given in fig. I I 5, it will also be seen that as we pass away from this point in either direction towards the living or dead area, we find an increasing positivity; the curve for the dead portion is, however, much steeper than that for the living. Thus two points, one $I^{\circ} 5 \mathrm{~cm}$. to the left in the dead tissue, and another $5 \mathrm{~cm}$. to the right in the living tissue, are iso-electric. But while the maximum positivity of the living 
is OI 8 volt, that of the dead is 0233 volt. Hence the dead tissue is here positive to the living, to the extent of .0053 volt.

We have seen that the prevailing idea is that the dead is negative to the living. But from the results here shown, we can see that this is not a complete statement of the case. Since then the electro-motive variation, instead of showing a

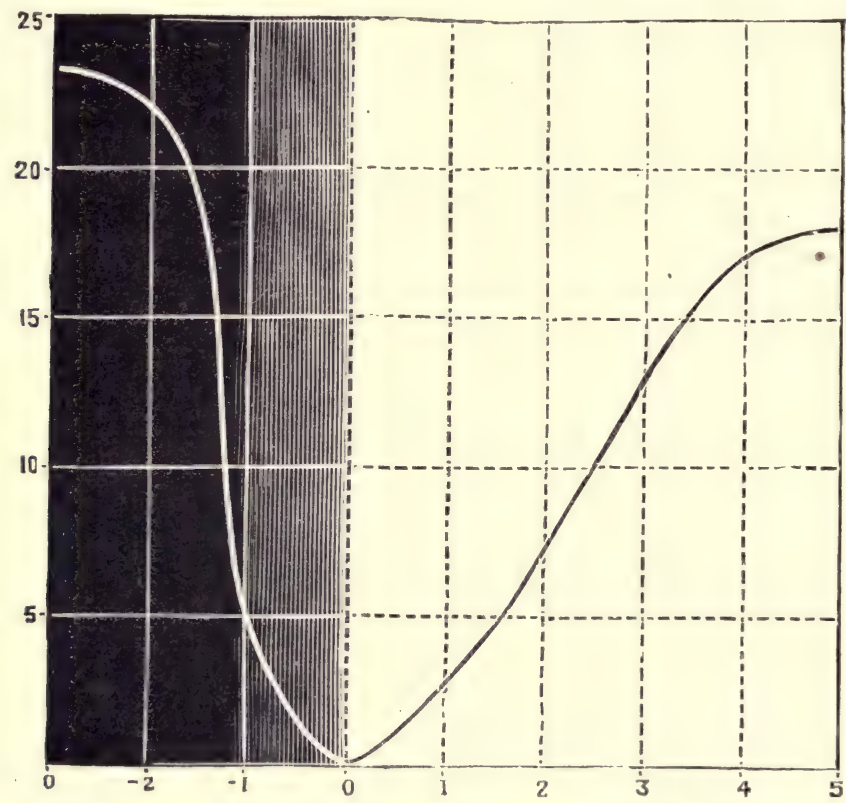

FIG. I I5. Distribution of Electric Potential in Petiole of Nymphrca alba, one end of which has been killed.

The point of maximum negativity is taken as zero, distances to the left or towards the dead taken as minus, to the right or living, as plus.

Ordinate represents potential difference in thousandths of a volt.

progressive change from the living to the dead, exhibits a maximum difference, followed by a reversal, it may be asked, what is the reason of this anomaly?

Much light is thrown on this subject from the results given by another line of inquiry, to be explained in detail in Chapter XVI. It is there shown that the plant-tissue on the first onset of death exhibits a sudden contraction, indicative 
of a strong excitatory reaction. This corresponds with the rigor mortis of the animal, and by means of suitable apparatus, the coicomitant mechanical response can be recorded. An electrical record of the same phenomenon may also be obtained, in the form of an electrical spasm of galvanometric negativity. Succeeding to this rigor of the dying tissue, a post-mortem relaxation takes place, with a concomitant change from galvanometric negativity to positivity.

Now in a tissue which has been killed unilaterally only, it will be understood that all possible gradations are to be expected. Passing from the completely dead to the fully alive, we must necessarily pass through various zones, beginning with the abnormally relaxed, through the intermediate highly contracted and rigored tissue on the deathfrontier, to the living, which is not so contracted as the dying, and not so relaxed as the dead. At the point where the onset of death is recent, the rigor, or excitatory contraction and galvanometric negativity, are at their maximum. Compared with this, the slightly tonically contracted living is positive, but not so positive as the abnormally relaxed dead.

The death-frontier, however, is not fixed. It is continually encroaching on the living. The line of maximum rigor and galvanometric negativity is thus also shifting in the same direction. Along with this, however, the opposite process of post-mortem relaxation is proceeding; so that a point which was, in consequence of rigor, maximally negative, becomes gradually converted to positive.

This positivity of dead tissue as compared with living, which has here been demonstrated in the case of the plant, I find to be also true of animal tissue, in those cases which I have investigated. Thus, while an injured and dying area in a frog's nerve is negative, an already dead area is positive, relatively to the living nerve. There is, moreover, an intermediate area, between the dying and dead portions of the nerve, which is iso-electric to the living. 
Hence, having one contact fixed on a living area and the other on (I) the dying, (2) the intermediate, and (3) the dead tissue, we shall obtain three different types of what is known as the 'injury-current.' In the first of these the second contact will be negative, a condition which has hitherto been assumed to be the sole characteristic of the current of injury. But there are two other cases to be considered. Of these, when the second contact is made at a point intermediate between the dying and dead tissues, we shall find it to be iso-electric with the first, or living contact. And thirdly, when the second contact is on a dead area, the latter will be positive to the first, or living contact. We thus find three cases of the current of injury-the first being negative, the second zero, and the third positive.

Taking the first of these-that in which the injured contact is negative-the action-current, in response to stimulus, will bring about a negative variation of the socalled current of injury. In the second, the result will be indeterminate, since the injury-current is zero. In the third, the response will be by a positive variation of the current of injury.

I give below three photographic records in illustration of these three cases, obtained with vegetable nerve. I may state here that I have often observed results precisely similar in the case of frog's nerve also. In the first record, in fig. i 6 , the thermal injury was moderate. The injured point was thus negative, and the current of injury is represented here by an up-line. The responses are seen to be by negative variation. In the second record the injury was greater, and the injured point was almost neutral; that is to say, on making contact there was a slight up-twitch, which subsided to zero. There is here, then, no current of injury. The subsequent responses are, however, down, the action-current being away from the living contact. In the third record the injury was so great as completely to kill the injured point, which thus became positive to the living. The 
reversed injury-current is represented as down, the subsequent excitatory responses are also down, and constitute a positive variation of the current of injury.

It will thus be seen that an identical excitatory reaction of the living tissue appears to give rise to directly opposite

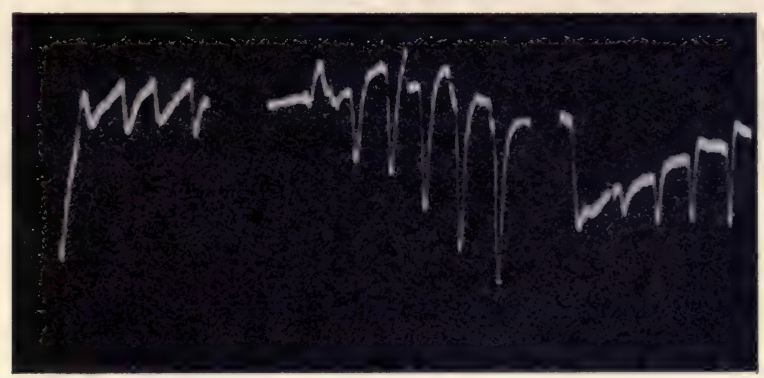

FIG. I16. Photographic Records of Responses of Vegetable Nerve, one end of which has been injured

In the first injury was slight; current of injury represented up, response by negative variation. In the second, injury greater; injured point neutral, response down. In the third, injured point killed; injury current reversed down, response by positive variation.

effects-namely, a negative or a positive variation of the injury-current.

I give below a short summary of the diversities of response which may occur when either the natural, or the injury-current, is taken as the current of reference.

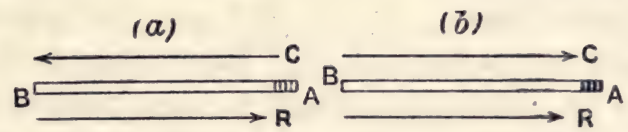

Fig. 117. Typical Cases of Variation of Current of Rest and Action.

Current. Specimen originally isotropic

(a) A, end slightly injured and negative ; C, current of injury ; $\mathrm{R}$, actioncurrent, a negative variation of C. (b) A, end killed and positive; $\mathrm{C}$, current of injury; $\mathrm{R}$, action-current, a positive variation of $\mathrm{C}$.

First-we take the case where the point A is slightly injured (fig. I I 7, a). The current of injury, $\mathrm{C}$, is $\mathrm{A} \rightarrow \mathrm{B}$, and the responsive current, $\mathrm{R}$, is $\mathrm{B} \rightarrow \mathrm{A}$, constituting a negative variation. 
But when $\mathrm{A}$ is killed, the current of injury $\mathrm{C}$ is $\mathrm{B} \rightarrow \mathrm{A}$, the responsive current is also $\mathrm{B} \rightarrow \mathrm{A}$, constituting a positive variation (fig. I $17, b$ ).

Second -we take an instance where, owing to some physiological difference, an intermediate point $\mathrm{A}$ is less excitable than $\mathrm{B}$ or $\mathrm{B}^{\prime}$ (fig. I I8, a). The primary natural current will here be from the less to the more excitable : that is to say, $\mathrm{C}$ will be $\mathrm{A} \rightarrow \mathrm{B}^{\prime}$ and $\mathrm{A} \rightarrow \mathrm{B}$. If stimulus be now applied at $\times$ on the right, an identical excitatory current $\mathrm{R}$, flowing away from the excited point from right to left, will cause seemingly opposite effects : that is to say, a negative variation of $A \rightarrow B^{\prime}$ and a positive variation of $\mathrm{A} \rightarrow \mathrm{B}$.

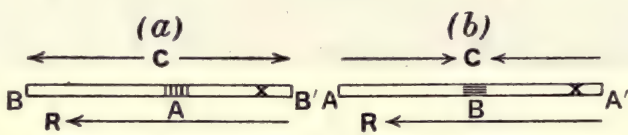

FIG. 118. Typical Cases of Variation of Current of Rest and ActionCurrent; intermediate point naturally less or more excitable than either of terminal.

(a) Intermediate A, less excitable, shown by vertical shading; current or rest $\mathrm{A} \rightarrow \mathrm{B}$ and $\mathrm{A} \rightarrow \mathrm{B}^{\prime}$; when right-hand point $\times$ excited, action-current $R$ from right to left, gives rise to negative variation of $A \rightarrow B^{\prime}$, and positive variation of $A \rightarrow B$. (b) Intermediate $B$, more excitable, shown by horizontal shading; current of rest $\mathrm{A} \rightarrow \mathrm{B}$ and $\mathrm{A}^{\prime} \rightarrow \mathrm{B}$; action-current on excitation at $x$, from right to left, giving rise to positive variation of $A^{\prime} \rightarrow B$ and negative variation of $A \rightarrow B$.

Again, we may have the intermediate point $\mathrm{B}$ naturally more excitable than $\mathrm{A}^{\prime}$ or $\mathrm{A}$. The natural current $\mathrm{C}$ will be $\mathrm{A} \rightarrow \mathrm{B}$ and $\mathrm{A}^{\prime} \rightarrow \mathrm{B}$ (fig. I $18, b$ ). Stimulation at $\times$ will now give rise to an excitatory current $\mathrm{R}$, from right to left. The results here will, however, appear to be exactly the reverse of those in the last case: that is to say, an identical current, $\mathrm{R}$, will give rise to a positive variation of $\mathrm{A}^{\prime} \rightarrow \mathrm{B}$, and negative variation of $\mathrm{A} \rightarrow \mathrm{B}$. Instances of these effects will be given in Chapter XVIII.

And, lastly, we may have a typically anisotropic tissue, composed of two halves, which are unequally excitable-as, for instance, the upper and lower halves of the pulvinus of Mimosa, or the muscle and gland in a muscle-and-gland 
preparation. Under normal conditions the primary or natural current $\mathrm{C}$ is from the less excitable $\mathrm{A}$ to the more excitable $\mathrm{B}$, represented by $\mathrm{A} \rightarrow \mathrm{B}$ (fig. I I9, $a$ ). The action-current $\mathrm{R}$, being in the opposite direction $\mathrm{B} \rightarrow \mathrm{A}$, constitutes a negative variation.

But owing to the after-effect of excitation, such as may occur in isolating the specimen by section, the normal resting current $\mathrm{C}$ is reversed to $\mathrm{B} \rightarrow \mathrm{A}$ (fig. I I $9, b$ ). Here the end $\mathrm{B}$ may still be the more excitable of the two, hence the action-current $\mathrm{B} \rightarrow \mathrm{A}$ will constitute a positive variation of the current of rest.

But when B becomes fatigued, its excitability is reduced below that of $\mathrm{A}$; hence the action-current is from the relatively more to less excitable, i.e. $\mathrm{A} \rightarrow \mathrm{B}$. In this case,
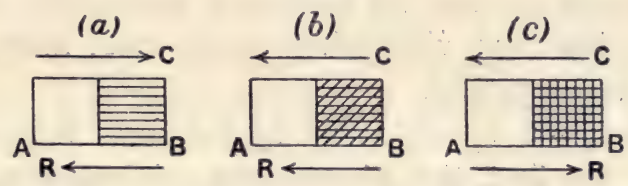

FIG. I 19. Typical Cases of Variation of Current of Rest and ActionCurrent. Anisotropic organ, B end originally more excitable than A

(a) Current of rest $\mathrm{A} \rightarrow \mathrm{B}$ : action-current, $\mathrm{R}$, in opposite direction ; response by negative variation. (b) Owing to excitatory after-effect, current of rest reversed to $\mathrm{B} \rightarrow \mathrm{A}$; $\mathrm{B}$ nevertheless more excitable than $\mathrm{A}$; actioncurrent, $\mathrm{R}$, is $\mathrm{B} \rightarrow \mathrm{A}$; response by positive variation. $(c)$ Current of rest reversed $\mathrm{B} \rightarrow \mathrm{A}$; action-current also reversed $\mathrm{A} \rightarrow \mathrm{B}$, by depression of excitability of $\mathrm{B}$, owing to fatigue ; response by negative variation.

on account of the reversal of both the current of rest and action-current, the latter appears to constitute a negative variation of the former (fig. I I9, c).

It will thus be seen how intricate and diverse are the responsive variations of the resting current, induced by stimulus. Sometimes negative, sometimes positive, it would appear as if there were no guiding principle to regulate these phenomena. The so-called explanations hitherto attempted have consisted in assigning the positive variation to a hypothetical process of assimilation, and the negative to dissimilation. Such explanatory phrases reach the climax of absurdity when we find ourselves compelled to ascribe one identical excitatory reaction now to assimilation and then to dissimilation. 
Indeed it must be said that, however suggestive the general theory of assimilation and dissimilation may have been found, its abuse has often stood in the way of physiological inquiry. The inquirer, when faced with any difficulty, instead of attempting to surmount it by patient inquiry, was tempted rather to evade it by invoking the aid of an hypothesis which could be made with equal ease to explain a given fact or its direct opposite. We must remember that in the investigation of obscure problems, the danger is always, instead of seeking an underlying law, to become satisfied with the mere registration of phenomena, and by naming these to imagine that they have been explained. The resulting chaos in the present case has served to deepen the impression that vital phenomena must always remain capricious and mystical.

But when we come to survey the facts that have been described, we find the phenomena of response, however diverse they may at first sight appear, to be in no way governed by chance or caprice. They are, on the contrary, definite and uniform under definite conditions.

As regards the so-called current of rest in a naturally isotropic tissue, of which one end has been subjected to injury, we must remember that the effect of injury is one of excitation, its sign, within limits, being contraction and galvanometric negativity. But we have seen that when a point is over-stimulated, fatigue-changes appear which give rise to a reversal of its normal sign of response, from contraction to expansion, from negative to positive (cf. fig. 64) The change at death, in which contractile rigor passes into post-mortem relaxation, is analogous to this. Thus when one end of the specimen is merely injured, that end becomes more or less persistently galvanometrically negative, the current flowing away from it. But when the same end is actually killed, the electrical change may be reversed, to one of galvanometric positivity.

In an isotropic tissue, then, we may, by moderate injury, bring about a state of anisotropy, under which the uninjured 
end is rendered relatively the more excitable, and galvanometrically positive, compared with the inexcitable injured end. In a naturally anisotropic organ, we have a state of things which is analogous. In this case, in the primary condition, the more excitable surface is galvanometrically positive. But under the excitation due to preparation, or accidental disturbance, this more excitable surface becomes the more excited, and, relatively to the other, galvanometrically negative. These varying changes in the direction of the so-called resting current, or current of reference, are the cause of the existing anomalies in the interpretation of response by the positive or negative variation.

But the direction of the action-current under normal conditions is always the same. On diffuse stimulation it is always from the more excited $\mathrm{B}$ to the less excited A. The differential excitability or anisotropy, may be either natural, or artificially induced, as by injuring one end of an isotropic tissue. There are two different conditions under which the normal effect may undergo reversal, those, namely, of great sub-tonicity or excessive fatigue. But the statement that the responsive current is always from the more excited to the less excited, remains universally true. Numerous illustrations, in verification of the cases laid down, will be met with in the course of subsequent chapters. 


\section{CHAPTER XV}

\section{EFFECT OF TEMPERATURE ON ELECTRICAL RESPONSE}

General observation of effect of temperature on plant-Effect of fall and rise of temperature on autonomous response of Desmodium - Effect of frost in abolition of electrical response-After-effects of application of cold, in Eucharis, Ivy and Holly-Effect of rise of temperature in diminishing height of responseThis not probably due to diminution of excitability-Similar effect in autonomous motile response of Desmodium-Enhanced response as after-effect of cyclic variation of temperature-Abolition of response at a critical high temperature.

WE have now seen that the physiological activity of a living tissue may be gauged by means of its electrical response. We know further that the influence of temperature is of importance in the maintenance of a proper physiological condition. There is a certain range of temperature which is favourable to this, and above or below these limits physiological efficiency is diminished. If the plant be kept too long at or above a certain maximum temperature, it is liable to undergo death. Similarly, there is a minimum point at which physiological activity is arrested, and below which death is apt to occur. The plant has thus two death-points, one above the maximum and the other below the minimum temperature. Some can resist these extremes better than others, and length of exposure is also a factor which should not be forgotten in the question of the ultimate survival of the plant under the given unfavourable conditions. Certain species are hardy, while others succumb easily.

An unmistakable indication of the effect of temperature on physiological activity is found in the variations induced by it in the autonomous motile pulsations of the telegraph plant, Desmodium gyrans. Here, too great a lowering of the 
temperature abolishes the pulsation. In fig. I20 are seen (I) the records of normal pulsations; (2) their arrest under the application of ice-cold water; and (3) their revival, as the plant regains the temperature of the room. In fig. I 2 I is shown the effect on similar pulsations of a rising temperature. The records in this case were obtained with a different

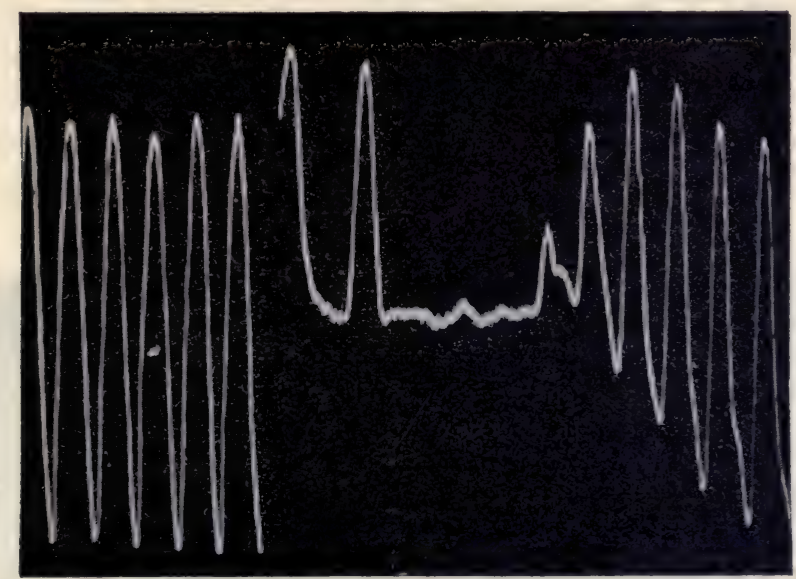

FIG. 120. Photographic Record showing Effect of Rapid Cooling, by Ice-cold Water, on Pulsations' of Desmodium gyrans

Normal pulsations recorded to the left. Effect of application of ice-cold water is seen in the production of diminished amplitude and abolition of pulsation. Gradual return to the temperature of the room revives the pulsation in a staircase manner, the period remaining approximately constant. Note that cooling displaces the pulsation in a downward or contracted direction. Gradual warming, conversely, is seen to produce the opposite displacement towards expansion. Up-records represent the fall of the leaflet, down-records its rise.

specimen, and it is seen that the pulsations are diminished in amplitude while their period is quickened, with rise of temperature. When the temperature is raised still higher, they come to a stop altogether.

We shall next proceed to observe the effect of temperature on the electrical response of plants. As regards the influence of cold, for example, I have found, during the course of a research carried out in England, that after frosty weather, 
the electrical responses undergo an almost complete abolition. During a certain week, for instance, the temperature was $10^{\circ} \mathrm{C}$., and the electrical responses then obtained from radish (Raphanus satious) were considerable, giving an E.M. response which varied from $\mathrm{O}_{5}$ to 'I volt. Two or three days afterwards, however, as the effect of frost, I found the electrical response of this plant to have practically disappeared. A few specimens were found nevertheless which were somewhat resistant. But even in these the average E.M. response had only a value of $\mathrm{OO}_{3}$ volt, instead of the normal mean of 075 volt. That is to say, their average sensitiveness had been reduced to one twenty-fifth. On now

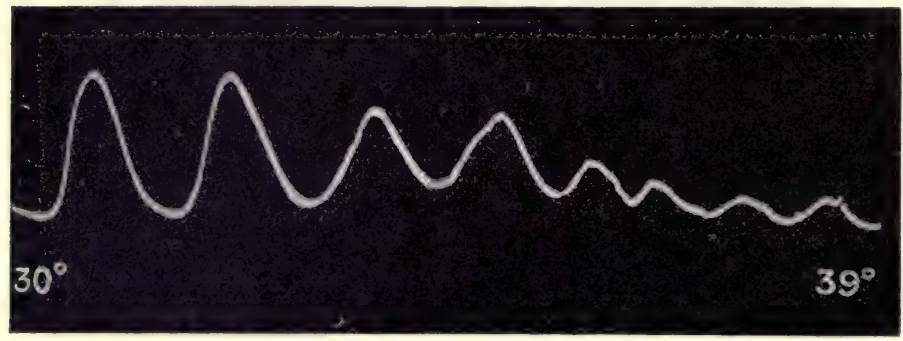

FIG. I2I. Photographic Record of Pulsations of Desmodium during Continuous Rise of Temperature from $30^{\circ} \mathrm{C}$. to $39^{\circ} \mathrm{C}$.

warming these radishes to $20^{\circ} \mathrm{C}$. there was an appreciable revival, as shown by their increasing response. But in those specimens which had been frost-bitten, warming effected no restoration. From this it would appear that frost killed some, which could not be subsequently revived, whereas others were reduced to a condition of torpidity from which, on warming, there was a revival.

I have also investigated the effect of an artificial lowering of temperature on the electrical response of plants. The Eucharis lily is particularly sensitive to cold. In this case I took the petiole, and obtained response at the ordinary temperature of the room, which was at the time $17^{\circ} \mathrm{C}$. I then placed it for 15 minutes in a cooling chamber at a 
temperature of $-2^{\circ} \mathrm{C}$. On now again trying to obtain response, it was found that it had practically disappeared. The same specimen was next warmed to $20^{\circ} \mathrm{C}$., and this induced a revival of response (fig. I22).

I was next desirous of studying the after-effect of lowered temperatures on different plants. For this purpose I chose three specimens (I) the petiole of Eucharis Lily, (2) the stem of Ivy, and (3) Holly. I took their normal responses at $17^{\circ} \mathrm{C}$., and afterwards placed them in an ice-chamber at a temperature of $0^{\circ} \mathrm{C}$. for 24 hours. The specimens were then taken out, and their responses under stimulation once more recorded (fig. 123). From these it will be seen that while the responsiveness of the delicate Eucharis Lily was completely abolished, that of the hardier plants, Holly and Ivy, exhibited complete revival.

One interesting fact

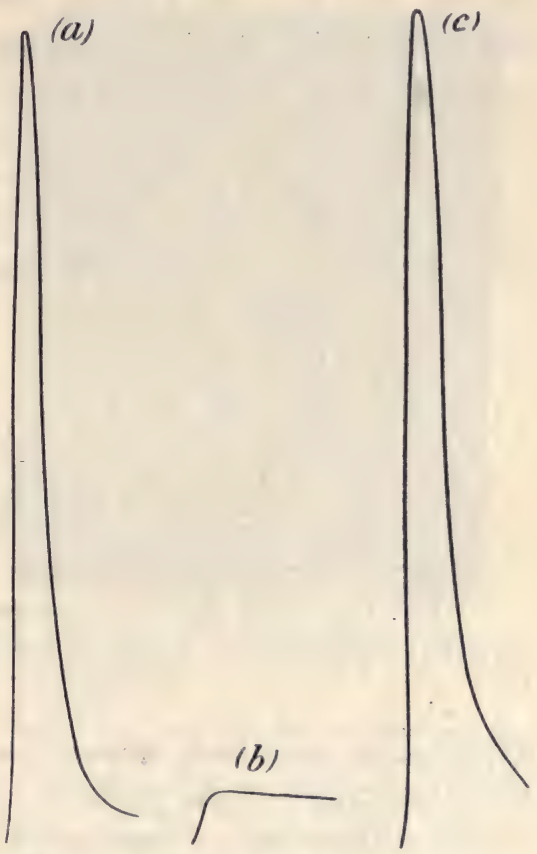

FIG. 122. Diminution of Response in Eucharis by Lowering of Temperature

(a) Normal response at $\mathrm{I} 7^{\circ} \mathrm{C}$.

(b) The response almost disappears when plant is subjected to $-2^{\circ} \mathrm{C}$. for fifteen minutes. which I have noticed

(c) Revival of response on warming to $20^{\circ} \mathrm{C}$.

is that when a plant approaches its death-point, by reason of excessively high or low temperature, not only is its response, of galvanometric negativity, diminished to zero, but it is even occasionally reversed to positive. This effect is due to the unmasking of the positive, by the abolition of the true excitatory component. 
We shall next study the effect on the electrical response of the plant of a rise of temperature. The great difficulty of this investigation lies in raising the plant-chamber to the various determinate temperatures required. I was able, however, to accomplish this by means of electric heating. A spiral of german silver wire was placed in the plant-chamber ( $c f$. fig. $2 \mathrm{I}$ ), and by varying the intensity of the current the temperature was then regulated at will. In the process of this electric heating a complicating factor was found in the excitatory action of any sudden variation of temperature. But no such excitatory disturbance occurs if the rise of temperature be not fluctuating, but gradual and continuous. I was able to secure this, by selecting at the beginning of the
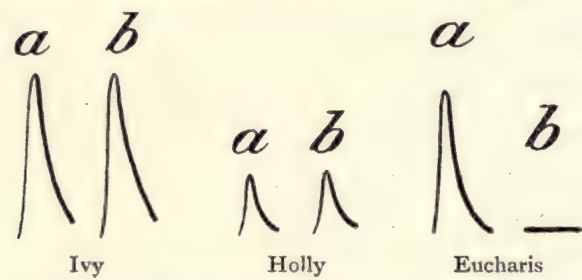

Eucharis

FIG. 123. After-effect of Cold on Ivy, Holly, and Eucharis Lily

$a$, The normal response; $b$, response after subjection to freezing temperature for twenty-four hours.

experiment, a suitable strength of current, such as to raise the temperature of the chamber continuously, at an approximately uniform rate. Care had also to be taken that thermal radiation from the wire should not strike the specimen, since such radiation, as we shall see later, acts as a stimulus. The interposition of a sheet of mica, however, obviated this difficulty, mica being opaque to thermal radiation.

While, under these conditions, the temperature was being raised, uniform vibrational stimuli were applied at intervals, and responses recorded, the temperature of the chamber at the moments of stimulation being carefully noted. In this way I obtained the following photographic record, with a petiole of Eucharis lily, affording a general idea of the effect of temperature on response. It will be seen that 
while the temperature was rising from $20^{\circ} \mathrm{C}$. to $22^{\circ} \mathrm{C}$. the amplitude of the response was increased. After this, however, it fell rapidly in height with rise of temperature and became very small, at or near $60^{\circ} \mathrm{C}$. On allowing the temperature to fall, however, the responses revived, with this peculiarity, that during the cooling, as compared with those given during the ascent of temperature, they were markedly enhanced (fig. 124).

The heating arrangements in this case were such that the temperature was made to rise somewhat rapidly. It will be noticed that response had not here disappeared, even at $65^{\circ} \mathrm{C}$., though, as we shall see in the next chapter, the deathpoint is at about $60^{\circ} \mathrm{C}$. This apparent anomaly is due to the fact that the plant, which is a bad conductor, was not allowed time fully to attain the temperature of its surroundings. We shall see that when the temperature is raised at a slower rate-about $\mathrm{I}^{\circ} \mathrm{C}$.

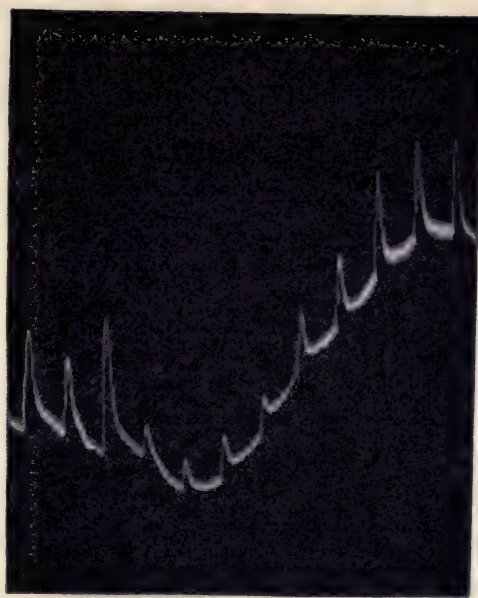

FIG. I24. Photographic Record of Responses in Eucharis Lily during Rise and Fall of Temperature

Stimulus constant, applied at intervals of one minute. The temperature of plantchamber gradually rose on starting current in the heating coil ; on breaking current, the temperature fell gradually. Temperature corresponding to each record is given below.

Temperature rising : (I) $20^{\circ}$, (2) $20^{\circ}$, (3) $22^{\circ}$, (4) $38^{\circ},(5) 53^{\circ},(6) 60^{\circ}$, (7) $65^{\circ}$

Temperature falling : (8) $60^{\circ}$, (9) $51^{\circ}$, (го) $45^{\circ}$, (I1) $40^{\circ}$, (12) $38^{\circ}$. in $\mathrm{I}^{\prime} 5$ minute - and when the specimen is not too thick, excitatory response disappears with the approach of death, at a temperature very near $60^{\circ} \mathrm{C}$.

I give below a record of the effect of temperature varying from $30^{\circ} \mathrm{C}$. to $50^{\circ} \mathrm{C}$. on the response of the stem of Amaranth (fig. 125). In order to obtain perfect results, it is necessary that the specimen should not exhibit any 
fatigue, and I have found that this plant is but little subject to it. It will be noticed how the response is continuously depressed, as the temperature rises from $30^{\circ} \mathrm{C}$. upwards. In this case, the thermal ascent took place at the relatively slow rate of $\mathrm{I}^{\circ} \mathrm{C}$. in $\mathrm{I}_{5} 5$ minute, so that there was time for the plant to attain the temperature of its surroundings. A tabular statement is given below, showing the effect of temperature on amplitude of response, in two different specimens of Amaranth:

SPECIMEN I.

Specimen II.

\begin{tabular}{|c|c|c|c|}
\hline Temperature & Height of response & Temperature & Height of response \\
\hline $30^{\circ} \mathrm{C}$. & 200 divisions & $30^{\circ} \mathrm{C}$ & I Io divisions \\
\hline $35^{\circ}$ & I 70 , , & $35^{\circ}$ & $90 \quad$, \\
\hline $40^{\circ}$ & $95 \quad$, & $40^{\circ}$ & 40 \\
\hline $45^{\circ}$ & $72 \quad$, & $45^{\circ}$ & 25 \\
\hline $50^{\circ}$ & $43 \cdot$, & $50^{\circ}$ & 10 \\
\hline
\end{tabular}

The same fall in amplitude of response, when a certain point has been reached in the thermal ascent, to which I

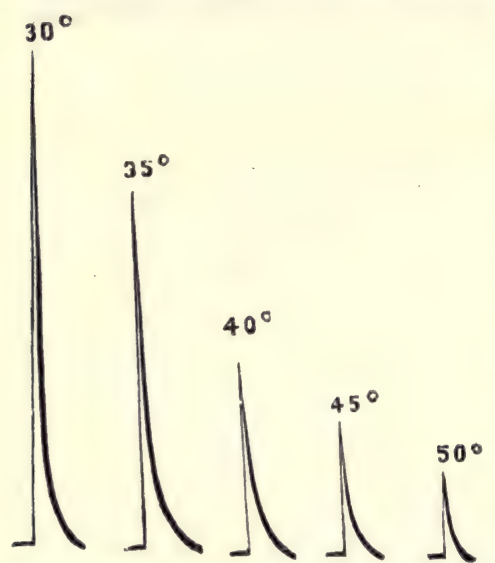
Response with Rising Temperature. (Stem of Amaranth)
FIG. 125. Diminished Amplitude of

have already referred, in the case of Eucharis lily, has also been noticed in that of muscle. From this it might be concluded that rise of temperature beyond $30^{\circ} \mathrm{C}$. or so, induced depression of excitability. But here we are met by an anomaly. For growth, which I have shown to be a phenomenon of excitatory response, increases, in the case of the plant, throughout the thermal ascent, up to an optimum point at or near $35^{\circ}$ C. Conductivity, again, which is, to a certain extent, correlated with excitability, undergoes enhancement with 
rising temperature. Thus in a certain specimen of Biophytum, for example, while the velocity of transmission at $30^{\circ} \mathrm{C}$. was $3.7 \mathrm{~mm}$. per second, it became enhanced to $9.1 \mathrm{~mm}$. - that is, nearly three times-when the temperature was raised to $37^{\circ} \mathrm{C}$. From these considerations, it would appear that the diminution in amplitude of electrical response, under a rising temperature below the rigor-point, might not always be due to a diminution of excitability, but to some other cause.

In connection with this, it must be borne in mind that two factors are included in the process of response: namely, the external stimulus which induces contraction, or galvanometric negativity, and the internal factor which brings about recovery. For I have already shown that whereas the action of stimulus induces one effect - the contraction, for example, of an excited tissue with galvanometric negativity -an increase of internal energy causes exactly the opposite - that is to say, the expansion of the tissue and galvanometric positivity. External stimulus and internal energy thus act antagonistically. A steady rise of temperature causes, as we have seen, an increase of internal energy. Hence, increased energy due to rise of temperature, enhancing the force of recovery, may cause a diminution of response, which is not due to diminution of excitability.

The inference that it is the increased internal energy due to rise of temperature which, by augmenting the force of recovery, diminishes the amplitude of response, appears the more probable from certain characteristics observed in the autonomous pulsation of Desmodium gyrans. If rise of temperature increased the force of recovery, we should expect, conversely, that a fall of a few degrees would have the effect of diminishing this force of recovery, and cor sequently enhancing the response. That this actually occurs will be seen in fig. I 26 , in the first part of which is given a series of responses at the temperature of the room, which was $29^{\circ} \mathrm{C}$. When the temperature of the plant-chamber was now lowered to $25^{\circ} \mathrm{C}$., the force of recovery would appear 
to be diminished, since the amplitude of response was considerably enhanced. That the general excitability of the plant was not increased by the lowering of temperature, is seen from the fact that the frequency of pulsation was reduced on cooling to about two-thirds of its original value.

The diminution of response with rising temperature may thus be due to an increase of internal energy, which tends

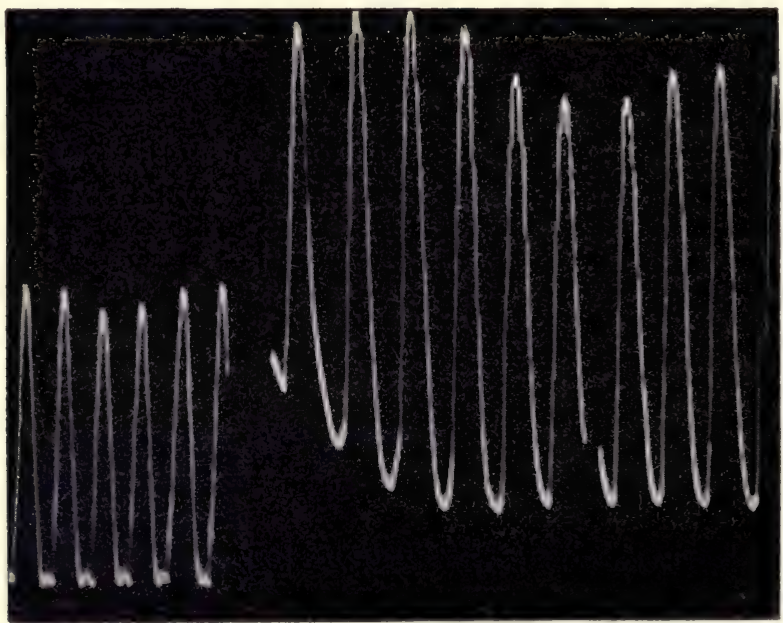

FIG. 126. Photographic Records of Autonomous Pulsations in Desmodium, showing Increase of Amplitude and Decrease of Frequency, with Lowering of Temperature

The pulsations to the left were recorded at the ordinary temperature of the room, $29^{\circ} \mathrm{C}$. Those to the right, when the temperature had been lowered to $25^{\circ} \mathrm{C}$.

to cause antagonistic expansion and consequent galvanometric positivity. This view finds support from the records seen in figs. I 29 and I33, given in the next chapter. The first of these (fig. 129) shows the expansion, with consequent physical elongation, of the filament of Passiflora under a rising temperature. In the second (fig. 133) is seen the increasing galvanometric positivity of a specimen of Amaranth under similar circumstances.

It is now clear that when the temperature of the tissue 
is below a certain thermotonic minimum, the effect of a rise of temperature will be to enhance the amplitude of response by removing molecular sluggishness. This fact has been illustrated in the gradually heightened mechanical response of the autonomous pulsation of Desmodium gyrans when a plant artificially cooled was allowed to return to the normal temperature of the room (fig. I20). If similarly a plant tissue be first cooled and then allowed to return to the surrounding temperature, its electrical responses to successive uniform stimuli being recorded throughout, a staircase increase of response will be observed during the return. When the temperature; however, is raised above a certain optimum, a depression of the amplitude of response begins, not by the depression of excitability, but by the increasing force of recovery due to an augmentation of the internal factor. True depression only takes place when the plant is approaching a condition of heat-rigor.

One very curious effect of temperature-variation which has been touched upon is the marked increase of sensitiveness which often makes its appearance as its after-effect. This is seen exemplified in the record given in fig. I24, showing the effect of a cyclic variation of temperature on Eucharis lily. In another experiment with Scotch kale, the response at the temperature of $30^{\circ} \mathrm{C}$. was eleven divisions, and at $50^{\circ} \mathrm{C}$. eight divisions, during the thermal ascent. During the descent, however, the amplitude at $50^{\circ} \mathrm{C}$. was sixteen, and at $30^{\circ} \mathrm{C}$. twenty-three divisions. The sensitiveness was thus doubled. This enhancement may be due in part to the increased molecular mobility consequent on the annealing effect, as it were, of temperaturevariation. But it may also be regarded as partly due to the difference of the antagonistic forces which the excitatory response has to overcome during ascent and descent. During the thermal ascent, the opposing expansive force is being rapidly accelerated. During the thermal descent, on the other hand, this is no longer the case, for the force of recovery is now undergoing a diminution. 
When the temperature is raised above a certain critical point, the plant is killed, and its electrical response disappears at the same time. This is demonstrated visually in the accompanying photographic record (fig. I27). In this case, normal responses were first obtained at the usual temperature of the room. Steam was next introduced into the

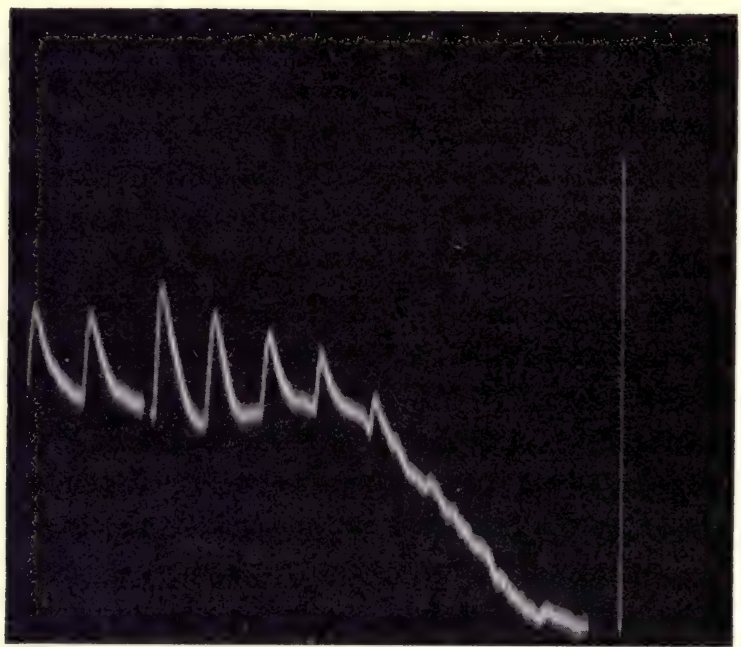

Before $\uparrow \quad$ After

FIG. 127. Photographic record showing effect of Steam in abolishing Response

The two records to the left exhibit normal response at $17^{\circ} \mathrm{C}$. Sudden warming by steam induced at first an inorease of response, but five minutes' exposure to steam killed the plant (carrot) and abolished the response.

Vibrational stimulus of $30^{\circ}$ applied at intervals of one minute; vertical line $=\cdot \mathrm{I}$ volt.

plant-chamber, and kept streaming in during the course of the experiment, electrical responses being recorded meanwhile at intervals of one minute. It will be seen that at first a transitory augmentation of excitability was induced. But this quickly disappeared, and in five minutes the plant was effectively killed, as is shown in the waning and final abolition of response. This experiment affords us a qualitative demonstration of the abolition of response at death under 
the influence of high temperature. In the next chapter we shall enter upon an exact determination of this critical point of death.

It is thus seen that temperature modifies the electrical response of plants. There is a temperature-minimum below which response is abolished. If the plant be kept too long at this temperature it is apt to be killed. In the case of a delicate species like Eucharis, which is highly susceptible of the injurious effect of cold, the electrical response is permanently abolished by long exposure. But hardier plants, like Holly and Ivy, show revival of electrical response, on a return to a favourable temperature. The electrical response disappears also at a certain maximum temperature constituting the death-point. 


\section{CHAPTER XVI}

THE ELECTRICAL SPASM OF DEATH

Different post-mortem symptoms-Accurate methods for determination of deathpoint-Determination of death-point by abolition or reversal of normal electrical response-Determination of death-point by mechanical death-spasmFrom thermo-mechanical inversion-By observation of electrical spasm : $(a)$ in anisotropic organs : $(b)$ in radial organs-Simultaneous record of electrical inversion and reversal of normal electrical response-Remarkable consistency of results obtained by different methods-Tabulation of observations.

IT will be seen from the last chapter that there is in the case of every plant a certain high temperature which is critical, since above it life passes into death. Much difficulty has been experienced in the exact determination of this critical point, because no sure criterion of death was hitherto available, such as would furnish an immediate and reliable indication of its occurrence. The various symptoms of death, such as drooping, withering, discoloration and the escape of coloured cell-sap, do not manifest themselves at the onset of death, but at some time indeterminately later. Even when a plant has been subjected to a temperature in excess of the fatal degree, it continues to appear fresh and living ; and it is not till after some longer or shorter interval that the death symptoms are seen.

To take, for example, the symptom of drooping, it is clear that the loss of turgidity on which this depends cannot at once make itself visible. In a thick tissue, again, death may take place in the superficial layers of the plant, the interior tissues, owing to feeble thermal conductivity, remaining comparatively unharmed. Or, if we employ the test of discoloration, which we shall find to occur some time after the initiation of death we find that the exact moment at 
which discoloration begins cannot be detected with sufficient precision. When we place the specimen in a thermal bath under a rising temperature, the beginning of discoloration after death is so slight as to be impossible of detection, and by the time it becomes marked, the temperature has already passed several degrees above the fatal point. I have found, for example, that the colour of the milk-white style of Datura alba has changed to brown by the time that the temperature of the bath has risen to about $64^{\circ} \mathrm{C}$. In the petals of Sesbania coccineum, again, a striking change of colour is detected, under similar conditions. Rich crimson here turns into pale blue at a temperature of about $67^{\circ} \mathrm{C}$. The filamentous corona of Passiflora quadrangularis, finally, in which the filaments are barred by purple rings, loses its colour normally at about $68^{\circ} \mathrm{C}$. In all these cases, the initiation of the loss of colour must have been imperceptible. Hence, all that can be determined from such experiments is that the death-changes must have commenced at some temperature lower than $64^{\circ}$ to $68^{\circ} \mathrm{C}$.

Before proceeding further, it is necessary to obtain a clear idea of what is meant by the death-point. In animals, an early symptom of death consists in the setting in of rigor mortis. But this does not synchronise throughout the body, certain parts of the organism undergoing the deathchange earlier than others. Thus the only definition of the death-point which can be made at all precise is that which regards it as the point of initiation of some unmistakable sign of death. I shall next proceed to describe several death-symptoms and the modes by which they may be detected with certainty.

With regard to such detection, I have pointed out elsewhere that, theoretically, it should be possible to make such a determination by watching the waning of some effect characteristic of the living condition, the death-point being known by its cessation at a given moment. Such a test, as we shall presently see, is afforded by the electrical responses. The ideally perfect method, however, would be by the 
detection of some effect which at the moment of death underwent a sudden reversal to its opposite. There would not here be even that minor degree of uncertainty which is incidental to the determination of the exact vanishing-point of a waning effect. And such methods are afforded by my discovery of the occurrence of mechanical and electrical spasms at the moment of death.

Turning now to the method of the waning effect, we have seen that response to stimulus by galvanometric negativity is distinctive of the living condition. When the plant is killed, this normal response disappears. At the moment of death from high temperature, therefore, we may expect to see the abolition of this normal excitatory response of negativity.

For this investigation I took a batch of six radishes. The specimens were kept for five minutes previous to each experiment in water at a definite temperature (say of $17^{\circ} \mathrm{C}$.), and were then mounted in the vibration-apparatus and their responses observed. Each specimen was next dismounted and replaced in the bath at a higher temperature (say of $30^{\circ} \mathrm{C}$.) for another five minutes. After this, a second set of responses, to the same stimulus as before, was taken. In this way observations were made with each plant, till the temperature at which response almost or altogether ceased was reached. I give below (p. 195) a table of the results obtained with the six radishes.

From these experiments it would appear that in these cases the responses disappeared at about $55^{\circ} \mathrm{C}$. It should be stated here that this investigation was carried out in the winter season in England, and it will be shown later that the incidence of cold has the effect of lowering the normal death-point by about $4^{\circ}$ or $5^{\circ} \mathrm{C}$.

I was next desirous of substituting, for this method of discontinuous observations, one which should be continuous. I, therefore, subjected the specimen-a stem of Amaranthto a continuous rise of temperature, and took records of responses to uniform stimuli after every few degrees of the ascent. I found here that not only was there a gradual 
Table showing EfFect of High Temperature in abolishing RESPONSE OF RADISH (Raphamus sativus)

\begin{tabular}{|c|c|c|}
\hline Specimen & Temperature & $\begin{array}{l}\text { Galvanometrie response } \\
\text { roo divisions }=0^{\circ} 7 \text { volt }\end{array}$ \\
\hline I & $\begin{array}{l}\mathrm{I} 7^{\circ} \mathrm{C} . \\
53^{\circ} \mathrm{C} .\end{array}$ & $\begin{array}{r}70 \\
4\end{array}$ \\
\hline${ }_{2}$ & $\begin{array}{l}\mathrm{I} 7^{\circ} \mathrm{C} . \\
53^{\circ} \mathrm{C} .\end{array}$ & $\begin{array}{r}160 \\
1\end{array}$ \\
\hline 3 & $\begin{array}{l}17^{\circ} \mathrm{C} \text {. } \\
50^{\circ} \mathrm{C} .\end{array}$ & $\begin{array}{r}100 \\
2\end{array}$ \\
\hline 4 & $\begin{array}{l}17^{\circ} \mathrm{C} . \\
55^{\circ} \mathrm{C} .\end{array}$ & $\begin{array}{r}80 \\
0\end{array}$ \\
\hline 5 & $\begin{array}{l}17^{\circ} \mathrm{C} . \\
60^{\circ} \mathrm{C} .\end{array}$ & $\begin{array}{r}40 \\
0\end{array}$ \\
\hline 6 & $\begin{array}{l}17^{\circ} \mathrm{C} . \\
55^{\circ} \mathrm{C} .\end{array}$ & $\begin{array}{r}60 \\
0\end{array}$ \\
\hline
\end{tabular}

decrease of response, tending towards its abolition, with rising temperature, but also that, at the death-point, it under-
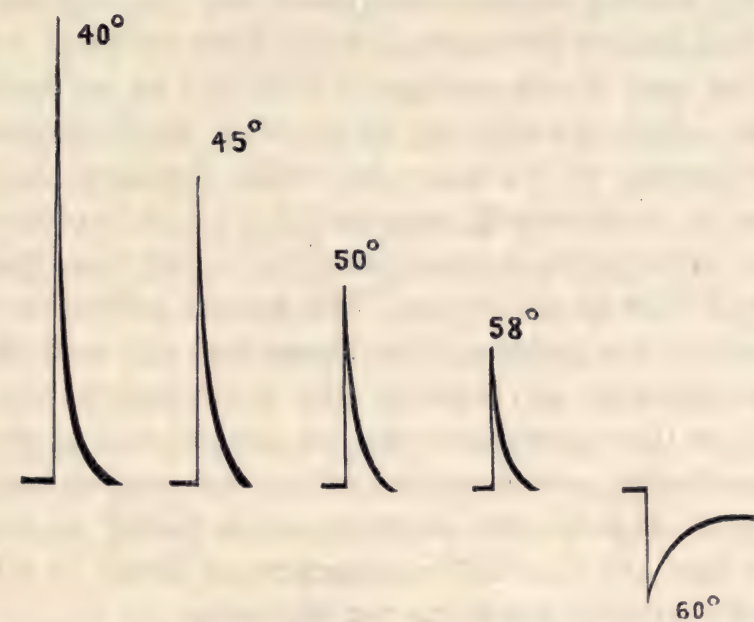

FIG. 128. Record of Electric Responses of Amaranth at various temperatures The response undergoes reversal to positive at the critical temperature of $60^{\circ} \mathrm{C}$.

went actual reversal from the normal galvanometric negativity to positivity (fig. I 28). This was due to the fact that 
on reaching the death-point, the contained positive component in response was unmasked by the abolition of the true excitatory effect. But this positive response disappears also after a short time. It will thus be seen that by this method the death-point is capable of determination within very narrow limits, having been, in the present case, near $60^{\circ} \mathrm{C}$. When the tissue is thin, this temperature soon proves fatal. But should it be thick, a very much longer exposure to it is necessary, if the interior of the tissue is to be killed effectively.

I have also discovered another method of obtaining the death-point with precision, in the symptoms afforded by mechanical responses. For I found that a death-spasm occurs at a certain critical moment in a plant, which is analogous to the death-throe of the animal. The experimental plantMimosa, for instance-was placed in a bath of water, whose temperature was being raised gradually, at a uniform rate of, say, $\mathrm{I}^{\circ} \mathrm{C}$. per one minute and a half, until the death-point was reached. During all this time there was no responsive fall of the leaf, for, we have seen, it is a sudden variation, and not a gradual rise of temperature, which acts as an excitatory stimulus. This gradual rise, on the other hand, increases the internal energy of the plant, by which the turgidity of the pulvinus is continuously augmented. In this process the increase of turgidity is more energetic in the more excitable lower half than in the upper. The greater expansion of the lower side of the pulvinus thus raises the leaf continuously. But immediately on reaching the death-point, there is a reversal of thls movement, and an abrupt fall of the leaf. This spasmodic movement is sudden and well-defined. In a vigorous Mimosa the death-spasm is found to occur at or very near $60^{\circ} \mathrm{C}$. This contraction of death is followed after some time by a post-mortem relaxation.

That the death-response is an excitatory phenomenon is seen from the fact that any circumstance which lowers physiological activity lowers the death-point also. Thus, after a spell of cold weather, I found that the death-point 
of Mimosa was lowered from the normal $60^{\circ}$ C. to about $53^{\circ} \mathrm{C}$. This latter value, it will be remembered, was approximately the same as that obtained with radish, in winter, by the method of electrical response. Again, I find fatigue to induce a lowering of the death-point, the extent of which depends upon the degree of fatigue. When this was moderate, I have found the death-point of Mimosa to be lowered to $56^{\circ} \mathrm{C}$.

This spasmodic contraction, indicative of the initiation of death, may express itself in diverse ways. For example, if the tubular peduncle of Allium be filled with water, and raised gradually in temperature, there comes a moment at which a sudden expulsion of the contained water occurs. A spiral tendril of Passiflora, under the same circumstances, exhibits a sudden uncurling. The florets of the ray, in certain Composite, show characteristic movements, either up or down. In all these cases alike, under normal circumstances, the death-point is found to be at or near $60^{\circ} \mathrm{C}$.

Turning next to the radial organs of ordinary plants, these also exhibit a sudden longitudinal contraction at the onset of death. I have shown elsewhere how, by means of the Morograph, an instrument which I devised for this purpose, a thermo-mechanical curve is recorded by the specimen, while it is being subjected to the continuous rise of temperature, culminating in the death-point. The ordinate of this curve represents the induced variation of length, and the abscissa the temperature. The expansion described in the case of Mimosa is seen here in the form of a gradual elongation, up to the moment of reaching the death-point. When this point is reached, however, a sudden contraction takes place, giving rise to an inversion of the curve. This turning point is very abrupt. The curve as a whole is thus one of life-and-death, in which the point of inversion separates the two. I give below a photographic record of this thermo-mechanical curve, obtained with the coronal filament of Passiflora. The death-point occurred here at $59^{\circ} 6^{\circ}$ C. (fig. 129). The thermo-mechanical curve is very 
similar in similar specimens under normal conditions. Fig. I 30 gives two records of two different styles of Datura alba, obtained from flowers of the same plant. The deathpoint is seen to have occurred at $60^{\circ} \mathrm{C}$. In recording the thermo-mechanical curve, there is found to be, normally speaking, a continuous expansion up to the death-point. In the case of vigorous specimens, in a good tonic condition,

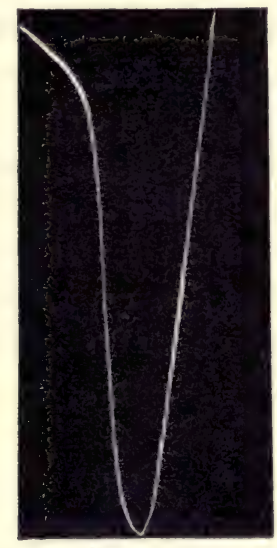

FIG. I29. Photographic Record of Thermo-mechanical Curve given by Coronal Filament of Passiflora

The first or down part of the curve shows expansion, but on reaching death-point, at $59^{\circ} 6^{\circ} \mathrm{C}$., there is a sudden inversion, due to spasmodic death-contraction. the inversion does not take place till about $59^{\circ} 6^{\circ}$ or $60^{\circ} \mathrm{C}$. But in less vigorous specimens, a certain hesitation, as it were, is seen to occur in the record at or near $55^{\circ} \mathrm{C}$. With vigorous specimens,

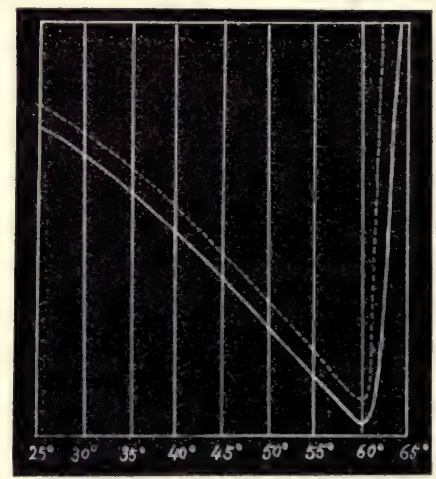

Fig. I30. Thermo-mechanical Curve of . Two Different Specimens of Style of Datura alba, obtained from Flowers of the same Plant

there may be the merest indication of this hesitation; in other cases, with less favourable tonic condition, the hesitation is prolonged, but the expansion finally proceeds, and the death inversion takes place at the usual temperature of about $60^{\circ} \mathrm{C}$. When the specimen, however, is enfeebled, or has been subjected to unfavourable circumstances, the point of transient instability becomes fatal, and the inversion takes place there. As an example of what has just been referred to-namely, the influence of unfavourable external circum- 
stances in lowering the death-point-it may be mentioned here that the sudden incidence of cold weather will lower it by some $4^{\circ}$ or $5^{\circ} \mathrm{C}$. Intense fatigue will lower it by as much as $19^{\circ} \mathrm{C}$.

I have already said that this spasm, taking place at the moment of death, is an excitatory response. On this theory it occurred to me that it should also be possible to determine the onset of death by an electrical spasm. It may be well at this point, therefore, to examine some of the conditions under which such a spasm, supposing it to take place, might be displayed most conspicuously. We may suppose a radial organ, with the usual electrical contacts, A and B, to have its temperature raised gradually up to the death-point. The excitatory effect of death may now be expected to cause the galvanometric negativity of a given point. But since these excitatory effects are equal and similar at A and B, they will balance each other, and there will be little or no resultant galvanometric response. In order to obtain a marked resultant effect, then, we must have an organ in which the excitabilities of the two points $\mathrm{A}$ and $\mathrm{B}$ are different. This difference of excitability, necessary to the exhibition of a resultant response, may be either natural or artificially induced. For the former, we may take a specimen which is not radial, but anisotropic, thus affording us two points of galvanometric contact, possessed of unequal excitabilities.

We have seen that the inner surface of the petiole of Cucurbita maxima was more excitable than the outer. The same is true of the hollow peduncle of Uriclis lily. There is also a great difference of excitability as between the upper and lower sides of the scale of the bulb of the same lily, in the season of flowering, the concave surface of this scale being more excitable than the convex. Any of these specimens described I find to answer admirably for the purpose of this investigation.

Taking the petiole of Cucurbita, then, I divided it longitudinally, and rejected one-half, thus obtaining a half-tube, of which the inner concave surface was more excitable than 
the outer convex. Electric contacts were now made through non-polarisable electrodes with equal and opposite areas on the two sides. These adjustments were made in a heating chamber containing electrical arrangements by which the temperature could be raised continuously. This was satisfactorily accomplished by an incandescent electrical lamp which was placed in a second chamber, vertically below the plant-chamber. There was a wooden partition between the two, by which the light of the lamp was excluded from the specimen (fig. I3I). For the radiation itself will be

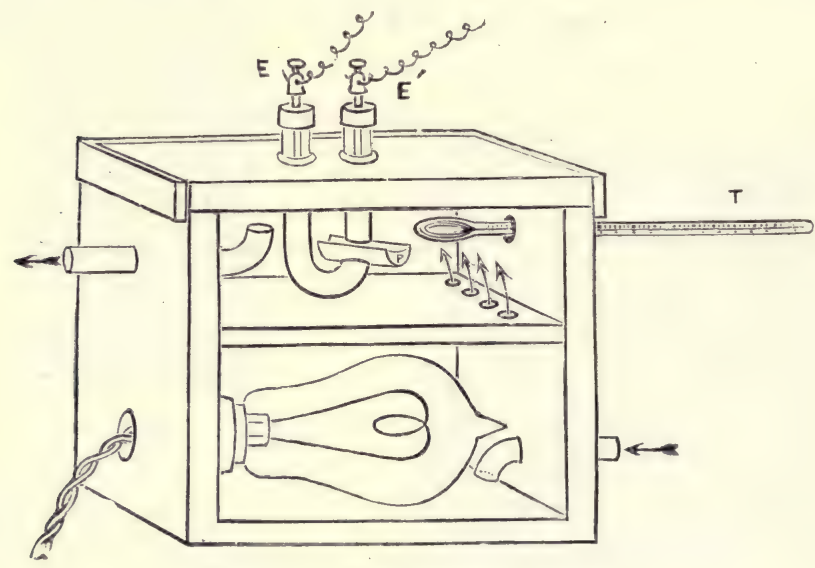

FIG. I3I. The Thermal Chamber

Electric lamp in the lower compartment raises temperature of the upper. E, $\mathbf{E}^{\prime}$, electrodes making contacts with the specimen; $\mathrm{T}$, thermometer.

shown to constitute stimulus, and the object in the present case was to eliminate all exciting factors except death itself. By means of side-openings, the heated air was enabled to pass into the plant-chamber, thus raising the temperature. A rheostat included in the lamp-circuit made it possible to adjust the rate of this rise of temperature, its average being about $I^{\circ}$ per minute.

The natural current through the petiole is, under normal circumstances, from the less excitable outer to the more excitable inner surface: that is to say, the inner is galvano- 
metrically positive. This is true when the excitation, due to section made for the purpose of preparation, has subsided. This stimulation, by causing greater excitation of the inner surface, is liable to induce there a temporary negativity. A gradual rise of temperature, as we saw, caused an increased turgidity of the more excitable lower side of the pulvinus of Mimosa, and this increased turgidity was exhibited mechanically by the erection of the leaf. But the electrical sign of increased turgidity is galvanometric positivity. We have also seen that electrical responses occur equally in motile and non-motile tissues. In the petiole of Cucurbita then, on its more excitable inner surface, we obtain, during the gradual rise of temperature, an increasing galvanometric positivity. This is true only, as has been said before, when the rise is continuous, and not marked by fluctuation. For any sudden variation will act as a stimulus, causing galvanometric negativity of the more excitable inner side. For this reason it is necessary that the rheostatic resistance interposed in the lamp-circuit, for the adjustment of the uniform rate of rise of temperature, should be made at the beginning of the experiment, such tissue being very sensitive to this particular stimulatory action. At the commencement of my investigation I experienced much trouble from the erratic movement of the galvanometer spot of light, and the obtaining of a steady electrical curve seemed at that time almost hopeless. Later on, however, I found that these fluctuations were traceable to temperature-variations, unavoidably associated with the attempt to regulate the rise of temperature by movement of the rheostatic slide. It is for this reason, then, that the adjustment must be made, once for all, at the beginning.

Carrying out the experiment in this manner, I obtained, with various anisotropic organs, a sudden inversion of the electric curve at the death-point. This death-point was found, in all vigorous specimens, from which traces of injury had been removed by previous rest, to occur accurately at $59^{\circ} 6^{\circ}$ or $60^{\circ} \mathrm{C}$. In these electrical curves, the same 
point of instability, already noticed in the thermo-mechanical curve, was often found to occur at or about $55^{\circ} \mathrm{C}$. And if the specimen were not in favourable tonic condition, or had been suffering from injury, the death-point was lowered to this degree.

I give below an electrical curve showing the point of inversion at death (fig. I32). It was obtained with the

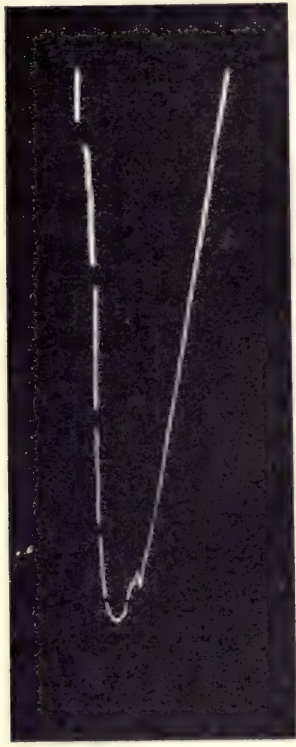

FIG. I32. Photographic Record exhibiting Electric Spasm in the Petiole of Musa

Sudden electric inversion takes place at the death-point, $59^{\circ} 5^{\circ}$. Record was commenced at $54^{\circ} \mathrm{C}$., and successive gaps in the record indicate $I^{\circ} \mathrm{C}$. rise of temperature. sheathing petiole of Musa. The inner or concave side of this petiole is more excitable, as we have seen, than the outer. These responsive electrical variations were very large, and could not be represented within the limits of the photographic plate. I therefore took a photographic record between the temperature of $54^{\circ} \mathrm{C}$. and $67^{\circ} \mathrm{C}$. only. The first part of the curve represents the increasing galvanometric positivity of the more excitable inner surface of the specimen. The same process of increasing positivity under the continuous rise of temperature, had been going on previously, it is to be understood, before arrival at $54^{\circ} \mathrm{C}$, at which the photographic record was commenced. This increasing galvanometric positivity corresponds to the gradual erection of the leaf in Mimosa, and to the expansion of a radial organ, such as a coronal filament of Passiflora, all alike being due.to the positive variation of turgidity. In order to give an indication of the particular temperature at each portion of the curve, the recording light was obscured for about I 5 seconds after each degree of temperature. The successive gaps, then, are one 
degree centigrade of temperature apart. These interruptions, however, were not made after the occurrence of the inversion. As soon as the death-point was reached, in the present case at $59.5^{\circ} \mathrm{C}$., there was a sudden inversion of the electrical curve (fig. 132), corresponding with the point of inversion of the thermo-mechanical curve (fig. 129). Each of these curves is seen to bear a striking resemblance to the other. In both cases, the inversion was due to the same fact of sudden excitation, finding expression in the one, in induced galvanometric negativity, and in the other, in mechanical contraction.

In the case of organs which are more or less radial, and in which there is little differential excitability, it is necessary to abolish the excitability of one contact, as, say, by previous scalding. For this experiment I took a leaf of Amaranth, and injured a portion of the lamina by immersion in boiling water. The two contacts were made, one with the petiole, and the other with the injured lamina. On raising the temperature continuously, the more excitable petiole became increasingly positive. The photographic record in this case was commenced only on reaching $55^{\circ} \mathrm{C}$., and the death-inversion took place at $59^{\circ} 5^{\circ} \mathrm{C}$. (fig. 133).

We have already seen that, besides this electrical reversal, there is another

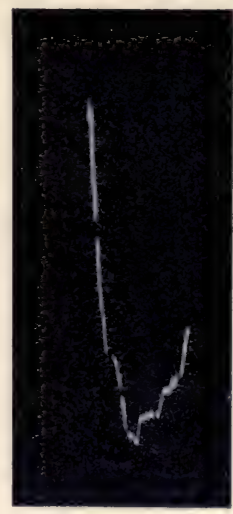

FIG. 133. Photographic Record showing Electric Inversion at Death-point, $59.5^{\circ}$, in the Petiole of Amaranth means of detection of the death-point, afforded by the conversion of the response to external stimulus from the normal negative into positive. Now it occurred to me that it would be interesting, if in the same specimen, both these tests could be applied at the same time. We could then see whether two methods so independent of each other furnished mutual corroboration or not. For this purpose I took a stem of 
Amaranth, and abolished the excitability of one of the two contacts - a lateral leaf-by scalding. The electrical curve, under a continuously rising temperature, was now taken in the usual manner. The existing electro-motive difference between living and injured contacts underwent the usual

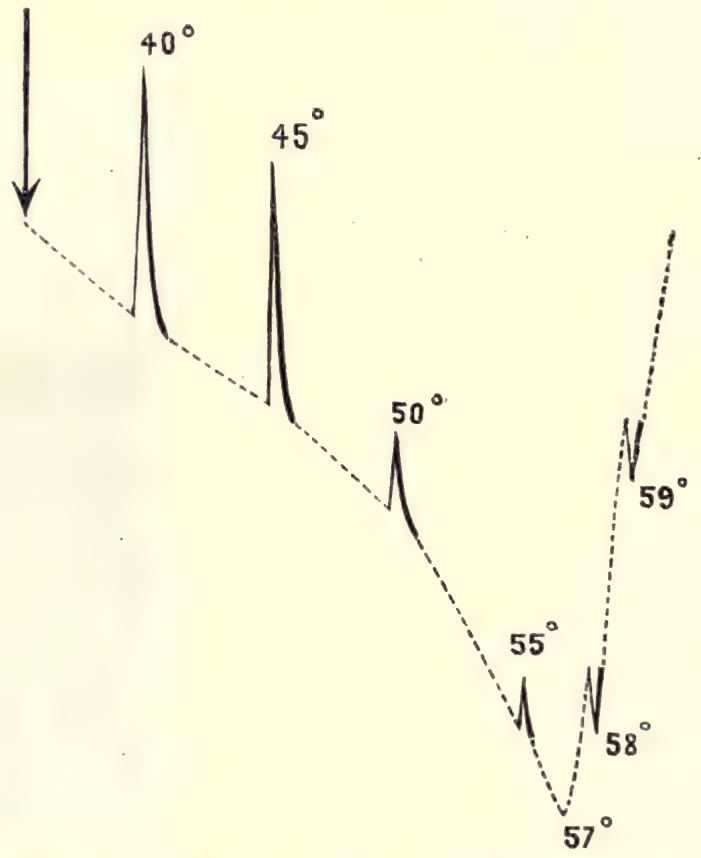

FIG. 134. Record showing Inversion of Electric Curve (represented by dotted line) and Simultaneous Reversal of Electric Response in Stem of Amaranth

$\downarrow$ indicates current of injury from injured to uninjured contact, which, reaching a maximum, undergoes reversal at death-point. Normal response up, also becomes reversed to down after death-point.

increase, reaching a maximum at the death-point. Meanwhile electrical responses to uniform vibrational stimuli were taken at intervals a few degrees apart. It will be seen (fig. I34) that the electrical inversion took place at $57^{\circ} \mathrm{C}$, this moderate lowering of the death-point being due, in all probability, to the slight depression caused by scalding at 
the distal contact, which had not had time to pass off. The test-responses to uniform mechanical stimulation which were being taken meanwhile show a continuous diminution towards the death-point. When this, however, had been passed, the response is seen to be reversed in direction to positive. As has been said before, this positive response also disappears after a time. We thus obtain a very striking demonstration of the fact that the reversal of the electrical curve and the reversal of the sign of response are concomitant.

It may be mentioned here, in anticipation of a future chapter, that the death-point may also be obtained by the sudden inversion of the curve of electrical resistivity, and that the value obtained in this way coincides with those already given.

I give below a table showing the death-points determined by various methods :

Table showing Death-Points Obtained by Different Methods

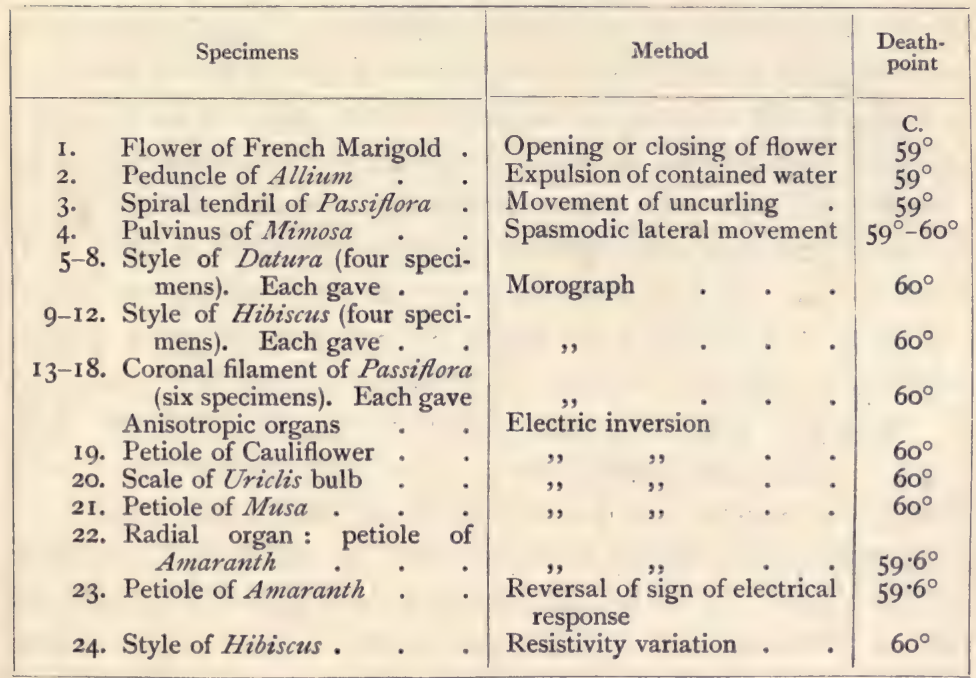

It is thus seen that employing different methods and using plant-organs which are equally diverse-flowers, bulbs, petioles, and others - a death-point is determined which 
is very definite and practically the same for all phanerogamous plants. Among the mechanical methods, that which depends on the thermo-mechanical curve, giving the death-point by a sudden inversion, is specially accurate. Of an equally precise character is the death-point obtained by the inversion of an electrical curve, and also that which is given by reversal of the electrical response. And it is in the highest degree remarkable that the points of inversion in the mechanical and electrical curves respectively, together with the point of reversal of the electrical response itself, should so exactly coincide. 


\section{CHAPTER XVII}

\section{MULTIPLE AND AUTONOMOUS ELECTRICAL RESPONSE}

Repeated responses under single strong stimulus-Multiple mechanical response in Biophytum-Multiple electrical responses in various animal and vegetable tissues-Continuity of multiple and autonomous response-Transition from multiple response to autonomous, and vice versa-Autonomous mechanical response of Desmodium gyrans and its time-relations - Simultaneous mechanical and electrical records of automatic pulsations in DesmodiumDouble electrical pulsation, principal and subsidiary waves--Electrical pulsation of Desmodium leaflet under physical restraint-Growth-pulsation -So-called current of rest in growing plants.

WE have seen that when a plant organ is acted on by a single stimulus of sufficient intensity, it exhibits a single excitatory effect, which may show itself in two independent ways, as mechanical and electrical response. We have also seen that part of the impinging stimulus may become latent, to find appropriate expression later. It was also shown that with increasing intensity of stimulus the amplitude of response reaches a limit. It may thus happen that a very strong stimulus, not finding adequate expression in a single response, will exhibit itself by means of repeated responses. The incident energy in such cases is held latent for a time, ${ }^{1}$ to manifest itself later in a rhythmic manner.

I have been able to demonstrate the occurrence of this multiple excitation, in response to a single strong stimulus, by several different and independent methods. The simplest and most striking of these depends on the recording of the motile effects in the leaflets of Biophytum. In fig. I 35 are seen no less than sixteen multiple pulsations resulting from a single strong thermal stimulation of the petiole bearing the leaflets.

1 For more detailed account see Bose, Plant Response, pp. 2'79-357. 
The average period of each pulsation is here about thirty seconds ; but this may vary in different cases from half of this to one minute. I have also been able to detect these multiple excitatory waves, during their transit through non-motile conducting tissues such as stems. The imperceptible volumetric changes which occur on the arrival of excitation were here detected electrically by variations of pressure induced in an enclosing microphonic contact. In fig. I36 is seen such

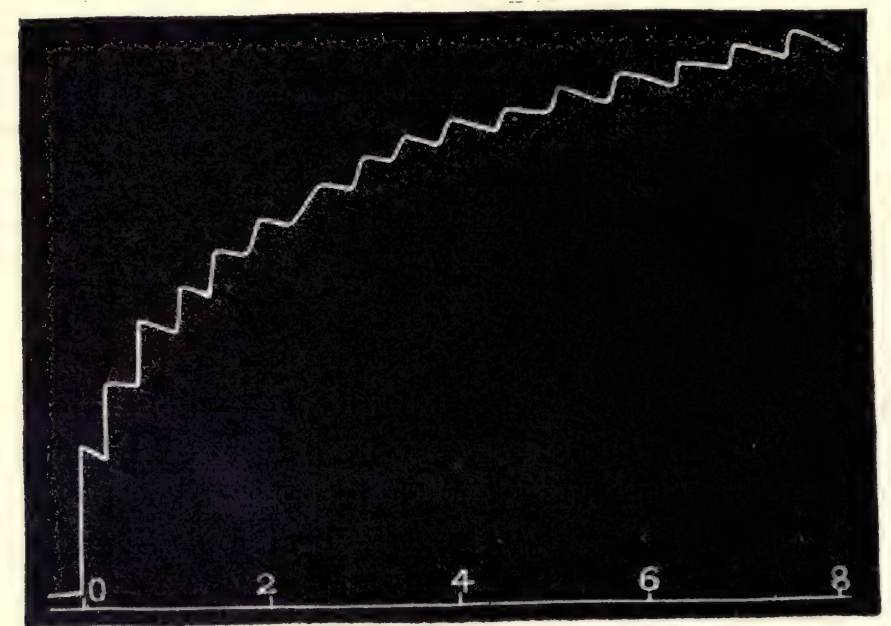

FIG. 135. Multiple Mechanical Response of Biophytum, due to a Single Strong Thermal Stimulus

multiple electro-tactile response in the stem of Mimosa, due to a single thermal stimulus.

I have also been able to record such multiple excitatory effects by means of electro-motive response. In fig. 137 is seen a photographic record of a series of such responses, given by the leaf of Biophytum, the individual thermal stimuli being here applied at intervals of five minutes. It will here be noticed that each single stimulus gave rise to from five to eight responses, the average period of which was thirty seconds. From the corresponding mechanical 
responses, it will be remembered that the average period of these had also this value.

These multiple responses to a single strong stimulus, while very strikingly manifested by such plant-tissues as the pulvinules of Biophytum, and in the animal, by the cardiac tissue, are also exhibited by almost all kinds of tissues under favourable circumstances. In fig. I 38 will be seen records which show this in the case of different vegetable organs under diverse forms of stimulation. In fig. I 39 is given a series of multiple responses which I have obtained from frog's stomach. I have also

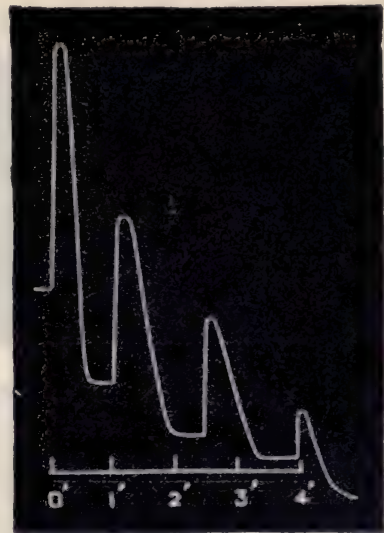

FIG. I36. Multiple Electro-tactile Response in Stem of Mimosa, due to Single Strong Thermal Stimulus

(Original record reduced to $\frac{1}{8}$.) detected the occurrence of multiple responses in nerves of animals, which will be described in a later chapter.

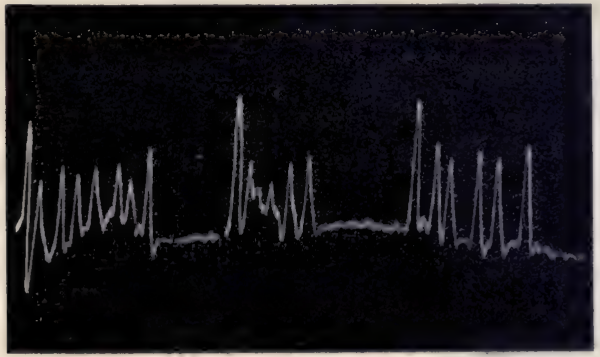

FIG. 137. Photographic Record of Multiple Electrical Response in Leaf of Biophytum

First series of eight responses to a single thermal stimulus; second stimulus, after interval of five minutes, evoked five responses; third stimulus, after second interval of five minutes, gave six responses. Average period of each response, thircy seconds nearly.

We have seen that multiple response takes place in consequence of some sufficient increase of internal energy. 
In the cases mentioned, this was derived from impinging stimulus. The internal energy of a tissue may, however, be

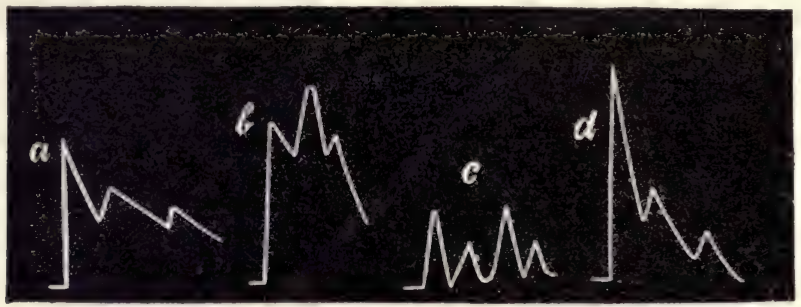

FIG. 138. Multiple Electrical Responses under Different Forms of Stimulus in Different Organs

(a) In Mimosa due to thermal, and (b) to chemical stimulation ; $(c)$ in peduncle of Biophytum, due to thermal stimulus ; [N.B. - This series persisted for two hours.] (d) in hypocotyl of Tamarindus indica, due to stimulus of cut.

increased, and multiple response may consequently be initiated in other ways, as, for instance, by adequately raising

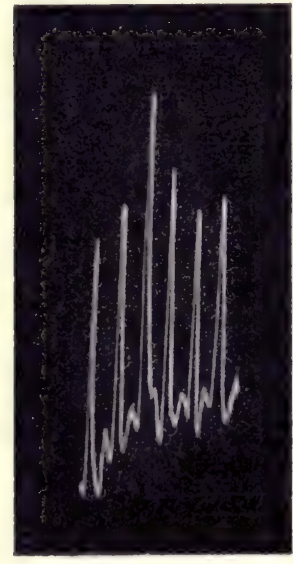

FIG. 139. Photographic Record of Multiple Electrical Response to Single Thermal Shock in Frog's Stomach the temperature of the plant. This is seen in the following record of pulsatory responses as induced in a young leaflet of Biophytum, when the temperature was raised to $35^{\circ} \mathrm{C}$. With the increase of internal energy, the turgidity of the tissue was enhanced, and the excessive hydrostatic tension thus brought about induced autonomous pulsations (fig. I40), as in a quiescent snail's heart similar pulsations are induced by increase of the internal hydrostatic pressure. I have elsewhere shown that the energy which expresses itself in pulsatory movements may be derived by the plant, either directly from immediate external sources; or from an excess of such 
energy already accumulated and held latent in the tissue, aided by the incidence of external stimulus; or from the excessive accumulation of such latent energy alone. There is thus a continuity between multiple and autonomically responding plants. Biophytum, which under ordinary circumstances belongs to the former of these classes, becomes converted into the latter under exceptionally favourable tonic conditions. That is to say, it responds by a single response to a single moderate stimulus, and by multiple

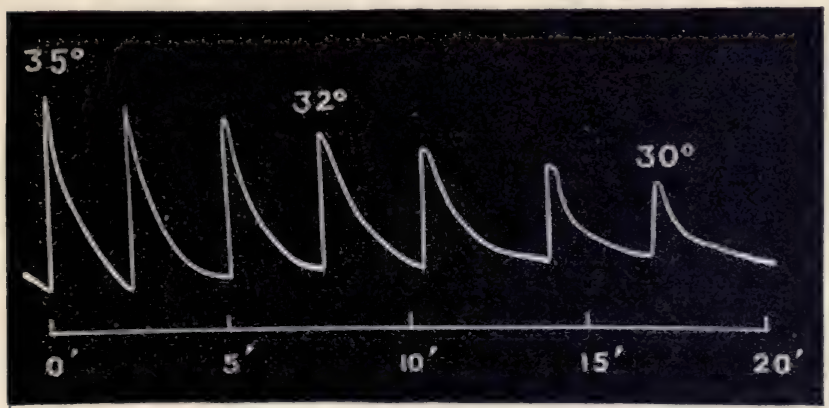

FIG. 140. Induction of Autonomous Response in Biophylum at Moderately High Temperature of $35^{\circ} \mathrm{C}$.

Note the diminution of amplitude of response with falling temperature. The pulsations came to a stop below $29^{\circ} \mathrm{C}$.

responses to a strong stimulus. Under exceptionally favourable tonic conditions, however, it exhibits spontaneous or autonomous responses. Desmodium gyrans, on the other hand, which ordinarily exhibits autonomous response, will, under unfavourable circumstances, cease to exhibit spontaneous movements. It then exhibits a single response to a single moderate stimulus, and multiple responses to a single strong stimulus :

When the leaflet of this plant, owing to deficit of internal energy, is in a state of standstill, a renewal of the supply of stimulus will restore it to a condition of autonomous response. This is seen in the following record (fig. 14I) of the response 
of the leaflet of Desmodium. The leaflet was in a quiescent condition, but under the action of stimulus of light, it exhibited multiple responses ; and these, owing to the increasing absorption of energy, showed a staircase enhancement of amplitude. On the cessation of light, the energy absorbed maintained the pulsation for some time.

It is thus the absorption of energy which is the cause of the so-called autonomous movements. The energy, as already stated, may be derived by the plant either directly from external sources; or from the excess already accumulated and held latent in the tissue, aided by incident external stimulus; or from an excess of latent energy previously accumulated.

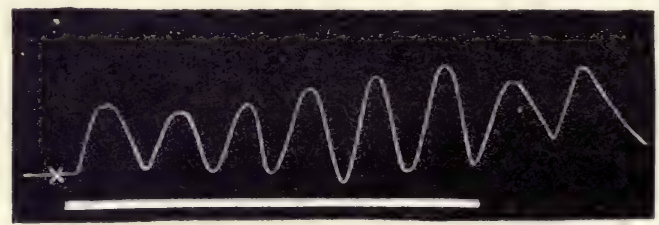

FIG. I41. Initiation of Multiple Response in Lateral Leaflet of Desmodium originally at Standstill

Light applied at $\times$ and continued till the end of the sixth response, as shown by the thick line. The responses show a staircase increase with increase of absorbed energy. Pulsations persist for a short time even on the cessation of stimulus.

It would be impossible to conceive of movement without an exciting cause. Only under the action of stimulus can a living tissue give responsive indications. An external stimulus may either give rise to an immediate responsive expression, or be partly or wholly reserved in latent form for subsequent manifestation. 'Inner stimuli' are simply external stimuli previously absorbed and held latent. A plant or animal is thus an accumulator which is constantly storing up energy from external sources, and numerous manifestations of life-often periodic in their character-are but responsive expressions of energy which has been derived from external sources and is held latent in the tissue. 
In Desmodium gyrans, as is well known, we have the typical example of autonomous response, its secondary leaflets executing periodic up and down or elliptical movements. The movement of the leaflet in some instances takes place by jerks, in others it is more uniform. The period of a complete up-and-down movement varies between two and four minutes. The length of this period is much affected by temperature, being less when this is moderately high. From the normal, or highest position, the leaflet sinks somewhat rapidly; having reached its maximum depressed position, it rests for a while. There is next a rather slow rise to its original position. This up-and-down motion is in some cases approximately straight. In others, the pulvinule of the leaflet is slightly twisted after its descent, and the corresponding curve described becomes more circular.

In view of certain peculiarities to be observed in the electrical response of Desmodium,

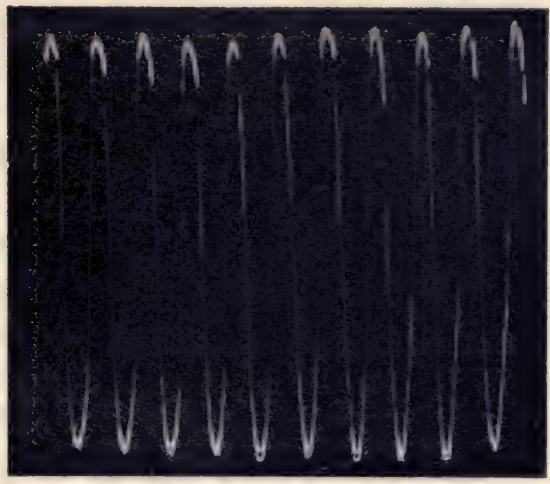

FIG. 142. Photographic Record of Autonomous Mechanical Pulsation in Desmodium Leaflet Period of each complete pulsation $=2.7$ minutes.

it is necessary here to enter into some detail regarding the time-relations of its mechanical response during the two phases of down and up movements. The great difficulty in recording the pulsatory movements of Desmodium lies in the extreme slenderness of the lateral leaflets. This is such that the friction of a light recording-lever against the recording surface is sufficient to bring these movements to a stop. This difficulty has, however, been overcome, as stated elsewhere, by means of the Optical Lever. ${ }^{1}$ Fig. ${ }^{142}$ 
gives a photographic record of a series of autonomous pulsations exhibited by a leaflet of Desmodium.

For the accurate observation of the rate of movement of the Desmodium leaflet during its different phases I have also been able to make records by means of a series of punctures produced by electrical sparks on a recordingsurface. The sparks occur at the short gap between the end of the recording arm of a very light aluminium lever and the drum, these being connected respectively with the two electrodes of a Ruhmkorff's coil. The electrical dis-

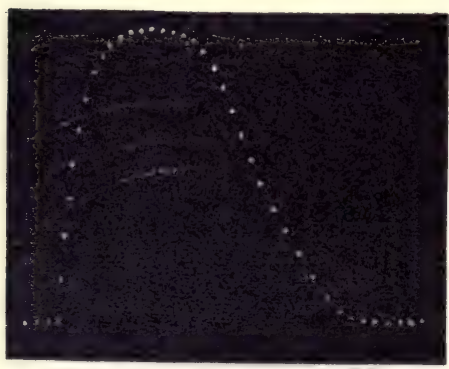

FIG. 143. Spark-record of Single Pulsation in Leaflet of Desmodium

Showing time-relations of down- and up-movements in single pulsation of leaflet of Desmodium. Upcurve represents down-movement of leaflet and vice versa. Interval between successive dots $=5$ seconds. turbance does not affect the plant, as the pulsating leaflet is separated from the other arm of the lever by a long silk thread. The primary current in the Ruhmkorff's coil is broken at intervals of five seconds. Hence successive punctures in the record represent intervals of five seconds each. I give here (fig. I43) a record obtained in this manner, of a single mechanical pulsation of a leaflet of Desmodium. It is to be understood that the up-movement in the record represents the down-movement of the leaflet.

These movements are produced by excitatory contractions of the lower and upper halves of the pulvinus alternately. An inspection of the record given shows that after a pause in the highest position a sudden excitatory impulse is developed in the lower half, which is gradually exhausted as the lowest position is reached. The maximum rate of movement to which this excitation gives rise is in this particular case $: 7 \mathrm{~mm}$. per second. After the lowest position is attained there is a pause. The up-movement 
then takes place more gradually and at a much slower rate. This movement is due to natural recovery, aided by a moderate excitatory contraction of the upper half of the pulvinus. I give herewith a table showing the characteristic rates of movement in the different phases of the entire pulsation.

Table showing Rates of Movement at Different Stages of Pulsation in Desmodium.

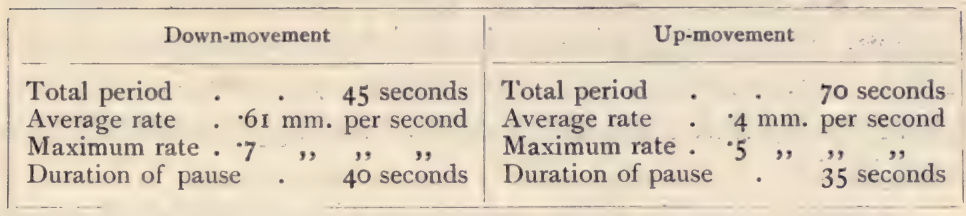

Several facts are brought out in this table which are of special importance, and first we observe that the excitatory impulse which causes the down-movement is brief and quickly exhausted. This is seen by the great distance covered during the first ten seconds, after which the movement gradually slows down. This indicates a short-lived impulsive action, the subsequent movernent of the leaflet being mainly due to inertia. There is then a pause in the down-position, after which the up-movement commences. It will be noticed here that this movement is more gradual and prolonged than the down-movement. From the indications given by these characteristic movements, we may conclude that the excitatory reaction by which the down-movement is caused is relatively more intense and more quickly exhausted than that which brings about the up-movement. We may gauge the relative intensities of the two impulses approximately, either from the maximum or the average rates of the down and up motions. The former gives the ratio of $\frac{7}{5}=\mathrm{I}_{4} 4$; the latter gives $\frac{6 \mathrm{I}}{4}=\mathrm{I}^{\circ} 5^{2}$. The intensity of the downward impulse may therefore be taken to be roughly one and a-half times as great as that which occasions the up-movement. 
The total duration of the down-movement is again much less than that of the up.

We have seen that a single excitation has a single concomitant electrical pulsation. We have also seen the multiple electrical responses corresponding to multiple excitations. It remains, then, to find out whether autonomous pulsations have any electrical concomitant, and if so, of what nature. I shall here, therefore, describe experiments for the recording of the electrical pulsation of the Desmodium leaflet. For this purpose I selected specimens in which the movement of the leaflet was not spasmodic, but gradual and continuous, and where the up and down movements were approximately in a straight line.

In order to obtain those responsive electro-motive changes which might accompany the automatic movements of the leaflets, it was necessary to make one of the electric contacts at a point on the tissue which was free from excitation, the second contact being made at a place where the excitatory reaction was at its maximum. I have shown elsewhere ${ }^{1}$ that the seat of autonomous excitation in Desmodium is neither central nor peripheral, but localised at the slender pulvinated joints to which the leaflets are attached, the latter thus serving merely as indicating flags. Acting on these considerations, I made one contact with the slender pulvinule of a lateral leaflet, the other being made with the common petiole. These electrical connections were made securely by means of cotton threads moistened with normal saline solution, and attached to non-polarisable electrodes. The electro-motive variations induced in the plant now gave rise to corresponding deflections in the galvanometer in circuit. On taking records of these electrical responses, I was surprised to find that, corresponding with each complete mechanical vibration, there was a double electrical pulsation-a large principal followed by a smaller subsidiary wave. In a given case, where the period of the complete mechanical vibration was about 3.5 minutes, the period of the principal of these two

\footnotetext{
1 Bose, Plant Response, p. 299.
} 
waves of electrical response was slightly less than I minute, and that of the subsidiary wave a little over 2.5 minutes. These double electrical pulses, corresponding to a single mechanical vibration, are at first very puzzling, and I undertook special investigations to ascertain the reason of this peculiarity. In order to obtain an insight into the relation between these mechanical and electrical responses it was necessary to take simultaneous records of the two on the same recording drum. This was accomplished by having the two recording spots of light-ore from the galvanometer and one from the optic lever-thrown on the same horizontal slit, in front of the revolving drum, round which was wrapped a sensitive photographic film. The galvanometer spot of light, and consequently the electrical response record, was the lower of the two. The vertical movement of the spot of light which records the mechanical response is to be understood as converted into horizontal by reflection from a second mirror suitably inclined. This experimental arrangement is similar to that employed for simultaneous mechanical and electrical records in the case of Mimosa, as shown in fig. 12 .

The record given in fig. 144 exhibits the simultaneous mechanical and electrical responses thus obtained. It will be seen that the minor electrical wave took place while the leaflet was moving up from $(a)$ and coming to its highest position at $(b)$. This was followed by a wave of higher amplitude but shorter period, which coincided with the movement of the leaflet again from its highest to its lowest positions. It will thus be seen that the subsidiary electrical wave of small amplitude and relatively long period coincided with the slow up-movement of the leaflet, and that the principal wave, characterised by large amplitude and short period, corresponded with the quick down-movement of the leaflet. These galvanometric deflections indicated, it must be understood, a condition of galvanometric negativity of the pulvinule at the moments of its excitatory up and down movements. The following considerations make it easy to 
understand why two electrical waves correspond to one mechanical pulsation. First, we know that an excitatory change at a given point will have, as its concomitant, an electro-motive variation of galvanometric negativity. Second,

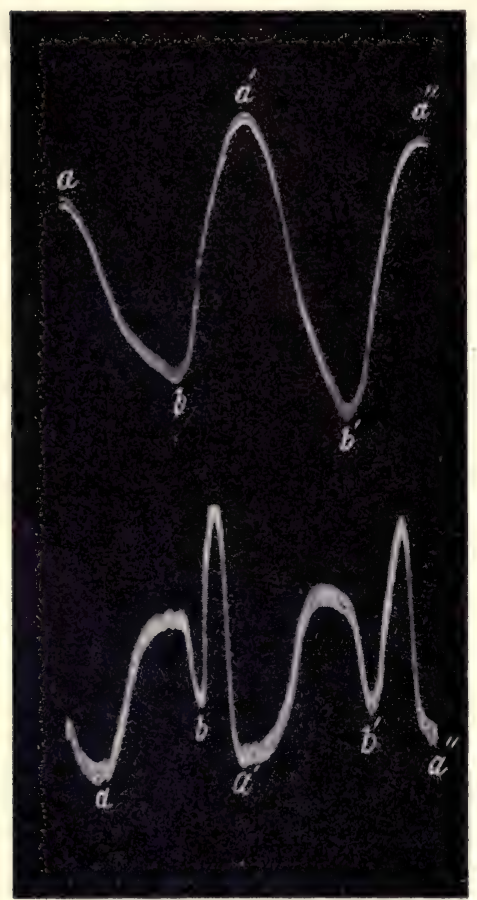

FIG. I44. Photographic Records of Simultaneous Mechanical and Electrical Pulsation of Desmodium Leaflet

$a b$ (upper figure) represents up-movement of leaflet; $a b$ (lower figure) corresponding electrical subsidiary wave; $b \quad a^{\prime}$ (upper figure) down-movement of leaflet; $b a^{\prime}$ (lower figure) corresponding principal electrical wave. the intensity of this electromotive variation depends on the intensity of the excitatory change. And lastly, on the cessation of excitation there is an electrical recovery.

Now we have seen from the spark-record (fig. I43) that the leaflet during one complete mechanical pulsation is subjected to two excitatory impulses, occurring in the upper and lower halves of its pulvinule alternately. It is these two excitations which give rise to the two electrical disturbances of galvanometric negativity. And the different amplitude and period in the two cases are fully accounted for by the different period and intensity of the two excitatory impulses. It will be remembered that the excitatory impulse which produced the up-movement was the feebler and more protracted of the two. It is consequently attended by an electrical disturbance of moderate intensity and corresponding persistence. On the cessation of the up-movement, as we have seen, there is a pause, and during this time we find that 
electrical recovery takes place; but before this is complete, and while there is still a certain residual galvanometric negativity, there occurs that more intense and short-lived excitatory reaction which finds mechanical expression in the downward-movement. The same reaction finds electrical expression in a brief and intense response of galvanometric negativity; on the expenditure of this excitatory impulse there is again an electrical recovery, which becomes practically complete.

In the instance given in the spark-record, it was found that the relative intensity of the down to the up impulse was approximately as $I \cdot 5$ is to $I$. And it is interesting to see that in the photographic record of the electrical responses (fig. I44), the ratio of the amplitudes of the corresponding electrical waves is also the same. In other instances, the relative intensity of the principal wave is still higher. I give below two tables showing the absolute values of the electromotive variations in two different cases.

TABLE I.

\begin{tabular}{|c|c|c|}
\hline $\begin{array}{l}\text { Number } \\
\text { of obser- } \\
\text { vation }\end{array}$ & $\begin{array}{l}\text { E.M. variation. } \\
\text { Principal wave }\end{array}$ & $\begin{array}{l}\text { E.M. variation. } \\
\text { Subsidiary wave }\end{array}$ \\
\hline I & 'oor4 volt & .00055 volt \\
\hline 2 & .0013, & .00051, \\
\hline 3 & .0014, & .00054, ,", \\
\hline 4 & .0015, & .00054, \\
\hline 5 & .0oI6, , & - \\
\hline
\end{tabular}

TABLE II.

\begin{tabular}{|c|c|c|}
\hline $\begin{array}{l}\text { Number } \\
\text { of obser- } \\
\text { vation }\end{array}$ & $\begin{array}{l}\text { E. M. variation. } \\
\text { Principal wave }\end{array}$ & $\begin{array}{l}\text { E.M. variation. } \\
\text { Subsidiary wave }\end{array}$ \\
\hline I & $\cdot 0024$ volt & 'oor6 volt \\
\hline 2 & .0025, & .0015 \\
\hline 3 & .0025, & .0016, , \\
\hline 4 & .0025 , , & .0017, \\
\hline 5 & .0026, & - \\
\hline
\end{tabular}

It might be thought that these two electrical waves had been induced by the mechanical movement of the leaflet as such. We have seen, however, that the electrical response is a concomitant of the excitatory condition, whether such excitation be followed by any mechanical response or not. This we saw in the absence of mechanical movement in the case of ordinary plants. The same was found also in the 
case of sensitive plants when responsive mechanical movements were prevented from taking place by physical restraint (p. 20). The mechanical and electrical responses are thus independent modes of expression of a single fundamental excitatory process. In order to demonstrate this in the case of the autonomous pulsation of Desmodium, I first obtained simultaneous mechanical and electrical responses of

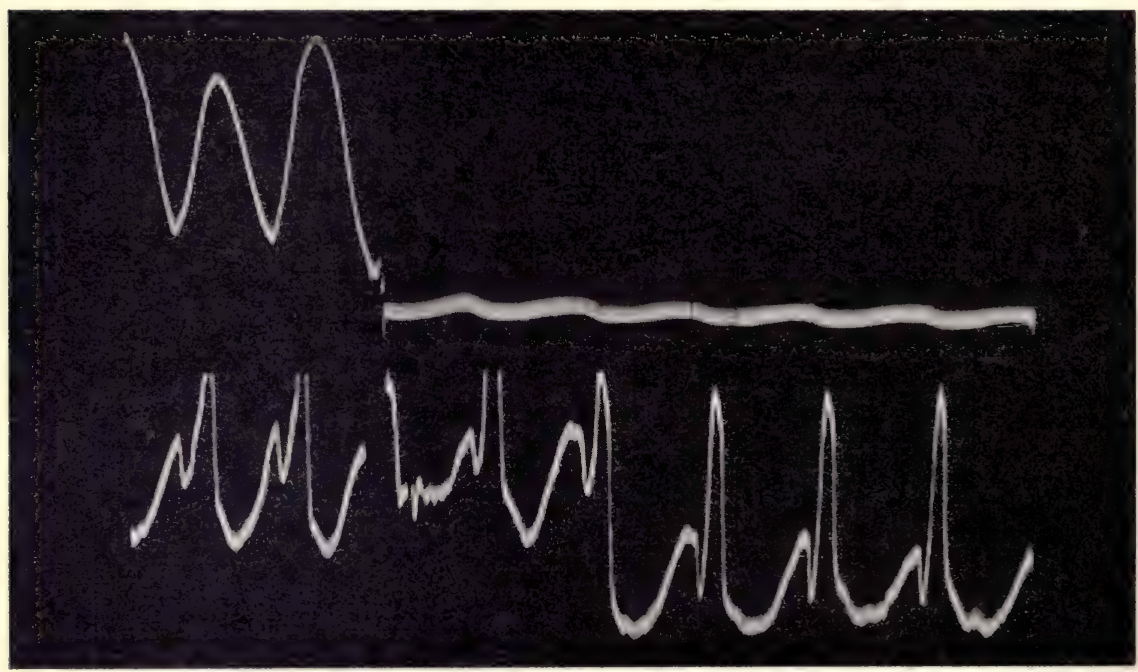

FIG. I45. Photographic Record of Simultaneous Mechanical and Electrical Pulsation in Leaflet of Desmodium, before and after Physical Restraint of Leaflet.

The first part of this record shows both mechanical and electrical pulsation. In the second part, leaflet was physically restrained, as seen in the mechanical record, becoming horizontal. Electrical pulsation now seen to persist with even greater vigour than before.

the leaflet (fig. 145). In the next part of the same record the mechanical movement of the leaflet was restrained, as seen in the upper mechanical record, which here becomes a straight line. But the lower record, which gives the electrical response, still shows the double electrical pulsation unimpeded. Indeed, so far from the mechanical response having been the cause of the electrical, we find that on its arrest, at least in this particular case, the latter becomes very 
much enhanced. In fact, it appears as if the fundamental excitatory reaction, being now deprived of one of its two modes of expression, exhibited the other with the greater energy.

We thus see that not only does the electrical response give us a means of detecting the action of external stimulus on a tissue, but that the same mode of indication enables us further to demonstrate the existence of those internal excitations which may find mechanical expression in the socalled 'autonomous' movements.

One such autonomous pulsation present in all plants is that of growth, and by means of the highly magnified record given by the Crescograph. I have shown this to consist of the additive effects of multiple minute pulsatory movements. We see this in fig. 146,

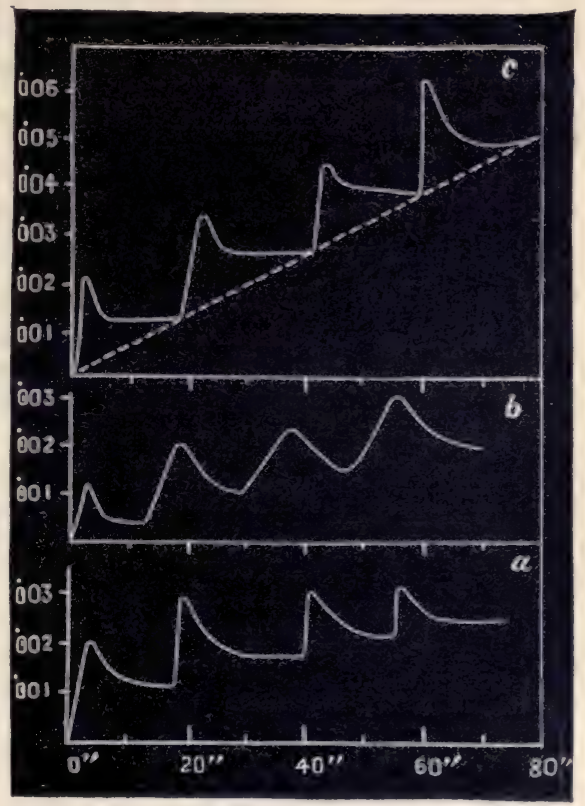

FIG. I46. Crescographic Record of Multiple Growth-responses in Peduncle of Crocus

The ordinate represents the extent of responsive elongations in $\mathrm{mm}$. ; the abscissa, time in seconds.

which gives a series of records of multiple growth responses obtained with the peduncle of Crocus at different times of the day. In the present case, the average period of each pulsation is twenty seconds. In the case of relatively slow pulsations like these, if one electrical connection be made with the growing-point, where such movements are in progress, and the other with an old leaf in which they have 
ceased, the galvanometer-spot of light is thrown into a state of oscillation, indicative of the local excitatory reactions at the growing-point.

These multiple pulsations of growth consist of alternating positive and negative turgidity-variations. In dealing with the concomitant electrical response, however, we have seen (p. 64) that the induced galvanometric negativity, owing to its greater intensity, always overpowers the galvanometric positivity, if the two occur in rapid succession. In growthpulsation, the constituent pulses are often extremely rapid. Hence a growing-point may be expected to exhibit, galvanometrically, a resultant negativity. This consideration may explain the observation of Johannes Müller-Hetlingen, otherwise unexplained, that the growing-points of both shoot and root, in the seedling of Pisum sativum, are negative, as compared with the indifferent cotyledons. 


\title{
CHAPTER XVIII
}

\author{
RESPONSE OF LEAVES
}

Observations of Burdon Sanderson on leaf-response in Dioncea-Leaf-andstalk currents-Their opposite variations under stimulus-Similar leaf-andstalk currents shown to exist in ordinary leaf of Ficus religiosa-Oppositedirectioned currents in Citrus decumana-True explanation of these restingcurrents and their variations-Electrical effect of section of petiole on Dionca and Ficus religiosa-Fundamental experiment of Burdon Sanderson on lamina of Dionca-Subsequent results-Experimental arrangement with symmetrical contacts-Parallel experiments on sheathing leaf of MusaExplanation of various results.

IT was pointed out in Chapter II. that progress in the investigation of the subject of excitatory phenomena in plants had been long delayed, in consequence of the prevalent idea that only motile plant-organs were 'excitable.' The attention of investigators was thus mainly confined within the narrow range of the so-called 'sensitive' plants, such as Dionce. It was also shown, in the same place, that the results already arrived at by observers in this field had not been altogether concordant, and presented many anomalies.

As it has now been demonstrated, however, in the course of previous chapters, that ordinary plants are fully sensitive, it will be well to proceed to show that the various effects observed in the 'sensitive' Dioncea may be still better studied in ordinary leaves. It will be possible, moreover, by following this line of inquiry, to determine those general laws, of which the peculiarities observed in Dioncea are only instances; and thus we shall be the better able to offer an explanation of such cases as now appear anomalous.

Before doing this, I shall briefly recapitulate the principal effects observed by Burdon Sanderson in the leaf of Dionae. 
These observations relate firstly to the existing current of rest in the petiole and midrib, and the variations of this resting-current, whether under excitation of the lamina, or by section of the petiole, or again, by the action of electrotonus; and secondly, to the induction of variations of a transverse current between the upper and lower surfaces, of the lamina. As regards the current in the petiole and its prolongation the midrib, which I shall distinguish as 'the longitudinal petiolar current,' Burdon Sanderson found this

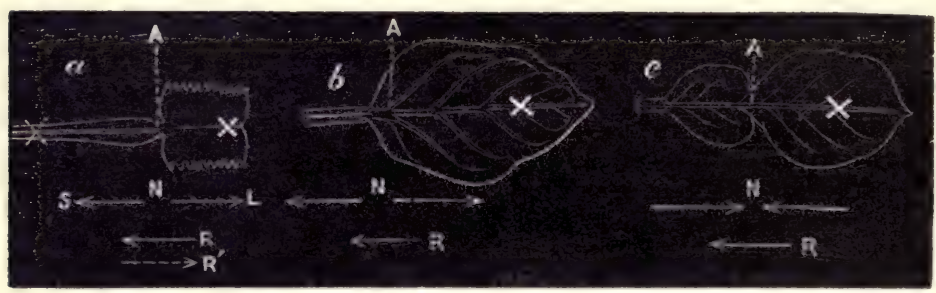

FIG. 14\%. Natural and Responsive Currents in Leaves

(a) Leaf- and stalk-currents in Dionea. Natural current, $\mathrm{N}$, flows outwards $\leftarrow \mathrm{A} \rightarrow$, s being stalk-current, and $\mathrm{L}$ leaf-current. Stimulation of lamina at $\times$ gives rise to responsive current, $\mathrm{R}$, from right to left, inducing negative variation of leaf-current and positive variation of stalk-current. When stimulus, however, is applied on left at $\times$, responsive current, $R$, is from left to right, inducing effects exactly opposite of former, viz. negative variation of stalk-current and positive variation of leaf-current; (b) Leaf- and stalk-currents in Ficus religiosa similar to those of Dioncea. Natural current flows outwards $\leftarrow \mathrm{A} \rightarrow$. Stimulation of lamina at $\times$ gives rise to responsive current, $\mathrm{R}$, inducing negative variation of leaf- and positive variation of stalkcurrents; (c) Leaf- and stalk-currents of Citrus decumana, opposite to those of Ficus and Dioncea, $\rightarrow \mathrm{A} \leftarrow$. Stimulation at $\times$ induces positive variation of leaf and negative variation of stalk-currents.

to flow in the midrib, from the end proximal to the stalk to the distal end. This he designated as the 'normal leafcurrent.' He further found that if electrical connections were made, so that one contact was near the lamina, and the other away from it, the stalk-current was opposite in direction to the leaf-current (fig. I $47(a)$ ).

On stimulation of the lamina, these resting leaf-and-stalk currents were found to undergo responsive variations. But these changes were exactly opposite to each other. That is to say, the leaf-current underwent a negative, and the stalk- 
current a positive, variation. No explanation has as yet been offered, regarding either the existence of these oppositedirectioned currents of rest, or the apparently anomalous result, that an identical stimulus would induce, in one case a negative, and in the other a positive, variation of them.

As regards these peculiar currents of rest we have seen (p. 176), that if an intermediate point be physiologically less excitable than either of the two terminal points, then a resting current will flow from the less to the more excitable. This is the particular current-distribution in the leaf of Dioncea. It is not a unique phenomenon, for I have noticed other such instances in ordinary leaves. The point of junction of the petiole with the lamina of Ficus religiosa, for example, is galvanometrically the most negative point in that petiole-and-midrib. The currents here also, then, as in the case of Dionca, flow outwards from the point of junction-the leaf-current towards the tip of the leaf, and the stalk-current in the opposite direction (fig. I $47(b)$ ).

We also saw, however, in the same place, that there may be instances in which an intermediate point is more excitable than either of the two terminal. When this is so, the currents of rest will be reversed in direction, and flow inwards. This I find to be the case in the leaf of Citrus decumana (fig. I47 (c)).

Next with regard to the excitatory variation of these resting-currents in leaf and stalk, we must remember that the effect of stimulation is to give rise to a true excitatory current, flowing away from the excited. If then there be already a resting-current, the responsive current will be added to this algebraically. When the lamina to the right is excited, the responsive current flows from right to left. This would naturally, in the case of Dioncea, induce a negative variation of the leaf-current, and a positive variation of the stalk-current (fig. I47 (a)). The same thing is seen on stimulating the lamina of Ficus religiosa, where also the excitatory current, being of opposite sign to the leaf-current, and of the same sign as the stalk-current, induces a negative 
variation of the former, and positive variation of the latter (fig. I47 (b)). The same stimulus thus induces effects which are apparently opposite. Or an interesting variation of the phenomenon may be obtained, on repeating the experiment with the leaf of Citrus. Here, on stimulating the lamina, we observe a positive variation of the leaf-current, and a negative variation of the stalk-current (fig. I46 (c)). This is because the currents of reference or resting-currents are the opposite of those in Dionae and Ficus religiosa.

Another series of variations exactly the reverse of these, and therefore at first sight anomalous, is caused by simply changing the point of application of stimulus, from the right end on the lamina, to the left end on the stalk. The direction of the excitatory current is thus reversed, being now from left to right (fig. I47 (a)). By algebraical summation, there now occurs a negative variation of the stalk-current, and a positive variation of the leaf-current, in Dioncea and Ficus religiosa, while the very opposite takes place in Citrus.

I shall here draw attention once more to those errors to which an investigator becomes liable when he infers that positive and negative variations must necessarily be the expression of assimilatory and dissimilatory processes. For we have just seen that the same responsive current, by algebraical summation with two opposite-directioned restingcurrents, may appear to be both positive and negative, at one and the same time. Again, with a single resting-current, it is possible to obtain either a positive or a negative variation, according as the same stimulus is applied to the right or the left. It is now abundantly clear that the one universal effect of stimulus is to give rise to a responsive current which flows from the more to the less excited portions of the tissue. If there be already an existing current, the responsive current is added to this algebraically, and induces, according to circumstances, either a positive or a negative variation. Much confusion, and many erroneous inferences would be avoided, if instead of looking at these variable indications attention were centred on the one constant criterion, namely 
that the excitatory current always flows from the more to the less excited portions of the tissue.

Another effect observed by Burdon Sanderson was, that on cutting the petiole across, the existing normal leaf-current was increased, the amount of this increase being determined by the length of the petiole cut off, in such a way that the shorter the petiole left, the stronger the leaf-current became. In Nature (vol. x. p. I28), he suggested an explanation of this phenomenon. In the leaf of Dioncea, as already said, there is a resting-current in the stalk, opposed in direction to that in the leaf. Thus 'the electrical conditions on opposite sides of the joint between stalk and leaf are antagonistic to each other ; consequently, so long as the leaf and stalk are united each prevents or diminishes the manifestation of electromotive force by the other.' He thus inferred that the progressive removal of the antagonistic element, by section of the stalk, would serve to enhance the intensity of the leaf-current.

Taking the ordinary leaf of Ficus religiosa, I have myself been able to obtain results precisely similar to those described in Dioncea, by making successive sections of the petiole, at shorter and shorter distances from the point of junction. The leaf-current at each section underwent an increment. The parallelism of the two sets of effects will be seen from the following table.

Ficus Leaf.

\begin{tabular}{|c|c|}
\hline Length of stalk & Galvanometric deflection \\
\cline { 1 - 2 } $7 \mathrm{~cm}$. & 16 divisions \\
\hline 4, & $36,$, \\
\hline 2, & $50,$, \\
\hline I , & 60, \\
\hline
\end{tabular}

Dionea Leaf (Burdon Sanderson).

\begin{tabular}{|c|c|}
\hline Length of stalk & Galvanometric deflection \\
\hline $2.5 \mathrm{~cm}$. & 40 divisions \\
\hline $1.25,$, & 50,3 \\
\hline $0.6 \quad$, & 65, \\
\hline $0.3,$, & 90 \\
\hline
\end{tabular}

Burdon Sanderson's suggested explanation that the successive augmentations of the leaf-current were due to successive removals of the antagonistic element, by section, is 
quite untenable. He failed to see that the effect was, on the contrary, due to the increasing excitatory action of the sections themselves. Similar results may be obtained, even without the bodily removal of the supposed antagonistic element, if, instead, we apply an increasing intensity of stimulus, as say, by contact of a hot wire at points nearer and nearer to that of junction. In the case of the transverse section, the cut acts as a stimulus, and the responsive current flows from the left to the right. Algebraical summation of this with the existing leaf-current, which is also from left to right, causes an increase, or positive variation of it, in a manner exactly the converse of the negative variation induced in the leaf, when the stimulus was applied on the lamina. As the section is made nearer and nearer to the point of junction, the degree of stimulation, and the consequent positive variation of the resting-current, must become greater and greater.

And lastly; in the case of the longitudinal leaf-current, Burdon Sanderson found that if a current from a battery were directed through a leaf-stalk, at the same time that the two ends of the midrib were led off to the galvanometer, the difference previously existing between the ends of the midrib would be increased, if the current led through the leaf-stalk were in the same direction with the leaf-current, and diminished, if it were in the opposite direction. A similar effect, as seen in the conducting tissues of ordinary plants, will be studied in detail, when we take up the question of the extra-polar effects induced by electrotonic currents (Chap. XXXIX.).

We have already seen that, by means of induced variation of the longitudinal stalk-current, under the stimulation caused by section of the petiole, it is easy to obtain an unmistakable indication of the nature of the true excitatory electrical change. Burdon Sanderson, however, laboured under the disadvantage, as already said, of having failed to recognise that a section acts as a stimulus. His investigation, therefore, on the character of the excitatory variation, 
was chiefly carried out by means of experiments on electrical variations induced in the lamina. These depended (I) on variations in the cross-difference of existing potential between the upper and lower surfaces, according to his 'fundamental experiment,' and (2) on electrical variations in the led-offs of symmetrical surfaces of contact on the under-side of opposite lobes. The results which he obtained, however, by these methods, appear to the reader to have been very conflicting, and in fact the experimental methods described by him would seem to have been open to many sources of complication of which he himself was unaware.

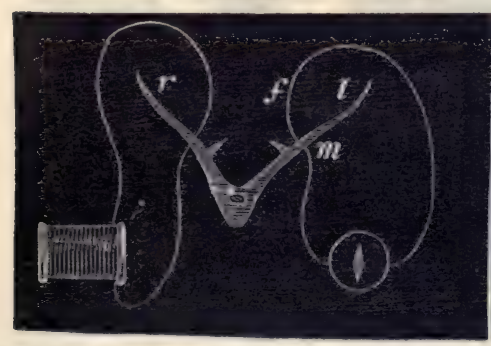

FIG. I48. Burdon Sanderson's Fundamental Experiment on Dioncea Leaf

Electrical stimulus applied on distal lobe, ", induces responsive effect on led-off circuit $f m$. Upper or internal surface, $f$, more excitable than lower, $m$.

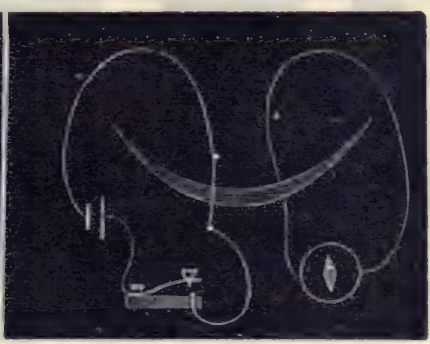

FIG. 149. Parallel Experiment in Sheathing Petiole of Musa

Thermal stimulus applied on distal side induces responsive effect on led-off circuit. Upper or 'internal' surface more excitable than lower.

I shall deal first with Burdon Sanderson's 'fundamental experiment,' of which the excitatory electrodes are seen on the left lobe, and the led-off on the right in fig. I48. In fig. I49 is given a diagram of a parallel experiment carried out by myself on the petiole of Musa. According to BurdonSanderson, as the result of excitation, a $\uparrow$ current is induced in the right lobe of Dioncea (fig. I50). This means, of course, that the upper or more excitable surface of the right lobe has become positive to the lower. This current, however, he termed 'excitatory,' regarding it as the analogue of the 'action-current' known to animal physiology. After this first phase, when a certain interval had elapsed, he 
observed a second phase to set in, in which the upper surface became relatively negative to the lower. This negative change, which he called the 'after-effect,' he described as taking place at that moment at which the mechanical effect of excitation also made itself evident.

This negative phase-called by him the 'after-effect'Burdon Sanderson regarded as connected with those electrical changes which had been observed by Kunkel to be induced by movement of water in the tissues. The first effect on the
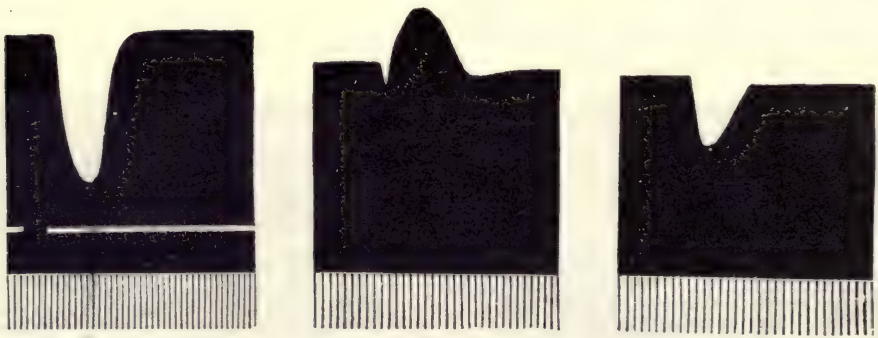

FIGS. I 50, 151, I52. Records; of Electrical Responses of Different Leaves of Dioniea according to Fundamental Experiment of Burdon Sanderson

Fig. I50. Positive response of certain leaves of Dionce. Time-marks 20 per second (Burdon Sanderson).

Fig. I5I. Diphasic response of leaf of Dioncea ' in its prime.' Positive followed by negative. Time-marks Io per second (Burdon Sanderson).

Fig. I52. Positive response of same leaf when 'modified' by previous stimulation. Time-marks Io per second (Burdon Sanderson).

The above records were obtained with capillary electrometer.

contrary, which immediately preceded this, and was characterised by relative positivity of the upper surface, he regarded, as already mentioned, as the true excitatory or action-effect. The following is from his summary:

' The first phase of the variation--the effect which immediately follows excitation, and has an opposite sign to the after-effect, and a much higher electro-motive force-does not admit of a similar explanation : for it cannot be imagined that a change which spreads over the whole lamina in less than one-twentieth of a second can be dependent on migration of water. The excitatory disturbance which immediately follows excitation 
is an explosive molecular change, which by the mode of its origin, the suddenness of its incidence, and the rapidity of its propagation, is distinguished from every other phenomenon except the one with which I have identified it-namely, the corresponding process in the excitable tissues of animals. Of the nature of this preliminary disturbance (to which alone the term excitatory variation ought to be applied, it alone being the analogue of the 'action-current' of animal physiology) we know nothing. ... The direction of the excitatory effect in the fundamental experiment is such as to indicate that in excitation, excited cells become positive to unexcited, whereas in animal tissues excited parts always become negative to unexcited. The apparent discrepancy will probably find its explanation in the difference of the structural relations of the electromotive surfaces.' ${ }^{1}$

From this quotation it will be seen that Burdon Sanderson had fallen into the basic error of mistaking what I have demonstrated to be the hydro-positive, for the true excitatory effect, and vice versa.

In a subsequent Paper again (Phil. Trans. vol. I79, I889) Burdon Sanderson published certain results, which differed from those referred to above. He had previously found that usually speaking the upper surface of each lobe was negative to the lower. Later, however, he came to the conclusion that in the leaf of Dioncea in its 'prime,' the upper surface was positive to the under. On repeating his 'fundamental experiment' moreover, with these vigorous leaves, he found that instead of the pronounced positive response which he had previously observed, he now obtained a short-lived positive effect succeeded by a strong negative (fig. I 5 I). He was unable to offer any definite explanation of this difference between the two sets of results, but suggested that it might arise, in some way, from changes of the resting-current. 
'In the leaf, observed facts show most conclusively that the two sets of phenomena-those of the excited and those of the unexcited state-are linked together by indissoluble bands : that every change in the state of the leaf when at rest conditionates a corresponding change in the way in which it responds to stimulation, the correspondence consisting in this, that the sign, that is the direction, of the response is opposed to that of the previous state, so that, as the latter changes sign in the direction from $\uparrow$ to $\downarrow$, the former changes from $\downarrow$ to $\uparrow . ' 1$

In making this statement, Burdon Sanderson was probably guided by the prevalent opinion that response takes place by a negative variation of the existing current of rest. We have seen, however, that this supposition is in fact highly misleading. For, owing to such fluctuating factors as age, season, previous history, or excitation due to preparation, the so-called current of rest may and frequently does undergo reversal. Thus a single excitatory effect might, as we have seen (pp. I75-I 77) under different circumstances, appear either as a positive or a negative variation of the existing current. The assumption of the universality of response by negative variation is thus seen to be unjustifiable.

Indeed, it would appear from the description of some of the experiments actually related by Burdon Sanderson himself, that response did not, even in these cases, always take place by negative variation of the existing current. For instance, while in the leaf of Dionea in its 'prime' (upper surface positive) the response is negative, and while this latter becomes reversed to positive, as he tells us, in consequence of 'modification' due to previous excitation (fig. 152), yet headmits that even in these circumstances the upper surface had first returned to positivity (ibid. p. 447). Thus, though the responses of the leaf in its 'prime,' and of the 'modified' leaf are opposed, yet the antecedent electrical condition of the modified leaf has not in this case undergone reversal.

Phil. Trans. I889, vol. 179, p. 446. 
The suggestion, therefore, that the reversal of response is due, in some way unexplained, to a reversal of the electrical condition of the leaf, cannot hold good. Nor does the use of the term 'modification' in any way assist in the elucidation of the phenomenon. A satisfactory explanation of this reversal of response, then, still remains to be found.

So much for the 'fundamental experiment.' The next experimental arrangement employed by Burdon Sanderson consists of a leaf which is led off by symmetrical contacts on the under surfaces of its two lobes (fig. I 53). If now the

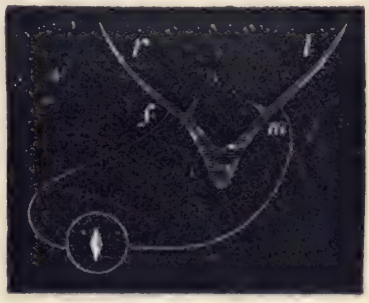

FIG. I53. Experimental Connections with Dioncea according to the Second Experimental Method of Burdon Sanderson right lobe was excited, by touching one of the sensitive filaments (on the upper surface) with a camel's-hair pencil, in the neighbourhood of the leading-oft contact, it was found that the under-surface of the right lobe became first positive, and subsequently negative (fig. I 54), relatively to the left (ibid. p. 440).

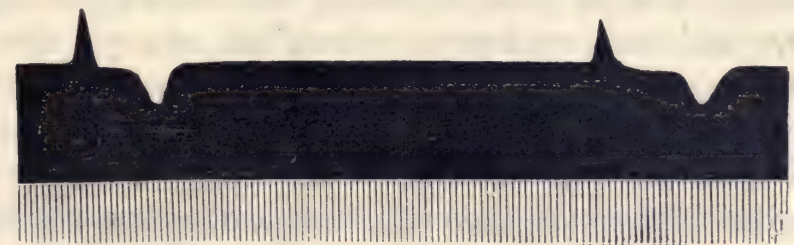

FIG. I54. Response of Under-surface of Leaf of Dionaa, with Electrical Connections as in Fig. 153

Mechanical excitation of upper surface of right lobe shows relative positivity of under surface of same right lobe (up curve), followed by its relative negativity (down curve). Time-marks 20 per second (Burdon Sanderson).

Summarising these various observations, then, we find results which are very much at variance. First, according to the 'fundamental experiment,' certain leaves are seen to give rise to the positive response; other leaves, in their prime,' give diphasic response, the upper surface becoming 
first positive and then negative. These latter again, after previous excitation, become so modified as to show only positive changes. And lastly, using the experimental arrangement of symmetrical contacts, a diphasic variation is obtained-positive followed by negative-on the undersurface, instead of the upper, of the lobe excited. No theory is advanced, however, by which a comprehensive explanation might be afforded of these apparently anomalous results.

But from the generalisations which I have already established, regarding the electrical signs of the hydro-positive and true excitatory effects respectively, and from the results of certain experiments on ordinary leaves which I shall presently describe, it will be found easy to arrive at a true explanation of the various observations related by Burdon Sanderson, which would otherwise have appeared inexplicable. The fact that hydrostatic disturbance induces galvanometric positivity, and that true excitation induces negativity, has already been clearly demonstrated under conditions from which all possible sources of complication had been eliminated (p. 6I). The experimental arrangement adopted by Burdon Sanderson, however, laboured under the double disadvantage, not only of a liability to confuse the hydropositive and true excitatory effects, but also of the complexity arising from the differential excitability of the responding organ. It is only indeed by the closest analysis that it is possible to discriminate, in his results, between such as are due to true excitation and those arising from the hydropositive effect.

The various electrical phenomena which are possible in an anisotropic organ in consequence of the hydro-positive and excitatory effects respectively, may be clearly exhibited, as I have already shown, by means of the mechanical response of the leaf of Mimosa. With regard to this, we have seen (pp. 59,60) that direct stimulation of the pulvinus induces a negative mechanical response, or fall of the leaf, by the greater contraction of the more excitable lower half of the organ. The corresponding electrical variation would 
thus consist in the greater galvanometric negativity of this more excitable lower, in relation to the less excitable upper half. If the stimulus, however, be applied at some considerable distance, so that true excitation cannot reach the responding point, then we have an erectile or positive mechanical response of the leaf. This is brought about by the relatively greater expansion of the more excitable. The corresponding electrical response will be the galvanometric positivity of this more excitable, in relation to the less excitable half of the organ. Between these two extremes lies that experiment in which stimulus is applied at some intermediate point, the consequence of which is that the hydro-positive wave, with its greater velocity, reaches the responding organ earlier than true excitation, thus bringing about a preliminary erectile or positive response, followed by the excitatory negative or fall of the leaf. The corresponding electrical response would therefore be diphasic, positive followed by negative.

But the occurrence of this second or negative phase is only possible when the conductivity is so great as to allow the wave of true excitation to reach the organ. We may imagine that in a very vigorous plant, with its great conductivity, we have found a point, at the maximum distance from which the true excitatory effect of a given stimulus is capable of transmission to the organ. With such a specimen, in its 'prime,' we shall observe a diphasic effect-preliminary positive followed by negative. But if we took a less vigorous specimen, and applied the stimulus at the same distance from the responding point, the true excitatory wave would fail to reach the responding organ, and we should see there, only the positive effect due to hydro-positive action. Hence, two different specimens, treated in exactly the same way, may exhibit two different effects, one diphasic, and the other positive alone; this difference being due to their unequal vigour, and concomitant inequality of excitability and conductivity. This will account for the diphasic and positive responses which were exhibited by the more and 
less vigorous leaves respectively of Dionce, when stimulation was applied on the distal lobe, according to the fundamental experiment of Burdon Sanderson.

We must next refer to the reason why a leaf that originally gives diphasic response-positive followed by negative - undergoes such 'modification,' in consequence of previous excitation, as thereafter to give only positive response. We have seen that the negative element of the diphasic response is due to the arrival at the responding point of the true excitatory wave originated at the distant point of stimulation. Now it has been shown (p. 65), that if by any means the conductivity of an intervening region should become diminished, we may expect that the hydro-positive effect will continue to be transmitted, although the passage of true excitation is partly or wholly blocked. By means of this selective block, I was able to unmask the hydro-positive component present in resultant response (cf. fig. 49).

I have shown elsewhere ${ }^{1}$ that the conducting power of a tissue will be impaired by the fatigue consequent on previous stimulation. Thus, in the petiole of Biophytum, I found that while the plant, when fresh, had a conductivity measured by the velocity of transmission of excitation, at a rate of I. $88 \mathrm{~mm}$. per second, the same plant, when partially fatigued by four successive stimulations, had its conductivity diminished, the velocity of transmisson being now only I.54 $\mathrm{mm}$. per second. The diminution in this case, then, was about I 8 per cent. I shall moreover show in a later chapter that in consequence of growing fatigue the passage of true excitation may at a certain stage be arrested, the hydropositive effect alone being then transmitted. It is thus easy to explain how it was that in Burdon Sanderson's experiment, of stimulus applied on the distal lobe, the wave of true excitation became blocked, and the 'modified' leaf gave positive response alone. These considerations will be found as I think, to offer a satisfactory explanation of the conflicting results arrived at by Burdon Sanderson.

1 Plant Resbonse, p. 244. 
I shall now, however, proceed to describe a series of experiments exactly parallel to the 'fundamental experiment' on Dioncea, carried out on ordinary plants. We have seen that the inner or concave surface of the sheathing petiole of Musa is relatively more excitable than the outer or convex. Thus it corresponds with the 'internal' or upper surface of the leaf of Dioncea. The more excitable internal surfaces of both these, again, correspond with the more excitable lower half of the pulvinus of Mimosa. In fig. I49 is shown an experimental arrangement with a specimen of Musa which will be seen to be parallel to that of Burdon Sanderson's fundamental experiment on Dioncea. In order to avoid any such disturbance as might conceivably arise from current-escape, if the electrical form of stimulus were used, I employed the thermal mode of stimulation. A momentary heating-current passed through a thin platinum wire gave the thermal variation required, and was found to furnish a very satisfactory form of stimulus. The led-off circuit was at first placed at a distance of $16 \mathrm{~mm}$. from the point of stimulation. As the stimulation was moderate, and as the conductivity of the tissue was not great, the effect induced at the responding circuit was hydro-positive, the more excitable concave surface becoming positive (fig. I $55(a)$ ). This resporise is the same as the positive responses given by the 'unmodifiable leaf' of Dioncea (fig. I 50), as well as that of a vigorous leaf which had been 'modified' by fatigue (fig. I 52). On next taking a second pair of led-off points, at the shorter distance of $8 \mathrm{~mm}$., the hydro-positive effect reached the led-off points earlier, and was followed by the true excitatory wave. This is seen as a preliminary positive response, followed by the excitatory negative (fig. I55 (b)). This again is the same as the di-phasic response of a Dioncea leaf in its 'prime' (fig. I 51). In the experimental arrangement with Musa the led-off circuit was now brought still nearer to a distance of $4 \mathrm{~mm}$. There was now little interval between the arrival at the led-off points of the hydro-positive and true excitatory effects; and since the latter is of predominant electrical 
expression, the former is masked by it, and we obtain here only the excitatory negative variation (fig. I 55 (c)).

It only remains to consider the responses which Burdon Sanderson obtained with symmetrical contacts (fig. 1 52) on the under-surfaces of the two lobes. In the next figure (fig. I 53) is reproduced his record of electrical response, obtained on mechanical stimulation of a sensitive filament situated on the upper surface of the right lobe, vertically above the right-hand led-off. This response is, as will be seen, di-

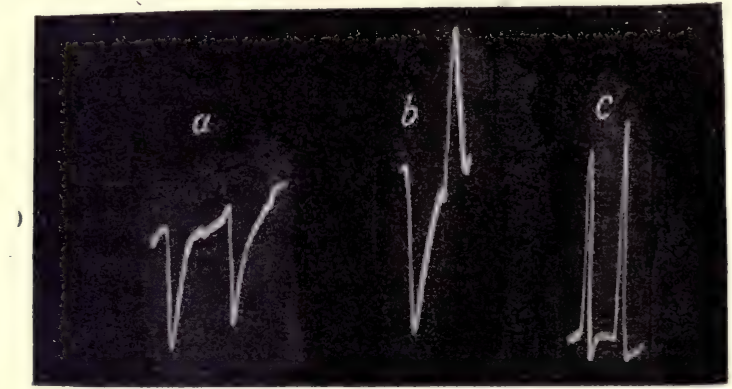

FIG. 155. Photographic Records of Positive, Diphasic, and Negative Responses of Petiole of Musa depending on the Effective Intensity of Transmitted Stimulus

(a) Here stimulus was applied at a distance and hydro-positive effect alone transmitted; (b) Stimulus was applied nearer, and the positive effect was succeeded by the true excitatory negative; (c) Stimulus was applied very near, with the result of true excitatory negative response.

phasic, its first phase being one of relative positivity of the under-surface of the excited lobe, and the second representing its subsequent relative negativity. This first phase is clearly due to the earlier transmission of the hydro-positive or indirect effect of excitation, from the stimulated point on the upper surface. It was supposed by Burdon Sanderson that the second phase of this response represented the later arrival of the same positive effect at the distal second contact, which would thus induce reversal. But it appears much more probable that this second phase of negativity is due to the arrival at the 
under-surface of the wave of true excitation, initiated vertically above. This relative negativity of the undersurface may or may not be helped by the induction of positivity at the distal, due to the transmission of the hydropositive effect. This view is supported by the fact that in a corresponding experiment on an ordinary leaf, in which the second contact was at a distance too great to allow of the effective transmission of any hydro-positive wave, the stimulation of the upper surface induced a similar diphasic response at a point diametrically opposite, on the under side. In this case the second or negative component of the response could not be due to anything but the subsequent arrival of the true excitatory wave with its concomitant negativity.

It is now clear that among the various results obtained from the study of the electrical responses of the leaf of Dioncea, there are some which do not represent true excitation at all, while in others it is only one of the two phases which is significant of this, the other being due to the hydro-positive effect. We have also seen that Burdon Sanderson at starting fell into the error of wrongly identifying the true excitatory electrical effect with that which was due to the hydro-positive effect, and vice versa. We have seen that there is not a single response given by the so-called excitable leaf of Dioncea, which cannot be obtained under similar conditions from the leaves of ordinary plants also. In fact it has been by means of experiments carried out on the latter that we have been enabled to unravel all the intricacies which were offered by the recorded responses of the lamina of Dioncea.

It has further been shown in the course of the present chapter that the leaf and stalk currents observed in Dioncea are also found in, for instance, the leaf of Ficus religiosa. These have been shown, moreover, to be due to physiological differences between an intermediate and the terminal points. The negative variation of the leaf-current, and the positive variation of the stalk-current, on the stimulation of the 
lamina, were both alike shown to be the result of the algebraical summation of a definite excitatory current with the two opposite-directioned resting-currents. The positive variation of the leaf-current, again, on section of the petiole, has been traced to the same cause, namely the stimulatory action of mechanical section, giving rise to an excitatory current which was summated with the existing leaf-current. Finally the positive response of the concave surface of Dioncea has been shown to arise, not from any specific difference between plant and animal response, but from the fact that in this particular case it was the indirect hydropositive effect of stimulus that was transmitted, inducing an action opposite to that of true excitation. 


\section{CHAPTER XIX}

\section{THE LEAF CONSIDERED AS AN ELECTRIC ORGAN}

Electrical organs in fishes-Typical instances, Torpedo and MalepterurusVegetal analogues, leaf of Pterospermum and carpel of Dillenia indica or pitcher of Nepenthe-Electrical response to transmitted excitation - Response to direct excitation-Uni-directioned response to homodromous and heterodromous shocks-Definite-directioned response shown to be due to differential excitability-Response to equi-alternating electrical shocks-Rheotomic observations--Multiple excitations-Multiplication of terminal electromotive effect, by pile-like arrangement, in bulb of Uriclis lily.

IT has been shown that by a study of the peculiarities of electrical response in plants, it is possible to obtain an insight into the obscurities of similar responses in the animal tissue. Among animal structures, there is one-the electrical organ of certain fishes-the explanation of whose action offers unusual difficulties to the investigator. But I shall attempt to show in the course of the present chapter, that there are also, on the other hand, vegetable structures, the study of which will be found to elucidate the electro-motive action here involved. Taking that of the Torpedo as type, we find that the electrical organ is disposed in the form of columns, each column consisting of numerous electrical plates, arranged in series, one over the other, like the plates in a voltaic pile. Each electrical plate consists of a rich plexus of nerve-fibres imbedded in a gelatinous mass. There are thus two surfaces, one nervous and the other nonnervous. Each disc then becomes electro-motive under the impulse from the nerve. Though the induced electro-motive force in each plate is small, yet in consequence of their serial arrangement in columns, the elements are coupled for intensity, and the resulting E.M.F. of discharge becomes high. 
Fritsch estimates the total number of these plates in some of the Torpedos to be over I 50,000.

From the point of view of their development, these electrical organs in general constitute modified muscles, containing nerve-endings. The electrical fish known as Malepterurus of the Nile is an exception to this rule, inasmuch as morphological evidence goes to prove that in its case it is glandular, rather than muscular, elements which have been so modified.

The peculiar characteristic of the discharge of electrical organs in general, is that it takes place in a definite direction at right angles to the plates. It was Pacini who tried to establish the generalisation that the direction of the discharge would be found to be dependent on the morphological character of the organ. He found that as a general rule the discharge takes place in a direction from that surface of the disc which receives the nerve (henceforth to be referred to as the anterior surface) to the opposite non-nervous, or posterior, surface. Thus in the Torpedo, where the plates are horizontal, and the anterior or nervous surface constitutes the ventral aspect of the disc, the discharge is from the ventral or anterior, to the dorsal or posterior surface. In Gymnotus again, the plates or discs are vertical to the long axis. The anterior or nervous surface is here towards the tail-aspect, and the discharge is from tail to head. If these cases had been all, Pacini's generalisation, as regards the direction of discharge-from the anterior nervous to the posterior non-nervous - would have been complete, and from it some attempt might have been made to offer an explanation of the phenomena. Unfortunately, however, this is not so, since Malepterurus presents a hitherto inexplicable cxception to the rule. In this fish, though the anterior or nervous surface is towards the tail-aspect as in Gymnotus, yet the discharge is in the opposite direction towards the head : that is to say, from the posterior surface to the anterior. The difficulties in the way of an explanation of the activity of these electrical organs of certain fishes are thus seen to be 
very great. Is the activity something specific occurring in these fishes alone, and unrelated to other electro-motive phenomena in the animal tissues? Or is it related to the electromotive action already observed in excited muscles? In support of the latter view, it is urged that most of the electrical organs consist of modified neuro-muscular elements. Against this argument, however, as we have just seen, is the instance of Malepterurus, in which, from a morphological standpoint, the organ is to be regarded as a modified gland, and therefore not muscular in character.

There are certain peculiarities, further, about the action of these organs which call for elucidation. Among these is the question of the character of the natural current of rest, about the significance of which there have been differences of opinion. There is also the fact that the organ, under a single strong excitation, gives rise not to one, but to a series of electrical responses.

We have seen that the apparently unique character of this group of organs constitutes an added difficulty in arriving at a correct theory on the subject. But it is clear that if we could succeed in discovering among vegetable organs any cases which showed similar characteristics, we should then be so much the nearer to the determination of that fundamental reaction on which the phenomenon in animal and vegetable alike depends.

In the typical case of Torpedo, it has been seen that the conducting nerve, when entering into an electrical plate, breaks into an extensive ramification, and thus forms the nervous surface, in contradistinction to the jelly-like substance in which it is imbedded, forming the opposite, and here indifferent surface of the plate. Now this arrangement is closely imitated by many ordinary leaves, in which the vascular elements break, on reaching the lamina, into a profuse arborisation.

I must here anticipate matters to say that I have discovered in the fibro-vascular bundles of plants (see Chap. XXXII.) elements which are in every way analogous to the nerves of 
animals. For an exact vegetal analogue to the electrical plate of Torpedo, we may take certain leaves in which the ventral, or anterior, surface is formed of a prominent network of highly excitable nervous elements, while the upper consists of an indifferent and relatively inexcitable mass of tissue. An example of this may be found in the leaf of Pterospermum suberifolium (Rox.) whose lower surface is characterised by a remarkably perfect venation, while the upper or posterior is dry and leathery. Thus the nerve passing into an electrical plate of Torpedo corresponds with the petiole attached to the leaf just described, since in the two cases alike, it is the ventral surface which contains the highly excitable nervous elements.

In the exceptional Malepterurus, on the other hand, it is, as we have seen, a modified gland, and not a modified muscle, which forms the posterior surface of an individual electrical element. Morphologically speaking, the vegetal analogue is found in such organs as the carpellary leaf of Dillenia indica, or the pitcher of Nepenthe, both of which are glandular on their upper or inner surfaces. In point of structure, then, these leaf-organs are analogous to single discs or elements of the electrical organs of Torpedo and Malepterurus respectively. But we have still, in the course of the present chapter, to inquire whether the electrical reactions are equally correspondent - that is to say, whether, on stimulation, the excitatory current in the type of vegetable organ represented by the leaf of Pterospermum is or is not, from the lower or anterior surface to the upper posterior, as in the electrical plate of Torpedo; and, conversely, whether in the type represented by the carpel of Dillenia or the pitcher of Nepenthe the excitatory current is from the posterior to the anterior surfaces, corresponding with the discharge in the electrical element of Malepterurus, from the posterior glandular to the anterinr non-glandular surface.

While dealing with the theory of the action of electrical organs, I shall be in a position to show that the characteristic 
reaction of each of these two types is governed entirely by the question of the relative excitabilities of the two surfaces. The physiological anisotropy on which the distinctive effect of the type depends is very pronounced in the representative cases of the vegetal analogues which have been named. In many other cases, however, though the results under normal conditions are fairly definite, and approach one or other of the two types, yet the characteristic responses are liable to be reversed under the physiological modifications induced by age and surrounding conditions. In this way it may be said of the leaf of water-lily (Nymphrea alba), of Bryophyllum calcineum, and of Coleus aromaticus that when vigorous, and in their proper season, their responses are of the first of these two types, while those of the bulb-scale of Uriclis lily, with its glandular inner surface, are of the second type.

The electrical organ of the fish may be excited indirectly by means of stimulus transmitted through the nerve; or direct stimulation may be applied, as by means of inductionshocks. Under either of these conditions the excitatory discharge is definite in its direction. In the case of Torpedo, as already mentioned, this is always from the ventral and anterior to the dorsal or posterior surface. Turning then to the corresponding vegetable organ of the first type, I shall show that transmitted stimulus induces an effect exactly similar; and I shall demonstrate this experimentally by means of the leaf of Nymphrea alba. Suitable galvanometric connections were made with the ventral anterior and with the dorsal posterior surfaces of the lamina. Thermal shocks, by means of the electro-thermic stimulator, were applied on the petiole, close to the lamina, at intervals of one minute, records being taken photographically of the resulting responses. It should be remembered here that excitation is transmitted to the lamina by the conducting nerve-like elements present in the petiole. The records (fig. I 56) show that the effect of this periodically transmitted stimulation was a series of responsive currents, whose direction was like that of the discharge in Torpedo, from the anterior surface to the posterior. 
Of great importance was the investigation carried out by $\mathrm{Du}$ Bois-Reymond on the effects induced in the electrical organ by the passage of currents in different directions. Polarising-currents in the direction of the natural discharge of the organ are distinguished, in the terminology introduced by Du Bois-Reymond, as homodromous, and those in the opposite as heterodromous. Polarisation-effects in the direction of the natural discharge he distinguishes as

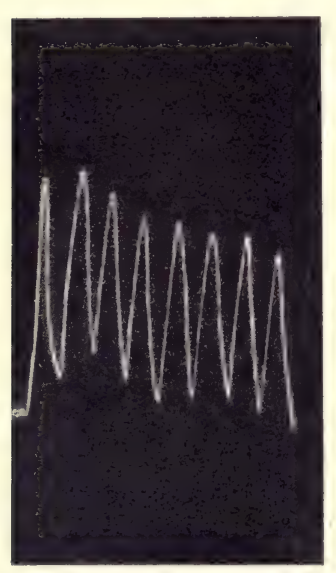

FIG. 1 56. Electrical Response of Lamina of Nymphica alba due to Transmitted Excitation from Petiole

Direction of responsive current from anterior or lower to posterior and upper surface.

'absolutely positive polarisation,' and against that direction 'as absolutely negative.' A polarisation-current in the same direction as the polarisingcurrent he calls 'relatively positive,' and in the opposite direction 'relatively negative' polarisation. It was found by him that polarisingcurrents of fair intensity and short duration, whether homodromous or heterodromous, would always give rise to polarisation-currents in the same direction as the natural discharge. He believed this to be due to the occurrence in the electrical organ of two different polarisationeffects, positive and negative. This will be understood from his own diagrammatic representation (fig. I 57) of the effect which he supposed to take place immediately on the passage of the polarising-current. In the upper figure the ascending arrow represents the homodromous polarising-current. This gives rise, according to $\mathrm{Du}$ BoisReymond, to two opposite polarisation-effects. The deflection seen in the galvanometer is the resultant of these, represented by the shaded part of the figure. The resultant of a homodromous current, then, is positive polarisation, both absolute and relative. The heterodromous current, on the other hand, induces absolutely positive and relatively 
negative polarisation. According to $\mathrm{Du}$ Bois-Reymond, further, heterodromous shocks induce no relatively positive polarisation, or only infinitely little (see down curve in lower part of figure). On sending congruent alternating currents from Saxton's machine, he obtained only the absolutely positive polarisation - effect. This he accounted for by supposing the relatively negative polarisations in both directions to cancel each other; the heterodromous positive to be so small as to be practically negligible ; and the homodromous positive therefore to be alone effective.

Du Bois-Reymond failed to recognise the element of excitation in these phenomena. What he calls positive polarisation has been shown by subsequent workers to be due to local polar excitation. But the question as to how polarising-currents in both directions could give rise to a single-directioned responsive effect has not up to the present, so far as I am aware, been explained fully and satisfactorily. The ex-

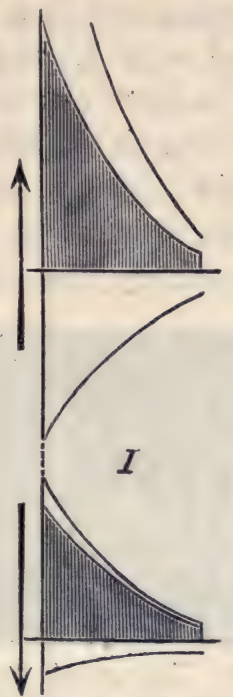

Fig. 157. Diagrammatic Representation by Di Bois-Reymond for Explanation of Electrical Response in Organ of Torpedo.

The natural discharge is here supposed to be from below to above. A homodromous current $\uparrow$ (upper half of figure) is supposed to induce two opposite polarisations, positive and negative. The resultant, represented by shading in figure, is absolutely and relatively positive. A heterodromous current $\downarrow$, on the other hand, is regarded as inducing a resultant absolutely positive and relatively negative polarisation (lower part of figure). periments carried out on leaves, which I am about to describe, will, however, throw much light on this subject.

It has already been shown, from anatomico-physiological considerations, that there are certain leaves which approximate to the character of single plates of such electrical organs 
as that of Torpedo. One such leaf, already mentioned, was that of Pterospermum. When induction-shocks are sent in both homodromous and heterodromous directions through such a leaf, between upper and lower surfaces, the leaf being, it is understood, in a normal condition, a responsive current is found to be evoked, always in one direction-that is to say, from the lower or anterior to the upper or posterior surface. This is strictly parallel to the electrical reaction observed by Du Bois-Reymond in Torpedo.

That this result is really due to the excitatory effect is proved by the fact that the same is found to occur

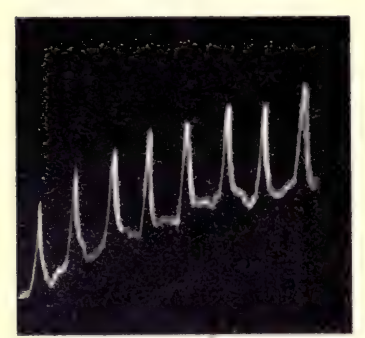

FIG. 1 58. Photographic Records of Responses given by Leaf ot Coleus aromaticus, when both Surfaces are Excited Simultaneously by Thermal Shock

Resultant responsive current from more excitable anterior to less excitable posterior surface.

when other forms of stimulation are used. Thus, if we place the leaf of Coleus aromaticus within a surrounding thermal helix, successive thermal shocks, acting simultaneously on both surfaces, give rise to responsive currents which are, as in the last case, from the lower anterior to the upper posterior surface. Fig. I 58 gives a series of such responses. From the fact which has already been fully established, that on the simultaneous excitation of two points the responsive current is always from the more to the less excitable, it is quite clear that in the present case it is the lower or anterior surface of the leaf which is the more excitable. These responsive currents, obtained under a nonelectrical form of stimulus, and similar to those evoked by electrical shocks, completely demonstrate the fact that the result is brought about, not by polarisation, either positive or negative, but by the differential excitability of the tissue itself. The response of electrical organs in general, then may be summarised in the following law :

The excitatory discharge is determined by the physiological anisotropy of the organ, its definiteness of direction being deter- 
mined by the fact that the responsive current is always from the more to the less excitable of the two surfaces.

Referring once more to the definite-directioned aftercurrent which we have seen to be induced as the result of polarising-currents, whether homodromous or heterodromous, it is now clear that these currents act as an electrical form of stimulus. The intensity of the after-current here seen in the galvanometer, however, is not wholly due to the excitatory electro-motive change, but in part also to physical polarisation, which is added to it algebraically. Thus, an exciting homodromous shock gives rise to an electrical after-effect, in which the excitatory current is opposed by a counter-current of negative polarisation. Under a heterodromous shock, on the other hand, the excitatory electrical change becomes summated with the negative polarisation, which is now in the same direction as itself. In these cases, though the preponderating nature of the excitatory effect determines the definite direction of the after-effect, yet it is difficult to know how much of the latter is actually due to excitatory action as such, and how much to ordinary polarisation helping or opposing this.

Very much greater complexities ensue again in practice rom the difference between anodic and kathodic actions on the two unequally excitable surfaces. In Torpedo, for instance, according to $\mathrm{Du}$ Bois-Reymond, the electrical organ responds better to a homodromous than to a heterodromous exciting current, while in Malepterurus, according to Gotch, the reverse is the case, the heterodromous being more efficient than the homodromous. Such diversity of results is probably to be accounted for by the considerations to which I have referred.

If we take, tor example, the simplest case, that in which the anterior surface is more excitable than the posterior, and if we suppose an induction-current of moderate intensity to be sent in a homodromous direction, we may assume that Pfliger's Law - the kathode excites at make, and the anode at break-will hold good. We shall here, for the sake of 
simplicity, neglect any effects that may accrue from anodemake and kathode-break. Under a homodromous inductionshock, then, two different excitatory electrical changes will be induced, on the lower and upper surfaces respectively, the consequent currents through the tissue being in opposite directions. On these, moreover, will be superposed again the polarisation-current. Calling the effect induced by anodebreak as $A_{b}$ and that of kathode-make as $\mathrm{K}_{m}$, we shall obtain a resultant consisting of $\mathrm{A}_{b}$ on the more excitable anterior surface, minus $\mathrm{K}_{m}$ on the less excitable posterior, minus the negative polarisation-effect. Under a heterodromous shock, on the other hand, we shall have $\mathbf{K}_{m}$ on the more excitable anterior surface, minus $\mathrm{A}^{b}$ on the less excitable posterior, plus the negative polarisation-effect.

Even this, however, does not exhaust the possibilities of complication. For I shall show in a subsequent Chapter, and have already shown elsewhere, that under a high E.M.F. Pfliger's Law does not apply. The relative excitatory values of anode and kathode may indeed undergo one or more reversals, according to the intensity of the acting electro-motive force. Thus, under a moderately high E.M.F. $\mathrm{i}_{\mathrm{n}}$ what I have designated the A stage, both the anode and kathode are found to excite at make, and either kathode or anode at break. In the B stage, under a still higher E.M.F., it is the anode which excites at make, and the kathode at break.

It will thus be seen what a number of complicating factors may be present when an organ is excited by currents of varying direction and intensity. If, then, we wish to study the purely excitatory reaction of an organ, as dependent solely upon its individual characteristics, uncomplicated by defects inherent in the method of excitation, we must see first that the applied stimulus is equal on both surfaces, and, secondly, that such factors as are not excitatory - that is to say, negative or counter-polarisation-are eliminated. These ends may be accomplished by subjecting the responding organ to symmetrical and alternating equal and opposite shocks, 
following each other in rapid succession. For the resultant negative polarisation will in practice be neutralised, if the primary polarising currents are similar, equal, and opposite. The stimulus applied on the two surfaces, moreover, will be equal, if the two rapidly succeeding and opposite-directioned shocks be so symmetrical as to be interchangeable. Whichever may be the factor of excitation will then act equally on both surfaces. The response, therefore, will now be determined solely by the natural difference of excitability as between the two surfaces.

It has been said that in order to accomplish these experimental conditions, the two opposite shocks should be equal in intensity and in point of time-relations. An ordinary makeand-break Ruhmkorff's shock does not fulfil this condition, since the break-shock is there the quicker and more intense of the two. Moreover, owing to the varying residual magnetisation in the iron core, successive shocks may not be equal. These defects are overcome by sending round the primary, with a constant rapidity, two equal and opposite currents in alternation. During one semi-cycle, then, the primary current varies from $+\mathrm{C}$ to $-\mathrm{C}$, and during the next from $-\mathrm{C}$ to $+C$, and since these two changes are effected with the same rapidity, the induced currents are. symmetrical, equal, and opposite.

Such reversal of current is accomplished by means of a rotating reversing-key. The key $\mathrm{R}$ is wound up against the tension of a spring $\mathrm{S}$, being maintained in this set position by the electro-magnet $\mathrm{E}$, acting on the armature. When the current in the electro-magnet is broken, the alternating double shock from the induction coil I is passed through the experimental leaf $\mathrm{L}$, by means of non-polarisable electrodes $N_{1} N_{2}$. In the case just described the sequence of the current through the primary coil was, say, right-left-right. In the next experiment, by means of the Pohl's commutator, $\mathrm{K}_{4}$, this sequence may be made left-right-left (fig. 159).

Empioying this method, I have carried out rheotomic observations for determining the time-interval after the shock 
at which the E.M.F. attained its maximum. The general arrangement here is similar to that described in Chapter IV. (cf. fig. 37). C is the compensator by which any existing electro-motive difference is compensated at the beginning of each experiment. The striking-rod A breaks the current in the electro-magnet $\mathrm{E}$, by which the rotating reverser $\mathrm{R}$ is actuated, which brings about equal and opposite shocks to the leaf. The galvanometric after-effect, at any short

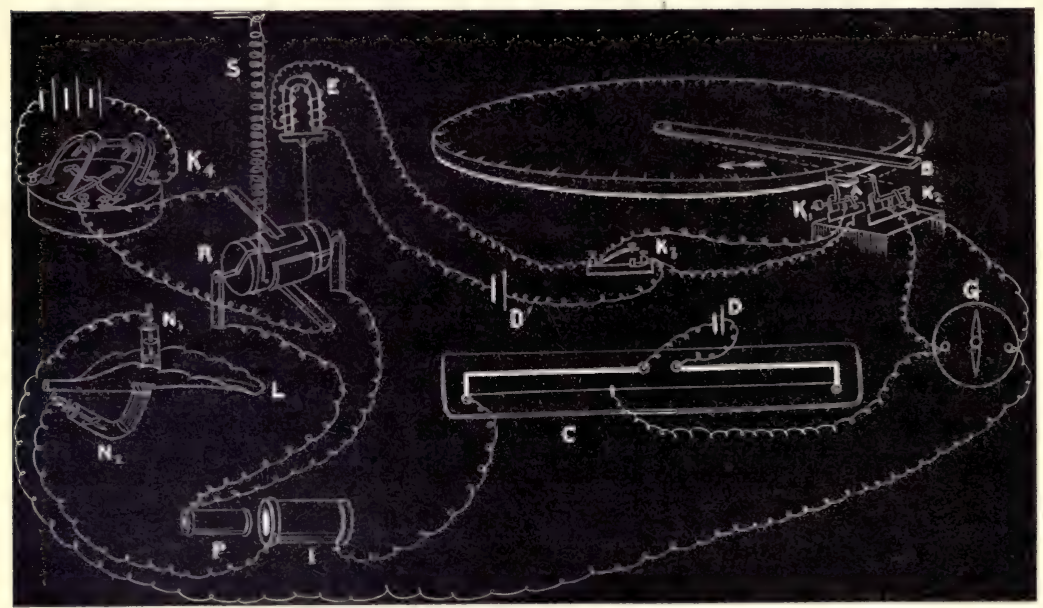

FIG. 159. Experimental Arrangement for Rheotomic Observations

$A, B$, striking-rods attached to revolving rheotomic disc; $\mathrm{K}_{1}$, key for electro-magnetic release of rotating reverser $\mathrm{R} ; \mathrm{K}_{2}$, key for unshunting the galvanometer when pressed by rod, $\mathrm{B}$, for a definite period; $\mathrm{K}_{3}$, key for preliminary adjustment ; E, electro-magnet with its armature by which rotating reverser, $\mathrm{R}$, is set against antagonistic spring, $\mathrm{S}$; $\mathrm{K}_{4}$, Pohl's commutator; C, compensator; $\mathrm{P}$, primary, and $\mathrm{I}$, the secondary, of the exciting induction-coil ; $\mathrm{N}_{1}, \mathrm{~N}_{2}$, non-polarisable electrodes, making electrical contacts with posterior and anterior surfaces of leaf.

interval after excitation, is obtained by the un-shunting of the galvanometer, caused by the striker $\mathrm{B}$ impinging against the key $\mathrm{K}_{2}$ (fig. I 59). We have seen that, owing to the presence of various complicating factors, as well as to the occurrence of negative polarisation, successive responses to homodromous and heterodromous shocks are unequal. By the employment of equi-alternating induction-currents, how- 
ever, we obtain true excitatory effects, unmodified by any such elements of uncertainty. In order to show how perfect the results obtained by this method become, I give here (fig. I60) the records of two successive excitatory responses obtained from a leaf of Bryophyllum calycinum, the responsive current being from the lower or anterior surface to the upper or posterior. In this mode of stimulation, by equal and opposite shocks, as already said, no advantage is given to either surface over the other. Nevertheless, I thought it well to take two successive records under shocks, in which the alternating currents in the primary circuit were first right-left-right, and then leftright-left.

In the electrical organ of Torpedo Gotch found the maximum electromotive change to be attained in about or second after the application of the excitatory shock. In leaves, again, I find the rapidity with which the maximum effect is attained to depend on the nature of the tissue, and also on the intensity of the

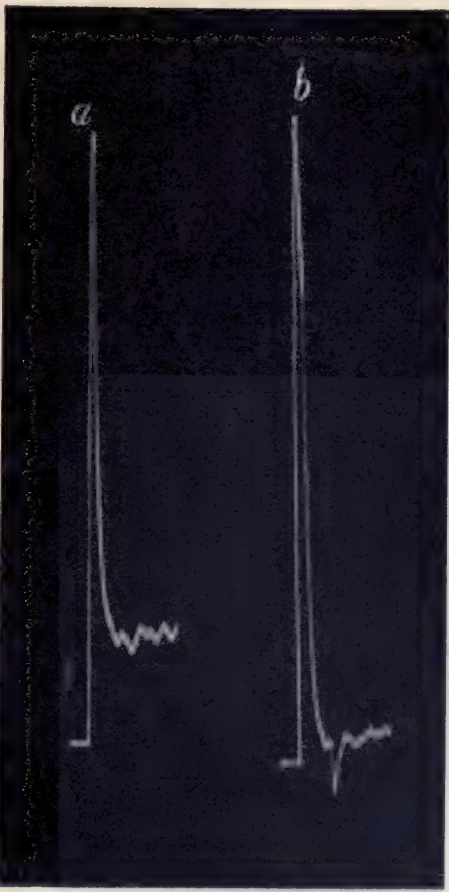

Fig. I60. Records of Two Successive Responses in Leaf of Bryophyllum calycinum under Equi-alternating Electrical Shocks

exciting shock. In sluggish specimens this may be as long as 2 second. It should be remembered that in the case of mechanical stimulation of moderate intensity also, this period was, similarly, about 2 second (p. 5I). With very vigorous leaves of Nymphaa alba, however, and employing a stronger electrical stimulus, the maximum effect was 
attained in a much shorter time -that is to say, in about O3 second. I give below a table showing the rheotomic observations made on such a leaf at gradually increasing intervals after the exciting shock. It should be remembered

TABLE OF RHEOTOMiC ObSERVATIONS.

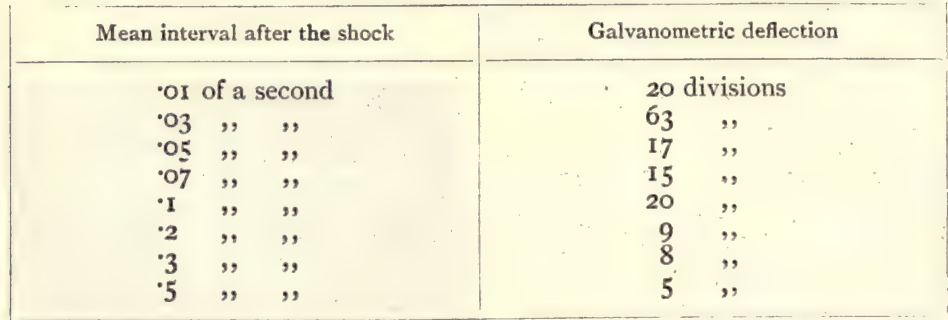

that the recording galvanometer was un-shunted for 'OI second, The curve given in fig. 16I has been plotted from

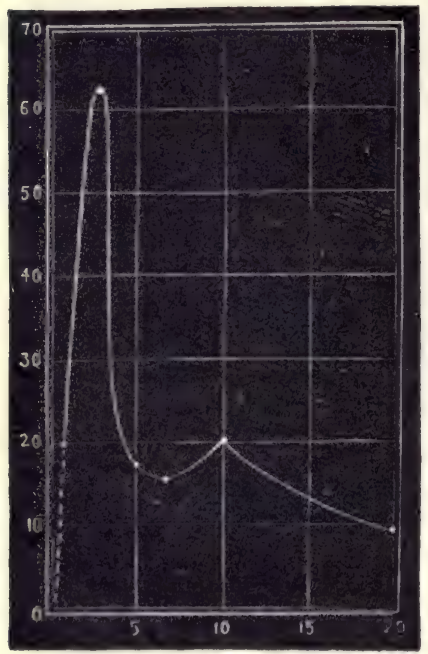

FIG. I6I. Response-curve from Rheotomic Observation on Leaf of Nymphcea alba

Ordinate represents galvanometer deflection; abscissa, time in hundredths of a second. these results. The maximum electro-motive change took place, as already pointed out, in $\mathrm{O} 3$ second after the application of stimulus. This curve shows multiple apices, as was also the case, it will be remembered, after a strong mechanical stimulation (cf. fig. 40). This point will be referred to in greater detail in the next chapter. In the course of half a second after the shock, the excitatory electro-motive change had subsided to about one-twelfth of the maximum.

It has been said that the excitatory current depends for its definiteness of direction on the physiological anisotropy of the organ. In those leaves in which the physiological differentiation of the upper and lower surfaces is not strongly marked, the differential 
excitability of the two is liable to undergo reversal, under the changing conditions of age, season, and fatigue induced by previous stimulation. In the leaf of Pterospermum, however, which I have here taken as the type corresponding with Torpedo, the normal differential excitability is generally very persistent. Here the excitability of the posterior surface, which is leathery, is slight, and practically negligible. But the anterior surface, with its rich and prominent venation, is highly excitable. The excitatory discharge of such a leaf is thus from the anterior to the posterior. I give in fig. 162 a series of its responses to equi-alternating electric shocks. It will be seen that these are very uniform, and exhibit practically no signs of fatigue.

We have thus found a vegetable organ whose responses are exactly parallel to those of a single plate of the electrical organ of Torpedo and its type. We shall next study the responsive peculiarities of the vegetable organ whose responses correspond with those of the organ of

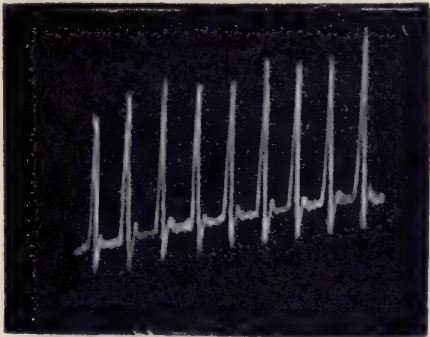

FIG. I62. Series of Responses given by Leaf of Pterospermum suberifolium to Stimulus of Equi-alternating Electrical Shocks at Intervals of I Minute

Malepterurus. It has been mentioned that the posterior surface of each single element of this electrical organ is regarded as consisting of a glandular, rather than a muscular, modification. Among corresponding leaf-organs, then, the carpellary leat of Dillenia indica might, as we also saw, be taken as the type, its posterior surface being glandular. Or the analogy will be still more perfect if we take as the vegetal type the pitcher of Nepenthe. Here the internal or posterior surface is richly provided with glands. The next point to be determined is whether, in these cases also, on excitation the responsive current is from the posterior surface to the anterior, as in the electrical element of Malepterumus. And on subjecting them to equi-alternating electrical shocks, I found 
this to be the case. The responsive current here flowed from the glandular posterior to the non-glandular anterior surface. From this experiment we see that a glandular surface is exceptionally excitable, a conclusion which will be found to be supported by the numerous experiments on glandular organs in general, to be described in Chapter XXIV. I give in fig. 163 a series of photographic records, obtained on excitation of Dillenia indica. In the next record (fig. 164) are seen the responses given by the pitcher of Nepenthe.

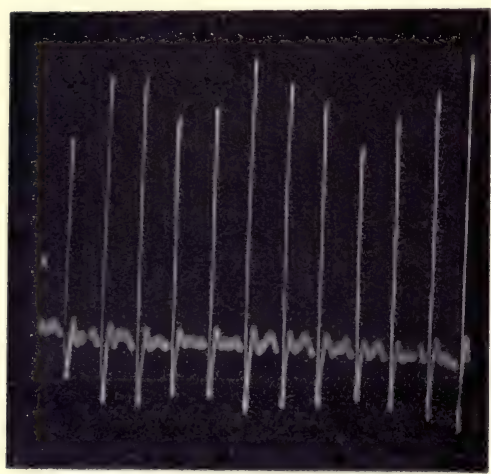

FIG. 163. Photographic Record of Responses of Carpel of Dillenia indica

Natural current from posterior to anterior, and responsive current from anterior to posterior surfaces.

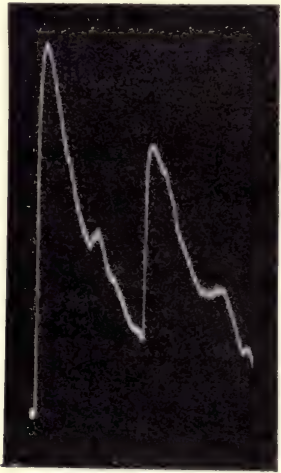

FIG. I64. Photographic Record of Normal Responses given by Pitcher of Nepenthe, under Equialternating Electric Shocks

Responsive current from internal glandular to external nonglandular surface. Note tendency to multiple response.

An interesting fact to be noticcd in the latter is the tendency to multiple response.

Similar results were also obtained on taking any single scale of the bulb of Uriclis lily about the time of flowering. In each of these the lower or outer surface is invested with a more or less dry and glistening membrane, while the upper or concave is moist and glanduloid. The moisture observed inside each scale is in fact exuded from this inner surface. On subjecting one of these scales, then, to the electrical 
excitation already described, it is found that a very strong responsive current is obtained, whose direction is, as in the last case, from the glanduloid to the non-glanduloid surface.

The effect of the serial arrangement, again, in enhancing the electro-motive force-as seen in the pile-like arrangement of the electrical organ of fishes-may be exemplified, in the parallel instance of the plant-organ, by means of the superposed scales of the bulb, as found in nature. The bulb may be divided longitudinally into halves, of which the right-hand half is mounted, for experiment, with the scales vertical. It will be understood that all the glanduloid surfaces here face the left, while the non-glanduloid are turned to the right. Thus the left aspect of this pile corresponds to the head aspect of the organ of Malepterurus. The latter, on excitation, responds by a current in the direction of head to tail-that is to say, from glandular to non-glandular; and similarly, in the pile-like half-bulb of Uriclis lily, the responsive current is from glanduloid to nonglanduloid-that is to say, from left to right.

Another interesting way to perform the same experiment is without making any section of the bulb. We take a bulb of Uriclis, with the peduncle rising out of the middle. When this hollow peduncle is cut across, it allows of an electrical connection being made with the centre of the interior of the bulb. An equatorial belt makes the second, or outer, connection. On subjecting this to equi-alternating shocks, the resulting response will be found to be from the inner surface to the outer, through the numerous intermediate scales, the individual effect in each being concordant and additive.

We have thus seen how the response of a leaf gives us an insight into the action of a plate of an electrical organ; how the differential excitabilities of the two surfaces give rise on stimulation to an induced E.M.F. as between the two; how a nervous and indifferent-tissued surface will give rise to a response in one direction, and a glandular and non-glandular 
in the other; and finally how, by a serial arrangement, the terminal electro-motive effect becomes enhanced. The light thus thrown on the two types of response, known to occur in the electrical organs of fishes, is evident. Further considerations, relating to the theory of electrical organs, will be given in detail in the next chapter. 


\section{CHAPTER XX}

\section{THE THEORY OF ELECTRIC ORGANS}

Existing theories-Their inadequacy-The 'blaze-current' so called-Response uni-directioned, to shocks homodromous or heterodromous, characteristic of electric organs-Similar results with inorganic specimens-Uni-directioned response due to differential excitability-Electrical response of pulvinus of Mimosa to equi-alternating electric shocks-Response of petiole of Musa Of plagiotropic stem of Cucurbita-Of Eel-The organ-current of electric fishes-Multiple responses of electrical organ-Multiple responses of Biophytum.

ONE of the most perplexing problems in connection with the phenomena of electrical organs is the question as to whether the activity of such organs is specific-that is to say, peculiarly characteristic of them-or falls into line with the other electro-motive reactions observed in animal tissue. Many arguments have been brought forward for and against the identity of these phenomena with the excitatory reactions of the nerve and muscle.

From the experimental results which I have described, however, it would already appear that such reactions as these of the electrical organ are not specifically characteristic, even of the animal structure, but may equally well be observed in plant tissues. It is therefore essential, if we are to determine that basic reaction which is common to all alike, that we should find a wider generalisation than has hitherto been contemplated. This basic reaction, as we have already seen, depends upon the differential excitability of an anisotropic organ, and this aspect of the case we are now about to study in greater detail. Before doing this, however, we shall briefly glance at various theories which have been suggested, but are generally admitted to be inadequate. 
Bell, for example, thought it might be possible to explain 'the discharge of the electrical organ solely by the negative variation of the nerve-current, concomitant with innervation. In this arrangement the dorsal surface of the electrical plate (of Torpedo) would, at the moment of innervation, become positive, the ventral surface negative, as actually occurs.'

As against this, it was pointed out by Du Bois-Reymond that this hypothesis in the first place predicates the existence of a current of rest, caused by the 'natural' cross-sections (acting like artificial sections) of the nerves in the plates, and accordingly heterodromous to that of the discharge. Instead of this permanent current-which must correspond in E.M.F. with the discharge, if the nerve-current is to disappear in the negative variation-there is only an inessential P.D. during rest, and the resulting 'organ-current' is always homodromous with the discharge. ${ }^{1}$

Even had these objections not existed, however, Bell's hypothesis would have failed to explain the electrical action of Malepterurus. Du Bois-Reymond himself tried to explain the action of the electrical organ, 'not by the negative variation of the nerve current, but by a process in the electrical plates transformed from muscles, comparable with the negative variation of the muscle-current, as set forth from the standpoint of the pre-existence theory.' Here, however, it is perhaps sufficient to point out that the pre-existence theory, on which the hypothesis was based, is now held to be invalidated.

There remains only the Chemical, or Alteration Theory, which associates all electrical changes with the corresponding chemical processes of assimilation and dissimilation. But it has not been made clear in what way these can bring about the characteristic discharge of the electrical organ.

There is another point, not altogether unrelated to this subject, which may be dealt with on the present occasion, I allude to the so called 'blaze current' of Dr. Waller. By this is meant an after-current in the same direction as the

1 Biedermann, Electro-Physiology (English translation), vol. II. p. 462. 
exciting current. It is, in, fact a new name for that phenomenon which Du Bois-Reymond indicated as 'positive polarisation-current.' Du Bois-Reymond had also shown that this particular effect was most markedly exhibited when the functional activity or 'livingness' was at its highest. Under opposite conditions, again, it would disappear. The intensity of this homodromous after-effect was thus dependent on the degree of vitality of the tissue under experiment. Hermann and Hering, however, afterwards showed that what Du Bois-Reymond called ' positive polarisation ' was in reality excitatory reaction. These excitatory effects are known to be caused by either the anode or the kathode ${ }^{1}$; and I have, in the course of the last chapter, demonstrated the fact that it is the differential excitability of a tissue which determines such uni-directioned response. It is difficult, therefore, to see the necessity of a new name for these phenomena. Dr. Waller himself, however, offers the following as an important reason :

' The great mass of living things, whatever else they may give and take from their surroundings, take oxygen and give carbonic acid; they may live slowly or they may live quickly-sluggishly. smoulder or suddenly blaze. A muscle at rest is smouldering : a muscle in its contraction is blazing; the consumption of carbohydrate and the production of $\mathrm{CO}_{2}$, never absolutely in abeyance, even in the most profound state of rest, are sharply intensified when the living machine puts forth its full power, and there is then a sudden burst of heat, and an electrical discharge. ... '2

This amounts to another way of saying that the cause of the excitatory galvanometric effect is some explosive dissimilatory change, a view which I have already shown in

1 "Within a given "physiological" range of strength of current the negative kathodic must, equally with the positive anodic, be designated an "irritative" after-current, due entirely to "polar current-action." --Bìedermann, ElectroPhysiology (English translation), vol. I. p. 448.

"Waller, Signs of Life, p. 74. 
previous chapters to be quite untenable. I shall presently describe experiments which will further show that galvanometric responses, not to be distinguished from this, take place when there is no possibility of any consumption of carbohydrates or production of $\mathrm{CO}_{2}$.

The fact, however, that the excitatory after-effects described, disappear on the death of the tissue, has led Dr. Waller to put forward the generalisation that this so-called 'blaze-current' is the final distinction between living and non-living matter. His formula, with regard to this, is, 'If the object of examination exhibits blaze in one or in both directions, it is living.' He admits, nevertheless, that a substance which is undoubtedly living will not always exhibit the 'blaze-current.' But it is contended that the occurrence of 'blaze' is an undoubted 'sign of life,' and that thus a strong distinction is to be made between vitalistic and non-vitalistic, or physical, reactions. Hence, as there is supposed to be no excitatory reaction possible in non-living or inorganic matter, it would follow that electrical shocks passed through such a substance, in either direction, should give rise only to those counter-polarisation currents which are known to physicists. In such cases, on reversing the direction of the shock, the direction of the after-current is also reversed; but in the living substance, it is maintained, the case is quite different. If the direction of the shock be here reversed, the after-current will still appear, with direction unchanged, because in this latter instance it is not generated by the shock, but is, on the contrary, an inexplicable function of the living material, set in action by it, in the same way as a loaded gun is fired by pulling the trigger. The possibility of obtaining from the given substance such a uni-directioned after-current, independently of the direction of the shock, is thus to be taken as the test and token of 'vitality.'

Now, while it is certainly true that the domain of physiological phenomena has not yet been so thoroughly explored as that of the physical, it is nevertheless equally true that no one could venture to claim that even physical phenomena 
had up to the present been exhaustively studied. It is, then, somewhat hazardous to declare that because a particular phenomenon has not yet been observed to occur in inorganic matter, it is by that fact demonstrated to be hyper-physical in its nature, and must be relegated to the different and mystical category of the exclusively vitalistic. The very foundation of such a statement would be swept from under it, the moment it was shown that the same phenomenon followed, under the same circumstances, in conditions which were admitted to be purely physical.

I have shown, it will be remembered, in the previous chapter, that the uni-directioned response to electrical shocks in either direction was due to the differential excitability of the structure. The response of any anisotropic organ would always be from the more to the less excitable, the more excitable becoming relatively galvanometrically negative. There may here be various cases of excitation, all giving results of the same type, say, a responsive current from B to A. The first is that in which, on excitation, both B and A become galvanometrically negative, A being the less so of the two. In the second case, the excitability of A being slight, or negligible, B alone becomes negative. And in the third case, excitation induces positivity of $\mathbf{A}$ and negativity of $\mathbf{B}$. In all these cases the relative negativity of $\mathrm{B}$ being greater, the responsive current will flow from $B$ to $A$. The resultant current is made up, in the first case, by subtracting the galvanometric negativity of $A$ from that of $B$; in the second case, it consists of the galvanometric negativity of $\mathrm{B}$, that of $\mathrm{A}$ being zero; and in the third case, it is produced by the addition of the effect at $\mathrm{A}$ to that at B. Examples of the last of these will be found in certain animal and vegetable skins, described in Chapter XXII.

These being the conditions, then, for the induction of the uni-directioned responsive current, it appeared to me probable that the same result could be obtained with inorganic substances, provided that the specimen were so 
prepared as to be anisotropic, one side having a greater potentiality of galvanometric negativity under excitation than the other. In that case, further, it was clear that the strongest resultant current would be obtained, if one surface of the structure became galvanometrically positive, and the other negative, on excitation. I have already stated, in Chapter I., that different inorganic substances give electrical responses of opposite signs. Thus the response of lead is positive, while that of brominated lead is negative. If, then,
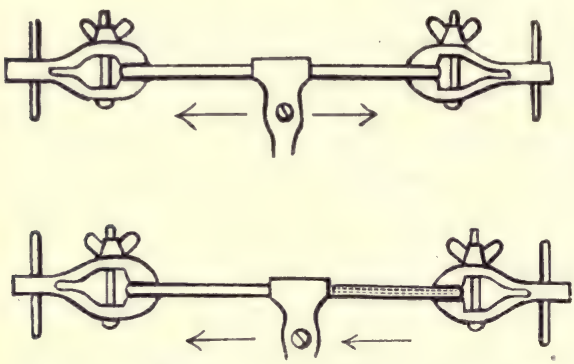

FIG. 165. Responsive Currents in Lead Wire Upper figure-Excited wire, galvanometrically positive. Simultaneous excitation of both ends balance each other; resultant response zero.

Lower figure-Left portion, lead wire, right portion, brominated lead wire, shown as shaded. Response of first positive, of second negative. Simultaneous excitation of the two induces resultant response which is additive, from the right to the left.

we take a lead wire A $B$, clamped in the middle at C, as represented in the upper diagram (fig. I65), and stimulate the right-hand end $B$, say by mechanical vibration, a responsive current will be induced, which will flow towards the stimulated, B thus becoming galvanometrically positive. The same will be the case with $A$, on stimulation. When both $\mathrm{A}$ and $\mathrm{B}$ are simultaneously stimulated, it is evident that the two responsive currents, being antagonistic, will cancel each other. But if the right-hand half $\mathrm{B}^{\prime}$ of the wire consist of brominated lead (lower diagram, fig. I65), while the left-hand half $\mathrm{A}^{\prime}$ is of lead like that used in the first case, then stimulation of $\mathrm{B}^{\prime}$ will cause a responsive current to flow away from the right-hand excited end, $\mathrm{B}^{\prime}$ thus becoming galvanometrically negative; $\mathrm{A}^{\prime}$, on the other hand, will give rise to a positive response towards the excited. Simultaneous excitations of $\mathrm{A}^{\prime}$ and $\mathrm{B}^{\prime}$ will not then be antagonistic, but additive in their effects. The resultant response will thus be from the negative $\mathrm{B}^{\prime}$ to 
the positive $\mathrm{A}^{\prime}$. We may next take a flat strip of lead, of which the lower surface $B$ is brominated. ${ }^{1}$ On mechanical stimulation of both surfaces by vibration it will now be found that the resultant responsive current flows across the strip, from the negatively-responding $B$ to the positivelyresponding A (fig. I66). It will be seen that here-as the molecular disturbance on which excitation depends is caused by a non-electrical form of stimulus-the electrical character of the response obtained is unexceptional in its freedom from any complication by the polarisation-

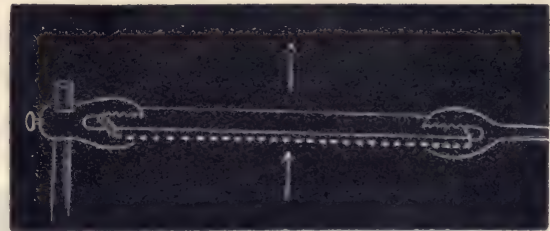

Fig. 166. Flat Strip of Lead, of which lower Surface is Brominated

Response of upper surface positive, of lower surface negative. Resultant response from lower to upper. factor.

Let us next consider what would be the effect of sending induction shocks across such a strip. If inorganic bodies be really inexcitable, then we can here obtain only the counter-polarisation current as the after-effect. That is to say, on sending a shock from below to above in an ascending direction, we should observe only negative polarisation as an after-effect, or a descending current from above to below. A descending shock should, on the other hand, give rise to an ascending after-current. But if inorganic substances should prove to be excitable, and if $\mathrm{B}$ were to become on excitation relatively negative to $A$, as we found in the experiments on mechanical stimulation, then, whatever the direction of the shock, we should have a uni-directioned response, from below to above, exactly like the excitatory discharge of the electrical organ of Torpedo, from the ventral surface to the dorsal.

1 In order to brominate this surface as required, it may be held exposed to vapour of bromine. Or an electrical deposit may be made by electrolysis, in a bath of potassium bromide solution. A certain depth of deposit and time of formation are important to the successful preparation of the plate. When these are secured, the proper condition of responsiveness is found to be long-enduring as will be seen from the photographic records. 
I give below (fig. I67) photographic records of the responsive after-effects actually obtained on carrying out this experiment on the lead strip prepared as described. It is here seen that whatever had been the direction of the shock, homodromous $\uparrow$ or heterodromous $\downarrow$, the responsive current was always one-directioned: that is to say; from the more negatively-excitable lower to the positively-excitable upper surface. If the test of the 'blaze-current' is to be accepted, then we shall be compelled to refer this metallic strip to the category of organic matter in a living condition!

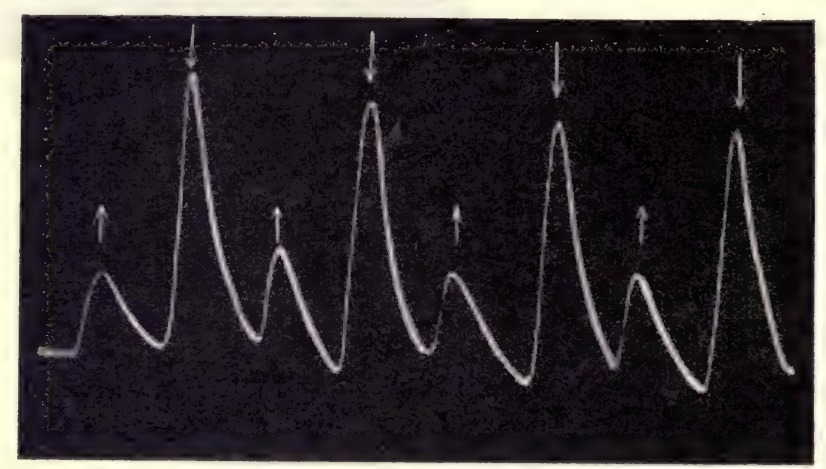

FIG. I67. Photographic Records of After-effect of Homodromous $\uparrow$ and Heterodromous $\downarrow$ Induction-shocks in Prepared Strip of Lead

Note uni-directioned response, whatever be direction of induction-shock.

Since it is admitted that the electrical response of living tissues is due to molecular excitation, and since we obtain similar electrical effects, as just seen, from the inorganic, it is clear that excitability is the property, not of the organic alone, but of all matter. It is also clearly seen that the one directioned response of an anisotropic structure-so characteristic, among other things, of the electrical organ of certain fishes - depends on its differential molecular excitability. The phenomenon is thus reduced to what are almost its last terms-namely, the molecular. It is therefore unnecessary to call in the aid of such indeterminate factors as vitalism, or assimilation and dissimilation. 
In taking these responses to homodromous and heterodromous shocks, the counter-polarisation-current is superposed upon the excitatory effect. For this reason we see, in fig. I64, that the homodromous response, in which negative polarisation was an opposing factor, is smaller than the heterodromous, in which polarisation conspires with the excitatory current. We have seen, in our experiments on the leaf-organ, that when this negative polarisation is annulled by taking responses under equi-alternating electrical shocks, successive responses, giving the true excitatory effect, become equal." " I give here (fig. 168) a series of responses of the prepared lead strip, under these conditions of equialternating shocks, in which they are seen to have become equal, the resulting responsive current being from the lower surface to the upper, as before.

Having thus demonstrated in various ways the nature of that fundamental condition, which

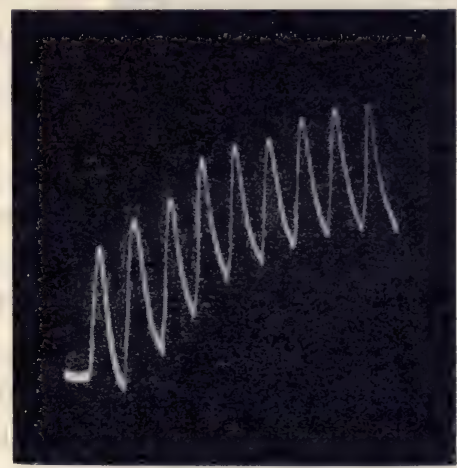

FIG. 168. Photographic Record of Responses to Equi-alternating Electric Shocks at Intervals of One Minute in Prepared Lead Strip

Responsive Current as before from lower to upper surface.

determines the excitatory discharge of the electrical organ, we may next take other organs which are known to be differentially excitable, and observe whether they also, under electrical excitation, give definite uni-directioned responses.

The differential excitability of the upper and lower surfaces of the pulvinus of Mimosa are well known. On subjecting this organ, then, to equi-alternating electrical shocks, I obtained uni-directioned responsive currents, their direction being from the more excitable lower to the less excitable upper half, of which a photographic record is given 
in fig. I69. In this particular specimen, owing to growing fatigue, the responses are seen to undergo diminution. In the sheathing petiole of Musa also, we found, as will be remembered, that the inner or concave surface was more excitable than the outer or convex. In taking records of the responses of such a specimen to equi-alternating electrical shocks, the direction of the responsive current was found to be from the more excitable inner to the less excitable outer surface.

It is now evident that in the

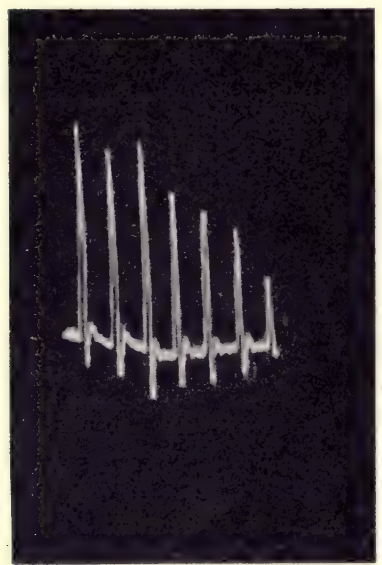

FIG. I69. Response of Pulvinus of Mimosa to Equi-alternating Electric Shocks

Responsive current from more excitable lower to less excitable upper. structure of any single element of an electrical organ, the essential question is not as to the correspondence of one part or another with muscle, nerve, or gland, but merely that of anisotropy. Any tissue which is anisotropic is potentially an electrical element. Thus a stem of Cucurbita originally radial, becomes physiologically anisotropic when it happens to assume the recumbent posture, owing to the a-symmetrical action of environmental stimuli. Such an anisotropic organ, when electrically excited, gives a onedirectioned responsive current from the lower or ventral to the upper or dorsal surface. A similar reaction is, curiously enough, shown by the body of the Eel, which also, on excitation, gives a responsive current from the ventral surface to the dorsal.

The responsive peculiarity of the constituent elements of the electrical organ is thus not unique. The extraordinary character of the organ depends merely upon the serial arrangement of innumerable such elements, connected with a nerve-trunk, by means of which a voluntary impulse is enabled to bring about the excitatory discharge of the electrical pile, and so convert it into a weapon of offence. 
The most difficult problem with regard to these electrical organs having thus been solved, it remains to say a few words concerning two other points: namely, the natural current of rest and the repeating character of the excitatory discharge.

As regards the first of these, Du Bois-Reymond found the current of rest-which he designated as the organ-current - to be in the direction of the electrical discharge. We have found, however, that, as a general rule, in the primary condition, the true natural current flows in the opposite direction to that of excitation, that is to say, from the less to the more excitable. In agreement with this, I find, in the leaf of Pterospermum, the electrical reaction of which is similar to that of the plate of Torpedo, that while its excitatory current is from the more excitable ventral to the less excitable dorsal, its resting-current is opposite to this-that is to say, from dorsal to ventral. With regard to the electrical organ, then, there can be little doubt that here also the true resting-current would have been found to be opposite in direction to the excitatory, if it could have been observed and recorded under an absolute condition of physiological rest. In a highly excitable structure, however, the shock of preparation, as we have seen and shall further see, leaves an after-effect which reverses the natural current of rest. For this reason, the current of rest observed in preparations of the electrical organ has not, in all probability, represented the true current, but rather the excitatory reversal of it. This view is supported by the fact that while in the organ-preparation of Torpedo, this reversed, or ingoing-current of rest is considerable, in the intact fish it is negligible. It must be borne in mind that the fish is spontaneously excitable, and also that there must remain a certain residual effect from the organ-discharges. Nevertheless Zantedeschi found a restingcurrent to occur in the intact fish in the reverse direction to that of the excitatory discharge. This was evidently the true resting-current. This view of the organ-current, of 
Du Bois-Reymond, as, in reality, a persistent after-effect of excitation, gathers confirmation from an observation made by Gotch, that it is considerable in Torpedo, in which the excitatory effect also is known to be very persistent; but in Malepterurus, where recovery from excitation is rapid, this particular organ-current is practically absent from excised preparations.

We may turn now to the repeated or oscillatory character observed in the electrical discharge by both Gotch and Schönlein. This is seen in the multiple apices of its rheotomic curve. Multiple apices are also found, as we have seen, in the rheotomic observations on vegetable organs, given in fig. 40. Gotch attempts to explain these repeated responses of the electrical organ-which he calls 'autoexcitation'-by the passage through the tissue of the intense current due to the response; this he regards as exciting the tissue again, and bringing about the repetition of the same effect. With regard to this hypothesis, however, it is unnecessary to suppose that it is the intense electrical current of the first response which is the exciting cause of the second, and so on. For we must bear in mind that multiple response is not exclusively characteristic of these electrical organs, with their high intensity of discharge-current, but is exhibited by tissues of various kinds. These, as we have seen in Chapter XVII., may be thrown into a condition of rhythmic or multiple excitation by any form of stimulus, provided that its intensity be beyond a certain value. It thus happens, as we have seen, that while a single moderate stimulus induces a single response, a single strong stimulus induces multiple responses.

Indeed this fact-that it is not the intensity of the first responsive current, causing a new excitation of the tissue, which is accountable for the second, and so onbecomes quite clear, as soon as we make quantitative observations of response, in those cases in which it receives undoubted visible manifestation, by the orderly fall of leaflets serially arranged-that is to say, in such plants as Biophytum. 
The sensitiveness of these leaflets is of that order which requires on an average an E.M.F. of 12 volts to cause excitation by kathode-make or anode-break. And this value is considerably higher for anode-break than for kathode-make. The lowest E.M.F. which I have found with highly excitable Biophytum to be effective in causing excitation, was 4 volts, and in less sensitive specimens it might be as high as 20 volts.

Now, on applying a strong stimulus, say by contact of hot wire, on the petiole bearing the sensitive leaflets, an excitatory wave is initiated, which, during its progress, brings about depression of the leaflets in serial order. After an interval of about half a minute, a second wave is found to be initiated from the original point of excitation, causing a second series of responses, shown by the same serial depression of the leaflets as before. And such recurrent excitations may take place from a single stimulus, as often as twenty times. On determining the E.M.F. value of each of these excitatory waves, however, I have found it to be of the order of oI volt. This, it will be seen, is only about four one-hundredths of the minimum E.M.F. necessary to induce excitation of the leaflet. It clearly follows that here the original stimulus is the cause of these multiple excitations, and not the first response the cause of the second.

The phenomenon of multiple response then, is, as we have seen, of very extensive occurrence, and not confined to electrical organs. We have seen it exhibited even by ordinary tissues, and we shall find in subsequent chapters that such repeated responses are actually induced in nervous and glandular tissues, under the action of an intense stimulus. This is interesting in view of the fact that the electrical organs of fishes are made up of either neuro-muscular or neuro-glandular elements. 


\section{CHAPTER XXI}

\section{DETERMINATION OF DIFFERENTIAL EXCITABILITY}

UNDER ELECTRICAL STIMULATION

Advantage of electrical stimulation, in its flexibility-Drawbacks due to fluctuating factors of polar effects, and counter polarisation-current-Difficulties overcome by employment of equi-alternating electric shocks-Methods of the After-effect and Direct-effect-Experiment of Von Fleischl on response of nerve-Complications arising from use of make and break shocks--Rotating reverser-Motor transformer-Response of Musa to equi-alternating shocksAbolition of this response by chloroform-Response records of plagiotropic Cucurbita and Eel-Differential excitability of variegated leaves, demonstrated by electric response.

WE have seen, in Chapter IX. that the differential excitability of any two points in a tissue can be detected, by observing the direction of resultant response, when both the points are simultaneously excited by an identical stimulus. It was seen in the same place also that the more excitable point becomes, under diffuse stimulation, relatively galvanometrically negative. I further described the various forms of quantitative stimulus which might be employed for this purpose, those, namely, of mechanical vibration and of stimulation by thermal shocks. By employing these non-electrical forms of stimulus the fundamental law of differential response was firmly established, in such a way as to exclude every source of uncertainty.

The electrical form of stimulation is characterised nevertheless by many advantages. Its intensity, for instance, is easily graduated. But its principal superiority lies in its great flexibility of application. Any two points, however remote or difficult of access they may be, may by this means be subjected to a required stimulus, if we can only apply two 
electrodal points to them. These qualities, however, are counterbalanced by many serious drawbacks. For the resultant electrical change, induced at a given point by an electrical shock, is the sum of a number of changing factors, which may be broadly classified as excitatory and polarising. Let us suppose the induction shock to enter at a point. We shall there have the physiological polar effects of anode-make and anode-break. There is again, on the cessation of the current, a counter electro-motive force due to polarisation. The physiological reaction, moreover, will depend on the excitability of the point.

Next, as regards the second or kathodal point, where the shock-current leaves the tissue. We have here the physiological effects of kathode-make and break, the factor of the excitability of the point, and the counter electro-motive force due to polarisation, all contributing to the resulting electrical change at that point. That relative electrical difference induced between the points $\mathrm{A}$ and $\mathrm{B}$, which determines the observed electrical variation, thus equals the sum of the fluctuating factors acting on A, minus the sum of the fluctuating factors acting on $\mathrm{B}$. These elements of variation are sufficiently complicated; but still another, as already said, is added to them, in the fact that, with regard to the excitatory polar effects of currents themselves, we have not to deal simply with the law enunciated by Pfliger, that the kathode excites at make and the anode at break. The complete law, as will be shown in a later chapter, is complicated by the fact that the result depends on the intensity of the electromotive force. With feeble and again with excessive E.M.F., the actual facts are the opposite of conclusions arrived at by Pfliger. Under these circumstances it would appear at first sight impossible that any reliable results could be obtained by the employment of the electrical form of stimulation. I shall now, however, proceed to show in what manner all these difficulties may be overcome, and the electrical form of stimulus made extremely reliable. This will perhaps be best understood if we take a concrete example. Let us suppose 
that a single electrical shock of moderate intensity enters an isotropic tissue at the point $\mathrm{A}$, and leaves it at $\mathrm{B}$; A will then be the anode, and B the kathode. Here the true excitatory effect is found to take place only at the kathode, probably because the anode-break excitation takes place at much higher intensities of E.M.F. than the kathode-make. At these higher intensities, then, the anode-break effect also will occur. At an excessively high E.M.F. again, these relations, for reasons already explained, may undergo reversal. The point of such reversal would depend on the nature and excitability of the tissue. Though, for all these reasons, the relative excitations of $\mathrm{A}$ and $\mathrm{B}$ remain a matter of doubt, yet we may be sure of the excitation of both points, if two, or any equal number, of exactly equal shocks be sent through the tissue in opposite directions, in rapid alternation. If, again, instead of two alternate shocks only, we give $n$ alternating shocks, absolutely equal, and if, further, the natural excitability of the two points A and B have been the same, then there will be nothing to distinguish the excitatory effect induced at A from that at $\mathrm{B}$. In other words, the two excitations will be exactly equal. These strictly equal and opposite alternating currents, moreover, can have no resultant polarisation-effects, for the effect arising from an induction shock, in either direction, will be counteracted by that caused by the opposite.

We thus see that by the employment of this method the only change induced at the two electrodes will be the excitatory change, the physical polarisation-factor being eliminated. Thus, on subjecting two points, $\mathrm{A}$ and $\mathrm{B}$, to equal stimulation, the induced galvanometric negativity, at both the points, will be equal, if the natural excitabilities of the two have been the same. But if the tissue be anisotropic, and the natural excitability of one point, say $B$, greater than that of $A$, then we shall obtain a resultant responsive current, which will flow in the tissue from the more excited $B$ to the less excited $\mathrm{A}$, the induced galvanometric negativity of $\mathrm{B}$ being relatively the greater. We have here what is merely a repetition, by electrical stimulation, of the results which 
were described in Chapter IX., as obtained by thermal and mechanical stimulus. How perfect and how consistent, due precautions being taken, these results may be rendered, will be seen from the numerous records in this and the following chapters.

I have postponed till now the consideration of the mode of application of these alternating shocks. The usual alternating current from a Ruhmkorff's coil would be entirely unsuitable for delicate and crucial experiments ; first, because the excitatory values of the slow make- and quick break-shocks are unequal ; and, secondly, because such currents leave their residual polarisation effects.

These defects I have been able, as stated in the last chapter to avoid, by quick reversals of the primary current which actuates an induction-coil. When the primary current is reversed from the plus to the minus direction, we obtain an induction-current due to magnetic variations of lines of force, from, say, plus $n$ to minus $n$. When the primary current is re-reversed, from minus to plus, we obtain an opposite induction-current, due to magnetic variation, from minus $n$ to plus $n$. It will be seen that if these reversals of the primary current are made with equal rapidity, the alternating induced currents will be equal and opposite. The reversals are accomplished by means of a Pohl's commutator, worked up and down by a crank, in connection with an electric revolving motor (fig. I70). The intensity of the induction-shock may be varied by sliding the secondary nearer to, or further away from, the primary. Having now described the general means of producing equialternating electric shocks, it still remains to explain two distinct methods of applying them, for the determination of the differential excitability of the tissue. These may be distinguished as : ( I ) the Method of the AFTER-EFFECT ; and (2) the Method of DiRECT-EFFECT. According to the first of these - the method of the after-effect-the tissue is excited for a definite length of time, and the excitatory effect observed, by connecting it immediately afterwards with the 
galvanometer-circuit. The manner in which this is done will be understood from fig. I 70 . We have a highly insulating electrical key, $\mathrm{K}$, of ebonite. $\mathrm{P}$ and $\mathrm{Q}$ are connected with two points $\mathrm{A}$ and $\mathrm{B}$ of the tissue, whose relative excitabilities are to be determined. A spiral spring keeps the key down, connecting the two points in the specimen with the galvanometer. Any existing difference of potential, as between the two points

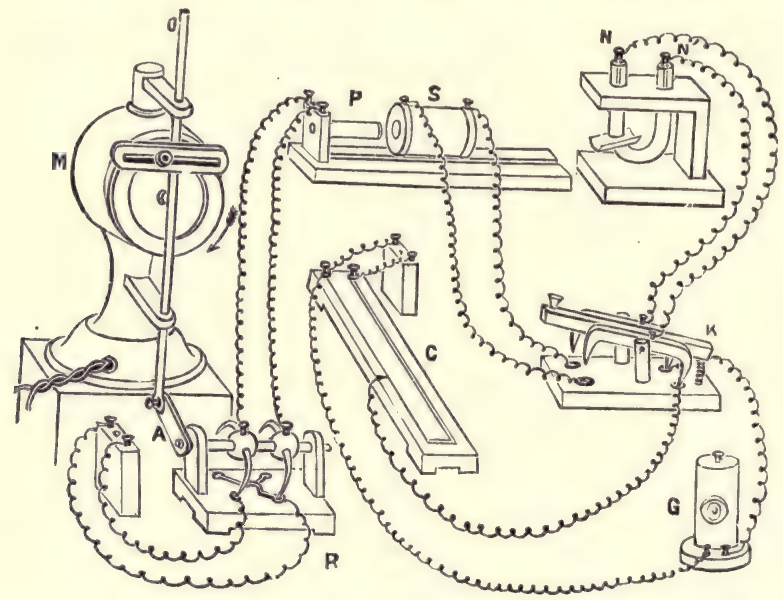

FIG. I70. Experimental Arrangement for Determination of Excitatory After-effect of Equi-alternating Electrical Shocks

M, electrical motor working Pohl's commutator for alternate reversal of current in primary, P. Note that the connecting-rod, A, works simply up and down, causing reversals of current; S, secondary coil ; $\mathrm{K}$, ebonite key kept down by elastic spring, the two surfaces of the specimen being thus in circuit with galvanometer, G. When key is pressed, these are put in circuit with exciting coil, s. When key is released, after-effect of excitation on specimen exhibited by galvanometer deflection. c, the compensator.

is balanced by the compensating potentiometer C. Under these circumstances, the galvanometer spot of light would remain steady, whether the key was up or down. By pressing the key $\mathrm{K}$, the galvanometer-circuit is broken, and the tissue is put in series with the exciting circuit $\mathrm{s}$, which forms the secondary of the induction-coil. When the key is again released, the galvanometer-circuit is rapidly made, at a definite short interval after the cessation of the exciting shock, and the resulting deflection of the galvanometer indicates the differ- 
ential excitation as between $\mathrm{A}$ and $\mathrm{B}$. In this way records may be taken of a series of the after-effects of brief excitations, at intervals of, say, a minute. The direction of this responsive current, which in the tissue is from the more to the less excitable, enables us to determine the relative excitabilities of the two points, $\mathrm{A}$ and $\mathrm{B}$.

Much more delicate is the second method, that, namely, which depends on the record of the Direct-Effect of equialternating shocks; but for its perfect working, certain difficulties have to be overcome. One of the first conditions to be fulfilled lies in the perfect equality of the alternating shocks. The importance of this will be understood on observing the effect of the alternating shocks given by a Ruhmkorff's coil, when actuated by a vibrating hammer. Here, the make- and break-shocks are of unequal intensity and duration, and the following sources of disturbance come into play: (I) a galvanometric drift in one direction or the other; (2) a resultant inequality of polarisation-effects; and (3) the inequality of the excitatory values of the two shocks.

The galvanometer-drift, owing to the inequality of the induction shocks, becomes very troublesome, when we have to employ, as is necessary, an instrument of high sensibility. If the differential excitability of the specimen be very great, this drift may be masked by the predominant excitatory effect. In other cases, however, the excitatory effect itself may be overpowered by the drift. The difference of intensity as between the make- and break-shocks in the Ruhmkorff's coil described, thus becomes a strongly disturbing element. The necessity to make the two shocks absolutely equal will be understood when we find that alternating telephonic currents, which are generally regarded as equal and opposite, induce a drift of the galvanometer in one direction or the other, on account of a slight difference of intensity between the two alternating currents.

The difficulty arising from inequality of polarisationeffects is too obvious to require further elucidation. Still 
more important, moreover, is the disturbing factor last mentioned, that of the inequality of excitatory values as between the make- and break-shocks themselves. This element of uncertainty is very clearly seen in the experiments of Von Fleischl on the response of nerve. The resulting deflection he found to be in the direction of the break-shock. The explanation of this phenomenon has hitherto been regarded as a matter of great difficulty, authorities being much divided on the subject. In these experiments, alternating currents from a Ruhmkorff's coil are sent through the galvanometer and the nerve, in series. The two points on the nerve, $\mathrm{A}$ and $\mathrm{B}$, are presumably of equal excitability. A make-shock of relatively lower E.M.F. passes, say, from A to B, followed by a break-shock of higher E.M.F. in the opposite direction, from $\mathrm{B}$ to $\mathrm{A}$. Confining our attention to the excitatory effects of these shocks, we have at A, during make and break, the following four effects : (a) feeble anode-make; (b) feeble anode-break; (c) strong kathode-make; and (d) strong kathode-break. At $\mathrm{B}$, on the other hand, at the same time, we shall have: $\left(a^{\prime}\right)$ feeble kathode-make ; $\left(b^{\prime}\right)$ feeble kathode-break; $\left(c^{\prime}\right)$ strong anodemake ; and $\left(d^{\prime}\right)$ strong anode-break. Now, since the excitatory value of anode-break is probably the stronger and more persistent, and since the intensity of this effect will depend, within certain limits, on the intensity of the anodic shock, it follows that the $\left(d^{\prime}\right)$ or strong anode-break effect at B will, as a general rule, be the most conspicuous of these. That is to say, as a result of all these excitatory effects in combination, greater galvanometric negativity will be induced at $B$ than at $\mathrm{A}$, the responsive current being thus from $\mathrm{B} \rightarrow \mathrm{A}$, in the same direction as the break shock, which was the actual result. In any case, whatever may have been the cause of this, it is clear that the employment of such unequally exciting shocks of make and break would be fatal to any attempt to determine accurately the natural difference of excitability as between the two points. I may state here, that when I have employed absolutely equal alternating shocks on a specimen 
of nerve, I have obtained no resultant deflection whatever, showing that such shocks induce exactly equal excitations in an isotropic tissue. But if the excitability of one of the two points be first abolished by killing, then a definite resultant responsive current is obtained, from the excitable living to the inexcitable dead. So perfect were in fact the results secured by means of these equi-alternating electric shocks, that I was desirous not only to detect, but also to record photographically, the responses thus obtained. In this a certain difficulty is experienced, inasmuch as the alternating shocks are apt to render the recording spot of light tremulous, and thus to spoil the photographic impression. This may, however, be overcome by making the alternation frequency so high, in reference to the period of the needle or suspended coil of the galvanometer, that the unsteadiness of the deflection ceases.

I shall now describe the practical means employed to obtain equi-alternating shocks of any frequency that may be desired. This I have been able to do in several ways, and, among others, by using a Rotating Reverser. This consists of an ebonite disc, on the periphery of which there are strips of metal of equal breadth, and separated from each other by equal distances. The odd strips ( $1,3,5$, and so on) are connected together and led to a metallic ring on the left of the disc. The same is done with the even strips, which are led to the right. The two electrodes of a battery are led through a key, $\mathrm{K}$, to these two metallic rings and are connected with them by means of brushes. Thus one ring, with all the odd strips, is connected, say, with the positive, and the other, with all the even strips, with the negative pole of the battery. The current is led off by a second pair of brushes, placed diametrically opposite to each other on the disc, in the primary circuit, P, of an induction coil (fig. I7I). Let us suppose the upper brush to be connected with an odd strip, the lower will then be connected with an even. The current in the primary coil now flows in one direction. When the disc is rotated, so as to bring the next pair of strips in 
contact with the brushes, the upper will then be connected with the even strip and the lower with the odd. Thus the direction of the current will be reversed, and rapid rotation of the disc will give rise to equi-alternating currents in the primary of the induction coil. This will in turn induce equi-alternating induction currents in the secondary, the intensity of which can, as already said, be varied within wide limits by appropriate changes of distance between the primary and the secondary. The number of strips in the apparatus used is fifty, and when the disc is rotated, by

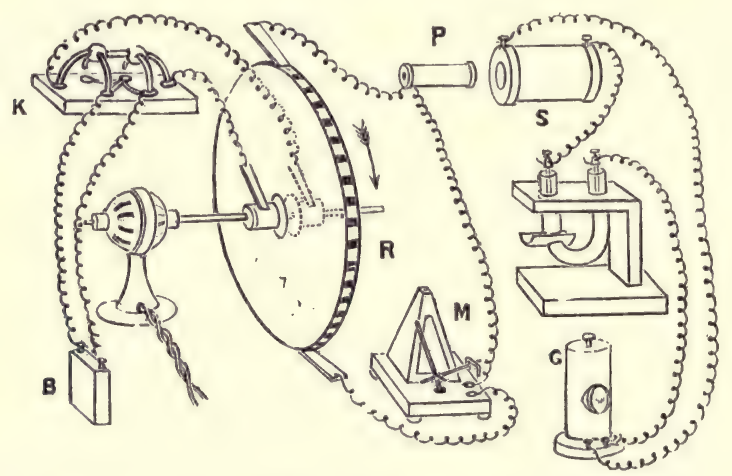

FIG. I7I. Method of Direct Effect of Excitation by Equialternating Shocks

$\mathbf{R}$, rotating reverser, in circuit with primary coil, $\mathrm{P}$. Duration of stimulation determined hy metronome, $\mathrm{M}$. $\mathrm{s}$, secondary coil in series with specimen and galvanometer.

means of an electrical motor, at a rate of one revolution per second, there will be fifty alternations of current in a second. The duration of the application of the stimulating shock to the tissue is regulated by a metronome, which completes the primary circuit for a definite short length of time. When the metronome, $\mathrm{M}$, is so adjusted as to complete the circuit for 5 second, then a stimulus of that duration will be imparted at each stroke. A second interrupting key, not shown in the figure, is included in the circuit. When this key is closed, a single beat of the metronome gives a stimulating shock of ' 5 second's duration. The key is now opened 
for one minute for recovery. In this way, records of response and recovery are obtained, at intervals, say, of one minute.

Another very effective means of producing equi-alternating shocks is by the employment of an alternatingcurrent dynamo, driven by an electrical motor, M (fig. I72). The alternating current is led to the primary of a Ruhmkorff $\mathrm{s}$ coil in the usual manner. The motor is driven by an electrical supply from the street mains, its speed being adjusted by a regulation of the current, which is effected by

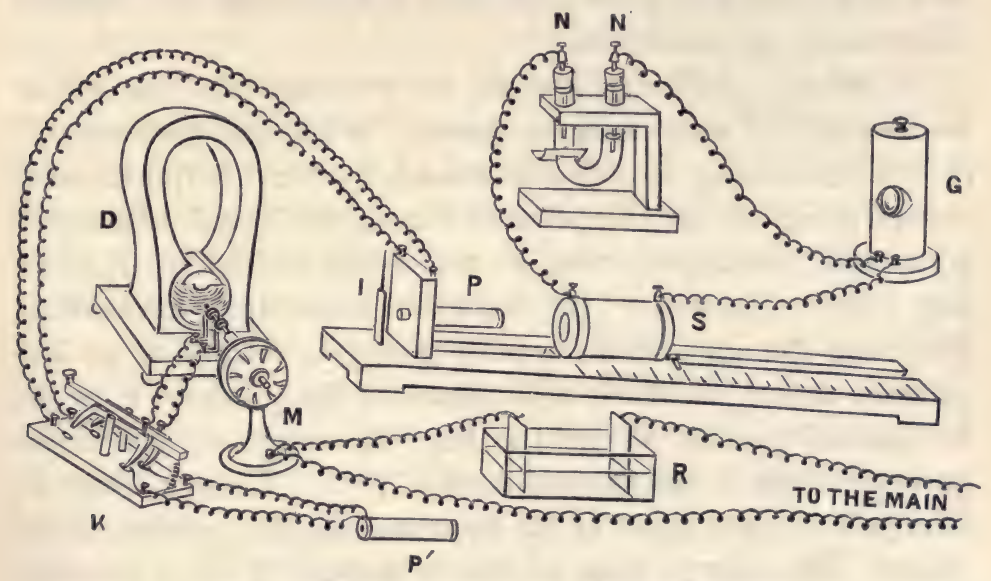

FIG. 172. Excitation by Equi-alternating Shocks

M, motor, rotating armature of alternating-current dynamo, D; R, liquid rheostat, in circuit with street-mains, for regulating speed of rotation of motor ; $\mathrm{P}^{\prime}$, idle coil ; $\mathrm{P}$, primary coil ; $\mathrm{r}$, resonating index ; $\mathrm{S}$, secondary coil, in series with specimen and galvanometer. Duration or excitation determined by pressure of key, $\mathrm{k}$.

an electrolytic rheostat, R. As the dynamo is provided with a permanent horse-shoe magnet, the intensity of the alternating current is determined by the speed of rotation of its armature. If the speed be kept always constant, the number of alternations will also be constant, and the exciting value of the electric shocks will depend simply upon the distance of the primary from the secondary. It is thus possible day after day to use the same intensity of stimulation, and thus to compare the relative excitabilities of 
different tissues. The constancy of the speed of rotation of the alternating-current dynamo is secured by means of the resonating index, I. This consists of a short steel spring with a long index. When the frequency of alternation is the same as the natural period of vibration of the spring, the resonator is thrown into strong sympathetic vibration. At first the rheostatic resistance, which determines the speed of the motor, is made slightly too large. The movable plate is now gradually brought nearer, till the proper speed has been arrived at, and this point is at once indicated by the induced vibration of the resonator.

A further difficulty has to be overcome in the maintenance of the uniformity of speed. When the open circuit of the alternating dynamo is closed, by the interposition of the primary of the Ruhmkorff's coil, the speed undergoes a sudden diminution, owing to the work which the dynamo has now to perform. In order to avoid this fluctuation, then, the dynamo circuit is kept closed by means of an idle primary coil, $\mathrm{P}^{\prime}$, which is a duplicate of the primary, $\mathrm{P}$, of the Ruhmkorff's coil. When the key, $\mathrm{k}$, is pressed, the alternating current is transferred from $\mathrm{P}^{\prime}$ to $\mathrm{P}$. There is thus no fluctuation in the speed of the dynamo, and the duration of the closure determines that of the stimulus. I may mention here, that instead of employing a separate motor to drive the alternating-current dynamo, I have sometimes used, with equal success, a motor transformer, giving rise to alternating currents. It is easy to construct a very compact and portable form of this latter apparatus.

In this manner we may apply uniform stimuli of equialternating shocks at regular intervals of time, say of one minute. The usual preliminary test of the successful elimination of all sources of disturbance may here be made in the following way. The kaolin ends of the non-polarisable electrodes are connected with each other, without the interposition of a specimen, and alternating shocks from the secondary are passed through the circuit. These should give rise to no deflection in the galvanometer. It may be 
said here that I use a D'Arsonval type of galvanometer, in which, instead of a suspended needle, we have a suspended coil. There is thus here not even the remote contingency of disturbance which might arise from the demagnetisation of the magnetic needle. Having thus tested, by null action, the symmetry of the electrodes and the galvanometer, the differentially excitable tissue, say the sheathing petiole of $M u s a$, is interposed, with its concave and more excitable surface upwards. On now applying excitation by equialternating shocks, the responsive current will be found to flow downwards, from concave to convex, giving a deflection of the galvanometer, say to the right. And this deflection will continue to be to the right, even if the battery current (fig. I 7 I) be reversed by means of key $\mathrm{K}$. The direction of the excitatory current, moreover, depending solely, as it does, on the relative excitabilities of the two surfaces of the specimen, will remain constant, even if the connections with the secondary coil, $\mathrm{S}$, be reversed. The zinc rod, $\mathrm{N}$, of the non-polarisable electrode in connection with the concave surface (fig. I72) has thus, up to the present, shown induced galvanometric negativity, the galvanometric deflection being to the right. But if we exchange the zinc rods of the nonpolarisable electrodes, it will then be $\mathrm{N}^{\prime}$ which will be connected with the more excitable concave surface, and it will now be this electrode $\mathrm{N}^{\prime}$ which will show galvanometric negativity. This reversal of the galvanometer deflection with the reversal of the electrodes affords additional confirmation of the greater excitability of the concave surface of the specimen of Musa.

In these experiments the existing current of rest may be balanced previously by a potentiometer. But this is not absolutely necessary. I give below a series of records obtained with a specimen of the sheathing petiole of Musa (fig. I73), in which we know the inner or concave surface to be more excitable than the outer or convex. The responsive current is seen under this form of electrical stimulus, as we found to be the case under mechanical and 
thermal stimulation, to flow from the more excitable concave to the less excitable convex. In order next to demonstrate the physiological character of these responses, I subjected the tissue to the action of chloroform, and the record in the second part of the figure shows the consequent depression of the response.

The great delicacy and pliability of this mode of application of stimulus enable us to attack many difficult problems,

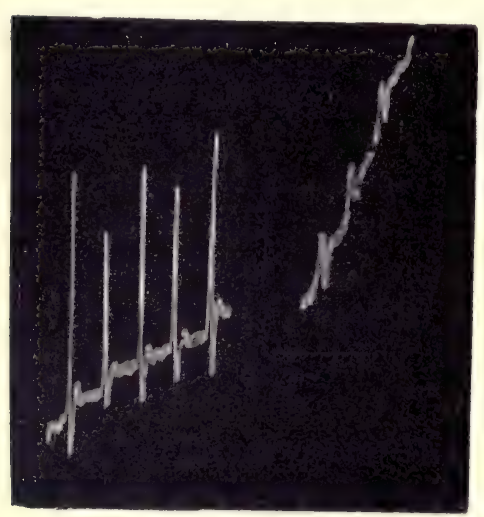

FIG. 173. Photographic Record of Response of Petiole of Musa to Equialternating Electric Shocks, before and after Application of Chloroform. on the difference of excitability between two points in a tissue, with perfect ease. To how many distinct investigations it can be successfully applied will be set forth in detail in succeeding chapters. As there is nothing to prevent the two exploring electrodes from being applied on any two points, however distant, of the same organism, it is seen that we have here a means of determining, not only the differential excitability of any two points of the same organ, but also that of any two organs of the same specimen. For the present I shall, however, content myself with giving a few instances only in illustration of the extreme delicacy of this method in detecting physiological differences as between two points.

We shall first turn our attention to those physiological modifications which are due to the a-symmetrical action of the environment on the organism, and here we shall select the case of the plagiotropic stem of Cucurbita. We have seen that in the recumbent stem of this plant the tissue of the upper side is rendered relatively fatigued by the continuous action of sunlight, and thus becomes permanently less excitable than the lower side. We have also found that 
while the natural resting-current was from the less excitable upper to the more excitable lower side, the responsive current under mechanical stimulation was in the opposite direction-namely, from the lower to the upper (p. I I2). Using now the electrical form of stimulus, we obtain results which are identical. Fig. I74 gives a series of such responses under equi-alternating electrical shocks. Curiously enough, as pointed out in the last chapter, I have detected a similar plagiotropy in the case of the eel. The head of the fish was cut off, and voluntary action thus eliminated; electrical connections were then made, after a period of rest, with the

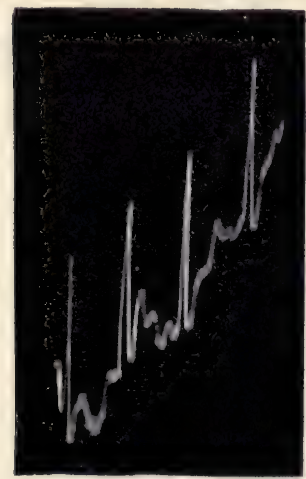

FIG. 174. Photographic Record of Responses of Plagiotropic Stem of Cucurbita to Equialternating Electric Shocks

Direction of responsive current from ventral to dorsal surface. dark dorsal upper surface, and the colourless skin of the ventral or lower. A natural current was now found to flow from the upper

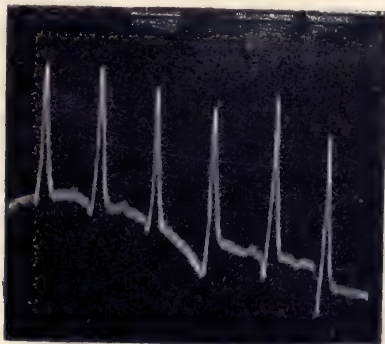

FIG. I75. Electrical Responses of Eel to Equi-alternating Electrical Shocks

Current of response from ventral surface to dorsal.

surface to the lower, as in the case of the plagiotropic stem of Cucurbita. Electrical excitation was now applied, and the result was a responsive current from the more excitable lower to the less excitable upper surface again, as in the case of Cucurbita. In fig. 175 is seen a series of records in illustration of this.

Another investigation which I thought might be interesting had reference to the variegated colouring of certain foliage leaves, A striking example of this is found in the 
tropical elephant creeper (Pothos), the rich green of which is barred by longitudinal streaks of milk-white. This distribution of colour is found even in the youngest and most vigorous leaves. The question whether such colouring was accidental or associated with physiological differences could, I thought, be determined by the delicate mode of investigation which was now at my disposal. On making electrical connections, then, with the green and white portions of a leaf, I found that the natural current of rest was from white to green through the tissue, and, on further testing the differential excitability in the usual manner, the responsive current was observed to flow from the green to the white. This showed that pallidity was here associated with a depressed physiological condition. 


\title{
CHAPTER XXII
}

\author{
RESPONSE OF ANIMAL AND VEGETAL SKINS
}

Currents of rest and action-Currents in animal skin-Theories regarding these - Response of vegetal skin-Stimulation by Rotary Mechanical Stimulator-Response of intact human skin-Isolated responses of upper and lower surfaces of specimens-Resultant response brought about by differential excitability of the two surfaces-Differences of excitability between two surfaces accounted for-Response of animal and vegetal skins not essentially different-General formula for all types of response of skinResponse of skin to different forms of stimulation gives similar resultsResponse to equi-alternating electric shocks : (I) Method of the After Effect ; (2) Method of Direct Effect-Response of grape skin-Similar response of frog's skin-Phasic variation of current of rest induced as result of successive stimulation in $(a)$ grape skin; $(b)$ frog's skin; $(c)$ pulvinus of Mimosa-Phasic variation in autonomous mechanical response of Des. modium gyrans-Autonomous variation of current of rest-True current of rest in skin from outer to inner-This may be reversed as an excitatory after effect of preparation-Electrical response of skin of neck of tortoiseElectrical response of skin of tomato-Normal response and positive aftereffect - Response of skin of gecko-Explanation of abnormal response.

IN this and the next few chapters it is my intention to make an inquiry into the responsive peculiarities of the skin, epithelium, and glandular tissues, alike in plant and animal. By the study of such simple cases as are found in plants, it should be possible to obtain' a clear insight into the various factors which go to make the corresponding phenomena in animal tissues so complicated and obscure as to be difficult of reconciliation with each other.

It is not possible in a short space to give any but the briefest summary of the work hitherto done on this extended subject in animal physiology. All that can be attempted is to indicate some of the leading theories and results, at the same time drawing attention to those outstanding questions which still remain open. Some of the methods which I have 
employed in the investigation of plant phenomena, moreover, have proved so highly satisfactory that records will be given of the results obtained by their means in the case of animal tissues also. And this will, I hope, show the great reliability and simplicity which it is thus possible to introduce into the investigation as a whole.

With regard to the electrical effects in animal skin, epithelium, and glands, the inquiry resolves itself into the determination of, (I) the direction of the current of rest; (2) that of the excitatory current ; and, lastly, (3) a consideration of theories regarding these. The first of these, the current of rest, was found by Du Bois-Reymond and Engelmann in the skin of frog to be 'ingoing'-that is to say, passing from the outer surface to the inner. Hermann also found a similar current in the skin of eel. He regarded the source of electro-motive action as lying in the partial mucin-metamorphosis of single cells. From the fact that in the toad, where the ingoing current is specially strong, the skin glands are vigorously developed, and from the discovery by Rosenthal that in the mucous glands of the stomach the current is also ingoing, it was assumed that the observed electro-motive forces were due to the glandular nature of the tissues. The skin current of the frog and of the fish, and the glandular current of the stomach, are thus usually regarded as due to the same cause.

There is, however, a serious discrepancy in this view, inasmuch as, while local stimulation of the upper surface of the frog's skin induces a positive change, a similar stimulation of an unmistakably glandular surface is found to bring about a negative. If then the electrical effect on the skin of frog be the same as on a glandular surface, the discrepancy of their responsive reactions becomes inexplicable.

As regards the excitatory change, very diverse results have been recorded when stimulus has been applied indirectly - that is to say, through the nerve. This fact is not to be wondered at, since the responsive effects are subject, as will be shown, to numerous modifying influences. It is generally 
supposed, in the preparations made for these experiments, that it is one surface only which is electro-motive. I shall show, however, that the responsive effects are brought about by the differential excitability of the two. This response, again, is modified by relative changes induced in the two surfaces. And, in addition to these, still further complications are introduced when the stimulus is indirect-that is to say, applied through the nerve. In this case, the relative excitations of the two surfaces will be determined by the particular distribution of the nerve-endings. Again, we shall see that, in an isolated preparation, the nerve itself is liable to undergo certain changes by which its transmitted effect may be modified even to reversal (p. 530). Thus, so long as it remains highly excitable, the transmitted effect is one of true excitatory galvanometric negativity. But with physiological depression, the conductivity of the nerve is very much lowered, and the effect transmitted becomes reversed to positivity.

For all these reasons, if we wish to study the specific reactions of skin, epithelium, and gland preparations, it is better to do so by observing them under direct stimulation.

Engelmann, in studying the responsive reactions to direct stimulation of frog's skin, found a negative variation of the current of rest. Since the latter was naturally 'ingoing,' as regards the upper or' epidermal surface, this meant that the responsive current was 'outgoing.' Reid, again, working on the skin of the eel, obtained ingoing response, or positive variations of the resting-current, by single induction shocks in either direction. Biedermann, in the mucous membranes of the tongue and stomach, obtained both positive and negative variations of the current of rest. Waller, using single induction shocks in either direction, found in the digestive mucosa both ingoing and outgoing responses, the former being much predominant.

Even under the simple conditions imposed by direct stimulation, then, the results obtained are seen to be inconsistent. They would appear to show both that the 
responsive effects in the different preparations are different and that, even in the same preparation, they may be reversed under unknown changes of circumstances.

It appeared to me, as already said, that much light might be thrown on the questions thus raised by means of an investigation carried out on plants. The most perfect method of experiment here would consist in observing the separate responsive effects on upper and lower surfaces of the preparation. Waller employed single induction shocks for this purpose, observing the after-effect. But in this case, the action of polarisation was not excluded. It would thus be more satisfactory, in order to eliminate this unknown element, to employ either a non-electrical mode of stimulus or an electrical form which would leave no resultant polarisation effect. The latter condition, as we have seen, was fulfilled by the employment of rapidly alternating currents, whose alternating components were absolutely equal.

As regards the application of a non-electrical form of stimulus, both thermal and mechanical forms may theoretically be employed. Engelmann and others used heated metals in the proximity of one of the electrodes, for the production of thermal stimulus. This, however, has the disadvantage of thermo-electrical variation, due to unequal heating of the two contacts. Besides this, there is also the effect of a rising temperature, which, as we have seen, is opposite to that of sudden variation, the latter alone constituting the excitatory effect. I have already explained how these difficulties may be overcome by using thermal shocks in which a sudden thermal variation is made to act on both contacts at once. The resultant response thus obtained was shown to be determined by the differential excitability of the two contacts under examination. As regards the mechanical mode of stimulation, previous observers have employed pressure or friction. Such stimulus, however, is at best merely qualitative. If it be applied at the contact itself, objections may be taken to the effect, as 
due to, or modified by, the variation of contact resistance. And if to avoid this the mechanical stimulus be applied, not at the electrode, but at a neighbouring point, the results will be quite different, according as the conductivity of the intervening tissue is great or slight. In the former case they will consist of the transmitted effect of true excitation ; in the latter of the indirect effect, whose electrical sign is the exact opposite.
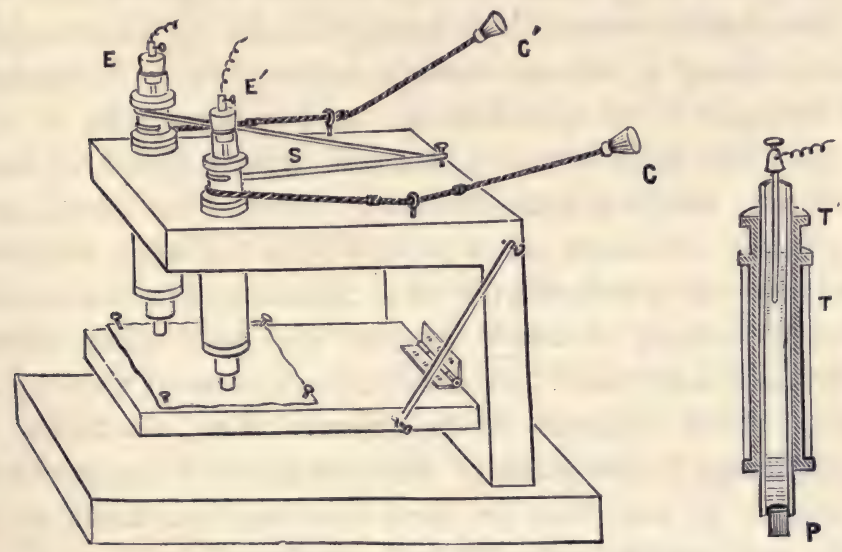

FIG. 176. Rotary Mechanical Stimulator

Specimen of skin pinned on hinged platform which is pressed against electrodes by elastic india-rubber. Electrodes rotated by cord, c c'. S, antagonistic spring, made of elastic. Enlarged view of electrode seen to the right. $\mathrm{T}$, outer fixed brass tube ; $\mathrm{T}^{1}$, inner rotating tube, holding non-polarisable electrode. P, pumice-stone.

A perfect method of direct mechanical stimulation has been described in Chapter III., the stimulus being vibrational. But for investigations on limp structures, such as skin, this method is not practicable, and the modification which I am now about to describe is necessary, in order to meet the difficulties of the case. The apparatus consists of a hinged platform, P (fig. I76), on which the specimen is securely pinned. The two electrodes, $\mathrm{E}$ and $\mathrm{E}^{\prime}$, rest with a definite pressure on the two points $\mathrm{A}$ and $\mathrm{B}$, whose excitatory reactions are to be studied. These electrodes have at their 
lower ends projecting pumice-stone cylinders of equal sections, soaked in normal saline. When the electrode $\mathrm{E}$ is rotated, the mechanical friction induces local excitation of the point A. B may also be subjected to similar isolated excitation in the same way. In order that such successive excitations may be quantitative and uniform, it is necessary first that a definite area at $\mathrm{A}$ or $\mathrm{B}$ should be stimulated. In other words, there must be no lateral slip. For this reason the electrodes are passed through tubular holders which, from the description presently to be given, will be seen to allow rotation about a definite vertical axis. The extreme bases of the pumice-stone cylinders are, as has been said, of equal section. The glass electrode tube is tightly fixed, by means of a cork, inside a brass rotating tube. The latter, again, plays inside an outer brass tube, which is fixed. The inner brass tube is provided with two collars, one below and one above, by means of which rotation can take place without up or down movement. A string is also wound round it, by pulling which rotation is produced. The electrodes are perpendicular to the plane of the platform which carries the specimen. It will thus be seen that any variation of the surface subjected to stimulation is prevented.

The next difficulty to be overcome is that of liability to variation in the pressure of the contact. It will be remembered that the platform is hinged. It is further held up against the electrodes by the tension of an elastic piece of india-rubber or a spiral spring of steel. This pressure can be regulated to a suitable value, and kept constant.

The final difficulty is to apply successive stimuli of equal value, and to render them capable also of graduation from low to high values. This could be secured by rendering the successive rotations of the exciting electrodes equal in number and in time of execution. The intensity of stimulus might then be increased by increasing the number of rotations or the pressure of the electrodes on the specimen.

In order to apply successive rotations of definite number, one end of the string wound round the inner brass tube is 
attached to a piece of stretched india-rubber, which is fixed by its other end to the apparatus. The second end of the string is tasselled, after being passed through a fixed ring. This position of the string is adjusted by means of a knot, so that the india-rubber at its other end is already in a state of tension. When the tassel is now suddenly pulled and let go, it gives rise to a number of rotations in the positive, followed by an equal number of rotations in the opposite direction, the latter work being performed by the stretched antagonistic spring. It should be remembered that a mechanical rotation, whether plus or minus, gives rise to the same excitatory reaction. Next, to make the number of rotations definite, let us suppose the inner brass tube to have a circumference say of I $\mathrm{cm}$. If the string be now pulled through a distance of $5 \mathrm{~cm}$., and let go, there will be five rotations in the positive, followed by five rotations in the negative direction. A second knot in the string, at the distance of $5 \mathrm{~cm}$. from the first, exactly limits the length of the pull; and increase or decrease in the intensity of the stimulus can be brought about by a change in the distance of the regulating knot.

The distance between the two electrodes being always the same, the resistance of the interposed tissue remains approximately constant. To nullify any accidental variation, a high and constant external resistance is interposed in the galvanometer circuit. When the excitatory electro-motive variation of the specimen is very great, it is possible to use an external resistance as high as one million ohms. It should, however, be remembered that even if there be any unavoidable variation of resistance, it will not in any way affect the discrimination of sign of the characteristic electro-motive response. For the excitatory effect at either electrode may be tested by repeating the experiment with the other. The experiments which will be described afforded definite and characteristic records, which were found capable of repetition. The physiological character of such responses was further demonstrated by repeating the experiment, after killing the 
tissue with boiling water, when these electro-motive variations were found to disappear. How very reliable these responses can be rendered is shown by the photographic record in fig. I 80, which is of very special interest, giving as it does the record of responses afforded by the intact human skin.

Turning now to the nature of the response of the skin, it has been found by Engelmann and others, as already said, that in the frog, while the natural resting current is from the outer surface to the inner, the responsive current is from inner to outer. Dr. Waller, again, undertook to analyse the constituent elements of this response, by passing induction shocks along each of the surfaces, first upper and then lower, and in both directions. He then observed the after-effect at one of the excited points, in relation to an indifferent point. In this way he found that an excited point on the upper surface becomes galvanometrically positive, the current being thus outgoing. The inner surface, however, he found to be ineffective. When an induction shock is passed across the tissue, the resultant response from lower to upper is thus, according to Dr. Waller, due to induced positivity of the upper surface.

With vegetable specimens, however, such as the outer skin of apple, the results obtained by him were opposite to those of the frog's skin. The responsive currents were here found to be ingoing, the excited point being galvanometrically negative. The explanation offered, in regard to these results, is that living tissues have the peculiarity of responding by 'blaze currents' to electrical shocks. The use of this phrase, however, as already said, offers no real explanation; but even apart from this point, the question remains, Why should the blaze currents, so called, be directly opposed in the cases of frog's skin and of the particular vegetable skins which are mentioned, respectively? In answer to this, the hypothesis put forward by Dr. Waller is, that the difference arises from the different natures of animal and vegetable protoplasms. ${ }^{1}$

1 -Vegetable protoplasm is in major degree an instrument of synthesis and accumulation, in minor degree the seat of analysis and emission. Animal 
I shall, however, be able to adduce facts and considerations from which it will be possible to arrive at a simpler and more conclusive explanation of these phenomena, on the basis of the differential physiological excitability of the two surfaces. I shall show, moreover, that the difference between animal and vegetable protoplasm, thus assumed to exist, has nothing to do with the question.

We have seen that when the physiological activity of a tissue is in any way impaired, its normal excitatory re-action of galvanometric negativity is depressed. This may even go so far as to cause an actual reversal of the response, to galvanometric positivity, as we found in the case of depressed tissues (p. 84). Taking a vegetable specimen, then, say a hollow petiole or peduncle, we find that the outer surface, which is habitually exposed to the manifold influences of the environment, becomes histologically modified, being much more cuticularised than the inner. Thus these outer and exposed cells generally become reduced in size, and thickwalled, with little protoplasmic contents. Hence, as regards functional activity, these epidermal cells are in a physiological sense very much degraded. We should then expect their excitability to be proportionately lowered in comparison with, say, the inner surface of the same tube, protected as that has been from outside influences. And the variation of physiological excitability thus induced may involve not only the surface, but also the subjacent layers to a certain extent.

Theoretically, then, the induced galvanometric negativity of the outer would be less than that of the inner surface, ${ }^{1}$ and simultaneous excitation of both, by whatever means produced, should give rise to a resultant responsive current

protoplasm is in major degree an instrument of analysis and emission, in minor degree the seat of synthesis and accumulation. The vegetable, in most immediate contact with inert things, combines, organises, and accumulates. The animal, in less immediate contact with inert matter, disrupts, utilises, and dissipates in their fragments organic compounds that it has received ready made from other animals and from plants.'-Waller, Signs of Life, p. 85.

1 This refers to normal skin, and not to that in which the surface is typically glandular. 
from inner to outer. The degree of this diminution of excitatory negativity in the outer surface, moreover, culminating as this may in actual positivity, will depend upon the extent of its transformation. In connection with this it should be remembered that, in order to bring out the differential excitabilities of the two surfaces, it is necessary to apply localised stimuli of an intensity not too excessive. For, if the stimulus be very strong, there is always a possibility of its affecting deeper layers of the tissue and thus causing complications in the resultant excitatory changes. The intensity of stimulus which may be safely

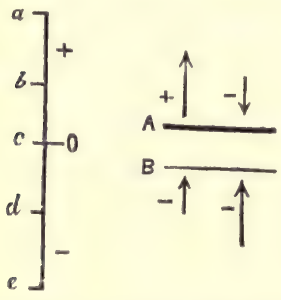

FIG. 177. Diagram Representing Different Levels of Excitability, Plus, Zero, and Minus

Diagram to right of figure shows how resultant up response (inner to outer) may be obtained when induced change at $\mathbf{A}$. is plus, and at $\mathrm{B}$ minus, or when induced change at $\mathbf{A}$ is less negative than at $\mathrm{B}$. used without bringing about such complications will depend on the conductivity of the tissue. Epidermal cells are, generally speaking, feeble conductors, but in this matter it must be understood that the differences in this respect between different tissues are not absolute, but a question of degree, and may to a certain extent be modified under different circumstances. Thus a feebly conducting tissue, under a favourable condition of temperature and strong intensity of stimulation, will become to a certain extent conducting. Highly conducting tissues like nerve, on the other hand, under unfavourable circumstances may, as I shall show later, be converted into very feeble conductors.

Returning now to the question of the responsive reactions of skin, we see the theoretical possibility of the following typical reactions. Let the scale of excitabilities be represented by diagram to the left of fig. 177. Now, if the transformation of the outer epidermal surface, $A$, be maximum, the sign of its reaction will exhibit the greatest extent of deviation 
from the normal negativity. That is to say, its response will become absolutely positive, as represented by $a$ above the zero line. The response of the inner surface, B, may be normal and strongly negative, as represented by $e$ below the zero line. When both these surfaces, then, are simultaneously excited, the excitatory positive variation, or 'outgoing' current at A, will conspire with the 'ingoing' current at $B$ and the resultant electro-motive difference will be $\mathrm{B}_{e}+\mathrm{A}_{a}$, the direction of the responsive current being thus from inner to outer. But the same resultant up-response will also be induced, even if the reaction of both surfaces be negative, provided only that that of the outer, $A_{a}$, be less negative than that of the inner, $B_{e}$, as explained by the diagram to the right of fig. 177 . The responsive current will then be represented as $\mathrm{B}_{e}-\mathrm{A}_{a}$, that is to say, as proceeding from the more negative $B$ to the less negative A. We have thus examined the two extreme cases possible under the following formula, in which the arrows show the direction of the responsive current, from the more to the less induced negative :

$$
e \rightarrow d \rightarrow c \rightarrow b \rightarrow a .
$$

I shall next proceed to demonstrate the existence of these two extreme types, taking vegetable skins as the experimental specimen. It was supposed by Dr. Waller, as will be recalled here, that owing to characteristic differences between animal and vegetable protoplasm the response of vegetable skin was opposite to that of animal skin : that is to say, the former was 'ingoing ' and the latter 'outgoing.' That this generalisation is not, however, justified, will be seen from the experiments which I am about to describe, carried out on the skin of grape.

These results, it should here be pointed out, are not dependent upon any one method of inquiry, for each problem was subjected to attack and analysis by four different modes of experiment. The first of these (I) was by the Rotary Method of Mechanical Stimulation. This method has the great advantage that by it the absolute 
response of each surface is displayed separately, without either being affected by the other. There is here, moreover, no complication due to the polarisation factor, inevitable when uni-directioned induction shocks are employed for excitation. Thus, after the individual responses of each surface have been analysed, we are able to arrive at a definite conclusion as to what would be the character of the resultant response if both the surfaces were simultaneously excited. This conclusion is then submitted to three other tests. Thus, (2) the two surfaces of the specimen are subjected simultaneously to the same thermal shocks, according to the method already described. Again, (3) the Method of the After Effect under equi-alternating shocks is employed. And, finally, (4) the Direct Effect of these equi-alternating shocks of moderate intensity is recorded. The results obtained by all these diverse methods are in complete concordance with each other, and fully support the theoretical inferences which have already been made.

I took the skin of a ripe muscatel grape, such as are available in Calcutta. On making the galvanometric connections with the outer and inner surfaces, a resting current, $C$, was found to flow in the skin from the outer to the inner, just as in the skin of the frog (right-hand diagram, fig. I78). The grape skin was now mounted in the rotary stimulating apparatus, first, for the stimulation of the outer or epidermal surface, with the outer layer placed upwards. The distance between the two electrodes was always the same-namely, $2 \mathrm{~cm}$. On now stimulating one of the two contacts, response took place by the induced galvanometric positivity of that point. That is to say, the current was 'outgoing,' into the galvanometer circuit, from the surface of the skin. When the second point was now stimulated the deflection previously obtained was reversed, the second contact thus also exhibiting galvanometric positivity on excitation.

The position of the skin in the apparatus was now changed, the inner surface being placed upwards. In this way points diametrically opposite to those in the last case 
were subjected to excitation. It was now found that the responses of the inner surface were normal - that is to say, of galvanometric negativity. I give here (fig. I 78, $a$ ) records of two successive sets of responses obtained from the external and internal points $\mathrm{A}$ and $\mathrm{B}$. These records clearly demonstrate that the resultant up-response, on simultaneous excitation of the outer and inner surfaces, is brought about by the induced galvanometric positivity of the outer added to the induced negativity of the inner surface. As the resist. ance of the circuit in the two successive experiments was maintained approximately the same, the amplitude of these responses gives a fairly accurate idea of the relative electrical effects induced on the two surfaces. The positive or 'outgoing' effect of the outer surface is here slightly greater than the 'ingoing' effect. The diagram in fig. $178, b$,

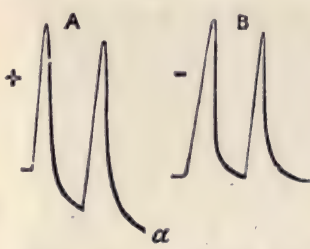

Fig. I78. Electrical Response of Grapeskin to Rotary Mechanical Stimulation

(a) A, positive response of outer surface ; B, negative response of inner surface; (b) c, current of rest, from outer to inner; R, excitatory response from inner to outer, consisting of summated results of positive response of outer with negative response of inner.

shows how the individual effects conspire to give rise to a responsive current from the inner to the outer.

In order to test the reality of the correspondence between this response of the grape skin and that of the frog, I now repeated these experiments, employing the same apparatus for mechanical stimulation, on the skin of the frog. From the records given in fig. 179 it will be seen that the isolated response of the outer surface is positive, or 'outgoing,' that of the inner being negative, or 'ingoing.' The amplitude of the former was, however, much greater than that of the latter. These responses disappeared altogether when the tissue had been killed by immersion in boiling water. From isolated responses obtained by means of induction shocks, Dr. Waller had been led to regard the outer surface of frog's 
skin as alone active, the inner being, to his thinking, ineffective. This particular result may possibly be accounted for by supposing that he used a stimulus intensity which was not sufficiently strong. In my own experiments I obtained clear dernonstration of the effectiveness of both surfaces in opposite ways electrically, though the effect obtained from the outer was undoubtedly the more intense of the two. By comparing these two experiments, then, on the grape skin and skin of frog, it will be seen that the inference that the vegetable protoplasm reacts in any way
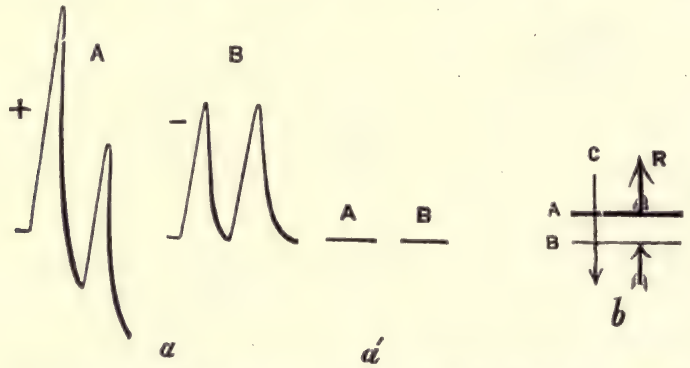

FIC. 179. Electrical Response of Frog's Skin to Rotary Mechanical Stimulation

(a) A, positive response of outer; $\mathbf{B}$, negative response of inner; $\left(a^{\prime}\right) \mathrm{A}$ and $\mathrm{B}$ exhibit abolition of response in skin on boiling; $(b) \mathrm{C}$, current of rest from outer to inner; $\mathrm{R}$, excitatory response from inner to outer, being summated effect of positive response of outer and negative response of inner.

essentially different from that of the animal is quite unjustified. How widely applicable is the method of mechanical excitation by rotary stimulus will be seen in an attempt, successfully carried out, to determine the very difficult question of the characteristic response of the intact human skin. This will be seen in the following record of the results obtained with the skin of a forefinger. The responsive electrical changes represented by the down records, exhibit induced galvanometric positivity of the excited surface (fig. I 80).

I shall next describe the results obtained by simultaneous excitation of the inner and outer surfaces of grape-skin. The responses now given, under stimulation by thermal 
shocks, are seen in fig. $18 \mathrm{I}$, the resultant current being seen to be 'up' - that is to say, from the inner to the outer.

On observing the excitatory after-effect of equi-alternating shocks, the results were found to be the same, the responsive current being now once more from the inner to the outer.

I next took a series of records of the direct effect of equi-alternating shocks, the results of which were precisely the same as before. On applying stimulation, by exactly equal and alternating shocks, we, as already explained,

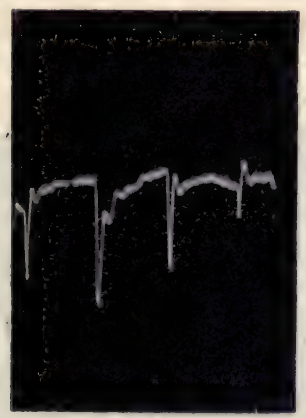

FIG, I8o. Photographic Record of Electrical Responses of Upper Surface of Intact Human Forefinger to Rotary Mechanical Stimulation.

Down responses here indicate induced galvanometric positivity.

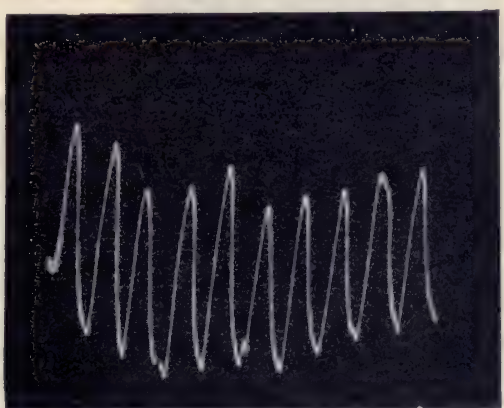

FIG. I8I. Photographic Record of Electrical Responses of Grape-skin to Thermal Shocks at Intervals of a Minute

Responsive current from inner to outer.

obtain a result which is due solely to the differential excitation of the two opposite surfaces. This is not complicated in any way by the factor of polarisation, although the latter could not have failed to be present if the exciting shocks had been one-directioned. Under the conditions of these equi-alternating shocks, then, a certain effect is often seen, in the phasic variation of the base-line, which is extremely characteristic. We have already seen (p. 98) that when a tissue is subjected to repeated or continuous stimulation, its condition undergoes a phasic or periodic variation. Thus from a neutral or positive condition, it may pass into one of maximum contraction or galṿanometrịc negativity, to 
be subsequently reversed to positive once more. Such phasic changes, moreover, may be repeated. They find visible indications in appropriate shiftings of the base line of the record. Similar effects are also shown in the differential response as seen in the records given in fig. 182 ; this feature

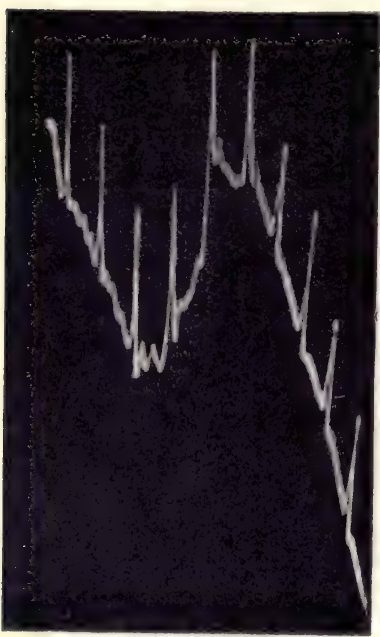

FIG. I 82. Photographic Record of Electrical Responses of Grape-skin to Stimulation by Equi-alternating Electrical Shocks at Intervals of a Minute

Responsive current from inner to outer. Note periodic variation of resting-current, causing shifting of base-line, down and up. is very noticeable. We here obtain the resultant response of the two surfaces of grape-skin, from the inner to the outer. As the result of the series of stimuli applied, the existing current of

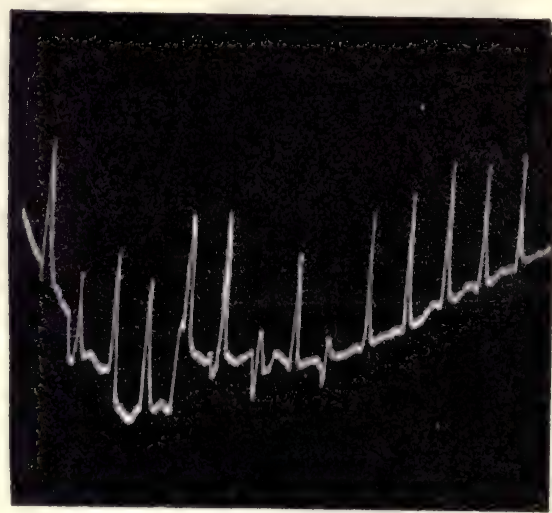

F1G. I83. Photographic Record of Series of Electrical Responses of Frog's Skin to Equi-alternating Electrical Shocks applied at Intervals of One Minute

Direction of responsive current from inner to outer. Note also variation of base-line.

rest undergoes a periodic variation. If this had remained constant, the base-line would have been horizontal. In the present case, the original current of rest was from outside to inside. This, at first, underwent an increase; then a decrease; to be followed, later, by another increase. Thus, in the course of about ten minutes, it exhibited an alternation of almost one whole cycle.

In the next figure (fig. 183) I give a series of results obtained 
with frog's skin in direct response to equi-alternating shocks. Here we find the usual 'up' responses, showing that, as before, the direction of the responsive current is from within to without; and here also we see the existing current of rest undergoing a periodic change.

It has now been fully demonstrated that the response of skin is determined by the differential excitabilities of its two surfaces, upper and lower, that of the lower being the greater. That the resultant responsive current from lower to upper, is in such cases brought about by the greater excitability of the lower, has been fully shown, in a previous chapter, by experiments on the pulvinus of Mimosa. I next made records of a long series of responses given by the last-named specimen, with the object of finding out whether or not these also exhibited a periodic variation of the resting-current similar to those just observed in the anisotropic skins of grape and of frog. Electrical connections were made with diametrically opposite points on the upper and lower surfaces of this

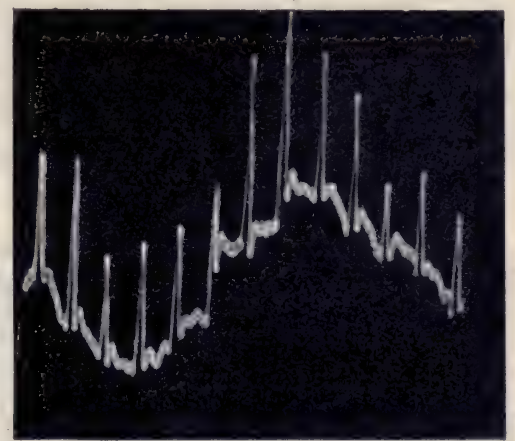

FIG. 184. Photographic Record of Transverse Response of Pulvinus of Mimosa to Equi-alternating Electrical Shocks

The direction of the responsive current is from the more excitable lower to the less excitable upper. Note the cyclic variation of the current of rest. organ, and they were subjected to equi-alternating shocks. Owing to the conducting power of the tissues, it was not now the upper and lower skin surfaces merely, but the upper and lower halves of the organ that became excited. And the responsive current was from the lower to the upper, as already demonstrated. In the particular record seen in fig. I 84 , the general resemblance to the responses of skin is sufficiently obvious. The interesting feature of this record is the periodic changes in the resting- 
current, which exhibit a complete cycle in the course of thirteen minutes. Thus, as a consequence of the after-effect of stimulus, a cyclic variation of relative conditions is induced, as between any two anisotropic surfaces, such as those of skin or pulvinus. This cyclic variation of relative conditions is indicated by the concomitant variations induced in the resting-current, shown in the shiftings of the base line.

I have been able, further, to demonstrate the interesting fact that such phasic variations are capable of exhibition even through mechanical response. I have already explained that autonomous pulsations, such as those of the lateral leaflets of Desmodium gyrans, may be regarded as the after-effeci of stimuli previously absorbed and held latent by the tissue. In taking the record of a series of such pulsations, I have often found phasic variations to occur, similar to those obtained with long-continued response of skin or pulvinus. If, for example, the lower half of the pulvinus of the lateral leaflet of Desmodium undergoes an increase of turgidity above the average, that half will become more convex, and the base-line of the record will be correspondingly tilted. The converse will take place under the opposite change. Thus the phasic variations shown in the record (fig. I85) clearly indicate that the relative turgidities of the two surfaces of an anisotropic organ may undergo a periodic change. The corresponding electrical expression of this we have seen in the variation of the current of rest. This variation may sometimes be so great as actually to reverse the normal current of rest. Thus, while under normal standard conditions the resting-current in the pulvinus of Mimosa is from the upper half to the lower, across the organ, this normal direction may sometimes be found to be reversed.

It may now be asked, What is it, in the case of the skin, which determines the respective directions of the restingcurrent and the current of response? We have seen that the current of rest in the frog's skin, from outer to inner, is generally attributed to the possession of glands by the outer, 
a supposition seemingly supported by Rosenthal's discovery, already referred to, that an apparently similar 'ingoing' current was to be observed in the mucous coat of the frog's stomach. Against this may be urged the conclusion, to which Hermann drew attention, that the skin glands are normally nearly closed to the external surface, and cannot therefore have any external galvanic relation. There are, moreover, other arguments. First, a similar current is observed in the case of grape-skin, where there is no special glandular layer. Second, the specific response of a glandular surface is

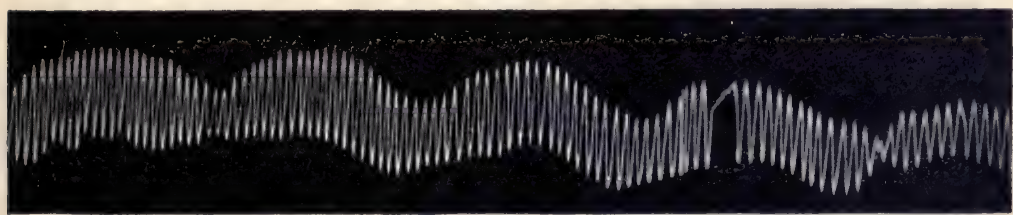

6 P.M.

9 P.M.

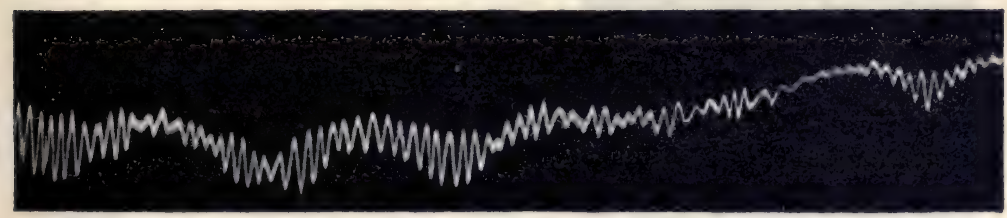

3 A. M.

6 A.M.

FIf. 185. Continuous Photographic Record of Autonomous Pulsation of Desmodium gyrans from 6 P.M. to 6 A.M.

The lower record is in continuation of the upper. Note phasic variation.

definite, and is by galvanometric negativity, whereas the response of the outer surface of frog or grape skin on excitation is by galvanometric positivity. And, thirdly, we shall see that the current observed in the mucous membrane of stomach is most probably not the natural current of rest, but the excitatory after-current.

It will be remembered, however, that we have always found the natural current to flow in the tissue from the less to the more excitable, and the current of response in the opposite direction. In the skin, owing to physiological and 
histological modifications, the outer surface is reduced in excitability. The epidermal layers have little protoplasmic contents, and may be transformed in various ways, becoming corneated or cuticularised. The extent of such transformation may be small or great, but the external layer will as a general rule become less excitable than the inner tissue. Hence, under normal conditions, we have a current of rest from without to within.

If the inner layer be only moderately excitable, or if its power of recovery from excitation be great, then the dis-

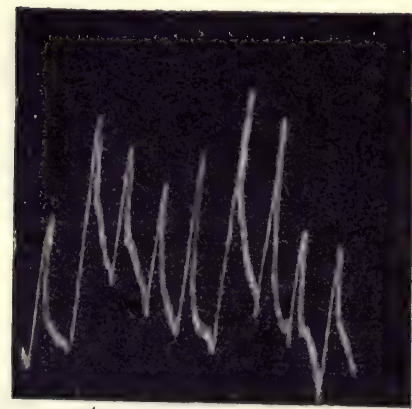

FIG. 186. Photographic Record of Electrical Responses in Skin of Neck of Tortoise to Stimulus of Equi-alternating Electrical Shocks at Intervals of One Minute

The direction of the responsive current was from inner to outer. The so-called current of rest was also in this case, owing to the excitatory aftereffect of preparation, from inner to outer. turbance caused by the preparation of the specimen will be slight, or will pass off quickly. It is to be remembered that as the inner surface is the more excitable, the responsive current due to the mechanical stimulus of preparation will be from inner to outer; and therefore its after-effect, proving in certain cases persistent, may give rise to a current apparently the reverse of the true normal current. Thus, the direction of the current of rest, which we should have inferred theoretically to be from the less to the more excitable, may occasionally be found reversed, owing to the excitatory after-effect of preparation. The current of rest, moreover, is liable to autonomous periodic variation, as we have seen.

The most satisfactory method of determining the relative excitabilities of two surfaces, then, lies in subjecting them to simultaneous excitation, and observing the direction of the responsive current. In the skin, unless the tissue was fatigued, I have always found this to be from the more 
excitable inner to the less excitable outer, even in those cases where the normal direction of the resting-current had been reversed, as an excitatory after-effect of preparation. This fact is well illustrated in the following record, taken with the skin of the neck of tortoise. As an after-effect of preparation, the resting-current so called was here reversed, flowing from inner to outer. But the excitatory responsive current was nevertheless from the more excitable inner to the less excitable outer (fig. I86).

It was stated at the beginning of this chapter that the resultant response from inner to outer merely expresses the general fact that the excitability of the inner is greater than that of the outer. And this will still remain true, even when the transformation of the external layer is not so great as actually to reverse its individual galvanometric response from negativity to positivity. The experimental results obtained with the skin of ripe tomato form a case in point. The natural

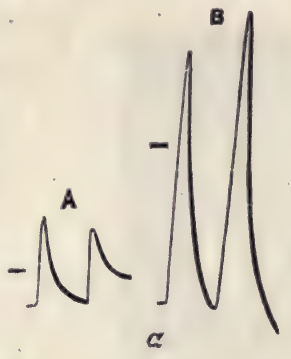

FIG. I87. Isolated Responses of Upper and Lower Surfaces of Skin of Tomato to Rotary Mechanical Stimulus

(a) A, negative response of feeble intensity in outer surface ; B, negative response of much greater intensity in inner surface ; $(b) \mathrm{C}$, current of rest from outer to inner. Resultant excitatory response from inner to outer, due to greater induced galvanometric negativity of inner.

current of rest is here, as usual, from the outer to the inner, and the excitatory responsive current in the opposite direction. But from the analysis of individual responses, on the outer and inner surfaces, obtained by means of the rotary apparatus for mechanical stimulation, it will be seen (fig. 187) that both the surfaces alike give the normal excitatory response of galvanometric negativity. This responsive negativity of the inner, $B$, is, however, very much greater than that of the outer. The resultant response, then, representing as this does the difference in 
degree between two negativities, is still from inner to outer, owing to the greater excitatory reaction of the inner.

That the direction of the resultant response is actually from the inner to the outer is seen in the series of records given in fig. I88. The stimulus consisted of equi-alternating electrical shocks, applied at intervals of one minute. The record shows negative responses, followed apparently by the positive after-effect. In order to observe the peculiarities of this response in greater detail, the record was taken on a faster-moving drum (fig. I89). From this

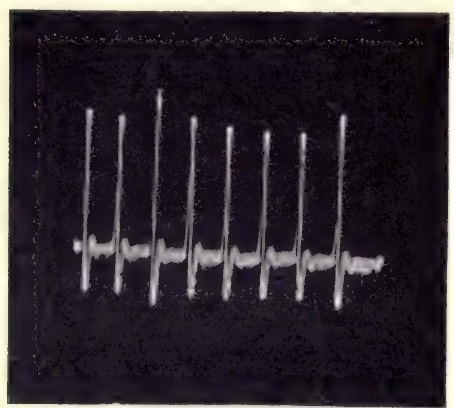

FIG. I 88. Photographic Record of Series of Responses in Skin of Tomato under Equi-alternating Electrical Shocks applied at Intervals of One Minute

Direction of resultant current from inner to outer followed by feeble opposite after-effect.

figure it will be seen that there was a short latent period of no responsive reaction. Response then rose to a maximum, and again subsided. After now reaching the zero position, the record proceeded in the positive direction, and again reverted back to zero. In similar records, the occurrence of this latent period, and positive after-variation, has been adduced by certain physiologists as affording visible demonstration of the existence of opposite processes of assimilation and dissimilation. It has been supposed that the various features of the response were the outcome of a sort of tug-of-war between the two opposed forces, the preliminary pause being the expression of a short-lived balance, while the subsequent negative and positive variations were to be regarded as indicating the predominance, now of the one process, and then of the other. That in the present case such an assumption is unwarranted will be evident when we observe the isolated responses of the upper and lower surfaces separately (fig. 187). In each of 
these we see the normal response of galvanometric negativity, followed by recovery, without evidence of any antagonistic process, such as might give rise to subsequent positivity. The difference between these two responses lies simply in their time-relations. On simultaneous excitation of the two, the predominant negativity of the inner gives the first half of the negative response. The persistence of the excitatory reaction of the outer, on the other hand, after the subsidence of the effect on the inner, gives rise to the apparently

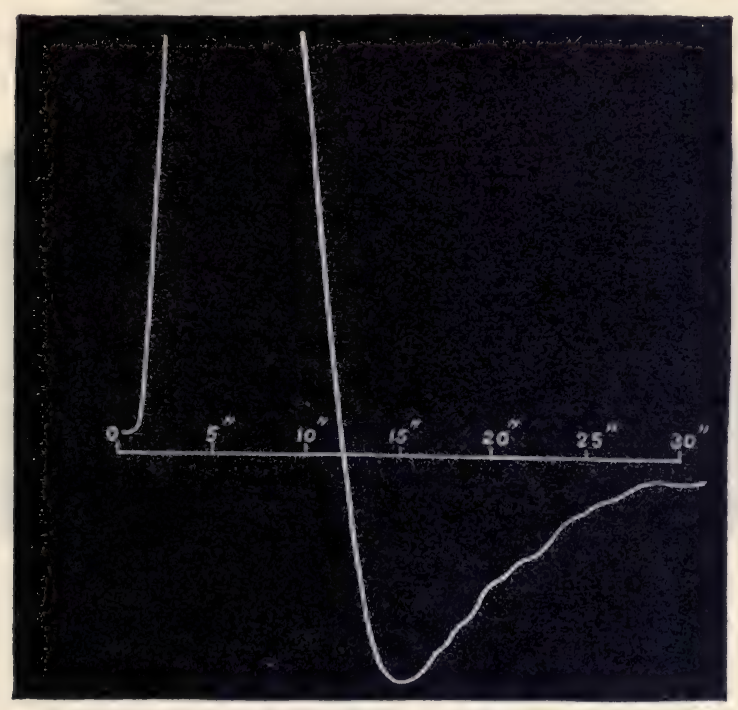

FIG. I89. A Single Response of Skin of Tomato to Equi-alternating Shock recorded on Faster Moving Drum

positive after-effect. Thus, here the supposed tug-of-war between two opposite processes of assimilation and dissimilation is, in reality, between two normal responses having different time-relations. It is from a failure to recognise the fact that the excitatory reaction is not confined to one, but takes place on both surfaces, that such erroneous assumptions as that referred to have often been occasioned. 
The result which I have described-namely, a greater responsive negativity of the inner than of the outer, giving rise to a resultant responsive current from inner to outer-is that which occurs in the majority of cases with tomato. But, as establishing a continuity between this response and that of grape-skin, I may mention the interesting fact that in a few instances I obtained records in which, while the inner surface on excitation exhibited a strong negativity, the outer, under the same stimulus, exhibited a feeble positivity.

The normal response of skin is sometimes, however, found to be reversed, and no explanations have yet been

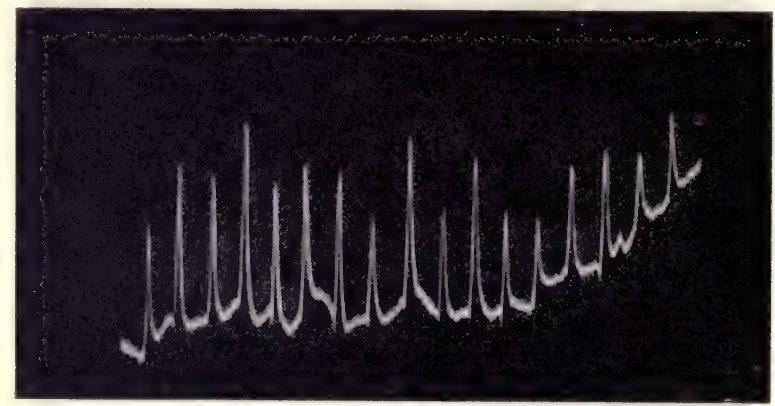

FIG. 190. Photographic Record of Series of Normal Responses in Skin of Gecko

Responsive current from inner to outer surface.

offered to account for this. But I have shown two definite conditions of universal application, which are liable to bring about the reversal of normal response. These are, on the one hand, sub-tonicity, and, on the other, fatigue. Should the condition, in a given case, be the former of these, then the impact of stimulus will of itself, by raising the tonic condition, restore the normal response. Thus in a case of abnormal positive response due to sub-tonicity, an intervening period of tetanisation will tend to convert the abnormal response to normal. An abnormal positive will thus pass into diphasic, and thence into the normal negative. 
For the following experiments, I took the skin of gecko, which can be detached from the body with very little injury. This animal offers remarkable facilities for many electrophysiological experiments. Its isolated tissues can be maintained in a living condition for a very long time. Its sciatic nerve affords us a specimen about $15 \mathrm{~cm}$. in length. Thus for electro-physiological investigation, it provides much greater advantages than the frog.

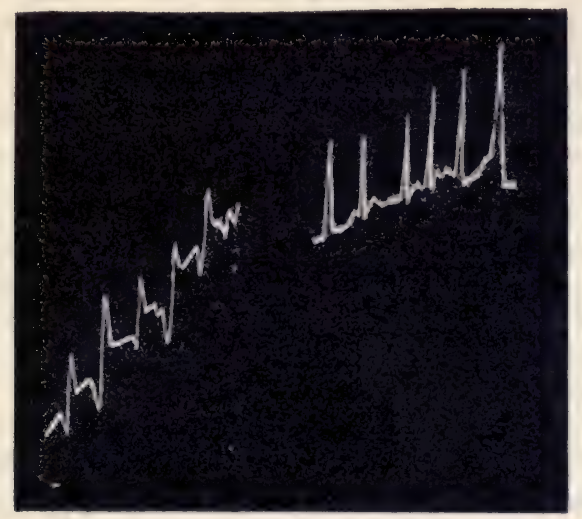

FIG. 191. Photographic Record of Abnormal Diphasic Responses in Skin of Gecko, converted to Normal, after Tetanisation

Taking a specimen of gecko skin, which was in a favourable tonic condition, I obtained the series of normal responses to equi-alternating shocks, which is given in fig. 190. The responsive current here flowed from the inner to the outer surface. I next took another specimen, which was in a less favourable tonic condition, and obtained records of its responses, here seen (fig. 19I) to be diphasic. An intervening period of tetanisation is seen, however, to restore the normal response. 


\section{CHAPTER XXIII}

\section{RESPONSE OF EPITHELIUM AND GLANDS.}

Epidermal, epithelial, and secreting membranes in plant tissues-Natural resting-current from epidermal to epithelial or secretory surfaces-Current of response from epithelial or secretory to epidermal surfaces-Response of Dillenia-Response of water-melon-Response of foot of snail-The socalled current of rest from glandular surface really due to injuryMisinterpretation arising from response by so-called 'positive variation' - Natural current in intact foot of snail, and its variation on sectionResponse of intact human armpit-Response of intact human lip-Lingual response in man-Reversal of normal response under sub-minimal or supermaximal stimulation-Differential excitations of two surfaces under different intensities of stimulus, with consequent changes in direction of responsive currents, diagrammatically represented in characteristic curves-Records exhibiting responsive reversals.

HAVING now seen how the responsive peculiarities of the epidermis may be elucidated by the responses of similar tissues in the plant, we shall next take up an inquiry as to the parallelism between the responses of epithelium and glands in animal and in vegetable tissues. And here, as in the last case, we shall find the obscurities of the one made clear by the study of the other.

If we take the hollow peduncle of a Uriclis lily, and, cutting this into longitudinal halves, take a portion from the upper end of one, we shall observe noticeable differences between the investing membranes of the outer and inner surfaces. On the outer, as we have seen elsewhere, the cells are dry, thick-walled, and cuticularised. This surface then is naturally distinguished as epidermal. The internal membrane of the hollow tube, however, is very thin, and its cells very little differentiated (fig. 192). The internal membrane may thus be distinguished as epithelial.

If now we examine this inner membrane continuously 
from the top to the bottom, at the point where the peduncle rises from the bulb, we shall find that the epithelial layer of the upper end passes imperceptibly into a markedly secretng (glandular?) layer at the lower. By this, secretion is constantly taking place, filling up the hollow tube with fluid. In one instance which I measured, the amount of this secretion was as much as Io grammes in the course of the day. These secreting cells in this, which may be called the glanduloid layer, are very thinwalled and excessively turgid, and, from an evolutionary point of view, these gradual transitions from epidermal to epithelial, and from epithelial to secretory layers, observed under conditions of such great simplicity, are extremely interesting.

When we come to test the electrical reactions of these tissues, we find, on making electrical connections with the external epidermis and the internal epithelium, that a natural current flows in the tissue from the external surface to the internal. This would indicate that the internal was the more excitable of the two. This conclusion is confirmed on the

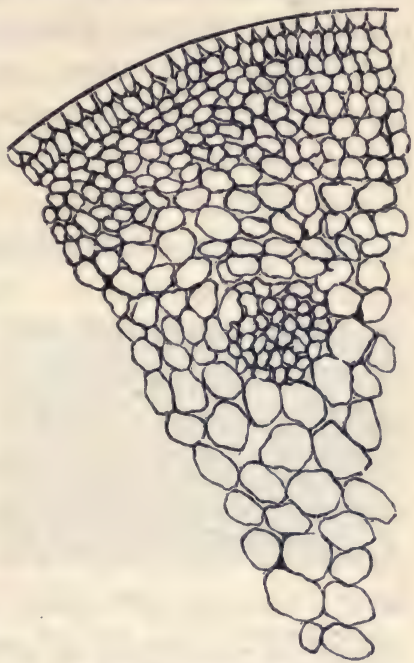

FIG. 192. Transverse Section of tissue of Hollow Peduncle of Uriclis Lily

Cells of epidermis are small and thick-walled, those of inner surface large and thin-walled. application of simultaneous excitation to outer and inner; for the direction of the responsive current is found to be from the internal surface to the external.

If, next, electrical connections be made with the epidermal and secretory layers, a current of rest is once more observed from the external to the internal. On excitation, a very strong electrical response is given, its direction being from the highly excitable secreting layer to the less excitable epidermal. From these experiments we see that the 
epidermal cells are, generally speaking, the least, and the secreting cells the most, excitable.

I shall show, moreover, that all the responsive characteristics of these secretory cells are to be found repeated in those admittedly glandular layers which occur in the lining of the pitcher of Nepenthe, and cover the upper surface of the leaf of Drosera. Before, however, entering upon the consideration of these highly differentiated organs of Nepenthe and Drosera, which are further characterised by some of the digestive functions, I shall first discuss in detail the reactions of a simpler type of vegetable organ. This is exemplified by a single unripe carpel of Dillenia indica, already referred to. When this is carefully removed from the inside of the pseudocarp, and opened, the inside is found to be filled with a gelatinous secretion. This is gently removed, and electrical connections are made with the inner and outer surfaces. It must be borne in mind that these vegetable organs, being not highly excitable, admit of experimental preparations being made with little or no excitatory effect of injury. Allowing now for a period of rest after making the preparation, it will always be found that the current of rest is from the outer layer to the secreting inner layer, which latter is thus, relatively speaking, galvanometrically positive. From the fact which we have generally observed, that the natural current of rest is from the less to the more excitable, it would appear, then, that the inner layer is here the more excitable. This conclusion, moreover, is in agreement with the inference already arrived at, in connection with the tissues of the Uriclis lily, that secreting cells as a rule are relatively the most excitable. This inference may, however, be subjected to the test of direct experiment.

I first tested the response of the same specimen by means of thermal shocks, applied to both surfaces simultaneously. The definite direction of the responsive current, from the inner to the outer, across the tissue, proved conclusively that the inner surface was the more excitable, becoming as it did, galvanometrically negative in relation to the outer. A similar 
effect was obtained as an after-effect of equi-alternating shocks. I next took records of the direct effect of equi-alternating shocks. The direction of the responsive current was found, as before, from the inner to the outer.

In the fruit of water-melon we obtain another specimen whose interior cavity is filled with secretion. On making a suitable preparation of this specimen, and arranging electrical connections with the outer epidermal and inner secretory surfaces, I found the responsive current, under equi-alternating electrical shocks, to be from the inner secretory to the outer epidermal. Fig. I93 gives a photographic record of these responses.

From the typical responsive effects thus obtained with vegetable specimens under the simplest conditions, we are enabled to see that the effect of localised stimulus depends on the characteristic response of the surface layers of the organ. When dealing with this question of the electrical reaction of epithelium and glands in animal tissues, Biedermann rightly came to the conclusion that it was the surface epithelial layer which was, in an electro-motive sense, most effective, the term,

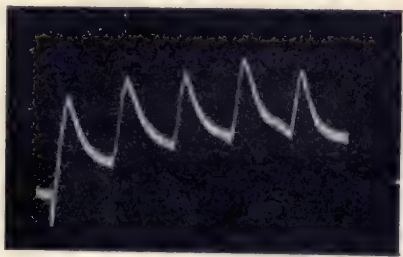

FIG. 193. Photographic. Record of Responses of Water-melon to Equi-alternating Electric Shocks

Responsive current from internal secreting to external epidermal surface.

in its widest sense, including the epithelium of glands and papillæ.

One complicating factor present in the electrical reactions of animal epithelia and glands, but relatively absent under the simpler conditions of the plant, is the effect of injury caused by the process of isolation. The very fact of making the necessary section involves a stimulus of great intensity, and unless the effect of this has thoroughly subsided, the after-effect of such stimulation may be so strong as to reverse the normal current of rest, and otherwise modify the excitability of the tissue. This is seen, for example, in an experiment carried 
out by Dr. Waller, ${ }^{1}$ on the isolated paw of a cat. The current of rest was found by him to flow from the surface of the pad to the section. From this he was led to the conclusion that this current could not have been due to injury, since in that case it would have flowed from the sectioned to the uninjured surface, and not in the opposite direction, as was found to be the case.

This misconception arises from a failure to realise that the so-called 'current of injury' is, in fact, an after-effect of excitation. In the case under consideration, the section, acting as an intense stimulus, simply induces greater excitatory reaction of the more excitable, which in this case happens, as we should have expected, to be the glandular surface. The injury-current here, then, is necessarily from the more excited glandular to the less excited non-glandular.

A precisely similar result was obtained in the case of anisotropic plant-organs, where excitation caused by injury of the less excitable side, becoming internally diffused, induced greater galvanometric negativity of the more excitable distal point (p. I62). For a typical experiment on a glandular preparation, showing the principal effects, and the complications that may arise owing to injury, we may take the detached foot of the pond-snail, the lower surface of which, as is well known, secretes a slimy fluid. On allowing for the necessary period of rest, and then making electrical connections, we observe a current of rest, so-called, which flows from the glandular to the sectioned end. This is not to be mistaken for the true natural current of rest, being in fact the after-effect of a greater galvanometric negativity at the more excited glandular surface, consequent on section. An independent experiment, in support of this induction, will be described presently. On now simultaneously exciting the two surfaces, by equi-alternating shocks, the responsive current is found to flow from the gland inwards, the more excitable gland becoming thus galvanometrically negative. The responsive current is in this case in the same 
direction as the so-called current of rest, constituting a positive variation of it.

From these experiments it is clear that the responsive current is due to the greater intensity of the induced galvanometric negativity at the more excitable glandular surface. It must, however, be noted here that this definite understanding of the phenomenon has been arrived at by fixing our attention on the relative excitatory reactions at the two contacts. If, instead of this, we had regarded it from the usual point of view, of variations of the resting-current only, we must have interpreted it as apparently an abnormal positive variation $;^{1}$ for the so-called resting-current, in such a case, on account of the excitatory after-effect of injury, must also, as we have seen, flow from the more excited gland to the less excited muscle. Great confusion, and resultant misinterpretation of observations, have arisen from not sufficiently recognising these facts, that the resting-current may be originated in either of two distinct ways, and that the excitatory effect may consequently be summated with it in different manners. The resting-current in the primary condition is, as I have demonstrated elsewhere, the natural current. This originates in the natural differences of excitability between different points, and is, in the intact specimen, through the tissue from the less to the more excitable. External stimulus now gives rise to a responsive current, which is in the opposite direction, and therefore constitutes a negative variation of this. This takes place because the more excitable point, which was naturally positive, has now become negative. But we may have a current of rest which is due to previous excitation, or injury, such as may be caused by the shock of the preparation. This current, though usually regarded as the resting-current, is not the true natural current of rest. It is really, as it were, the responsive current become persistent. Succeeding stimuli, inducing responsive galvanometric

1 We shall find in Chapter XXVII. that similar misinterpretations have arisen with regard to the responsive current in the retina, p. 417. 
negativity of the more excitable, will now give rise to a current in the same direction as this resting-current, thus constituting a positive variation of it. It is only when fatigue has set in at the more excitable, and induced great depression of excitability there, that the response-current may undergo a reversal, its direction now being from what was originally the less excitable, to the originally more excitable (p. I77). An example of this I found in the sectioned foot of the large Indian garden-snail. Here the excitatory action on the glandular surface, due to the shock of preparation, was extremely great, as evidenced by the profuse secretion which occurred immediately. Owing to this over-stimulation, fatigue was induced, with consequent great depression of excitability. Hence the responsive current was now found to be reversed, having, with reference to the glandular surface, become outgoing instead of ingoing.

It has been stated above that the ingoing current of rest, observed at the glandular surface under preparation, was not the true natural current, but due to the excitatory after-effect of injury. This I was able to verify by observing the current of rest under natural conditions, without excitation. The snail was allowed to crawl on a glass surface, in the middle of which was a strip of linen moistened in normal saline. This brought the glandular surface into electrical connection with one of two non-polarisable electrodes. When the snail had of its own accord come to a temporary standstill on this piece of linen, the other electrode was quietly placed against the skin of the upper side of the protruding body. The natural current of rest was now found to be outgoing, as regards the glandular surface of the foot, the more excitable being thus galvanometrically positive. The absolute electro-motive difference was found to be + -OOI 3 volt. The foot was now sectioned, and the difference of potential between the same points was found to have undergone a reversal. The supposed resting-current was now ingoing, through the glandular surface. Thus, owing to the excitation consequent on preparation, the more excitable surface, originally positive, 
had become negative. The induced variation from the original condition, in the present case, was from + - OOI 3 volt to $-\mathrm{OO} 2 \mathrm{O}$ volt. It will thus be seen that any irritation is liable to change the natural positivity of a highly excitable glandular surface to negativity. The supposed similarities between the ingoing responsive currents of frog's skin, and the glandular surface of the stomach, are therefore not real. That the two cases are quite different is proved indeed by the fact that local stimulation of the surface of the skin induces galvanometric positivity, whereas a similar stimulation of the glandular surface induces negativity.

In experimenting on animal tissues, it is therefore advisable, wherever possible, to use intact specimens. The numerous experimental difficulties with which we are in that case confronted, may be overcome by the method of simultaneous and equi-alternating shocks which has been described. How practicable this method has been rendered will appear from the experiments which 1 have yet to describe on human subjects.

We have seen that a protected surface is likely, other things being equal, to be more excitable than an exposed one. Partly owing to this fact, and partly also to its richer possession of imbedded glands, it appeared to me probable that the inner surface of the armpit would prove electrically more excitable than a corresponding area, on, say, the upper and outer surface of the same shoulder. In the records which I succeeded in obtaining (fig. 194), this

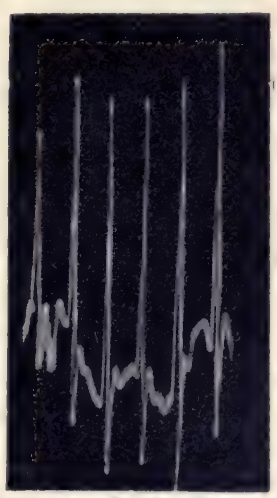

FIG, I94. Photographic Record of Electrical Responses of Intact Human Armpit

Responsive current from armpit to shoulder. supposition was fully borne out. Equi-alternating shocks of one second's duration were applied at intervals of one minute, and the direct effect photographically recorded. The resulting responses were found to be ingoing as regards the armpit, thus proving that that surface was the more excitable. 
In order next to show that epithelial cells in the animal are relatively more excitable than epidermal, as we have already found to be the case in vegetable tissues, I performed the following experiment on the human lip. Here it was important that the electrical connections should be maintained steady. A light spring-contact key was therefore made, as seen in the lower part of fig. 195. The lower contact of this spring-clip consisted of an amalgamated plate of zinc leading to the lower electrode. Over this were tied

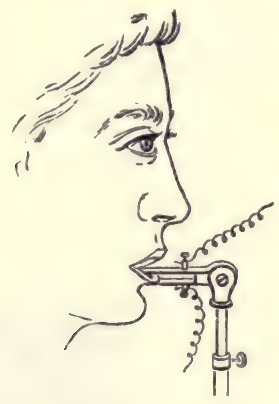
four thicknesses of blottingpaper soaked in zinc sulphate solution. On this again were placed three more thicknesses of blotting-paper, soaked in normal saline. The zinc plate which formed the upper limb of the clip, in connection with the second electrode, was similarly covered with separate layers of

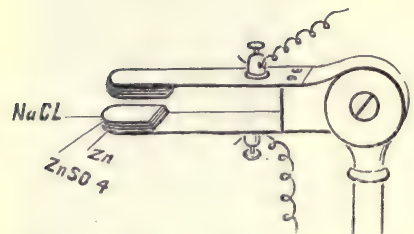

FIG. 195. Experimental Arrangement for Response of Human Lip.

Lower figure gives an enlarged view of the spring-electrodes. blotting-paper, soaked in zinc sulphate and normal saline respectively. The protruded lower lip was now placed in the clip, as shown in the upper figure, in such a way that the latter made a gentle but secure contact. A galvanometer and a source of equi-alternating currents were also placed in the circuit. Of the two electrodes, the upper was in connection with the epithelial, and the lower with the epidermal surfaces. The natural current was now found to flow in the tissue, as in the corresponding cases of plant specimens, from the epidermal to the epithelial. The perfect steadiness of the contact was evidenced by the stillness of the deflected galvanometer spot of light. On now applying the alternating excitatory shock, the responsive current was found to be in the oppo- 
site direction to the natural current, thus demonstrating the fact that the epithelial layer was here, as in the plant, the more excitable of the two. The regularity of this effect will be seen from the series of photographic records given below (fig. 196), in which is exhibited a slight staircase effect.

We next proceed to deal with the response of the glandular organ, the tongue. The tongue of the frog has formed the subject of a very extended series of researches, by Engelmann and Biedermann. On very careful isolation, entailing as little injury as possible, it was found by these workers that the natural current was 'entering': that is to say, it flowed acrosis the tongue from the upper surface to the lower. Both electrical and mechanical stimulation was found by these observers to cause a negative variation of this natural current.

As isolation of such a highly excitable organ as the tongue may, however, give rise to unknown excitatory after-effects, it appeared to me very desirable that an investigation on this subject should be carried out on the intact human tongue. In connection with this, I must point out that both the surfaces of the tongue are excitable. Our inquiry, therefore, is into the relative excitabilities of its upper and lower surfaces. Here

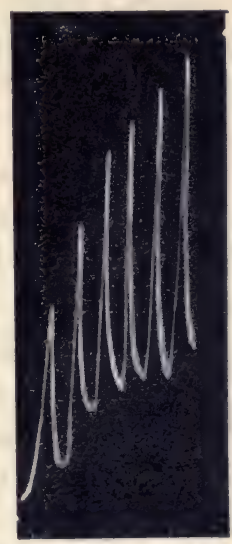

FIG. I96. Photographic Record of Electrical Response of Intact Human Lip

Responsive current from epithelial to epidermal surface. the experimental difficulty lies in this very high excitability of the organ, on account of which-except when in a quiescent state and with a very steady contact-the galvanometer spot of light is apt to be erratic in its movements. Much of this difficulty is overcome, however, by holding the protruded tongue lightly clamped between the teeth. The upper and lower surfaces may then easily be held in the clip-key already described. From this double support of the clip and the teeth it is, with a little practice, possible to arrange matters in such a way that the galvanometer spot is 
practically stable. The current of rest in the intact human tongue is then found to be from the upper to the lower surface, as in the frog. This, according to our previous results, would indicate that the upper surface is the less excitable. This inference finds independent verification, when we subject the organ to the stimulus of equi-alternating shocks. A very strong responsive current is now found to flow through the tongue, from the lower to the upper surface.

The tongue is so extremely sensitive that its characteristic response can be evoked even with very feeble stimulus. I have already explained that the alternating currents induced by speaking before a telephone are not exactly equal and opposite, the current being slightly stronger in one direction. Hence, if such currents be made to play upon an organ in which the excitability is only moderately differential, the preponderance of one of the two elements of the alternating shocks is then likely to mask the true excitatory effect. But the differential excitability of the tongue is so great that the responsive current is always from below to above, whether the exciting current be made to act in a favourable or unfavourable direction. Thus, if one speak, even in a very ordinary voice, into an exciting telephone, which is in series with the rest of the circuit, with its poles direct or reversed, a definite lingual current is induced in response. This, as already said, is always in direction from the lower surface to the upper-surely a curious instance of the speech of one inducing lingual response in another, by direct, and not by provocative action!

The results which have been described are the normal effects given in response to stimulus of moderate intensity. By moderate stimulus is here meant that intensity of current which is obtained when the primary coil is slightly within the secondary. By feeble, on the other hand, is meant the intensity produced when the primary is at a distance from the secondary. Excessively strong stimulus again occurs when the primary is pushed fully within the secondary. I shall now proceed to describe occasional variations which 
may be observed when the stimulus is either very feeble or excessively strong.

We have seen (p. 83) that when the intensity of stimulus is below the critical degree which is sufficient to induce response, its effect is to increase the internal energy of the tissue. We have also seen that the sign of this increased internal energy is galvanometric positivity, being thus opposite to the excitatory effect. Hence, in a differentially excitable tissue, we may expect to find instances in which stimulus that falls below the threshold of true excitation will act by inducing a greater galvanometric positivity of the more excitable, whereas, under normal intensity of stimulus, the more excitable would have become galvanometrically negative. We can thus see the possibility of response being reversed under very feeble stimulus.

It must be remembered that the excitability of both the contacts is a factor in the response, which has hitherto been overlooked. A second very important factor, which has not yet been taken into consideration, is the difference between the characteristic curves of the tissues at the two different surfaces. By characteristic curve is here meant the curve which shows the relation between intensity of stimulus and response. This difference will be better understood from the diagram of the theoretical curves given below (fig. 197). This exhibits all the cases that can possibly exist.

Let the curve A $a a^{\prime} a^{\prime \prime}$ represent the characteristic curve of the surface A. Let the curve $B \quad b b^{\prime} b^{\prime \prime}$ similarly represent the characteristic curve of the surface $B$. Of these two surfaces, B is under moderate stimulation, normally the more excitable. In the middle portion of the curve, representing response under moderate intensity of stimulus, the induced galvanometric negativity of $\mathrm{B}$ is thus greater than that of $\mathrm{A}$. Under moderate excitation, therefore, the current is $b^{\prime} \rightarrow a^{\prime}$ through the tissue in the direction from $\mathrm{B}$ to $\mathrm{A}$. But below the threshold of true excitation, B would be positive, and A 
relatively negative to it. Hence there would here be a reversal of response, the direction of the responsive current $a \rightarrow b$ through the tissue being now from A to B. This current will be recorded by the galvanometer, provided the induced difference between $\mathrm{A}$ and $\mathrm{B}$ be sufficiently great.

Having thus inferred the different effects possible under sub-minimal and moderate stimuli, we shall next consider the differential effect which may sometimes be induced by excessively strong stimulus. In the middle part of the curve,

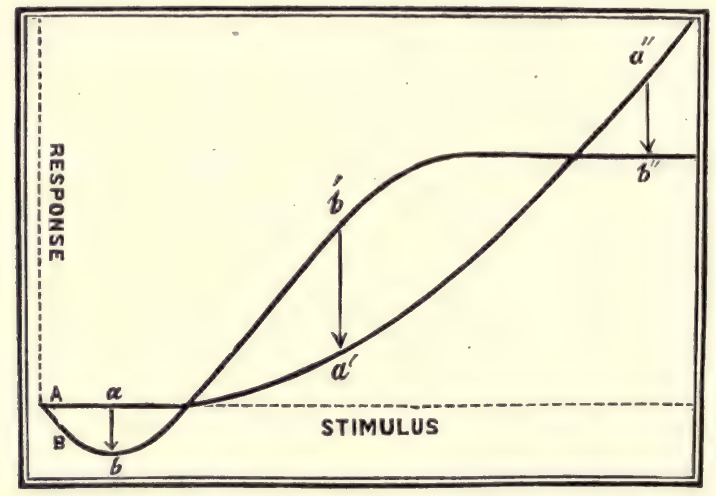

FIG. 197. Possible Variations of Responsive Current, as between Two Surfaces A and B, shown by Means of Diagrammatic Representations of Characteristic Curves

A, $a, a^{\prime}, a^{\prime \prime}$, characteristic curve of surface A; B, $b, b^{\prime}, b^{\prime \prime}$, that of $\mathbf{B}$. Under moderate stimulation, $\mathbf{B}$ is the more excitable, its induced galvanometric negativity being greater, and the direction of current from $b^{\prime}$ to $a^{\prime}$, as in the middle part of the curve. Under sub-minimal and super-maximal stimulation the direction of the responsive current is reversed to $a \rightarrow b$ and $a^{\prime \prime} \rightarrow b^{\prime \prime}$ respectively.

B $b b^{\prime} b^{\prime \prime}$ is seen to be very much steeper than A $a a^{\prime} a^{\prime \prime}$; that is to say, the excitatory effect increases very rapidly with the stimulus, in the more excitable of the two surfaces. But this increase may sooner or later reach a limit, that curve tending to become horizontal, aided in this process, possibly, by growing fatigue. The curve A $a a^{\prime} a^{\prime \prime}$, however, though not so steep, may yet continue to rise throughout a longer abscissa, representing increasing intensity of stimulus. In such a case, there would be a second crossing-point, and 
a second reversal of normal response into $a^{\prime \prime} \rightarrow b^{\prime \prime}$, under excessively strong stimulation. We are thus enabled to see the theoretical possibility of the reversal of normal response under the two conditions of sub-minimal and super-maximal stimulation. All these phases may not be displayed in the same specimen; but it may be possible to find different specimens exhibiting one or the other.

In some cases the difference $a-b$ is too small to allow of an appreciable galvanometric effect, and in their higher parts the curves do not cross. In such specimens, then, there is no response under sub-minimal stimulus, and only normal response under increasing intensities, however strong. The only exception to this will take place when fatigue supervenes, a case which will be dealt with presently. I find that this type of response is the most common.

We have next to consider those cases in which in the sub-minimal region, the difference $a-b$ is appreciable, the reversal to normal $b^{\prime} \rightarrow a^{\prime}$ taking place under higher intensities of stimulus. There need not in such an instance, be any second reversal. Here, then, the normal response under moderate or strong stimulus is reversed when the stimulus is sub minimal. An example of this will be given presently.

Lastly, there may be a type of response in which in the sub-minimal region the difference $a-b$ is slight, and the normal $b^{\prime} \rightarrow a^{\prime}$ is reversed to $a^{\prime \prime} \rightarrow b^{\prime \prime}$ in the region of excessive stimulation. I shall be able to give an example of this also.

I have been able, by taking different specimens, to demonstrate the occurrence of these theoretical reversals of response, under sub-minimal and super-maximal stimulation. I have not yet been able to find a single specimen exhibiting both reversals, but it is not impossible that this exists.

The type in which response remains normal throughout a wide range of stimulus-intensity is too numerous to require special illustration. But this normal response may be reversed under fatigue. Generally speaking, a highly 
excitable tissue may be expected to show earlier or greater fatigue, than, other things being equal, a less excitable tissue. ${ }^{1}$ Thus, under strong or long-continued stimulation, the excitability of the originally more excitable $\mathrm{B}$ may be depressed, so as to fall below that of $\mathrm{A}$, with consequent reversal of response. This will be seen clearly in a typical experiment on the pulvinus of Mimosa. Electrical connections were here made with the upper and less excitable surface $A$ and the more excitable lower surface $B$, records being then taken of normal responses to equi-alternating

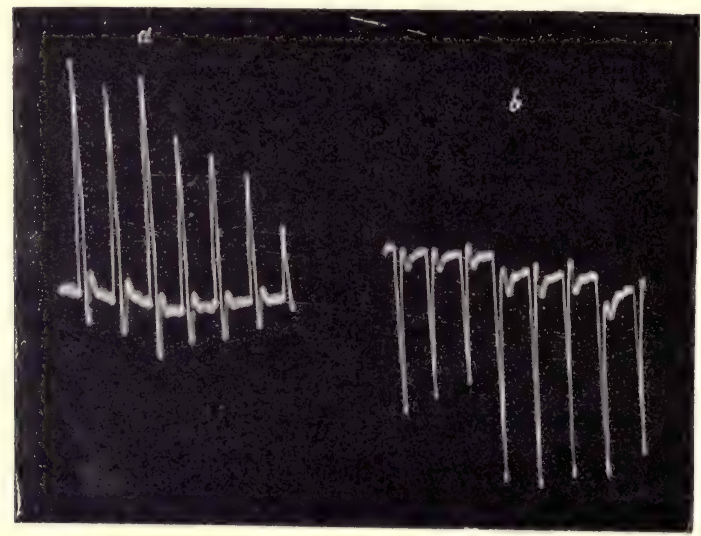

Fig. 198. Photographic Record showing Reversal of Normal Response in Pulvinus of Mimosa due to Fatigue

(a) Series of normal responses, direction of current being from more excitable lower to less excitable upper; (b) Reversed responses in same specimen, due to previous tetanisation, causing fatigue.

electric shocks. The stimulus employed was of moderate intensity, the secondary being placed slightly overlapping the primary. The responses (fig. $198, a$ ) are seen to be normal, the responsive current being from the lower to the upper. They also show signs of slight fatigue, their amplitude undergoing diminution. The secondary was then pushed over the primary, and the tissue subjected to the consequent intense stimulus for two minutes continuously.

1 In this matter, the nature of the tissue must be taken into consideration, nerve, for example, being less subject to fatigue than muscle. 
The secondary was next brought back to its original position and a record once more taken of its successive responses to stimuli of the same intensity as before. It will be seen (fig. $197, b$ ) that the response is completely reversed by the relative depression of the excitability of the lower surface B. Similar reversal under fatigue will be shown in the glandular organ of Drosera in the next chapter (fig. 208). I have obtained other interesting variations of normal response induced by fatigue. Thus, taking the carpel of Dillenia indica, and making electrical contacts with its inner and outer surfaces, the responses under moderate stimulus were found to be normal-that is to say, from inner to outer - the secondary being here understood to be partially overlapping the primary at a distance of six divisions of the scale. The secondary was now pushed home, and the tissue subjected for a short time to strong and continuous stimulation. Moderate fatigue was thus induced. When the secondary was now brought back to the distance of six divisions of the scale the response was found to be reversed. Thus, moderate fatigue had here been sufficient to bring about the reversal of the relative excitabilities of the two surfaces of the carpel of Dillenia indica, when the testing stimulus was of original intensity. But when the intensity of stimulus was increased, by pushing in the secondary to a position marked three divisions on the scale, the response became once more normal. Thus, fatigue had in this case modified the excitability of the two surfaces in such a way that an intensity of stimulus, which was formerly effective to induce greater excitation of the originally more excitable, was now ineffective ; and its greater excitation, with the restoration of normal response, could now only be evoked under stronger stimulus.

We shall next describe the reversal of normal response under sub-minimal stimulation. For this we shall once more select the carpel of Dillenia indica. A record of its normal response-the direction being from inner to outer-is given in the first series of records in fig. 199. After taking 
this record the intensity of stimulus was reduced by pulling out the secondary further away from the primary. The responses were now found reversed, as seen in the subsequent series.

We have last to consider the reversal induced by intense stimulation. Such instances must not be confused with the effect of fatigue. The two

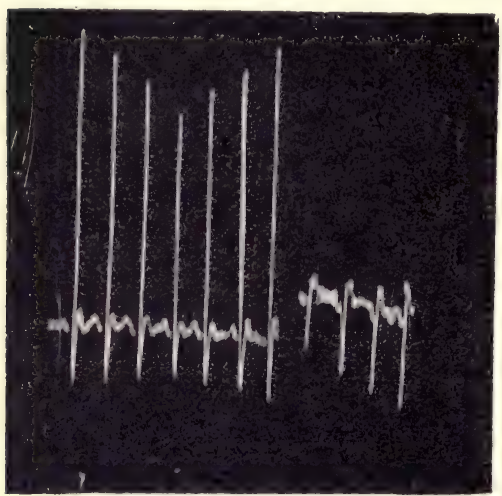

FIG. 199. Photographic Record showing Reversal of Response in Carpel of Dillenia indica, under Sub-minimal Stimulation

The first series show normal electrical responses under moderate stimulus, responsive current from internal to external surface; the second series exhibit reversed response under subminimal stimulation, current from external to internal surface. can be distinguished by the fact that the fatigue-reversal takes place after a series of normal responses, whereas the true reversal, due to strong intensity of stimulus, which we are now discussing, is exhibited at the very beginning. Such an effect I have observed in the response of the human lip. The direction of the responsive current was normally, under moderate stimulus, from the epithelial to the epidermal surfaces. Under very strong stimulus, however, this normal direction was found to be reversed.

But the employment of excessively strong stimulation introduces other complicating factors. The applied stimulus may be supposed to be localised only when it is of moderate intensity. With intense stimulus the subjacent tissues are liable to be involved in giving rise to excitatory response; and it then becomes a difficult problem to discriminate how much of the observed effect is due to the superficial layer, and how much to others more deeply situated. 


\section{CHAPTER XXIV}

\section{RESPONSE OF DIGESTIVE ORGANS}

Consideration of the functional peculiarities of the digestive organs-Alternating phases of secretion and absorption-Relation between secretory and contractile responses. Illustrated by $(a)$ preparation of Mimosa; $(b)$ glandular tentacle of Drosera--General occurrence of contractile response-True current of rest in digestive organs--Experiments on the pitcher of Nepenthe-Three definite types of response under different conditions--Negative and positive electrical responses, concomitant with secretion and absorption-Multiple responses due to strong stimulation - Response in glandular leaf of DroseraNormal negative response reversed to positive under continuous stimulation -Multiple response in Drosera-Response of frog's stomach to mechanical stimulation-Response of stomach of tortoise-Response of stomach of gecko , -Multiple response of frog's stomach, showing three stages-negative, diphasic, and positive-Phasic variations.

HAVING now dealt with the responsive characteristics of the skin, epithelium, and glands, alike in plant and animal, the next subject to be taken up is that of the response of the digestive mucosa. And here we have to determine, first, whether or not there is, broadly speaking, any continuity between the responses of digestive organs and those which we have just been studying; and, secondly, to what extent the functional specialisation of the tissue has acted in accentuating certain of its responsive peculiarities.

Surveying the function of digestion as a whole, we see that it consists, briefly, of two different processes-those, namely, of a previous secretion, by which food is rendered soluble, and of a subsequent absorption, by which the dissolved foodstuffs are absorbed. In the membrane of the simplest digestive organ, then, the epithelial lining, using that term in its most inclusive sense, must be endowed with the two properties of secretion and absorption under different 
circumstances. The question then suggests itself, what are the circumstances which determine this outflow or inflow? In the digestive processes, moreover, in plant and animal alike, each of the reactions referred to, whether of secretion or absorption, must be more or less long-continued. Thus these responsive actions, instead of being single and spasmodic, are likely to be multiple and long-sustained. The characteristic response to stimulus of a secreting organ is understood to be by secretion. Is this reaction essentially different from those fundamental processes which underlie the responses of contractile organs, or are we to regard contraction and secretion as but different expressions of a single responsive phenomenon?

In order to test this question, of the connection between responsive secretion and contraction, it will be well here to draw attention to certain experiments of Sachs, on the response of Mimosa, though our inferences will be somewhat different from those which their author intended. If we take a longitudinal slice of the lower half of the pulvinus of Mimosa and keep it in a moist chamber for some time till the tissue has recovered from the excitation due to section, and if we then subject it to fresh excitation, water will be found to ooze out, or undergo secretion from the excited tissue. We may explain this occurrence in either of two ways : first, that, in consequence of the molecular changes induced by stimulus, contraction and permeability-variations take place in the cells, the expulsion of water being an expression of the active process of contraction ; or, secondly, that the oozing-out of the water is a passive process, due to permeability-variation of the turgid cells alone, without contraction.

The two theories may be distinguished broadly as those of active contraction and passive secretion. In the thin section of the Mimosa pulvinus it is the exudation of water that is noticeable, and not any marked movement characteristic of contraction. But in the intact pulvinus, owing to its anisotropic structure, the greater contraction of the more 
excitable lower half is exhibited in a marked manner by downward mechanical movement, magnified as this is by the long petiolar index. The intact organ, moreover, is invested with an impervious skin, hence the excitatory exudation, or expulsion, of water, being internal, is not seen outwardly. Thus a single identical reaction may appear from different points of view, as either secretory or contractile.

The occurrence of contraction is thus most easily demonstrable when it is accompanied by conspicuous movement. This, however, demands considerable physiological anisotropy, the differential contraction then giving rise to a very marked lateral movement, as in Mimosa. In radial organs of plants, on the other hand, owing to balanced contractions of opposite sides, there is no marked responsive movement. Hence ordinary plant-organs have hitherto been regarded as non-contractile and insensitive. But I have shown that all these radial organs exhibit longitudinal contraction, to be detected and recorded by means of suitable magnifying devices. All motile responses are brought about, it must be remembered, by transference or redistribution of fluids. Now, in organs invested with impervious membranes the effect of fluid-transference is manifested by mechanical movement; whereas, in naked tissues, the fluid-transference is directly visible as secretion.

From the very important series of researches carried out by Darwin, on the excitatory reactions in the tentacles of Drosera, we know that the pedicel, carrying the gland on its summit, is somewhat flattened, and that it is this anisotropic lower part which is alone capable of movement. The glandcells on the head of the tentacle have been shown by Gardiner to be provided with delicate uncuticularised cellwalls, which are curiously pitted on their upper or free surfaces. The terminal organ, or head, which is radial, would thus seem to be peculiarly fitted for the exudation of liquid on excitation. In the anisotropic motile portion of the pedicel, on the other hand, the responsive reaction manifests itself by bending. It would thus appear that the same 
excitatory reaction may exhibit itself in different parts, even of the same organ, by mechanical movement and secretion respectively, according to the facilities which one or the other portion offers.

That the phenomenon of contraction is behind the excitatory expulsion of water in a vegetable organ, would appear highly probable from certain results obtained in the electrical response. The stimulation of an ordinary vegetable tissue gives rise to two distinct electrical effects at a distance. The first of these is the arrival of the hydro-positive effect of galvanometric positivity, with positive turgidity-variation. The second is the wave of true excitation, with its characteristic of negative turgidity-variation and concomitant galvanometric negativity. The first, consisting, as this does, of a hydrostatic blow delivered at a distance, can only, it appears to me, be ascribed to an active process of contraction, causing the squeezing-out of water in the excited region. A passive escape of fluid, due to mere permeability-variation, could not, as I think, originate that impulsive hydrostatic shock which is transmitted to a distance. For such a result to take place an active expulsion would seem to be requisite.

In view of these facts, is it necessary to hold the doctrine of discontinuity, or, when there is evidence in its favour, are we to believe in the continuity of these apparently different reactions? The excitatory reactions of different classes of tissues have hitherto been regarded as different, chiefly because some were looked upon as motile, and others as non-motile; muscle, for example, was held to be typical of the first, and nerve of the second, of these classes. In this case of the nerve, it has been believed that there was no visible manifestation of the excitatory change. I shall, however, be able to show that even this supposition is incorrect, since the excitatory reaction in the nerve is in fact attended by contraction. The electrical indication of galvanometric negativity which is concomitant with contraction in contractile tissues, is also obtained in the case of excited 
glands. The visible changes which occur under stimulation in these three types of tissues would thus appear to differ only in degree.

We may now turn more especially to the question of the electrical reactions of the digestive mucosa. As regards the natural current of rest, we have seen that Rosenthal and others found that this current was ingoing-that is to say, the mucous layer was negative, as compared to the muscular coat of the stomach. Biedermann had also noticed a strong current of rest between the glandular surface of Drosera and the stalk. But it will be shown that the glandular coat of the stomach is more excitable than the muscular layer. Hence we should have expected that the natural current of rest would have been from the less excitable to the more excitable, the mucous layer in a state of rest being thus relatively galvanometrically positive. The opposite direction of the current which has been observed, would rather appear to be ascribable to the excitatory after-effect of preparation. I have already described how the glandular foot of the snail, under conditions of perfect rest, is galvanometrically positive. But the excitation caused by preparation renders this highly excitable glandular surface negative That the fact of cutting open the stomach, to make the experimental preparation, similarly, would cause intense excitation with galvanometric negativity, was to have been expected. I shall be able, indeed, to show, by means of experiments to be described presently, that the shock consequent on this preparation is to give, not one, but a prolonged series of multiple electrical responses.

I have almost invariably found, in making electrical contacts after section, with the inner and outer surfaces of frog's stomach, that the multiple responses caused by section, persisted for more than an hour; and until these had subsided no fresh experiment could be undertaken, to obtain records of the response of the stomach to external stimulus. I have also found that many of these frogs were in the habit of swallowing stones and pebbles, the persistent 
mechanical irritation of which would contribute to the negativity of the mucous lining.

Finding, then, that it would be impossible to obtain the natural current, in a stomach which had to be cut open, I next turned my attention to stomachs which are naturally open. These are seen in the upper concave surface of the leaf of Drosera, for instance, which is provided with glandular tentacles. I here made electrical connections with the upper and lower surfaces respectively. But the tentacles excited by the contact of the electrode bent and clasped it round, an excitation which was seen in the galvanometer as negativity of that surface. From this may be gauged the difficulties which attend the observation of the true natural current of rest in such an excitable organ as the stomach. The demonstration, however, of the galvanometric positivity of the snail's foot, and of the inner glandular surface of the carpel of Dillenia indica, lead to a strong presumption in favour of the true resting-current in the stomach being from the non-mucous layers to the mucous.

Having thus seen the difficulties imposed by the high motile excitability of the tentacles of Drosera, I next turned my attention to other specimens. We have seen that there is a secretion of fluid at the lower end of the hollow interior of the peduncle of Uriclis lily, and that the secreting inner layer is here galvanometrically positive in a state of rest. As this tube, however, is closed, it cannot be regarded as subserving the absorption of food-material. But the same limitation does not apply to those modified foliar structures, the pitchers of Nepenthe ${ }^{1}$ (fig. 200). These, as is well known, are open. They have a histological differentiation, moreover, of their lining membrane, actual glands being present (figs. 201, 202), which are admitted to be comparable to those of the animal digestive organ, though of a much simpler type. A fluid is secreted by these glands, and insects entrapped in the pitcher are in it dissolved or decomposed. The products

1 I have to thank the authorities of the Botanical Garden, Sibpur, for supplying me with these valuable specimens. 
are subsequently absorbed by the tissue, as in corresponding cases, by the stomach of animals. From the study of the responsive peculiarities of so primitive a type of stomach, we might then expect to gain much light on the action of more complex and highly specialised digestive organs. In order first to obtain the true current of rest, I took a young pitcher which had previously been kept free from all disturbance. I next made electrical connections, by means of non-polarisable electrodes, with the inner (glandular) and outer surfaces of this pitcher respectively. As some excitatory reaction may be induced in a highly excitable organ, even by the contact of normal saline, the cotton threads in connection with these nonpolarisable electrodes were moistened with the natural secretion of the pitcher itself. On carrying out the experiment under these ideal conditions, I found, as I had expected, that the current of rest flowed from

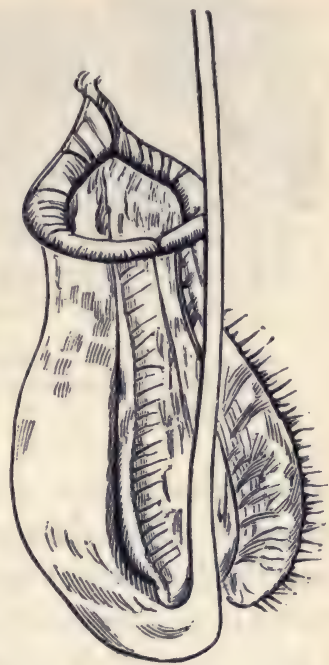

FIG. 200. Pitcher of $\mathrm{Ne}$ penthe, with lid removed. the outer non-glandular to the inner glandular surface, the latter thus being galvanometrically positive.

In investigating next the excitatory reaction, I obtained three different types of responses-negative, diphasic, and positive-characteristic of certain definite conditions. Before entering upon the details of these experiments, it is advisable to discuss here the probable significance of the negative and positive electrical reactions observed.

We have seen that a slice of tissue from the' pulvinus of Mimosa excretes water under excitation. The electrical reaction under these circumstances is one of galvanometric negativity. But during the process of recovery, when the tissue is absorbing water, this negativity diminishes, a change that is tantamount to that increase of positivity with which 
we are already familiar, as the invariable accompaniment of a positive turgidity-variation.

Thus, if galvanometric negativity is to be taken as the concomitant of the expulsion or secretion of fluid, it would

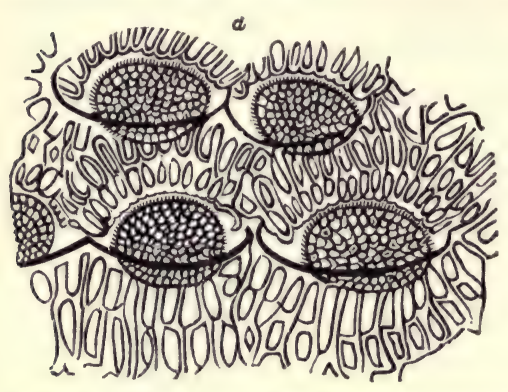

FIG. 20I. Glandular Surface of a Portion of the Living Membrane of the Pitcher of Nepenthe. appear that the opposite process of absorption would be indicated by the responsive galvanometric positivity.

Again the fresh pulvinus of Mimosa responds, when excited, by a mechanical fall, a negative turgidity-variation, and by galvanometric negativity. But after long-continued stimulation, these normal responses are found to undergo reversal. The pulvinus expands; water must be re-absorbed, and the leaf is re-erected. The normal galvanometric negativity is now reversed to positivity. It will thus be seen that while, in a fresh tissue, stimulus gives rise to expulsion of

\section{(}
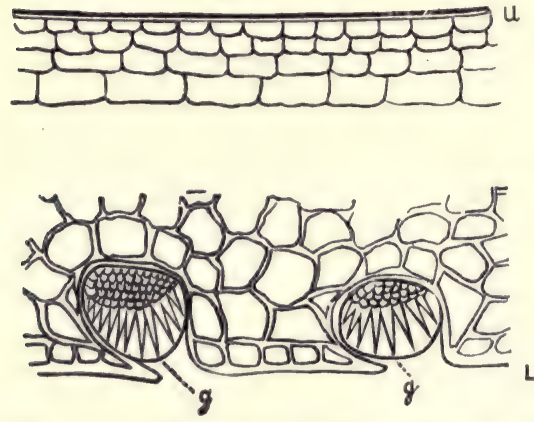

FIG. 202. Transverse Section of Tissue of Pitcher of Nepenthe.

$u$, outer surface; $\mathbf{L}$, inner surface ; $g$, glands present in the internal surface. reactions of the outflow and inflow of fluids appear to us of little consequence, except in the form of those appropriate fluid-the electrical indication of this process being galvanometric negativity-in a tissue which has already, on the other hand, been under continuous stimulation there will be a tendency towards the phasic reversal of response to galvanometric positivity, indicative of the process of absorption.

In the case of motile tissues, these excitatory 
motile responses which they occasion. But in glandular organs, they become possessed of much greater significance, since they constitute the main function of such structures.

Electrical responses, in every way analogous to those which have been described, are obtained from the glandular surfaces of digestive organs. That is to say, the glandular surface when fresh exhibits responsive galvanometric negativity on excitation. In Drosera, for example, under these conditions secretion is seen to take place. Thus the negative phase of response, in this as in the case of Mimosa, is associated with expulsion of fluid or secretion. After continuous stimulation, again, the responsive phase here, as in Mimosa, is found to be reversed to positive, indicative, as there is every reason to believe, of absorption. From a consideration of the functions of the digestive organ, we should be prepared, as already pointed out, to expect the occurrence of two alternating processes. In the fresh state, ingestion of food, acting as a stimulus, would naturally induce excitatory secretion; and this excitatory secretion must be followed later by the absorption of dissolved food. These alternating phases of secretion and absorption indubitably occur. We shall also find, in the electrical response-records, a phasic alternation of negative and positive under appropriate conditions.

We have seen that there is a continuity between the different reactions of non-glandular and glandular tissues. We have also seen that, as in the one case, so too in the other, a phasic change takes place from negative to positive. In the digestive organ, however, we have to deal mainly with the fluctuations of fluids-secretion and absorption-and the attendant electrical variations, which consist of two opposite phases, positive and negative. As far as I have found it possible to test the matter experimentally, it has invariably been the case that the negative electrical phase was associated with secretion; and everything points to the probability that the converse of this - the association, namely, of the positive electrical phase with the process of absorption-holds equally good. 
We now return to the question of the normal response of the pitcher in its three different conditions. Of these, in the youngest, no flies are present; such a specimen will be known as 'fresh.' In others, somewhat older, are a few insects. These pitchers may be regarded as moderately excited, owing either to the struggles of the insects or to the supply of food, or to both. Still another class is found, in which the glandular part of the inner surface of the pitcher is practicaily coated with captured insects, and has thus already

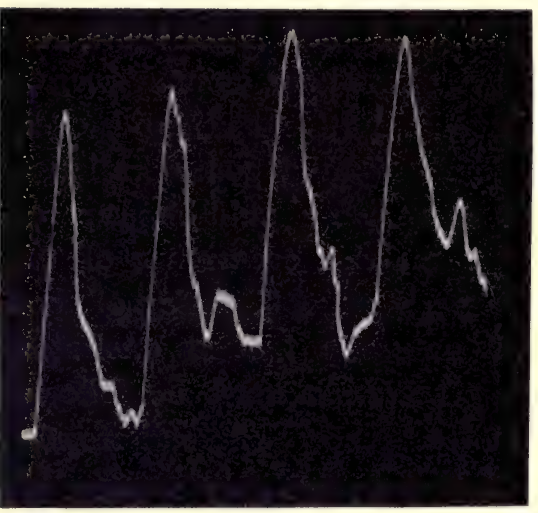

F1G. 203. Photographic Record of Series of Normal Negative Responses of Glandular Surface of Nepenthe in Fresh Condition to Equi-alternating Electric Shocks given at Intervals of Two Minutes

Responsive current from internal glandular to external non-glandular surface. Note occurrence of multiple response and trend of base-line upwards. been subjected to longcontinued stimulation.

The responses of these three classes of specimens are in each case, as I shall show, very characteristic.

I shall first describe experiments carried out on fresh specimens. Records were made of their responses to equialternating shocks of moderate intensity, at intervals of two minutes. The responsive current was here found to flow from the internal glandular to the external nonglandular surface. Fig.

203 gives a series of such responses. It was said at the beginning that the responses of digestive organs were likely to be multiple. This is seen to be true even under the moderate stimulus applied in the present case. But under the action of stronger stimulus, such as that of a thermal shock, the response is found to consist of a long and multiple series, records of which will be seen later.

Another peculiarity to be noticed, in the series of responses given in fig. 203, is that the base-line of the record 
trends upwards. This indicates that the glandular surface, by the residual effect of stimulation, is being rendered more and more galvanometrically negative. This explains why the internal surface of the stomach has been found by different observers to be negative, a condition of more or less persistent negativity being thus clearly due to the excitatory after-effect of preparation. Had it not been for the exceptional opportunity afforded by the open pitcher of Nepeinthe, it would have been impossible to make galvanometric connections with the intact inner glandular surface and thus to ascertain that such a surface is naturally galvanometrically positive.

I may here point out the very interesting modification of response which occurs in the same specimen under a long-continued series of stimulations. This modification, due to fatigue so-called, makes its appearance first in diminution of the height of the responses. Some of the constituent multiple responses due to a single stimulus are then found to be reversed to positive, and after this they show a tendency to become more or less completely reversed.

It is also interesting to find that the same modifications make their appearance, in the same order, in those pitchers which have been subjected to continuous stimulation, to a greater or less extent, by the supply of insects. That is to say, a pitcher containing a few insects is found to give responses, the multiple constituents of which are sometimes positive and sometimes negative. This intermediate phase is seen well illustrated in the record given in fig. 204. But in the pitcher whose inner surface is already thickly coated with insects, and which has long been exposed to the continuous action of such stimulation, the characteristic response is found to be the reverse of that of the fresh specimen. It will be seen from the record in fig. 205 that in such a case the individual effect of a single stimulus is a series of multiple responses which are positive. In this record a curious effect is again seen, that of the shifting of the base-line, now 
downwards. This indicates an increasing positivity of the glandular surface.

The results which have thus been described, in the case of the fresh pitcher, and of one subjected for a long period to the stimulus of food, are fully compatible, it will be observed, with the theory of digestion as a diphasic process, in which galvanometric negativity is associated with a predominant secretion, and the subsequent galvanometric positivity with a predominant absorption by the glandular memibrane.

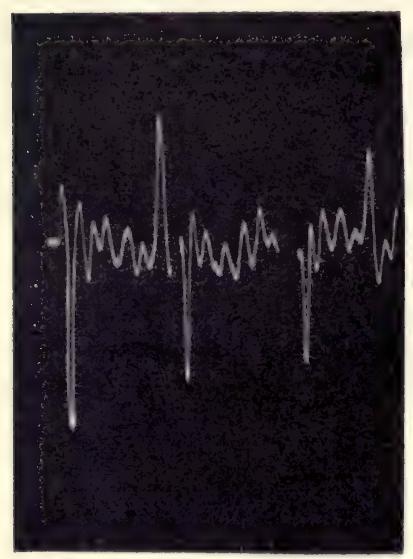

FIG. 204. Photographic Record of Responses of Pitcher in Intermediate Stage, having Attracted a Few Insects

Note here the occurrence of two phases in constituent responses, positive being predominant.

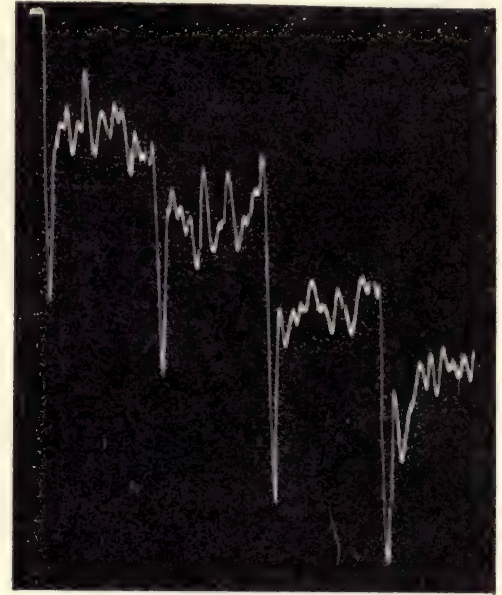

FIG. 205. Photographic Record of Responses of Pitcher in Third Stage, the whole Glandular Surface thickly Coated with Insects. Stimuli applied at Intervals of two Minutes

The response here is in the positive phase, direction of current being from nonglandular to glandular. Note also the multiple character of responses to single stimuli.

It is also important to notice that while in the fresh condition the glandular surface is positive, and in the moderately stimulated condition negative, yet positivity of the glandular surface is not always to be taken as a sign of its fresh condition. For we have here seen that under longcontinued stimulation, the electrical condition is apt to be reversed to one of positivity. 
It has been stated that on account of the highly excitable nature of the digestive organ, a single stimulus, if strong,

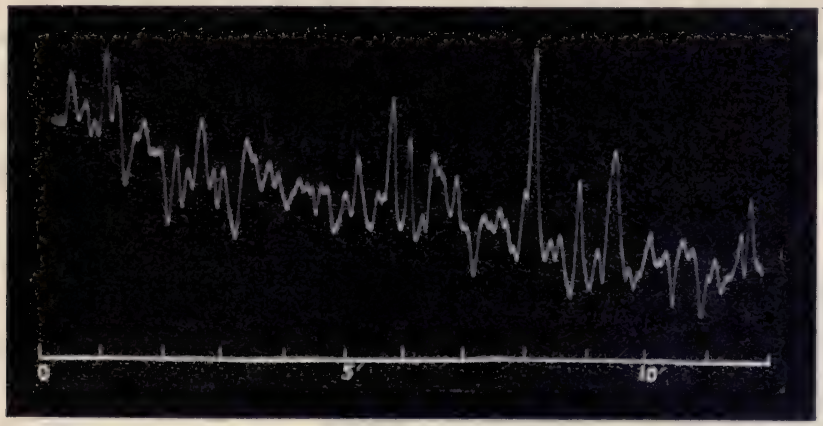

FIG. 206. Multiple Response of Pitcher of Nepenthe, in First or Fresh Stage, to Single Strong Thermal Shock

The constituent responses are both negative and positive, the former being stronger.

would give rise in it to a multiple series of responses. The two following records (figs. 206 and 207) illustrate this fact in two different specimens which were in somewhat different conditions. The stimulus employed in each case was a single strong thermal shock, and the multiple responses were found to persist, in both, for quite an hour. In the first of these figures, the constituent responses of the series were both negative and positive, the former being predominant. In the second record (fig. 207) the

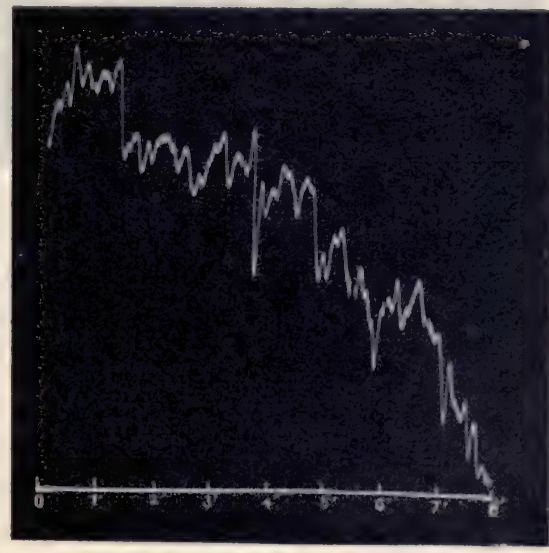

FIG. 207. Multiple Response of Pitcher of Nepenthe, in Third Stage, to Single Strong Thermal Shock

The constituent responses are here predominantly positive. positive phase is predominant in the responses, and the trend of the base-line downwards shows increasing positivity of the glandular surface. 
In taking up this investigation on the pitcher of Nepenthe it appeared to me that much light would be thrown, by the study of this simple organ, on the many. difficulties connected with the response of the more complex digestive organs of the animal. This surmise has proved to be fully justified, for in the experiments which I have carried out in the latter field, the results are a mere repetition of these typical effects seen in Nepenthe under corresponding circumstances.

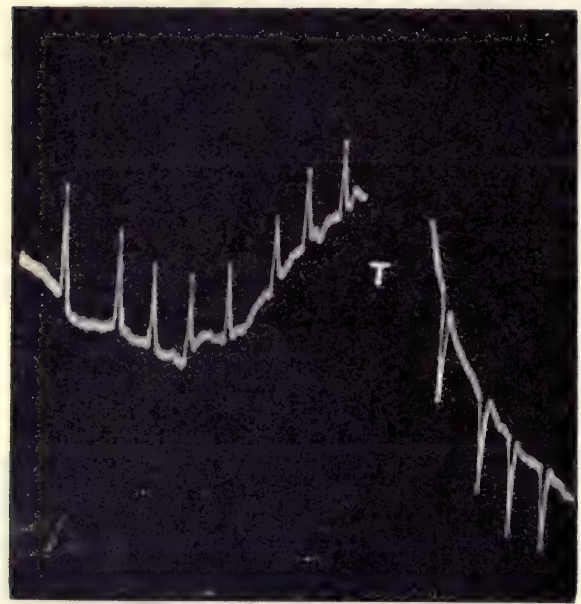

FIG. 208. Photographic Record of Responses in Fresh Leaf of Drosera to Equi-alternating Electrical Shocks

The first series show normal responses. Current from upper glandular to lower non-glandular surface. In the second series normal response is reversed to positive, after tetanisation, T.

Before passing from Nepenthe to the study of digestive tissues in animals, it will be well to deal here with the more complex type of vegetal digestive organ seen in the plant Drosera. I took for my experiment a specimen of the Indian Drosera longifolia, the upper surfaces of whose leaves are covered, as is well known, with glandular tentacles. Here, as in the case of the pitcher of Nepenthe, the response of leaves which are fresh and have not been subjected to previous excitation, is by induced negativity of the glandular surface, and this is reversed to positive under long-continued stimulation. These two phases are seen in figure 208, in which the first series is a record of normal responses of galvanometric negativity, to equi-alternating shocks applied at intervals of one minute; and the second, the reversed responses exhibited by the same leaf, to the same stimulus, when it has, in the meantime, been subjected to tetanising shocks for three minutes continuously. It is 
curious and interesting to note here, as in the case of Nepenthe, the trend of the base-line up, when the response is the normal negative, and down when it is the reversed positive, indicating in the one case increasing negativity, and in the other increasing positivity.

As in the Nepenthe, so also in the leaf of Drosera, specimens which are not fresh-that is to say, previously unexcited---are apt to exhibit the positive phase of response. I give below a series of multiple responses (fig. 209) induced in such a leaf by a strong stimulation. The stimulus was in this case given by sectioning the leaf, and the response therefore illustrates the fact that preparation itself acts as a stimulus. In the present case, electrical connections with the galvanometer, were made with the upper and lower surfaces of the leaf on the plant, intact. On now cutting the petiole across, a long series of multiple responses, lasting for about 45 minutes, was found to be set up. These pulsations were at first rapid, and then slowed down gradually, the average period of a single pulsation being about 30 seconds. Only a portion of the record is shown in fig. 209.

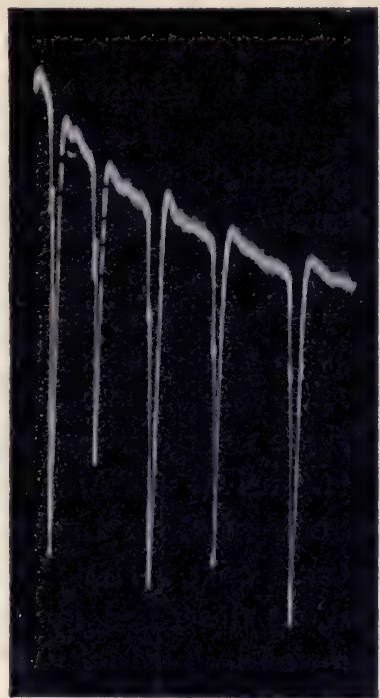

FIg. 209. Photographic Record of Multiple Response of Leaf of Drosera in Positive Phase

Stimulus was caused here by section of the petiole.

Having thus seen the typical responses exhibited by the digestive organs of plants, we shall now pass to the consideration of the reactions induced in animal stomachs. Here, again, two different subjects of inquiry arise, the direction, namely, of the natural current of rest, and that of the action or responsive current. As regards the first of these, it will be remembered that Rosenthal found it to be strongly 'ingoing'-that is to say, from the mucous to the 
muscular coats of the stomach. From this it was supposed, as we have seen, that the mucous coat of the stomach of the frog had the same electro-motive reaction as its outer skin. We shall find, however, that there is in reality no such similarity between the two, inasmuch as, while the excitatory reaction makes the outer skin galvanometrically positive, its effect on the mucous surface under normal conditions is to induce galvanometric negativity. In the case of Nepenthe, further, we have seen that the natural current of rest is from the non-glandular outer to the glandular inner surface, and that this is liable to reversal, as an excitatory after-effect of preparation. The ingoing current, therefore, observed in the preparation of frog's stomach, is to be regarded, not as the natural current of rest, but as the excitatory after-effect due to isolation.

With regard, next, to the current of action, Biedermann states that direct electrical excitation, by rapidly alternating shocks, induces a negative variation usually preceded by a positive swing. Since the so-called current of rest is ingoing, a 'negative variation' of it evidently means an outgoing current-that is to say, galvanometric positivity of the mucous coat. Hence the responsive action of the mucous coat, as described by Biedermann, is a transient negativity followed by positivity.

In dealing with this question of the electrical response of the digestive organ, we must be prepared, as the result of previous experiments on plants, to meet with variations of the excitatory effect, due to the phasic condition of the tissue. And first, for the clear demonstration of the effect of excitation on the mucous surface, uncomplicated by changes induced at the second contact, I employed the Rotary Method of Mechanical Stimulation of the given area. The rotating electrodes were applied to the inside of a properly mounted frog's stomach, and experiment commenced some time after the cessation of the multiple response due to preparation. The following record (fig. 2IO) exhibits the first four of these responses to individual mechanical stimuli, 
applied at intervals of one minute. The responsive variation took place by the induced galvanometric negativity of the excited area. Under long-continued stimulation fatigue was found to be induced, the responses becoming diminished and even tending to a reversal from the normal to positive.

In order to show that the inner mucous surface is relatively more excitable than the muscular coat, I next subjected the two to simultaneous excitation by equi-alternating electrical shocks. And for the sake of establishing a

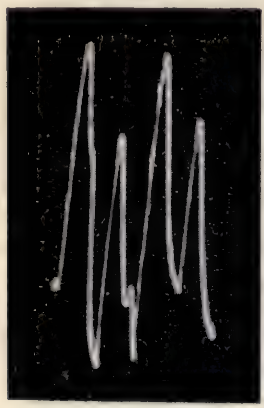

FIG, 210. Photographic Record of Normal Negative Responses of Frog's Stomach to Mechanical Stimula. tion

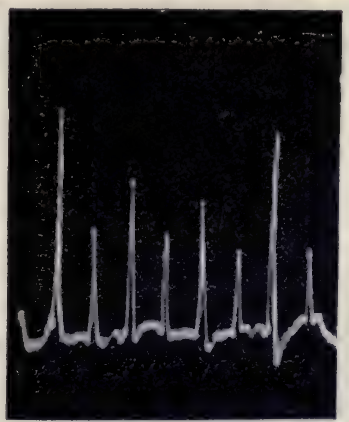

FIG. 211. Photographic Record of Normal Negative Responses of Stomach of Tortoise to Stimulus of Equialternating Electric Shocks applied at Intervals of One Minute

generalisation as to the reaction of the stomach, I now took a different specimen-namely, the stomach of tortoise. The responses in the figure (fig. 2II) showed relative galvanometric negativity of the inside of the stomach.

We have seen that fresh vegetable stomach responds by normal negativity, but that, under continuous stimulation, a phasic change is induced, by which response is reversed to positive (cf. fig. 208). I shall next demonstrate the corresponding effect in the animal stomach. Taking a preparation of the stomach of gecko, I obtained normal responses, whose direction was from the glandular internal to the muscular 
external surface. After an intervening period of tetanisation, however, the responses are seen to be reversed (fig. 2I 2).

The next record has been selected for the purpose of showing the gradual process of transition from the normal negative to the reversed positive response. The specimen taken was frog's stomach. At the commencement of the experiment the galvanometer spot was quiescent, but when the specimen was subjected to a single strong thermal shock, a prolonged series of multiple responses was initiated, persisting for more than an hour. Of this series I here re-

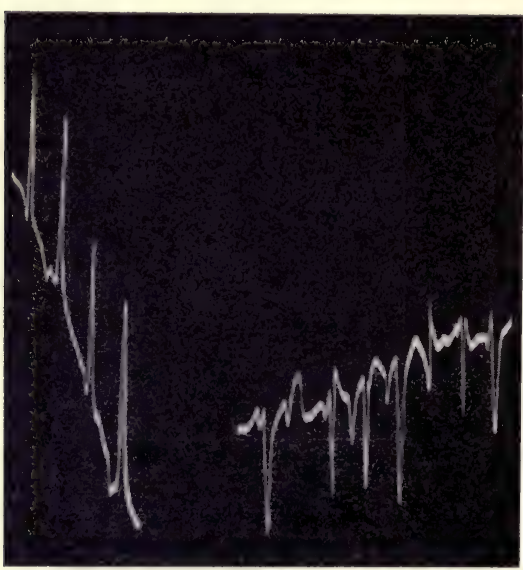

FIG. 212. Photographic Record of Normal Response in Stomach of Gecko to Equialternating Shocks, seen to be reversed after Tetanisation produce four different portions (fig. 213). The first of these (a) consists of pulses of galvanometric negativity of the internal surface. The recoveries are here incomplete, and the base-line shifts upwards, showing an increasing negativity of that surface. The negative pulses are then reversed to positive, through an intermediate di-phasic (b). In the first part of this pronouncedly positive response $(c)$ the base line is horizontal. It then begins to shift downwards $(d)$, thus exhibiting a decreasing negativity-or increasing positivity-of the internal surface. In this periodic variation of the electrical condition we have a significant parallel to the records which we have already seen in Nepenthe and in Drosera (figs. 203, 205, 208, and 209).

It has already been pointed out that, in view of the functional peculiarities of the digestive organ, it might be expected that the alternate reactions of secretion and absorption would neither of them be single and spasmodic, but each long- 
sustained. In this connection it is suggestive that the digestive organs should show so strongly marked a characteristic of multiple response. It would thus appear, as already said, that mechanical stimulation during ingestion of food gives rise to the responsive reaction of secretion, evidenced electrically by response of galvanometric negativity of the internal surface. There then sets in the opposite phase, associated with the

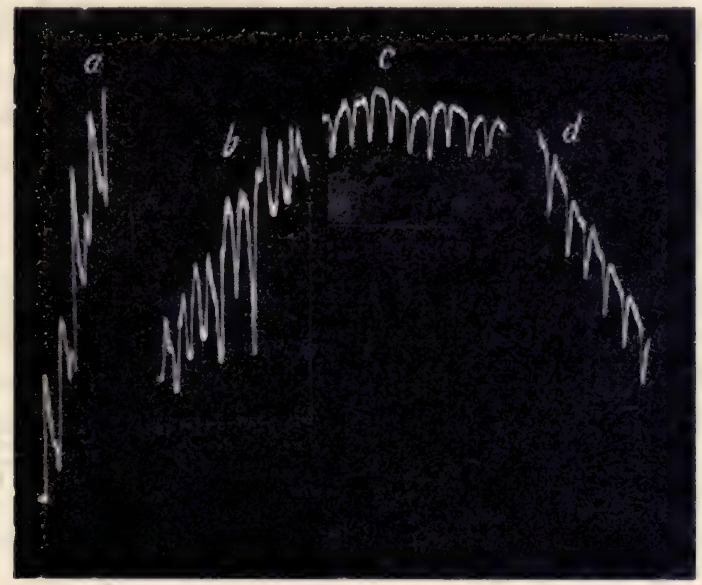

FIG. 213. Photographic Record of Multiple Responses in Stomach of Frog to a Single Strong Thermal Shock

Four parts are given of this long series. (a) Negative series; (b) Alternating negative and positive constituent responses ; $(c)$ Positive series ; (d) Positive series. Note trend of base-line upwards in negative $a$, and downwards in positive $d$.

reversal of electrical response, probably indicating the absorptive process. This reversal of response, to galvanometric positivity, may be the work of three different factors, which may or may not be mutually dependent. In the first place, we have seen that long-continued stimulation was apt of itself, other things being equal, to give rise to a reversal of response. Secondly, after secretion has reached its maximum, the empty mucous cells in contact with fluid would naturally tend to reabsorb. And, lastly, we have seen that an increase of internal energy, in whatever way produced, 
tends to give rise to a responsive reaction, whose sign is opposite to that of excitation-expansion instead of contraction. Now such an increase of internal energy could not fail to be the result of the absorption of the chemicallydissolved food.

Another interesting consideration to be remembered in connection with digestive organs is that periodically-acting forces give rise to an induced periodicity, which persists for a time, even in the absence of the periodically-exciting cause. A well-known illustration of this is met with in the nycti- tropic movements, so-called, of plants, induced as these are by the periodic variation of night and day. These movements persist for a certain length of time, even when the plant is kept in continuous darkness. Similarly, animals accustomed to the supply of food at regular intervals would undoubtedly exhibit alternating phasic changes apparently spontaneous, in the condition of the digestive organ in consequence of the original periodicity of the exciting cause. Such an organ, therefore, must necessarily exhibit periodic electrical variations. 


\section{ABSORPTION OF FOOD BY PLANT AND ASCENT OF SAP}

Parallelism between responsive reactions of root and digestive organ-Alternating phases of secretion and absorption-Association of absorptive process with ascent of sap-Electrical response of young and old roots-Different phasic reactions, as in pitcher of Nepenthe-Response to chemical stimulationDifferent theories of ascent of sap-Physical versus excitatory theoriesObjections to excitatory theory-Assumption that wood dead unjustifiedDemonstration of excitatory electrical response of sap-wood-Strasburger's experiments on effect of poisons on ascent of sap-Current inference unjustified.

WE have seen in the last chapter that in the digestive process as a whole there must be alternating phases of secretion and absorption. The secretion of dissolving fluids, by which insoluble substances are rendered soluble, we found to take place under stimulation, and to be succeeded by a process of absorption, by means of which the now dissolved foodmaterial found access into the organism. These functions, though seen characteristically in the digestive organs of animals, are also to be observed in some plants, such as the pitcher of Nepenthe, or the leaf of Drosera. Here, situated externally, we find what are practically open stomachs, digesting, as do those of animals, solid organic food. But plants in general have to depend on the supply of inorganic food-material, often presented in solid or insoluble forms, for their nourishment. In this case also it is obvious that the same sequence of solution by dissolving fluids, and subsequent absorption, must be gone through. And the organ by which this takes place must evidently be the root. In this regard the well-known experiments on the corrosion of marble by the root of a growing plant are sufficient to show 
that these organs secrete acids, by means of which insoluble substances are made soluble. It is equally clear, further, that the inorganic solids so dissolved are afterwards absorbed by the plant. Thus it will be seen that these alternating processes of secretion and absorption of food-material, as they take place in the vegetable organism, are not very different in their essential features from the ordinary phenomenon of digestion as known to us. The chief distinction between the two would now seem to lie in the fact that in the animal the supply of food is in the main organic, and in the plant inorganic. Even here, however, we meet with connecting links in the form of insectivorous plants, in whose case the organic supply is obtained by means of the digesting leaf, and the inorganic through the roots. We may regard digestion, therefore, in its widest sense, as a process of absorption of insoluble food rendered soluble, whether such food be organic or inorganic. Apparently, then, in the case of the plant the root functions as a digestive organ. But whether or not this analogy is merely superficial can only be determined by an experimental inquiry into the parallelism which may or may not exist between the various excitatory reactions of the root on the one hand and a typical digestive organ on the other.

In order to obtain the large quantity of inorganic material which is necessary to the nutrition of a tree, for instance, it is clear that fresh quantities of charged fluid must be constantly taken up. In order further that this process may be maintained continuously it must be possible to get rid of the useless water, which is accordingly passed off, chiefly from the transpiring leaves, in the form of vapour. The absorption of food and the ascent of sap, or transpiration-current, would appear therefore to be related phenomena. I shall, in the course of the present and following chapters, then, take up in detail the consideration of these two aspects of the problem, which will thus constitute two main lines of inquiry:

(I) Whether or not the excitatory reaction of the root has any similarity to that of digestive organs in general; and 
(2) whether or not the ascent of sap is fundamentally due to similar excitatory reactions.

With regard to the latter of these questions it may be stated here that the nature of the efficient cause of the ascent of sap is universally regarded, in plant physiology, as constituting a problem of the greatest obscurity. The various non-physiological theories which have hitherto been advanced are admitted to be inadequate, as we shall see later. We are thus confronted either with an insoluble problem or with the necessity of finding physiological reactions which will account for the ascent of sap.

As regards the latter of these alternatives, however, objections apparently very serious have been brought forward. Against the physiological character of the action it has been urged (a) that wood, being supposed to be dead, could take no part in the ascent of sap. It is known moreover $(b)$ that killing the roots with boiling water does not prevent the ascent of sap. And, lastly $(c)$, in the wellknown experiments of Strasburger it was found that strongly poisonous solutions can be carried to the tops of trees. From these facts it has been held to be proved that the ascent of sap cannot be dependent on the livingness of the tissues concerned.

But if, on the other hand, it could be shown that these objections were not valid, and if, further, some crucial experiment were devised to demonstrate that excitatory action was attended by a concomitant responsive movement of water in the tissue, it might then be claimed that the physiological theory of the ascent of sap had been established on a firm basis. The attempt to do this will form the subject of the next chapter.

The first question that falls within the scope of our investigation, then, is as to whether the reactions of the root are or are not similar to those of digestive organs in general. We have seen, in the case of the latter, that as there are two opposite activities, of secretion and absorption, so also there are two opposite responsive phases, negative and positive, the 
former being the more characteristic of the fresh condition, and the latter of a specimen which has been previously subjected to continuous stimulation. Before giving any account, however, of these electrical responses, it will be interesting to demonstrate here the occurrence of secretion in young or fresh specimens, when subjected to excitation. The fact that young rootlets secrete, on excitation by contact, has already been seen in the well-known experiment on the corrosion of marble, mentioned above. But I shall now describe a new experiment, in which this fact is even more convincingly demonstrated. I took a specimen of Colocasia, growing in marshy soil. The plant was lifted bodily, with earth adhering, and placed in water, so as to expose the roots gradually, without causing injury. It was then kept overnight, with the roots in normal saline solution, which was slowly absorbed by the tissues. Next morning, again, it was carrefully washed till there was no trace of salt adhering. One of the very young roots was now immersed in very dilute solution of silver nitrate. If the previous washing had oeen effective, there ought now to be no white precipitate, or only the merest trace, formed in the silver solution. The two electrodes of a Ruhmkorff's coil were next connected, one with the silver solution, "and the other with the stem of the plant. On now passing tetanising shocks, the immersed root became excited, and secreted its contained salt solution, this being seen in the silver nitrate as streams of white precipitate.

Turning next to the electrical mode of investigation, we have found that in the digestive organs, the galvanometric negativity, which is the characteristic response of a specimen in the fresh condition, becomes reversed to positivity under continuous stimulation. In the case of Nepenthe, very young pitchers exhibited this normal response of negativity, which was converted, under continuous stimulation, into diphasic, tending towards positivity. Older specimens, again, previously stimulated by the presence of excitatory food-material, 
were found to be in the positive phase, giving rise to response by galvanometric positivity.

In the case of the root, it is interesting to find that there is a similar alternation of responsive phases. For this demonstration I again took the root of Colocasia, and recorded its responses to equi-alternating electric shocks. Among the mass of roots there are naturally some which are dead and decaying. One of these was selected for one electrical contact, while the other was made with a young and vigorous root. The responsive reaction, under these conditions, was found to take place by galvanometric negativity (fig. 2 I4). Under long-continued stimulation, however, I have often found this normal response by galvanometric negativity to be reversed to its opposite, positivity. From this we may pass to the consideration of response in older roots, where the phasic reaction is typically positive.

Now we have seen in previous chapters, as will be remembered, that there are two different conditions

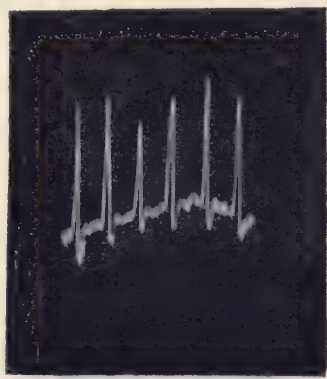

FIG. 214. Photographic Record of Normal Negative Response of Young Root of Colocasia under which the positive may be substituted for the normal negative response. The first is that of reversal under longcontinued stimulation, which we have just seen. And the second occurs when the stimulus falls below the critical level which is necessary to the evoking of true excitation. In this latter case, as we saw further, the incident stimulus increases the internal energy, and causes expansion, positive turgidity-variation, and galvanometric positivity. It would thus appear that one identical stimulus may induce one effect, that of galvanometric negativity, in a highly excitable tissue, and the opposite, or galvanometric positivity, in a tissue that is less excitable. In connection with this question the experimental results which I am about to describe are very significant. 
We have seen that a young root of Colocasia, when fresh, gives the normal response of galvanometric negativity. Taking next an older root of the same plant, and employing the same intensity of stimulus as before, I found the responses to take place, generally speaking, by galvanometric positivity (fig. 215). This would appear to suggest a tendency towards specialisation of function, galvanometric negativity being associated, as we have seen, with secretion, and positivity, in all probability, with the opposite-namely, absorption. A similar specialisation of certain cells for secretion and others for absorption is manifested more

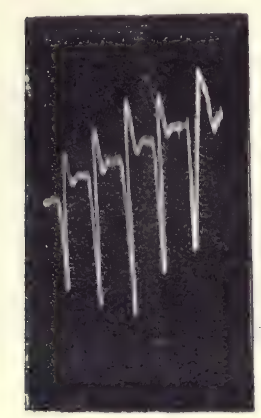

FIG. 215. Tholographic Record of Positive Response in Older Root of Colocasia unmistakably in the digestive organs of the higher animals.

Thus in the young roots the predominant reaction would seem to be secretion, reversed under continuous stimulation to absorption. In the older roots, on the other hand, the predominant reaction must be supposed to be absorptive. Here, then, judging from the electrical indications, we would seem to have proof of that physiological activity in virtue of which water is taken up by the root, thus giving rise to the so-called "rootpressure.' We can also see how, by the summated activities of numerous roots, this 'root-pressure' is kept approximately constant for a certain length of time. Taking longer periods into account, further, we can see that this physiological activity is likely to undergo periodic change, a fact which is evidenced by the known periodic variation of root-pressure. The question, however, of the actual influence of excitation on the process of the ascent of sap will be dealt with in the next chapter.

One form of stimulus to whose action the roots must often be subjected is that of the chemical substances present in the soil, and I undertook to test the electrical variations 
induced by these. The results obtained, at least with the specimens which I have tried, are in general parallel to those obtained by the electrical form of stimulation. Thus, in young roots, in the majority of cases, when subjected to the action of so dilute a solution as 5 per cent. of sodium carbonate, an electrical change of galvanometric negativity was induced. The continued action of this solution, however, tended to induce a reversal to galvanometric positivity. But the reactions of older roots were different-that is to say, in the greater number of the latter cases, a solution of 5 per cent. induced galvanometric positivity; and it required a much stronger solution, of from 5 to 10 per cent., to bring about the reaction of galvanometric negativity.

We have thus seen that in the root, as in the digestive organ, there are alternating phases of secretion and absorption, and that it is by means of the secreted fluid that solid inorganic substances are rendered soluble, for subsequent absorption as food. We have seen moreover that the electrical reactions in the two cases are similar; that in the young root, as in the young glandular organ of Nepenthe, the characteristic response is by galvanometric negativity; and that long-continued stimulation induces diphasic variation, with a tendency towards the reversal to positive. We saw further that older roots, like the glands in the older pitchers of Nepenthe, have a phasic reaction which is predominantly positive. And now, having thus completed our first line of inquiry, we shall turn to the second--the question, namely, as to whether the ascent of sap is or is not essentially due to physiological reaction.

The possible explanations of the ascent of sap may be grouped broadly under two different heads, as either physical or physiological. Under the former of these must be named such theories as those of atmospheric pressure, capillarity, osmosis, and evaporation from leaves. Under the latter, the physiological, the movement of water is regarded as mainly due, in some hitherto undefined way, to excitatory actions by which the sap is propelled in a uni-directioned 
manner, this primary movement being aided by accessory factors.

Among the physical theories which have been propounded for the explanation of the ascent of sap those of atmospheric pressure and of capillarity are admitted to be inadequate. But that of osmosis and transpiration, put forward by Dixon, Joly, and Askenasy, is of much greater weight. According to this, the ascent is brought about by transpiration from leaves. The fluid in the mesophyll cells of the leaves becomes concentrated by evaporation; thus osmotic attraction is set up by the leaves, and the suction thereby exerted is supposed to be transmitted backwards as far as the roots, through cohering columns of water. The difficulties in the way of this theory lie (I) in explaining how a slow osmotic action could produce so rapid a water-current ; and (2), in the absence of any conclusive proof that, under actual conditions within the plant, the water-column could have sufficient tensile strength. Even apart from these objections, however, the fact remains that energetic watermovements take place in the plant in the entire absence of transpiration. For example, sap exudes from the cut end of a tree which may exert a pressure as great as that of a column of liquid I 3 metres in height.

It is thus seen that there is an independent activity or some kind which maintains the movement of water through the plant. That this activity, moreover, is not resident in the root merely is seen from the fact that exudation of water takes place from the tips of grass-blades when their cut stems have been placed in water. Criticising the theory of transpiration, Strasburger rightly remarks that transpiration only makes a place for inflowing water, but cannot furnish the force necessary to convey a large volume of fluid rapidly for a considerable distance through wood. From the consideration of these and other facts, Pfeffer, in his summary, was led to the conclusion which he states as follows: 'A satisfactory explanation of the means by which the transpirationcurrent is maintained has not yet been brought forward. If 
no vital actions take part in it, then it is obvious that we have only an incomplete knowledge of the causes at work and of the relationship of the different factors concerned.' ${ }^{1}$

The inadequacy of these theories to explain the ascent of sap has, then, been freely admitted. With special reference, further, to that of osmosis, I shall myself be able to show that the movement of water often takes place in the plant, in a direction contrary to what it would be if osmosis alone were involved. The fact that the absorption of water is not a merely passive process, but a phenomenon connected with irritability, will be further shown in the depression of the rate of water-movement by such conditions as depress irritability, whereas the opposite circumstance will be found to enhance it.

We are thus driven to examine the possibility of a physiological explanation of the ascent of sap. On such a theory it must be supposed to be brought about by the action of stimulus, inducing reactions expressed in the responsive movement of water. The objections made to the physiological explanation have already been recapitulated. They are ( $\mathrm{I}$ ) that the movement of water is known to take place rapidly, and by preference, through woody tissues which are supposed to be dead; and (2) that when the roots have been killed by hot water, or when poison is supplied, the transport of sap continues to take place. I shall now proceed to examine these arguments, and to show that the objections raised, though apparently so strong, are not really valid.

We shall first refer to the argument which has been based upon the fact that in trees conduction takes place very rapidly through woody portions which are regarded as dead. It is not, it must be noted, implied by this that the presence of wood is essential to the ascent of sap, inasmuch as even in trees there are tracts of living cortical tissue in the roots which have to be traversed before the water can reach the woody tissues. In seedlings of Gramince

1 Pfeffer, Physiology of Plants (English translation), 1903, p. 224. 
only one or two days old, again, water ascends, and is excreted at the tip of the yet unopened leaf. Transpiration is here at its minimum, and the fibro-vascular elements at this early stage cannot be regarded as 'dead wood.' Finally, in herbaceous plants, where woody elements are insignificant, the ascent of sap is seen to take place.

Assuming, for the sake of argument, that in the case of the tree, the mass of wood in the interior were dead, it might still conceivably be of use in the irrigating system as a central reservoir. This would certainly be advantageous to rapidity of transit. At the lower end of the tree, the wood abuts upon the delicate parenchymatous tissues of the root, and at the upper upon those of the leaves. According to the physiological theory, then, it might be supposed that it was by the multiple activity of the cells of the root that water was pumped into the wood; and that at the other end the central reservoir was able to furnish a supply to make up for the constant loss by transpiration. Laterally also, in the stem itself the cortical tissues could draw upon this central supply. Under such an arrangement no part of the plant could be very far away from the reservoir.

As a matter of fact, this sketch corresponds roughly to the working of the tree as an hydraulic machine. The system is, however, somewhat more complex than has been indicated. Besides the central, we have also to remember the presence of lateral reservoirs, in the parenchymatous tissues of the cortex. But the transport of water through these is not, of course, so rapid as through the central, more specifically conducting, system. In the case of herbaceous plants, where the quantity of wood is insignificant, we may regard the central channels as abolished. Here we have soft cortical tissues extending continuously from root to leaves through the stem, and it is obviously through these that the ascent of water takes place. In woody trees, then, there is no reason to suppose that the cortical tissues could not play a similar part in the conveyance of water. The difference is, that in this case there is also an added and 
better channel available, which will naturally come into requisition where quick transit is required.

In a woody trunk, then, we have (I) the outer cortical cylinder of water-conducting tissues, by which the ascent of sap takes place slowly. We have (2) the highly-conducting central woody tissue, which not only allows of water ascending rapidly through it, but is also (3) in lateral communication with the outer cylinder. The hydraulic system thus consists of a large central canal, as it were, connected with innumerable lateral reservoirs, which are the cells of the cortex. When a demand arises for rapidity of water-supply on account of transpiration, we can now see that no less than three different factors are brought into requisition. First there is the rapid upward transit through the wood; secondly, the slow ascent through the cortex; and thirdly, the lateral supply from the cortex by way of the nearest wood.

As regards the last of these, the cortical tissues in contact with the wood act in a manner not very unlike that of the roots towards the soil. That is to say, under different circumstances, they absorb water from it, and excrete water into it, these alternating processes being by no means accidental, but guided by appropriate excitatory reactions.

Turning our attention for a moment to the movements of Mimosa leaf, we find that on excitation the expelled water makes its way to the fibro-vascular tissue. There is here, in the excitable tissue, unlike the case of secretory organs, no external vent, and we see the necessity of a central reservoir to which water excitatorily expelled may find access. On the subsidence of excitation, the water is re-absorbed by the organ, causing expansion and re-erection of the leaf. Such movements of inflow and outflow evidently take place in the trunk of the tree itself. Under the stimulus of sunlight, the excited cortical tissue will squeeze water inwards into the central reservoir. If this takes place, the effect will be seen in a diametric contraction. At the time when transpiration is most rapid, under the action of sunlight, there is thus 
besides the water coming from the roots, an additional supply available from the lateral reservoirs. The loss of water thus sustained by the cortex during the day is made up again at night, when it will suck water outwards from the central reservoir. We have here a case analogous to the action of the excitatory tissue of the pulvinus of Mimosa expelling water into the wood on excitation, and reabsorbing it on the cessation of excitation. The occurrence of these reactions in the cortex explains the observation made by Kraus that the organs of the plant diminish in bulk from morning to afternoon, the reverse process taking place from afternoon to morning.

We have thus seen how important a factor is excitatory reaction in the observed movements of water, even on the supposition that the woody tissue, being dead, is a merely passive agent. The question has still to be attacked, however, whether this assumption, so generally made, is correct, that the wood used for conduction of water is dead. This supposition has arisen from the chemical transformation undergone by the protoplasm in woody vessels. We have seen, however, in the case of the epidermal cells of the skin, that it is possible for chemical transformation to occur, without necessarily being accompanied by the death-change.

Before proceeding to inquire whether the conducting woody channels are really dead, it is desirable to say a few words as to the particular tissues in the wood, which are most effective for this purpose. Many experiments have been carried out to determine this. Among other things various staining fluids have been employed. But an objection raised in the case of some of these has been that the water of such solutions travels faster than the dye dissolved in it. For my own part, I have found the employment of dilute solution of phenolpthaline to be exceedingly delicate and useful for the purpose of this investigation. It is perfectly colourless, and the staining appears only after appropriate development. The cut end of the stem is placed in this dilute solution and left for some time. Transverse 
sections of the stem at different heights are then made and placed in the field of a microscope. There is now nothing distinguishing to be seen, as the solution sucked up by the stem was colourless. Dilute solution of potash, however, will act on this as a developer. The particular tissues, therefore, through which the solution has been conducted, on now being subjected to the action of this agent, become of a rich crimson colour. By this means it is easy to see, as already determined by various observers, that it is the younger, or 'sap-wood,' which is concerned in the work of the rapid conduction of water, the older, or 'heart-wood,' being ineffective for this purpose. If, however, the wood taking part in the ascent of sap had been dead, and acted as a passive agent merely, it is difficult to understand the reason of this selective action of the younger, and presumably more living, woody tissues.

It occurred to me, finally, that as electrical response is an indubitable concomitant of the excitatory reaction of living tissues, the question as to whether sap-wood was alive or dead could be subjected to a decisive test. For this purpose I took various strips of sap-wood from different woody plants. The cortical tissue was in each case carefully removed, and the specimens were placed in water, allowing them a period of rest. The first experiment was to observe whether local stimulation by the Rotary Mechanical Stimulator did or did not evoke electrical response. I found from this, that mechanical excitation of the sap-wood induced considerable excitatory response of galvanometric negativity. I then subjected the same tissue to the action of boiling water for a length of time, and again tested its electrical reaction by the same method. The wood was found to be very resistant to the action of heat, and it was only after long immersion that the responses were entirely abolished. Drying was in fact found, significantly enough, to be an easier method than the application of heat, to kill, and therefore to abolish the responsiveness of, the wood. If the wood be first dried, and then soaked in water, it entirely ceases to manifest electrical 
response. The ordinary wood of commerce exhibits no response. The familiar fact that the cut end of a woody stem, when not placed in water immediately, ceases to suck up water, has been supposed to be due solely to the intervention of air-bubbles. From the experiment which I have described, however, it would appear that the death of the exposed tissue by drying must be included here as a factor in this abolition of suction.

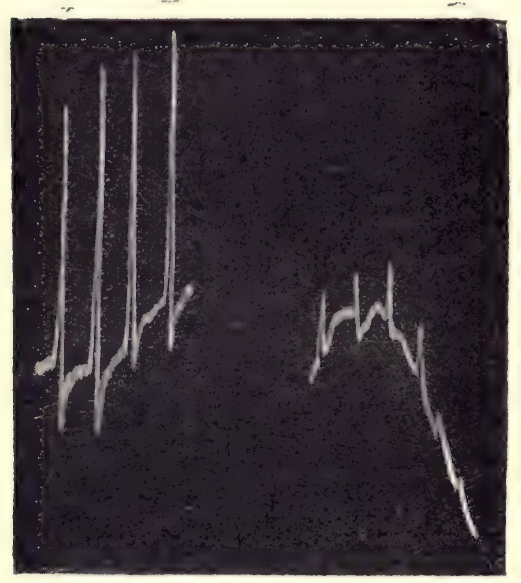

FIG. 216. Photographic Record of Electrical Response of Sap-wood

The normal negative responses seen in the

first series are depressed after application of chloroform in the second.

With sap-wood I was also able to obtain the indication of galvanometric negativity in response to thermal stimulation. Hot platinum wire was applied at a distance of $5 \mathrm{~mm}$. from the proximal contact. Response was thus due to the transmitted effect of excitation.

I was next desirous to obtain photographic records of the normal response of living wood and its variations under chemical agents. For this purpose I employed both the electrical and vibrational modes of stimulation. For the first of these, the strip of sap-wood was cut in the form of a two-pronged fork, of which one prong was killed by exposure to the drying influence of the air, while the other was kept alive by immersion in water. The specimen was now placed in water as a whole, in order to moisten the dried half. After this, electrical connections were made in the usual manner with the killed and unkilled ends of the specimen. On next subjecting it to equi-alternating shocks' response was obtained as induced galvanometric negativity of the living prong. These responses are seen in fig. 216 in the first series of records to the left. Chloroform was next 
applied, and we observe the consequent depression of response; when the chloroform was blown off the responses were found to undergo revival.

In the next experiment, a specimen of living wood was mounted in the vibrational apparatus, and its normal responses taken. I next applied copper sulphate, and the record shows the consequent abolition of response (fig. 217). I have thus been able to establish the fact that the woody vessels of the sap-wood are not dead but living, and hence fully susceptible of physiological reaction. This will, I think, be found to dispose of one of the difficulties raised in regard to the physiological theory of the ascent of sap.

We come, secondly, to the objections that have been based on the ground of the ascent of sap through a tree whose roots have been killed by boiling water, and, further, on the experiments of Hartig and Strasburger. These observers set cut ends of trees in tubs of poisonous

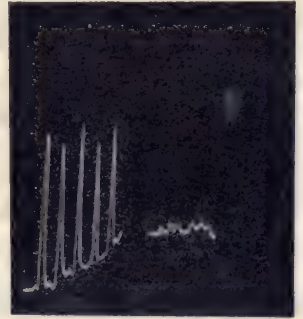

FIG. 217. Photographic Record showing Normal Responses ot Living Wood to Vibrational Stimulus, and the Abolition of Response by a Toxic Dose of Copper Sulphate

solutions, such as copper sulphate, which were found, in spite of their toxic character, to ascend to the leaves. It is clear that if such violent protoplasmic poisons ascend the trunk, they must kill all the cells lying in their path. And from this it was inferred that the living cells in the stem could not be necessary to the rise of sap. Strasburger was thus led to the conclusion that 'the supposition that the living elements in any way co-operate in the ascent of the transpiration current is absolutely precluded.' ${ }^{1}$

It does not, however, appear that this inference on the part of Strasburger was justified, for we must remember the fact that any cut piece of stem when placed in water is found to exhibit suctional activity. Hence the active cells concerned-if the process is to be regarded as due to such-

1 Strasburger, Text-book of Botany (English translation), 1903, p. I88. 
must be distributed throughout the plant. Death of a given zone, then, would arrest the activity of that particular zone but not that of another zone higher up. Thus a poisonous solution would only abolish activity in those cells which it had already reached. The activity of cells above would remain unaffected. That the death of cells below offers no resistance to the passage of water, when suctional activity is maintained above I have demonstrated elsewhere, and shall again show in the course of the next chapter, in cases in which the lower part of the plant was killed by boiling water. Under the action of poisons, similarly, I have been able to show that a poison can pass easily through killed tissues owing to the suctional activity of cells higher up. This was demonstrated by means of experiments on Desmodium gyrans, where the cut end of the petiole was placed in copper sulphate solution. It is fortunate that in this case, during the ascent of poison, we have areas whose activity is manifested visibly by the rhythmic motile indications of the pulvini of the inserted lateral leaflets. That copper sulphate solution arrests rhythmic activity, and induces death, is seen by the rapid stoppage of pulsation when we apply it directly on the pulvini of the pulsating leaflets. When it is applied, however, at the cut end of the petiole, the arrest of pulsation only takes place after sufficient time has clapsed for the poison to ascend through the intervening distance. This shows clearly that successive zones are killed one after another, and that the death of a point below does not stop the suction above. From this experiment it is evident that the application of poison, at the root, or the cut end of a stem, need not be expected to arrest suction until the whole plant has been killed, and from Strasburger's account of his experiment it is evident that the movement of water did come to a stop when the poison reached the top of the tree.

We thus see that the objections which have been raised, with regard to the physiological nature of the ascent of sap, are not valid. I shall therefore proceed in the next chapter to describe crucial experiments in demonstration of the fundamentally excitatory character of this process. 


\section{CHAPTER XXVI}

\section{THE EXCITATORY CHARACTER OF SUCTIONAL RESPONSE}

Propagation of excitatory wave in plant attended by progressive movement of water-Hydraulic response to stimulus-The Shoshungraph-Direct and photographic methods of record-Responsive variations of suction under physiological modifications induced by various agents-Effects of lowering and raising of temperature-Explanation of maintenance of suction, when root killedEffect of poison influenced by tonic condition-Effect of anæsthetics on suctional response-Excitatory versus osmotic action--Stimulation by alternating induction-shocks-Terminal and sub-terminal modes of applicationThree modes of obtaining response-records, namely (I) the unbalanced, (2) the balanced, (3) the over-balanced-Renewal of suction previously at standstill, by action of stimulus-Reponsive enhancement of suction by stimulus-After-effect of stimulus - Diminution of latent period as after-effect of stimulus-Response under over-balance-Response under sub-terminal stimulation-Variation of response under seasonal changes.

In the last chapter it was shown that the various objections hitherto urged against the excitatory nature of the ascent of sap were not justified. In the course of the present chapter, therefore, I shall adduce proofs that the water-movement in the plant is the result of stimulatory action. Instead of vaguely referring the phenomenon to physiological activity, moreover, we shall attempt, proceeding from the basis of other excitatory reactions, already clearly established, first to see whether inferences based on these are capable of explaining the present problem, and secondly, to subject those inferences themselves to the test of experimental investigation.

We cut a certain length of the stem of Mimosa, and keep it immersed for some time in a very dilute solution of common salt, until the tissue has become charged with this. The specimen is then taken out, and thoroughly rinsed with clean water. It is now held vertically, with the lower end dipped 
in a highly dilute solution of silver nitrate. A portion of the tissue higher up is now excited by contact with a hot wire. The excitation thus induced is then found to travel through the intervening distance, with a velocity characteristic of the conducting power of the tissue. The arrival of the excitatory wave at the lower end is attended by an expulsion of the cell-sap containing the salt solution previously absorbed. This expulsion is instantly made visible by the formation of a dense white precipitate of silver chloride. From this experiment it is seen that the passage of excitation is attended by a forward movement of water in the direction of propagation.

The next point to be realised is that a strong or a longcontinued stimulus will give rise in the tissue, not to one, but to a multiple series of propagated waves. If the tip of a leaf of Biophytum be strongly excited, we see successive waves of excitation, marked by the serial fall of the motile leaflets, proceeding again and again in the centripetal direction, from the terminal excited point. If, similarly, the end of the root be excited, by any means, an excitatory movement of water will be induced, proceeding away from this end, in an upward direction. It must be remembered, however, that excitation proceeds in all directions from the excited point. If then the point of excitation be terminal, it is evident that the direction of propagation, being away from this, will be upwards. But if the tip of the root be highly excitable, then, owing to local excitation, there will also be a certain amount of secretion into the soil. Even highly excitable tissues, however, after continuous stimulation, show a tendency, as we have seen, to the reversal of their character istic response. This secretion at the terminal point of the root will tend to become changed into absorption. Again, looking at the succession of excitatory waves propelling water upwards, we can see that these will leave a deficit of cell-sap behind, which will further act rather for the absorption than for the secretion of fluid. And in addition to these, if there be any other directive influences, such as 
evaporation from the leaves, they will tend to help the particular uni-directioned flow. We have seen that these conclusions are confirmed by the results obtained in the eiectrical response of the roots. We there saw that very young roots give at first negative responses, which are afterwards reversed to positive, under continuous stimulation. Older roots, as we also saw, give response by positivity. We saw, further, that there was much reason for regarding negative response as associated with the secretion, and positive with the absorption of fluid.

Thus the serial propagation of excitation from cell to cell, with the concomitant movement of water, will normally be upwards. In this connection it is very significant that the younger portion of the fibro-vascular bundle is the preferential channel for the conduction, at once of water and of excitation. It is thus seen that a one-directioned movement of water may be produced by the multiple excitatory activity of the tissue. And just as the multiple activity of certain tissues, say, for instance, the leaflets of Desinodium, may be gauged by their multiple mechanical movements, so in the rate of the water-movement we have a means of measuring the intensity of the multiple rhythmic activity of those which are concerned in the ascent of sap. This would be analogous to the measurement of the rhythmic activity of the heart, by a determination of the rate of flow of the circulating blood. In the case of the plant, however, this rate of movement might be measured, either by means of the propulsion of water forwards, or by the suction exerted behind.

In order to demonstrate the fact that the water movement in the ascent of sap is mainly dependent on excitatory reactions, it is necessary to have at our disposal some means of rapidly observing and recording the variations induced by physiological changes in the rate of ascent. For this purpose I was successful in devising the Shoshungraph or suction-recorder, described in detail in my book on 'Plant Response.' ${ }^{1}$ 
This instrument consists of (I) an arrangement by which the specimen may be subjected rapidly to the action of different excitatory or depressing agents; (2) a potometric tube for the measurement of changes of suctional activity, under different external conditions; and (3) a contrivance by means of which the movements of the water-index, with their time-relations, are recorded. The principal parts of the apparatus are seen in fig. $218 . \mathrm{V}$ is the plant-vessel, in which the specimen is mounted, with or without roots, by means of a watertight india-rubber cork. $\mathrm{R}$ is the reservoir, which may be filled with hot or cold water, or with the required chemical solution. By appropriate manipulation of stopcocks, by means of key $\mathrm{K}$, the water in the plantvessel may be replaced quickly by any of these, and the effect of the changed condition on the rate of water-movement observed.

A direct record of the rate of movement may be made on the revolving drum, actuated by clockwork, as shown in the figure. For this purpose, a pen, 1', is fitted over the potometric tube, by means of a brass collar, which has a rectangular opening, kept always coincident with the waterindex. The collar, carrying the pen, is attached to a thread, which passes round small pulleys. One end of this thread carries a counterpoise, $\mathrm{M}$, and the other is wound round a wheel, W, which can be so manipulated as to make the pen follow the movements of the water-column. When the water-index is followed in the way described, a direct record of the water-movement in the plant is obtained. A curve is thus traced, the ordinate of which represents the quantity of water sucked up, and the abscissa the time. The slope of the curve thus gives the rate of movement. As long as suction is uniform, this slope remains constant. If, however, any exciting agent increases the rate of suction, there is an immediate flexure in the curve, which thus becomes steeper. A depressing agent lessens the slope, and when suction is abolished, the record becomes horizontal. For the detection of the slightest variation in the rate of suction, the Method of 
Balance is employed, which depends upon an application of compensation, by which, under normal conditions, the waterindex is kept stationary, though the suctional movement in

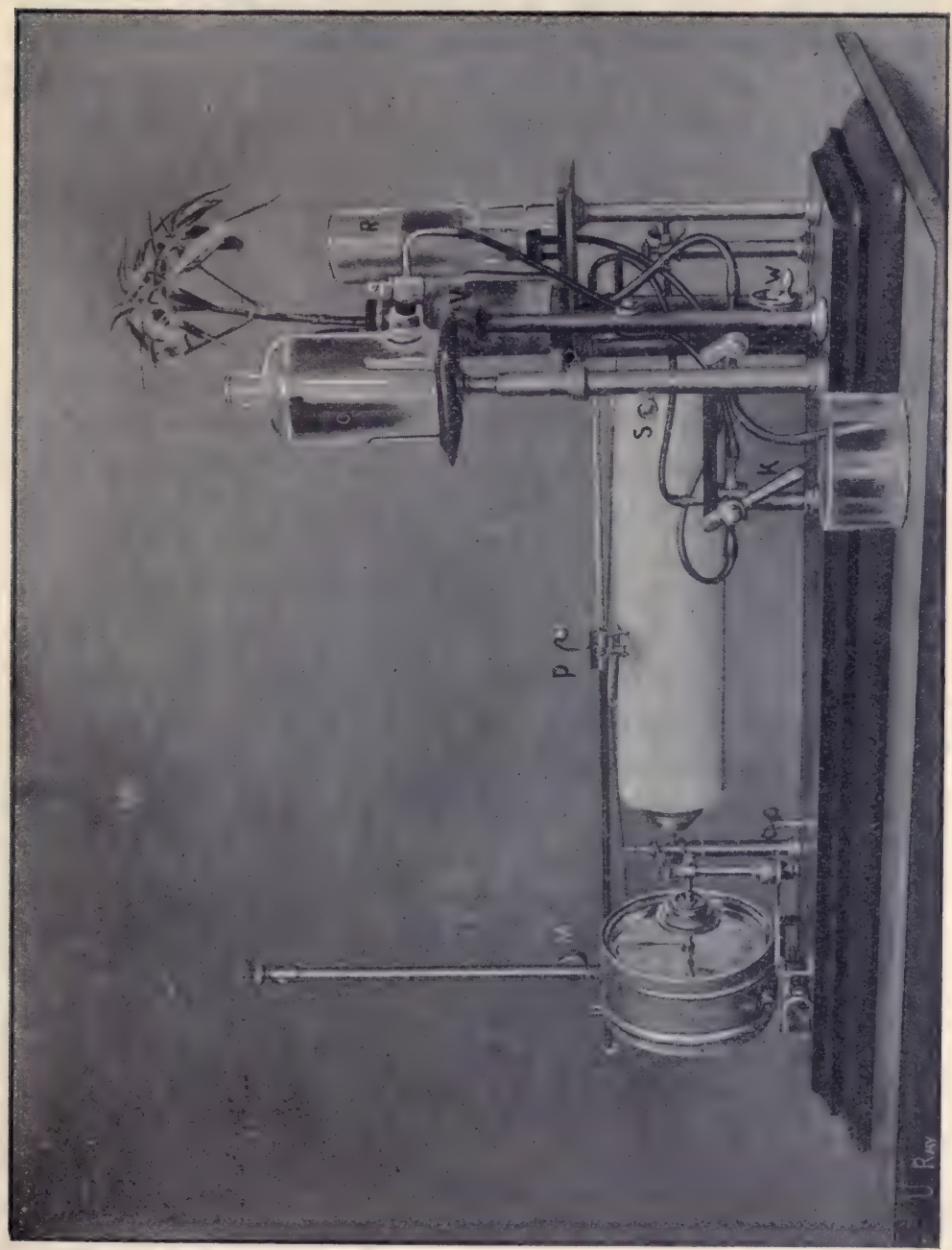

FIG. 218. The Shoshungraph

$\mathrm{v}$, plant-vessel ; R, reservoir ; C, compensator, whose balancing height is adjusted by rack and pinion, s; $\mathrm{k}$, key for manipulation of four-way stop-cock; $\mathrm{P}$, recording pen, with counterpoise $\mathrm{M}$, manipulated by wheel, w. The drum/is rotated by the clock at uniform speed. 
the plant is in no way disturbed. The balance is obtained by allowing water to erter the plant-vessel, from the compensator, $\mathrm{C}$, at a rate exactly equal to that of its withdrawal by suction. Thus, under a condition of balance, the record becomes horizontal. An exciting agent now produces an inclination of the record upwards, or a depressing agent a declination downwards. This was the instrument employed by me for the obtaining of records in a simple manner of suctional response and its variations, under different physiological modifications. A fuller account of the method and the results obtained by it will be found in my book on 'Plant Response.' As the subject of the ascent of sap is, however, of extreme importance, I thought it desirable to see to what extent the sensitiveness of this instrument could be raised, and also to devise means, in connection with it, for the automatic record of results obtained. For the latter purpose I employed photography. As the water-index, whose excursions in the potometric tube are to be recorded, is transparent, it is necessary to provide an additional opaque index, which shall move in and out with it. This consists of a short length of mercury, lying in contact with the end of the water-column. The potometer tube is placed in the field of a magic lantern, and the index is focussed on a moving photographic plate by means of an objective. The sensitiveness of this method of record may be increased in two ways. First, the bore of the capillary tube may be made finer and finer. But this cannot be carried to an extreme, as the capillary offers great resistance to the free movement of the index. Secondly, the sensitiveness may be increased to any extent by the employment of a highly-magnifying and short-focus objective. By the combination of both these devices, we are able in practice to arrive at an extraordinary degree of sensitiveness, qualifying us to attack some of the most difficult problems with the greatest ease. For our present purpose, however, it is not by any means necessary to approach the limit of this sensitiveness. The photographic records given in the course of the present chapter 
were obtained with a tube having a bore I square $\mathrm{mm}$. in section, and employing an ordinary magic lantern objective, which casts an image without any magnification. The Method of Balance again, as we have seen, affords us another opportunity of arriving at an experimental adjustment of great sensitiveness.

The experimental delicacy obtained by high magnification can only be used to the greatest advantage when coupled with the Method of Balance, if we have means at our disposal for securing the utmost possible perfection of the balance. We have seen that the balance is adjusted when the rate at which water is removed from the plantvessel by suction is exactly equal to the rate of its inflow from the compensating vessel, C. This compensation is roughly effected by the rack and pinion, s, which serves to regulate the flow by raising or lowering the compensating vessel (fig. 2I8). For the purpose of the final adjustment the narrow bore of the thick india-rubber tubing, which connects the compensator with the plant-vessel, is capable of gradual constriction. This must be accomplished by equal compression on all sides, as bilateral compression alone would act to induce a discontinuous closure and sudden arrest of flow. If the compressing arrangement be something after the model of the iris-diaphragm, then the bore, which regulates the flow, may be constricted gradually and continuously. With such an arrangement it is easy to arrive at a balance so perfect that the index appears to be quite stationary. Under-balance would now make it move, say to the right; and over-balance to the left.

We shall now proceed to show that those agents which exalt physiological activity also act to enhance suction; and that those which induce physiological depression will also depress suction. One of those which enhance the multiple activity of the tissue is, as we know, the rise of temperature ; whereas cooling, or lowering of temperature, tends to depress it, even to the extent of abolition. Thus an automatically vibrating leaflet of Desmodium has its vibration-frequency. 
enhanced by a rise, and depressed or arrested by a fall, of temperature. A similar effect is seen to occur in the suctional response of plants. Thus, in a given specimen of Croton, application of cold water at $4^{\circ} \mathrm{C}$. to the root was found to arrest the suction in the course of 8 minutes. This arrest by cold was not permanent, for the normal rate of suction reappeared on the return of the water to a normal temperature. On applying water of raised temperature to the root, on the other hand, the rate of suction was immediately found to be enhanced. This is illustrated in the following record (fig. 2 I9), in which the normal rate of suction at $23^{\circ} \mathrm{C}$. was

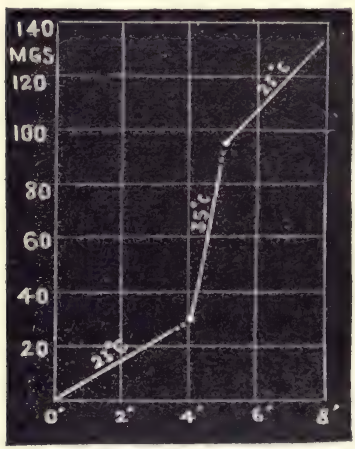

FIG. 219. Curve showing Normal Suction at $23^{\circ}$ C., Increased Suction at $35^{\circ}$ C., and the After-effect persisting on Return to Normal Temperature
7 cubic $\mathrm{mm}$. in volume, or $7 \mathrm{mg}$. in weight per minute. The ap. plication of water at $35^{\circ} \mathrm{C}$. now induced a steep rise of the curve, inclicating an enhanced rate of suction of $58 \mathrm{mg}$. per minute, or more than eight times the rate at $23^{\circ} \mathrm{C}$. On now once more substituting water at $23^{\circ} \mathrm{C}$, the rate is seen to become lowered, but not to fall so low as at the beginning. It now fell from 58 to $14 \mathrm{mg}$. per minute, instead of returning to the original $8 \mathrm{mg}$. This is due to the fact that the internal energy of the tissue has been raised in the meantime by the absorption of warm water, the enhancement of the normal rate being a persistent after-effect of this.

We shall next take up the apparently anomalous case, in which, when the root has been killed by pouring boiling water over it, the suction of the plant is nevertheless maintained. In such an experiment the normal record was first taken, and boiling water was then passed through the plantvessel continuously for some time till the roots were killed. On allowing the water in the vessel to return to the temperature of the room, it was found that suction was taking place 
at an even greatcr rate than ordinary. This result would at first appear to show that protoplasmic activity had nothing to do with the ascent of sap, and the objection would have been fatal if the activity which produces suction had been confined to the roots alone. But in reality, as we have already seen, such activity is present, to a greater or less extent, throughout every zone of the plant. The only part which is killed, however, in the experiment just described, is that which is actually immersed in, or in immediate contiguity with, the boiling water; and the unkilled tissues above continue their suctional activity unabated. The increase in the rate of suction is to be explained by the fact that the entrance of water, instead of being effected through the extremely attenuated channels of the root-hairs, now takes place through the whole mass of the root, acting virtually as a wet rag tied round the base of the living stem. The mass of water which it is thus possible to suck up directly through the broad-sectioned stem is evidently much greater than could have been taken in through the resistant, organically-conducting channels of the rootlets.

The fact thus demonstrated, that the local death of a given zone does not per se arrest the suctional activity of the tissues above it, explains why a poison may be carried to the top of a tree. It is evident that only when it has thus been conveyed, and when all the tissues have thus been killed, could a permanent arrest take place. And Strasburger himself admits that arrest under these conditions does occur.

With reference to the effect of poison, again, it is important to bear in mind that its toxic effect depends, to a certain extent, on the tonic condition of the tissue. Thus a Desmodium leaflet which was moderately vigorous had its pulsatory movement arrested soon after the local application of the copper sulphate solution. In more vigorous leaflets, however, the arrest did not take place till after a considerable length of time. Indeed, in some cases, after a preliminary arrest, the leaflet is able to shake off the effects of the poison 
absorbed, by some process of accommodation. I have shown elsewhere $^{1}$ that the effect of poison on the response of growth is modified to a remarkable extent by the different tonic conditions of the tissue. The experiments in question were carried out on similar specimens of Crinum lily, in which the only difference induced depended on the fact that one set had been kept at a temperature of $30^{\circ} \mathrm{C}$., and were thus in moderate tonic condition, while the others had been maintained at $34^{\circ} \mathrm{C}$., bringing about, as I have shown, an optimum tonic condition. The application of a 5 per cent. solution of copper sulphate to one of the first of these was found to induce the rapid decline and final arrest of growth, while a similar application, on a specimen in the optimum condition of the second set, induced a preliminary exaltation followed by a slow depression and ultimate arrest of growth, the last-named, however, being reached only after the lapse of a considerable time.

The difference of effect under different tonic conditions was still more strikingly exhibited by the application of a smaller dose-namely, of a I per cent. solution. This was found to induce a depression of growth, which was ultimately fatal to the plant, in the case of specimens kept at $30^{\circ} \mathrm{C}$. But when the same dose was applied to a plant which had been kept at $34^{\circ}$ C., the effect was seen in a marked exaltation of the rate, for a fairly long time, after which it shook off the effect of poison altogether, resuming its normal rate of growth.

Thus the effect of poison on the various activities of the plant is seen to depend not only on the amount of the agent, and the duration of application, but also on the tonic condition of the tissue. Strong and prolonged applications will abolish all active processes, by inducing the death of the plant. In accordance with this, I find that in certain plants under the action of copper sulphate the arrest of suction is more rapid than in others. All alike, however, exhibit permanent arrest sooner or later.

1 Bose, Plant Response, pp. 487-488. 
The question of the excitatory nature of the ascent of sap may again be tested by the application of anæsthetic agents, such as solution of ether. The record is first taken of the actual rate of suction, and then by quick manipulation of the double key, the water in the plant-vessel down to the base of the specimen is replaced by ether solution. The original rate of suction had in a particular case been 40 cubic mm. per minute. After the application, however, this became depressed, and in the course of four minutes underwent a preliminary arrest. This short arrest was succeeded by reversal, or expulsion, which lasted for fourteen minutes, and then gave place to what was practically permanent arrest (fig. 220).

I have occasionally observed an interesting variation in the arrest of suction induced by ether. Shortly after the application just described an arrest of suction is induced. This, however, is only preliminary, suction after an interval being renewed at a very slow rate. When this has proceeded for some time, the process undergoes a

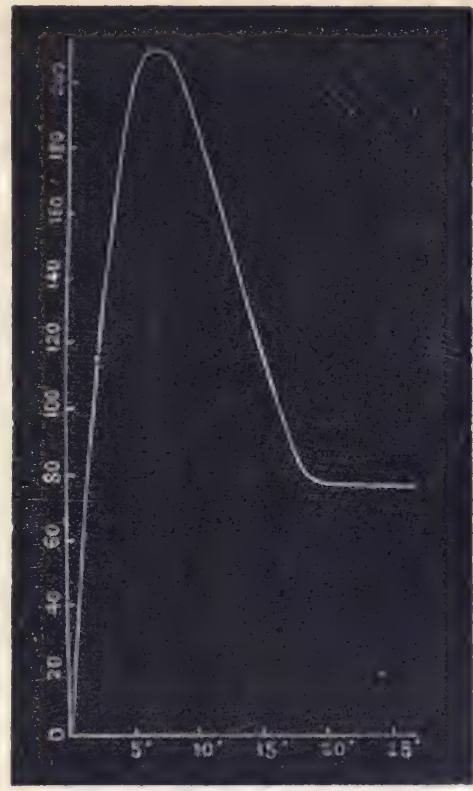

FIG. 220. Action of Anæsthetics in Abolition of Suction

Solution of ether substituted for water at point marked with dots... Suction abolished within fifteen minutes. gradual and final arrest.

It has already been said that though the excitatory reaction is to be regarded as the fundamental cause of the transport of water in the plant, yet there are other factors which undoubtedly contribute to that result. One of these may be the favourable disposition of osmotic substancesfor example, the concentration of cell-sap consequent on 
evaporation, in the leaves. That this osmotic effect is, however, merely secondary, and that the ascent is chiefly due to excitatory action, is seen in certain experiments which may be mentioned here, in which the ascent takes place with even greater vigour than before, when it is opposed by an osmotic influence.

The various solutions of salts are very unequal in their physiological action : some, like potassium nitrate, are neutral, ${ }^{1}$ but others, as strong solutions of sodium chloride, are ex-

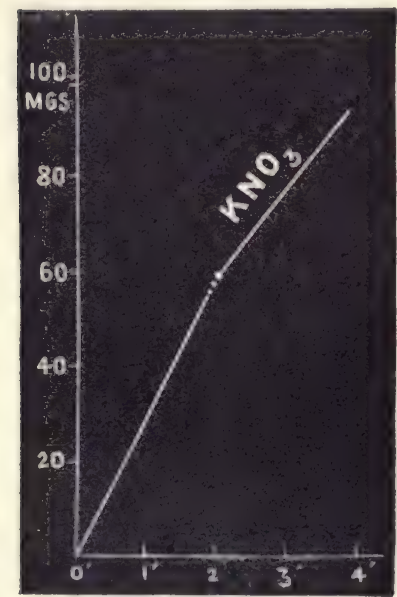

Fig. 22I. Effect of Strong $\mathrm{KNO}_{3}$ Solution

The first record shows the normal and the second the depressed rate of suction caused by the reagent. citatory. Thus the action of a strong solution of potassium nitrate is physiologically more or less neutral, while its osmotic action, at the same time, is pronounced. A strong solution of common salt, on the other hand, is both excitatory and osmotic.

If then we apply $\mathrm{KNO}_{3}$ solution to the cut end of a stem, water will be osmotically withdrawn from the plant, in opposition to the normal ascent of sap. There will thus be a depression of the rate of suction after the application, as seen in the following record (fig. 22I), which I obtained with a cut

branch of Croton. If, however, in a similar experiment, a strong solution of $\mathrm{NaCl}$ be applied, two antagonistic reactions will be set up. One, due to the osmotic action, will oppose suction, and the other, due to the excitatory nature of the reagent, will accelerate it, while the resultant effect will be modified by the excitability of the experimental plant. In fig. 222 is shown the effect of $\mathrm{NaCl}$ solution in

1 It should be mentioned here that even such neutral salts, in strong solution, induce physiological depression. 
increasing the suction of a specimen of Croton in a favourable condition of excitability. We have here a very great enhancement of the ascensional movement caused by this solution, which would, acting osmotically, have retarded the normal rate. The physiologically excitatory action of strong sodium chloride, however, is not permanent, and the enhanced excitability is followed by depression. The effect on suctional response in such cases, then, is modified by the factor of time, the first enhancement being followed by a fall below the original normal rate of suction.

The experiments which I have here described show how intimately suctional response is connected with excitatory réaction. Having thus seen that any physiological modification of the tissue is attended by an appropriate change in the rate of suction, it only remains to demonstrate finally the fact that stimulation is attended by a responsive movement of water in a tissue. In order to do this, we should have at our disposal some means of applying stimulus which is capable of

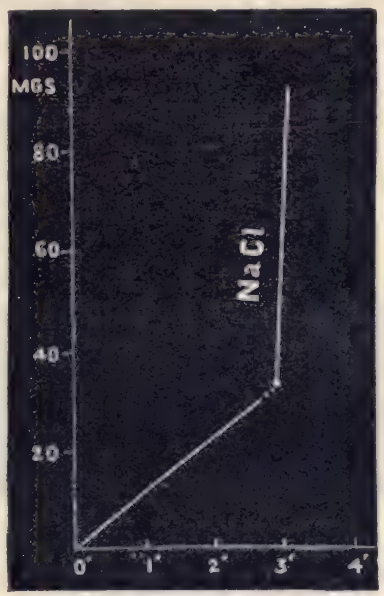

FIG. 222. Effect of Strong $\mathrm{NaCl}$ Solution

The first record shows the normal and the second the exalted rate of suction caused by the reagent. measurement, capable of graduation, and not in itself of a nature to disturb the delicate balance of the shoshungraphic record. The end sought after was first to record the normal rate of suction, and then to observe the effect immediately induced in this by the application of the stimulus, the process of record being uninterrupted meanwhile. The only form of stimulus which would comply with these conditions is the electrical, given by tetanising induction shocks, of longer or shorter duration. The possible objections to the use of this form of stimulus are as follows : 
I. The water in the plant-vessel may be supposed to undergo decomposition by electrolysis. There may also be a certain evolution of heat in the plant-vessel. 2. In a sluggish tissue, such as that of the plant, the excitatory value of induction-shocks may not prove sufficient to modify suctional response.

With regard to the first of these objections, it is to be borne in mind that the shocks, being alternate, will produce but little polarisation effect. The heating effect of a current so small in quantity, moreover, is also likely to be very

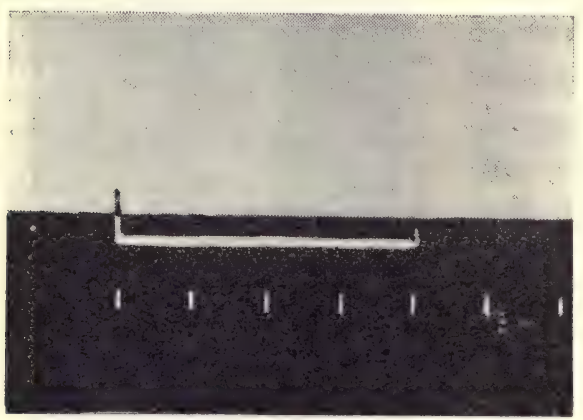

FIG. 223. Record of Blank Experiment showing Absence of any Disturbance of Record from Induction-shocks as such

Photographic record of excursion of mercury index in Shoshungraph. The thick white line shows duration of application of shock. Time-marks in this and other photographic records represent intervals of five minutes. slight. The extent of the disturbance from these causes can, however, be determined by a blank experiment. The electrodes, by whose proper application the plant is excited, are allowed to hang down in the plantvessel without direct attachment to the plant itself. The alternating current will now pass through the water, only a very small fraction of it, incapable of producing effective excitation, passing through the plant. The Shoshungraph is adjusted for the balanced condition, given as we have seen, a horizontal line of record, and that intensity of induction-current which is to be used in subsequent experiments is now passed through the plant-vessel for twenty minutes continuously. It will be seen from the record (fig. 223), that no disturbance was induced by this in the balanced line, thus proving that such disturbing effects, if they exist, are in practice negligible. In actual experiments, where the excitatory effect is to be studied, 
the duration of application is often less than a minute, and seldom exceeds five minutes. It should also be borne in mind that individually and collectively the effects of the disturbing causes enumerated would, if anything, be towards expulsion from the plant-vessel. We shall see, however, that the typical responsive effect is movement in the opposite direction, indicative of an enhancement of suction. With regard to the second cause of misgiving, as to whether planttissues may or may not be made to exhibit excitatory variation by means of induction-shocks, I have found that some specimens, notably those of Croton, are sufficiently susceptible to this form of stimulation. For this purpose it is necessary to use very strong induction-shocks from a large coil. This necessity is further increased by the fact that much of the induction-current is uselessly and unavoidably shunted by the water in the vessel.

This electrical stimulation may be applied to the cut end of the branch, immersed in the water of the plant-vessel, in either of two different ways. The first of these may be described as the Terminal Mode of Application, its object being to localise the excitation more or less at the lower end of the specimen. This is done by tying two small pieces of platinum, in connection with the electrodes, to diametrically opposite sides of the base of the stem by means of a thread. Or two pins, in connection with the electrodes, may be pricked into the lower section of the specimen near its circumference. This transverse mode of stimulation, across the diameter of the stem, is not, theoretically, so effective as longitudinal stimulation would be, but under the particular experimental conditions nothing better could be devised (fig. 224). The second mode of applying this electrical stimulus I shall distinguish as Sub-terminal. Here, two pins in connection with the electrodes pierce through the stem, one above the other, in planes at right angles to each other (fig. 225). After arranging the electrical connections in any one of the ways enumerated, the specimen is adjusted in the Shoshungraph, and allowed a period of rest for the 
passing off of excitatory effects of preparation. The record of normal suction is then taken by means either of the ordinary recorder or of photography. The variation induced in the record after the application of stimulus, then, exhibits the cffect of excitation.

In order to test the results in as many ways as possible, I arranged for three different methods of record. The first was without balance. Here the slope of the suction-curve indicates the normal rate of suction, the enhanced rate under stimulus being indicated by flexure and increased steepness.

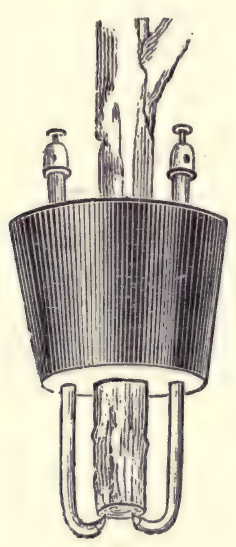

FIG. 224. Terminal Mode of Application of Stimulus

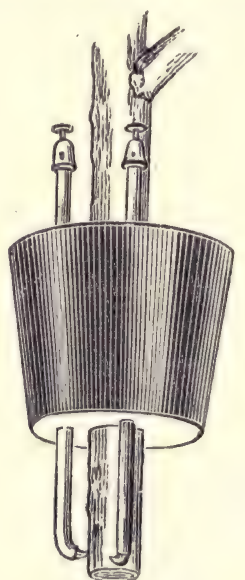

FIG. 225. - Sub-terminal Mode of Application of Stimulus

A diminution in the rate of suction, or an expulsive effect, would, on the other hand, be indicated by a corresponding diminution of the slope, or reversal of the curve.

The second method of record is carried out under exact balance, and is, as already explained, an extremely delicate means of detecting variations in the rate of suction. If this rate be enhanced the curve rises suddenly from the balanced horizontal line, a depression inducing, on the contrary, a downward movement. The last method is that of Overbalance, where, owing to a supply of water from the com- 
pensator at a uniform rate in larger quantity than necessary, a movement of the index is induced in a direction opposite to that of suction. Here the slope of the curve, due to overbalance, is down, and should the stimulus cause any enhancement of suction, the steep down-curve must be replaced by a less steep, or horizontal, or up-curve. The Method of Balance is, as I have said, the most delicate, but this Method of Overbalance enables us to detect the after-effect of stimulus in a striking manner.

As regards the form of this response by movement of water, reference has already been made to previous results, in which we saw that the excitatory effect in the plant, as studied by electrical response, was of two different types, according to certain phasic conditions. Highly excitable roots, we found, gave response by galvanometric negativity, indicating secretion or expulsion of water. Less excitable roots, on the other hand, gave response by positive variation, most probably indicating responsive absorption. These two opposite effects actually occur, as I find, under different phasic conditions, in the suctional responses which I am about to describe.

I shall deal first with the results which I invariably obtained in carrying out experiments on Croton and certain other plants during the month of February. The Indian winter was just over, and the spring had not yet fully set in. The nights were still cold, though the days were growing warmer. The plants, therefore, owing to these peculiar climatic conditions, must be regarded as having been somewhat sub-tonic. My first experiment related to the initiation of suctional activity in a specimen which, in this respect, had been previously at standstill. Stimulus might here be expected to renew that multiple activity on which suctional response, according to our theory, depends. We may here refer once more to the initiation of multiple activity by stimulus in a leaflet of Desmodium previously at standstill.

I have shown that when this plant is deprived of its store of latent energy by unfavourable conditions, then the 
multiple movement of its leaflets is arrested. If the plant, for instance, be kept for some time in a dark room, the leaflets cease to pulsate. But if now an electrical shock of moderate intensity be given to the pulvinus, the incident stimulus, by its excitatory action, gives rise to a number of responsive movements, which again come to a stop as soon as the imparted energy is exhausted. Or we may, in such a case, employ the stimulus of light. A record of the subsequent effect has already been given on a previous page (cf. fig. I4I).

It will there be noticed (I) that the quiescent leaflet is thrown into pulsatory movements after the lapse of a short latent period; (2) that the increasing absorption of stimulus has the effect of augmenting the amplitude of response; and lastly (3) that, owing to the presence of latent energy derived from the impinging stimulus, the activity of the leaflet continues for some time, even on the cessation of stimulus itself. It is, in fact, by that enhancement of the tonic condition, which comes about by the continuous absorption of energy from the environment, that the apparently autonomous response of the leaflet is maintained. There is, as has been said before, no essential difference between multiple and autonomous response. A tissue, which was responding autonomously, comes to a state of standstill when its store of latent energy falls below par. Conversely, by the accession of energy from external stimulus, activity is resumed, multiple passing into autonomous response.

Returning, then, to the hydraulic mode of response, as observed in variations of suction, we might expect that the irnpact of stimulus would initiate this in a tissue at suctional standstill. For this experiment I took a specimen of Croton and mounted it, with terminal electrodes, in the Shoshungraph. At this time it showed a moderate rate of suction. It was then kept undisturbed in a dark room for forty-eight hours, at the end of which time, owing to the run-down of its latent energy, the suctional activity was found to be arrested. But on the application of electrical stimulation, the suctional 
activity was again renewed, after a latent period of two minutes, and found to persist for a considerable time, even on the cessation of stimulation (fig. 226). We have here, then, an exact parallel to the renewal of the so-called autonomous response of Desmodium leaflet referred to above. ${ }^{1}$

We have thus studied the phenomenon of the variation of suction by renewal when found at zero. We shall next con-

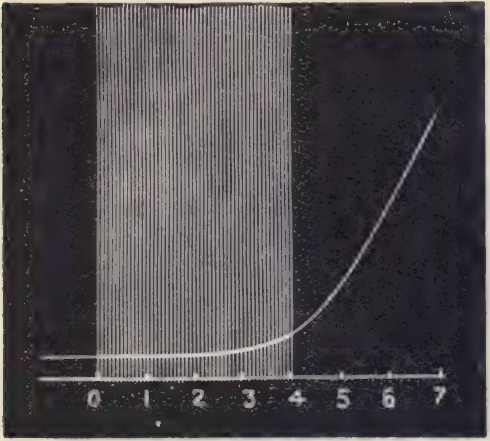

FIG. 226. Renewal of Suction, Previously at Standstill, by Action of Stimulus

The half-shaded portion of figure represents time of application of stimulus. Suctional response is seen to be initiated after a latent period of one minute, and to persist after the cessation of stimulus.

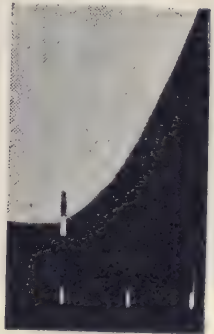

F1G. 227. Photographic Record of Effect of Stimulus in Enhancing Rate of Suction

This record was taken under balanced conditions. Vertical line represents moment of

it application of stimulus for 30 seconds.

sider the case of a variation induced in the existing rate by the action of stimulus. The normal rate is exactly balanced, a condition which is represented in the photographic record by the straight line which results from the stationary position of the mercury index. Stimulus of 5 seconds' duration was now applied, and the responsive acceleration is seen as a steep rise in the record (fig. 227). This responsive acceleration persists

I If a cut branch of any plant be kept in water for several days, its suctional activity, as is well known, disappears. This is commonly attributed to the blocking of the cut end by mucilage and bacterial growths, since the making of a fresh section is found to renew the activity. This making of a fresh section, 
during a period that depends on the intensity of stimulus and the condition of the tissue, after which it declines slowly. After recovery, however, the rate is generally somewhat higher than at first. This is due to the persistent after-

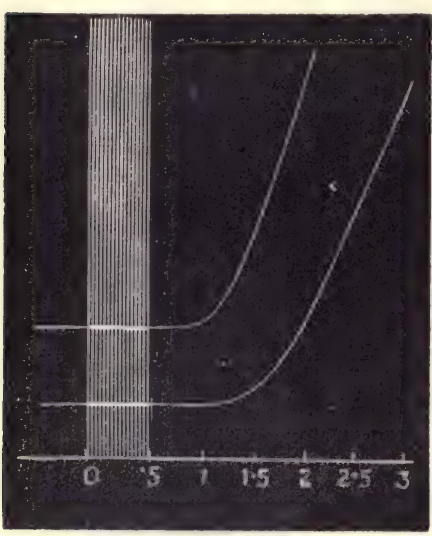

FIG. 228. Variation of Latent Period as After-effect of Stimulus

The record was taken under balanced conditions. Half-shaded portion represents application of stimulus for 30 seconds. Lower record shows latent period to be 45 seconds. After re-balance, stimulus of 30 seconds was once more applied. Upper record now shows reduction of latent period to 30 seconds. effect of stimulus absorbed. If the enhanced suctional rate be now again balanced, and stimulus applied once more, there will be a still further enhancement of the rate. In this way, owing to the successive increase of latent energy, the suctional activity is enhanced till it reaches a limit, after which there is but little additional effect to be induced by stimulus.

There is another and interesting effect which is often observed, in consequence of energy absorbed from previous stimulation. This is the diminution of the latent period, after which response takes place. This will be seen in the following record (fig. 228). The initial rate of suction was in this case balanced, as usual, and stimulus of thirty seconds' duration was applied. The responsive acceleration is seen to take place forty-five seconds after the cessation of stimulus. The enhanced rate was balanced, and a stimulus of thirty seconds' duration however, does not decide the question; for in making it, is involved the strong mechanical stimulus of a cut. The outgrowths may, no doubt, obstruct the passage of water, and yet the total abolition of suction not be due to this cause alone. The more effective cause is, in fact, the run-down of energy, as proved by the experiment described above. In another experiment I took a cut stem in which suction had come to a standstill, and, without disturbing the mucilaginous end, I supplied it with water somewhat above the ordinary temperature. This thermal stimulation at once initiated renewed suctional activity with great vigour. 
was applied once more. It is here seen that this stimulus induces a further acceleration. The latent period, however, of this second response is reduced from forty-five seconds, which was its value in the first case, to thirty seconds.

This variation of latent period is brought out still more clearly by the application of a stimulus of shorter duration, in which case the latent period is more prolonged, and its variations, therefore, more easily observed. In order to show this, I took a fresh specimen of Croton, and, after the initial balance, applied stimulus of five seconds' duration. It will be seen from the photographic record (fig. 229) that the

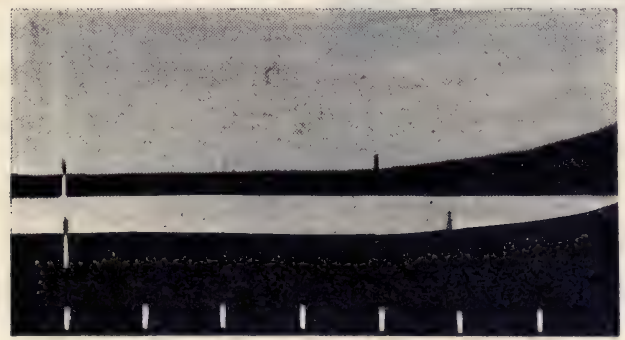

FIG, 229. Photographic Record showing Variation of Latent Period as After-effect of Stimulus

Stimulus applied was for 5 seconds. Moment of application represented

by vertical line. Lower record shows latent period to be 25 minutes. After re-balance, stimulus of 5 seconds was again applied. Upper record now shows reduction of latent period to 20 minutes.

latent period was here very long, being as much as twentyfive minutes. After re-balance stimulus was once more applied, lasting, as before, for five seconds. The latent period in the second case is seen to be reduced to twenty minutes.

As an interesting and independent verification of the enhancement of suction by stimulus, I now took a number of response-curves, using the Method of Over-balance. Here, it will be remembered, the normal over-balance is indicated by a down-curve, and acceleration of suction by diminution of the slope, or even by reversal, of this curve. In fig. 230 is seen a record obtained in this manner the first down part 
of which shows the curve of over-balance. Stimulus of halt a minute's duration was now applied, and it will be noticed

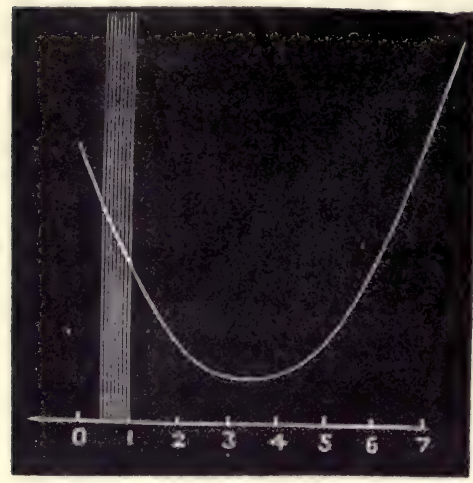

L FiG. 230. Suctional Response under Over-balance

Stimulus of 30 seconds neutralises over-balance and reverses curve. that on account of the responsive acceleration the slope becomes increasingly diminished, till, after an interval of one minute and a half, the curve becomes horizontal. After this it is reversed to the upward direction. It will thus be seen that the responsive acceleration has here induced a rate of suction which is not merely sufficient to compensate the over-balance, but greatly exceeds it. In the next photographic record (fig. 23I) I have been successful in showing the immediate and persistent after-effects. In order to do

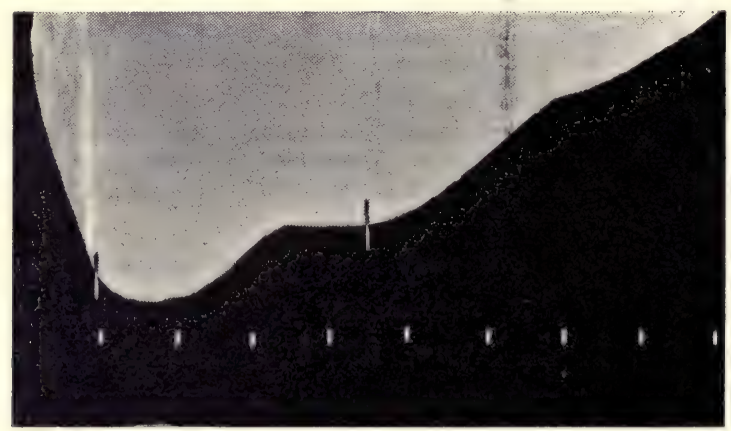

FIG. 231. Photographic Record of Effect of Stimulus on Over-balance

First stimulus for 2 seconds, represented by first vertical line, neutralises and reverses over-balance. Horizontal record after reversal represents persistent after-effect. Second stimulus for 2 seconds, represented by second vertical line, gives rise to up-record, the persistent aftereffect being represented by a curve of diminished slope.

this within the limited range of a photographic plate, I employed stimulus of the short duration of two seconds. 
The first part of the record shows the normal down-curve of over-balance. Stimulus of two seconds' duration was now applied, at the place marked in the record with a vertical line. This is seen to induce a growing diminution in the slope of the curve culminating in reversal; and afterwards, owing to the persistence of the after-effect of stimulus, the record becomes horizontal. A second stimulus of two seconds' duration was now applied. This is seen to induce a further enhancement of the rate, which is shown by the upcurve. The slope of this curve undergoes a slow decline with the waning of the immediate effect of stimulus. But on account of that component of the stimulus which remains latent in the tissue, there is induced a more or less persistent after-effect, which is greater than the after-effect due to the first stimulus. For while the after-effect of the first stimulus was seen to make the record horizontal, the second aftereffect renders the curve slightly ascending. From these and other facts previously enumerated it will be understood that the effect of latent stimulus derived from external sources is to increase suctional activity up to a certain limit.

Having now described the various effects induced in a slightly sub-tonic tissue, under the simplest mode of stimulation, namely, the terminal, we shall proceed to inquire as to what are the effects induced under a somewhat more complex mode of stimulation. This is the case with sub-terminal stimulus, where the point stimulated is not on the external extremity, but within the tissue, though near the lower end. Under these conditions the excitatory wave will proceed in two opposite directions, upwards and downwards. The short terminal zone, however, being close to the directly stimulated area, will be more intensely affected than the extended upper region. For this reason there is likely to be a predominant expulsion of water from the lower end. Under continued strong stimulation, however, the more intensely excited lower zone may become fatigued. The natural upward suction, probably enhanced by the action of 
stimulus, may now be expected to reassert itself, thus converting expulsion into renewed suction.

In any case, whatever the explanation, I find that the result of this mode of stimulus is, first, a movement of expulsion, followed, under continued intense stimulus, by renewal of the upward suction. These various effects are seen in the following photographic record, where the up-curve represents the normal unbalanced suction

(fig. 232). Continuous

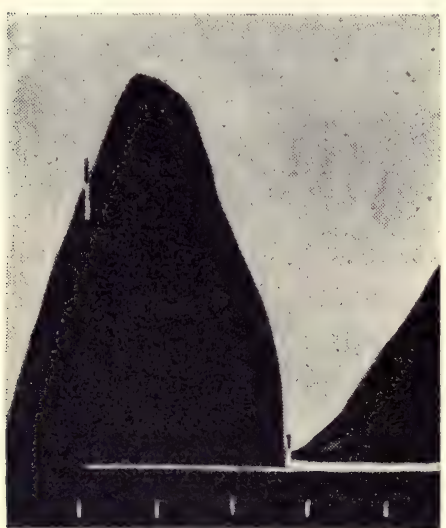

FIG. 232. Photographic Record of Response to Continuous Sub-terminal Stimulation

Response was taken without balance Continuous stimulation applied from moment represented by vertical line. This induced diminution, arrest, and reversal of response to expulsion. Stronger stimulation applied at second vertical line. This induced a second reversal to suction. Thin white line shows duration of application of stimulus of moderate intensity; and subsequent thick line, of greater intensity.

six to eight volts. It will be seen how this reversed the expulsion, converting it into renewed suction.

We have seen, as already stated in the electrical response of roots, that while less excitable old roots will generally give positive response, highly excitable young roots give 
negative. We saw, further, that there was reason to associate this positive response with the process of absorption, and the negative, conversely, with that of expulsion or secretion. With various kinds of tissues, moreover, we have found, and shall see further, that as a general rule positive response is obtained, either when the tissue is sub-tonic, and very slightly. excitable, or when the stimulusintensity is feeble, and when the tissue is fatigued by overstimulation. Negative response, on the other hand, is characteristic of highly excitable tissues. Under natural conditions, then, when the roots are subjected to the moderate stimulation of such factors as contact with soil, water, and food, we might expect their response to be positive or absorptive. In cut branches, also, in which the tissue is not extremely excitable, a simple terminal application of stimulus induces, as we saw, the absorpto-positive effect, either by initiating suction, or by enhancing that which was already taking place.

But in highly excitable young roots, in contact with a stimulating supply of inorganic food, the characteristic response, as we have seen, is by secretion. The electrical response also of young roots we found to be negative. Between these two extremes of positive and negative, then, there must be an intermediate case in which the responsive action to external stimulus will be zero.

Applying this to the parallel case of suctional response in cut branches, we should expect to meet with two different cases besides that already given. In one, where the tissue is very highly excitable, the response under simple terminal stimulation will be negative or expulsive. In tissues, however, which are not so highly excitable, but more excitable than those sub-tonic specimens whose characteristic responses I have already described, it might be possible to find cases in which the suctional response to stimulus will be zero. In the sub-tonic tissues referred to, we have already seen that in consequence of increase of internal energy by external stimulus, the suctional response tends to reach a 
limit, after which further stimulation would produce little or no effect.

Thus we see that if a tissue from any cause be sub-tonic, even moderately strong stimulus will induce positive or absorptive response. If it, on the other hand, be highly excitable, the response may be expected to be negative or expulsive. Between these two, in the intermediate state of excitability, the effect will be neither one nor the other, that is to say, zero. These results concern the application of somewhat strong stimulus, such as that of electrical shocks. Feeble stimulus will generally evoke response by absorption. We can also clearly see that the tonic condition, and therefore the excitability, of a tissue will vary with the seasons, being low at the end of winter, and high in spring or summer. Thus the same strong stimulus which in the one season will induce absorption, might be expected in the other to provoke expulsion. In experimenting on suction during the period of seasonal variation, I obtained results which verified these inferences.

These experiments, as will be remembered, were begun in February, when the spring had scarcely commenced, and the plants were in a sub-tonic condition. Under these circumstances, we saw that the terminal application of stimulus uniformly evoked positive response, by enhancement of suction. By the end of February, however, when warmer weather prevailed, and the vigour of the plants was evidently greater, I was surprised to find that the same stimulus, applied in the same way, to similar specimens of Croton evoked little or no response. A week later-that is to say, in the beginning of March, when the Indian spring was well advanced, and the physiological activity of the plants high-I found that the response which had thus seemed to disappear was renewed, but had become reversed in sign. Strong terminal stimulation now, as a rule, evoked responsive expulsion.

From the considerations enumerated at the beginning of these investigations, it was seen that the various physical 
theories brought forward to account for the ascent of sap were admittedly inadequate. The further objections, urged against the fundamentally excitatory nature of the processes involved, on the ground of the important part played in the ascent by sap-wood, generally regarded as dead, I have also shown to be untenable. The sap-wood I have shown to be not dead, but living, and to exhibit the normal response of living tissues to excitation. The long persistence of suction, when the roots are killed with hot water, or the cut specimen placed in poison, was shown to be accounted for by the fact that the death of any individual zone does not arrest suction in those above. I have shown, moreover, that those agents, such as rising temperature, which exalt the general physiological activity of the tissue, enhance suctional activity also. Those which, like cold or anæsthetics, act, on the other hand, to depress the general physiological activity, will depress and arrest its suctional activity also. And, finally, the fact that the water-movement is a form of excitatory response has been fully demonstrated by the experiments described and the records given in the course of the present chapter. The physiological theory of the ascent of sap may thus be regarded as established. 


\section{CHAPTER XXVII}

\section{RESPONSE TO STIMULUS OF LIGHT}

Heliotropic plant movements reducible to fundamental reaction of contraction or expansion-Various mechanical effects of light in pulvinated and growing organs-Electrical response induced by light not specific, but concomitant to excitatory effects-Electrical response of plant to light not determined by presence or absence of chloroplasts-Effect of unilateral application of stimulus on transversely distal point-Positive response due to indirect effect and negative to transmission of true excitation-Mechanical response of leaf of Mimosa to light applied on upper half of pulvinus-Mechanical response consists of erection or positive movement, followed by fall or negative movement-Electrical response of leaf of Mimosa to light applied on upper halt of pulvinus; induction in lower half of pulvinus of positivity followed by negativity-Longitudinal transmission of excitatory effect, with concomitant galvanometric negativity-Direct effect of light and positive after-effectCircumstances which are effective in reversing normal response-Plants in slightly sub-tonic condition give positive followed by negative responseExemplified by $(a)$ electrical and $(b)$ growth response-Examples of positive response to light-Periodic variation of excitability-Multiple mechanical response under light-Direct and after effect-Multiple electrical response under light, with phasic alternations of $(-+-+)$ or $(+-+-)$-After effects; unmasking of antagonistic elements, either plus or minus-Three types of after-effects.

THE first important point that arises, in connection with the response of living tissues to light, is the question whether such response is peculiar in its character, or fundamentally similar to that which is evoked by other forms of stimulus. The mechanical movements of plants under light are apparently so diverse that it would at first sight appear almost impossible to derive them all from any common fundamental reaction. Thus, some plant organs are found to turn towards the light, others away from it, and others again to remain perpendicular to it. Thus three different typical effects-positive, negative, and dia-helio- 
tropic-are induced in different cases by the same stimulus. These effects, moreover, are found to occur in growing as well as in pulvinated organs. This inconsistency of effects has been a source of great perplexity, inclining observers to the belief that the action of any given plant organ under light is determined, not by some definite reaction; but by its own power to decide what is for its individual advantage.

I have shown elsewhere, however, ${ }^{1}$ that as regards mechanical response, the reaction of plant organs to the stimulus of light is extremely definite. This, like other forms of stimulus, induces negative turgidity variation and contraction, as well as consequent retardation of growth in growing organs. Such excitatory effects, moreover, if the tissue be of fair conducting power, may be transmitted in either a transverse or a longitudinal direction. The intensity of this transmitted excitatory effect is thus dependent, as I have shown, on the intensity and duration of stimulus and on the conductivity of the tissue. If neither the intensity of the stimulus nor the conductivity of the tissue be great, it will be the indirect or hydro-positive effect which will reach the distant point, there to induce a positive turgidity variation and expansion. The foregoing observations relate to tissues in a normal condition of excitability. When the tissue is sub-tonic, however, the absorbed stimulus, as we have seen, increases the internal energy and brings about a responsive expansion.

I have also shown that the various mechanical movements induced by the unilateral action of light, depend (I) upon whether the stimulus remains localised on the proximal side of the organ or is conducted to the distal; and (2) on the relative excitabilities of proximal and distal. I have shown, moreover, that all the diverse effects induced by light are demonstrably traceable to the action of these various factors in varying combination. And, finally, certain highly excitable tissues, owing to excess of energy derived from

\section{Plant Response, pp. 551 to 685.}


continuous stimulation, exhibit alternations of phase, negative and positive, or vice versa, constituting multi-phasic or oscillatory response. On these considerations it is possible to summarise the principal effects caused by light, as follows :

\section{Mechanical Effects of Light on Pulvinated and \\ Growing Organs.}

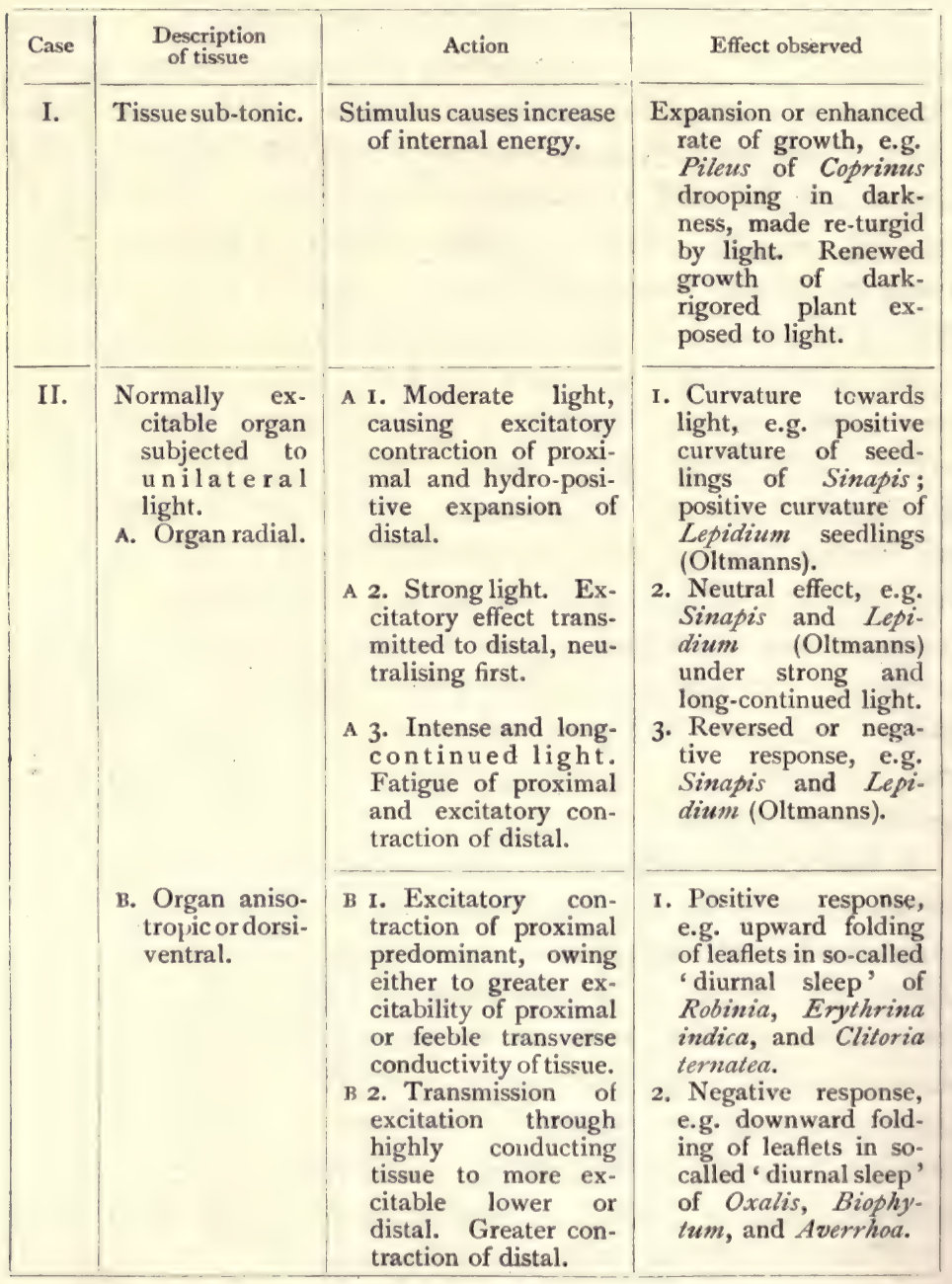




\begin{tabular}{|c|c|c|c|}
\hline Case & $\begin{array}{l}\text { Description } \\
\text { of tissue }\end{array}$ & Action & Effect observed \\
\hline III. & $\begin{array}{lr}\text { Tissues which } \\
\text { exhibit mul- } \\
\text { tiple or auto- } \\
\text { nomous re- } \\
\text { sponse. }\end{array}$ & $\begin{array}{l}\text { Considerable absorp- } \\
\text { tion of energy, im-- } \\
\text { mediate or prior. }\end{array}$ & $\begin{array}{l}\text { Initiation of multiple } \\
\text { response in Desmo- } \\
\text { dium gyrans pre- } \\
\text { viously at standstill; } \\
\text { multiple response } \\
\text { under continuous } \\
\text { action of light in } \\
\text { Biophytum ; photo- } \\
\text { tactic movements } \\
\text { of swarming spores ; } \\
\text { cf. multiple visual im- } \\
\text { pulses in retina. }\end{array}$ \\
\hline
\end{tabular}

Turning now to the electrical responses induced by light, our investigation is resolved into the inquiry whether these are not the electrical concomitants of those excitatory effects which we have already been able to analyse through mechanical response. But, before proceeding to this question, I shall first briefly refer to certain 'electrical effects of light upon green leaves' which have been observed by Dr. Waller.'

His experiments were performed by making galvanometer connections with two halves of the same leaf, one being strongly illuminated and the other unilluminated. With leaves of different plants he obtained opposite electrical effects under the action of light. From the leaves of Iris, for example, during illumination, he obtained response of galvanometric negativity, with reversal, or positivity, on the cessation of light as its aftereffect. With leaves of Tropaolum and Mathiola, on the contrary, he obtained positive response during illumination and subsequently negative. Beyond the suggestion that negativity may be associated with dissimilation and positivity with assimilation, Dr. Waller offers no explanation of this opposition of effects observed by him. He states, however, that he regards the presence of 'chloroplasts' as essential to these electrical reactions under light, inasmuch as petals, he found, gave no response. Even in the case of the green leaves 'of ordinary garden shrubs and trees,' moreover, he found no 
response. This he ascribes to their 'low average metabolism.' It will thus be seen that no satisfactory explanation is offered either of the mutual opposition of the electrical effects observed or of the way' in which the presence of 'chloroplasts' acts as a determining factor.

To turn now to the subject of my proper inquiry, it has to be determined whether those electrical responses which may be observed in vegetable tissues under the action of light are or are not another expression of the same excitatory reactions under light which I have already demonstrated by means of mechanical response. And it may be as well to say at the outset that it is the excitability of the tissue, and not the presence or absence of 'chloroplasts,' that is the critical factor in determining this electrical response. For I have obtained strong responses under light from pulvini, stems, and other tissues which are relatively deficient in 'chloroplasts'; and again, while the lamina of a plant rich in 'chloroplasts' would give but moderate response to light, the petiole of the same plant, characterised by less 'chloroplasts,' would often give much stronger response. The etiolated stem of celery, moreover, gives strong electrical response. And, finally, it is an error to suppose that petals of flowers are irresponsive to light, for I have obtained strong response from petals of Sesbania coccineum and from Eucharis lily. Animal nerve, again, in which there is no chlorophyll, gives response to light.

As I have already shown the typical heliotropic effects exhibited by plants to be brought about by differential cxcitatory action on the proximal and distal sides of the same organ, I shall now proceed to exhibit the electrical counterparts of these experimentally. As I wish, moreover, to show that the general electrical response to the unilateral action of light is fundamentally the same as that induced by other forms of stimulus, the first experiment to be described will be one depending on the unilateral application of a nonluminous stimulus, say thermal.

If on one side of a growing organ we apply a series of 
thermal shocks, by means of the electro-thermic stimulator, the proximal side will undergo contraction, while the expelled water, by its hydro-positive effect, will induce expansion on the distal side. By means of these two conspiring actions the organ will be bent towards the source of stimulus. The electrical variation on the distal side will therefore be positive; but if the stimulus applied be sufficiently strong and long-continued, true excitation will be transmitted across the tissue to the distal side. This will neutralise the first mechanical movement, and the corresponding electrical effect will be a reversal of the previous

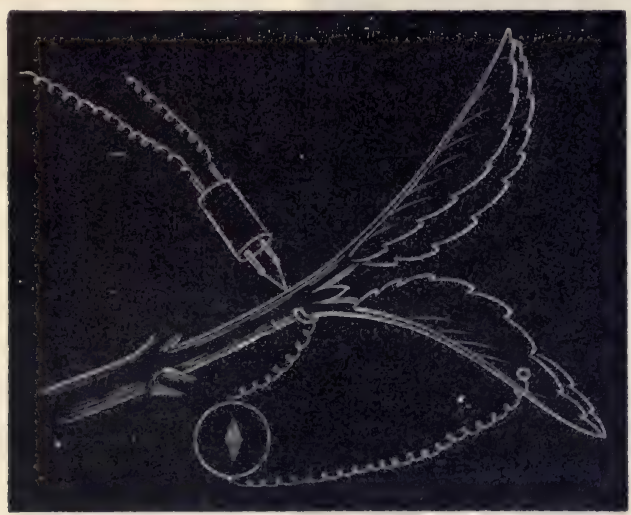

FIG. 233. Experimental Arrangement for Detection of Electrical Change induced at the Point transversely Distal to Point stimulated

Upper point stimulated by thermal shocks from electro-thermic stimulator, the lower being the transversely distal point.

positive into the excitatory negative. Similar electrical effects will also be observed if the organ be restrained from movement, or if it be so old as to have lost its power of motility.

Taking now a young stem of Bryophyllum, I applied a series of thermal stimuli at the proximal point (fig. 233). Periodic closure of the electrical circuit by means of a metronome caused rapidly succeeding thermal shocks to act on the upper or proximal side. By adjusting the heating current the stimulus was at first made moderate. It will be seen 
from the photographic record (fig. 234) that this gave rise electrically to an increasing positive effect at the diametrically opposite point. This clearly shows that the latter underwent a positive turgidity variation, in consequence of the forcing-in of water expelled by excitatory contraction from the upper side. It is at this stage the indirect and not the true excitatory effect of stimulus that is being transmitted to B.

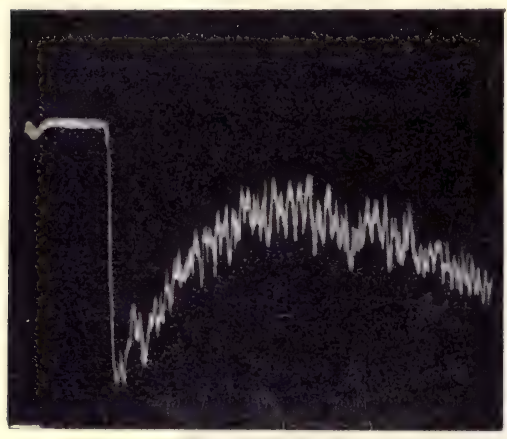

FIG. 234. Record of Response to Moderate Unilateral Stimulation under the Experimental Arrangement described

Response of distal point by increased galvanometric positivity due to hydropositive effect. Note initiation of multiple response.
By this experiment it is also demonstrated that the positive curvature induced by the unilateral application of any stimulus is the joint effect of the direct excitatory contraction of the proximal side and the indirect or hydro-positive expansion of the distal.'

Another interesting phenomenon to be observed in this curve is that, after the maximum effect has been reached, there is a series of oscillatory multiple responses. In this result there may possibly be two factors in operation: first, after the maximum hydro-positive tension has been set up there may be a gradual percolation of the true excitatory effect, with its opposite reaction, the unstable balance thus produced manifesting itself in oscillations; and, secondly, we know that increased hydrostatic tension has the effect of initiating multiple responses, as seen

1 In records of such a response as I have just described, when exhibited by highly excitable tissues, a preliminary negative twitch, of momentary duration, may sometimes be observed. This is not due to the conduction of true excitation, but to pseudo-conduction. The sudden blow delivered by the hydrostatic wave on its arrival at the distal point is, in a highly excitable structure, sufficient of itself to induce a short-lived excitatory effect. Thus this does not represent the true transmission of excitation, but its initiation de novo by a secondary mechanical cause (p. 446). 
in the snail's heart, under a sufficiently high degree of internal hydrostatic pressure.

As regards conduction in general, we know that a strong stimulus is transmitted to a greater distance than a weak. In the next record (fig. 235) this may be seen in an interesting manner. At first a moderate stimulus was employed, and this gave rise to $(a)$ a maximum positive variation of the distal point $B$. The stimulus was then increased and we observe that $(b)$ the excitatory effect, now reaching $B$, causes a reversal of the curve, owing to induced galvanometric negativity. If at the beginning we had used a stimulus of fairly strong intensity the first effect would have been a positivity of $B$, due to the indirect effect of stimulus; and, secondly, the excitatory effect would have reached the point gradually, neutralising and afterwards reversing the first. I shall now describe the corresponding effects, both mechanical and electrical, which are induced by stimulus of light. We take a Mimosa plant, and subject only the upper half of

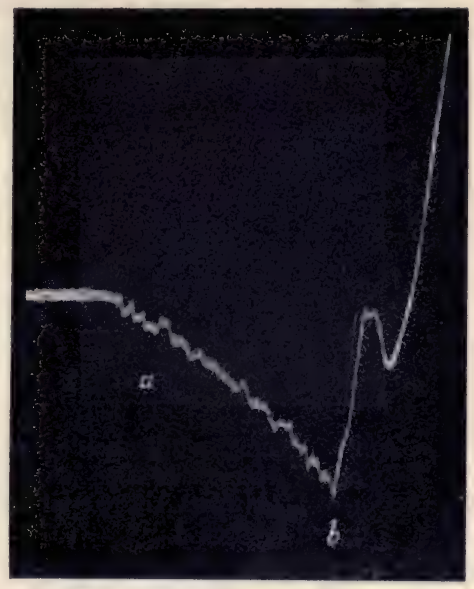

FIG. 235. Record of Different Specimen under same Experimental Arrangement when Stimulus is first Moderate and then Increased

(a) Positive response, due to hydropositive effect; this is converted to negative in $(b)$ due to transmission of excitatory effect under stronger stimulation.

one of its primary pulvini to the action of sunlight. The effects thus induced are (I) the local contraction of the excited upper half, and the expansion of the lower half by the hydropositive effect; (2) the gradual percolation of true excitation to the lower half, and consequent initiation of excitatory contraction there; and (3) the continued action of excitation and increasing contraction on the more excitable lower half. All these effects are exhibited in the mechanical response 
shown in fig. 236, where the first is seen in the up-curve, which thus indicates the joint action of contraction in the upper and expansion in the lower, giving rise to an erectile movement. After an interval of one minute the excitatory effect is seen to have reached the lower half, giving rise now to a reversed or down movement, which, on account of the greater excitability of the lower, is seen to carry the leaf downwards, much below its original position. The dotted portion of the curve shows the after effect on the cessation of

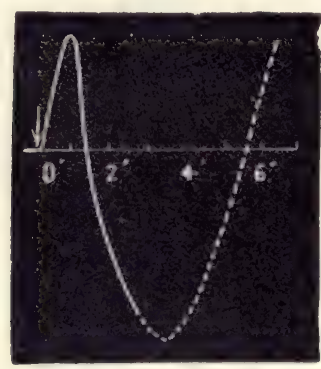

FIG. 236. Mechanical Response of Pulvinus of Mimosa to Continuous Action of Light from Above applied at Moment marked $\downarrow$

Positive heliotropic movement caused by excitation of upper half neutralised by transmission to distal side, and ultimately reversed owing to greater excitability of lower half. Dotted line represents recovery on cessation of light. Note final erection of leaf above original position as after-effect of absorbed stimulus. the stimulating light. It is here interesting to observe that it is possible to obtain an after-effect which is positive and of opposite sign to the true excitatory effect, this positivity being due to the increased internal energy consequent on the absorption of stimulus.

In fig. 237 are shown the electrical effects consequent on stimulation by light in another specimen of the pulvinus of Mimosa. The electrical contacts are made in this case, one with the lower half of the pulvinus and the other with a distant indifferent point. Stimulus of light is applied, as in the last case, on the upper half. This experimental method is free from the objection which has been urged against Dr. Waller's experiments on green leaves, that the result was complicated by the direct action of light on the electrode itself. It should be pointed out, however, that the presence of such photo-electric action is more important theoretically than practically, being of relatively small amount. A further complication which arises from the direct action of light on 
one of the electrodes lies in rise of temperature. Though in all such experiments the incident light should pass to the organ through a thick stratum of water, which absorbs its heat-rays, yet the absorption of light by the tissue must necessarily occasion a slight rise of temperature. In connection with this should be remembered the fact I have elsewhere demonstrated, that though sudden variation of temperature acts as an excitatory agent, yet a slow and gradual rise, enhancing the internal energy, brings about only a slight positivity, opposite to the effect of true excitation. In this particular experiment, however, as the electrical contacts are not directly acted on by light, we obtain results uncomplicated by such disturbing factors.

The first electrical effect brought about in the lower half of the pulvinus by application of light on the distal upper half is seen in fig. 237 as an increasing galvanometric positivity. This is concomitant to the hydropositive effect at the lower

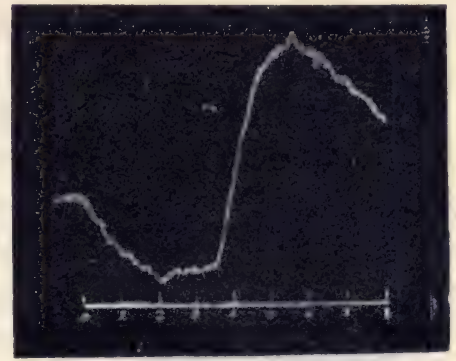

FIG. 237. Electrical Response in the Lower Half of the Pulvinus of Mimosa due to Stimulation of Distal Upper Half by Light

Observe the first phase of positivity, due to hydro-positive effect, converted subsequently into negative by the transmission of true excitation.

half, which, conspiring with the contraction of the upper, produces that up-movement of the leaf seen in the previous figure. The excitatory effect next reaches the lower half, and we there obtain increasing galvanometric negativity in consequence. This corresponds with the mechanical movement of depression. From this experiment it is clear that light, like other forms of stimulus, induces, as its true excitatory reaction, galvanometric negativity, the indirect or hydropositive effect being one of galvanometric positivity.

In the last case, then, we obtained a transverse transmission of the true excitatory effect. Similar effects are 
also obtainable by longitudinal transmission. In order to do this an organ must be selected which is a fairly good conductor. I have thus been able to observe a series of responses to transmitted stimulus of light, using such specimens as the petiole of Bryophyllum. Light was here applied at a distance of $5 \mathrm{~mm}$. from the proximal contact, and this gave rise to a series of true excitatory responses of galvanometric negativity.

Having thus established unmistakably the negative sign of the excitatory electrical variation induced and transmitted under stimulus of light, I shall

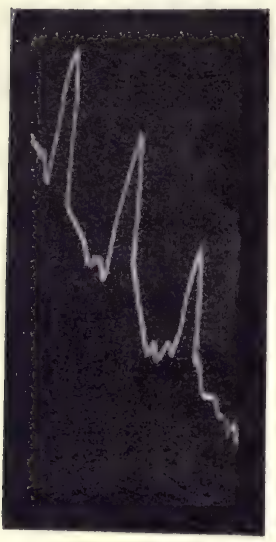

FIG. 238. Photographic Record of Series of Negative Responses of Petiole of Bryophyllum to Stimuli of Sunlight of Five Seconds' Duration applied at Intervals of Two Minutes

Observe the positive after-effect, due to increase of internal energy, which causes downward trend of base-line. next proceed to give records of experiments in which light was applied directly. The effect observed in these cases is naturally much larger, as there is no enfeeblement by transmission. Fig. 238 shows a series of such responses, obtained at intervals of two minutes, by the application of sunlight - previously passed through a stratum of waterduring five seconds only in each case, on the petiole of a vigorous leaf of Bryophyllum. It will be noticed that after each response of galvanometric negativity there is an after-effect of positivity, in consequence of which the base line of the series, instead of remaining horizontal, trends downwards. This power of holding stimulus latent, for the increase of internal energy, we shall later see to be important, as heralding the initiation of multiple response. The exhibition of these after-effects, due to increase of latent energy, is also to be observed in the record given already of the mechanical response of Mimosa 
(fig. 236), where the leaf, after its excitatory fall, was reerected, on cessation of stimulus, above its original height. This, as we saw, was due to a certain portion of the incident stimulus becoming latent, and thus increasing the internal energy.

We shall next take up the subject of the occurrence of positive response, as sometimes induced by light. This may be the result of various different causes. There is one fact, however, in connection with the action of light which it is important to bear in mind. Thus, if we subject the lower half of the pulvinus of Mimosa, for instance, to the action of sunlight, its responsive fall will be gradual, unlike the sudden depression caused by thermal or mechanical stimulation. This is because light, usually speaking, constitutes a stimulus of only moderate intensity. We have seen that a stimulus which falls below a certain critical level of excitatory intensity will evoke positive, instead of negative response. We have also seen that from a sub-tonic tissue the positive response is more easily obtained than from one which is highly excitable. Now, as the excitatory efficiency of a mechanical stimulus is very great, and as that stimulus is also incapable of finely graduated decrease, it follows that, in order to exhibit positive response under such stimulation, it is necessary that the tissue stimulated should be extremely depressed, or even moribund. Under such conditions I have shown (p. 83) that it is possible under feeble stimulus to obtain positive response, which, under stronger, will pass into the normal negative.

The stimulus of light, then, whose action is very moderate, discriminates more finely between tonic gradations of the tissue than can other forms of stimulus. If this tonic condition be very favourable, and the excitability high, the response will be by normal galvanometric negativity. If the tonic condition, however, be less favourable, the response is liable to be positive. This latter fact will be very strikingly demonstrated, in a later chapter, by experiments carried out on nerves. It will there be shown that while highly excitable 
nerve gives the normal negative response, the same tissue, if its tonic condition be below par, gives a more or less persistent positive response. It is only when the tonic condition of the nerve has again been raised, by long-continued stimulation, that it will once more give normal response. Fatigue is another condition which is liable to give rise to the abnormal positive response.

The use of the stimulus of light carries with it, also, a further limitation. A mechanical stimulus, say vibrational, throws into activity the whole mass of tissue, not only in its superficial, but also in its deeper lying strata. Now we have seen that the epidermal layer of living tissues is less excitable than those which are deeper seated. It may even, in fact, on loca! excitation, give positive response (p. 298). It is to be noticed, moreover, that light acts from outside, its excitatory influence affecting the outmost tissue first, and only by gradual percolation passing to the subjacent. Owing to these two facts, then, of the moderateness of this stimulus and the superficial character of its action, the tissue, if not highly excitable, is apt, under its application, to give positive response. We have seen, further, that various circumstances, such as age and season, have an important effect in varying the excitatory reaction of a tissue. We saw the effect of age exemplified in the responses given by two different specimens of roots (p. 353), in which a young root gave negative and an older positive responses. Again, we shall see presently that there is a diurnal period, on account of which the state of turgor, the excitability, and the sign of response, are all alike liable to undergo periodic variations. Under the stimulus of light these varying excitabilities may be expected to find varying expressions.

That sub-tonicity tends to make the response, under moderate stimulation, positive, is seen in the fact that an etiolated petiole of celery gives positive response under light Again, I have noticed that leaves of Bryophyllum, which usually give normal negative responses, sometimes exhibit positive, if the plant, during the previous night, have been 
subjected to unusual cold. Even in such a case, however, though the first responses are positive, successive exposures to light, by raising the tonic condition, are found to restore the response to the normal negative.

The same facts receive interesting illustration in the response of growth. If the growing organ be in a normally excitatory condition, the stimulus of light, inducing negative turgidity variation, causes retardation of growth. If the tissue, however, be in an extremely sub-tonic condition, light stimulus, by increasing the internal energy, gives rise to the positive effect, that is to say, the initiation, or enhancement, of the rate of growth. If the growing tissue, again, be only slightly sub-tonic, we shall have a preliminary positive, or enhancement, followed by the negative response, or retardation of the rate of growth. This is seen in the following record, which I obtained from a slightly

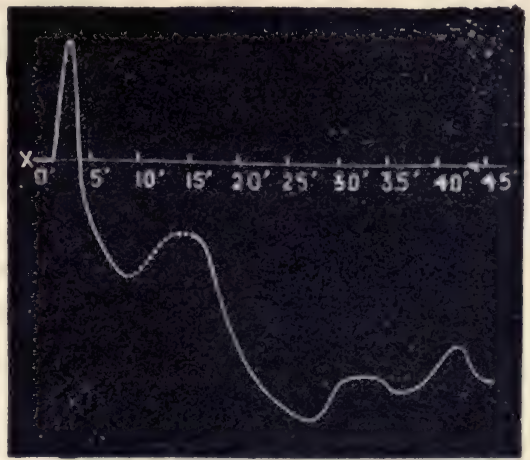

FIG. 239. Record of Responsive Growthvariation taken under condition of balance in slightly Sub-tonic Flower-bud of Crinum Lily under Diffuse Stimulation of Light

Continuous lines represent the effect during application of light, the dotted line on withdrawal of light. The plant was originally in a sub-tonic condition, and application of light at $\times$, after short latent period, induces preliminary acceleration of growth. After this follows the normal retardation. On withdrawal of light, in the dotted portion of the curve is seen the negative after-effect, followed by return to the normal rate of growth. A second and long-continued application of light induces retardation, followed by oscillatory response. sub-tonic flower-bud of Crinum lily, in the induced variations of the normal rate of growth under the stimulus of light (fig. 239). This record was made with the Balanced Crescograph, where the normal rate of growth is recorded as a horizontal line, enhancement or positive variations of the rate being represented by up-curves, and retardation, or negative variations, by down-curves. It will be noticed that 
the first effect of light on this sub-tonic tissue was to induce a positive response, followed subsequently by the normal negative. Continuous stimulation is seen later to give rise to oscillatory responses.

Having thus shown the continuity between the normal negative and positive responses, I give below a record of the

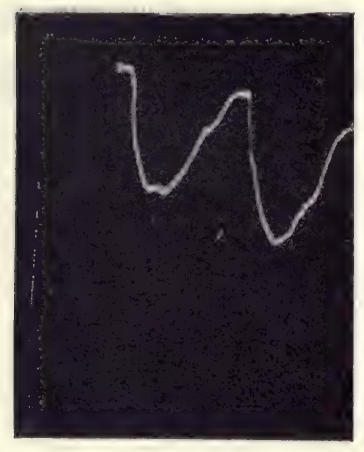

FIG. 240. Photographic Record of Positive Response of the Petiole of Cauliflower to Light of Five Seconds' Duration applied at Intervals of Two Minutes response to light of a petiole of cauliflower (fig. 240), a specimen which usually, though not always, exhibits galvanometric positivity. Each stimulus, by exposure for five seconds; was in this case applied after an interval of two minutes. It should be mentioned here that the same tissue which gives positive response to the moderate stimulus of light will show the normal negative when subjected to a stronger stimulus, such as the mechanical.

The effects studied up to the present have consisted of single responses induced by light. But this stimulus also induces multiple response, as we saw in the oscillatory variations of growth in the Crinum lily in fig. 239. The same phenomenon is observed in the case of motile response. For example, I took a plant of Biophytum, in which the leaflets are outspread, in the presence of diffuse light, and threw upon it direct sunlight. A series of multiple responses was now induced, under the continuous action of stimulus, a record of which is given in fig. 24I. The up-curves here represent excitatory downward movements, and the downcurves their partial recoveries. Owing to the incomplete character of these recoveries, the leaflets, as the result of a series of such responses, are finally closed downwards. In the outspread diurnal position the lower half of the pulvinus of the leaflet is somewhat more turgid than the upper half, and in 
consequence of repeated responses there is also a relatively greater contraction and loss of turgidity in that lower half. The leaflets are thus closed by the additive effect of multiple normal negative responses or downward movements.

When a Biophytum plant is kept in the dark the leaflets undergo a closure which is outwardly similar to that induced by light, but actually arises from a cause precisely opposite. Under strong stimulus of light a general contractile effect drives the water inwards to the interior of the plant, and, the loss of turgor in the lower halves of the pulvini being, as wc have just seen, greater than in the upper brings about the closure of the leaflets. But in complete darkness the reverse process is set up. The water returns to the pulvini, making them over turgid. Under this condition of excessive turgor, however, the upper half of the pulvinus becomes more turgid than the lower, and, being thus rendered more convex, closure of the leaflets is brought about. Exposure to light in this condition induces a greater loss of turgor by the upper than by the lower half, and we consequently obtain the

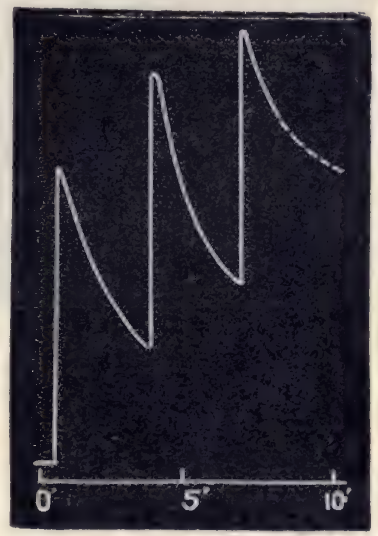

Fig. 24I. Multiple Mechanical Response of Leaflet of Biophytum under the Con. tinuous Action of Iight outspread or erected position of the leaflets by a series of small positive movements. Thus, as the result of internal changes, the response of an organ may undergo reversal from the ordinary negative to positive. The change from light to darkness, by which we have here seen the character of response to be so greatly modified, occurs in the diurnal periodic alternation. And we can see that, in consequence of such an imparted periodicity, an alternation of phase is impressed upon the organism, which will carry with it a periodic variation of excitability. If this diurnal periodicity acted alone its after-effect would be comparatively simple, 
but it is complicated by other periodicities, such as that of temperature variation, which do not always coincide in maxima and minima with these variations of light.

We have seen from mechanical indications that multiple excitations are induced by light. We have, therefore, to determine whether similar multiple excitations can be detected electrically. Fig. 242 is a photographic record of a series of

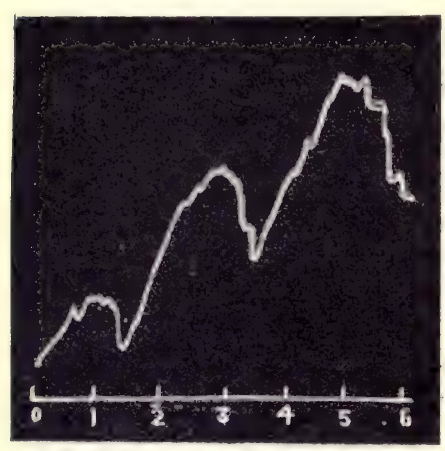

FIG. 242. Photographic Record of Multiple Electrical Response in Leaf of Bryophyllum under Continuous Action of Light such electrical responses obtained from the lamina of a leaf of Bryophyllum subjected to the continuous action of light. We see here an alternation of phase in the response, negative being followed by positive in each case throughout the series. This constitutes a parallel case to that of the mechanical response in fig. $24 \mathrm{I}$, inasmuch as, owing to incomplete recovery from each negative phase, the base line is gradually tilted upwards. But this need not always occur, for the two phases may be equal, and in that case the base line remains horizontal.

We have seen, in the chapter on Multiple and Autonomous Response, that these effects are due to the absorption of an excess of energy. When this absorption is great the energy may find an outward expression, even after the cessation of the stimulus. An example of this was seen in the mechanical response of a Desmodium leaflet (fig. I4I). The plant was at first in a sub-tonic condition, and the autonomous pulsation of its lateral leaflets had come to a standstill. One of these was now exposed to the continuous action of light and its record taken. It will be noticed that under this stimulus of light multiple responses were initiated, which persisted for a time as an after-effect, even on the cessation of light. In taking electrical records of the after-effect 
of stimulation by light, I have obtained similar multiple after-effects. And in this connection I have discovered certain characteristic peculiarities of the electrical after-effect which would appear to throw much light on the obscure subject of after-effects in the retina. The electrical after-effect of stimulus of light varies greatly in different conditions of the tissue, but is capable of classification under three different types.

The first of these types refers to those multiply responding tissues which give the usual single response of galvanometric negativity under short exposure to light. When such specimens are subjected to the continuous action of this stimulus they give multiple responses whose phases alternate in the sequence of minus-plusminus-plus $(-+-+)$. Confining our attention to the first two pairs of such phasic alternations under continuous stimulation of light (fig. 243), we observe that, in the first period, excitatory negativity attains its maximum at $b$, after which the phase becomes reversed to positive, in which process the curve may arrive at the original base line, or stop short of this or go beyond it. The maximum of this phase, $a^{\prime}$, we shall designate as maximum positivity. Under the still-continued action of the stimulus the phase now changes once more to negative, and so on.

It has already been stated (p. 100) that these periodic alternations in phase were brought about by antagonistic reactions becoming effectively predominant by turns. Thus, as was there pointed out, in those cases which normally give 
negative response, the internal energy may be so increased by the continuous absorption of impinging stimulus as to induce a coritinuously increasing antagonistic reaction of positivity. Hence, in such cases, negativity is gradually diminished, and ultimately reversed. After the attainment of maximum positivity, however, the negative element once more becomes predominant.

With a fresh specimen, exhibiting multi-phasic responses under the continuous action of light, I find it easy to obtain a convincing demonstration of the presence of the positive factor by a sudden cessation of stimulus at an appropriate moment. If the stimulus be stopped at $b$, the increasing internal energy and natural tendency to recovery will conspire with each other, and the after-effect will, generally speaking, be $b a^{\prime}$. That is to say, this effect is the same as that of natural recovery.

Wheu the second or positive phase has reached its maximum $a^{\prime}$, the response under the continued action of stimulation is once more reversed to negative $a^{\prime} b^{\prime}$, as we have seen. At this point of reversal $a^{\prime}$, the positive, is balanced by the negative. If then at this point the impinging stimulus be suddenly withdrawn, the positive element, finding itself unopposed, will overshoot the line of balance, and the curve will proceed in the positive direction towards $c$ (fig. 243). This phasic sequence (Type I.) then, during stimulation, and as an after-effect of it, should thus be $(-+\therefore)$, the dotted sign representing the after-effect. That this is actually so, is seen in the following series of photographic records (fig. 244), where the dotted portion of the record exhibits the after-effect. The intensity of this positive after-effect varies with the freshness and vigour of the specimen. The influence of fatigue, in the gradual diminution of the effect, is seen in the two subsequent series $(b)$ and $(c)$ obtained from the same specimen. This diminution culminates in the gradual diminution of the positive after-effect, and its conversion into a negative after-effect, as seen in (d).

We thus arrive at an intermediate case (Type II.), exem- 
plified by specimens which are not very fresh. The sequence is here $(-+\ldots)$. In some instances, if the stimulus be stopped at the end of the first negative phase, we obtain a small increment of negativity as the after-effect. The sequence here, then, is $(-\ldots)$.

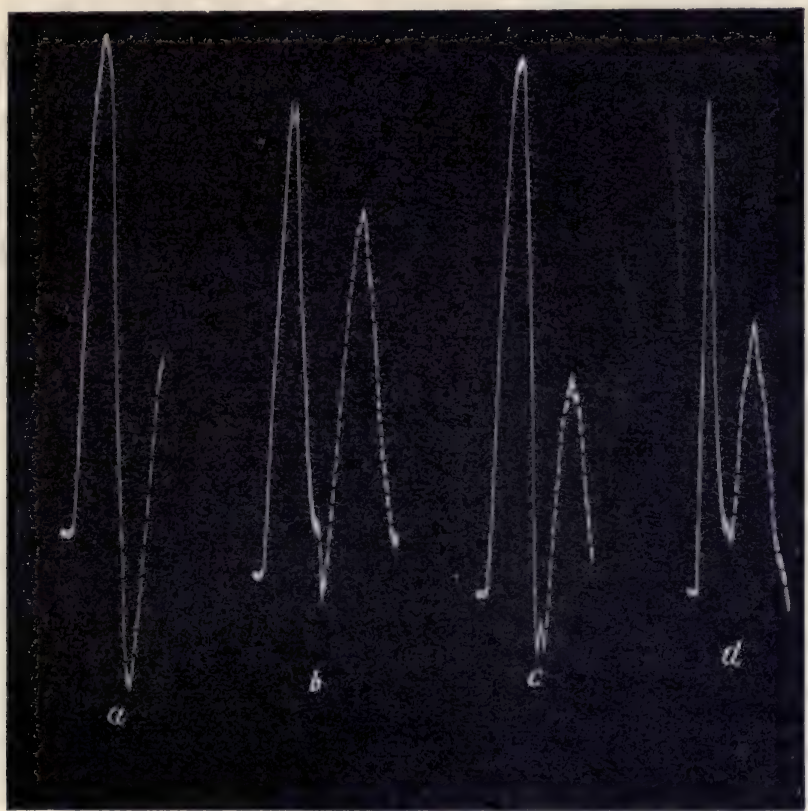

FIG. 244. Photographic Record of Phasic Alternations, showing Direct and After Effects of Light in Type I., represented by Bryophyllum

$a$ : First record of the series. Positive after-effect represented by dotted curve is here strong. $b$ and $c$ : Less strong positive after-effects due to fatigue. In $c$ light was stopped slightly beyond the second phase of maximum positivity; $d$, owing to fatigue, after-effect converted into negative. Dotted portions of curve represent, as usual, the after-effect on cessation of light.

Turning to Type III., we take specimens whose characteristic response to short-lived stimulus of light is by galvanometric positivity, the multiple phases in which, under its continuous action, may be expected to be in the sequence represented in figure 232 , that is to say $(+-+-)$ As the responses here are the exact opposite of those in 
Type I., we may expect to obtain the unmasking of the negative element in the response, as the after-effect, on the withdrawal of stimulus. Here there should be cessation of stimulus at the end of the second phase, in this case, maximum minus. The negative element, thus freed from its opposing positive, will demonstrate its presence by over-shooting in the

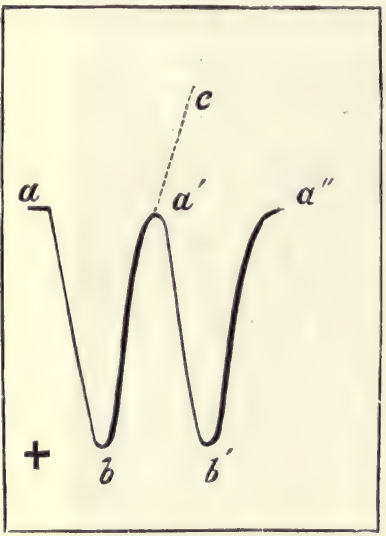

FIG. 245. Diagrammatic Representation of Phasic Alternations and After Effect in Type III.

During action of light the phasic alternations are $a b, b a^{\prime}$, $a^{\prime} b^{\prime}, b^{\prime} a^{\prime \prime}(+-+-) ; b$ is here the maximum positive, and $a^{\prime}$ the maximum negative. Withdrawal of stimulus at point of reversal $a^{\prime}$ causes unmasking of negative element, which is exhibited by the overshooting of the curve in the negative direction, $a^{\prime} c$. negative direction (fig. 245). This is seen in the next two figures (246 and 247). The specimen taken was the petiole of cauliflower, in that particular condition which gives the positive as its immediate response. The alternation of plus-minus-plus-minus, under continuous stimulation, is seen in the first part of fig. 246. Stimulus was now stopped at a point short of the maximum negativity, and we see the consequent overshooting in the negative direction. In the next figure (fig. 247), is seen a single alternation of plus-minus during stimulus, in a different specimen, with its after-effect and recovery from that effect. The impinging stimulus was in this second case stopped at the exact point of maximum negativity, and the overshooting curve shows, by its abrupt steepness, its sudden freedom from the restraint imposed by the opposite element of positivity. Thus, in this instance of Type III., we arrive at the typical formula of $(+-\ldots)$. It may then be said that under this head, of alternation of phase, we have examined representative cases of the two extreme Types I. and III., between which lie many variations, classified as intermediate, or Type II. 
It has thus been seen that the electrical response of plants to light is not essentially different from their response to other forms of stimulus. The various effects observed are the electrical concomitants of those excitatory effects which we had already seen to be exhibited in mechanical response. In a

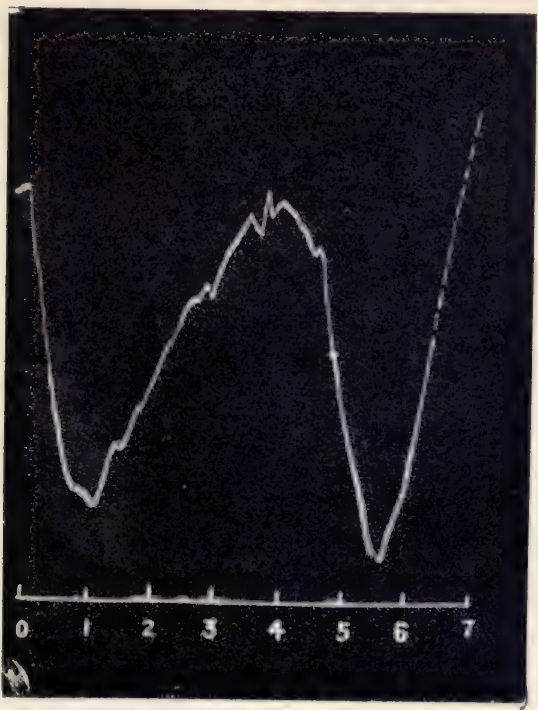

FIG. 246. Photographic Record of Phasic Alternation, showing Direct and After Effects in Type III., represented by Petiole of Cauliflower

Continuous line represents effect during application of light. Stimulus was withdrawn slightly before the attainment of the second maximum negativity, resulting in overshooting of curve in negative direction. Dotted portion of record represents aftereffect on cessation of light.

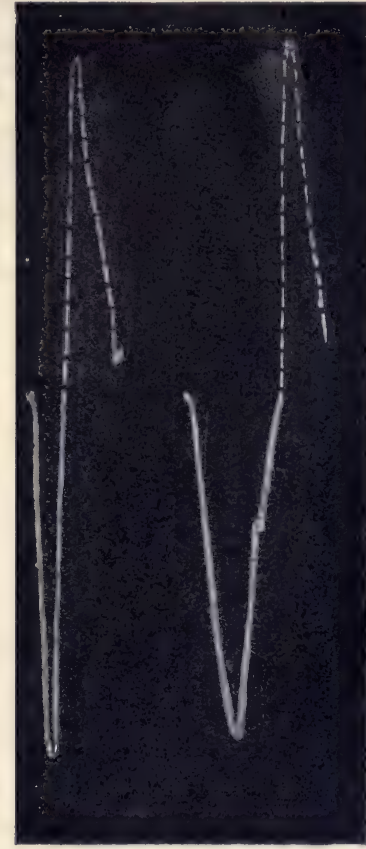

Fia. 247. Photographic Record of Pair of Responses obtained with a Second Specimen of Cauliflower, representative of Type III.

The stimulus of light was in these cases withdrawn exactly on the attainment of the negative maximum. Dotted portions of the record exhibit the after-effect.

highly excitable tissue the direct effect gives rise to galvanometric negativity. The indirect effect of stimulus, however, is one of galvanometric positivity. Positive response may also be obtained from a tissue which is not highly ex- 
citable or in a sub-tonic condition or fatigued. The presence or absence of chlorophyll is not a determining factor in electrical response. As in the case of mechanical and growth responses, so also in the electrical, continuous stimulation gives rise to multiple responses, as its direct or aftereffect. The sequence of phases, under the direct action of continuous stimulation, may be $(-+-+)$ or $(+-+-)$. Taking into account both the direct and after-effect, the observed results may be classified under three types. In the first type, that of fresh and highly excitable tissues, the formula is $(-+-i-)$. In the second or intermediate type, it is $(-+\ldots)$ or $(-\ldots)$. And in the third type we have the formula $(+\cdots)$. 


\section{CHAPTER XXVIII}

\section{RESPONSE OF RETINA TO STIMULUS OF LIGH'T}

Response of retina-Determination of true current of rest-Determination o, differential excitabilities of optic nerve and cornea, and optic nerve and retina - The so-called positive variation of previous observers indicates the true excitatory negative-Retino-motor effects-Motile responses in nerveVarying responsive effects under different conditions-Reversal of the normal response of light due to (1) depression of excitability below par ; (2) fatigue-The sequence of responsive phases during and after application of lightDemonstration of multiple responses in retina under light, as analogous to those in vegetable tissues-Three types of after-effect-Multiple afterexcitations in human retina-Binocular Alternation of Vision-Demonstration of pulsatory response in human retina during exposure to light.

THE nature of the electrical reaction of the retina under stimulus of light constitutes a problem which has attracted many investigators, chief and earliest among these being Holmgren, Kuihne and Steiner, Dewar and McKendric. The results obtained by these workers have been confirmed by the later researches of others; but while their general observations are fairly concordant, the way in which the phenomena they have described are related to the excitatory reaction is a question which has hitherto remained undetermined.

Observed results, in fact, would seem to show that the electrical reactions of the retina are of a nature quite different from those exhibited by other animal tissues. For whereas nerve or muscle, for example, responds on excitation by negative variation, the retina, under the same normal conditions, is said to exhibit a positive variation. The subject is very much complicated, moreover, by the confusion which has unfortunately arisen as to the meaning of the terms positive and negative. These positive and negative variations are so named in reference to the existing current of 
rest, which, as we have already seen, does not constitute a very definite or unvarying standard. A still further source of complication is introduced again when, under certain internal changes in the retina itself, the normal response is found to be reversed.

The present inquiry, then, resolves itself into the following questions :-(I) What is the true current of rest, and do the various currents which have been observed really fall under this head or not? (2) What is the galvanometric character, positive or negative, of the excitatory reaction of the retina-that is to say, is the excitatory phenomenon in the eye similar to, or different from, that of other tissues? (3) May we not discover, in the case of the retina, those multiple excitatory reactions which we have already found to be induced by light in vegetable tissues? (4) And, lastly, what are the after-effects of this particular stimulus in the retina?

Of these we shall deal first with the question of the direction of the current of rest. In an eyeball which is isolated entire it has been found that the nerve is negative to the cornea, and the current of rest is therefore believed to flow through the eye from nerve to cornea. In an isolated nerve-retina preparation, on the other hand, the rod-surface of the retina is negative to the nerve, the current of rest being thus from retina to nerve. These observed currents, however, may not be true currents of rest, but rather excitatory after-effects, due to injury in preparation. In such cases we have seen that the naturally more excitable becomes persistently negative. We have also found that the true current of rest flows from the less to the more excitable, the latter being thus galvanometrically positive. In order, therefore, to determine the true nature of the resting-current, we have first to determine which of each two surfaces, nerve and cornea, and nerve and retina, is the more excitable.

I have already described how, by means of equi-alternating electrical shocks, the differential excitability of a preparation can be determined. We saw also that under such conditions the resulting responsive current flows from 
the more to the less excitable. The experimental arrangement used in this investigation is shown in fig. 248. In such an experiment, equi-alternating shocks are given to the preparation by means of a secondary coil included in the circuit. The existing current, from nerve to cornea, may be balanced previously by a potentiometer. Whether the record be taken under balanced or under ordinary conditions, it is found that in a normal eyeball the responsive current is from the nerve to

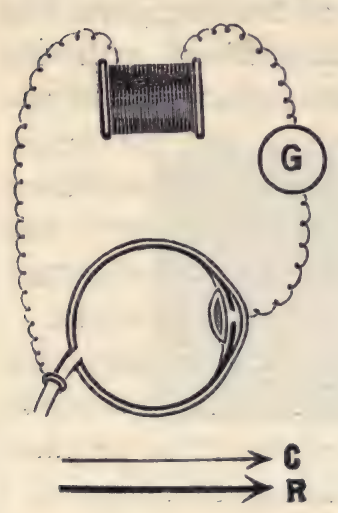

FIG. 248. Experimental Arrangement for Determination of Differential Excitability of Optic Nerve and Cornea

C, injury current, or so-called current of rest; $\mathbf{R}$, responsive current. the cornea, showing that the nerve is relatively the more excitable of the two. Fig. 249 gives a series of such responses. It will also be noticed that the excitatory current is in the same direction

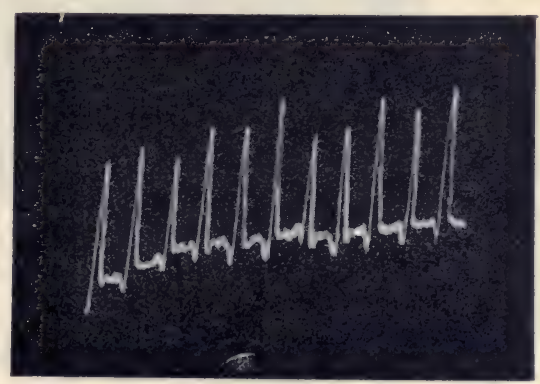

FIG. 249. Series of Photographic Records of Excitatory Responses in Frog's Eye to Equi-alternating Electric Shocks at Intervals of One Minute

Current of response from nerve to cornea.

as the existing current, and thus constitutes a positive variation of it. This positive variation of the current in the eyeball thus indicates a true excitatory reaction of the optic nerve.

From the fact that the nerve is more excitable than the cornea, it is clear that the sectioning of it for isolation of the eyeball, acting as an intense stimulus, will result in its excitatory negativity, which will persist for a time and slowly disappear. Owing to this fact, a current flows from the more excited nerve to the less excited cornea. That this current 
is not the true resting-current, but rather an excitatory effect consequent on preparation, is supported by the known fact that it undergoes a decline more or less rapid. On the other hand, from the fact just demonstrated that the nerve is the more excitable, we should expect that, under natural or primary conditions - that is to say, in the absence of excitatory disturbance-the resting-current would be from the less to the more excitable, or in other words, from cornea to nerve. This conclusion I was able to verify by carefully dissecting away

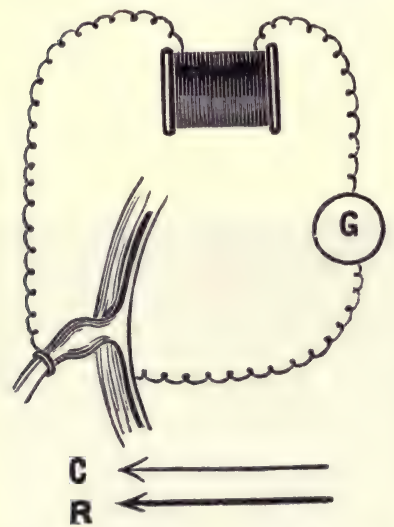

FIG. 250. Experimental Arrangement for Demonstration of Differential Excitability as between Retina and Optic Nerve

C, so-called current of rest; $\mathrm{R}$, responsive current. the socket of the frog's eye, and making connections with the longitudinal surface of the undetached nerve and the cornea. Under these ideal conditions the true resting-current was detected, and was, as expected, from the cornea to the nerve.

In order, next, to determine whether the current, observed to flow: in a nerve-retina preparation from retina to nerve, is a true resting-current, or merely an excitatory after-effect, I proceeded to determine, in the manner already described, which of the two surfaces was the more excitable. Under the excitatory effect of equi-alternating shocks, the responsive current was found to flow from the retina to the nerve, thus proving that the retina was the more excitable (fig. 250). The first series of responses in fig. $25 \mathrm{I}$ gives a record of these effects with a moderate intensity of stimulation, while the second series shows the responses under an intensity nearly twice as great. The so-called current of rest observed in the nerve-retina preparation is thus to be taken as due to that after-effect of preparation which is inseparable from the isolation of such a highly excitable tissue. It will also be 
noticed that the true excitatory effect on the retina is in the same direction as the existing injury-current, and thus constitutes a positive variation of it.

Thus, as regards forms of stimulus other than light, such as, for example, the electrical, the responsive reaction of the retina is by induced galvanometric negativity. This at once disposes of the doubt that the general reaction of the retina might be of a different sign from that of any other tissue. But we have still to determine whether or not the stimulus of light, in particular, induces the same normal negative change. The next question to be taken up, then, is that of the true nature of the responsive variation induced in the

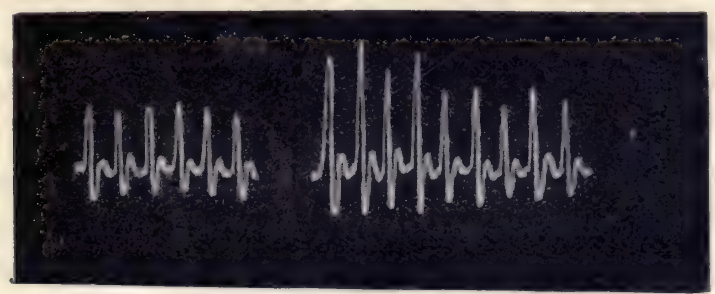

FIG. 25I. Series of Photographic Records of Excitatory Responses in Frog's Retina to Equi-alternating Electric Shocks at Intervals of One Minute

Responsive current from retina to nerve. First series show response to stimulus of moderate intensity ; the second series to stimulus of intensity nearly twice as great.

retina by light. We have seen that the effect recorded as normal by numerous observers, whether in the eyeball or in the isolated retina (Kühne and Steiner) was a positive variation. But since we now know that retinal response to stimulation in general is not unlike that of other tissues, and since, with regard to the stimulus of light in particular, we have seen it induce true excitation in vegetable tissues, we might expect the responsive reaction of the retina to light to take place by galvanometric negativity. We must then accept one of two conclusions. Either the positive change is a misnomer for the phenomenon observed in the eye, or the inference which we have drawn from the analogy of vegetable tissues is not justified. 
As, in reference to this latter point, however, it may be urged that the retina is exceptionally sensitive to light, while the reactions of vegetable tissues are generally sluggish, it may be worth while to point out here that vegetable tissues are not so insensitive as is generally supposed, but are often, on the contrary, highly susceptible to the action of light. It was, for instance, found by Darwin that the cotyledons of Phalaris canariensis were, in the course of some hours' exposure, curved towards a small lamp, placed at a distance from them of 12 feet. The intensity of the stimulus of light was in this case extremely feeble. I have myself observed, again, the remarkable sensitiveness to light of the terminal leaflet of Desmodium, which, on the mere striking of a match in its vicinity, was thrown into a state of pulsatory movement.

I shall, in the course of the present chapter, describe certain definite effects on the retina, which will prove, in an unmistakable manner, that, when exposed to light, it undergoes a change of galvanometric negativity. It is now therefore necessary to show clearly that what has been described as the positive variation is really due to the excitatory negative change of the retina. What has to be demonstrated, then, is the way in which this simple underlying reaction of negativity comes to appear as a positive variation of the opposite-directioned currents of rest in the eyeball and isolated retina respectively.

As regards experiments on the eyeball, with contacts at nerve and cornea, we have seen that the existing injurycurrent is from nerve to cornea, and that this undergoes a positive variation when the nerve is stimulated in any way. This is because the added negativity induced in the nerve gives rise to an increase, or positive variation of, the existing current (fig. 248). Now, when light falls on the eye it acts on both the cornea and the retina. The former, however, is relatively inexcitable, especially to so moderate a stimulus as that of light. But the retina is excited, and its excitatory condition is rapidly transmitted to the optic nerve, which 
thus becomes galvanometrically negative. The so-called positive variation observed under such an experimental arrangement, then, is really the result of the true excitation of the retina conducted to the optic nerve.

In experiments on nerve-and-retina preparations, with contacts on the nerve and the retina, the existing injurycurrent is from retina to nerve. When both nerve and retina are excited simultaneously, by equi-alternating electrical shocks, we have seen that, on account of the greater excitability of the retina, the resultant responsive current, here differential, is from retina to nerve, constituting a positive variation of the existing injury-current (p. 419). In the case of stimulation by light, however, it is the retina which is directly excited. The added negativity thus induced in the retina gives rise to an increase or positive variation of the existing injury-current (fig. 250). Hence, in all these cases, the socalled positive variation really indicates the normal excitatory negative effect. The apparent anomaly involved in the supposition that the response of the retina to light was positive, and thus of opposite character to that of other excitable tissues, is thus seen to be due to a misinterpretation of observed results. It is unfortunate that, as a consequence of this misinterpretation, the effects described by different observers, of the response of the eye as 'positive,' are really to be understood as 'negative,' and vice versa. When quoting these results, therefore, I shall always give the actual effect indicated in italics and in parentheses.

Another observation which lends independent support to the view that the retina exhibits the true excitatory reaction, is found in the fact-noted by Van Genderen-Stort and Engelmann-that the cones exhibit a motile effect by retraction under light. It has been supposed from this that the optic nerve contains not merely sensory, but also retinomotor fibres. But I shall show that it is not that the endings only of the optic nerve, but also that nerve itself, which exhibit true excitatory contraction under stimulation (cf. figs. 324,404$)$. All nerves, in fact, will be shown in a later 
chapter to exhibit normal excitatory contraction, and it will be by the study of these motile responses in nerves, and their variations under different condition, that we shall be able unerringly to relate the abnormal responses sometimes seen in the retina to those changes of condition to which they are due.

With regard to these abnormal responses, I have already shown that various tissues exhibit them under the two different conditions of sub-tonicity and fatigue. With regard to nervous tissue in particular, however, I may refer here, by anticipation, to results which will be given in detail in Chapter $\mathrm{XXXV}$. concerning the mechanical response of nerve and its variations. In normal conditions of excitability the nerve gives response by contraction, and this is its true excitatory response, concomitant to the electrical response of galvanometric negativity. This excitatory response, then, whether by contraction or by negativity, will here, as in preceding chapters, be designated 'negative.' We have seen that the maintenance of the normal condition of a highly excitable tissue depends on its supply of energy. Hence, when such a tissue is isolated from the organism of which it forms a part, it is liable to fall below its normal tonic level. This depressed condition, however, does not connote any permanent chemical depreciation, but only a temporary depression of its fund of energy. Under such an induced lowering of the tonic condition, the response is reversed to positive. But under continuous stimulation, excitability is again enhanced, and the abnormal positive is converted to normal negative.

Thus we obtain, in a somewhat sub-tonic tissue, the following results :

(I) Under a short-lived or instantaneous stimulus, whose effective value falls below the true excitatory level, response is positive (expansion).

(2) Under the continuous action of stimulus the effective value is at first below, but after a time rises above, excitatory efficiency. In consequence of this we obtain a first phase of 
response which is positive, followed by a second which is negative (expansion followed by contraction).

The second condition to induce a reversal of the normal response occurs in consequence of the fatigue due to previous over-stimulation.

(3) A reversed positive response (expansion) in consequence of fatigue.

The various anomalies which occur in the response of the eye will all be found resolvable into one or other of these cases. I shall first describe certain experiments which will demonstrate the reversal that is brought about by induced sub-tonicity. We have seen that the normal response of an eyeball, with two contacts at nerve and cornea, consists of a current from the nerve to the cornea. I have already given records of such normal responses, obtained by subjecting the preparation to equi-alternating electric shocks (fig. 249). In certain cases, however, in which the isolated eyeball of the frog had fallen into a sub-tonic condition, the response was found to be reversed, the nerve, under excitation, becoming relatively positive instead of normally negative. It has already been said that such a reversal is due to great depression in the condition of the nerve. In dealing with these cases, therefore, it occurred to me that it ought to be possible to restore the normal response by the application of some exciting reagent-say, dilute $\mathrm{Na}_{2} \mathrm{CO}_{3}$ - to the nerve in its depressed condition. As the result of this application I found the reversed response to be restored to the normal.

I obtained results precisely similar to these with isolated retina of the frog. The normal responses-a current from retina to nerve (fig. 238)-under equi-alternating shocks, were here found, in a depressed specimen, to be reversed. But the application of $\mathrm{Na}_{2} \mathrm{CO}_{3}$ solution on the retinal surface brought the responses back to the normal. These abnormal responses, then, rectifiable to normal, are those due to subtonicity. And under fatigue finally, induced by longcontinued, or over stimulation, I find the normal response of the eye to be reversed. 
This is the place in which to refer to various anomalous effects observed by previous investigators, and to offer satisfactory explanations of them from the results which I have already demonstrated. I shall therefore give a brief summary of these from the admirable account of Biedermann. ${ }^{1}$

(a) 'In light, frogs-i.e, such as have been exposed for hours to the full effect of daylight-the positive fore-swing of the negative variation concomitant with the impact of light is entirely wanting, or appears as a trace only.' 'Since this positive variation of the existing current really means, as I have already shown, the 'true excitatory negative,' and the negative variation conversely, the occurrence of positive response, this observation means that a frog's eye, previously exposed for a long time to light, gives positive response.

This, then, is a simple instance of the reversal of response from negative to positive under fatigue, which I have already dealt with above as case (3).

(b) 'The fact that the three phases of the retinal action current, due to transitory illumination, appear in sensitive preparations, even when, as with the electric spark, the impact of light is momentary, shows that the medium negative (positive) phase must not be regarded merely as the consequence of permanent illumination, since it is just this phase which alone appears in less excitable preparations with instantaneous light stimuli.'

With regard to this it may be said that the medium positive phase here referred to, as given by the excitable retina under continuous stimulation, is not the same as that positive response which 'alone appears in less excitable preparations with instantaneous light stimuli.' The former is due to fatigue-reversal, while the latter is an instance of the abnormal positive response of a sub-tonic tissue to feeble stimulus. This will be understood from the following considerations. In the retina, under continuous stimulation, the first phase of response is the true excitatory negative. This gives place to a second, or positive, due to fatigue-decline.

\footnotetext{
1 Biedermann, Elcctro-Physiology (English translation), vol. ii. pp. 474-477.
} 
And this is again succeeded by a transitory negative effect on the sudden cessation of light. These constitute the three phases of retinal action-current just referred to, under continuous stimulus. This sequence is wrongly represented in symbols as $(+-+)$, since the actual changes concerned are $(-+-)$.

Now in highly excitable tissues, under instantaneous stimulation, we observe a sequence of response apparently similar, the first being normal negative, the second positive, owing partly to recovery and partly to the positive aftereffect; and the last phase representing a return from this positive. These three phases, therefore, though apparently similar, are not really the same as those just referred to under continuous stimulation, where the 'medium negative (positive) phase' was the result of fatigue-reversal. The socalled negative (positive) effect which alone appears on the instantaneous stimulation of relatively inexcitable tissues is, again, the positive response of a sub-tonic tissue to a stimulus deficient in true excitatory value, already described (p. 422) as case (I). Similar positive responses of vegetable tissues in a sub-tonic condition under the action of light were seen in fig. 240.

We come next to the question whether or not we may discover multiple responses in the retina analogous to those which have already been demonstrated in the case of vegetable tissues. The occurrence of such an effect has not hitherto been suspected. We have seen that, under the continued action of light, vegetable tissues exhibit multiple responses; and since we have found a general close analogy to exist between the responses to light of the retina and of these, we should expect that similar multiple responses would be found in the eye also. The reason why these were not hitherto detected lay in the inevitable depression of excitability in the isolated retina or eyeball. In the retina of certain fishes, where the excitability does not appear to decline so rapidly, I have often obtained records of multiple responses For example, in the retina of Wallago attu fish the stimulus of 
light applied for three seconds gave as its after-effect multiple responses which lasted for ten minutes, the average period of each oscillation being twenty seconds. I have also obtained such multiple responses from the eye of vigorous bull-frogs, a photographic record of one of these results being given in fig. 252. We shall also see at the end of this chapter that it is quite easy to detect the occurrence of these multiple responses in the intact human eye under stimulus of light.

As we have seen that multiple response is the expression of energy previously absorbed, and held latent in the tissue for a time, and since, as has been stated, the retina itself exhibits multiple response, it is easy to see that this organ, on the cessation of light, will show after-effects.

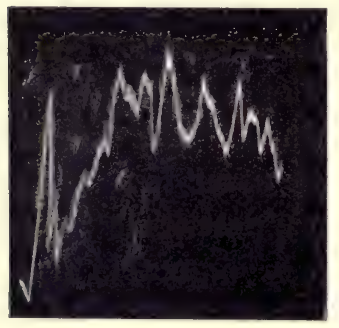

FIG. 252.-Photographic Record of Multiple Response of Retina of Frog under Continuous Action of Light

In my experiments on the effects of light on vegetable tissues I found, as has already been said, three different types of direct- and aftereffect. The first of these related to those highly excitable tissues which under continuous stimulation gave normal responses $(-+-+)$. In such cases, if the stimulus was stopped on the attainment of maximum positivity the immediate after-effect was an increase of positivity. The formula was thus $(-+\cdot)$. In the third type, with sub-tonic tissues, the sequence, under continuous stimulation, was $(+-+-)$, and on the stoppage of stimulation at maximum negativity this negativity became suddenly augmented. The formula here was thus $(+-\cdots)$. In the second or intermediate type, again, the formula of the direct and aftereffects was either $(-+\cdots)$ or $(-\cdots)$.

I shall now discuss in some detail the various types of after-effects met with in the retina, corresponding, as I shall be able to show, with those met with in vegetables tissues. After-effects like those of Type I., had not hitherto been noticed in the retina for the reason that their demonstration 
can only be obtained in a tissue of normal high excitability, and not in one which has undergone depression in consequence of isolation. I was fortunate enough, however, to meet with a species of fish, Ophiocephalus marulius, whose vitality is so exceptional that it lives for days when taken out of water. When the fish is pithed, its heart continues beating vigorously for many hours. I made a preparation of the eye of this fish and carried out experiments on it under the action of light.

In fig. 254 is given a record obtained with this specimen during the application of light and on its cessation. It will

FIG. 253.

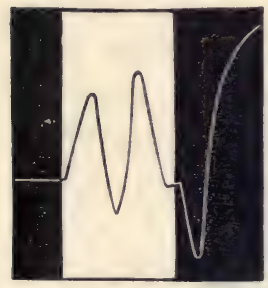

FIG. 254.

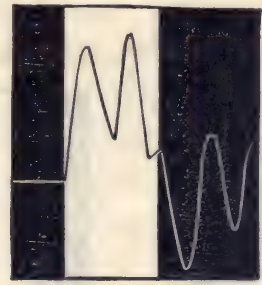

FIG. 255 .

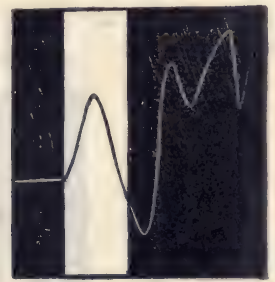

Figs. 253, 254, 255. Parallel Records of Responses given by Plant and Retina, during and after Illumination, illustrative of Type I $(-++)$. In all these cases up-curve represents induced galvanometric negativity ; down-curves, positivity. White background in this and following records represent light, and shaded, darkness.

Fig. 253. Response of petiole of Bryophyllum. Light was cut off on attainment of maximum positivity in the second of the multiple responses.

Fig. 254. Similar effect in response of retina of Ophiocephalus fish.

Fig. 255. The same with another specimen. Light was here cut off after the first oscillation.

be seen that during the application of light the sequence was $(-+-+)$. It will be noticed that, after completing two oscillations, and after the response-curve was even slightly reversed at its maximum positive phase the light was withdrawn. The immediate effect was a sudden increase of positivity, followed by a series of after-effect oscillations. In the next figure (255), obtained with a different specimen of the same fish, light was withdrawn at the exact moment of maximum positivity, and the result is seen to be similar to the last-namely, an immediate enhancement of positivity, 
followed by an after-oscillation. The essential similarities between these and corresponding records obtained on a fastmoving drum, of the response of the petiole of Bryophyllum under light (fig. 253), are sufficiently obvious.

When the excitability of the tissue is not so high, we may obtain after-effects of Type II., in which the formula is $(-+\cdots)$ or $(-\cdots)$. This was exemplified in vegetable tissues (cf. fig. $245 d$ ). In Ophiocephalus, I was able to obtain this result also, when the specimen was slightly fatigued (fig. 256). With the eye of the frog, Kühne and Steiner obtained the record given in fig. 257 , which is seen to be parallel to that given in fig. 256 , its true significance being shown in the formula $(-+\cdots)$.

Fig. 256 ,

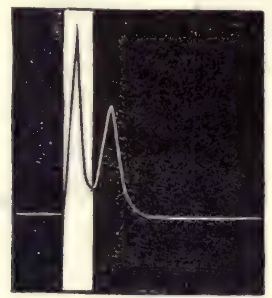

FIG. 257 .

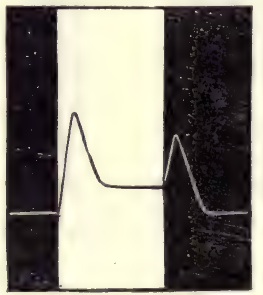

Figs. 256, 257. Parallel Records given by Plant and Eye, during and after Illumination, as illustrative of Intermediate Type II. $(-+\cdots)$

Fig. 256. Response of retina of Ophiocephalus when slightly fatigued.

Fig. 257. Response of frog's eye (Kühne and Steiner).

A sub-case of Type II. is represented again, by $(-\cdots)$, where on the sudden cessation of light, there is a transient increase of excitatory response. This, as we saw, was due to the abrupt withdrawal of the antagonistic influence of a reversing force. I may state here that I have been able to demonstrate an exactly parallel effect with nerve of frog, where the excitatory negative effect during stimulus undergoes a brief and sudden augmentation on its cessation (p. 536). This sudden augmentation on the stoppage of stimulus has been taken as a proof of the existence of antagonistic processes of assimilation and dissimilation, rather than as due to molecular derangement by external stimulus and its after-effect. 
That, for its explanation, however, it is not necessary to postulate the two processes of assimilation and dissimilation is clearly seen from the fact that I have obtained an exactly similar effect in inorganic substances, such as silver bromide, a record of whose response is seen in fig. 258 .

We next turn to what has been designated as Type III., in which the sequence of responses, owing to the depressed condition of the tissue, is reversed, the formula here being $(+-+-)$, while the direct and aftereffects are represented by $(+-\cdots)$. This result was already obtained in the sub-tonic tissue of the petiole of cauliflower, a record of this being given in fig. 259. I was able to detect similar effects in a retina of Ophiocephalus, in which response had become reversed under the sub-tonicity due to long isolation (fig. 260). These results will explain the somewhat anomalous response which Kühne and Steiner obtained with the isolated retinæ of certain fishes (fig. 26I).

Returning now from the question of multiple excitations during and after the exposure to light-in prepared specimens, where results must be modified to an unknown extent by the effects of isolation and preparation-to that of the responses of the intact eye, where alone we may expect to obtain the truly normal effects, we may attack

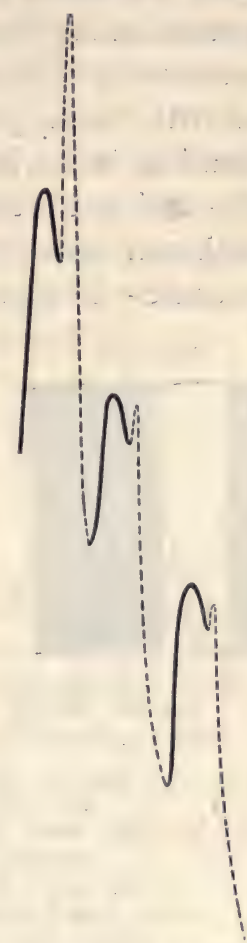

FIG. 258. After-effect of Light on Silver Bromide

The thick line represents response during light (half a minute's exposure), and dotted line the recovery during darkness. Note the terminal positive twitch. the problem by means of visual sensation itself. Multiple excitation, as the after-effect of strong lurninous stimulation, is here somewhat easy of demonstration. But its exhibition 
during the action of light presents unusual experimental difficulties.

It is a well-known fact that if, after looking at a bright object for some time, we close the eyes, we see the image repeated many times. The occurrence of these after-images is somewhat different under a transitory flash of illumination, and with more persistent exposure to light. As the visual sensation is to be regarded as the excitatory effect, there will-owing to the fact that this excitation must reach its maximum some time after the cessation of an instantaneous exposure-be a persistence of the excitatory after-effect, with

FIG. 259.

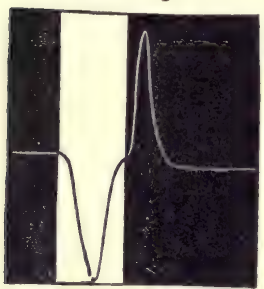

FIG, 260.

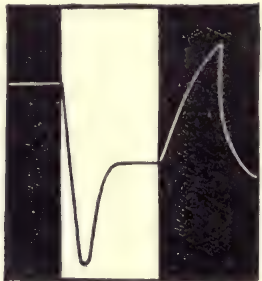

FIG. 26I.

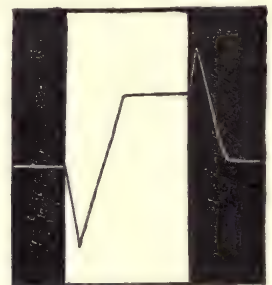

Figs. 259, 260, 26r. Parallel Records of Responses given by Plant and Retinæ, during and after Illumination, illustrative of Type III. $(+-\ldots)$

Fig. 259. Response of petiole of cauliflower. Light was here cut off on attainment of maximum negativity.

Fig. 260. Response of retina of Ophiocephalus fish when depressed.

Fig. 26I. Response of isolated retina of fish as observed by Kithne and Steiner.

N.B.-The true excitatory negative response was by these observers described as the 'positive variation.'

its corresponding visual sensation. This is not so, however, when the eye is closed after looking at a bright object for a considerable time. In this case there is a positive reboundopposite to the true excitatory negative effect-with concomitant sensation of darkness. The next rebound is negative, giving rise to a repetition of the original sensation ; and these alternating phases may be repeated many times. With the eyes closed, the negative or luminous phases are the more prominent.

The same phenomenon may be observed in a somewhat different manner when, after: staring at a bright object, we 
look towards a well-lighted wall. The dark phases will now become the more noticeable. If, however, the wall be dimly lighted, both the dark and bright phases will be noticed alternately. It has been suggested that these phenomena might be due to some obscure form of fatigue, but the regular alternations observed clearly demonstrate them to be a case of oscillatory changes and multiple response.

The demonstration of multiple responses in the human retina, as an after-effect, is thus seen to be easy. But their occurrence during continuous exposure of the eye to light is more difficult to prove. This arises from the fact that the waxing and waning of the effect are so gradual as not to be noticeable, unless against some definite standard of comparison. I shall now prove that, during the continuance of constant light, pulsatory visual effects are produced. These pulsations go unnoticed in visual sensation, not only for want of a standard of comparison, but also because of the remarkable fact which I have discovered, that while the impressions of each individual retina undergo variation, the sum total of the two remains always approximately constant. I have been able to provide the necessary comparison-standards by having two distinguishable images produced in the two eyes, the fluctuation of the visual excitation in one eye being thus capable of detection, by comparison with that in the other. It would have been impossible to detect this fluctuation, had the excitatory variation taken place in the two eyes simultaneously, i.e. if the maximum excitation in the one had occurred at the same moment as the maximum excitation in the other. But I have found that, as regards excitation there is a relative difference of phase, of half a period, between the two retinæ, so that the maximum effect at a given moment in one eye corresponds to the minimum in the other. This constitutes the phenomenon which I have designated the Binocular Alternation of Vision. It is owing to this fact that the periodic excitations in each retina are unmistakably brought out by the following experiment, which consists in looking through a stereoscope that holds, 
instead of photographs, incised plates with two inclined cuts, the right eye seeing the slit inclined to the right, while the left sees that inclined to the left. When the observer looks through the stereoscope turned to the bright sky, his two eyes are acted on by strong stimulus of light through these divergently-inclined slits. . The resultant sensation forms the image of an inclined cross (fig. 262). On now closing the eyes the multiple after-effect is observed as recurring images. The relative difference of half a period, already referred to, is here perceived in a very interesting manner, for the afterimage is not now the complete inclined cross. Instead of this, each of the two arms is seen in regular sequence alternately.

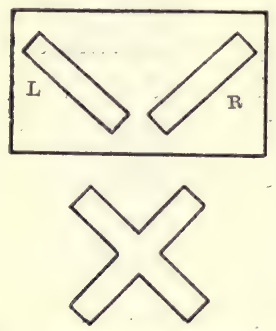

FIG. 262. Inclined Slits for Stereoscope and Composite Image formed in the Two Eyes
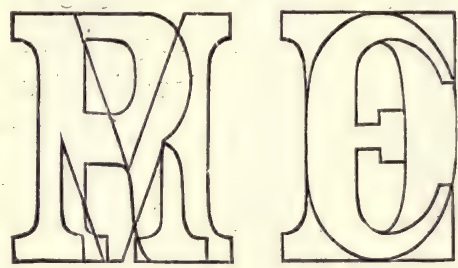

FIG. 263. Composite Indecipherable Word, of which Components are Seen Clearly on Shutting the Eyes

That is to say, when the one is at its brightest the other has completely disappeared, and vice versa. The impression in each eye thus undergoes a periodic fluctuation. Some yery curious effects can thus be exhibited, when, instead of the two inclined bars, the incisions to be looked through compose a word. For instance, two letters forming half of the word may be seen by one eye, and the other two by the other. The result of this is that, as long as the eyes are open, we obtain an indecipherable image, due to superposition, like the following (fig. 263).

But when the eyes are closed, then, owing to binocular alternation of the multiple after-effect, the blur disappears, and the word is spelt out in repeated succession in the field 
of dark vision, as RO ME RO ME, and so on. In this curious instance one sees better with eyes closed than open!

The next problem that presents itself is that of demonstrating the pulsatory nature of the visual sensation in each eye, under the constant action of light. The stereoscope, with its two inclined slits, is now taken and turned towards the bright sky, and when the design is looked at steadily it will be found that, owing to pulsatory excitation in each eye, and the binocular alternation of vision, when one bar of the cross begins to be dim the other becomes bright, and vice versa. The success of this experiment is determined by the fact that the image in each eye forms a comparison-standard for the other. And as the changes in the two eyes proceed in opposite directions, the visual fluctuations during the continuous action of light, are brought out with the greatest distinctness. It may be stated here that the period of this visual oscillation has an average value of about four seconds. It is, generally speaking, shorter with young people, and longer with old. Even in the same individual, again, it is modified, according as the previous condition has been one of rest or activity. I give here a set of readings given by an observer :

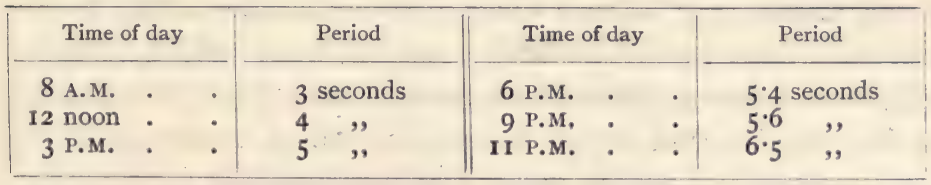

In this connection it may be interesting to mention that the period of a single oscillation in a multiple response, measured in frog's retina, under experimental conditions, was ten seconds. In the case of the retina of Wallago, the average period was twenty seconds. 


\section{CHAPTER XXIX}

\section{GEO-ELECTRIC RESPONSE}

Theory of Hydrostatic Pressure and Theory of Statoliths-Question regarding active factor of curvature in geotropic response, whether contraction or expansion-Crucial experiment by local application of cold-Reasons for delay in initiation of true geotropic response-Geo-electric response of shootDue to active contraction of upper side, with concomitant galvanometric negativity-Geo-electric response of an organ physically restrained.

IN the case of the action of external stimulus on plant organs it is possible, given the direction of incident unilateral stimulus, to predict the nature of the responsive movement. I have shown elsewhere, in my work on Plant Response, that all the actual movements of a plant organ can be deduced from the simple law that it is the more excited side that becomes concave. But though this law is sufficient guide in dealing with the action of a known external stimulus, yet the problem becomes much more obscure when we have to account for any movement which occurs in response to a stimulus whose seat is internal. An example of this class is afforded in the responsive curvature associated with gravity. Thus, a shoot laid horizontally will curve upwards till the free end becomes vertical. In connection with this subject there are two different points to be elucidated. First, is the question as to the mode in which gravity exercises stimulation; and second, that of the method by which, in answer to this stimulus, a definite responsive curvature takes place. As regards the first of these, it may be said that the only conceivable way in which gravity could produce stimulation is by some differential effect of weight acting on the responding cells. According to this, the necessary differential weight-effect may be due to the weight of cell- 
contents, whether of the sap itself or of those heavy particles like starch-grains, which are contained in it. The former, or Theory of Hydrostatic Pressure, was suggested by Pfeffer and supported by Czapek; the other, or Theory of Statoliths, has been advocated by Noll, Haberlandt, and Nemec.

In the case of a multicellular plant laid horizontally (fig. 264), $\mathrm{E}$ and $\mathrm{E}^{\prime}$ may be regarded as areas in which stimulation is caused by the weight of the particles. It is obvious that the effects produced on the upper and lower sides of the shoot are antagonistic; yet, in spite of this, we obtain a resultant curvature upwards. This shows that the excitation of one side must be greater than that of the other, the particular directioned mechanical effect being due to this fact.

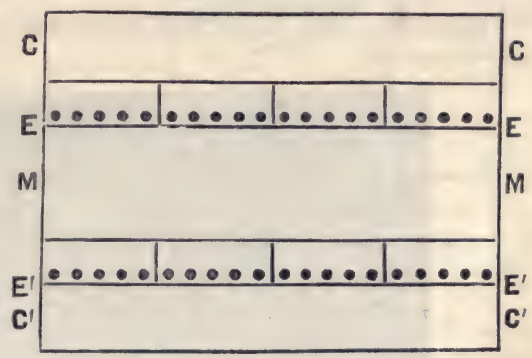

FIG. 264. Diagrammatic Representation of a Multicellular Organ laid Horizontally and Exposed to Geotropic Stimulus.

On the upper side the statoliths act on the inner, and on the lower side on the outer, tangential wall (after Francis Darwin).

It is clear, then, that the induced curvature upwards of the horizontally-laid shoot is due to the effective action of the weight particles on either the upper or the lower side of the shoot. We can see that if it is the upper which is the more effective, then curvature must take place by excitatory contraction; if, on the contrary, the lower be the more effective, the curvature is then to be regarded as the result of responsive expansion. There has been considerable uncertainty as to which of these is actually the case, the prevailing view being that it is the expansion of the lower surface which is the active factor. ${ }^{1}$

That it is, however, the excitatory contraction of the upper side which is the active factor in this curvature I have already demonstrated by alternate unilateral applications of

${ }^{1}$ For more detailed account see Plant Response, pp. 495 to 5 II. 
cold. It is known that the continuous application of cold diminishes or abolishes the excitability of a tissue. If, then, it is by excitatory action on the upper side that curvature is induced, it will be found that the local application of cold on that side will retard or arrest this responsive curvature, the application of cold on the lower side producing practically no effect. If, on the contrary, this curvature should be mainly due to some excitatory action on the lower side, we may then expect to find that the application of cold on that side has

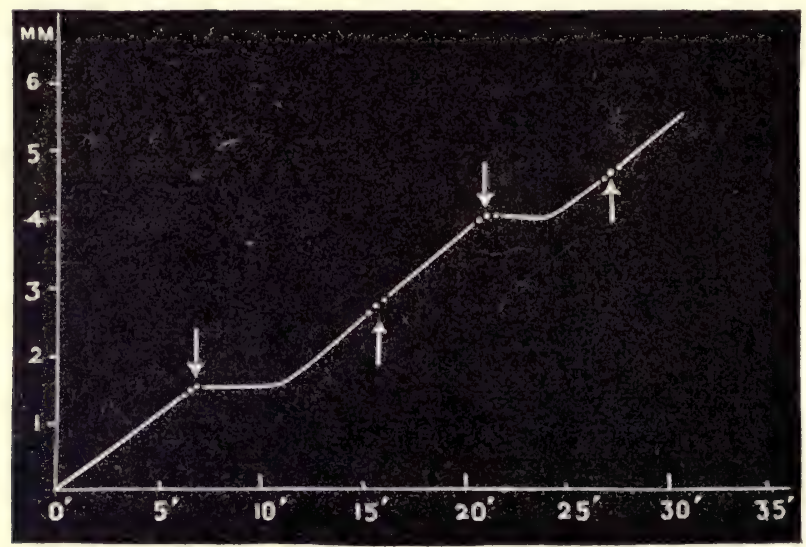

FIG. 265. Effect on Apogeotropic Movement of Temporary Application of Cold on Upper and Lower Surfaces respectively

Application above is seen to produce arrest of movement, while application below has no perceptible effect. Ordinate of curve represents up-movement of tip of organ in $\mathrm{mm}$; abscissa represents time.

the effect of arresting the growing curvature, its application on the upper being more or less ineffective. On carrying out these experiments it was found that cold had the effect of arresting curvature only when it was applied on the upper side of the shoot (fig. 265). This conclusively proved that gravitational stimulus, acting on the horizontally-laid shoot, induced response by excitatory contraction of the upper side. This fact, of response by contraction, is fully concordant with what we know of the effect on the plant of other forms of stimulation. This question I intend, however, in the course 
of the present chapter, to submit to independent examination by means of electrical response.

I have already shown that the unilateral pressure of particles on the growing organ is effective in inducing curvature in such a way that the side acted upon becomes concave. This experiment was carried out by subjecting one side of a growing organ to the pressure of iron particles, which were pressed against it by the action of an electro-magnet on the opposite side (fig. 266). The magnetic particles in this case functioned as virtual statoliths, and a curvature was induced by the excited side becoming concave (fig. 267).

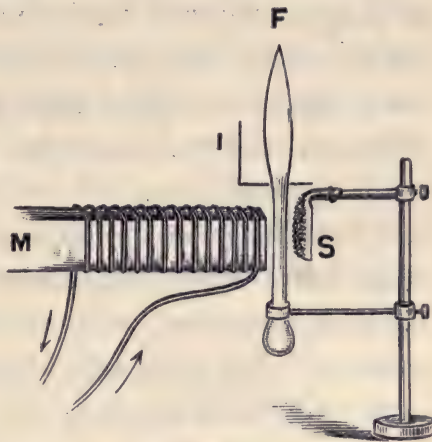

FIG. 266. Diagrammatic Representation of Experiment showing Curvature Induced by Unilateral Pressure Exerted by Particles

F, flower-bud of Crinum; s, indiarubber strip studded with iron particles attracted by electromagnet, M, causing unilateral pressure on growing region; 1 , index attached to flower.

There is, however, one difficulty in connection with the statolithic theory of stimulation. When the stem is held erect the particles rest on the bases of the cells, and their general distribution on the two sides of the organ is symmetrical. Asymmetry of distribution is induced, however, when the shoot is laid horizontally, and stimulation might be ex- FIG. 267. Record of Responsive Curvature pected to follow within

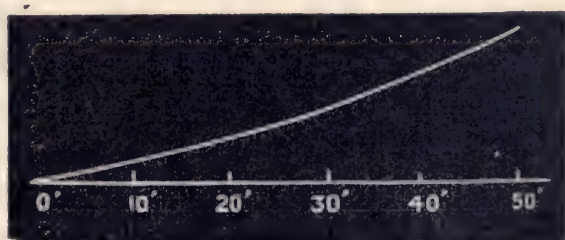
Induced in Bud of Crinum Lily by Unilateral Pressure of Particles

a very short time, since

the displacement of particles from base to side cannot take long. This being so, the geotropic curvature of the shoot upwards should take place within a short time. But, instead of this, we find that the curvature is at first downwards ; and 
it is not till after the lapse of a period, which is sometimes as much as an hour in duration, that there is a reversal of this downward movement into the normal apo-geotropic movement. Fig. 268 gives a curve which exhibits this preliminary movement persisting for nearly forty minutes before its ultimate reversal into the usual normal apogeotropic curvature upwards.

This delay in the appearance of the characteristic response may, however, be explained from the known fact that steady tension increases, whereas compression retards, the rate of growth. Thus, in a horizontally-laid shoot, there is a tendency to curve down by its own weight, the upper side being

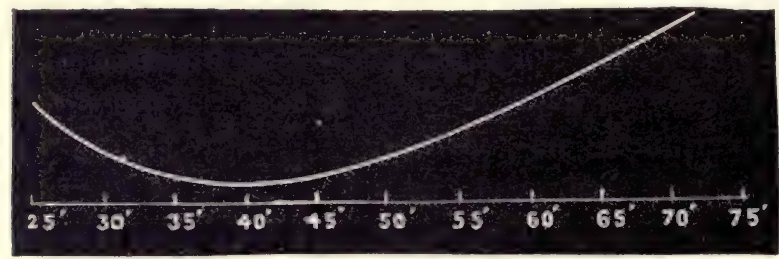

FIG. 268. Record of Apogeotropic Response in Scape of Uriclis Lily'

The up curvature due to apogeotropic action proper commenced forty minutes after the specimen was laid horizontally.

in this way subjected to tension, and the lower to compression. The effect of these is an increased rate of growth on the upper, associated with a decreased rate on the lower, sides of the specimen, giving rise to a downward curvature. The fact that geotropic stimulus has to overcome this action before its own characteristic effect can be exhibited may account for the observed delay in its appearance. A crucial experiment in support of this explanation will be given presently.

We have seen in the last chapter that the presence of internal excitation is capable of detection by electrical indications, and by taking advantage of this fact we have an independent means of coping with the obscurities of the present problem. I have demonstrated, by means of experiments already referred to, that it is the upper side which, 
under the action of gravity, undergoes excitatory contraction. It follows that, as an indirect effect of such contraction, the water expelled from the upper will reach the lower side of the shoot, and cause an increased turgidity and expansion of that side. The apogeotropic curvature is thus brought about directly by the contraction of the upper, and indirectly by the expansion of the lower, side of the horizontally-laid shoot. This being so, we may expect, in accordance with our previous investigations on the true excitatory and hydrostatic effects respectively, that that side will become galvanometrically negative which is excited by geotropic stimulus. And it is found that it is in fact the upper side of the horizontally-laid shoot which exhibits galvanometric negativity; the lower, in which the positive turgidity-effect had been indirectly induced, being found to show galvanometric positivity.

But this result is only obtained simultaneously with the induction of the normal apogeotropic curvature. And, before this, other disturbing effects may occur. We take an erect stem and make two galvanometric contacts, one on each side of its growing region. The stem is next laid horizontally. As regards the electrical effects which are now exhibited three distinct phases are to be distinguished. First, in consequence of the mechanical disturbance due to the laying of the organ on one side, there will be an excitatory electrical effect on both upper and lower sides, and the resultant responsive current will be determined by the difference of excitability which may happen to exist, in this particular individual, between the two. This variable stage is terminated in the course of about ten minutes. We next have a steady condition, brought about by the effects of tension and compression already described as acting on the upper and lower sides respectively. This gives rise, as we have seen, to increased growth, with its attendant positive turgidity, on the upper as compared with the lower side.

Hence, during this stage, when the curvature is proceeding downwards, we may expect that the upper side will 
be galvanometrically positive in relation to the lower, the direction of the current in the horizontal shoot being from below to above. And finally, in the third stage, we have the geotropic stimulation effectively overpowering and reversing this downward movement. And since it is the upper side that is now geotropically excited to an effective extent, we find that that side becomes galvanometrically negative. Thus, the electrical indication, like the mechanical, gives in this ultimate stage the characteristic response of the

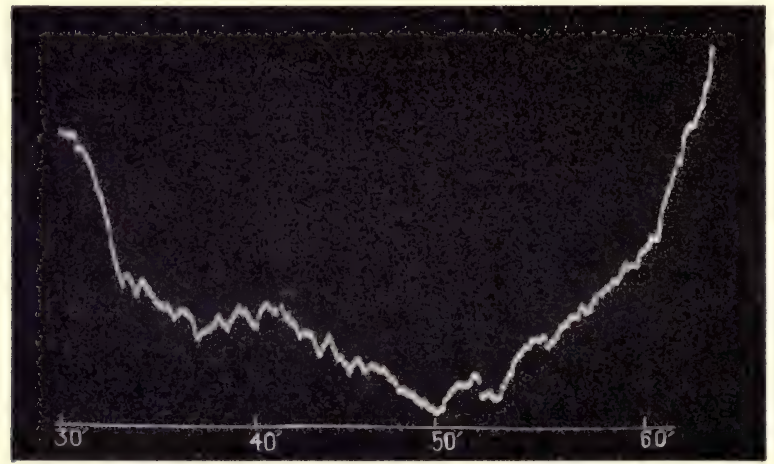

FIG. 269. Photographic Record of Geo-electric Response in the Scape of Uriclis Lily laid horizontally

In the first phase of response, the current is from the upper surface to the lower, the upper being galvanometrically positive. After fifty minutes, the excitatory geotropic effect reverses the current, which is now ascending, or from below to above, the excited upper-surface being galvanometrically negative. (Compare corresponding mechanical record, fig. 268.)

plant-tissue to gravitational stimulus. The excitatory effect is now exhibited mechanically by the contraction and concavity, and electrically by the galvanometric negativity of the upper side of the horizontally laid shoot (fig. 269). These similarities between the mechanical and electrical records will be seen on comparing this figure with fig. 268. We have thus seen that, owing to secondary mechanical disturbances, the proper exhibition of the true geotropic response is delayed. And from this it is difficult to say how quickly the geotropic response follows the dis- 
placement of the hypothetical statolithic particles. I have been able to overcome this difficulty, which at first appears very great, in the following way. It has been shown that the state of excitation, even when all mechanical expression of it is restrained, may be detected by galvanometric negativity. Those secondary effects, due to mechanical disturbance, which mask for a time the excitatory effect of gravitational stimulus, may thus be eliminated completely by restraining all movement of the shoot. The problem thus resolves itself into the fixing of an experimental shoot, say the peduncle of Uriclis lily -in such a way that mechanical

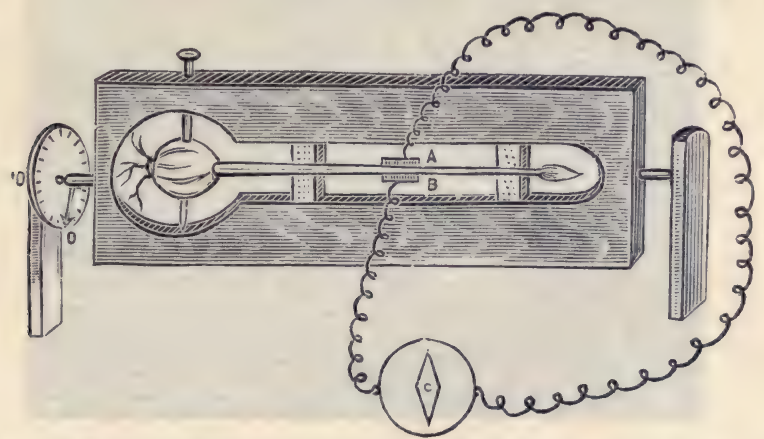

FIG. 270. Experimental Arrangement for Subjecting Organ to Geotropic Stimulus, Mechanical Response being Restrained

response is completely restrained. The next point is to subject the specimen, at a given moment, to the stimulus of gravity, and record the consequent electric response.

I shall now describe the experimental method by which these conditions were successfully met. It is clear that when any two points are acted on symmetrically by the force of gravity, there is no resultant geotropic action. This is the case in regard to two diametrically opposite points, A and B, situated laterally on an erect shoot. When the shoot is laid horizontally, two lateral points are again acted on symmetrically by the force of gravity, and there is thus no differential action as between the two. But if the shoot be now rotated on itself, so that one of these points is diametrically 
above, and the other below, a differential action will be induced as between the upper and lower sides, the upper being relatively the more excited. In the following experiment I took a specimen of Uriclis lily, and fixed the entire plant horizontally, as seen in the figure (fig. 270). The pivoted support allowed the responsive points $\mathrm{A}$ and $\mathrm{B}$ to be at first lateral. Owing to symmetry there was now no

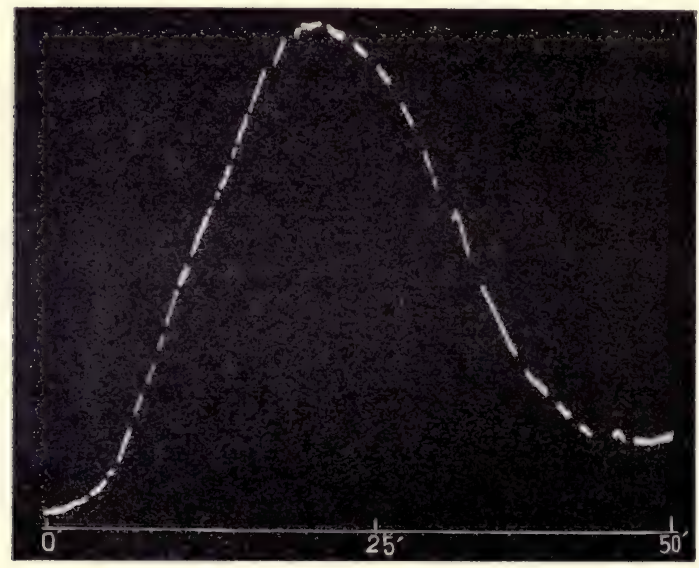

FIG. 271. Geo-electric Response of the Physically Restrained Scape of Uriclis Lily

Up-curve represents responsive current from upper to lower surface during action of geotropic stimulus, Down-curve represents recovery on cessation of stimulus. Response commenced after latent period of one minute ; after-effect persisted for two minutes. Breaks in curve are due to obscuration of recording spot of light at brief intervals.

differential action of gravity, nor consequent electrical variation, as between the two. The galvanometric record was now a horizontal line. The specimen, on its support, was next quickly rotated through $90^{\circ}$. The statolithic particles were thus displaced, falling on the inner tangential wall of the upper side, and outer tangential wall of the lower. An electrical response was perceived in about one minute, which went on augmenting with time, the upper side being increasingly galvanometrically negative (fig. 27I). The fact 
already demonstrated by the alternate cooling of the upper and lower surfaces is again seen here : namely, that it is the upper surface which exhibits the true excitatory effect, by induced concavity in the case of mechanical and by galvanometric negativity in that of electrical response. It will also be seen that when secondary disturbing causes are removed, the response to gravity is immediate, showing that there is no anomalous delay between the displacement of the hypothetical particles and the consequent response. By now rotating the specimen back through $90^{\circ}$, the action of gravity is virtually removed. The after-effect persists for two minutes, and after this the response curve shows the usual recovery. 


\section{CHAPTER XXX}

\section{DETERMINATION OF VELOCITY OF TRANSMISSION OF EXCITATION IN PLANT TISSUES}

Transmission of excitation in plants not due to hydromechanical disturbance, but instance of transmission of protoplasmic changes-Difficulties in accurate determination of velocity of transmission-A perfect method-Diminution of conductivity by fatigue-Increased velocity of transmission with increasing stimulus-Effect of cold in diminishing conductivity-Effect of rise of temperature in enhancing conductivity-Excitatory concomitant of mechanical and electrical response-Electrical methods of determining velocity of transmission-Method of comparison of longitudinal and transverse conductivities - Tables of comparative velocities in animal and plant-Existence of two distinct nervous impulses, positive and negative.

WHEN a point in the tissue is stimulated the state of excitation is often found to be transmitted to a distance. This is well seen in the case of sensitive plants, where the excitation applied at one point is found to give rise to motile responses of the distant leaf or leaflets.

In this transmission of excitatory impulses to a distance in the plant we have a phenomenon which would seem to be analogous to nervous transmission in the animal. For certain reasons to be given presently, however, it has usually been supposed that there is actually nothing in common between the two. 'The nervous system belongs exclusively to the animal organisation, and, indeed, to the more highly developed Metazoa only. Plants, unicellular animals, and the lower Metazoa have no nerves, and if in exceptional cases (as in the excitatory movements of many plants) there are forms of activity which resemble the vital manifestations of the animal organisation, as effected by nerves, it is easy to prove that the resemblance is merely superficial.' ${ }^{1}$

1 Biedermann, Electro-Physiology, English translation, 1898, Vol. ii. p. 32. 
This assumption, that there could be nothing in common between the transmission of excitatory impulses in plant and animal, was thought to derive support from Pfeffer's experiment on the effect of anæsthetics on conduction in Mimosa. The anæsthetisation of the pulvinus is found to abolish its motile excitability. The effect of strong stimulus was nevertheless found by Pfeffer to be transmitted across the anæsthetised area, giving rise to the depression of leaves beyond. It was natural to infer from this that, as the motile excitability of the pulvinus was abolished by the anæsthetic, so must the protoplasmic conductivity also have been abolished. It was therefore inferred that-unlike the conduction of stimulus in animal tissues, where such transmission is known to take place by the propagation of protoplasmic changes-the apparent conduction of excitation in a plant was purely hydromechanical.

But I have shown elsewhere, and shall demonstrate again in Chapter XXXIII. by different means, that though excitability and conductivity are related phenomena, yet the variation of the one is not necessarily identical with that of the other. Thus a certain degree of anæsthetisation may be sufficient to induce arrest of motile excitability, and yet may not always abolish conductivity. ${ }^{1}$

Another objection which has been urged against the theory of the transmission of protoplasmic changes through the plant is based on certain experiments of Haberlandt. In these the excitation in Mimosa is said to have been propagated over dead tracts of the petiole, these portions having been killed by scalding. But it is extremely difficult to ensure the death of interior tissues by such means as superficial scalding. I have found that a portion of a plant tissue which had been subjected locally to the action of boiling water afterwards exhibited signs of true excitatory electrical response. It is only by prolonged immersion in boiling water that the electrical response is totally abolished. Only after such treatment, therefore, can one be quite 'sure that the 
interior tissue is really killed by scalding, and unless this is thoroughly done it is easy to see that the inner cells may continue to conduct excitation.

There is, moreover, another possibility, that of pseudoconduction, by which the effect of stimulus might appear to be transmitted across dead areas. In Haberlandt's experiment, even if the intervening tissues had been killed, there would still be two masses of tissue, separated from each other by an intervening area of dead tissue. A strong stimulus applied to either of these might then cause an excitatory expulsion of water, capable, when transmitted across the dead area, of imparting a mechanical blow to the second living tissue, sufficient to set up excitation de novo in that portion of the petiole.

I shall, however, be able to show that conduction is brought about in the plant by the same transmission of excitatory protoplasmic changes which occurs in the animal ; and that those agencies - such as cold, anæsthetics, fatigue, and the polar effects of currents - which induce its variation in the one case, have the same identical effect in the other also. The electrical responses, again, afford us a crucial method of distinguishing between hydrostatic and excitatory effects. For, had the transmission of excitation taken place in plants by means solely of the propagation of hydrostatic disturbance, its electrical sign would then have been one of galvanometric positivity alone. But we have found, on the contrary, that the sign of the true excitatory reaction is always of galvanometric negativity. The distinct characters of these true excitatory and hydrostatic waves have already been demonstrated in various experiments, in some of which the hydrostatic has been seen as a positive twitch preceding the true excitatory negative, while in others the transmission of the negative was abolished by the application of a selective block, such as chloroform, thus bringing about the exhibition of the positive alone (p. 66). So true indeed is it that excitatory changes are propagated in the plant as in the animal, that I have actually been able to isolate certain 
tissues whose responsive peculiarities are indistinguishable from those of animal nerves. These must therefore be regarded as vegetable nerves (Chapter XXXII.).

The determination of the velocity of transmission of excitation in sensitive plants may be made by applying a stimulus at any point and observing the interval which elapses before motile effects are visible at a given distance. It is, however, impossible by such means to obtain accurate and consistent results until certain factors of variation are successfully eliminated. These are (I) indefinite changes of excitability owing to injury caused by stimulus at the point of application ; (2) changes of conductivity caused by fatigue; and (3) the unknown effects of varying intensities of stimulus on velocity of transmission.

As a result of investigations on this subject I found that the velocity of transmission can only be regarded as a determinate quantity when the intensity

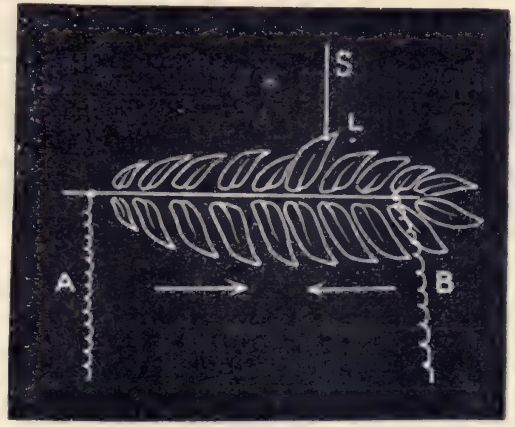

FIG. 272. Diagrammatic Representation of Electrical Connections for Determination of Velocities of Centrifugal and Centripetal Transmissions

$\mathrm{A}$ and $\mathrm{B}$ are exciting electrodes, and $\mathrm{L}$ the indicating leaflet. of stimulus has a definite value. Excessive stimulation, again, is found, by causing injury, to modify the excitability and conductivity of the tissue. These difficulties, however, are overcome by the employment of a stimulus which does not cause injury, and which is capable of repetition at uniform intensity. One such form of stimulation is obtained by the use of discharge from a condenser previously charged to a known.voltage (fig. 272). As regards those changes of conductivity which are due to fatigue, I have found that fatigue is removed, and conductivity fully restored, after the lapse of a definite period of rest, varying in duration in different plants from four to 
ten minutes. In the case of Biophytum, for instance, the required interval was found to be about five minutes.

Taking a specimen of Biophytum, and employing the method of determination which has been described, I found successive values which were very consistent; and, having thus secured conditions which made it possible to obtain exact results, I proceeded next to investigate the effects of various factors - such as fatigue, intensity of stimulus, and temperature-in modifying the velocity of transmission. Before proceeding to describe these results in detail, however, it should be mentioned that, though the velocity of transmission of excitation is constant in the same plant under uniform conditions, yet this is not necessarily the case, if the direction of conduction be reversed. In the petiole of Biophytum, for example, the centrifugal velocity is always higher than the centripetal, being about fifty per cent. greater.

Specimen I

\begin{tabular}{|c|c|c|c|c|c|}
\hline \multicolumn{3}{|c|}{ Direction } & Distance & Time & Velocity \\
\hline Centripetal & . & . & $22.5 \mathrm{~mm}$. & I $1 \cdot 2$ seconds & $2 \mathrm{~mm}$. per sec. \\
\hline Centrifugal & . & . & $45 \mathrm{~mm}$. & $15 \cdot 2 \quad$, & $2.9 \mathrm{~mm} . \quad$, \\
\hline
\end{tabular}

Specimen II

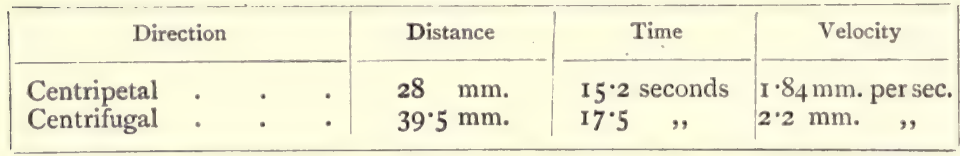

In order to study the effect on velocity of progressive fatigue, we may gradually shorten the interval of rest. The velocity of transmission in the petiole of Biophytum when fresh was found to be $1.88 \mathrm{~mm}$. per second in the centripetal direction. Before making a second experiment on the same specimen, an intervening period of rest of three minutes was allowed. This was found to reduce the velocity slightly, it being now I.86 mm. per second. The following table shows the results obtained by a series of five experiments on the 
same specimen. The distance through which the transmission was observed was $27 \mathrm{~mm}$.

Table Showing Variations of Velocity of Transmission and of Amplitude of Response with Increasing Fatigue

\begin{tabular}{|c|c|c|c|c|c|c|c|c|}
\hline \multicolumn{3}{|c|}{ Intervals of rest } & \multicolumn{2}{|c|}{ Time } & \multicolumn{2}{|c|}{$\begin{array}{l}\text { Amplitude of } \\
\text { response }\end{array}$} & \multicolumn{2}{|c|}{ Velocity } \\
\hline The plant fresh & . & . & $14: 3$ & conds & 34 & visions & $1.88 \mathrm{~mm}$. & gersec. \\
\hline 3 minutes & . & - & $14 \cdot 5$ & ", & 20 & , & I $.86 \mathrm{~mm}$. & ", \\
\hline 2 minutes & - & $\cdot$ & $15 \cdot 7$ & , & $14 \cdot 5$ & , & $1.72 \mathrm{~mm}$. & , \\
\hline I minute & - & - & $16 \cdot 4$ & , & 25 & ", & $1.64 \mathrm{~mm}$. & , \\
\hline$\frac{1}{2}$ minute & • & . & $17 \cdot 5$ & " & $1^{\circ} 0$ & ", & I. $54 \mathrm{~mm}$. & ", \\
\hline
\end{tabular}

It will thus be seen that the fatigue due to having only half a minute's rest reduced the normal velocity of the specimen by 18 per cent.

The effect of intensity of stimulus on velocity of transmission was next studied. The stimulus employed was that of condenser discharge, increased intensity being obtained by an increasing voltage of charge. In this way it was found that velocity increased with increasing intensity of stimulus. This fact is shown in the following table, which gives the results of an experiment on a petiole of Biophytum.

Table Showing Increase of Velocity with Increasing Stimulus

Specimen I.-Centripetal Transmission

The distance traversed by stimulus was $27 \mathrm{~mm}$.

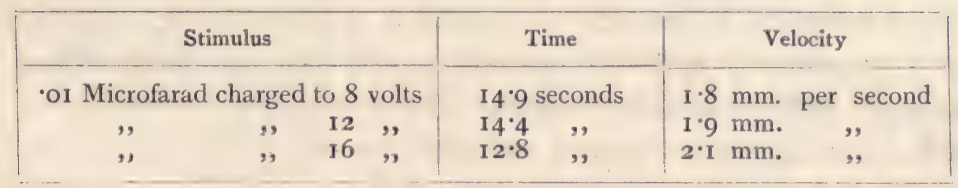

Specimen 11.-Centrifugal Transmission

The distance traversed by stimulus was $38 \mathrm{~mm}$.

\begin{tabular}{|c|c|c|c|c|c|c|}
\hline \multicolumn{3}{|c|}{ Stimulus } & \multirow{2}{*}{\multicolumn{2}{|c|}{$\frac{\text { Time }}{\text { I I } 6 \text { seconds }}$}} & \multicolumn{2}{|c|}{ Velocity } \\
\hline \multicolumn{3}{|c|}{ or Microfarad charged to 8 volts } & & & $3: 27 \mathrm{~mm}$. per & second \\
\hline , & , & 16, & $10 \cdot 2$ & , & $372 \mathrm{~mm}$ & \\
\hline , & , & 243 & $10 \cdot 1$ & , & $3.76 \mathrm{~mm}$ & , \\
\hline , & , & 32, & $9^{\circ} 9$ & , & $3.83 \mathrm{~mm}$ & , \\
\hline
\end{tabular}


With regard to the effect of temperature, I found that cold reduced the velocity of transmission. Thus, in one experiment, slight cooling reduced it to one-third, and when carried still further, it abolished the conductivity altogether. A rise of temperature, on the other hand, had the effect of enhancing velocity of transmission. The following table shows that a rise of temperature from $30^{\circ} \mathrm{C}$. to $35^{\circ} \mathrm{C}$. doubled the velocity, and that at $37^{\circ} \mathrm{C}$. the rate was almost three times that at the first temperature. The velocity was in this case determined in the centrifugal direction.

Table Showing the EFfect of Rise of Temperature on Velocity.

Distance traversed by stimulus $4 \mathrm{l} \mathrm{mm}$.

\begin{tabular}{|c|c|c|}
\hline Temperature & Time & Velocity \\
\hline $\begin{array}{l}30^{\circ} \mathrm{C} \text {. } \\
35^{\circ} \mathrm{C} \text {. } \\
37^{\circ} \mathrm{C} \text {. }\end{array}$ & $\begin{array}{l}\text { I I seconds } \\
5.5 \quad, \\
4.5 \quad,\end{array}$ & 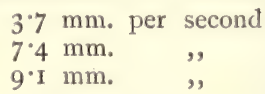 \\
\hline
\end{tabular}

Transmission of excitation, as I have shown elsewhere, and shall show again, is depressed or abolished by the action of anæsthetics. We shall also see, further, that the polar effects of currents on the velocity of transmission are the same in the plant as in the animal, being opposite, according as it is the anode or kathode. In the case of a so-called 'sensitive' plant, by taking advantage of the motile indications afforded by the leaf or leaflet, it is possible to determine the velocity of transmission of excitation and its modifications. With ordinary plants, however, no such indications being available, it is obvious that we must find some other means of detecting and observing the excitatory wave during transit. One such I have described elsewhere as the Electrotactile Method. It is found that the passage of the excitatory wave, even through an ordinary tissue, brings about minute form-changes. These give rise to pressure-variations as between two enclosing contacts. And this variation of pressure, in turn, can be recorded by means of a sensitive electrical device. 
There is, however, a more direct way of detecting the excitatory wave during its passage through a vegetable tissue. In this-the Electro-motive Method-the galvanometer takes the place of the motile leaflet. It has been shown that when the plant tissue is directly excited, the state of excitation is invariably accompanied by an electromotive variation, the excited point becoming galvanometrically negative. Hence any excitatory wave which is transmitted through the tissue will always have an electromotive wave as its strict concomitant. The moment, therefore, at which excitation reaches any given point, may always be determined by observing the arrival at that point of the excitatory electrical disturbance of galvanometric negativity. In order to prove that the arrival of excitation at the given point is attended by this specific electrical response, we may perform an experiment on a plant such as Biophytum, which is provided with motile leaflets. One of the indicating leaflets is attached to the optic lever, its base being connected with one of the electrodes of the galvanometer, while the second is attached to a distant point on the leaf. The two spots of light, one from the optic lever indicating the mechanical response, and the other from the galvanometer, indicating the electrical, are so adjusted as to lie one above the other, on the same revolving-drum. On now applying a stimulus, say thermal, at a distant point, it will be found, after the lapse of a definite interval; that both spots of light are deflected at the same time, showing that both alike give an outward indication of that state of molecular disturbance which is synonymous with excitation. These manifestations, of both kinds, would therefore take place at an identical moment, if only the inertia of the two indicators were absolutely the same. But, just as the same impulse would be indicated at slightly different times, if one indicating-lever were light, and the other heavy, so here also there may be a slight difference as regards time between the appearance of the mechanical and electrical 
responses, according as the virtual inertia of the one indicator exceeds that of the other.

In determining velocity of transmission by the Electromotive Method, a previous experiment gives us the loss of time due to the inertia of the galvanometer. This, deducted from the observed interval between the application of stimulus and response, gives the time required for transmission through the given distance. In this manner I have been able to determine the rate of transmission of excitation in ordinary plants. I give below a table which shows these velocities as determined by me in the case of sensitive plants, and of ordinary plants, and for the purpose of comparison, those obtained by other observers, in the nerves of some of the lower animals, from which it will be seen that all these are more or less of the same order.

Tables giving Velocities of Transmission of Excitatory Wave

(a) Animal.

\begin{tabular}{|l|l|l|}
\hline \multicolumn{1}{|c|}{ Subject } & Velocity \\
\hline $\begin{array}{l}\text { Nerve of Anodon } \\
\text { Nerve of Eledone (observed by Uexkiili) }\end{array}$ & - & Io mm. per second \\
\hline
\end{tabular}

(b) Sensitive Plants.

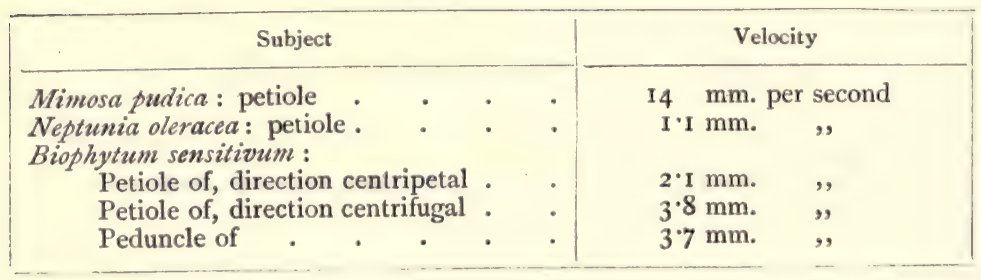

(c) Ordinary Plants.

\begin{tabular}{|c|c|c|c|c|c|c|c|}
\hline \multicolumn{5}{|c|}{ Subject } & \multicolumn{3}{|c|}{ Velocity } \\
\hline Fern : isolated nerve of & - & - & - & . & $5^{\circ}$ & $\mathrm{mm}$. & per second \\
\hline Ficus religiosa: stem. & . & - & - & - & $9 \cdot 4$ & $\mathrm{~mm}$. & , \\
\hline Cucurbita: tendril : & · & - & - & . & 5 & $\mathrm{~mm}$. & , \\
\hline Jute : stem : & - & - & - & $\cdot$ & 3.5 & $\mathrm{~mm}$. &,, \\
\hline Artocarpus: petiole . & - & - & - & - & $\cdot 54$ & $\mathrm{~mm}$. &, \\
\hline
\end{tabular}


Since the conduction of excitation takes place by the transmission of protoplasmic changes, it is evident that it must occur most easily along those paths in which there is greatest protoplasmic continuity. It is clear, then, that certain elements in the fibro-vascular bundles will furnish the best conducting medium. Cells of indifferent tissue, on the other hand, like the parenchyma of the leaf, are divided from each other by more or less complete septa, the fine filaments, by which neighbouring cells may be protoplasmically connected, being so minute that the conduction of stimulus through such imperfect channels must be comparatively feeble. Such tissues are, therefore, indifferent conductors of excitation, the stimulus remaining more or less localised in them. Plant-organs, then, which contain fibro-vascular elements, such as the stem, peduncle, and petiole, are for that reason relatively good conductors. Conductivity in such an organ, again, is, as we should expect, much greater along the length than across.

I shall now describe an important method by which the relative conductivity of a tissue in different directions may be experimentally determined, verifying by its means the difference in the power of a tissue to transmit stimulus longitudinally and transversely. For this purpose I took a thick peduncle of Musa, and made two electrical connections, of which one was at a fixed point $\mathrm{B}$, transversely situated as regards $\mathrm{C}$, the point of application of stimulus. The second point, A, was longitudinally above $\mathrm{C}$, and its distance from it could be varied in successive experiments (fig. 273).

If we now take a point, $\mathrm{A}$, in such a position that $\mathrm{CA}$ is equal to $\mathrm{CB}$, then, on account of the better conductivity along $\mathrm{CA}$, the excitation will reach the A contact earlier than that at $\mathrm{B}$, making that point galvanometrically negative. The direction of the first responsive current, therefore, will be from $A \rightarrow B$ in the tissue. If, next, the longitudinal contact be moved to $\mathrm{A}^{\prime \prime}$, that is to say, so far that the excitation reaches the $\mathrm{B}$ contact first, then the responsive current will 
be reversed, flowing now from $\mathrm{B} \rightarrow \mathrm{A}^{\prime \prime}$. A point of transition, or of balance, $A^{\prime}$, may now be found by searching, at which the movement of the exploring contact, nearer or further, will give rise to opposite responsive currents. The conductivity along the longitudinal direction will then be, to that in the transverse direction, as the balancing-distance $\mathrm{CA}^{\prime}$ is to $\mathrm{CB}$. With a given specimen of the peduncle of Musa the transverse distance $\mathrm{CB}$ was $3.7 \mathrm{~cm}$., and the longitudinal balancing-distance $\mathrm{CA}^{\prime \prime}$ was determined at $\mathrm{IO}_{4} 4$ $\mathrm{cm}$. Hence the longitudinal velocity was $2 \cdot 8$ times that in the transverse direction.

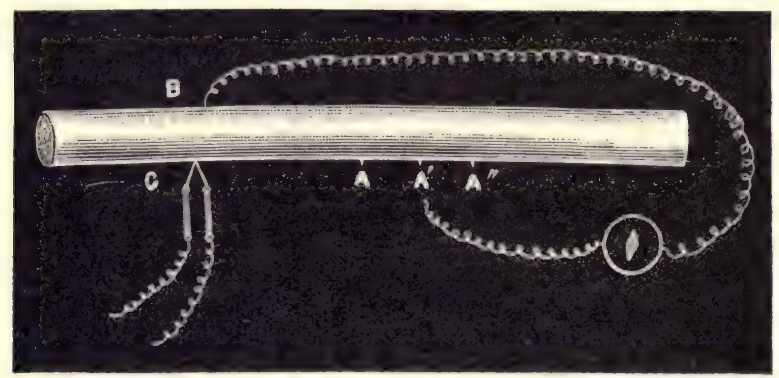

FIG. 273. Experimental Arrangement for Comparing the Relative Conductivities in Transverse and Longitudinal Directions

C, point of application of stimulus; B, permanent transverse contact ; A, A', A', exploring points of longitudinal contact for obtaining balance.

It has been shown that different tissues in the plant may possess extremely different powers of conducting stimulus. In animals there are specialised channels of conduction known as nerves, and in plants also I have been able to discover similar conducting tissues, which can be isolated for the study of their responsive peculiarities. Experiments on this subject will be related in detail in Chapter XXXII. It may be said here, however, in anticipation, that the velocity of transmission of true excitation through these nervous channels is, generally speaking, fairly high, being at the rate of about $50 \mathrm{~mm}$. per second in the case of isolated 
nerve of fern. This, for the relatively sluggish vegetable tissue, is undoubtedly very high.

In connection with this question of velocity of transmission, a fact not hitherto taken into account is, that there are two distinct kinds of nervous impulses, travelling with different velocities-namely, the hydro-positive and the true excitatory negative. Of these the velocity of the former is greater. In the nerves of higher animals, where the velocity of transmission of true excitation is also great, it is not generally easy to distinguish one from the other, so rapid is their succession. But their occurrence as distinct waves, even in animal tissues, I shall be able to demonstrate in a subsequent chapter. In plants, however, where the velocity of transmission of true excitation is not very high, it generally lags perceptibly behind the positive wave (p. 59). Burdon Sanderson, in his determination of the velocity of transmission of excitation in Dioncea, arrived at the exceptionally high result of $200 \mathrm{~mm}$. per second. I have shown, however, that the wave whose velocity he measured was not of true excitation, but of hydro-positive disturbance (p. 231).

In the present chapter it has been my object to demonstrate the reality of true excitatory propagation in plants similar to that in the animal. The examples given will be found more fully described on referring to my book on 'Plant Response.' I shall, however, in the course of the present work describe new and extremely delicate means by which the modifications of conductivity may be studied in plants unde varying physiological conditions. 


\section{CHAPTER XXXI}

\section{ON A NEW METHOD FOR THE QUANTITATIVE STIMULATION OF NERVF.}

Drawbacks to use of electrical stimulus in recording electrical response-Response to equi-alternating electrical shocks-Modification of response by decline of injury-Positive after-effect-Stimulation of nerve by thermal shocksEnhancement of normal response after tetanisation--Untenability of theory of evolution of carbonic acid-Abnormal positive response converted into normal negative after tetanisation-Gradual transition from positive to negative, through intermediate diphasic-Effect of depression of tonicity on excitability and conductivity-Conversion of abnormal into normal response by increase of stimulus-intensity-Cyclic variation of response under molecular modification.

IN the study of the electrical effects of excitation on the nerve, the chief experimental difficulty lies in the selection of a form of stimulus which can be made quantitative. In such investigations it is usual to employ the electrical form of stimulus, because of the great facilities which it offers. A marked drawback to its use, however, lies in the fact that unless extraordinary precautions are taken it is liable to lead to serious error. It must be remembered that for the detection of responsive variations in the nerve an extremely sensitive galvanometer has to be employed. The excitatory effect which is to be detected being indicated by the relatively feeble electrical response, and the form of stimulus being also electrical and being of high intensity, the results are liable to be disturbed in an unknown manner by leakage of the stimulating current.

In some cases it is possible to take the bold step of including the experimental nerve itself in a circuit in which the exciting coil and the galvanometer are in series. Under these circumstances, and employing strictly equi-alternating 
shocks, we have seen that the resultant response is due to the differential excitabilities of the two nerve-contacts $\mathrm{A}$ and B. If, for instance, we wish to obtain the responsive reaction of one point only, say A, uncomplicated by that of $\mathrm{B}$, it is only necessary to abolish the excitability of the latter. This can be done to a greater or less extent by injury, as, say, by making a transverse section, or by scalding. Response will then take place by the induction of relative galvanometric negativity at A. In fig. 274 is seen a series of records obtained in this manner. The responses here apparently indicate growing fatigue of the nerve. They also exhibit the positive aftereffect.

With reference to the method of obtaining response by injuring one contact, commonly employed, it may be said that the assumption that the excitability of the injured point is totally abolished is not justified; for I have found that though recent injury causes a great depression of excitability, yet after a lapse of time the injured point tends to recover its excitability to a greater or less extent. In such a case we may expect two different effects to be exhibited in the responses. The resultant response being due, as we have

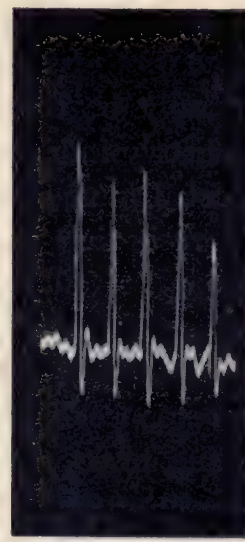

FIG. 274. Response of Frog's Nerve under Simultaneous Excitation of both Contacts, by Equi - alternating Electrical Shocks, one Contact being Injured

Note the positive aftereffect. seen, to the differential excitability of $A$ and $B$, the gradual restoration of the excitability of $\mathrm{B}$ will progressively diminish the amplitude of the resultant response, thus giving it the appearance of fatigue. Under these conditions, and after a sufficiently long interval, response may almost disappear. This appears to me to be the true explanation of the gradual fall in the amplitude of response, when the specimen is a nerve, having one contact at the transverse section. It also explains why, in such a nerve, a fresh section, causing 
renewed depression of excitability, is necessary in order to obtain renewed amplitude of response.

The second effect due to this depression, without abolition of the excitability of $\mathrm{B}$, is seen in the diphasic character of the responses. The positive after-effect observed in the record shown in fig. 274 may thus be ascribed to the later induction of negativity at the depressed point $B$. The electrical response of the nerve is apparently liable in this way to great variations, when the method of record employed is differential. But it must be remembered that true characteristic variations of the response as determined by physiological modification can only be obtained by finding some means which shall be strictly independent of this differential factor. With this object, I have succeeded in devising a new mode of observing and recording the direct effect of stimulus on the nerve, uncomplicated by the differential factor. In a subsequent chapter we shall, using this method, be able to determine the conditions which induce the characteristic variations in the response of nerve, from the staircase increase to the fatiguedecline, or even reversal, through the intermediate phase of uniform reponses.

The method which has just been described, of exciting the nerve at both contacts by equi-alternating shocks, is not applicable, however, where the object of investigation is the conductivity of an intervening tract of nerve between the exciting and the led-off circuits. Here the employment of electrical shocks as exciting stimulus gives rise to disturbing unipolar effects, which persist even when the physiological conductivity of the intervening tract is destroyed as, say, by ligature or by crushing. Thus-

'If the nerve of a frog's leg is laid across two electrodes connected with the poles of a secondary coil, so as to close the induction circuit, a ligature being then applied to the myopolar tract, tetanus may still be observed in the isolated leg, on making the lead off from it at a certain distance of coil. ... These unipolar effects 
may obviously be very disturbing, and are indeed productive of fallacies in vivisection and also in experiments with the galvanometer, if not avoided by due precautions. Hering has pointed out that in experiments such as the investigation of the negative variation of nerve-currents, in which galvanometers and exciting circuits are separated by a long tract of nerve, the most complete insulation of the two circuits is no guarantee against the overflow of induced electricity through the interpolar part of the nerve into the galvanometer circuit. .. This kind of unipolar stimulation is an obvious danger in all experiments on action-currents and negative variation in nerve, while it shows what narrow bounds restrict the intensities of current that may be safely used in these experiments.' ${ }^{1}$

From this it will be seen how important it is to have at our command some non-electrical form of stimulation, when the response to be recorded is electrical. Heidenhain employed a mechanical form of stimulation, by which the nerve was subjected to blows from an ivory hammer, which was kept vibrating by means of an electro-magnetic arrangement The employment of this mode of stimulation would therefore eliminate all that uncertainty-arising from the possible escape of current-which is inseparable from the use of electrical stimulus. Though this method must be regarded as one of great value, yet it is impossible to say how far the excitability of a given point in a structure so delicate as nerve will remain unmodified under the repeated action of such blows. In any case, it appeared desirable to inquire whether there was no other non-electrical form of stimulus that could be rendered practicable.

Besides the mechanical, the only remaining non-electrical forms of stimulus are the chemical and the thermal. Of these, the former is obviously incapable either of repetition or

1 Biedermann, Electro-Physiology (English translation), r898, vol. ii. pp. 222223. 
of being rendered quantitative. As regards the latter, I have already shown its practicability for experiments on excitatory phenomena in vegetable tissues. Thus a single loop of platinum wire may be made closely to surround the experimental tissue. A definite current sent through the platinum loop for a given length of time will now subject the encircled area to a sudden thermal variation, which acts as a stimulus. Successive closures of the circuit for a definite length of time are ensured by means of a key actuated by a metronome. The intensity of stimulus may be graduated in a predetermined manner by the adjustment of the heating-current. Excitation may then be caused either by one or by a summated series of thermal shocks.

I was now desirous of determining whether this form of stimulation would prove advantageous to experiments on the nerve, and in the course of the investigation I found it to be extremely convenient and appropriate. With good specimens of nerve I have been able, using thermal stimulus, to obtain long-sustained records of perfectly regular responses. As regards its pliability and facility of application this form of stimulus is quite unique. How many difficult problems are made possible of attack by its means will be realised in the course of the two following chapters, where the responsive variations of different conducting tissues under changing conditions are subjected to investigation.

In order to obtain the electrical responses of animal nerve-that of frog, for example-the distal contact is killed and appropriate electrical connections made with the galvanometer. The heating current is then adjusted for the desired amount of excitation. The thermal variation, it must be remembered, should not be so great as to injure the tissue in any way. The platinum loop is not in this case in contact with the specimen, and this is the mode generally employed. Should a more intense stimulation be desired, however, the nerve may be allowed to rest on the platinum loop. In such a case care must be taken to see that the rise of temperature is not so great as in any way to injure the 
tissue. The nerve, as usual, must be enclosed in a moist chamber, a convenient form of which, as employed in practice, will be seen in fig. $29 \mathrm{I}$.

I shall next give a few records in illustration of the ease and efficiency with which this mode of stimulus may be applied. These records will show the characteristic variations of response given by the nerve under different conditions. When making records of electrical responses with frog's nerve, under electrical stimulus, Dr. Waller obtained responses of three different types. The first of these was the normal, and consisted of negative responses; the second was diphasic; and the third was the abnormal positive. This last he regarded as characteristic of stale nerve.

These normal negative responses of the first of the three classes were found by him to undergo enhancement after a period of tetanisation; while the third, that of the abnormal response of stale nerve, underwent a change into diphasic, or a reversal to normal, after tetanisation.

From the fact that carbonic acid enhances the normal negative response of nerve, Dr. Waller has suggested that the enhancement of response in normal nerve after tetanisation, and the tendency of the modified nerve to revert to the normal, are results of the hypothetical evolution or carbonic acid in the nervous substance, due to metabolism accompanying excitatory reactions. It must be said, however, that no trace of the presence of carbonic acid has yet been detected in such cases. I shall be able to show, moreover, that these effects are in no way due to the evolutions of carbonic acid, but take place in consequence of molecular changes induced in the responding tissue, which find concomitant expression in changes of conductivity and excitability.

I shall now give records of responses of these various types obtained under the action of thermal stimulus. In order to exhibit the effect of tetanisation I give, in fig. 275, a series of normal responses by induced, galvanometric negativity, given by nerve of frog in its normal excitatory condition. This nerve was then subjected to tetanic thermal 
shocks, after which its responses to individual stimuli of the former intensity were recorded once more. The subsequent responses show, as is seen in the record, an enhancement of amplitude.

The next series of responses, in fig. 276, exhibits abnormal galvanometric positivity. It may be mentioned here that

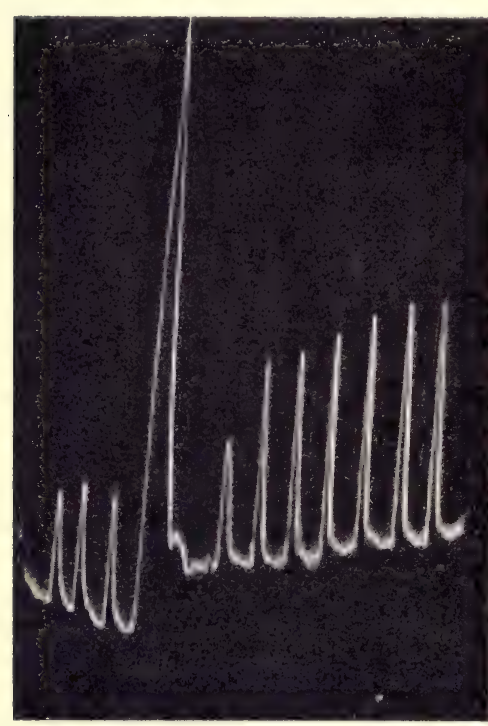

Fig. 275. Enhancement of Amplitude of Response, as After-effect of Thermal Tetanisation, in Frog's Nerve

The first three responses are normal. Brief thermal tetanisation is here applied, and the responses subsequently obtained under original stimulation are seen to be enhanced. these abnormal responses are not, as supposed by Dr. Waller, exclusively characteristic of the stale condition of the nerve. For employing other and more delicate methods of record I have found even fresh nerves, under certain conditions, to exhibit this effect. Neither is this positive response due in general to any chemical degradation. Instead of this, as we shall see in the present and succeeding chapters, it may be attributed to the run-down of the latent energy of the specimen, a process which becomes accelerated in isolation. When such a depressed specimen is supplied again with the requisite energy, it becomes normally, or even supernormally, excitable. The first part of the following record (fig. 276 ) gives a series of abnormal positive responses obtained from a specimen of frog's nerve, which was in a somewhat sub-tonic condition. After the application of tetanic thermal shocks it will be noticed that the responses in the second part of the figure have become converted into normal. 
Between these two extremes of normal negative and abnormal positive responses there lies the intermediate diphasic. All these-positive, diphasic, and negative-may be exhibited in the same specimen, in the course of a sustained record of responses to single stimuli, without tetanisation. This fact is illustrated in fig. 277 , where the first series shows the unmixed abnormal positive. The response then passes by a gradual transition into diphasicpositive followed by negative - and this phase, lastly, is succeeded by a series of purely negative responses.

We come next to the explanation of these phenomena. We have seen that on account of isolation the tonic condition of a highly excitable tissue will undergo a gradual decline. On account of this its excitability and conductivity will fall below par. We have also seen that in this depressed condition the normal response by negativity tends to be reversed to positivity. With regard to the conduction of excitation it may be said that this condition of

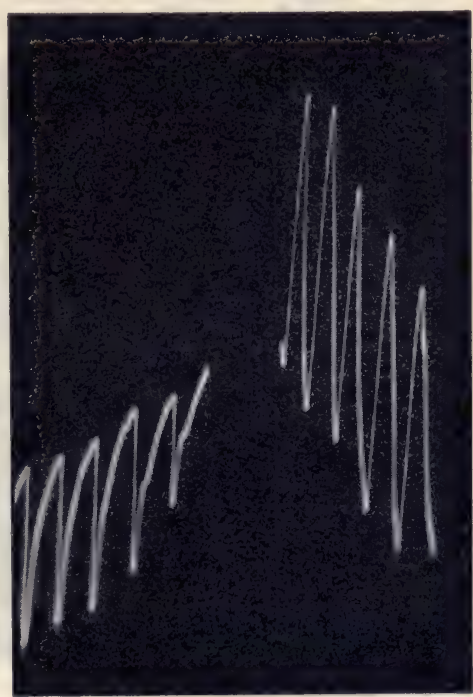

FIG. 276. Conversion of Abnormal Positive into Normal Negative Response after Thermal Tetanisation

Abnormal positive response to left converted into normal negative on right, after intervening tetanisation.

depression will lower the power of the tissue to conduct true excitation. Thus a stimulus of given intensity, capable under normal conditions of transmission to a certain distance, will, when the tissue is thus depressed, fail of conduction to the same distance. It will now, therefore, be the hydro-positive effect of stimulus which will make its appearance alone at the distant responding point. And the electrical expression of this will be galvanometric positivity. 
We have also seen that a tissue which is not in the highest tonic condition may have its tonicity increased by the action of impinging stimulus, with consequent enhancement of its excitability. I shall also demonstrate, in Chapter XXXIV, that the effect of an impinging stimulus on a sub-tonic tissue is a similar enhancement of conductivity. The result of this will be either (I) that a tissue which has already conducted a moderate intensity of

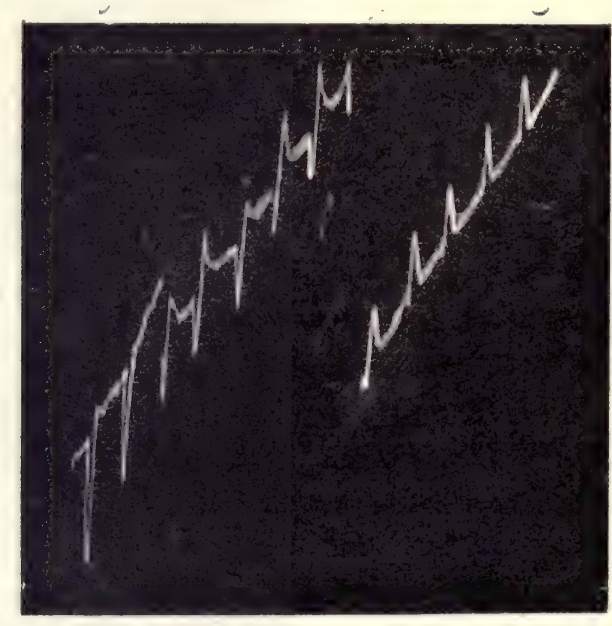

$=$

Fig. 277. Gradual Transition from Abnormal Positive, through Diphasic, to Normal Negative Responses in Frog's Nerve

C. similar effect in response of skin of gecko, fig. I9I.

stimulus to a distant point will show, after continuous stimulation, an enhariced power of conduction; or (2) that in a very sub-tonic tissue, in which true excitation has at first failed to reach the responding point, the true excitatory negative is subsequently transmitted instead of the hydropositive alone.

Under actual experimental conditions, where the stimulus is applied at a distant point, the twofold effects of exaltation of excitability and conductivity under tetanisation both come into play. In normally responding nerve, the increased con- 
duction of excitation, and the enhanced excitability of the responding-point, give rise to an increased amplitude of response after tetanisation, as already seen in fig. 275. In a depressed nerve, as the transmitted effect is positive, and the tendency of the responding point itself, owing to sub-tonicity, is to the abnormal positive, the record will exhibit the abnormal positive alone, as in fig. 276. But under a series of successive stimuli, the conductivity and excitability of the tissue are both gradually raised, and the effect of this is seen in the consequent gradual restoration of the normal negative response, through the intermediate diphasic (fig. 277). Or, if we do not wish to trace out the intermediate steps of transition, we may tetanise the depressed nerve for a certain length of time, and record only the terminal change to the restored normal negative, as is seen in fig. 276 .

Taking one of the extreme cases-say that in which the response to transmitted stimulus is positive, and is converted into normal negative after tetanisation -we see that the first result is due to inefficient conductivity, allowing only the hydro-positive effect to cause response. After this, increasing conductivity, making an increasing transmission of true excitation possible, gives rise to a diphasic, and ultimately to the normal negative response. This result is analogous to the three types of responses-positive, diphasic, and negative - which we have already obtained with the imperfectly conducting tissue of the petiole of cauliflower and the tuber of potato (figs. 47, 48). We there saw that where excitatory efficiency of transmitted stimulus was sufficiently great, it gave rise to the normal negative response. When this, however, was not so great, we obtained the diphasic. Finally, when the true excitatory effect could not be transmitted, only the abnormal positive response appeared. That gradation by which the transmitted stimulus was made fully, partially, or non-effective, to induce true excitation, was simply and most conclusively carried out in the case of the potato, by removing the point of stimulation to an increasing distance from the responding point. In the cases 
described, then, the three types of response are exhibited by the same tissue, in indubitable relation to the variation of its effective conductivity. If, then, results exactly parallel can be demonstrated to occur in the case of nerve also, it follows that there is no necessity there to make any such hypothetical assumption as that of the evolution of carbonic acid, suggested by Dr. Waller, in explanation of the conversion of abnormal response to normal.

In order to show how a varying conduction will give rise to these three types of responses, I shall now describe an

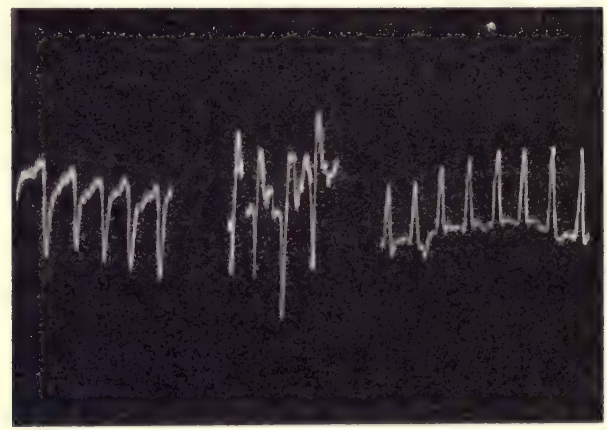

FIG. 278. Abnormal Positive Response converted through Diphasic to Normal Negative under the increasingly Effective Intensity of Stimulus, brought about by Lessening the Distance between the Responding and Stimulated Points

experiment which I carried out with a frog's nerve in somewhat subtonic condition. Here, when the stimulator was placed at some distance from the responding point, the response was the abnormal positive (fig. 278). When the effective intensity of transmitted stimulus was now slightly increased by moving the point of application a little nearer, the response became diphasic; and finally, when the stimulator was placed still nearer, the response became normal negative. Thus with an identical specimen we may obtain at will either negative, diphasic, or positive response, by making changes only in the effective intensity of stimulus employed. We have also seen, moreover, that if we kept 
the stimulator at a certain distance from the responding point, such as at first to cause only positive response, successive stimulations would then act to enhance conductivity gradually, and thus give rise to the appropriate changes, diphasic and negative in the response.

The ultimate cause of these variations must therefore lie in the molecular condition of the tissue. Under varying circumstances, this undergoes a cyclic change, the responsive reaction at any given moment constituting an indication of the particular molecular condition of the tissue. A more complete demonstration of this, carried out by an altogether different method, will be given in a subsequent chapter. My principal object in this chapter has been to prove the efficiency of the thermal shock as a mode of stimulation of nerve. Its wider applicability, in the case of other related investigations, will be treated in the two succeeding chapters. 


\section{CHAPTER XXXII}

\section{ELECTRICAL RESPONSE OF ISOLATED VEGETAL NERVE}

Specialised conducting tissues-Isolated vegetal nerve-Method of obtaining electrical response in vegetal nerve-Similarity of responsẹs of plant and animal nerve: $(a)$ action of ether- $(b)$ action of carbonic acid- $-(c)$ action of vapour of alcohol $-(d)$ action of ammonia-(e) exhibition of three types of response, negative, diphasic and positive- $(f)$ effects of tetanisation of normal and modified specimens-Effect of increasing stimulus on response of modified tissue.

IT has been shown in the previous chapter that the state of excitation is transmitted to a distance in vegetable tissues. It has also been proved that such transmission is not due to the propagation of hydrostatic disturbance but to that of protoplasmic changes, precisely as in the case of animal tissues. It is obvious, further, that such transmission will be the more perfect the less the interruption of protoplasmic continuity. Hence tissues like stems and petioles, which contain fibro-vascular elements, are found to be good conductors of excitation, whereas indifferent tissues, such as those of leaves and tubers, are relatively feeble as regards this power, excitation in their case remaining somewhat localised.

Even with regard to stems and petioles themselves, a contrast is found to exist in this respect between the fibrovascular elements and the ground tissue. Thus, in the case of a petiole of cauliflower, I made two experimental preparations. In the first, the ground tissue was cut away, leaving the fibro-vascular elements; and in the second, a column of ground tissue was left outstanding, denuded of fibro-vascular elements. The former of these was found to transmit excitation to a certain distance, whereas in the latter the transmission was practically absent. In the case of a third 
preparation I bifurcated the specimen, stripping away from one of the two limbs the fibro-vascular elements, and from the other most of the ground tissue. Galvanometric connections were now made with the free ends of the fibro-vascular and ground tissues respectively, and stimulus was applied by means of transverse cut, or by application of a hot plate across the area of union. The transmitted effect was now perceived as galvanometric negativity, at the end of that strip which was composed of fibro-vascular elements.

In studying this subject of conduction, I found the transmitted effect of excitation to be universally well exhibited in the petioles of ferns, successive responses, obtained at a distance from the point of stimulation, being in their case singularly perfect and uniform.

From this I was led to the conclusion that the disposition of the conductors must here be particularly well
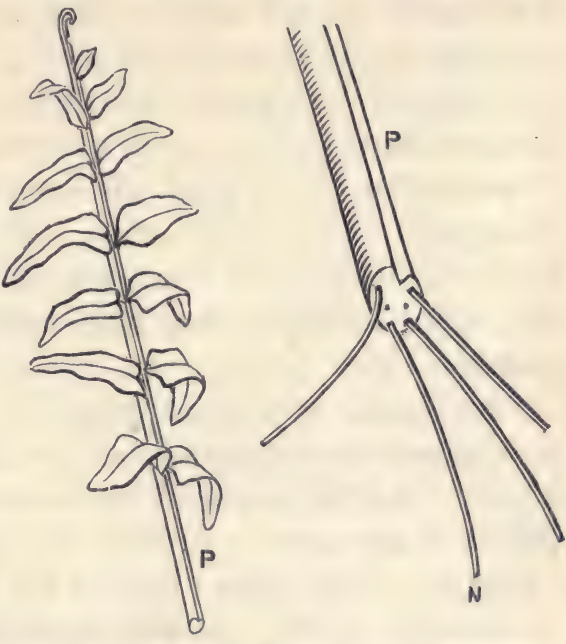

Fig. 279. Frond of Fern with Conducting Nerves $\mathrm{N}$ exposed in Enlarged Figure to Right adapted to their purpose. I had long been desirous of isolating whatever elements in the vegetable tissue were to be regarded as performing the function of nerves, and it appeared to me that I had here found a good subject for this investigation ; and accordingly, on carefully breaking the hard casing of the petiole, and pulling it away in both directions, I was able to isolate the conducting fibro-vascular threads, which were long, soft, and white in colour, remarkably similar in their appearance to animal nerves (fig. 279). These threads vary in number with different species of ferns, and resemble animal nerves in general appearance. It is sometimes 
possible to detach one of them having a length of $20 \mathrm{~cm}$. or more.

Now the essential feature of a nerve is its protoplasmic continuity, which is ensured by its fibrous structure. And in what I have called the vegetable nerve we find the same characteristic to hold good. On viewing this structure, as it appears on making a transverse section of the petiole, we find it enclosed within sheath-like sclerenchyma. It mainly consists in itself of a bundle of fine fibres with a few vessels in the centre. But however remarkable these external resemblances may seem, they are by no means so startling as the more fundamental similarities which are demonstrated so soon as we proceed to subject this vegetable structure to those tests of electrical response which are characteristic of animal nerve. It may be said that for the following investigation the nerves of the common maidenhair fern (Adiantum) and Nephrodium molle were found most suitable.

In obtaining a plant nerve for purposes of experiment it is possible to dissect it out and at the same time to avoid injury. It is then placed in normal saline solution for about half an hour, so as to remove all traces of excitation due to handling. When the external temperature is not high, the excitability of the isolated plant nerve is found to remain relatively unaffected for a considerable period, but in the hot weather it undergoes rapid decline; and the only way in which I could overcome this difficulty was by placing the specimen in normal saline solution which was ice-cold. The experimental precautions to be taken are precisely the same as those observed in corresponding experiments with animal nerve; that is to say, the specimen should be placed in a moist chamber. For the process of drying is found to induce a transient increase of excitability followed by a permanent abolition of responsiveness, in the one case as in the other. In order to obtain responses, one end of the specimen may be killed by the local application of hot salt solution. The galvanometric connections are then made, one with the killed, 
and the other with the unkilled portions of the specimen higher up. In order to ensure that the electrical indication be a true responsive reaction, it is well to use a non-electrical form of stimulus. One of the most perfect forms-as we have seen in the previous chapter, on excitation of animal nerve - is the thermal, and this may be applied in precisely the same manner, that is to say, by means of a platinum wire, surrounding, but not necessarily in contact with, the given area of the specimen, this wire being heated periodically in the manner previously described, by means of a metronome closing an electric circuit. With a good specimen, a single thermal shock, lasting for less than a second, will be found sufficient to induce a considerable electrical response, or a response of still greater amplitude may be obtained by the summated effects of several such stimuli. One of the most noticeable differences between this plant nerve and other vegetable tissues lies in its greater excitability. For example, while a single thermal shock of less than one second's duration is sufficient, as has been said, to evoke immediate and considerable response from the isolated nerve, we find that, in order to evoke similar response from the petiole of the fern as a whole, it is necessary to submit it to the same stimulus some twenty times in succession, the response even after this taking place with relative sluggishness.

A still further characteristic is its indefatigability. A long series of responses to uniform stimuli, such as would in the case of ordinary tissues bring about marked fatigue, will in that of nerve induce little or none. Rapidly succeeding tetanising shocks, moreover, such as in other tissues induce rapid'decline, induce, generally speaking, but little of such an effect on the response of nerve. In the case of this vegetable nerve also the same statements hold good. A long continued series of responses shows little fatigue. After tetanisation, moreover, we find that the responses of nerve, whether animal or vegetable, become enhanced.

In the matter of the effects induced by chemical reagents on animal and vegetable nerves, a further remarkable 
parallelism is to be observed. The completeness of this may be seen in greater detail in the next chapter. I shall, at the present point, confine myself to giving a few typical cases. Ether, for example, when acting on animal nerve, induces a preliminary exaltation of excitability, which is followed under its long continued action by depression. On blowing off the ether vapour again the original state of excitability is restored. In fig. 280 are seen the similar effects of this reagent on vegetable nerve, where $(a)$ exhibits the normal response, $(b)$ the immediate exaltation due to ether, $(c)$ the

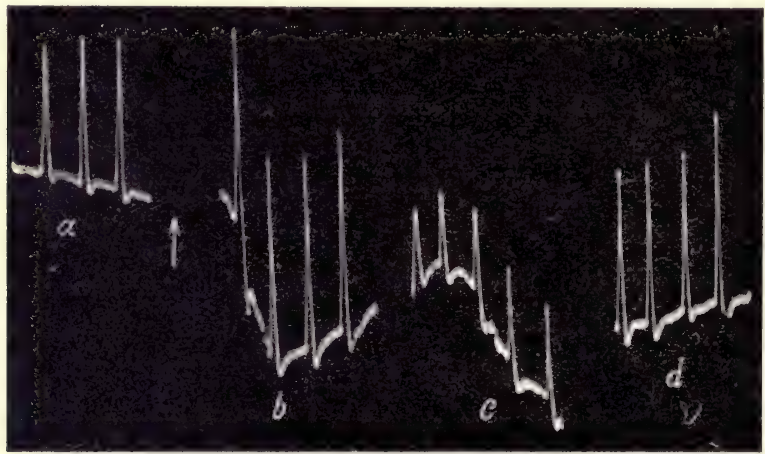

FIG. 280. Photographic Record of effect of Ether on the Electrical Response of Plant-nerve

(a) Normal response: application of ether at point marked with $\uparrow$; (b) Enhanced response in first stage of action of ether; (c) Subsequent depression; (d) Restoration of normal response after blowing-oft of ether.

subsequent effect of depression, which becomes marked after continuous action during twenty-five minutes, and (d) the restoration of the original condition on the blowing-off of the ether.

Carbonic acid is known, in the case of animal nerve, to have the effect, in the first stage, or in small quantities, of inducing exaltation, which passes under its prolonged action, or, in the case of a stronger application, into depression. A similar effect is seen in fig. 28I, where (a) shows the normal response of a vegetable nerve, and $(b)$ the preliminary exaltation due to carbonic acid introduced into the vegetable nerve- 
chamber. This is seen to increase continuously for some twenty minutes in (c). But after the expiration of half an hour depression makes its appearance $(d)$. This becomes still more marked, after the fortieth minute, in $(e)$.

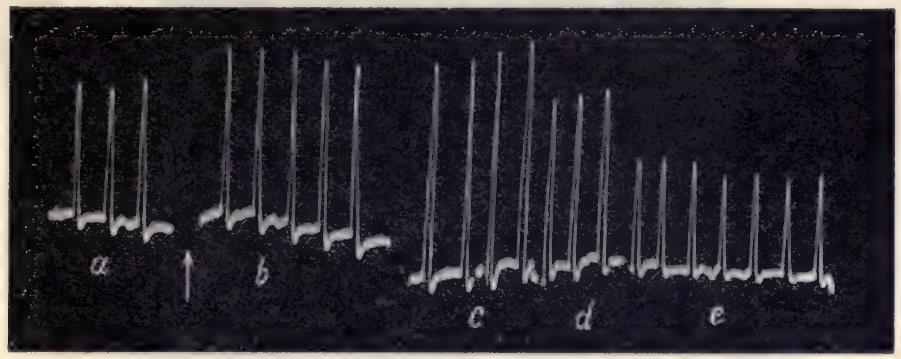

FIG. 281. Photographic Record of Effect of $\mathrm{CO}_{2}$ on Electrical Response of Plant-nerve

$a$, normal responses; $b$ and $c$, enhanced response during first stage of action ; $d$ and $e$, subsequent growing depression.

Alcohol vapour in strong, or long-continued applications, induces marked decline of response in animal nerve. Parallel effects are seen in the case of vegetable nerve in fig. 282 .

The effect of ammonia on animal tissues is characteristically different, according as the subject of experiment is

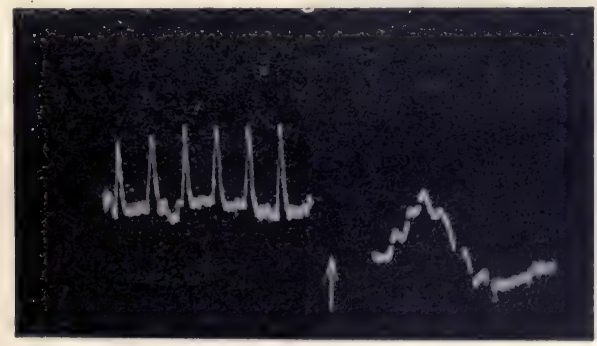

FIG. 282. Photographic Record of Abolition of Response by Strong Application of Alcohol

nervous or ordinary tissue. While the excitability of the muscle, for example, is but little affected by its application, that of nerve is quickly abolished. In order to see whether the same characteristic difference would be exhibited, as 
between ordinary vegetable tissues and vegetable nerve, I first studied its effect on the ordinary tissue of the petiole of

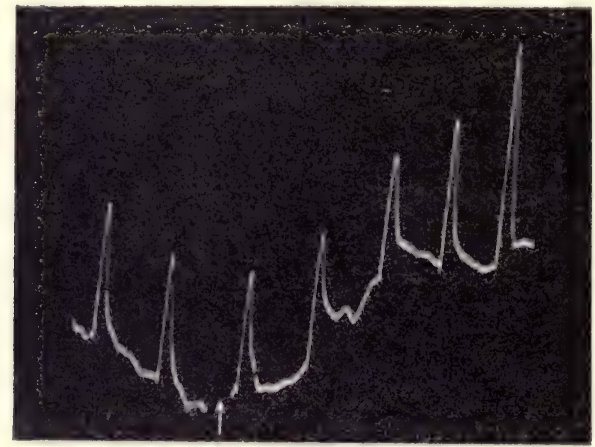

FIG. 283. Photographic Record of Effect of Ammonia on Ordinary Tissue of Petiole of Walnut

Note that the effect of ammonia here is practically negligible.

walnut. It will be seen from fig. 283 that ammonia here induced practically no change in the excitability. But when

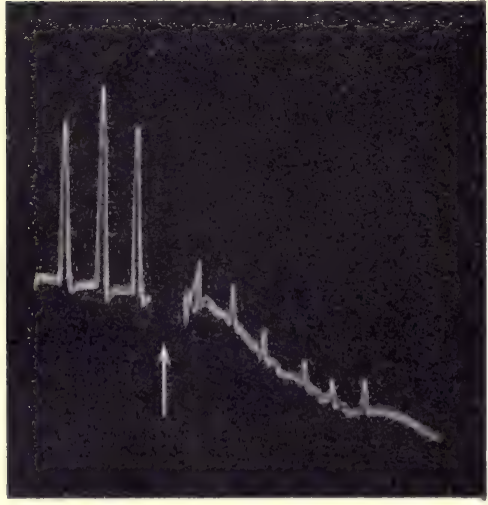

Fig. 284. Photographic Record of Effect of Similar Application of Ammonia on Plant-nerve

The response here is rapidly diminished and finally abolished.

the same reagent was applied to the isolated nerve of fern the response underwent depression, followed by total abolition, in the course of five minutes (fig. 284).

One very curious characteristic of the electrical response of frog's nerve is the occurrence, as referred to in the last chapter, of three distinct types of responses, according to its condition. Thus, as has already been said, while highly-excitable nerve exhibits the normal negative response, the same nerve, when it has become sub-tonic, will give a mixed or diphasic response; and a nerve which is 
modified to a still greater extent will show a purely abnormal or positive electrical response. In the case of vegetable nerve, I find exactly the same three types of response repeated, under the same conditions. This will be seen in the three sets of records given in fig. 285. The normal responses, which are negative, are here represented as 'up,' while the abnormal positive is represented as 'down.'

Still more remarkable is the parallelism observed between the effects of tetanisation, on animal and vegetable nerve, both normal and modified. In the case of fresh frog's nerve the responses are, as we have seen, enhanced, after

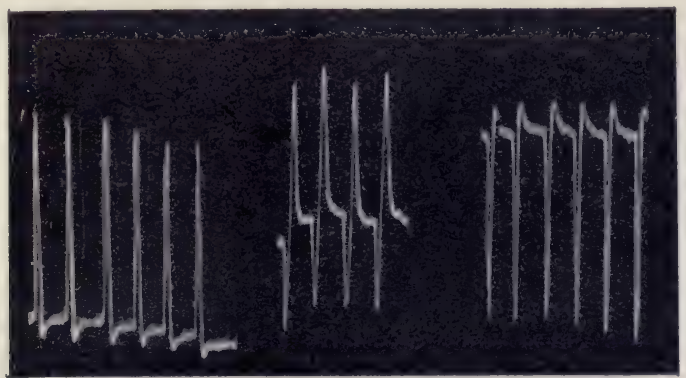

FIG. 285. Yhotographic Record of Exhibition of Three Types of Response, Normal Negative, Diphasic, and Alnormal Positive, in Nerve of Fern under Different Conditions

a period of tetanisation. The effect of tetanisation on vegetable nerve is precisely similar, as is seen in fig. 286 . In the case of the modified frog's nerve, moreover, it is found that the abnormal positive response tends, after tetanisation, to become normal. This is seen in the abnormal response, whether positive or diphasic, being converted to the normal negative type. I have obtained exactly parallel effects in the case of modified vegetable nerve. In fig. 287 we see the abnormal diphasic response of vegetable nerve converted, after tetanisation, into normal negative.

Thus, as in the response of animal nerve, so also in that of the vegetable, tetanisation is found to have the effect of enhancing the normal, or converting the abnormal into 
normal response. The abnormal response of nerve we found to be due to the joint depression of conductivity and excitability, on account of which the positive alone, instead of the true excitatory negative, was exhibited. In experimenting with frog's nerve we saw that abnormal response might, at will, be converted into normal through the intermediate diphasic by appropriately increasing the effective intensity of stimulation. A simple means of effecting this was to bring the stimulator gradually nearer the responding point.

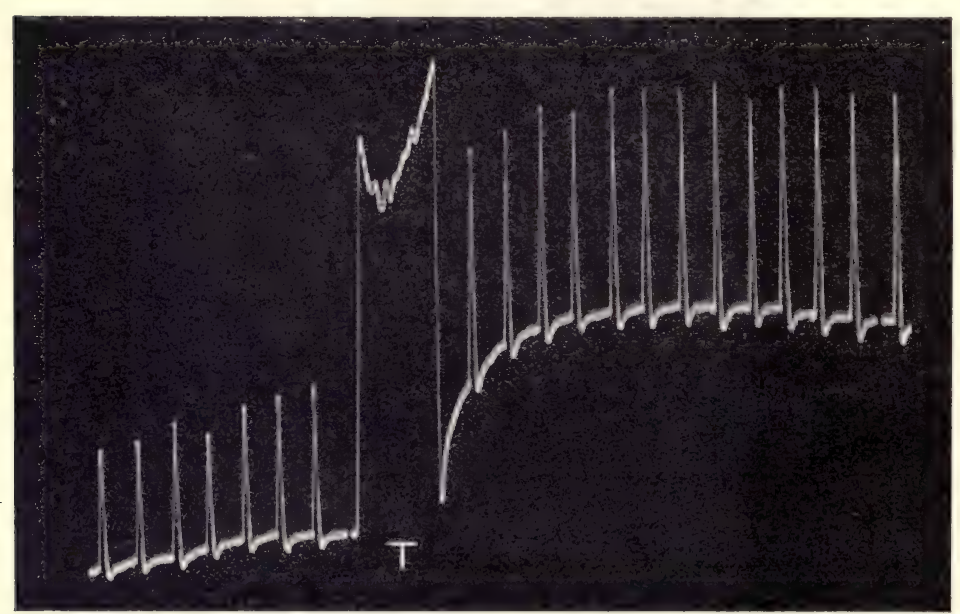

FIG. 286. Photographic Record of Effect of Tetanisation in Inducing Enhancement of Normal Negative Response in Nerve of Fern

The first series of responses seen to be enhanced after intervening tetanisation, $\mathrm{T}$.

In the response of vegetable nerve effects exactly parallel are to be observed.

With a given specimen of vegetable nerve, the stimulator had at first been placed at a distance of $2 \mathrm{~cm}$. from the proximal galvanometric contact, and the responses then taken were found to be of the abnormal positive type. The stimulator was now brought nearer, the distance being reduced to $\mathrm{I} \mathrm{cm}$., and the next pair of responses is seen to be diphasic, consisting of a positive twitch followed by the 
normal negative response. The distance was next reduced still further, namely, to $5 \mathrm{~cm}$., with the result that the

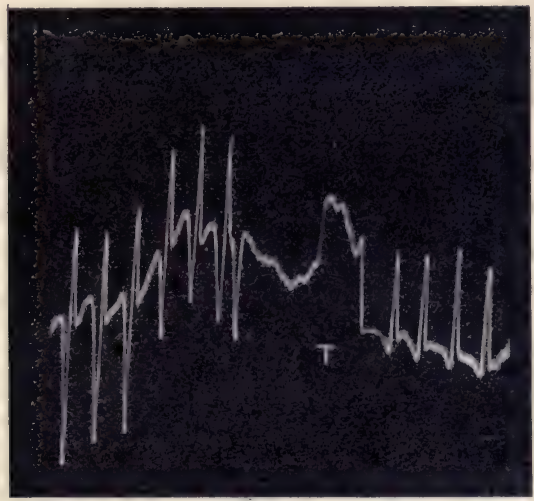

FIG. 287. Photographic Record of Conversion of the Abnormal Diphasic into Normal Negative, after Tetanisation, T, in Nerve of Fern

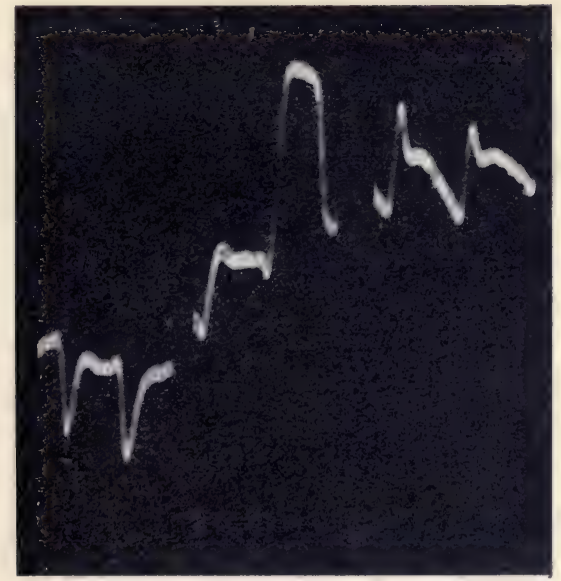

FIG. 288. Photographic Record showing how the Abnormal Positive Response is converted through Diphasic into Normal Negative, by the Increasing Effective Intensity of Stimulus, due to Lessening the Distance between the Responding and Stimulated Points

responses now became normal negative (fig. 288). It is thus seen that there is a continuity of response in the same 
tissue, as between the abnormal and normal; through the intermediate diphasic.

From the various experiments, then, which have been given in this chapter, it will be seen that the response of the isolated vegetable nerve is in every respect similar to the corresponding responses of animal nerve. And we shall also see how, by means of the study of this vegetable nerve, we are enabled to elucidate many obscurities in the responses of the corresponding animal tissue. We shall in the next chapter enter in detail into the question of the modifications induced in the conductivity and excitability of vegetable nerve under the action of various external agencies, and these will be found to exhibit the strictest parallel with corresponding variations induced in the animal. 


\title{
CHAPTER XXXIII
}

\author{
THE CONDUCTIVITY BALANCE
}

Receptivity, conductivity, and responsivity-Necessity for distinguishing these-

Advantages of the Method of Balance-Simultaneous comparison of variations of receptivity, conductivity, and responsivity-The Conductivity Balance-Effect of $\mathrm{Na}_{2} \mathrm{CO}_{3}$ on frog's nerve-Effect of $\mathrm{CuSO}_{4}-$ Effect of chemical reagents on plant nerve-Effect of $\mathrm{CaCl}_{2}$ on responsivity-Responsivity variation under $\mathrm{KCl}$-Comparison of simultaneous effects of $\mathrm{NaCl}$ and $\mathrm{NaBr}$ on responsivity - Effects of $\mathrm{Na}_{2} \mathrm{CO}_{3}$ in different dilutions on conductivity-Demonstration of two different elements in conductivity, velocity, and intensity-Conductivity versus responsivity-(a) effect of $\mathrm{KI}-(b)$ Effect of $\mathrm{NaI}-$ Effect of alcohol on receptivity, conductivity, and responsivity-Comparison of simultaneous effects of alcohol- $(a)$ on receptivity versus conductivity- $(b)$ on receptivity versus responsivity.

We know that when any point in a tissue is acted on by external stimulus, it receives the stimulation and is thrown into a state of excitation. This excitation is then conducted along the length of the tissue, and may be made outwardly manifest at some distant point by means of a suitable indication such as motile or galvanometric response. There are thus three different aspects of the excitatory effect to be distinguished from each other, namely, first the excitatory effect at the point of reception of stimulus, which I have elsewhere designated receptive excitability, or simply Receptivity: secondly, the power of transmission of excitation, or Conductivity: and thirdly, the excitatory effect evolved at the distant responding region, which I shall henceforth term Responsivity. Though these three aspects of the excitatory reaction are all alike dependent upon the molecular derangement caused by stimulus, it is nevertheless important to consider them separately, since their variation is not always the same under the same circumstances. We 
have seen, for example, that a rise of temperature, by increasing molecular mobility, enhances conductivity. But this increase of molecular mobility and internal energy also goes to augment the force of recovery, and, owing to this, the amplitude of excitatory response may be decreased. Thus, while a rise of temperature increases conductivity, it may appear to decrease responsive excitability. So much for the necessity of a distinction between conductivity and responsivity. The term 'excitability' is commonly used for receptivity and responsivity indifferently. But I shall show in the course of the present chapter that it is important to make a distinction between these, since the same external agent may effect the two differently. ${ }^{1}$ In the following investigation, receptive excitability, or receptivity, will be represented by $\mathrm{R}$, conductivity by $\mathrm{C}$, and responsive excitability, or responsivity, by $\mathrm{E}$.

In determining the effect of any external condition such as the application of a chemical reagent on responsive excitability, in the case of animal nerve, it is usual to take a series of normal responses, and then to record the modified responses after the application of the reagent. By comparing a number of such series of records, representing the action of various reagents on different specimens, the relative effect of each chemical may be inferred. The drawback to this method lies, first, in the fact that by the addition of the chemical reagent the resistance of the electrical circuit undergoes an unknown change, thus inducing a variation in the amplitude of response, which is not necessarily due to the excitatory electromotive change per se. It is true that this difficulty may to a greater or less extent be obviated by interposing a high external resistance in the circuit, but this, by reducing the deflection, necessarily reduces the sensitiveness of the method also. Different specimens again cannot but be characterised by slight individual peculiarities, and the experimental arrangements therefore can only be considered to be perfect when we are able to compare the effects of two 
agents on an identical specimen. Again, in a series of chemical compounds which differ but slightly in effect from one another, an arrangement has to be devised by which the most minute excitatory variations will be conspicuously displayed. The same delicacy of experimental adjustment also becomes necessary when we wish to investigate the varying effects of time and quantity in the application.

Similar considerations are involved when we attempt to observe the effects of various agents on conductivity and receptivity; and still more complicated are the difficulties to be overcome when we have to study the property of conductivity versus responsivity or receptivity, or of receptivity versus responsivity, under the action of the same external agent. The methods hitherto available are neither perfect nor delicate enough for a complete and satisfactory determination by their means of the various problems which arise in this connection. I shall now, however, describe a very perfect and delicate method carried out by an experimental arrangement which I have devised, and shall designate as the Conductivity-Balance, by which the variation of an affected region may be continuously compared with a normal area as regards each of the three different aspects of the excitatory reaction, namely, receptivity, conductivity, and responsivity. In this method, moreover, the result is unaffected by any variation of resistance in the circuit that may be induced by changed conditions. It also enables us to solve the various difficulties encountered in comparing the relative changes induced in conductivity with those induced in receptivity or responsivity, or in the two last in respect to each other, under the influence of a given reagent.

In fig. 289 is given a diagrammatic representation of the principal parts of this Conductivity-Balance. The thermal stimulator produces stimulation of the enclosed area of the specimen. The excitatory wave travels along both arms of the balance, through the conducting region $\mathrm{C}$ and $\mathrm{C}^{\prime}$, and induces excitatory electromotive effects at the two responsive points $\mathrm{E}$ and $\mathrm{E}^{\prime}$. The excitatory electrical effects at $\mathrm{E}$ and $\mathrm{E}^{\prime}$ 
are opposed, and when these are equal, and balance each other, the galvanometer indication is then reduced to zero. $\mathrm{E}$ and $\mathrm{E}^{\prime}$ are usually at a distance of about $4 \mathrm{~cm}$. from each other. When the stimulator is brought too near to the left contact $\mathrm{E}^{\prime}$, the excitatory effect of galvanometric negativity which is induced there is relatively greater than at E. The balance is thus disturbed, and the resultant responsive deflection is then, say, downwards. When the stimulator is placed, on the other hand, too near the contact $\mathrm{E}$, to the right, the resultant galvanometric deflection will be up. ${ }^{1}$ By suitable movement of the stimulator, to and fro between these two extremes, a point may be found where the excitatory effects at $\mathrm{E}$ and $\mathrm{E}^{\prime}$ will exactly balance each other. I give here (fig. 290) a record taken

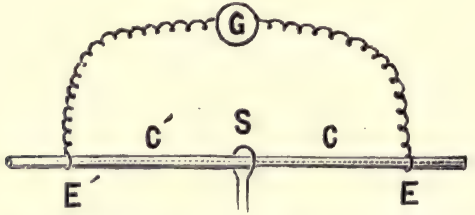

FIG. 289. Diagrammatic Representation of the Conductivity Balance

$\mathrm{s}$, thermal stimulator; $\mathrm{c}$ and $\mathrm{c}^{\prime}$, the conducting arms of the balance; $\mathbf{E}$ and $\mathbf{E}^{\prime}$, responding points. Differential excitatory electrical effects at $\mathbf{E}$ and $\mathbf{E}^{\prime}$ recorded by galvanometer, G.

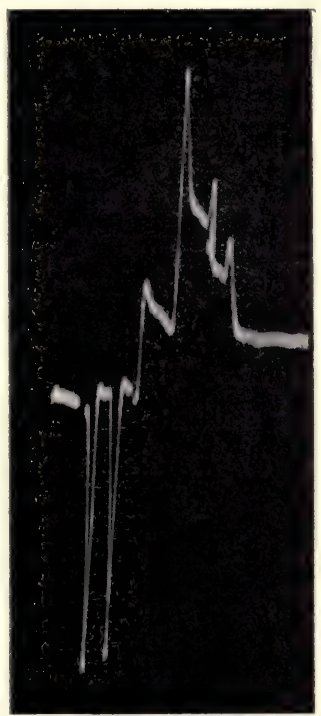

FIG. 290. Photographic Record made during Preliminary Adjustment for Balance of Nerve of Fern

The first two down-responses show over-balance, when $S$ is too near the left, E' being relatively more excited. The up-responses indicate over - balance caused by $\mathrm{s}$ being too much to the right. The horizontal record shows attainment of exact balance.

during this preliminary stage of adjustment. The first two down-responses were obtained when the stimulator was too far away from the balancing-point to the left. The next two

1 It is to be understood that what is said here refers to nerve in a normal condition of conductivity. 
up-responses were obtained when it was contrariwise too far to the right. More careful adjustment reduced this upmovement, as seen in the next two responses, and finally, when the exact balancing-point was reached, the effect was null, as seen in the horizontal record.

In studying the question of the variation of responsive excitability induced by any given reagent, the agent is applied at the point $\mathrm{E}$ to the right. Any variation of excitability will then upset the balance. If the reagent be of a stimulatory character we shall obtain a resultant up-response, but if it be of a depressing nature, $\mathrm{E}$ will be rendered relatively the less excitable of the two points, and the response will consequently be down. It will thus be seen that that upsetting of the balance by which either up- or down-responses are induced is due simply to the relatively excitatory or depressing effect of the reagent, and is completely independent of any variation of resistance which might be brought about by its application. In the course of the following investigation, it is to be understood that the electrical connections are so made that the greater excitation of the right-hand contact is always represented by up-response, and vice versâ. If it be desired to make a comparison between the excitatory reactions of two reagents, then the two are applied simultaneously, one at $\mathrm{E}$ and the other at $\mathrm{E}^{\prime}$. The resulting record then affords us a continuous graphic illustration of the relative and varying effects of the two.

If, again, it is the influence of any agent on conductivity that is to be studied, we first take a balanced record and then apply the given reagent on an area of about $\mathrm{I} \mathrm{cm}$. at $\mathrm{C}$ on the conducting arm. In this case, the responsive excitabilities of the two points $\mathrm{E}$ and $\mathrm{E}^{\prime}$ are the same, but if the effect of the agent have been to induce increased conductivity of $\mathrm{C}$, then the excitation transmitted to the right-hand side, E, will be greater, and the response caused by the upsetting of the balance will be upwards. Conversely, a down-response will indicate that the effect of the agent has been to depress the conductivity. Again, we can compare the relative effects in 
conductivity-variation induced by two different agents which are applied simultaneously, one on the arm $\mathrm{C}$ and the other on $\mathrm{C}^{\prime}$.

It is possible again to compare the variation of conductivity with that of responsivity, by applying one agent at a responding region, say $\mathrm{E}$, and the other on the opposite arm of the balance at $\mathrm{C}^{\prime}$. The mode of investigation of receptivity changes will be described presently.

In fig. 29I we have the complete apparatus. The animal or vegetal nerve, $\mathrm{N} \mathrm{N}$, rests on non-polarisable electrodes of

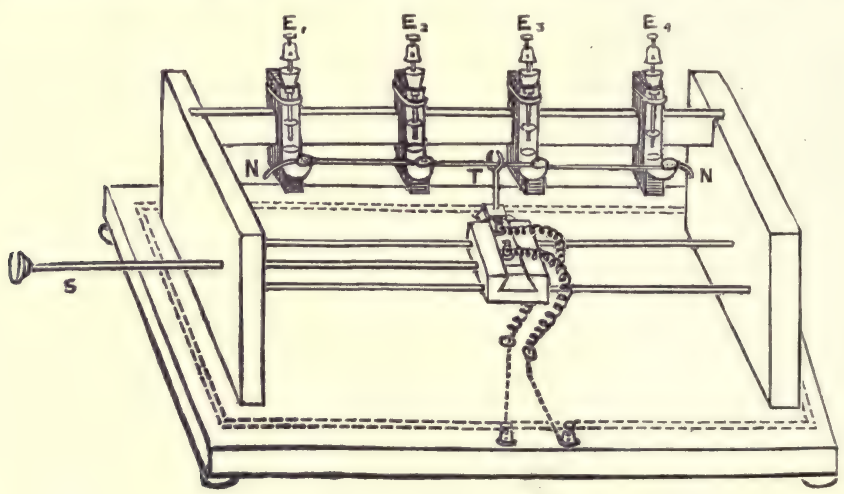

FIG. 291. Complete Apparatus of Conductivity Balance

The nerve $\mathrm{N}$ supported on electrodes $\mathrm{E}_{2}, \mathbf{E}_{3}$. The two other electrodes $\mathrm{E}, \mathrm{E}_{4}$ are not used in this experiment, but are employed for experiments on electrotonus; $\mathrm{T}$, thermal stimulator, the relative lengths of the arms of the balance being adjusted by the slide $\mathrm{S}$.

a U-shape. For the present experiments, two electrodes, $\mathrm{E}_{2}$ and $\mathrm{E}_{3}$, are sufficient, their mutual distance being capable of any variation by movement along a sliding-bar. The same apparatus might be used for experiments on electrotonus, in which two additional electrodes would be required. The position of the electrothermic stimulator $\mathrm{T}$ is capable of very careful adjustment for purposes of balance, by means of the sliding-rod s. A glass cover, not shown in the figure, fits into the groove which is represented by a double dotted line surrounding the apparatus, and thus enables the chamber containing the nerve to be kept in a properly humid condition. 
In all these experiments by balance, it is to be borne in mind that adjustment is always made for perfect balance at the beginning of the record, and represented by a short, more or less horizontal, line.

In order to show the typical effects of induced variations of excitability, in upsetting the balance, I shall first give records of experiments carried out on the nerve of frog. Dilute sodium carbonate is known to be an agent which enhances excitability. A long-continued application, or the

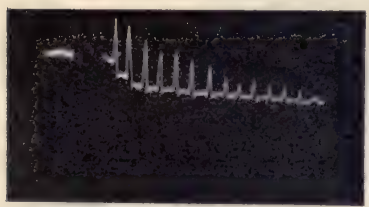

FIG. 292. Effect of $\mathrm{Na}_{2} \mathrm{CO}_{3}$ Solution on Responsive Excitability of Frog's Nerve

In this and following records the horizontal line at the beginning indicates exact balance. The upsetting of the balance in the up-direction represents either the enhanced responsivity of the right-hand responding point $\mathrm{E}$, or the increased conductivity of the right-hand arm c. Down-curves represent corresponding absolute or relative depressions. $\mathrm{Na}_{2} \mathrm{CO}_{3}$ applied to $\mathrm{E}$ is seen to exalt the responsivity of that point. application of a stronger dose, may, however, bring about a depression. When a dilute solution of $\mathrm{Na}_{2} \mathrm{CO}_{3}$ was ap-

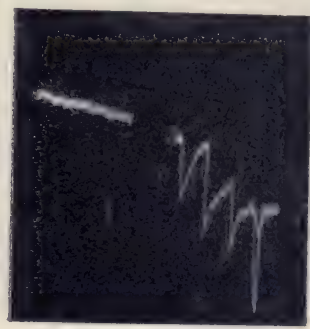

FIG. 293. Effect of $\mathrm{CuSO}_{4}$ on Frog's Nerve

The down record shows depression of excitability.

plied at the responsive point $\mathrm{E}$ on the right side, the upsetting of the balance upwards immediately indicated the greater excitability induced by the reagent. The longcontinued action of this reagent, however, showed that the enhanced excitability was undergoing a gradual decline (fig. 292).

In order to exhibit the characteristic upset caused by a depressing agent, I employed on another specimen a toxic solution of copper sulphate, applying it at $\mathrm{E}$ on the right. The previous state of equilibrium is seen by the horizontal line at the beginning of the record, and the 
subsequent depression of excitability at $\mathrm{E}$ is shown by the upsetting of the balance downwards (fig. 293).

I shall next take up the determination of the changes induced by chemical agents on the excitability of plant nerve and shall begin by describing the different effects which occur on the application of calcium and potassium salts. For this purpose, deci-molecular solutions were employed. Fig. 294 shows the effect of $\mathrm{CaCl}_{2}$ on

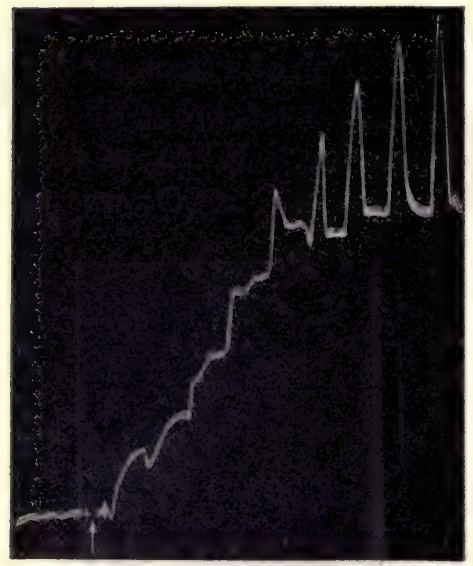

FIG. 294. Photographic Record showing Enhancement of Responsivity by Application of $\mathrm{CaCl}_{2}$

$\mathrm{CaCl}_{2}$ applied to $\mathbf{E}$ is seen to exalt the responsivity of that point. vegetable nerve, the solution being applied at $\mathrm{E}$ on the right-hand side. It will be noticed that this caused an upset of the balance, showing an increase of excitability that becomes considerable after the expiration of five minutes. In the case of $\mathrm{KCl}$, however, this effect was reversed, that is to say, a depression was induced. This is seen in fig. 295, where the balanced record gives way, first to diphasic, and afterwards to a down-response, indicating an effect of depression at E. These two experiments show the effect of the basic moiety in inducing changes of responsive excitability.

I shall next describe experiments by which the simultaneous effects of two different reagents on the responsivity of a given tissue may be compared. For this purpose, one agent is applied at one end of the balance $\mathrm{E}$, the other being administered at $E^{\prime}$. In the case of animal nerve, it was shown by Griitzner, that both $\mathrm{NaCl}$ and $\mathrm{NaBr}$ induce excitatory effects, that induced by $\mathrm{NaBr}$ being relatively the greater. But the continued action of either of these reagents 
induces depression, which sets in earlier in the case of $\mathrm{NaBr}$. The effect of these two reagents on vegetable nerve is pre-

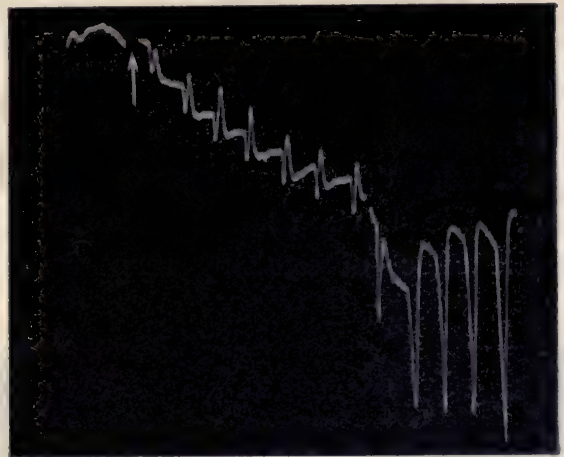

FIG. 295. Photographic Record showing Depression of Responsive Excitability by Application of $\mathrm{KCl}$

cisely the same, as will be seen from an inspection of the record given in fig. 296. The $\mathrm{NaBr}$ was applied on the

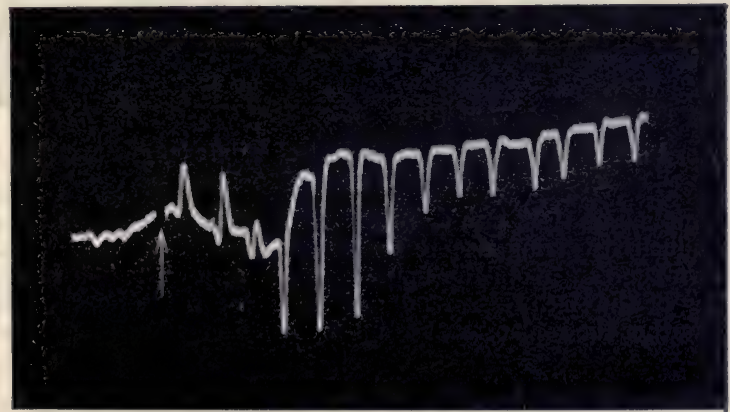

FrG. 296. Photographic Record exhibiting Comparative Effects of $\mathrm{NaCl}$ and $\mathrm{NaBr}$ on Responsivity

$\mathrm{NaCl}$ was applied on $\mathbf{E}^{\prime}$ and $\mathrm{NaBr}$ on $\mathrm{E}$, the formula being $\mathrm{E}_{\mathrm{NaCl}}^{\prime} \mathrm{E}_{\mathrm{NaBr}}$.

The record shows the greater and earlier effect of $\mathrm{NaBr}$ at $\mathrm{E}$ in causing relative excitation followed by relative depression.

right-hand side $\mathrm{E}$, and $\mathrm{NaCl}$ on the left-hand $\mathrm{E}^{\prime}$, a process which is expressed, for the sake of brevity, by the formula $\mathrm{E}_{\mathrm{NaCl}}^{\prime} \mathrm{E}_{\mathrm{NaBr}}$. The greater and earlier. excitatory effect of 
$\mathrm{NaBr}$, applied on the right-hand side, is shown by the resultant up-responses. But after a time, $\mathrm{E}$ being now depressed by the continued action of $\mathrm{NaBr}$, the effect of $\mathrm{NaCl}$, applied on the left, becomes relatively predominant, a fact demonstrated by the upset of the balance in the opposite direction, with concomitant down-responses.

We shall next take up the subject of variations induced in conductivity. We have seen that dilute solutions of $\mathrm{Na}_{2} \mathrm{CO}_{3}$ have the effect of exalting responsive excitability.

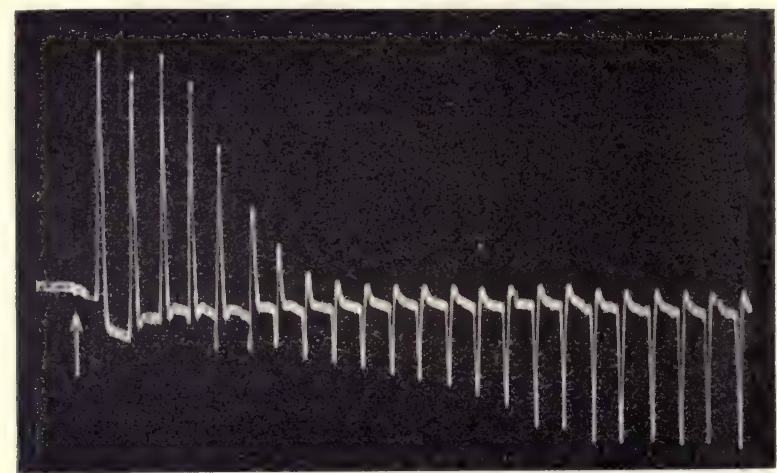

FIG. 297. Photographic Record of Effect of Dilute ( 5 per cent.) Solution of $\mathrm{Na}_{2} \mathrm{CO}_{3}$ on Variation of Conductivity

Reagent applied on right arm C. Record shows immediate enhancement of conductivity giving rise to up-curves, followed by depression, seen in down-curves. Note the appearance of a down-twitch at the beginning of the sixth response due to the later arrival of excitation at E. Note further the replacement of up- by increasing down-responses.

Long-continued applications, or strong solutions, however, have the effect of inducing a depression. Similarly, I find that this reagent has the effect of enhancing conductivity, provided the solution is sufficiently dilute. In the case of the petioles of ferns, a 2 per cent. solution was found to induce a preliminary exaltation of excitability, followed by a depression (p. 136). In dealing with the conductivityvariation in certain isolated vegetable nerves, however, a 2 per cent. solution was found to induce a depression of conductivity, but a 5 per cent. solution caused an enhancement 
of conductivity, followed, after long-continued action, by depression.

These facts are illustrated in an extremely interesting manner in the records given in figs. 297 and 298. In both these cases the solution was applied on the right arm of the balance at $\mathrm{C}$, the difference being only that in the first experiment the strength of solution was ${ }^{5}$, and in the second 2 per cent. An inspection of fig. 297 shows that the application of the first induced a great and immediate enhancement of conductivity, causing resultant up-responses, which were particularly marked during the first four minutes. This increased

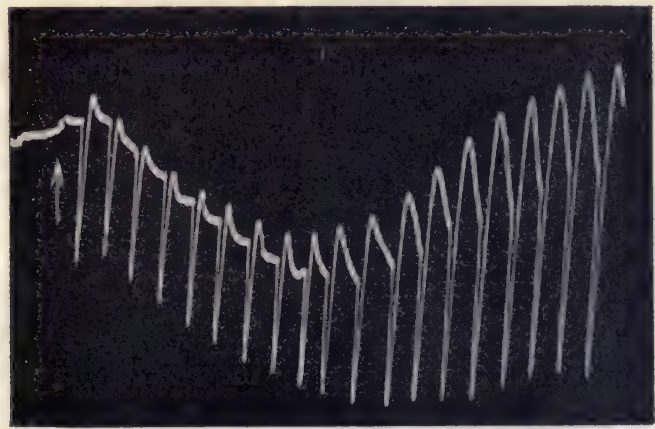

FIG. 298. Photographic Record of Effect of Stronger Dose (2 per cent.) of $\mathrm{Na}_{2} \mathrm{CO}_{3}$ Solution on Conductivity.

The solution was applied on the right arm of the balance c. Note growing depression and appearance of diphasic effect.

conductivity is then seen to undergo a continuous decrease and reversal into growing depression, as seen in the substitution of increasing down-responses. This record deserves special attention, inasmuch as it affords us an insight into a phenomenon which could not otherwise have been suspected. Greater conductivity is usually associated with increased velocity of transmission. It would appear, however, that the term conductivity really covers two different phenomena which may not always be concomitant. That is to say, an increase of conductivity may mean either a greater speed of transmission of excitation or a greater intensity of the 
excitation transmitted. In the first four records of the present series the induced enhancement of conductivity is shown by the occurrence of up-responses only. The fifth record, however, shows a marked preliminary twitch in the negative direction, followed by an up-response of some amplitude. This shows that the excitatory effect reached the right end E later than the left, though the intensity still remained greater. The continued action of the reagent subsequently reduced the intensity also, so that this diphasic ultimately became converted into a purely monophasic down-response, gradually increasing to a maximum. In fig. 298 we observe the depression of conductivity by a stronger dose of 2 per cent. solution of $\mathrm{Na}_{2} \mathrm{CO}_{3}$, applied on the right-hand side at $\mathrm{C}$. Here, again, we can see the separated effects of the two elements of conductivity-that is to say, the intensity of the effect transmitted and the speed of transmission. In the first few responses of this series we see the diminished intensity of transmission to the right giving rise to resultant responses which are entirely downwards. Later, this transmission of enfeebled excitation becomes delayed also, and by the phasedifference thus induced we obtain the growing diphasic effects which have already been fully explained on p. I44, fig. IOO. Owing now to this growing difference of phase, the two opposed effects no longer neutralise each other to the same extent as before, and we obtain increasing amplitude of both the constituent phases. The down-curve in the diphasic response represents the earlier arrival, and relatively greater intensity, of effect at the left contact $\mathrm{E}^{\prime}$. And the up-curve shows the later arrival of the less intense effect at the righthand contact $\mathrm{E}$. It is thus clearly seen that conductivity includes two different elements of speed and intensity which may not in all cases be coincident.

I shall next describe experiments which will demonstrate the variation of conductivity versus that of responsive excitability under the action of the same reagent. In animal nerve responsive excitability is diminished by the action of strong solutions of neutral salts, and potassium salts induce greater 
depression than corresponding sodium salts. But neutral salts, generally speaking, affect conductivity to a much slighter extent than responsivity. There is, however, a very curious exception to this rule in the case of animal nerve, where 6.I per cent. of $\mathrm{NaI}$ is found to affect the conductivity to a much greater extent than the responsive excitability. I find a remarkable parallelism to these effects in the case of vegetable nerve, which is capable of striking demonstration by the comparative method of simultaneous variations of conductivity and excitability already described. In order to demonstrate these contrasted effects of KI and $\mathrm{NaI}$ on conductivity and excitability, I shall here give an account of two different experiments. In the first, after obtaining the preliminary balance, KI was applied at $\mathrm{C}$, on the right arm, the same reagent being also applied at the end $E^{\prime}$ of the left arm, this process being represented by the formula $\mathrm{E}_{\mathrm{KI}}^{\prime} \mathrm{C}_{\mathrm{Kr}}$. The record seen in fig. 299 shows, by its resultant up-responses, that a greater depression of responsivity at $\mathrm{E}^{\prime}$ than of conductivity at $\mathrm{C}$ has been induced. In the next experiment (fig. 300) $\mathrm{NaI}$ was applied instead of $\mathrm{KI}$, on $\mathrm{C}$ to the right, and $\mathrm{E}^{\prime}$ to the left, the formula thus being $\mathrm{E}_{\text {Nat }}^{\prime} \mathrm{C}_{\text {Nar. }}$ The resultant responses were now downwards, showing that there was a relatively greater depression 
of conductivity than of responsivity. In respect of conductivity and responsivity, therefore, the effects of these two drugs, KI and NaI, are seen to be opposite.

In order to observe the effect of alcohol on nervous tissue, by means of the conductivity balance, I first experimented on the nerve of frog. A 5 per cent. solution was applied at the responding point $\mathrm{E}$. This is seen (fig. 30I) to induce

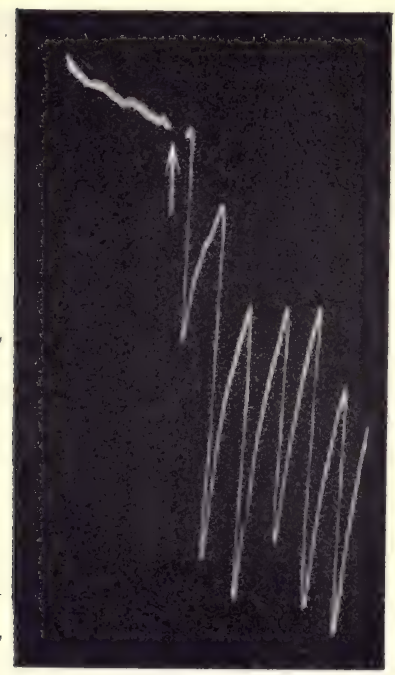

FIG. 300. Responsivity versus Conductivity under $\mathrm{NaI}$

The formula in this case is $\mathrm{E}^{\prime}{ }_{\mathrm{NaI}} \mathrm{C}_{\mathrm{NaI}}$. Photographic record shows an effect opposite to that of $\mathrm{KI}$ as previously described, there being now a relatively greater depression of conductivity than of responsivity. a depression of responsivity. A more dilute solution generally induces a preliminary exaltation followed by depression.

We said in the previous chapter that when alcohol vapour was passed into the chamber of the vegetable nerve the responses underwent a rapid abolition. This result, however,

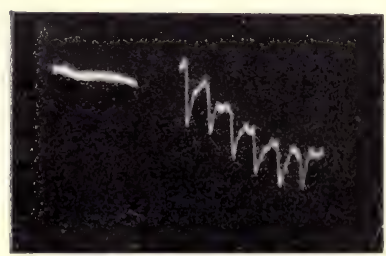

FIG. 30r. Effect of Alcohol on the Responsivity of Frog's Nerve

Upsetting of the balance in the downward direction shows depression.

was due to the joint action of the variations of receptivity, conductivity, and responsivity, some of which may possibly have been in the positive and others in the negative direction. In order to determine the effect of each of these we must, then, perform separate experiments. Such a determination I have made, using the method of the so-called 'negative variation,' in which the proximal galvanometric 
contact was on an unkilled, and the distal on a killed area. The first of these experiments was on variation of receptivity. The thermal stimulator was provided with mica shields, so that the receptive area was strictly circumscribed at the centre of the thermal platinum loop. Normal responses were first taken; the receptive area was next touched with I per cent. solution of alcohol, and the modified responses were recorded. The results are seen in fig. 302 , which gives

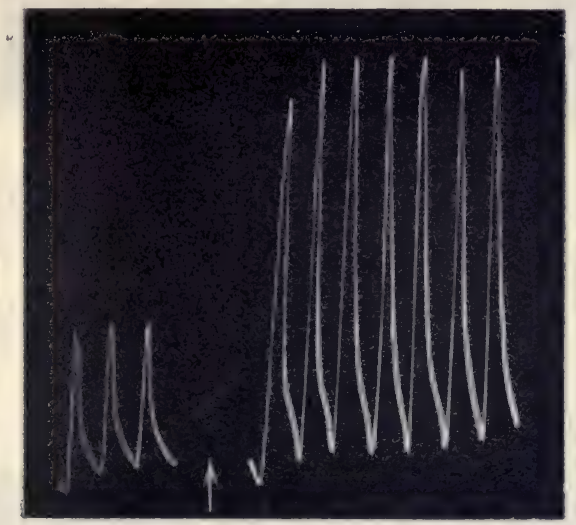

FIG. 302. Photographic Record of Effect of Alcohol Vapour on Receptivity

The three normal responses to the left are seen to be exalted after application of ether on-the receptive point.

a striking demonstration of the increased receptivity induced by dilute alcohol.

The effect on conductivity, however, is in curious contrast to this. On applying I per cent. solution in the conducting region between the stimulator and the proximal contact, a very great diminution of the conducting power is observed, as seen in fig. 303. It may be stated here that a similar enhancement of receptive excitability, and depression of conductivity, are found to be the result of the action of alcohol in animal nerve also. In the next experiment, it is the variation of responsivity under the action of dilute alcohol which is tested. After taking the normal 
responses as usual, a I per cent. solution of alcohol was applied at the proximal contact. It will be seen from the record in fig. 304 that the immediate effect was a depression

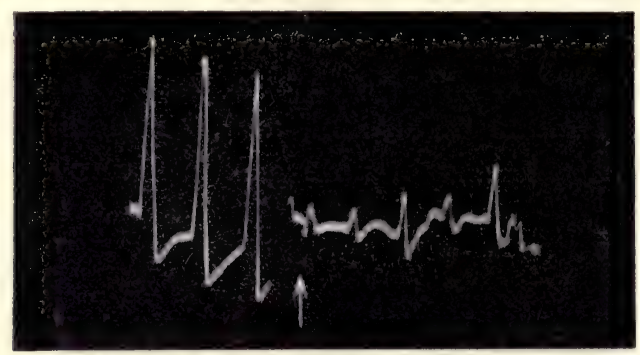

FIG. 303. Photographic Record of Effect of Alcohol on Conductivity

The three large responses to the left show the normal effect of transmitted excitation. Responses almost abolished, as seen on the right, by depression of conductivity.

of the amplitude of response. This subsequently becomes converted into a diphasic response, consisting of a preliminary positive followed by the normal negative; and finally the

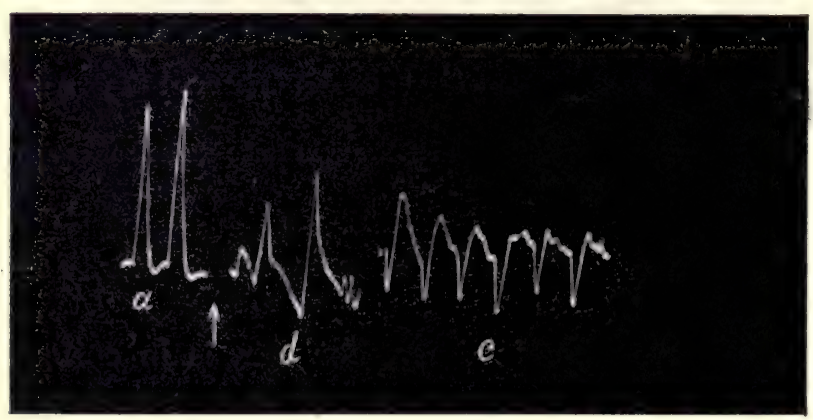

FIG. 304. Photographic Record showing Effect of Alcohol on Responsivity

$a$, normal responses, depressed, after application of alcohol, to $d$; and converted later to abnormal positive responses $c$.

response was totally reversed to positive, by the abolition of the true excitatory effect.

It is thus seen that while dilute alcohol exalts the receptive excitability, it induces a depression of both conductivity 
and responsivity. I shall now describe further experiments by which the relative effects of alcohol are compared, as between conductivity and receptivity, and as between receptivity and responsivity.

For the purposes of such a comparison, a new balancing arrangement has to be employed (fig. 305). Here, two electro-thermic stimulators are in series, so that excitations may be produced at two different points simultaneously. The galvanometer contacts $\mathrm{E}^{\prime}$ and

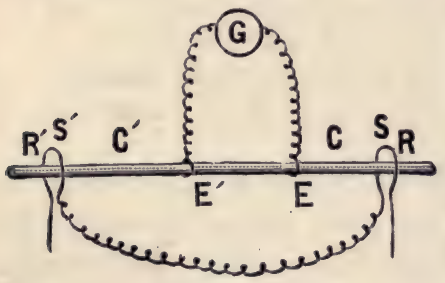

FIG. 305. Diagrammatic Representation of Experimental Arrangement for Demonstration of Receptivity versus CoNDUCTIVITY, or of RECEPTIVITY versus RESPONSIVITY

$\mathrm{S}$ and $\mathrm{s}^{\prime}$ are exciting thermal loops in series; $R$ and $R^{\prime}$, the enclosed receptive points; $\mathrm{C}$ and $\mathrm{C}^{\prime}$, conducting arms; $\mathbf{E}$ and $\mathbf{E}^{\prime}$, the responsive points.

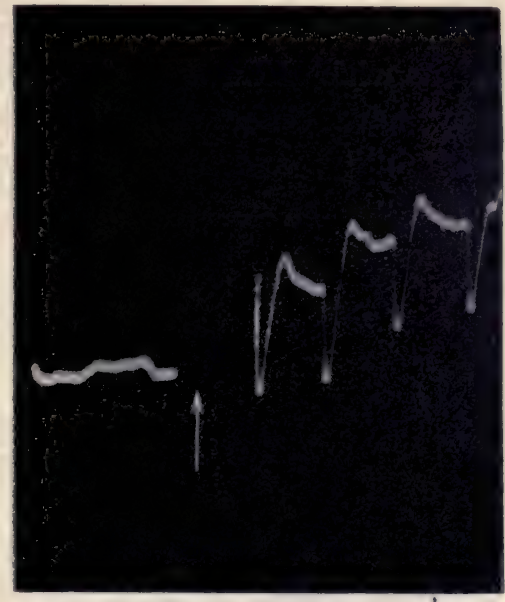

Fig. 306. Receptivity versus ResponsIviTy under Alcohol

Alcohol was applied at the receptive point to the left $\mathbf{R}^{\prime}$, and the responsive point to the right $\mathbf{E}$. The formula was $\mathrm{R}_{\text {alc. }}^{\prime} \mathbf{E}_{\text {alc. }}$ The photographic record shows the relative enhancement of receptivity.

$\mathrm{E}$ are made with two points intermediate between the stimulators. The distance of one of the two stimulators is kept constant, at, say, $2 \mathrm{~cm}$. to the left of $\mathbf{E}^{\prime}$, while the other is moved nearer to, or further from, E, until a balance is obtained. A I per cent. solution of alcohol is then applied to the left receptive point, $\mathrm{R}^{\prime}$, and the right conducting area, $C$, the formula now being $R_{\text {alc. }}^{\prime} C_{\text {alc. }}$ The fact that the receptive excitability is heightened by this reagent; and conductivity depressed, receives independent confirmation from the upset of the balance, giving rise to a downward response. 
The next experiment consists of a comparison of the simultaneous variations of receptivity and responsivity. Alcohol is applied at $R^{\prime}$ and $E$, the formula thus being $R_{\text {alc. }}^{\prime} E_{\text {alc. }}$ And we find here in confirmation of our previous results that, on account of the opposite effects of this agent on the receptive and responsive excitabilities, the resultant response is downwards (fig. 306), showing that the receptivity has been relatively exalted. Thus the experiments which I have here described show that the same agent may have different effects on receptive and responsive excitability, and thus accentuate the necessity of clearly distinguishing between the two. 


\section{CHAPTER XXXIV}

\section{EFFECT OF TEMPERATURE AND AFTER-EFFECTS OF STIMULUS ON CONDUCTIVITY}

Effect of temperature in inducing variations of conductivity : $(a)$ by Method of Mechanical Response; $(b)$ by Method of Electric Balance-Effect of coldEffect of rising temperature-The Thermal Cell-After-effect of stimulation on conductivity-The Avalanche Theory-Determination of the after-effect of stimulus on conductivity by the Electrical Balance-After-effect of moderate stimulation-After-effect of excessive stimulation.

IN studying the effect of temperature in inducing variations of conductivity, we may use either of two different methodsin the first place the method of mechanical, or in the second that of electrical response. For the first of these it is necessary to have what is generally known as a 'sensitive' plant, the leaves or leaflets of which afford conspicuous motile indications of the arrival of the excitatory wave from a distance. In such a case the time-interval between the application of stimulus and the response of a leaflet at a known distance gives us a measure of the velocity of conduction; and if we carry out successive experiments at different temperatures we have a means of determining the effect of temperature on conductivity. Employing this method, I have elsewhere given a determination of the effect of temperature on the velocity of transmission in Biophytum sensitivum. It was there shown that lowering of temperature reduced the velocity of transmission even to the extent of abolition, when the cooling was sufficiently intense. With moderate cooling the velocity was found to be decreased to about one-third. The effect of rise of temperature was, on the contrary, an increase of 
velocity. When it rose from $30^{\circ} \mathrm{C}$. to $35^{\circ} \mathrm{C}$, , for example, the velocity was doubled.

By employing the electrical method of response, however, we are rendered independent of the use of sensitive plants, and by means of the Conductivity Balance we are enabled to demonstrate the slightest variation of conductivity, as between the left arm of the balance, which is maintained at standard temperature, and the right, which is subjected to the given change.

Thus in a definite experiment on a nerve of fern the temperature of the room was $30^{\circ} \mathrm{C}$. After first obtaining the balanced record, the temperature of a portion of the right arm of the balance was lowered. This one-sided cooling was effected by supporting the right arm of the nerve, through a certain length, in the concavity of a $\mathrm{U}$-tube through which cold water at $15^{\circ} \mathrm{C}$. was passed. Stimuli were now applied at intervals of one minute. Previously, as will be understood, such stimuli, owing to balance, had induced no resultant effect. But now, on account of the depression of conductivity on the right side, brought about by cooling, the balance was disturbed, and the resultant down-response seen in fig. 307 shows the diminished conductivity of the right arm. On the "cessation of the flow of cold water the balance was gradually restored, in concomitance with the return to the original temperature.

I next investigated the results of a rise of temperature, and here I specially desired to observe the conductivity variations, not at any one degree, but throughout a graduated and continuous rise. I was confronted at the outset of this investigation by the difficulty arising from the fact that there was no convenient and satisfactory means for the local variation of the temperature of the nerve, in definite and known degrees. In connection with this there was also the further difficulty that a sudden variation of temperature will, in itself, act as a stimulus. Hence, in studying the effects of temperature per se, it is essential that there should be no such sudden variation. These difficulties were overcome by 
the employment of an electrical arrangement to bring about the graduated and continuous rise of temperature.

A certain length of the vegetable nerve on the right arm of the Conductivity Balance was thus raised continuously in temperature, and its conductivity compared with that of the left arm of the balance, the latter being maintained at the temperature of the room, which happened at the time to be $33^{\circ} \mathrm{C}$. The device by means of which this was accomplished

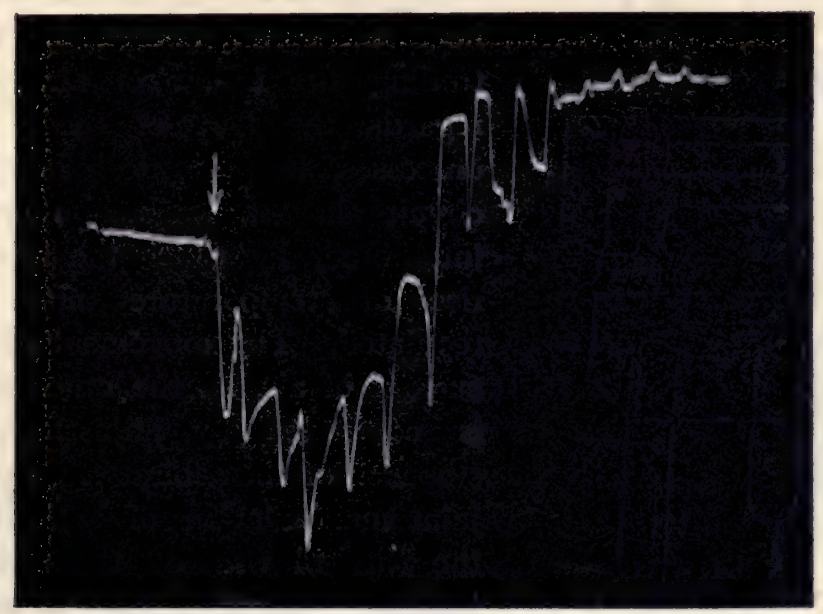

FIG. 307. Photographic Record showing Effect of Cooling on Conductivity of Plant-nerve

Balance was obtained at starting, when temperature of both arms was $30^{\circ} \mathrm{C}$. On cold being applied on right arm, the balance was disturbed, showing diminished conductivity on that side. On restoration of normal temperature, the balance is seen at the end of the record to be again restored.

will be understood from fig. 308. A piece of cork has a small chamber cut into it measuring $\mathrm{I} \mathrm{cm}$. each way. In this is placed moist blotting-paper, which keeps it damp, and across it passes a length of $\mathbf{I} \mathrm{cm}$. of the right arm of the vegetable nerve in the Conductivity Balance. This corkchamber has inlet and outlet tubes $t$ and $t^{\prime}$. The first of these contains a spiral, $\mathrm{H}$, of platinum, which can be heated to a suitable degree by means of an electrical current, the 
intensity of which is capable of careful adjustment. The cork chamber is closed with a cover, through which passes a thermometer, $T$, for the indication of the temperature within. The tube $t^{\prime}$ is connected with an aspirator, and air is thus sucked in by $t$, and, passing through the platinum spiral, is warmed, and raises the temperature of the nerve in
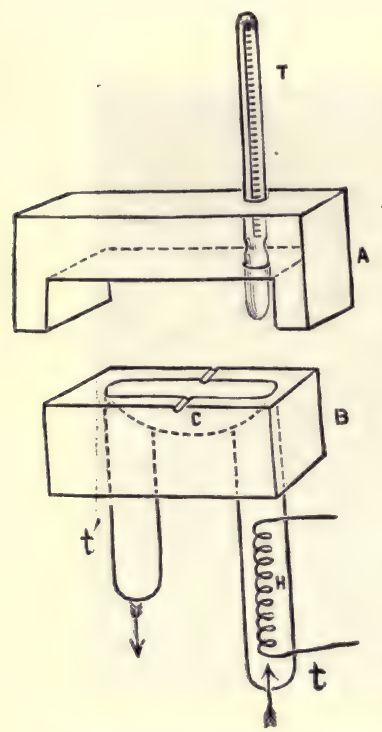

FIG. 308. The Cork Chamber for Gradual Raising of the Temperature of one Arm of the Balance

$A$ and $B$, the two halves of the chamber; $T$, thermometer; $t$ and $t^{\prime}$, inlet and outlet tubes; $\mathrm{H}$, thermal coil for heating. the chamber. This rise of temperature is adjusted (I) by regulating the electrical current which heats the spiral, and (2) by controlling the inflow of air. As regards the first of these two processes, the electrical heatingcircuit has a carbon rheostat interposed, by which the rate of rise of temperature may be regulated. The movement of the current of air, on the other hand, is controlled by adjusting the stopcock of the aspirator. By the joint manipulation of both these the rate of rise of temperature inside the chamber may be made perfectly uniform, and in my experiments this rate was approximately $\mathrm{I}^{\circ} \mathrm{C}$. per minute.

As already said, I selected a piece of vegetable nerve and took a balanced record. After this the temperature of the thermal cell on the right-hand side was raised continuously, the response-record being taken at each degree of the rise, till a temperature of $50^{\circ} \mathrm{C}$. had beenat tained. From the record given in fig. 309 it will be seen that the conductivity was always greater at temperatures up to $47^{\circ} \mathrm{C}$. than it was on the left-hand side, which was all the time maintained at the constant temperature of $33^{\circ} \mathrm{C}$. At $48^{\circ} \mathrm{C}$., 
however, the reversal of response showed that the conductivity was now being depressed. And at still higher temperatures it was found to undergo a very great depression, as is seen by the abrupt downward movement of the curve. It is thus seen that, by means of the Method of Balance, this very difficult problem of the variation of conductivity under variation of temperature is made capable of exact study.

I shall next describe the results of an investigation into the after-effects of stimulus on conductivity and excitability,

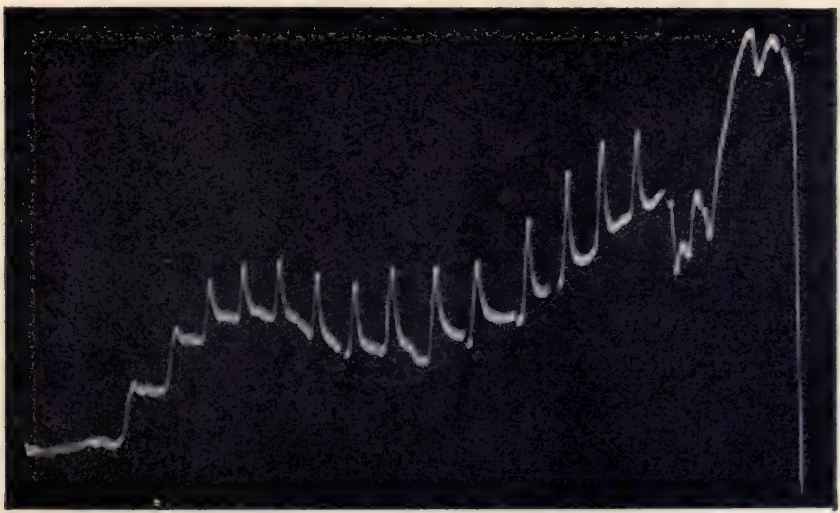

FIG. 309. Photographic Record Showing Effect of Rising Temperature on Conductivity

Balance obtained at starting at $33^{\circ} \mathrm{C}$. Successive responses recorded at each degree $\mathrm{C}$. of rise of temperature. Record shows increasing conductivity up to $47^{\circ} \mathrm{C}$. A depression of conductivity is seen by reversal of curve to set in at $48^{\circ} \mathrm{C}$., and this becomes extremely pronounced at $50^{\circ} \mathrm{C}$.

a subject of much difficulty and of considerable theoretical importance. It has been found in Animal Physiology that the sciatic nerve of a frog, for instance, is not equally excitable throughout its length. When such a nerve, with its attached terminal muscle, is cut off from the spinal cord, it is seen to be more excitable the further from the muscle is the point on the nerve that is subjected to stimulation. From this fact that excitation increased with the distance of the point excited from the motor organ, Pfluger was led to 
the 'Avalanche Theory,' namely, that during the passage of excitation down the nerve it actually gathers strength. But it is clear that this cannot be true, since we have seen that, other things being equal, excitation is always greater the nearer the point of stimulation to the responding region, and on this fact have depended all those experiments already described, which involved a delicate balance of equal excitations. It follows that the observed enhancement of excitability of a point on the nerve which is distal from the muscle, and in the neighbourhood of a section, must be ascribed to some other cause. In reference to this Heidenhain, indeed, explained the greater excitability of higher tracts of divided nerve by the proximity of the artificial section. For the lower end of the nerve at once exhibits the same marked activity as the upper end if a section be made lower down. Excitability is, in fact, raised near the section, wherever the section may be. The distance travelled by the excitation could not, therefore, be the determining factor in the magnitude of effect. For so far from increasing it, this, as a matter of fact, causes diminution. It is to be remembered that though the excitability is increased near the point of section, yet at the section itself it is almost abolished, otherwise there could not have been any response by so-called negative variation. The question now arises, Why should the excitability be raised near the point of section?

It has been supposed that this was due to certain electrical changes induced by section, which in turn gave rise to electro-tonic variations of excitability. We shall see, in Chapter XL, that the passage of an electrical current through a living tissue induces changes of excitability. And this phenomenon is known as the electro-tonic effect. Now any 'injury,' such as a mechanical or thermal section, is known to induce galvanometric negativity, or anodic change, at or near the point of section. But it is the kathode-effect which is excitatory. And the observed greater excitability of the nerve near a point of section is supposed to be due to katelectrotonus, produced within a certain tract from the cross- 
section by internal short-circuiting of the nerve-current. That, this explanation, however, does not meet all the requirements of the case will appear from certain experiments which I shall describe, where, under exactly similar electrotonic changes due to section, a result directly the opposite of this, that is to say, of depression, is seen to be induced.

All these various facts will be found fully reconcilable, however, on the basis of a proposition which I shall establish, namely that in a nerve, moderate stimulation enhances excitability and conductivity, while excessive stimulation has the opposite effect of bringing about the depression of both. It is indeed natural to expect that while moderate stimulation, by increasing molecular mobility, would bring about one effect, excessive stimulus, by inducing overstrain, would result in exactly the opposite. Before proceeding to give an experimental demonstration of this hypothesis, we shall first consider the explanation which it affords of the peculiar excitatory changes observed in the case of cross-sectioned nerve. In the first place we know that a cut acts as a stimulus. And since we found that the effect of stimulus decreases with the distance from the point of stimulation, it would appear that at the section itself the stimulation would be excessive; moderately strong at a certain distance from it; and practically negligible when very far away. In complete accordance with this is the resulting increase of excitability which has been observed near the point of section, while at the point itself the nerve is relatively inexcitable.

The fact that stimulation, when not excessive, increases the conductivity and excitability, we found illustrated in the staircase increase of electrical response, and in the enhancement of amplitude after tetanisation, in vegetable and animal nerves (figs. 275 and 286). The same fact will be demonstrated later by means of the mechanical response of nerve. I shall now describe certain experiments which demonstrate it once more in a new and interesting manner. 
A vegetable nerve was adjusted for balance, with the ends projecting some distance beyond the electrodes. In order to show that the effect of injury is due to stimulus as such, and not to any particular form of it, I now made a thermal instead of mechanical section, by applying salt solution heated to about $60^{\circ} \mathrm{C}$. in the region $\mathrm{A}$, at a distance of I cm. to the right of $\mathrm{E}$ (fig. 3IO). The effect of this stimulation was to induce a moderate excitation of the right arm of the balance, relatively to the left. If this moderate stimulation were to induce any increase of excitability and conductivity, that fact would be demonstrated by

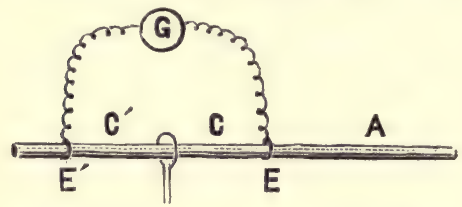

FIG. 310. Experimental Arrangement for Studying After-effect of Stimulus on Conductivity and Excitability

The stimulator adjusted to obtain balance between $\mathbf{E}$ and $\mathbf{E}^{\prime}$. Stimulus of moderate or strong intensity is applied to a point on the right of $\mathbf{E}$. Upsetting of the balance in an upward direction shows an enhancement, and in a downward direction, depression, of conductivity and excitability.

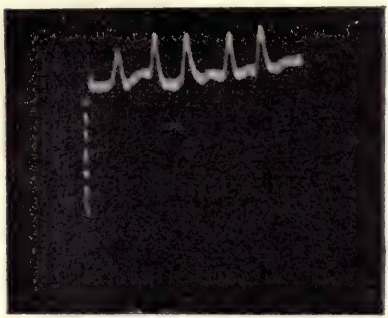

FIG. 3II. Photographic Record Showing Effect of Moderate Stimulation in Enhancing Conductivity and Excitability

otted line at beginning shows the resting-current, as a persistent effect of stimulation. The upsetting of the balance upwards constitutes a positive variation of the restingcurrent, and indicates enhanced conductivity and excitability.

the upsetting of the balance, the resultant response being upwards. That this is what actually occurs will be seen from the records in fig. 3 II. It will be noticed that in consequence of stimulation to the right of $\mathrm{E}$, that point became, more or less permanently, galvanometrically negative. This is represented by the dotted line upwards at the beginning of the record. It must be remembered that before the application of the thermal section, the right and left hand excitations, proceeding from the electro-thermically stimu- 
lated point in the middle, were exactly equal and balanced. The fact that after this application, however, there are resultant responses which are upwards, shows, as already said, that by the moderate stimulation of the right-hand side, both excitability and conductivity have been increased. The resultant upward response here is, then, in the same direction as the so-called 'current of injury,' and forms, as it were, a positive variation of it.

In another experiment, in which I wished to try the effect of excessive stimulation, instead of applying a hot solution at $60^{\circ} \mathrm{C}$., I produced greater injury and consequent excessive stimulation, by scorching the nerve at the same point as before, with a red-hot platinum wire. In this case resultant response was downwards, showing that the excitability and conductivity of the right-hand side of the balance had been depressed by over-stimulation.

I was next desirous of demonstrating that the excitability of the over-stimulated or excited point undergoes depression. For this purpose I took a fresh specimen and first ob-

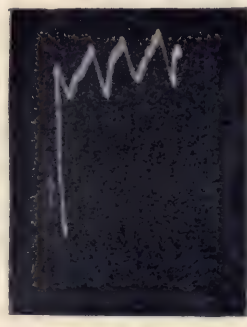

FIG. 312. Photographic Record showing Effect of Excessive Stimulation in Depressing Excitability and Conductivity

Up-line at starting shows the resting-current due to after-effect of stimulation. The upsetting of balance in a downward direction constitutes a negative variation of the resting-current, and shows depression of conductivity and excitability.

tained a state of balance. Similar excitation of $E^{\prime}$ and $E$ produced a balanced or null effect. The point $\mathrm{E}$ was then injured by touching it with a hot platinum wire. On now proceeding to take records, it is seen that the responses were downwards, showing the depression of excitability at the injured E (fig. 3I2).

The fact that galvanometric negativity had been induced at $\mathrm{E}$, by reason of injury, is demonstrated at the beginning of the record as an up-line. The subsequent resultant responses due to simultaneous excitation of $\mathrm{E}$ and $\mathrm{E}^{\prime}$ are 
seen to be a negative variation of the resting-current due to injury. It is thus seen that while simultaneous-excitations of two normally excitable points $\mathrm{E}$ and $\mathrm{E}^{\prime}$ are prevented by balance from giving rise to any response, the excitatory response becomes manifest when the balance is disturbed by the injury of either point of galvanometric contact ; and that, under these circumstances, the response is a negative variation of the current of injury. This experiment is important as giving a theoretical insight into the so-called response by negative variation. It also shows how limited is the applicability of the assumption that response is always by negative variation. For, in the similar experiment, previously described, under moderate injury, the response was by positive variation of the resting-current.

It is further seen from these experiments that the enhancement of excitability, under the stimulation due to moderate injury, could not be caused by the suggested electrotonic effect. For the same anodic change induced by injury at $\mathrm{E}$ causes, in the case of moderate injury, an enhancement, and under greater injury a depression, of excitability. It is thus clear that the modifying influence is the effective intensity of stimulation. This fact, that moderate stimulation enhances, and excessive stimulation depresses excitability, will be further demonstrated in a future chapter, by the independent method in which the effects of electrotonus are completely eliminated. 


\section{CHAPTER XXXV}

\section{MECHANICAL RESPONSE OF NERVE}

Current assumption of non-motility of nerve-Shortcomings of galvanometric modes of detecting excitation-Mechanical response to continuous electric shocks-Optical Kunchangraph-Effect of ammonia on the mechanical response of nerve-Effect of morphia-Action of alcohol-Of chloroformAbnormal positive or expansive response converted into normal contractile through diphasic, after tetanisation-Similar effects in mechanical response of vegetable nerve-Mechanical response due to transmitted effects of stimulation-Determination of velocity of transmission-Indeterminateness of velocity in isolated nerve-Kunchangraphic records on smoked glassOscillating recorder-Mechanical response of afferent nerve-Record of mechanical response of nerve due to transmitted stimulation, in geckoFatigue of conductivity-Conversion of normal contractile response into abnormal expansive, through diphasic, due to fatigue.

I HAVE already referred to the distinctions which are commonly insisted on, as between the reactions of different animal tissues. Certain of these are regarded as motile and others as non-motile. From an evolutionary point of view, however, it is difficult to conceive of such a hard-andfast distinction. It would be easier, believing in continuity, to suppose that a certain responsive reaction, characteristic of the simplest living substance, had become accentuated in some tissues, and not so accentuated in others, according to their different functional requirements. Thus the belief held so implicitly by physiologists that nerves exhibit no motile response whatsoever ${ }^{1}$ becomes questionable, and is seen to require investigation. After submitting it to this, moreover, one finds it difficult to understand how such an

1 'Nerves are irritable; when they are stimulated, a change is produced in them; this change is propagated along the nerve, and is called a nervous impulse ; there is no change of form in the nerve visible to the highest powers of the microscope.' (Kirke's Handbook of Physiology, 15th edition, p. 105.) 
idea ever gained currency, unless, indeed, it was due to the tyranny imposed on our thought by these arbitrary classifications themselves.

Before entering, however, on the question whether the excitatory reaction in nerve finds motile expression or not, we shall first examine the only method at present available for the detection of the condition of excitation. Since excited nerve has hitherto been supposed to exhibit no visible change, it followed that the only method possible for the detection of the excitatory change was the electrical. Investigations on nerve, therefore, had perforce to be carried out by this means, through the medium either of the capillary electrometer or of the sensitive galvanometer. But the electrical method labours under certain inherent disadvantages, and first of these is the objection which it raises to the free employment of the most convenient form of stimulus, that, namely, by induction shocks. For we have seen that unless extraordinary precautions are taken, we have here, owing to the possible escape of current, an element of error and uncertainty in the results. If, on the other hand, it should become possible to obtain mechanical response from the nerve, this particular form of stimulation might be employed without misgiving.

The second limitation which the electrical mode of detection imposes upon us is that arising from the differential character of the response which it indicates. For stimulus induces electrical changes at both the contactsproximal and distal--the record made being finally due to the algebraical summation of the two. It is true that the excitability of one contact is artificially depressed by injury. But it is often difficult to say how far this injury has been effective in completely abolishing the excitability of this point. The depression of excitability, due to partial injury, will sometimes disappear to a certain extent, with lapse of time, and much uncertainty sometimes occurs as to whether a certain curious variation in the response of the nervenegative followed by positive-is due to this or some other 
cause. With mechanical response, however, provided this could be rendered practicable, no such difficulty need arise. For in that case it would be the direct effect of the excitatory change, uncomplicated by any other disturbance, which would be recorded.

Finally, as regards the detection of the excitatory change itself, the galvanometer is unable to indicate any change below a certain high intensity of excitation. Thus it gives no indication when excitation is due to one or to a few shocks : it can only detect an excitatory effect which is much stronger than this, having been brought about by the superposed effects of tetanic shocks of a certain duration. In order to obtain even such effects, a galvanometer of very high sensitiveness is necessary. That of a fairly delicate instrument, detecting a current of about $\mathrm{O}$ I ampère, will have to be exalted some ten millions of times before it can give efficient indications of excitatory effects in nerves; and in such a degree of galvanometric sensitiveness we approach a limit which cannot be very much exceeded.

Returning now to our original question, we have first to determine whether excitation causes any motile effect in nerve. Under observation, it is easily seen that when the nerve is excited by tetanic electrical shocks it increases in thickness and at the same time shortens in length. We have here a phenomenon in every way analogous to the thickening and shortening of muscle under excitation. The contraction which occurs in nerve, moreover, is of an order by no means microscopic. I give here a record (fig. 313) of the contractile response of nerve under continuous stimulation by fairly strong tetanising electric shocks. This record was obtained by means of the ordinary lever-recorder, the magnification employed being only three times. The induced contraction in this particular case was about 14 per cent. It will also be seen that this contraction reached a limit, at which state of maximum contraction the nerve remained for a considerable time. After this we observe a tendency 
to decline, owing to fatigue. In some other cases, moreover, I have obtained a contraction of as much as 20 per cent.

If we wish to obtain a series of successive responses, however, it is desirable to avoid over-stimulation of the tissue. In order, then, to obtain a response-record under moderate stimulation, we have to employ a higher magnification. This magnification, if made about 200 times, is more than sufficient for all practical purposes, and the photographic records given in the course of the present chapter are of this order. With long specimens of nerve, however, a magnification of fifty times would be enough, and in the

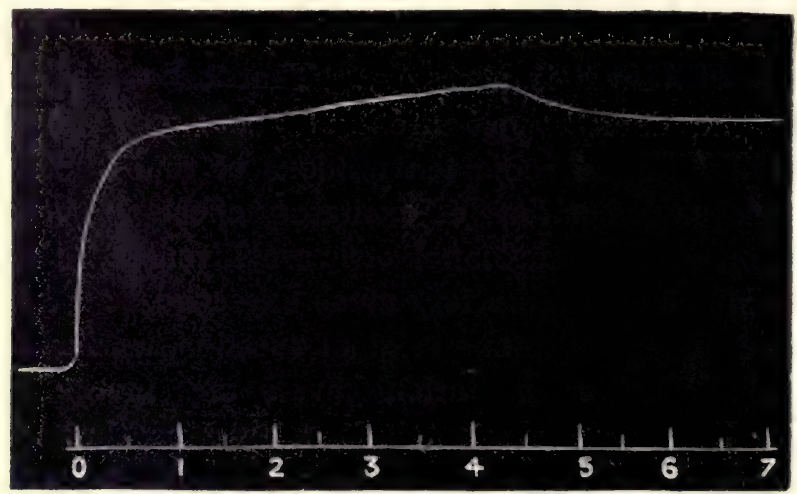

FIG. 313. Record of Contractile Response in Frog's Nerve under Continuous Electric Tetanisation.

Magnification, three times.

course of the next chapters, I shall give certain records on this scale, obtained directly on a smoked glass surface. The apparatus used for the purpose was the Kunchangraph (Sanskrit, kunchan, contraction), which I had already devised and employed in recording the contractile responses of planttissues. This apparatus, as adapted for the purpose of recording mechanical response in nerves, consists of, first, a nerve-chamber, $\mathrm{N}$; secondly, a modified Optical Lever, $\mathrm{O}$; and thirdly, a photographic recorder, D (fig. 3I4).

Of these, the nerve-chamber consists of a small rectangular ebonite box, the front of which is closed by a semi-cylindrical 


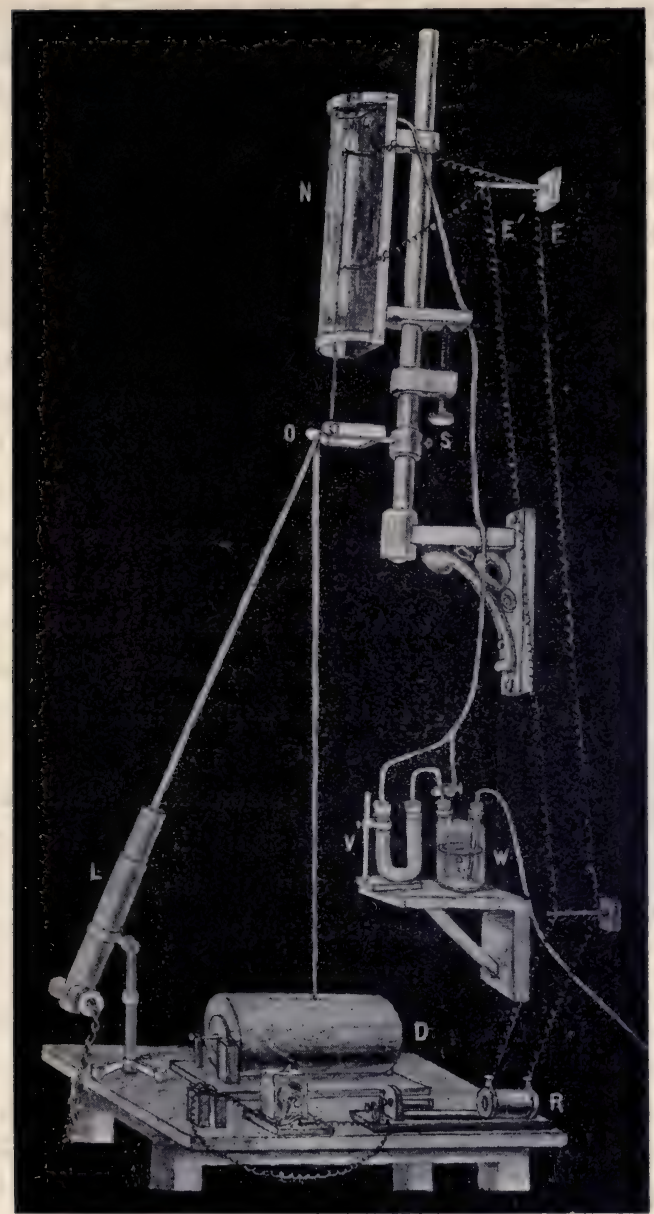

FIG. 3I4. Optical Kunchangraph for Record of Mechanical Response of Nerve.

$\mathrm{N}$, nerve chamber containing nerve with electrical connections, $\mathrm{E} \mathrm{E}^{\prime}$. Thread tied to lower end of nerve, and attached to short arm of optic lever, o. Beam of light from L reflected from mirror of optical lever, $\mathrm{O}$, falls on recording-drum, D. Adjustment of reflected spot of light made by micrometer screw, s. Periodic electric stimulation at intervals of one minute is automatically made by means of key regulated by clock-work. Air bubbles through water at w, and is led on by india-rubber tubing, $\mathrm{T}$, to nerve-chamber, thus kept humid. By proper manipulation of stop-cock any vapour-as ckl oroform-contained in vessel $\mathrm{v}$, may be passed through nerve-chamb er, subsequent responses showing effect. 
glass cover. The nerve is placed vertically within this, and held, at its upper end, by a clamp. The lower end of the nerve is connected with the short arm of the Optical Lever by means of a thread, which passes through a hole in the floor of the chamber. A second thread of cotton moistened with saline solution hangs loosely from the end of the nerve, and is connected with the electrode $\mathrm{E}^{\prime}$. When the electrodes $\mathrm{E}$ and $\mathrm{E}^{\prime}$ are put in connection with the secondary of an induction coil $\mathrm{R}$, the entire length of the nerve is subjected to direct excitation. When, on the other hand, we wish to study

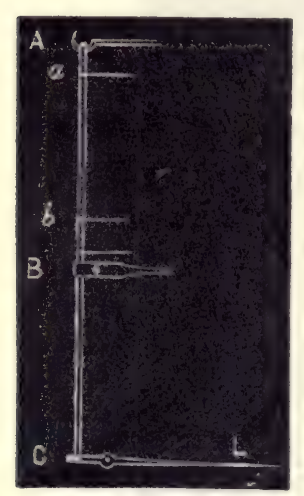

FIG. 315. Diagrammatic Representation of Arrangement for Obtaining Transmitted Effect of Stimulus. L, indicating lever the effect of transmitted excitation, the nerve is lightly clamped at $B$ (fig. 3 I 5). Excitation is then induced in the portion of the nerve $\mathrm{A} a$, and after transmission through the intervening tract, causes the motile effect in the responding portion of the nerve $\mathrm{B} \mathrm{C}$.

One precaution which I find to be very necessary is the maintenance of the properly humid condition in the nerve-chamber. This is specially important in the warm weather which characterises the greater part of the year in India. The usual means of keeping the chamber moist, by a large quantity of blotting-paper soaked in water, is not sufficient to bring about the maintenance of the normal excitability of the nerve for any length of time. This need was met by keeping moist vapour in uniform circulation through the nerve-chamber. An air-bag is kept under suitable pressure, and the air, bubbling through water in the vessel $\mathrm{w}$, is made to enter the nerve-chamber through an entrance-pipe, and to escape by an exit-pipe. In warm weather it is well to keep fragments of ice in the water-vessel. By proper manipulation of the stop-cock of the air-bag, a gentle stream of cooled and humid air is kept in constant circulation through 
the chamber. Observing these precautions, I have been able to obtain responses from a given nerve for as much as three hours continuously, whereas, without this care, they would have come to a stop in a very short time. By a modification of this arrangement, we are also enabled to study the effect on the excitability of the nerve of various gases and vapours contained in a second vessel, V. A series of responses is first taken, under normal conditions-that is to say, when the nerve is surrounded simply by a moist atmosphere. On now turning a three-way tap in a given direction, the water-vapour can be made to pass through the vessel v, filled with the given gas or vapour, before reaching the nerve-chamber. The series of responses then obtained will show either the immediate or the after-effect of the reagent at will. For it is easy, by means of the three-way cock, to shut off the gas and re-establish the first or normal condition, after which the responses will afford an indication of the nature of the after-effect.

The lower end of the nerve, as has been said, is attached to the arm of the lever which passes through the fulcrum-rod. A light mirror is fixed on the fulcrum-rod, its face being downwards. The pull caused by the excitatory contraction of the nerve causes rotation of the fulcrum-rod, and this in turn gives rise to a deflection of the spot of light reflected from the mirror. A responsive relaxation of the nerve would give rise, on the other hand, to a deflection of the spot of light in the opposite direction. The long arm of the lever, it will be noticed, is here the ray of light. The responsive movement of the spot of light is recorded on a moving photographic plate vertically below the mirror, and whose movement, regulated by clockwork, is in a direction at right angles to that of the spot of light. The photographic plate, or the film wrapped round the drum, moves under a fixed wooden cover, not shown in the figure, which is provided with a narrow incised slit. The length of this is parallel to the direction of the movement of light, and at right angles to that of the plate or film. The advantage of having the plate 
vertically below the mirror lies in the fact that a lighted candle may be placed in the dark room without spoiling the record by diffuse illumination. The only way in which such diffuse light could now find access to the plate would be by reflection from the ceiling. But if the ceiling of the experimental room is blackened, or a black cover placed over the nerve-chamber at a certain height, even this possibility is eliminated. The advantage which the observer enjoys, when, instead of groping in semi-darkness, he can work in a fairly well-lighted room is obvious. By making the arm of the lever to which the nerve is attached sufficiently short, and by placing the recording plate sufficiently far away, a wide range of magnification, from several hundreds to several thousands, may be obtaincd. It may sometimes be desirable to subject the nerve to a certain amount of tension, and this is secured by placing a small weight on the arm of the lever. With high magnification, due adjustment, which is very troublesome, lies in bringing the spot of light conveniently over the recording plate. This difficulty is obviated, however, by means of a fine micrometer screw $\mathrm{S}$ which moves the whole nerve-chamber up or down, in relation to the Optical Lever. The adjustment of this screw in a righthanded manner then moves the spot of light in one direction, say to the left, while its left-handed rotation moves it to the right. This movement can be made very fine, and the spot adjusted to any part of the photographic field.

It remains to deal with the possible disturbances incidental to the high magnification employed. Apprehension, in this matter, is often more fanciful than real. Disturbances might no doubt occur, however, when proper conditions are not secured for the experiment. If the nerve-chamber, for example, be supported on a different stand from that of the Optical Lever, then the slightest tremor of the common pedestal would result in relative movements of the two supports, causing constant disturbance of the spot of light. Under these conditions, heavy stone pedestals, erected on steady foundations, afford no security against the ground- 
vibrations of a busy city. But when both the nervechamber and the Optical Lever are fixed to the same supporting-rod, relative movements, due to external disturbance, are practically eliminated. This common supportingrod may be screwed securely to a wall. With these precautions, I have been able to take records, without the least disturbance from the adjacent electric tram line. As a matter of fact, when the magnification required is only of a few hundred times, nothing but gross carelessness could allow any source of disturbance to remain. It is only when the magnification has to be pushed to the order of a hundred thousand that unusual care is necessary to avoid errors of disturbance. One precaution which should, however, be taken, is that arising from disturbance of the mirror by convection currents of air. The remedy for this is obvious, namely, a suitable glass cover.

This is the order of magnification which is necessary for the recording of response under a degree of stimulation usual in making observations of excitatory electrical variation with a very sensitive galvanometer. But while the sensitiveness of the galvanometric method of detecting response is here nearing its limit, that of the mechanical method is in its first stage only, and how greatly the sensitiveness of the latter may be exalted when required will be shown in the next chapter.

I shall now explain how easy it is to study the physiological variations induced in the animal nerve under various agencies by means of the mechanical response. The following experiments were performed on specimens of the sciatic nerve of frog. A well-known reagent for abolition of excitability of the nerve is ammonia. Its effect on mechanical response is seen in fig. $3 \mathrm{I} 6$. In all the following experiments, the stimulus applied was by fairly strong tetanising electrical shocks, which were usually of two seconds' duration. Two series of records were taken, successive responses being recorded at intervals of one minute, before and after the application of the chemical reagent. In fig. 316 , the normal 
responses seen in the first series are found to be abolished when the nerve has been subjected to strong vapour of ammonia for some time. It should be mentioned here that this abolition takes place under the action of a strong dose. When highly diluted with air, the vapour may cause a temporary exaltation.

In the next figure (fig. $3 I 7$ ) is shown the effect of morphia. After the application of this solution for a certain length of time, the response is seen to

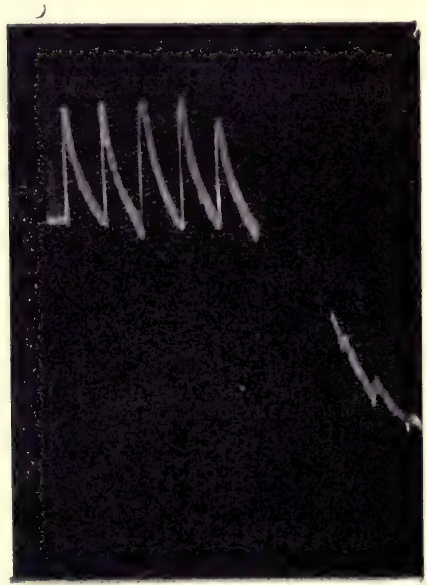

FIG. 316. Photographic Record of Effect of Ammonia on Mechanical Response of Frog's Nerve

First series of responses are normal. Second series show effect of ammonia in practical abolition of response. be abolished. The strength of application which brings about this abolition I find to vary according to the condi-

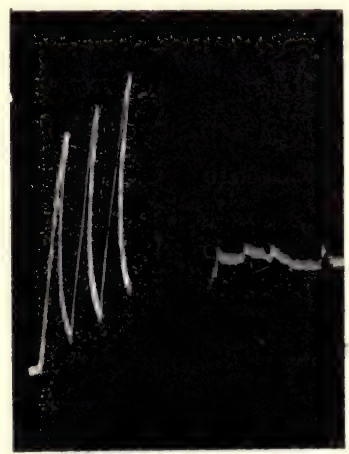

FIG. 317. Photographic Record showing Abolition of Mechanical Response of Frog's Nerve by Action of Solution of Morphia

tion of the nerve. Another agent by which the mechanical response of the nerve is found to be abolished is aconite. And it is of special interest to note that I have often found this to act as an antidote, for the revival of response previously almost completely abolished by morphia. The condition of the nerve here also appears to be a determining factor in the mutually antidotal action of these two poisons.

A strong application of alcohol after long-continued action 
abolishes the response of nerve. But its preliminary effect is often one of exaltation, as seen in fig. 318.

I shall next describe the effect of chloroform, which displays many interesting features. We have seen that when a tissue is excited by impinging stimulus, two opposite effects are induced : one of these is the increase of energy, by the absorption of stimulus, and the other is the expenditure of energy by excitatory response. The former, as we have seen, finds expression in galvanometric positivity and expansion. The latter, on the other hand, is exhibited as galvanometric negativity and contraction. In the record of excitatory response, the former of these elements is generally masked by the predominant negative or contractile effect. We have also seen that this hidden positive may be unmasked in either of two ways : first, by retarding the expression of one effect in relation to the other ; or, second, by abolishing the excitatory negative altogether. In the first of these cases, the negative response is converted into diphasic, say positive followed by negative. In the latter, the response becomes positive, by the suppression of the negative. An example of this unmasking of the positive element, by

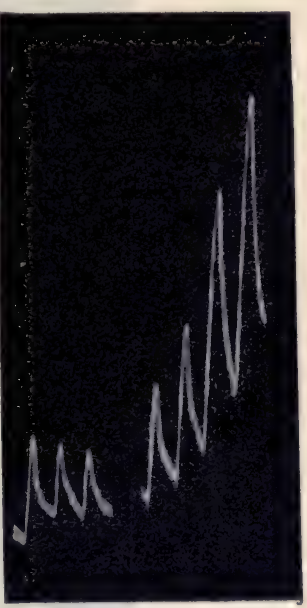

FIG. 318. Photographic Record showing Preliminary Exaltation in Mechanical Response of Frog's Nerve after Application of Alcohol suppression of the negative, we have already seen to occur under the action of chloroform (cf. fig. 49). This demonstration was made on a vegetable tissue, the test employed being electrical.

The experiment which I am now about to describe is interesting from the fact that effects parallel to those there seen in a vegetable tissue are in it shown to occur also in the highly specialised animal nerve. The unmasked electro-positive effect, moreover, is here seen to correspond with an expansive 
response of the tissue. A record of the various phases in the effect of chloroform on the mechanical response of nerve is found in fig. 319. It will be seen here that the first effect of chloroform was to cause a great enhancement of excitability, which in this case lasted for about a quarter of an hour. I have given only two responses of this series. After this, the responses began to decline, and another very

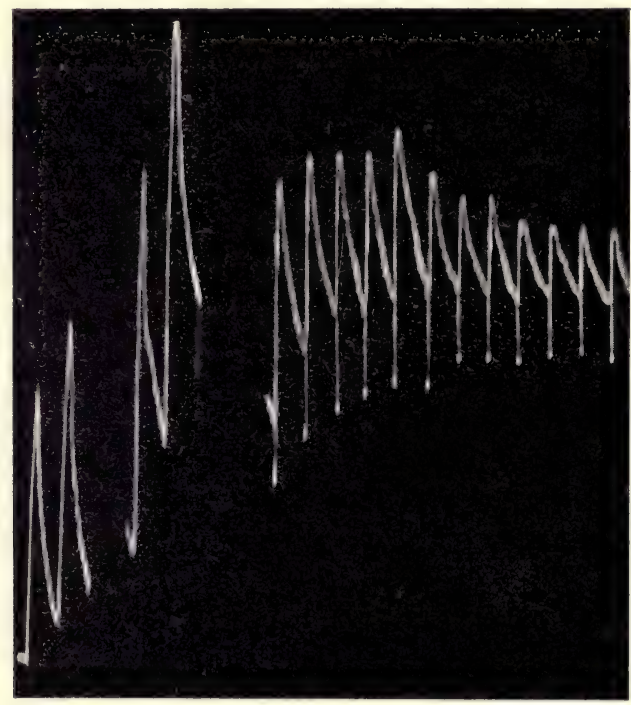

FIG. 319. Photographic Record showing Effect of Chloroform on Mechanical Response of Frog's Nerve

First pair of responses, normal ; second pair, preliminary exaltation on application of chloroform; last series exhibit subsequent effect of chloroform in unmasking the positive component as diphasic response. Expansion is here followed by contraction. Note regular waning of both components with growing anæsthetisation.

interesting reaction made its appearance. The impinging stimulus had hitherto induced only an immediate contractile response. But by the action of the chloroform the excitatory effect was delayed, and the positive, or mechanically expansive response was unmasked in the form of a preliminary downward twitch. Response was now, therefore, diphasicpositive followed by negative. Immediately on the applica- 
tion of stimulus, as may be seen from the record, there is a sudden expansive movement downwards, followed by an equally rapid reversed movement of contraction upwards, and this followed again by a slow recovery. Each of the successive stimuli evokes the same diphasic responsive sequence. It must be noted that the downward twitches are the preliminary, and not the after-affect. It is also interesting to note, as the tissue approaches death, under the continued action of chloroform, how regularly in both negative and positive directions the responses decrease in amplitude.

We shall next undertake an independent investigation into the causes which bring about the three types of response -abnormal positive, diphasic, and normal negative-known to be exhibited in the electrical response of the animal, and already demonstrated as occurring also in that of the vegetal nerve. While discussing these three types of electrical response and their variations in Chapter XXXI. it was stated that the differences of effect involved were due to changes in conductivity and excitability, brought about by varying tonic conditions. It was also explained, in the same place, that the continued isolation of so highly excitable a tissue as nerve, from its accustomed supply of energy, would be sufficient of itself to depress its tonic condition below par, with concomitant depression of its conductivity and excitability. The result of this depression of excitability will be to render inefficient a stimulus which was formerly efficient, to evoke the true excitatory reaction of galvanometric negativity. The absorbed stimulus will now induce only a responsive positivity. The depression of conductivity also would cause the transmission of the hydro-positive, instead of the excitatory negative, wave. Owing, then, to the joint action of these two factors, stimulus induces a positive response-the so-called 'abnormal' - at a distant responding point, when the tonic condition of the tissue has become depressed. Absorption of stimulus, however, by supplying the requisite energy, raises the tonic condition, with consequent restoration of conductivity and excitability. As 
a result of this, the abnormal positive will pass into normal negative response, through an intermediate diphasic, after the impact of a series of stimuli, or after tetanisation. The enhancement of conductivity and excitability thus conferred on the tissue by the absorbed stimulus will now act by still further tetanisation, to bring about the enhancement of the normal negative response. Starting thus, with the most depressed condition of the tissue, and subjecting it to continuous action of stimulus, we obtain four typical stages: (I) the abnormal, passing after short tetanisation into (2) the diphasic; this in its turn giving place to (3) the normal negative alone; which finally becomes (4) the enhanced negative.

In studying electrical response, both of animal and vegetal nerves, under appropriate experimental conditions, we have already seen various examples of these different types of response and their transformations. But under such modes of experiment as have been described, the effects were, as already stated, due to joint changes in excitability and conductivity. I shall now, however, describe a still simpler experimental arrangement, in which the stimulus is applied directly on the tissue, and the responsive variations are, therefore, due to variations in the excitability alone. These changes, moreover, will be recorded by means of their direct mechanical expression, namely, contraction, or its opposite expansion.

With regard to abnormal response, I have already stated that this is brought about, not by 'staleness,' or the moribund condition, with its concomitant chemical changes, but by the run-down of the energy of the tissue in isolation. On investigating this subject, by means of mechanical response, with its superior sensitiveness, this conclusion finds independent support of the strongest character. On taking even the freshest specimen, I generally find that its responses at first are the abnormal positive. These gradually pass into moderate negative through diphasic. This is due to the raising of the tonic condition by the absorption of 
the stimulus, and after a series of stimulations, the isolated tissue, which was originally depressed, has its tonic condition so much heightened, that the responses are enhanced to an unprecedented magnitude. A specimen, in fact, which was at first almost irresponsive, may generally be brought to any state of exalted excitability desired, with concomitant increase in amplitude of response, by merely subjecting it for a certain length of time to the action of impinging stimulus.

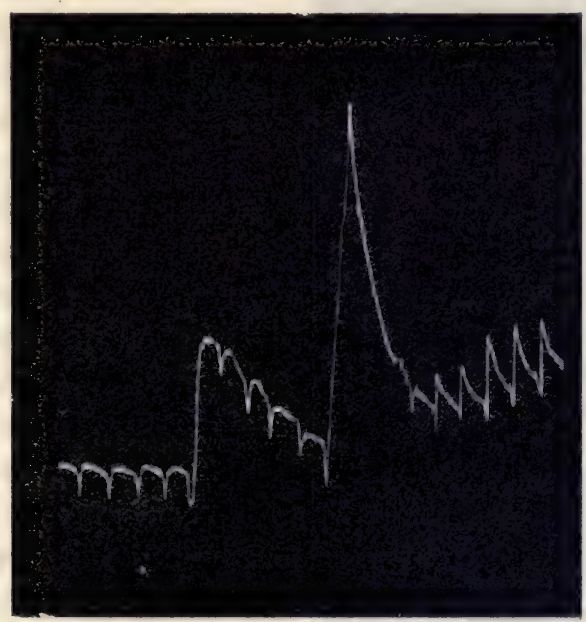

FIG. 320. Photographic Record showing Abnormal Positive converted into Negative Response after Tetanisation

First series, abnormal positive ; second series, persistence of this positive after very brief tetanisation; third series, conversion to negative, after a tetanisation of longer duration.

I shall now describe in detail some of the principal experiments. Selecting a specimen of frog's nerve, I took a series of responses to electrical shocks, of three seconds' duration, at intervals, in each case, of one minute. The testing stimulus was kept always the same throughout the experiment, except for certain intervening periods of tetanisation. The variations seen in the responses thus give a visual demonstration of the variations in excitability. The record of these is given in fig. 320. The responses in 
the first series are by the abnormal positive variation, that is to say, by expansion. The tissue here being sub-tonic, the impinging stimulus could not induce the true excitatory effect. The tissue was now subjected to short-lived tetanisation. But the absorbed stimulus was not yet sufficient to induce the normal responsiveness.

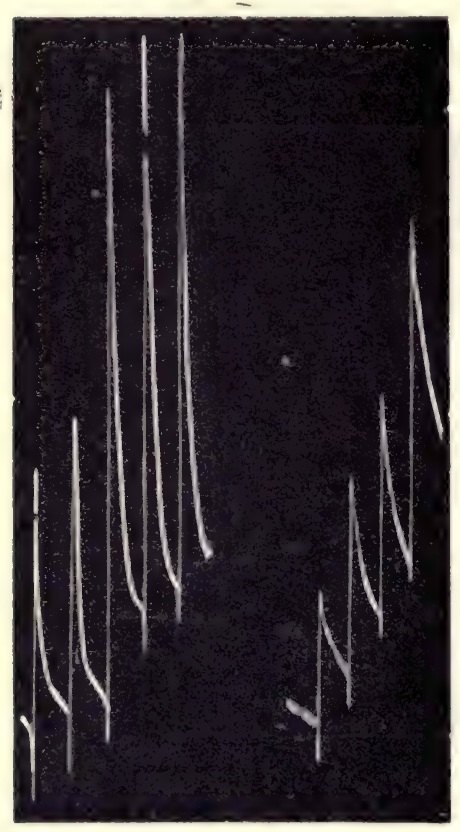

FIG. 321. Photographic Records showing Gradual Disappearance of Positive Element in Diphasic Mechanical Responses of Frog's Nerve and Plant-nerve

Note also the staircase increase.
The next series of records, therefore, still exhibited the abnormal positive response. Tetanic shocks of longer duration were next applied. This gave rise to a shortlived positive twitch downwards, succeeded by large contractile response upwards. After the cessation of the second tetanisation, the absorbed energy is seen to have brought the tissue to a condition of more or less normal responsiveness. This is seen in the third series, where the first responses are diphasic, but the positive component (the downward twitch) becomes perceptibly smaller and the negative larger, in each of the succeeding responses. It should also be noticed that the recovery from positive is much quicker than from negative response. This fact is important, in connection with certain psycho-physiological phenomena to be described in a later chapter.

The effect of successive stimuli, in enhancing normal response, when the nerve is not yet in maximum tonic condition is illustrated in a still more striking manner in the record given in fig. $32 \mathrm{I}$, obtained with a different specimen 
of frog's nerve. In this, also, the first series of responses was purely positive. But the record shown here begins at the point where, in consequence of previous tetanisation, response has become diphasic. Here it will be noticed that the true excitatory effect of contraction is undergoing a continuous increase, while the abnormal positive is decreasing. The excitatory response, indeed, becomes so great as to be incapable of record within the plate.

I have already shown how similar in every respec are the responsive characteristics of the vegetal nerve to those of the animal. This fact finds an interesting illustration in the various phases of its mechanical response. That is to say, plant nerve in a sub-tonic condition gives positive, passing into diphasic and normal negative response, under tetanisation. On arriving at this second stage of diphasic response, successive responses undergo enhancement in a manner precisely the same as holds good in the corresponding cases with frog's nerve. This is sufficiently illustrated in the two records given side by side in fig. $32 \mathrm{I}$, the first of which, as already said, is of frog's nerve, and the second, of nerve of fern. We see here again, as already in numerous cases before, how the responsive peculiarities and their modifications in the one are in every respect paralleled by those of the other. The only difference between them lies in the degree of their excitability, that is to say, two stimuli of equal intensity will in general induce a more intense excitatory effect in the nerve of frog than in that of fern; or in order to obtain from both an equal intensity of response, we must, in the case of fern, employ a stronger stimulus. We have seen that in consequence of the absorption of stimulus, not only does the abnormal positive phase disappear, giving place to the normal negative, but the subsequent negative responses themselves also show an enhancement in a staircase manner. I give here (fig. 322) another record showing the mechanical response of frog's nerve to undergo this staircase enhancement. From this effect then it is easy to understand that an 
intervening period of tetanisation will markedly enhance the negative response.

We have now seen that, by the direct mode of investigation afforded in mechanical response, we are able to trace out the causes which determine the three types of response found in nerves. It has been shown that all these are brought about by the varying tonic condition of the tissue. From this it is easy to understand that the three types of electromotive responses in nerve are also due to the same

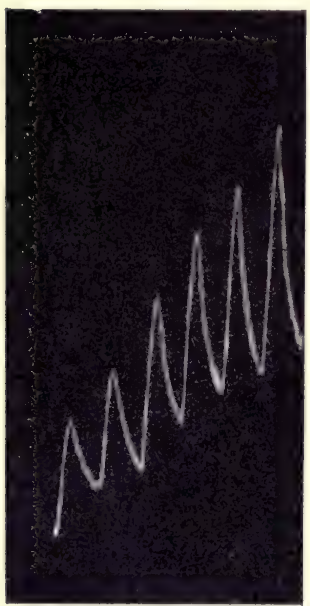

FIG. 322. Photographic Record showing Staircase Effect in $\mathrm{Me}$ chanical Response of Frog's Nerve

cause. In the experimental method there employed, the variations of conductivity appropriate to the tonic condition are superposed on parallel modifications of the excitability. Thus not only is the responsive change of a sub-tonic responding point positive, but the effect which is transmitted to it through sub-tonic conducting tissues is also pasitive; after tetanisation, however, the tonic condition of the tissue is raised. The power of transmitting true excitation, previously in abeyance, is now not only restored, but gradually enhanced to a degree, depending within limits, on the amount of tetanisation. The excitability also undergoes a similar transformation, from the abnormal positive to normal negative, which latter again becomes enhanced to a degree that depends, within limits, on previous excitation. These effects, seen in electrical response to transmitted stimulation applied at a distance, I find repeated also in the mechanical response of nerve, under similar circumstances. That is to say, an isolated nerve, by the very fact of its being cut off from its normal sources of energy in the body of the intact animal, is apt to be rendered sub-tonic, and under these conditions no true excitation is transmitted, and it is only when the tonic 
condition of the tissue has been raised, by the application of fresh energising stimulus, that the conducting power can be gradually restored.

This leads me to what is theoretically a very interesting mode of determining the velocity of transmission in nerve, by the mechanical response of the nerve itself, which will be understood from the diagram already given (fig. 3I5). In that figure, A B C is the nerve, so clamped at B as to prevent any mechanical slip, but not tightly enough to obstruct the transmission of excitation. The nerve, when brought to a normal excitatory condition, is first excited at A $a$ by a pair of electrodes in connection with an induction coil. The transmitted excitation, reaching $\mathrm{B}$ C, induces a contractile mechanical response there, observed by the highly magnifying optic lever. Records of the transmitted effect of stimulus obtained in this manner will be given later in the chapter. The interval of time, $t$, between the application of stimulus and the initiation of response is accurately determined by the usual methods. Stimulus is next applied at $\mathrm{B} b$, and the interval of time $t^{\prime}$ between stimulus and response again determined. The difference $\left(t-t^{\prime}\right)$ is the time required for the stimulus to travel the intervening distance $a b$. By this means, I found the velocity of transmission in a certain specimen of nerve of fern to be $50 \mathrm{~mm}$. per second.

It is thus easy, by means of two successive experiments, to eliminate from the observation the element of the latent period. It is to be understood that the molecular change, ultimately to be expressed as contraction, begins to be initiated as soon as excitation reaches the responding area. As the contractile effect exhibited by the nerve is relatively small, we can see that a certain time will elapse before it becomes sufficient to be perceptible, unless the magnification employed is very high. With a magnification of the order of 100,000 times, however-which, as I shall show, is quite practicable-this loss of time is much lessened.

It was while working out this investigation that I realised how indefinite must be any determination of the velocity of 
transmission in an isolated nerve. The conductivity, even in the intact organism, we have seen to be liable to modification from various factors such as fatigue, and it is easy to understand that it will become still more fluctuating when the conducting tissue is isolated. The inevitable changes consequent on separation from the natural sources of energy at once begin to take place. As the result of this sub-tonicity, even a typically conducting tissue, like nerve, will cease to be the conductor of true excitation, and there will then be, properly speaking, no physiological distinction between such a structure and a non-conducting tissue. By the absorption of stimulus, however, a transformation sets in, and the nonconducting becomes gradually reconverted, first, into a feebly, and then into a very highly conducting structure. The possible variations in conductivity, therefore, are not a matter of some few units per cent. quantitatively, but even considered qualitatively range from non-conductivity to the highest conductivity. And even, further, when the nerve has been once more rendered conducting, its velocity of transmission will vary greatly with the tonic condition conferred by previous stimulation. Over-stimulation, again, by inducing fatigue, diminishes the power of conduction of true excitation. This fact I shall be able to demonstrate by special experiments.

That such changes are not peculiar to the isolated nerve, where the manifestation can be traced unmistakably to its true cause, is seen in those cases of living animals where, owing to mal-nutrition, or for other reasons, the tonic condition of the nerve falls below par, with growing nonconductivity and paralysis as the effect. And here it may be said that the transformation again from non-conducting or feebly-conducting to the normal state of conductivity may in general be brought about by the same means as are employed with the isolated nerve, namely, by the frequent repetition of tetanising electric shocks.

The photographic method of recording the response of nerve, employed in the Kunchangraph, has the advantage 
that, as the record is made by the moving spot of light, the recording-point, as it were, encounters no friction, and the characteristic form of the response curve is thus unmodified. But prolonged work in the photographic dark-room is very fatiguing to the observer. I was, therefore, desirous of so perfecting the ordinary mode of record by the movement of the tracing-point of a lever over a smoked surface, that it would be adequate for most purposes. The difficulties involved in carrying this out lie, first, in the obtaining of a sufficiently high magnification, and, second, in the overcoming of friction at the writing point. A long lever, such as is necessary for high magnification, entails a heavy weight. But this can be obviated by employing a light and thin aluminium wire, $50 \mathrm{~cm}$. in length. The fulcrum-rod, to which the lever-index is attached, has a diameter of $2 \mathrm{~mm}$. A thread attached to the contracting nerve is wound once round this fulcrum-rod. The radius of the latter being I $\mathrm{mm}$., the magnification produced by this arrangement is 500 times. The magnification may in this manner be raised as high as $\mathrm{I}, 000$, by taking a longer lever. For the tracing point the end of the lever is bent at right angles, and a fine bristle attached. Even this degree of magnification is not always necessary, as I have already said. The records which immediately follow have a magnification of only fifty times.

The next difficulty, as already stated, lies in the friction to be overcome. The friction offered by a writing-surface of smoked paper is too great to be employed. A surface of plate-glass, coated with a thin and uniform layer of smoke, offers considerably less resistance. But even this retards the free movement of the tracing-point. I was therefore led to the construction of my Oscillating Recorder. The glass plate, on which the record is made, is carried on a primary frame, which is moved at a uniform rate, regulated by clockwork, on wheels, over rails. The plate is mounted on this primary frame in a secondary frame, which is held away from the primary, at a certain fixed distance, by means of spiral springs. This secondary frame, by means of an electro- 
magnetic arrangement, can be maintained in a state of toand-fro oscillation, always strictly parallel to the primary. The recording-index moves in a vertical plane, and the smoked plate backwards and forwards, at right angles to this, the extent of its oscillation being about I mm. The recording point is adjusted, barely to touch the smoked surface. Thus the oscillation of the plate brings it periodically in contact with the tracing-point, which is thus practically free to execute its movements unimpeded. When the oscillation frequency of the plate is sufficiently high, and the speed of the recording-surface low, the curve of record

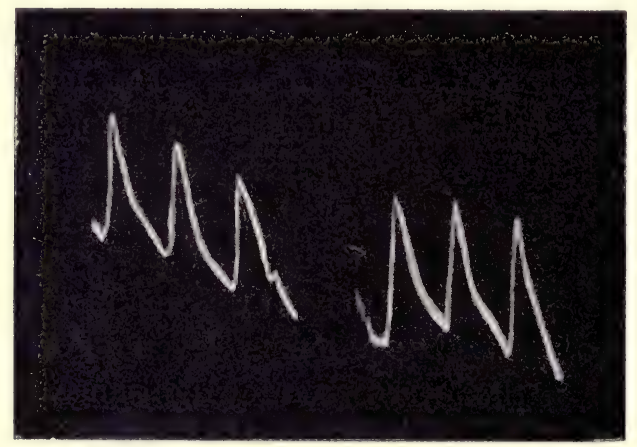

FIG. 323. Pho ographic Reproduction of Record of Mechanical Responses of Frog's Nerve (left-hand record) and Plant-nerve (right-hand record) obtained on Smoked Glass Surface of Oscillating Recorder

appears as continuous. In other experiments, where the determination of time-relations is important, a high speed can be given to the plate by the regulation of the clockwork, and the record will then appear as a succession of dots. From these, and a knowledge of the oscillation-frequency of the plate, the time-relations of different parts of the curve can be determined with accuracy. I give here two different series of uniform mechanical responses recorded with this instrument, obtained from the nerves of frog and of fern respectively (fig. 323 ).

I have also been able, by means of this instrument, to demonstrate a very important fact, namely, that the responses 
of the afferent or sensory nerves are in every way the same as those of the efferent, or motor. The numerous records already given are of the latter. For the demonstration of the former I took the optical nerve of Ophiocephalus, and recorded its responses to uniform electrical stimuli, on a smoked surface. The following (fig. 324) is a photographic reproduction of the record. Owing to sub-tonicity, the first response is seen here to be abnormal positive. Successive stinulation converts this, through diphasic, into normal negative, in a manner exactly the same as has already been observed in the sciatic nerve of frog. Another interesting record obtained with the optical nerve is given later (fig. 404).

$I$ also give in the next figure (fig. 325) a series of effects of transmitted stimulation, which show in a very interesting manner the effect of fatigue in the modification of the conductivity of a nerve. It is customary to suppose that the nerve is indefatigable. But I shall be able to show that not only is the conductivity of a nerve liable to fatigue, but its excitability also. The demonstration of the latter will be given

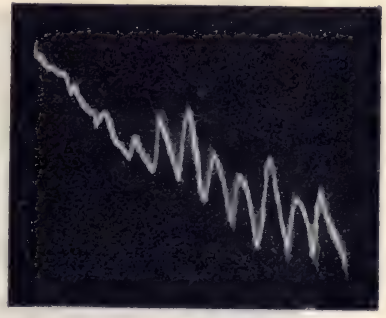

FIG. 324. Record of Mechanical Responses to Electrical Stimulus obtained on Smoked Glass, and given by the Optic Nerve of Fish Ophiocephalus

Note the abnormal positive responses passing through diphasic into normal negative.

in a succeeding chapter. For the demonstration of the effect of fatigue on conductivity I selected a length of IO $\mathrm{cm}$. from the sciatic nerve of gecko. This was attached for experiment to the Kunchangraph, in the manner diagrammatically represented in fig. 315. The length BC, which showed contraction, in response to stimulus transmitted from A, measured $5 \mathrm{~cm}$. The two exciting electrodes, A $a$, were $2 \mathrm{~cm}$. apart. The intervening tract, through which excitation was transmitted, was, therefore, $3 \mathrm{~cm}$. At the beginning of the experiment, owing to the depression of tone which the nerve had undergone, from 
isolation, its conductivity was below par, and the responses obtained were positive. After a series of stimulations, however, the true excitatory wave was transmitted, with the concomitant negative or contractile responses. In order to demonstrate the effect of fatigue on conductivity, the recording of this series was commenced only after many normal responses had already been given. In the series recorded we can see that the responses at first exhibit periodic fatigue. The accentuation of fatigue is then manifested by a rapid decline in the amplitude of the responses. A remarkable change next begins to appear. It has been

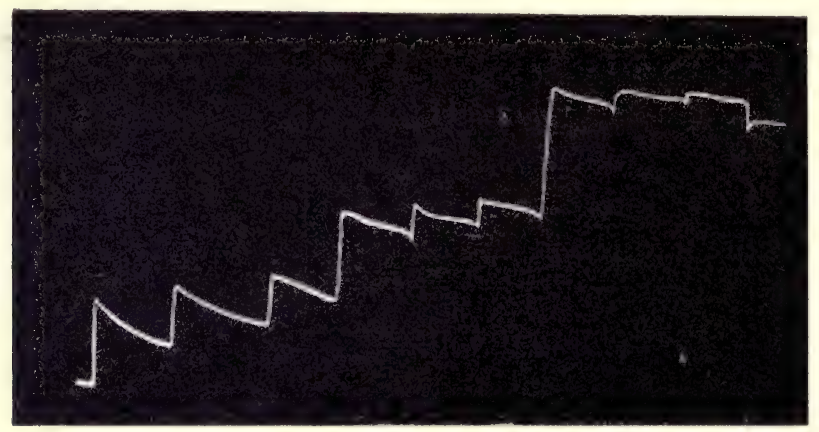

FIG. 325. Record, obtained on Smoked Glass, or Transmitted Effect of Stimulation on Nerve of Gecko

Note here the progressive effect of fatigue, seen first as periodic fatigue ; second as diphasic effect ; and third as reversal into abnormal positive.

shown that the true excitatory negative response contains a masked positive element. Owing now to growing fatigue, the exhibition of the negative is delayed, and the positive thus shows itself as a preliminary down-curve in a diphasic response. Afterwards, the excitatory negative is completely abolished, and the positive response by expansion alone remains, as seen in the last of the series. Ultimately, when the nerve is killed, by excessive stimulation, even the positive response disappears.

We may notice here the interesting fact that nerve, which is regarded as a conductor, par excellence, will sometimes 
become a non-conductor. Conduction, therefore, is not alone dependent on anatomical structure, but requires also a certain molecular condition. A nerve, whose continuity remains uninterrupted, may nevertheless undergo paralysis and cease to conduct. Recovery may then, in many instances, be brought about by tetanisation.

Thus, by means of mechanical response, obtained with a magnification of only fifty times, we have been able to demonstrate not only those results which may be observed by the most sensitive galvanometer, but also others which were never so detected. The magnification thus employed in the Kunchangraph, however, is here, as already stated, only in its lowest terms. When this is further exalted, still further and important phenomena regarding the excitatory changes in nerve are revealed, and some of these will be described in the next chapter. 


\section{CHAPTER XXXVI}

\section{MULTIPLE RESPONSE OF NERVE}

Great sensitiveness of the high magnification Kunchangraph-Individual contractile twitches shown in tetanisation of nerve-Sudden enhancement of mechanical response of nerve on cessation of tetanisation-Secondary excitation-Multiple mechanical excitation of nerve by single strong stimulation Multiple mechanical excitation of nerve by drying.

WE have already seen that, in order to detect the excitatory changes in nerve by the electrical method, the moderate sensitiveness of an ordinary galvanometer has to be exalted more than a million times. Galvanometric indications, moreover, are liable, as we have seen, to be complicated by the occurrence of differential effects at the two contacts. In the Kunchangraphic method of record, however, there is no possibility of such complications, for the response curve here represents the direct effect of stimulus. We also saw that, according to this method, a very moderate magnification would give us all the variations that could be detected by the most sensitive galvanometer, and, besides this, owing to its simplicity, it makes it possible to observe other phenomena, whose occurrence the galvanometer could not satisfactorily have demonstrated.

Such a magnification, however, as I have already said, is in its first stage only. With due precautions it is possible to obtain a Kunchangraphic magnification of a hundred thousand times. It will easily be seen that this places at our disposal an instrument of incomparable sensibility, by whose aid many of the phenomena of the nervous change, hitherto beyond our power of observation, may be brought within the sphere of investigation. 
This magnification, of the order of a hundred thousand times, may be accomplished in either of two different ways. A magnified image of the end of the long lever may, in the first place, be thrown on a distant screen. By this compound magnification the sensitiveness of the record may be raised to the extent desired. Or, in the second place, we may employ a battery of two levers in series. The first of these gives a magnification, say, of five hundred times, and is connected with a second optical lever, by which a multiplying magnification of two hundred times is easily obtained. It is unnecessary to point out that special care should in this second case be taken to ensure the steadiness of the support. With due precautions it is, however, not difficult to secure the entire elimination of all disturbing elements.

When the spot of light from the second lever is thus thrown on a distant screen, it is very interesting to watch the various changes induced in the nerve by the environmental conditions. An isolated nerve in a moist chamber, cut off from its natural sources of energy, becomes increasingly sub-tonic. This process is attended by an abnormal relaxation, which causes a steady movement of the spot of light in one direction. When the nerve has become very subtonic, the effect of stimulus, as that of electric shocks, is to enhance the tonic condition, and by this the downward drift of the spot of light is retarded or arrested. In cases of extreme sub-tonicity there is no further response, beyond this arrest. But where the sub-tonicity is less pronounced, stimulus will induce the abnormal positive response ${ }^{1}$ by a sudden positive variation of the drift, which is followed by recovery in the opposite direction. The after-effect of absorption of stimulus is further effective in causing the gradual retardation and final arrest of the downward drift. By such absorption of stimulus the tonic

1 The abnormal positive response is also obtained from 'nerve in ordinary tonic condition, it should be remembered, by the application of excessively feeble stimulus. 
condition is raised, and the normal excitability consequently enhanced. From this point onwards the responses are contractile or normal negative. At this stage the response of the nerve exhibits the staircase increase, the nerve itself showing a certain amount of tonic contraction. The excitability of the nerve then attains a maximum, and the successive responses become uniform. Long and intense stimulation will, after this, bring on fatigue. This stage is characterised, again, by a growing relaxation of the nerve as a whole, and its responses may become, first, diminished in amplitude, second, of a diphasic character, and, thirdly, reversed to the abnormal positive, according to the amount of fatigue which supervenes. We may thus, for the sake of convenience, distinguish four stages in the response of nerve. The first of these is the initial phase, SUB-TONIC RELAXATION, and the characteristic response to individual stimuli is here abnormal positive. The second phase is that of the TRANSITION to normal response. The characteristic responses to individual stimuli here show a staircase increase, with more or less permanent contraction as its after-effect. If at this stage the nerve is allowed to remain long without stimulation, it slowly reverts to the first stage of sub-tonic relaxation, with its growing relaxation and abnormal positive response. Stimulation, however, brings it back once more to the second or transition stage. In the third stage of UNIFORM responsiveness, the responses are normal and take place by equal contraction. In the fourth, or FATIGUE stage, there is a tendency, as already said, to relaxation on the part of the nerve as a whole, and it thus outwardly mimics the stage of sub-tonicity. The responses now, therefore, diminish in amplitude, and show the diphasic or the abnormal positive character. Further characteristics of these four stages, and their relations to each other, will be treated in detail in Chapter XLI.

A few words may be said about the mechanical response of the nerve, when it is in a favourable condition of excitability. 
We have seen that in order to obtain a galvanometric record of the electrical response of nerve, one or even a few shocks will not be sufficient to induce the necessary electromotive change. For this, tetanic shocks of a certain duration are necessary, and the responsive electromotive change is not immediately perceptible. In consequence of this intensity of stimulation, moreover, complete electrical recovery can only take place after a perceptible interval. With the low magnification Kunchangraph, too, tetanic shocks of something like a second in duration are necessary, and complete recovery here also requires a period of about one minute. But with the greater sensitiveness available in the highly magnifying apparatus, response with a highly excitable specimen of nerve is obtained with even so short-lived a stimulation as that of two or three vibrations of the vibrating interrupter of the secondary coil, lasting less than one-tenth of a second. The responsive contraction of this short-lived stimulus, and its recovery, are also quick. It is in consequence of the rapidity of this response and recovery that the responsive contractions due to the rapidly intermittent tetanising shocks do not become fused, but show themselves in the response-curve as consisting of successive twitches, corresponding to the component shocks. Owing to the high amplitude of these responses, and the trend of the base-line either up or down, it is difficult in practice to obtain photographic records of these effects. But it is easy enough to obtain definite visual demonstration of various characteristic effects in the response by the employment of the following device. The spot of light from the optic lever is made incident on a revolving mirror, and reflected from it to a large white screen at some distance. During a period of repose the quiescent spot traces a more or less horizontal line of light. This may trend either in a downward or an upward direction continuously, according as there is induced a continuous sub-tonic relaxation or a growing contraction, due to the after-effect of stimulus. Somewhere between these two extremes may be obtained a condition of more or less 
stability, where the record made by the spot of light appears as a horizontal line. Under normal conditions, then, the response to excitation is a sudden movement, due to contraction, say upwards, followed by recovery down. In the response-curve projected on the screen, the vertical movement or ordinate represents the amplitude of the response, and the horizontal abscissa the time. Under tetanisation a series of curves corresponding in frequency to the frequency of the shocks is observed as serrations.

Another very interesting observation often made in the mechanical response of nerve is that of the after-effect on the cessation of continuous stimulation by tetanic shocks. It has been found, it will be remembered (p. 428), that the response of the retina to the action of continuous light often shows on its cessation a sudden transient increase. This phenomenon has been regarded as peculiar to the retina. But I have found exactly parallel effects to occur in the mechanical response of nerve. Under continuous stimulation there is a tendency to the attainment of a maximum contraction, which suddenly, on the cessation of stimulation, overshoots, to be followed by the usual recovery. I have already referred to the two different effects of an opposite character caused by incident stimulus, namely, the effect of negativity, and its converse positivity. In the case of mechanical response, it is the former which is effective in inducing contraction, while the latter is associated with expansion, and is a factor in recovery. It will also be seen, in a general way, that by the antagonistic action of these two elements, and by the differing relative intensities of their after-effects, many diverse results may be exhibited. In the case of the after-effect in question, which occurs by a sudden positive variation of the contraction, the excitatory effect would appear to be predominant. Even when, after this brief positive variation, recovery is taking place, the excitatory element, with its contractile tendency, appears to persist; for if a second stimulus be applied, some time before the recovery is complete, the consequent contractile response takes place almost 
instantaneously. But when the recovery is once complete, a similar stimulation will not induce a similar immediate response. Instead of this there will be a brief period of hesitation or latency before its initiation.

Another interesting phenomenon, which I was first able to observe by the help of the highly magnifying Kunchangraph, was the occurrence of multiple response in nerve under intense stimulation. I was led to this discovery by an investigation which I had undertaken for the demonstration of the identity of response in animal and vegetable nerves. After showing the extended parallelism which exists between the two, under similar conditions and variations of conditions, as already described, I was desirous of seeing whether a plant nerve could be substituted in certain experiments for the animal nerve. In accordance with this I used the vegetal nerve in the experiment known as secondary contraction. Here a nerve-and-muscle preparation of frog is taken, and a second piece of frog's nerve is suitably laid, with one end lying upon the end of the other nerve. On excitation of this second detached nerve, say by electric shocks, excitatory electrical variation is found to cause responsive contraction in the muscle of the first preparation. In my own rendering of this experiment I employed, instead of the second piece of frog's nerve, a length of nerve of fern. In order that the experiment should not be open to any objection arising from the escape of stimulating current, I employed a non-electrical form of stimulus. This was done by touching the plant nerve with a strongly heated wire. The terminal muscle would then, under favourable conditions, begin to respond by strong spasmodic contraction. When this had subsided, a new series of tetanic contractions began; and this was repeated at short intervals, for nearly half an hour. When this series of spasms had come to a stop, I have often succeeded by a fresh application of the hot wire to the vegetal nerve in obtaining a second series of such repeated responses. It thus appeared that the strongly excited plant nerve gave rise to a series of multiple 
excitations, the indications of which were afforded by the nerve-and-muscle preparation.

The only perplexing feature of these responses was the abnormally long period of ten to fifteen seconds which was generally found to elapse between the application of the strong stimulus to the plant nerve, and the response subsequently given by the terminal muscle. Here it must be remembered that the excitation applied at one end of the plant nerve has to travel the entire length before its excitatory electrical variation can be communicated to the nerve of the frog-preparation. The transmitted excitatory variation in the primary has, moreover, to reach a certain intensity before it can effectively excite the secondary preparation. We know, further, that an isolated piece of nerve is liable to fall into a sub-tonic or depressed condition, in which its conducting power is much lowered, to be gradually restored again under strong or long-continued stimulation. These considerations will probably be found to account for the delay in the occurrence of the first of these responses. It would thus appear from the last experiment that a nerve, when subjected to a single strong stimulus, will give a multiple series of responses. In order to test this by direct experiment I employed the highly magnifying Kunchangraph, and subjected an experimental nerve of frog to a single strong thermal stimulation. This gave rise, at first, either to an abnormal positive response or to a moderate negative. But there followed, after a longer or shorter pause, a series of multiple contractile responses, which generally grew in intensity for a considerable time. There were in the series a number of short pauses, each followed by a veritable storm of excitation, in which individual responses were so rapid that the up or down movement of the spot of light appeared as brief flashes, in which all distinctness was obliterated. This experiment conclusively shows that the nerve, like certain other tissues, is susceptible of multiple excitation.

If the nerve in a nerve-and-muscle preparation be allowed to dry, the muscle is seen to be thrown into a series 
of spasmodic contractions. This also we may regard as caused by multiple excitations induced in the nerve. And the correctness of this supposition I have been able to verify by experiment. As the individual responses in these multiple series were of fairly large amplitude, I expected to be able to obtain a record of such a series by an ordinary magnification on smoked glass. In order to obtain this record under normal conditions a stream of air, bubbling through water, was passed through the chamber at a uniform rate. Owing to the rundown of the latent energy in the nerve, we are able to observe a consequent growing relaxation. By the manipulation of a stop-cock the air is passed through a calcium chloride tube, instead of a vessel containing water. In this way the nerve is quickly subjected to dry air instead of moist vapour. This substitution is represented in the record by an upward arrow $\uparrow$, and it will be noticed how at this point

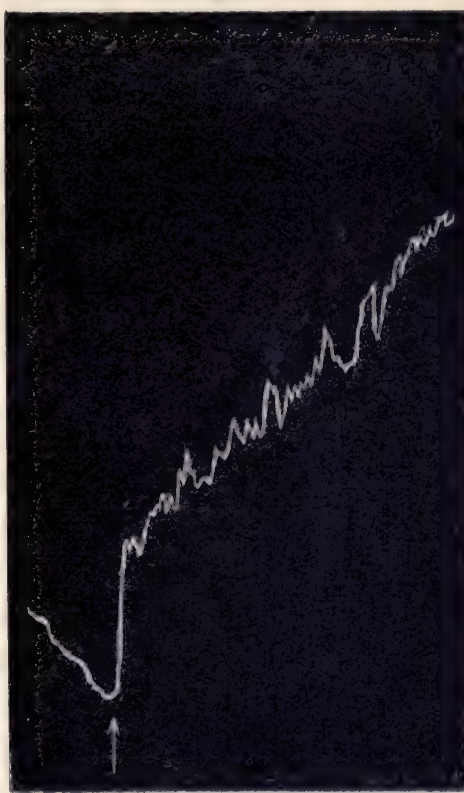

FIG. 326. Initiation of Multiple Response by Drying of Nerve

The nerve, owing to growing subtonicity, was showing a growing relaxation, as seen in the first part of the record. Air passed through $\mathrm{CaCl}_{2}$ tube, and, thus dried, was now passed through nerve-chamber at point marked with upward arrow $\uparrow$. This gave rise to a large contractile response, followed by subsequent multiple responses. Original record on smoked glass here reduced photographically to $\frac{1}{3}$.

the relaxation is suddenly converted into excitatory contraction (fig. 326). Under this process of drying, this single contractile movement is followed by a long-continued series of multiple responses, here seen to fall into a somewhat irregular periodicity. 


\section{CHAPTER XXXVII}

\section{RESPONSE BY VARIATION OF ELECTRICAL RESISTIVITY}

Variation of resistance in Dionce, by 'modification'-Excitatory change, its various independent expressions - Characteristic difficulties of investigationMorographic record by variation of resistivity - Inversion of curves at deathpoint-Similarities between mechanical, electro-motive and resistivity curves of death-The true excitatory effect attended by diminution of resistance - Response of plant nerve by resistivity variation-Independence of resistivity and mechanical variations-Responsive resistivity variation in frog's nerve, and its modification under anæsthetics.

IT was noticed by Burdon Sanderson that leaves of Dionce after 'modification' exhibited a diminished electrical resistance; and this 'modification' he found to be most easily induced after the passage of an electrical current through the tissue. Subsequent observers have also noticed a diminution of resistance in many cases when a tissue has been subjected to electric shocks. These diminutions of resistance are observed as more or less permanent after-effects. The experiments in these cases depend on obtaining the galvanometer deflections caused by a small E.M.F., before and after the modification. The larger deflection due to the same E.M.F. after modification shows that the resistance of the tissue has undergone a diminution.

This method, however, is open to several objections. The passage of constant or induction currents through the tissue would not only give rise to polarisation effects, but would also induce an unknown electromotive variation at the two contacts on the surfaces of the tissue, to an extent depending on their differential excitability. The observed deflection by a small testing E.M.F. is thus affected, not 
only by the variation of resistance, but also by varying polarisation and excitatory electromotive effects.

The question still remains, What is the nature and significance of this induced variation of resistance? As the effects which have been referred to are generally seen to be induced after excitation, and to constitute its after-effect, does it follow that the diminution of resistance takes place as a remote consequence of other excitatory changes? Or is it but a different manifestation of that fundamental molecular change, induced by excitation, of which the electromotive variation and change of form are other and independent expressions?

I showed in the first chapter of this book that one identical molecular change may be detected in different ways, according to the method of observation. Thus the same excitatory change is shown both in change of form and in electromotive variation. That either manifestation takes place in entire independence of the other is shown, for instance, when the mechanical response of Mimosa or Desmodium is restrained, under which condition the electromotive response proceeds as before. Excitatory changes, similarly, may express themselves independently either by electromotive variations or by changes of electric resistance. It was, in fact, by means of the latter method, that of the variation of resistance, that I first demonstrated the responsive molecular changes which take place in inorganic matter. ${ }^{1}$

If living tissues, therefore, really respond to excitation in a manner similar to the inorganic, it should be possible to obtain from them response-records by a new method, that of Resistivity Variation alone. In order to demonstrate this inference, it will be necessary to show that such variation of resistance takes place immediately on excitation, and not as an after-effect. We must, however, ascertain whether this method of Resistivity Variation does or does

1 Bose, De la Généralité des Phénomines Moléculaires produits par l'Electricité sur la matière Inorganique et sur la Matière Vivante. (Travaux du Congrès International de Physique. Paris, 1900.) 
not give us those two opposed effects, positive and negative, which we have already seen to be exhibited by living tissues in other forms of response whether mechanical or electromotive. Of these, again, supposing them to occur, it will also be necessary to determine whether it is the increase or decrease of resistance which corresponds to the negative and positive mechanical and electromotive responses respectively ; and finally it must be determined what are the effects of the various physiological modifications, induced by different agencies, on the response by resistivity variation.

In this investigation many serious experimental difficulties have first to be overcome. These will be dealt with in series in the detailed description of the method to be employed. It will be well, however, to see in what important respects the conditions for the obtaining of response here are unlike those of the electromotive variation. In the latter case, if we employ an isotropic tissue, diffuse stimulation will induce similar excitatory electromotive variations in every part of the tissue. The differential electromotive variation, therefore, on which the recording of response depends, will, under these circumstances, be impossible. In this case, therefore, it is necessary to injure or kill the tissue at one of the two contacts. Such artificial induction of anisotropy would not, however, be necessary under an experimental method which was not dependent on any differential action. Thus an isotropic tissue would give response by longitudinal contraction when the stimulus was diffuse. Similarly, though an isotropic tissue fails to give an electromotive response under diffuse stimulation, yet we may expect it to exhibit response by variation of resistance. The recording of excitatory response by resistivity variation has thus one advantage over that of the electromotive variation, inasmuch as the record is not affected by complications due to differential action, but is the expression of the direct effect of excitation. The question which we have next to determine, then, is whether or not the excitatory variation of the living tissue is attended by any variation of its resistance, 
and whether, if so, such variation is or is not of two opposite signs, according to the tonic condition of the tissue concerned. In subjecting this question to experimental investigation, it is well to employ a non-electrical form of stimulation, in order to avoid any possible disturbance of the galvanometer record from polarisation or current escape.

The first point to be decided is the character, positive or negative, of that resistivity variation by which the true excitatory change finds expression. We have already seen that when a tissue is subjected to a gradually rising temperature it exhibits response, which is expressed mechanically as increasing expansion, and electrically as increasing positivity. When the temperature, however, has reached the definite critical point of death, we have seen that there is a sudden excitatory effect induced, attended by a reversal of the sign of response. This is expressed mechanically by a sudden contraction, and electrically by a change to galvanometric negativity. I have already explained in Chapter XVI. that, in mechanical and electrical morographic curves, the abrupt point of inversion represents the death-point. I have also shown that this death-response is a true physiological response; that the temperature at which it takes place is definite in all phanerogamous plants, being at, or very near, $60^{\circ} \mathrm{C}$. in normal specimens ; and that it displays depression, by transposition to a lower temperature, when the tissue is physiologically depressed by such influences as fatigue. ${ }^{1}$

From these facts we might expect, if a tissue showed response by variation of resistivity, that up to $60^{\circ} \mathrm{C}$., or so, there would be a continuous one-directioned change of resistance, succeeded on reaching $60^{\circ} \mathrm{C}$. by an abrupt reversal to the opposite-directioned change. In that case, it would be the second of the two, which would be indicative of true excitation. To carry out this experiment I took a radial and physiologically isotropic pistil of Hibiscus, and mounted it on two non-polarisable electrodes. The specimen

1 Bose, Plant Response, p. 177. 
was now made the fourth arm of a Wheatstone's bridge (fig. 327), by which electrical resistance is usually determined. The plant specimens employed generally possessed high resistance, of the order of several hundreds of thousands of ohms. In the Wheatstone's arrangement employed by me, $P$ and $Q$ represented the ratio-arms; $R$ a standard megohm,

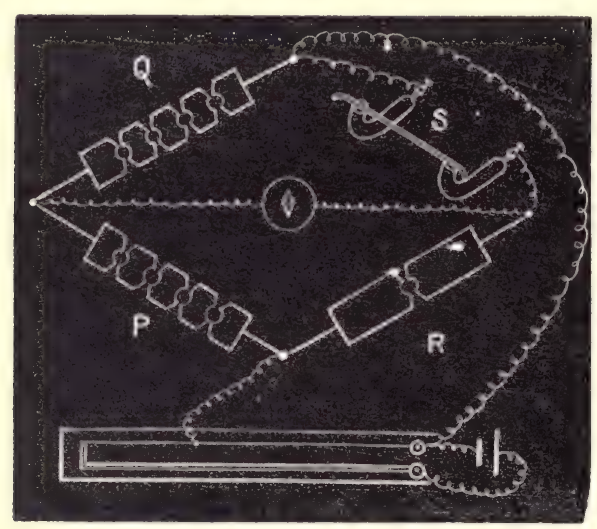

FIG. 327. Diagrammatic Representation of Experimental Arrangement for Recording Response by Resistivity Variation

$\mathrm{P} Q$, ratio arms of Wheatstone's bridge; $\mathrm{R}$, standard I or 5 megohm;

S, specimen.

or half-megohm; and $\mathrm{s}$ the specimen whose variations of resistance were to be determined. It is now evident that when the bridge is balanced, $S=\frac{Q}{P} R$.

The ratio-arms, $\mathrm{P}$ and $\mathrm{Q}$, consist of resistance-boxes, which allowed a variation of from I to IO,000 ohms. In order to obtain balance, of course, the ratio of the two had to be suitably adjusted. A highly sensitive galvanometer was used, and the electromotive force employed to obtain balance was only 05 volt. This low E.M.F. was obtained by the use of a suitable potentiometer slide. It will be seen that, owing to the very low E.M.F. and the high resistance in the circuit, the current flowing through the specimen was rendered extremely feeble. This was done in order to avoid 
any complication such as might result from the passage of a strong current.

In order to subject the specimen to a gradual and continuous rise of temperature, it was placed in the thermal chamber, which has already been described (fig. I3I). Before the gradual raising of the temperature is initiated, an exact balance is first obtained, the galvanometer spot of light being thus adjusted to zero. This position can be maintained for an indefinite length of time, provided the specimen is subjected to no variation of temperature. We have already seen that no resultant electro-motive variation is induced, in consequence of stimulus, in a physiologically isotropic tissue. Any change now recorded under a gradual rise of temperature, by the movement of the galvanometer spot of light, must, therefore, be due to a resulting variation of resistance. The movement of the galvanometer spot of light is recorded in the usual manner, on a photographic plate, a down-record representing an increase of resistance, and an up-record a diminution. In order that the curve should also give indications of different temperatures, light is cut off for a short time at every $2^{\circ} \mathrm{C}$. of rise of temperature. Thus each of the successive gaps in the record indicates a temperature-ascent of $2^{\circ} \mathrm{C}$.

Taking now the specimen of pistil of Hibiscus, balanced as described, it was seen, on beginning gradually to raise the temperature, that the balance was upset, while the growing deflection of the galvanometer spot indicated an increasing resistance. The method of experiment, which has been described, proved now so delicate that it was impossible to record the entire curve within the range of the photographic plate. It was, therefore, necessary to choose for record only that part of the deflection which included the interesting and significant point of inversion. The photographic record thus commences at $56^{\circ} \mathrm{C}$., it being understood that there has been, before this, a larger and continuously growing deflection downwards, indicative of increasing resistance. During record the deflection continues to increase, till the 
critical point is reached. And here, though the temperature still goes on ascending at the same rate as before, we see a sudden reversal in the characteristic curve of resistivity, showing that the hitherto increasing has suddenly become a decreasing resistance. This abrupt inversion represents the

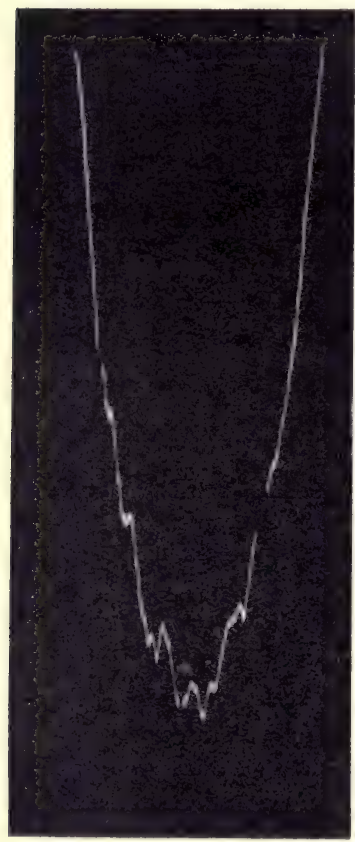

Fig. 328 .

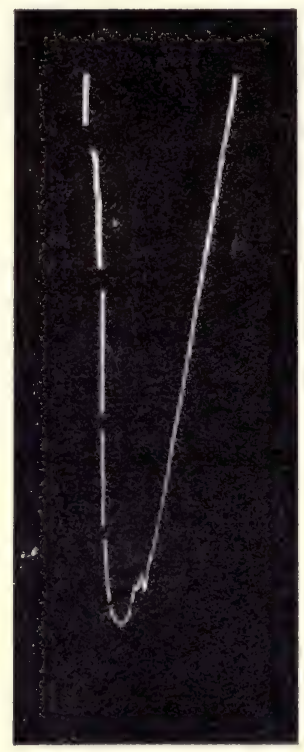

FIG. 329.

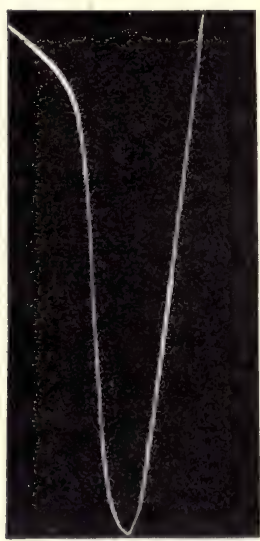

FIG. 330,

FIG. 328. Photographic Record of the Morographic Curve taken by Method of Resistivity Variation in Pistil of Hibiscus. Critical point of inversion at $60 \cdot 8^{\circ} \mathrm{C}$.

FIG. 329. Photographic Record of the Morographic Curve taken by Method of Electro-motive Variation in Petiole of Musa. Critical point of inversion at $59^{\circ} 6^{\circ} \mathrm{C}$.

FIG. 330. Photographic Record of the Morographic Curve taken by Method of Mechanical Response in Filament of Passiflora. Critical point of inversion at $59 \cdot 6^{\circ} \mathrm{C}$.

excitatory effect which occurs at the point of initiation of death, and is in the present case at $60 \cdot 8^{\circ} \mathrm{C}$. (fig. 328 ).

It is astonishing to find that the morographic curves obtained from different specimens, by three methods so different as the mechanical, the electro-motive, and that of 
resistivity, should bear so strong a resemblance to each other, as is here seen to be the case, in the three records given side by side (figs. $328,329,330$ ). The excitatory effect may thus be manifested by contraction, galvanometric negativity, or diminution of resistance. We have already seen that the electromotive is not a consequence of the mechanical response, but is exhibited independently, when physical movement is restrained. The response by resistivity variation likewise, is, as we shall see, an independent expression of the fundamental molecular change due to excitation.

Having thus established the fact that true excitatory response is exhibited by diminution of resistance, we have next to ascertain whether this method of resistivity-variation is capable of being employed in the study of excitatory phenomena in general, with as great facility as those mechanical and electro-motive method's with which we are already familiar. In order to determine this question I employed the same Wheatstone's bridge arrangement as before. As it was important, for reasons previously given, to use a non-electrical form of stimulus, I employed those thermal shocks which we have already found to be so reliable. The thermal loop of platinum wire enclosed the specimen as before, without being in contact with it. A short-lived passage of heating-current, controlled by a metronome, would now give rise to that sudden thermal variation which we have seen to be effective in causing stimulation It should be remembered that the rise of temperature, as such, induces a responsive increase of resistance. But as, on the other hand, the sudden thermal variation acts as a stimulus, it should induce the excitatory response, by a transient diminution of resistance. In the following experiments, I employed the physiologically isotropic nerve of fern. The resistance of this tissue, when balanced, was found to be 400,000 ohms. It should be stated here that this specimen was in a very good tonic condition, and might bè expected therefore to give normal response. It was now subjected to a series of stimuli of uniform intensity, at intervals of five 
minutes. The consequent responses are seen to be uniform, and to take place by that diminution of resistance which we already know to constitute the normal mode of response (fig. 33I). In order to obtain some idea of the magnitude of these resistance variations the balance was upset at the end of the response record, to the extent of 4,000 ohms. The deflection seen to the right of the figure represents the effect of this variation of resistance. The normal resistance of the tissue, including that of the non-polarisable electrodes was, as stated before, 400,000 ohms. The resistance of the electrodes themselves was 50,000 ohms. That of the tissue

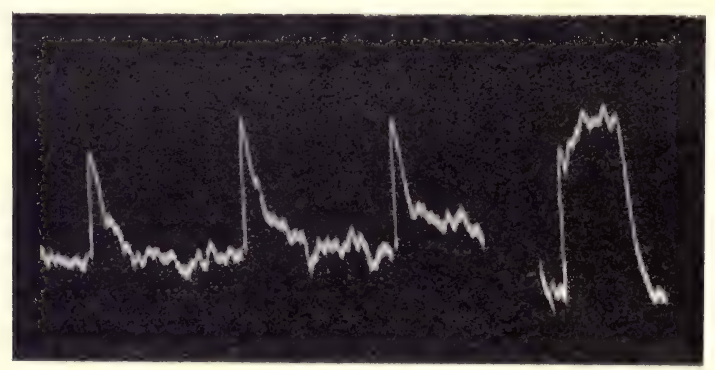

FIG. 33I. Response Records by Resistivity Variation, in the Nerve of Fern ; Stimuli at Intervals of Five Minutes

Kesponse to stimulation is by the negative variation or diminution of resistance. The record to the right shows deflection due to variation of resistance of $4,000 \mathrm{ohms}$.

alone was thus 350,000 ohms. The variation of resistance induced by stimulus, therefore, was, in the present case, approximately I per cent.

In order next to determine whether the resistance variation was a consequence of the responsive change of form, or an independent expression of the fundamental molecular change, I clamped a nerve of fern suitably, at its two ends, to prevent any possible change of length, the two non-polarisable electrodes being led off in such a way as to include a certain length of the specimen. On carrying out the experiment in this manner I obtained response by diminution of resistance, exactly as in the last 
case. It is thus seen that the response, by resistivity variation, is an independent expression of the excitatory variation.

In taking records of the responses of animal and vegetable nerves, by the methods of mechanical and electromotive variations, we saw that, while the normal response was negative, this was liable to become reversed to positive, under two different conditions-namely, sub-tonicity and the fatigue due to excessive stimulation. Similar reversals are observed under similar conditions, when the method of resistivity variation is employed. We saw, further, that the abnormal positive response, due to sub-tonicity, could be gradually converted into normal negative, through intermediate diphasic, by tetanisation - further tetanisation acting, moreover, to exalt this feeble into enhanced negative response. Parallel results are observed in the case of resistivity variation. The initial abnormal response, by increase of resistance, is found, after short tetanisation, to be converted into diphasic-an increase of resistance or positive response preceding the true excitatory or negative effect of diminution of resistance. Further tetanisation brought about the disappearance of this preliminary positive, and the enhancement of the negative, response.

I used the same method, finally, for the observation of response and its modifications, by means of anæsthetics in animal nerve. For this purpose I took a nerve of frog, and subjected it to chloroform. It will be remembered that in studying the effect of anæsthetics by the electro-motive variation method, we found it, first, to reverse the normal response to positive, and finally to induce an abolition, which might prove to be either temporary or permanent. The same thing is seen under the resistivity variation method. In the first series of records, given in fig. 332, we find normal responses by diminution of resistance, to a series of stimuli applied at intervals of two minutes. After the application of chloroform, the normal responses are seen to have disappeared. Stimulus now evokes 
either no response or an occasional flutter, in the positive direction.

We have thus seen, in the course of the present chapter, that, in addition to the mechanical and electro-motive modes of response, there is also a third mode available-namely, that by Resistivity Variation. We have also seen that the results obtained under these three methods are identical. It has been shown that the normal excitatory effect is in

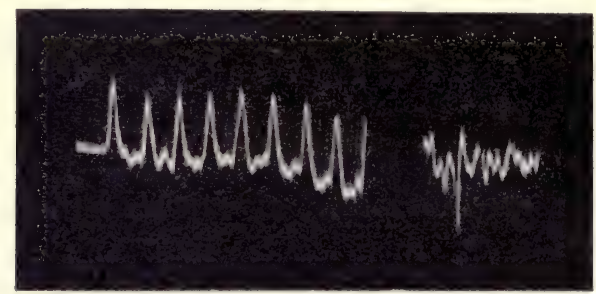

HIG. 332. Effect of Chloroform seen in Modification of Resistivity Variation in Frog's Nerve

The normal responses seen to the left by diminution or negative variation of resistance were evoked by stimuli at intervals of one minute. Those to the right exhibit the effect of chloroform. The normal response is thus abolished, and we have either no response or only an occasional flutter in the positive direction.

all three cases negative, consisting of mechanical contraction, galvanometric negativity, or diminution of resistance, as the case may be. In recording the morographic curve by these three methods, we find that up to the critical point of death, at or near $60^{\circ} \mathrm{C}$., we obtain expansion, galvanometric positivity, and increasing resistance. At that point, however, there is a sudden reversal of the curve, indicating conversion to negative, contraction, galvanometric negativity, and decrease of resistance. 


\title{
CHAPTER XXXVIII
}

\author{
FUNCTIONS OF VEGETAL NERVE
}

Feeble conducting power of cortical tissues-Heliotropic and geotropic effects dependent on response of cortical tissues only-Phenomenon of correlation - Excitability of tissue maintained in normal condition only under action of stimulus--Physiological activities of growth, ascent of sap, and motile sensibility, maintained by action of stimulus-Critical importance of energy of light-Leaf-venation a catchment-basin-Transmission of energy to remotest parts of plants-Plant thus a connected and organised entity.

In the animal body, different kinds of tissues are possessed of different degrees of conductivity, the nerve being specialised for the rapid transmission of stimulus. And it is now seen that in the plant also we have a similar state of things, cortical tissue, for example, though excitable, having feeble conductivity, whereas the vegetal nerve possesses this power in high degree. The question next arises: What is the function subserved in the economy of the plant by a tissue so highly specialised for the rapid conduction of stimulus? The various growth curvatures, by means of which plants place themselves under the directive action of light and gravity, are of advantage to the organism. But in bringing about these movements, the plant-nerve takes little or no part. And this is the case, even when the responsive curvature takes place at a certain distance from the point of stimulus. Here the transmission takes place slowly, through the feebly-conducting cortical tissues. For example, in Avena, curvature in consequence of such transmitted effect is observed, even when the fibro-vascular bundles have been cut across.

If, indeed, the highly conducting nervous elements had been concerned, these curvatures in response to unilateral 
stimulus could not well have taken place. This will be clear if we consider the case of a radial organ, such as the stem, unilaterally acted on by light. Here a positive heliotropic movement is induced, by which the growing organ is placed in the most favourable position as regards illumination. The peculiarity of this phenomenon lies in the responsive contraction of the side acted on by stimulus, with consequent concavity and curvature towards light. This heliotropic movement continues until the organ has placed itself in the direction of incident radiation. When this orientation has become perfect there is no further movement, because the proximal and distal sides are now equally stimulated. Had the cortical tissue, on whose differential responsive action the curvature depended, been as highly conducting as the vegetable nerve, this particular-directioned movement would have been an impossibility, for the stimulus, instead of remaining localised on one side, would in that case have become diffused, with the result of inducing antagonistic effects on the proximal and distal sides, under which there could have been no resultant curvature. Indeed this neutralising action of conduction, in nullifying responsive curvature, is seen even when unilateral stimulus is excessively strong. For under these circumstances stimulus is conducted transversely, through the imperfectly conducting tissue, with the result of undoing the previous curvature. And it is obvious that had the conductivity of the tissue been higher, this neutralisation would have taken place, even under feeble stimulation.

I have also shown elsewhere that, in the responsive movements of leaves, conduction through nervous elements plays little or no part. For the blade of the leaf may be acted on by light without showing any responsive movement. Hence the lamina is not to be regarded as the perceptive organ. The organ by which, on the contrary, the responsive movements of the leaves are determined is the pulvinus or pulvinoid. This is at once perceptive and motile. When such an organ, then, is acted on directly and locally by light, 
a responsive movement is induced. These are facts which can be demonstrated by shielding the lamina and pulvinus alternately from the action of light. When the lamina alone is exposed there is no action; but when it is the pulvinus to which light is admitted, there is an immediate responsive movement.

The lamina, it is true, is provided with a fine fibro-vascular network containing the nervous strands. But the stimuli received by this extensive system are ultimately conducted along the thicker channels of which it forms the terminal ramification, and serve to stimulate the plant as a whole. When such stimuli reach the petiole, then, they cannot act in that direct and unilateral manner in the motile organ which is required for the responsive movement of the leaf. The petiole, it is true, from its dorsiventral character, is unequally excitable on its two sides. But since, in the process of the transference of stimulus from the nervous to the ordinary elements there is a great loss, and since, moreover, the motile tissues in the case of most petioles are very sluggish, the diffusely transmitted stimulus induces practically no directive effect. Nor could there, in any case, have been any comparison between the effective strength of an external stimulus acting directly and unilaterally, and a transmitted stimulus acting diffusely.

It will thus be seen that conduction through specialised nervous elements is by no means the essential factor in bringing about those numerous directive curvatures which subserve so many important functions in the life of the plant. The question, therefore, as to what is the part in the economy of the organism played by the vegetable nerve still remains to be answered.

One very obscure problem in connection with Vegetable Physiology is that of Correlation. Thus various complex activities may be set up in one part of the plant, when another part, more or less distant from it, is subjected to the variation of some excitatory influence. In this way every part of the plant-organism would appear to be en rapport with 
the rest, and this intimate connection between outlying areas becomes comprehensible, when we are made aware of the easy communication afforded by the existence of specialised conducting elements.

I shall now proceed to deal with the importance of stimulus, and its conduction to the interior of the plant, as the essential factor in sub-

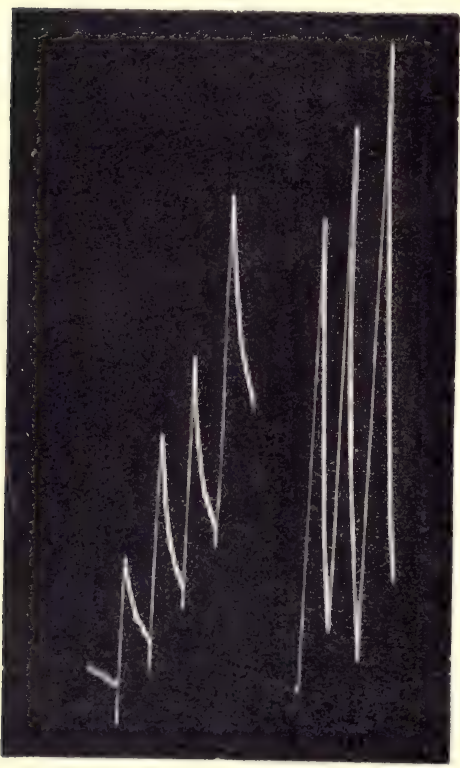

FIG. 333. Phutographic Record of Effect of Tetanisation in Enhancing Mechanical Response of P'lant-nerve

The first series of responses heightened to second series, after intermediate tetanisation. serving the various lifeactivities of the organism. That the reception of stimulus is important, in maintaining the excitability of a plant, is easily seen in the case of Mimosa, when deprived of light, for example. Under these conditions, its motile excitability is found to disappear. And this is only restored on re-exposure to light. We have seen again, in the course of the last chapter, that the isolated vegetable nerve, deprived, as it is, of normal favourable conditions, becomes sub-tonic or moribund, and then its ordinary responsive power is abolished or even reversed. Under these circumstances the normal excitability is found to be restored by the continued action of stimulus. Abnormal positive response is thus found to be converted into normal negative. Again, after an intervening period of stimulation, response of ordinary amplitude is found, as in fig. 333 , to become enhanced. It is thus seen that a tissue, when cut off from the supply of stimulus, loses its normal excitability, and that a more or less continuous supply of 
stimulus is essential to the maintenance of the proper excitatory condition of a tissue.

It is known that in the animal, when the conductivity of a nerve is abolished by nerve degeneration, the connected muscles also rapidly waste away. Thus the maintenance of the proper excitability of various tissues is dependent on their constant reception of stimulus or energy through the mediation of the attached nerve. It is therefore highly probable that the excitability of the indifferent vegetable tissues is kept at its normal level by the reception of energy of stimulus through the conducting nerve.

I shall next briefly refer to a fact which I have demonstrated fully elsewhere, that all the principal physiological activities of the plant, such as autonomous movement, ascent of sap, and growth are fundamentally excitatory phenomena. Thus, for example, the autonomous rhythmic movements of the lateral leaflets of Desmodium gyrans come to a standstill when their store of latent energy is exhausted. And it is only by the accession of fresh stimulus from outside that these multiple responses can be renewed. We describe the state of the plant, when its internal energy is below par, by saying that it is sub-tonic, the normal tonic condition, or health of the plant, being dependent on the sum total of stimulation previously absorbed by it. Turning next to the question of the ascent of sap, I have shown that the most important factor in bringing this about is the multiple rhythmic activity of certain interior tissues of the plant. Under such circumstances as cause the tonic condition of the tissues to fall below par, the rate of the ascent of sap will be lowered, or it may possibly even be brought to a standstill, owing to the depression of excitability induced. On now supplying fresh stimulus, we find the excitability to be renewed and the normal rate of ascent restored (p. 383). Another instance of this is seen in the pileus of Coprinus which droops when kept too long in the dark, but recovers its normal turgidity on exposure to light.

Growth, again, I have shown to be a result of multiple 
rhythmic excitation. When the tonic condition of the plant falls below par, growth is arrested, however large the amount of formative material present. But if stimulus be applied, while the plant is in this state of growth-standstill, there is a renewal of responsive growth.

The importance of the absorption of stimulus of light to the response of growth is seen in the development of certain seedlings. These, when grown in the dark, become diseased and perish. The growth and the development of organs cease, even though the cotyledons still contain considerable quantities of unused formative substance. They become moribund, as the expression of their loss of tonic condition, which, as we have seen, depends upon the supply of stimulus from outside. And in the present case the critical element is the stimulus of light.

This fact that the stimulus of light may enhance the excitability of a tissue, is shown in the following photographic records of the mechanical response of vegetal nerve. The first three responses show the extent of normal response to a given electrical stimulus applied in the dark. The specimen was then subjected to the light of an electrical arc lamp, and the next series show the consequent enhancement of response, under the same stimulus as before. Light was now cut off, and after an interval response once more taken. This is seen to have been of the same amplitude as at the beginning (fig. 334). Thus light is seen to enhance excitability.

In the cases which we have just been considering we have, for the sake of simplicity, confined our attention to a single factor-namely the photo-tonic-among the many which finally determine the general tonic condition of the plant. And we have found that when the organism is deprived of this source of stimulus, its motile activity, its suctional activity, and its response of growth, all disappear. When the plant, however, is restored to the direct action of light, all these various activities reappear. Now it is clear that this illumination cannot directly penetrate to many of those interior tissues, whose activity is nevertheless essential to the 
maintenance of life. How, then, is the external stimulus conveyed to these? It is evident that this can only be accomplished through the agency of the nervous elements.

This fact, of the transmission of the excitatory effect of an external stimulus from one part of the plant to another at a distance, there to maintain the tonic condition, is again still more clearly seen in the well-known experiment on the sensitiveness of Mimosa when partially kept in the dark. If one branch of this plant be covered by a dark box, while the rest of it is exposed to light, it is found that the leaves of the

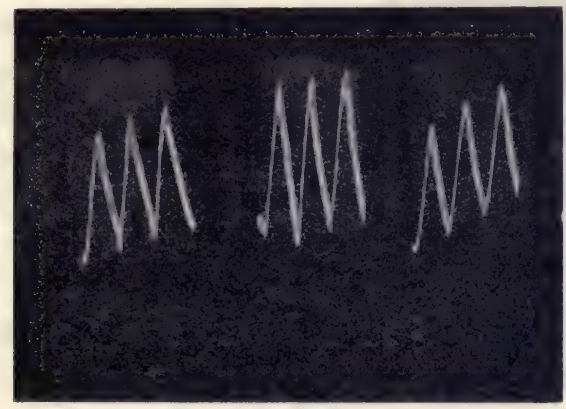

FIG. 334. Photographic Record showing Enhancement of Excitability under Action of Light in Nerve of Fern

First series, normal mechanical responses to electrical stimuli, in dark; second series, the same, taken under light; third series, taken after withdrawal of light.

first undergo no loss of motile sensitiveness. It is thus evident that the photo-tonic stimulus has been transmitted from the illuminated to the unilluminated portions of the plant, through conducting channels, in order to maintain the normal excitatory condition.

Next comes very interesting experiment of Sachs, in which a long shoot of Cucurbita was made to grow inside a dark box, the rest of the plant being exposed to light. The covered part of the plant, under these circumstances, showed normal growth of stem and leaves. Normal flowers and a large fruit were also produced in the same confinement. The tendrils inside the box, moreover, were found to be fully 
as sensitive as those outside. The transmission of stimulus by the plant, in such a way as effectively to maintain such complex life-activities as motility and growth, even in the absence of direct stimulation, is thus fully demonstrated, And we may gather an idea from this fact of the fundamental importance, to the life of the plant, of those nervous elements by which this is rendered possible.

One of the most important functions of the venation ot the leaf, not hitherto suspected, is now made clear to us.

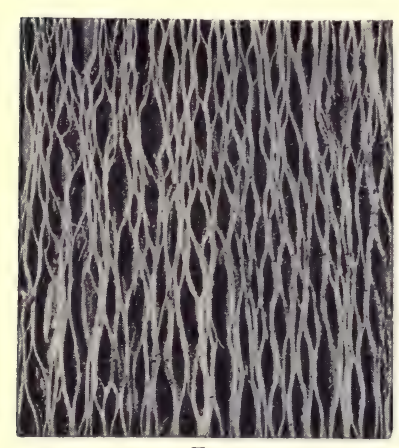

FIG. 335. Distribution of Fibrovascular Elements in Single Layer of Stem of Papaya

There are at least 20 such layers engirdling the stem. Among external stimuli, none perhaps is so essential, or so universally and easily available to green plants, as energy of light. And we now see that the fine ramification of fibrovascular elements over as wide an area as possible in the leaf, provides a virtual catchmentbasin for the reception of stimulus. The expanded lamina is thus not merely a specialised structure, for the purpose of photo-synthesis, but also a sensitive area for the absorption of stimulus, the effect of which is gathered into larger and larger nerve-trunks, in the course of its transmission downwards into the body of the plant.

And even in the interior of the plant the distribution of these is such that no mass of tissue is too remote to be excited by the stimulus conducted through the nervous elements buried in them. How reticulated they may be, even in the trunk, is seen in the accompanying photograph of the distribution of fibro-vascular elements in the main stem of Papaya (fig. 335). This network, of which only a small portion is seen in the photograph, girdles the stem through. out its whole length, and in this particular case there werc as many as twenty such layers, one within the other. 
It is thus seen how all parts of the plant are, by means of nerve-conduction, maintained in the most intimate communication with each other. It is, then, in virtue of the existence of such nerves, that the plant constitutes a single organised whole, each of whose parts is affected by every influence that falls upon any other. 


\title{
CHAPTER XXXIX
}

\author{
FLECTROTONUS
}

Extra-polar effects of electrotonic currents on vegetal nerve-Electrotonic variation of excitability-Bernstein's polarisation decrement-Hermann's polarisation increment-Investigation into the law of electrotonic variation of conductivity - Investigation on variation of excitability-Conductivity enhanced when excitation travels from places of lower to higher electric potential, and depressed in opposite direction-When feeble, anode enhances and kathode depresses excitability-All electrotonic phenomena reducible to combined action of these factors - Explanation of apparent anomalies.

WHEN an electrical current is led through a portion of a nerve-entering, say, at $\mathrm{A}$, and leaving by $\mathrm{K}$-it is found that electro-motive changes are induced by it in the extra-polar

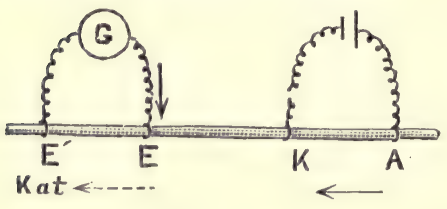

FIG. 336 .

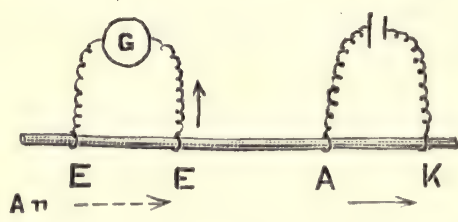

FIG. 337.

Extra-polar Kat- and An-electrotonic Effects

Fig. 336 shows kat.electronus, E near $\mathrm{K}$ being galvanometrically negative. Fig. 337 shows an-electronus, E near A being now galvanometrically positive.

regions. On the kathodic side, the electric potential near $K$ is found to be lowered in reference to a point further away. On the anodic side similarly, the electric potential of a point near $\mathrm{A}$ is found to be raised. These changes induced in the electric potential are indicated by the galvanometric negativity of the point near the kathode, and positivity of that near the anode (figs. 336, 337). In the tissue itself the current is assumed to flow in a direction contrary to that in 
the external galvanometric circuit, as indicated by the dotted arrow. ${ }^{1}$

In the medullated animal nerve, the electrotonic currents increase with the intensity of the polarising current. In the vegetal nerve, I have obtained exactly similar results. Taking a fresh and vigorous specimen, the polarising electrodes were placed at a distance of $2^{\circ} 5 \mathrm{~cm}$. from each other, the pair of extra-polar electrodes, where the electrotonic effects are observed, being separated from these by $2 \mathrm{~cm}$. and divided from each other by $2 \mathrm{~cm}$. also. The value of the acting polarising E.M.F. could be varied by the use of a

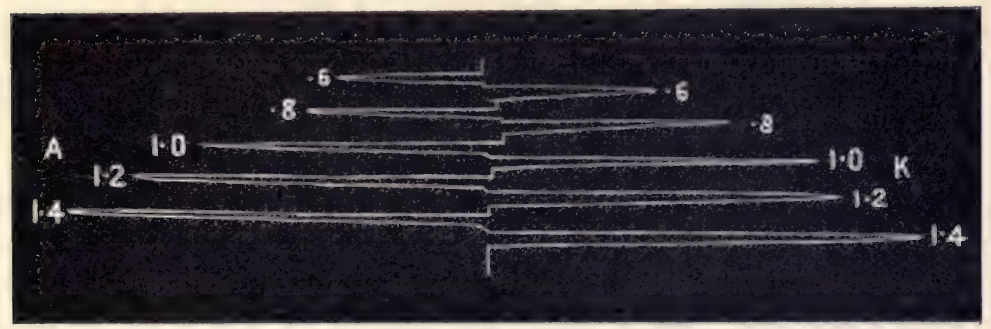

FIG. 338. Extra-polar Electrotonic Effects under an Acting E.M.F. which rises from $\cdot 6$ to $I \cdot 4$ Volts

A, an-electrotonic deflections seen to left ; $\mathrm{k}$, kat-electrotonic to right.

potentiometer arrangement. In order to induce in the extrapolar electrodes, an-electrotonic and kat-electrotonic effects alternately, the current in the polarising circuit can be sent in one direction or the other by means of a reversing-key. The record of the galvanometer deflection, in the extra-polar circuit, gives a measure of the electrotonic effect induced. In fig. 338 is seen such a record of effects both an-electrotonic and kat-electrotonic, taken while the acting E.M.F. was increased from 6 to 14 volt, by steps of 2 volt at a time.

1 Certain considerations, which need only be referred to here, cast some doubt on the validity of this assumption. But as it is so widely current in physiological literature, I shall confine myself, in dealing with the subject of the electrotonic current and its variations, to those indications in the external circuit which are afforded by the galvanometer. 
I give here a table which shows the galvanometric deflection corresponding to each particular E.M.F.

Table of Galvanometric Deflections and Corresponding E.M.F.

\begin{tabular}{|c|c|c|}
\hline Volts & An-electrotonic effect & Kat-electrotonic effect \\
\hline 6 & 50 divisions & $5^{8}$ divisions \\
\hline$\cdot 8$ & $65 \quad$, & $78 \quad$, \\
\hline$I^{\circ} 0$ & 107, & I IO \\
\hline$I \cdot 2$ & $126 \quad$, & I 24 \\
\hline$r \cdot 4$ & $150^{\circ}$ & I 48 \\
\hline
\end{tabular}

In this particular experiment, it will be seen that the an-electrotonic and kat-electrotonic effects are practically equal. But, to be more accurate, the an-electrotonic are slightly lower with low E.M.F., and slightly higher with high, than the corresponding kat-electrotonic deflections. A constant electrical current is thus seen to induce electromotive variations, outside its poles, in the vegetal nerve.

We next turn to the question of the variation of excitability induced in a tissue, by the passage of a constant current. On this subject the most important contributions have been made by Bernstein and Hermann. Bernstein, experimenting on the sciatic nerve of frog, found that excitation induced a polarisation decrement. This experiment is illustrated in the following diagram (figs. 339,

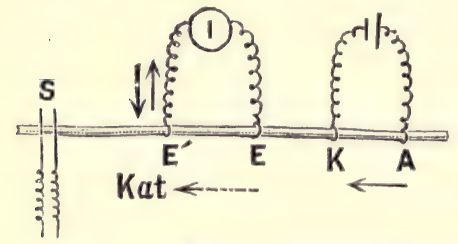

FIG. 339 .

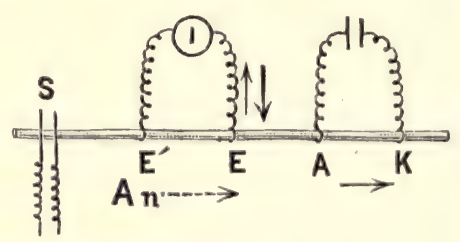

FIG. 340 .

Figs. 3.39, 340. Diagrams illustrating Bernstein's Electrotonic Decrement Fig. 339 shows decrement of kat-electrotonic, and fig. 340 of an-electrotonic currents, under stimulation at $\mathrm{S}$. In this and following figures the inside thin arrow indicates direction of polarising current, the outside thick arrow the direction of responsive current.

340), In fig. 339 the kathodal effect is seen induced in the extra-polar circuit. When the nerve is now excited by tetanising electric shocks, a diminution of the extra-polar current is induced. When the anodal effect is induced in 
the extra-polar circuit, by reversal of polarising current, the an-electrotonic current, opposed in direction to the former kat-electrotonic, also undergoes diminution on excitation of the nerve (fig. 340). It has been suggested that this diminution of electrotonic current was due to a supposed diminution, during excitation, of the susceptibility of the nerve to polarisation.

But this explanation is negatived by an experiment of Hermann, showing the occurrence of polarisation increment during excitation. In figs. $34 \mathrm{I}$ and 342 we have a polarising and exciting circuit in series, excitation being caused by the

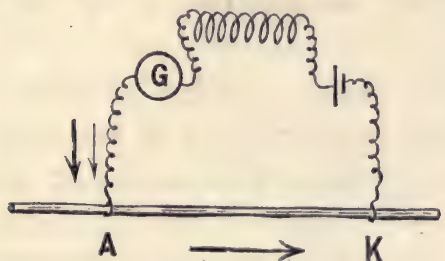

FIG. 34I.

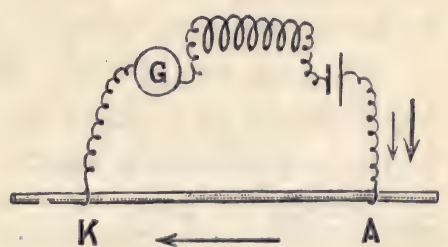

FiG. 342.

Figs. 34 I, 342. Diagrams representing Hermann's Polarisationincrement under Tetanising Shocks

Inside thin arrow indicates the direction of polarising current; the outside thick arrow, the direction of excitatory current.

secondary coil of an inductiorium. With such an arrangement, the polarising current, whether from left to right or from right to left, is found, during excitation, to undergo an augmentation. Hermann refers these facts to alterations of intensity in the negative wave of excitation, during its passage through the nerve, when the latter is polarised. ' It is, indeed, more pronounced at any point of the nerve, the more strongly positive and weakly negative the polarisation of the latter, i.e. it increases when it is becoming algebraically more positive, and diminishes when it advances upon more negative points' (Hermann's Law of the 'Polarisation Increment' of excitation). ${ }^{1}$

It would thus appear that the observations hitherto made, as to the effects of electrotonus on excitatory response, are of

1 Biedermann, Electro-physiology (Engl. transl.), vol. ii. p. 315. 
a somewhat discordant character. I shall, however, be able to show that their complexity is due to the combination of the different effects of the polarisation current on conductivity and on excitability. These separate effects may, according to circumstances, either conspire or act antagonistically. Hence the great variety of results, which appears at first sight incapable of a consistent explanation.

In order, then, to discover the laws by which an electric current induces a variation of conductivity and excitability, we must first determine the pure effect of the current on conductivity, apart from any excitatory variation; and, secondly, its effect on excitability, uncomplicated by any variation of conductivity.

To take conductivity first: the ideally perfect arrangement would be to have the polarising electrodes, in relation to the region whose conductivity-variation is to be tested, at a distance so great that they could exert no predominant an- or kat-electrotonic influence upon it. In a led-off circuit, moreover, a differential action, unless proper precautions are taken, is exerted on two electrodes placed side by side. It is, therefore, desirable to remove one of these outside the sphere of action. Such, then, being the conditions to be observed, in order to eliminate the effect of the poles themselves, and thus determine the influence of the direction of current on conductivity alone, I took a petiole of fern $20 \mathrm{~cm}$. in length, and connected its ends through a reversing-key with a Daniell cell (E.M.F. = I volt). The responding galvanometer-circuit had one electrode about the middle of the petiole, near the insertion of a certain lateral leaflet, the other electrode being connected with the lamina of the same leaflet, whose midrib, however, was cut across to prevent transmission of the excitatory effect (figs. 343, 344). It is thus seen that the led-off electrodes are at a relatively great distance from the polarising electrodes, and further, owing to one electrode being placed out of the way, on the lateral leaflet, and the other symmetrically between anode and kathode, it is clear that anodal or kathodal action is 
reduced to nil. The excitation from the stimulator is transmitted across the intervening conducting region, either along the slope of a falling electrical potential, that is to say, from a galvanometrically positive to a galvanometrically negative point, or against that direction, namely, in an electrically uphill manner, from the galvanometrically negative to the galvanometrically positive. Now, if the direction of an electrical current have an effect on the conduction of excitation, this fact will be detected by the modification induced in the normal response during the passage of the current:

The results of the present experiment will be found to determine this question. Excitation was induced by means

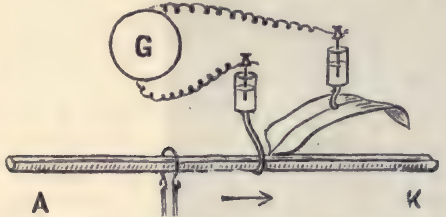

FIG. 343.

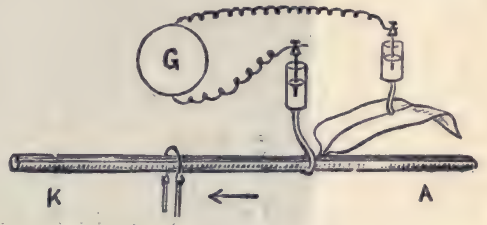

FIG. 344.

Figs. 343, 344. Experiment with Petiole of Fern demonstrating Variation of Conductivity by Polarising Current

of the thermal stimulator, and the normal responses taken, shown in fig. $345 a$, as 'up.' The polarising current was now sent from left to right; hence excitation will now be transmitted through the intervening conducting region in an electrically downhill manner, or in the direction of the falling potential - that is to say, from the region of the anode to that of the kathode. It will be seen presently that conduction is retarded or abolished when excitation is made to travel electrically downhill from the anode to the kathode. ${ }^{1}$ If this be so, we shall expect to detect the fact by the diminution of the amplitude of the normal response, or even by its actual reversal. For we have seen that when the excitatory reaction of galvanometric negativity is sufficiently, retarded, its opposite, the positive effect, often makes its appearance alone.

1 These remarks apply to a feeble or moderate rate of fall of potential. 
In fig. $345 b$, this is seen to have actually occurred. There will be other cases where, the depression of conductivity induced being not too great, it will be possible to watch the gradually lessening amplitude of response until it ends in actual reversal. We have next to determine the effect on conductivity, of the passage of excitation in an uphill direction-that is to say, from the kathodic to the anodic region. For this purpose the polarising current was reversed

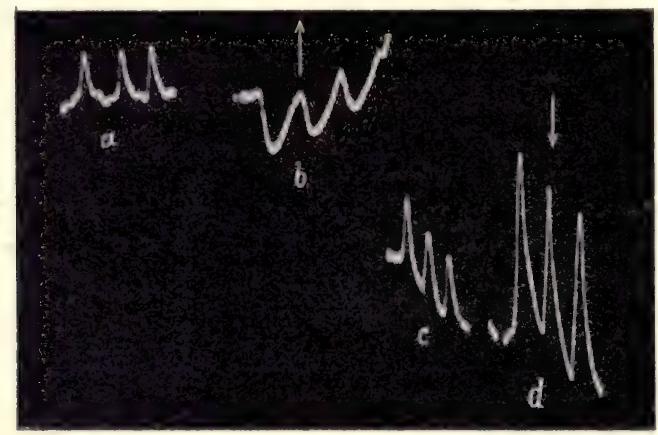

FIG. 345. Photographic Records of Responses taken in last Experiment, when Excitation was transmitted with and against the Polarising Current

$a$, Normal response of petiole of fern to transmitted excitation; $b$, Reversal of response when excitation was travelling electrically downhill or with the current; $c$, Normal response once more; $d$, Enhanced response due to increase of conductivity when excitation travels electrically uphill, or against the polarising current. Upward arrow $\uparrow$ indicates that polarising current is in same direction as normal response. Downward arrow $\downarrow$ shows polarising current in opposite direction. The same in the two following figures.

by means of a reversing key, the left-hand end of the petiole being now made the kathode. Before doing this, however, I stopped the current, and took a second set of records of normal responses. It will be seen that by reason of the cessation of the previously acting left-to-right current, leaving an after-effect, these were slightly enhanced above the first normal responses (fig. $345 \mathrm{c}$ ). On now reversing the current, conductivity was found to be enhanced, as seen in the greater amplitude of response (fig. $345 d$ ). 
I next undertook an investigation into the effect on conductivity, of variations of intensity in a moderate polarising current. This is shown in fig. 346 , in which excitation travels electrically downhill-that is to say, from the anodic to the kathodic region. In $a$ we have the normal response before the passage of the current. In $b$ we have the responses reduced by the diminution of conductivity consequent on the application of ' I volt for polarisation. On the application of 5 volt in $c$, there was a tendency towards

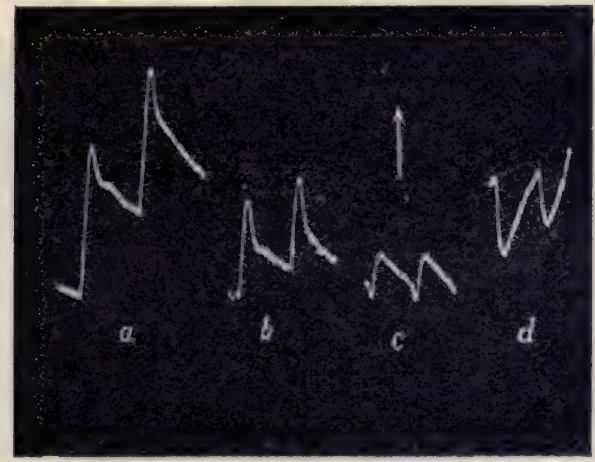

FIG. 346. Photographic Record of Modification of Conduction during Passage of Excitation from Anodic to Kathodic Region, under Increasing Intensity of Polarising E.M.F.

$a$, Normal response ; $b$, Diminished response where terminal E.M.F. was I volt; $c$, Response still further diminished and rendered diphasic under 5 volt ; $d$, Response reversed under I volt.

reversal, the response being now diphasic, positive followed by negative. Finally, on the application of I volt in $d$, we see the response reversed to positive, the conduction of the true excitatory effect being here altogether abolished.

In a second set of experiments, carried out on a fresh specimen, I investigated the effect of an increasing intensity of the polarising current, when the excitation was made to travel, electrically uphill, from the kathodic to the anodic region. It will be seen, from figure 347 , that the application of a polarising E.M.F. of 'I volt increased the conductivity, as seen in the heightened responses shown in $b$, as compared 
with the normal responses in $a$. The application of higher polarising E.M.F. of ' 5 , I, and $I^{\prime} 5$ volts respectively, now induced appropriate increments of conductivity, as seen in $c$, $d$, and $e$. I was unable to use an E.M.F. of higher than I.5 volts because the galvanometer spot of light became unsteady. It is to be borne in mind that the specimens in these experiments were $20 \mathrm{~cm}$. long, and the maximum potential gradient employed was only 07 volt per $\mathrm{cm}$. In experimenting on electrotonic effects, it must be remembered that the E.M.F. employed is, generally speaking, feeble.

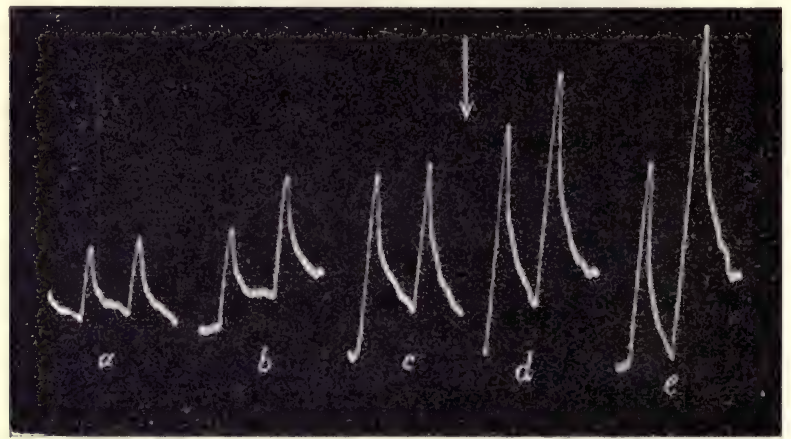

FIG. 347. Photographic Record showing. Enhanced Conduction from Kathodic to Anodic Region

$a$, Normal responses; $b, c, d$, e, Responses gradually enhancing under increasing polarising current.

From the results described, then, we arrive at the following law of the effect of a moderate or feeble constant electric current on conductivity.

A moderate polarising E.M.F. induces variations of conductivity. The conductivity is increased in the direction from the kathodic to the anodic region, and depressed in the opposite.

Having thus demonstrated the pure effect of a constant electric current on conductivity, we have next to study the unmixed effect of polarisation on excitability. We have seen that when two equally excitable points in the same circuit are simultaneously excited by an identical stimulus, there is no resultant response, since the two excitatory 
effects balance each other. The galvanometric effect is then zero. But if one of the two have its excitability enhanced in any way, this balance will be disturbed, and a resultant current will flow through the circuit, the more excitable contact becoming galvanometrically negative. I now took a long piece of isolated vegetal nerve and connected it with the galvanometer at $\mathrm{E}$ and $\mathrm{E}_{1}^{\prime}$, a secondary coil, giving equi-alternating electric shocks, being also in the circuit (fig. 348). The two longitudinal contacts $\mathrm{E}^{\prime}$ and $\mathrm{E}$ being more or less equally excitable, there was at first no resultant response to stimulation. $E^{\prime}$ was now made the anode, an E.M.F. of 'I volt being used for the purpose, and a permanent current was found to flow in the galvanometer in the direction of $E^{\prime} G E$ shown by the thin inner arrow, $\mathbf{E}^{\prime}$ being galvanometrically positive. On now applying equi-alternating shocks, the balance was found to have been disturbed, and the re-

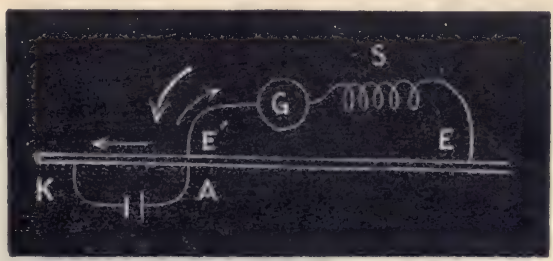

FIf. 348.

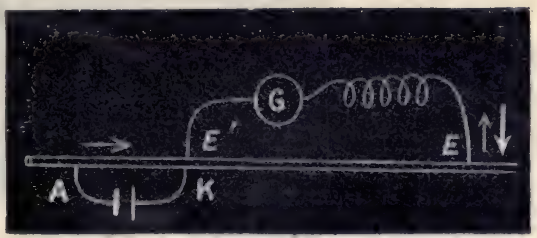

FIG. 349 .

Figs. 348, 349. Experimental Arrangement to Exhibit the Enhancement of Excitability at Anode, and its Depression at Kathode, when the Acting E.M.F. is feeble

Fig. 348 shows enhancement of excitability at anode; Fig. 349, the depression of excitability at kathode.

Inside thin arrow indicates direction of polarising current. Outside thick arrow, direction of excitatory current. Note that in both there is a so-called polarisation-decrement. sponsive current to be in the opposite direction, namely, $\mathrm{EGE}^{\prime}$, as shown by the thick arrow; $\mathrm{E}^{\prime}$ now undergoing an excitatory negative variation. This shows that the excitability of $\mathrm{E}^{\prime}$ has become enhanced by being made anode. $E^{\prime}$ was next made kathode, in consequence of which the permanent current in the galvanometer was now in the direction of $\mathrm{EGE}^{\prime}$ (fig. 349). $\quad \mathrm{E}^{\prime}$ is now the kathode, $\mathrm{E}$ 
in relation to it being anode. On excitation the responsive current was in the opposite direction to the permanent current, in consequence of the induced depression of the kathode $\mathrm{E}^{\prime}$ or the induced enhancement of excitability at the relative anode E. In fig. 350 are given the records of these effects. Two different experiments were carried out, on two different specimens of plant nerve, whose records are given in $a$ and $b$, fig. 350 . In each of these we observe, first,

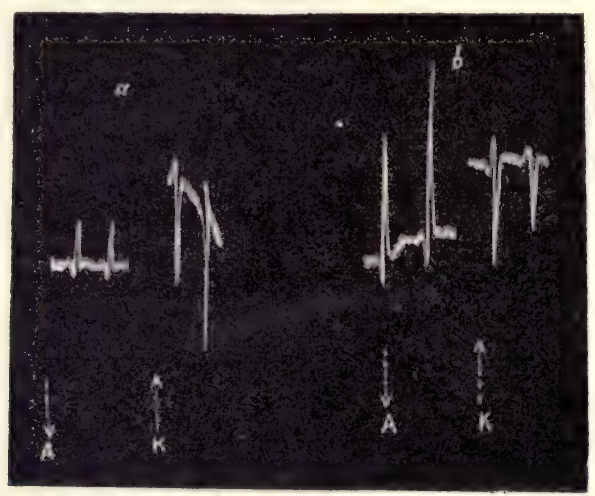

FIG. 350. Photographic Records of Response, illustrating the Enhancement of Excitability at Anode, and Depression at Kathode, under Feeble Acting E.M.F. in two Specimens of Nerve of Fern $a$ and $b$

Before application of polarising current there was no resultant response in either case. When $\mathrm{E}^{\prime}$ was made anode, there was an up-response, indicating enhanced excitability of that point. The dotted arrow seen below shows the direction of polarising current. The responsive current is then opposite in direction to the polarising current. When $\mathbf{E}^{\prime}$ is made kathode the resultant response is down, showing depression of the excitability of the point. The responsive current is here also opposite in direction to the polarising current.

the enhanced excitability due to $E^{\prime}$ being made anode, which gives rise to up-responses, opposite in direction to the permanent current, shown by the dotted arrow below. The second pair of responses in each case shows the depression of excitability at the kathodic point $\mathrm{E}^{\prime}$, which is tantamount to enhancement of excitability at the relative anode E. An inspection of figs. 348 and 349 will show that the responsive current is always in a direction opposite to that of the existing polarising current, thus constituting the so-called polarisation 
decrement. But we shall presently find that the direction of the responsive current is the only constant factor here, de. termined as this is by the relative excitabilities of the two electrodes. An identical variation of excitability may, as I shall show, appear under different circumstances, either as a polarisation-increment or as a decrement.

I have obtained results precisely like the foregoing, with the sciatic nerve of frog. It should be mentioned here that such effects are obtained without much difficulty in the first stages of polarisation. But, if this be prolonged, there is a certain liability to reversal.

From the experiments which have been described on variations of excitability by the polar action of currents, we arrive at the following law :

A feeble E.M.F. induces modifications in the excitability of a tissue: the anode enhances and kathode depresses excitability.

This result is startling, contravening, as it does, Pfliger's Law. A factor that had not been taken into account was the range of E.M.F., within which this law might be applicable. In the present case, the acting E.M.F. is relatively feeble, and we shall see later that Pflüger's Law does not apply above or below a certain medium range.

Having thus obtained the isolated effects of electric currents on conductivity and excitability respectively, I next took up those more complex cases in which both effects were present in various combinations. This problem was attacked by means of the Conductivity Balance. In this experiment, carried out on the petiole of fern, the led-off points $\mathrm{E}$ and $\mathrm{E}^{\prime}$ were at a distance of $6 \mathrm{~cm}$. from each other. The distance of each of the polarising electrodes $\mathrm{A}$ and $\mathrm{K}$ outside the led-off circuit $\mathrm{E}^{\prime}$ and $\mathrm{E}$ was $2 \mathrm{~cm}$. (figs. $35 \mathrm{I}$ and 352). The thermal stimulator $\mathrm{S}$ was so adjusted before the passage of the current that the excitations at $\mathrm{E}^{\prime}$ and $\mathrm{E}$ were exactly equal, as seen in the balanced horizontal record n fig. $353 a$. It should be mentioned here that the galvanometer connections were so arranged that an increased excitability of the left-hand contact $\mathrm{E}^{\prime}$, would be shown by 
means of 'down' and that of the right hand contact $\mathrm{E}$ by means of 'up' responses. An E.M.F. of '5 volt was now

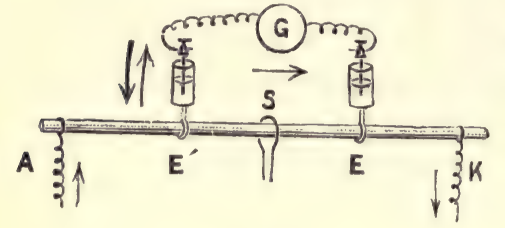

FIG. 35 I.

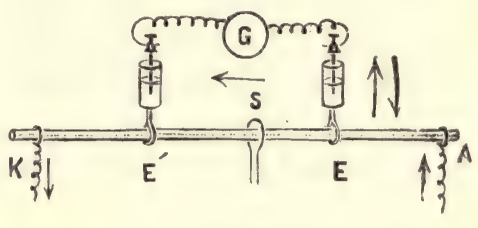

FIG. 352.

Figs. 351, 352. Experimental Arrangement demonstrating the Joint Effects of Variation of Conductivity and Excitability by Polarising Current

employed to induce polarisation, the anode being in the first case to the left. Here we have a greater excitability induced

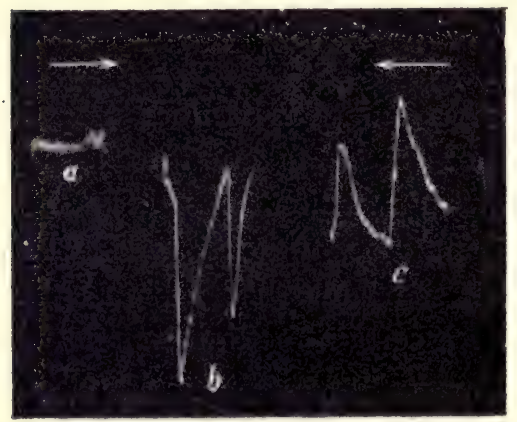

FIG. 353. Photographic Record of Response under the Arrangements given in Figs. 351, $35^{2}$ in Nerve of Fern

$a$, Balanced record before passage of polarising current.

$b$, Resultant response downwards when polarising current is from left to right, as shown by arrow $\rightarrow$. This shows excitability of $\mathrm{E}^{\prime}$ and conductivity in direction $\mathrm{SE}^{\prime}$ to be relatively enhanced.

$c$, Resultant response upwards when polarising current is from right to left $\leftarrow$. This shows excitability of $\mathrm{E}$ and conductivity in direction SE to be relatively enhanced. at $\mathrm{E}^{\prime}$ by the proximity of the anode, that of $\mathrm{E}$ being depressed by the proximity of kathode. Of the two waves of excitation, moreover, which proceed in opposite directions from the stimulator $\mathrm{S}$, that towards $\mathrm{E}^{\prime}$ is moving electrically uphill, or towards the anode, and owing to increased conductivity in that direction the excitation is better conducted than in the case of the second wave, which is proceeding electrically downhill towards the kathode at E. It must also be remembered that not only is the intensity of excitation which reaches $\mathrm{E}^{\prime}$ greater than that which reaches $\mathrm{E}$, but also that the point $\mathrm{E}^{\prime}$ is itself rendered more excitable by the 
contiguity of the anode, while $\mathrm{E}$ is depressed by that of the kathode. Hence, by the concordant action, at each end of the balance, of conductivity and excitability changes, and owing to the opposite nature of these changes at opposite ends, the original balance is disturbed, and we obtain resultant down responses, showing the greater excitation and galvanometric negativity caused at $\mathrm{E}^{\prime}$ than at $\mathrm{E}$, when anode is to the left and kathode to the right. This is exhibited in the first pair of down responses in figure $353 b$. When, however, the polarisation current is reversed (fig. 352), the excitation at the right-hand side, $\mathrm{E}$ being now near the anode, is relatively the greater, and we find the resultant responses to be upwards, as seen in the third record in fig. $353 \mathrm{c}$. To go back to the question of the relative directions of electrotonic and responsive currents, we find in that case, when the anode is to the left, that $\mathrm{E}^{\prime}$ is galvanometrically positive, while the excitatory change makes it galvanometrically negative. This means that the excitatory response takes place by the so-called polarisation decrement. When the anode again is to the right, the galvanometrically positive $\mathrm{E}$ tends by excitation to become galvanometrically negative. This will be clearly understood from the arrows which accompany the diagram, in figs. $35 \mathrm{I}, 352$. The inner and thin arrows represent the direction of the polarising current, and the thick outer arrows the responsive current. These results are tantamount to an example of the so-called polarisationdecrement. In order to show, however, that the same excitatory reaction might appear as a polarisation-increment, I shall describe another experiment.

The experimental tissue is here the isolated nerve of fern, and the method employed is again that of the Conductivity Balance. In this case, however, it will be noticed (figs. 354 , $355)$, that the galvanometer is included in series with the polarising E.M.F., instead of being placed as a shunt, as in the last case. The stimulator was first adjusted at balance. The left electrode was now made kathode, the right being anode, the E.M.F. employed being 22 volt (fig. 354). On account 
of the increased excitability of $\mathrm{A}$ at the anode, and also of the greater intensity of excitation conducted towards it, the balance was disturbed, and the resultant response took place by the enhanced galvanometric negativity of that point. The responsive current thus constituted an increment of the polarisation-current, as seen from the arrows; of which the thin inner represents the polarisation and the thick outer the responsive current. On now reversing the current again, the right-hand end being made anode and more excitable, the resultant response was found to take place by the enhanced negativity of that point, thus again constituting a polarisation increment (fig. 355). I give here two different

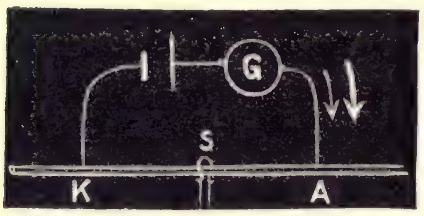

FIG. 354.

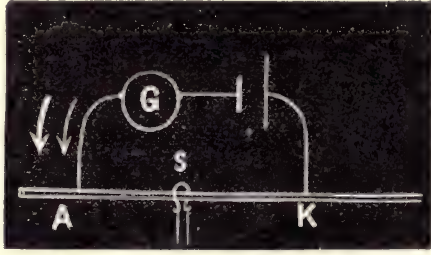

Ii IG. 355 .

Figs. 354, 355. Experimental Arrangements for Showing so-called Polarisation-increment by the Joint Effect of Increased Excitability at Anode and Enhanced Conduction of Excitation electrically Uphill

sets of photographic records, obtained with the nerves of fern and frog respectively. Balance was first obtained at the beginning of the record, but on the passage of the polarisation current, this balance was found to be disturbed. When the right end of the balance was made anode, the resultant response on excitation was up, demonstrating the enhanced excitability of the anodic point. When the righthand end, however, was made kathode, the balance was upset in the opposite direction, that is to say, down, showing that the left-hand anodic point was now the more excitable. Fig. 356 gives a record of these responses as obtained from the nerve of fern, and fig. 357 from the nerve of frog. The responsive currents in these cases, it should be noted, are in the same direction as the polarising current. 
On referring to the experiments on polarisation increment and decrement which have just been described it will be noticed that the excitatory reaction is the same in both cases, taking place by the enhanced galvanometric negativity of the more excitable anodic point. The seeming difference in the electrotonic variation in the two cases lies simply in the fact of the different dispositions of the galvanometer. This occupied, in the first case, the position of a shunt in the polarisation-circuit, while in the second it was placed in series.

From the investigations which have been described, we shall now find ourselves in a position to explain the various

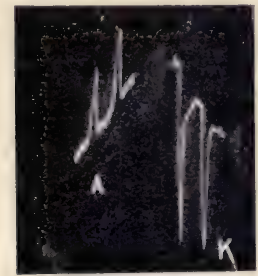

FIG. 356.

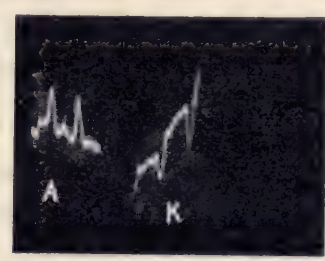

FIG. 357.

FIG. 356. Photographic Record of Responses in Nerve of Fern, under Anodic and Kathodic Action, as described in Figs. 354 and 355. The upsetting of the balance is upwards, when the right-hand end of the balance is made anode, proving the enhanced excitability thus induced. Resultant response downwards when the right-hand end of the balance is made kathode.

FIG. 357. Photographic Record of Similar Effects in Nerve of Frog.

experiments of Hermann and Bernstein, and to show that these, although apparently conflicting, are really mutually consistent. First, then, to take Hermann's experiment, and referring back to figs. $34 \mathrm{I}$ and 342 , in which the galvanometer is placed in series in the polarisation-circuit, we find this to be an instance in which we have to deal almost exclusively with the effect of anode and kathode on excitability. Simultaneous excitation of the anodic and kathodic points by alternating induction currents, induces greater excitation, and consequent enhanced negativity of $\mathrm{A}$. The responsive 
current, being thus concordant with the electrotonic current, causes an increase of it, the so-called polarisation-increment.

In Bernstein's experiment on polarisation-decrement, we are confronted with a question of greater complexity, for here we have to deal with changes of conductivity and excitability at the same time. We shall first take the case (fig. 339) in which one electrode of the led-off circuit $\mathrm{E}$ is under kat-electrotonus. $\mathrm{E}^{\prime}$ is therefore relatively anodal, and consequently more excitable. The excitation from the stimulator $\mathrm{S}$ which reaches $\mathrm{E}^{\prime}$ is in this case impeded in reaching $\mathrm{E}$ by the fact that it has to travel electrically downhill - that is, from the anodal $\mathrm{E}^{\prime}$ to kathodal $\mathrm{E}$. Thus, owing to the greater excitation which reaches $\mathrm{E}^{\prime}$, and owing also to the greater excitability induced in it by the fact that it is relatively anode, excitation induces a relatively greater galvanometric negativity of that point. The thick arrow in the figure indicates the excitatory current, which is opposite in direction to the polarisation current, which latter is indicated by the thin arrow. In the second case, when the polarisation current is reversed (fig. 340), E is anodal, and therefore relatively more excitable, and $\mathrm{E}^{\prime}$ kathodal, and therefore less excitable. Unlike the last case, the excitation from $\mathrm{S}$, in order to reach $\mathrm{E}$, has now to travel electrically uphill from the kathodal to the anodal points. The excitation of $\mathrm{E}$ therefore is in this case not impeded. Hence greater excitability of $\mathrm{E}$ makes that point, on stimulation, galvanometrically negative, and the responsive current, represented by a thick arrow, brings about a diminution of the polarisation-current.

It has thus been shown, in the course of the present chapter, that the same electrotonic effects are exhibited in the case of the plant, as in that of animal, nerves. It has been shown that various apparently anomalous results may be brought about by simple combinations of two different factors. Thus, the so-called polarisation increment and decrement are not mutually conflicting. They are, on the contrary, due to the distinct and definite effects induced by electrotonus on conductivity and excitability respectively. 
As regards conductivity, it has been shown that excitation travels best in the direction electrically uphill-that is to say, from a place of low to one of high electric potential. In consequence of this fact a moderate excitation becomes enhanced when travelling from the kathodic to the anodic region. Conductivity is depressed, on the other hand, from anode to kathode. An excitatory impulse is thus retarded in travelling electrically down-hill. For these reasons, a normal negative excitatory effect may, during transmission, undergo either diminution of intensity, or actual reversal to positive.

With reference, again, to electrotonic variations of excitability, we have seen that under feeble E.M.F. it is the anode that exalts, and the kathode that depresses. This conclusion is obviously opposed to the generalisation known as Pfluger's Law, the extent of the applicability of which will be discussed in detail in the following chapter. 


\section{CHAPTER XL \\ INADEQUACY OF PFLÜGER'S LAW}

Reversal of Pflitger's Law under high E.M.F.-Similar reversals under feeble E.M.F.-Investigation by responsive sensation-Experiments on living wounds-Under moderate E.M.F., intensity of sensation enhanced at kathode, and depressed at anode-Under feeble E.M.F., sensation intensified at anode and depressed at kathode-Application of electrical currents in medical practice.

IN studying polar variations of excitability in nerves, in the last chapter, we found that, during the passage of the current, it was the anode which enhanced excitability and the kathode which induced depression. Now this conclusion, as will be remembered, is directly opposed to what is known as Pfliger's Law, the universal applicability of which has hitherto been regarded as beyond dispute. Pfliger's Law lays it down that the kathode excites at make, and the anode at break; and that, moreover, during the passage of a constant current, excitability is raised at or near the kathode, and depressed at or near the anode. We are next, then, led to inquire: Under what conditions is this law applicable, and when does it fail to hold good? Now, as regards the effects at make and break, I have shown elsewhere, in the course of experiments on plants, that these are not determined by anode and kathode alone, but also by the intensity of the acting electro-motive force. Thus, in the case of the sensitive Biophytum, in a given experiment it was found that, using the moderately strong E.M.F. of 24 volts, the excitatory wave at make was found to be initiated at kathode, and to travel in both directions, causing depression of nine pairs of leaflets. The forward half of this wave of excitation, stopped only at 
one pair of leaflets before the anode. There was no action at the anode itself at make. After a suitable interval, during which the leaflets re-erected themselves, the current was interrupted. There was now no action near the kathode at break; but excitation was induced at the anode, as was shown by the fall of three pairs of leaflets in its vicinity (fig. 358). The experiment was now repeated by reversing the direction of the current. The poles being thus reversed, eight pairs of leaflets fell at the new kathode, in and out. There was no effect, however, at the new anode at make. But at break, excitatory reaction was initiated at the anode, and none at the kathode.

These are the normal effects, falling under Pfluiger's Law, which holds good within a certain medium range of E.M.F. But when the E.M.F. is much higher, I find that these normal effects become reversed. Thus, employing an E.M.F. of 220 volts, it was found that excitation took place at the anode at make, the excitatory depression of

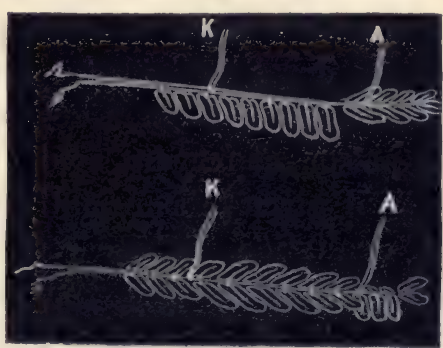

FIG. 358. Make-kathode and Breakanode Effects in Biophytum

Upper figure shows effect at make, excitation being produced at kathode. Lower figure shows effect at break, excitation being now produced at anode.

the leaflets passing slowly thence towards the kathode. At break, excitatory action was initiated at the kathode, the wave of excitation then passing towards the anode. I thus found, by the employment of a very high E.M.F., that the normal polar effects were completely reversed. Intermediate between these two extremes of normal and reversed action, I obtained a transitional phase, in which both anode and kathode were seen to excite at make. At break also there was here occasional excitation, at either anode or kathode. Similar reversals and transitional effects have also been noticed, in the case of certain protozoa by Kühne and Verworn. Thus Pelomyxa is excited by the anode at make, 
and by the kathode at break. Actinospharium, again, shows excitation on make, at both anode and kathode, and on break at the kathode only.

Having thus demonstrated the fact that an excessively strong E.M.F. induces a reversal of the normal polar effects, it may not appear improbable that there should be a similar reversal of these effects when the intensity of E.M.F. is varied in the opposite direction, that is to say, when it is very weak. I have already drawn attention, in many places, to the importance of this factor of intensity in determining the excitatory effect of a stimulating agent. A chemical reagent, for instance, when administered in moderate or very dilute doses, will induce one effect, say that of exaltation, and, in greater quantities, the very opposite, or depression. A poisonous reagent, again, which usually induces depression, will, if given in sufficiently minute quantities, have the effect of exaltation. These reversals, under varying intensities of the external agent, are noticeable again in different physicochemical phenomena. Thus it is well known that in the formation of the photographic image, while a moderate intensity of light gives us the normal 'negative,' a stronger intensity will produce a 'positive,' and a still more intense light, bring about a re-reversal. We may thus have a series of recurrent reversals.

Returning, then, to the question of polar action on excitability, we find that the typical results of Pfliger with nerve and muscle preparations, were obtained when using a moderately strong E.M.F. In this, which is sometimes distinguished as the third stage, excitatory contraction of the muscle is induced, only on the closure of the descending current, or opening of the ascending. In the former case, the kathode is nearest the muscle, and as there is no intermediate block the excitation is clearly due to the make-action of the kathode. In the second case, similarly, the break of the anode, which is now near the muscle, causes excitation. With very weak E.M.F., however-that is to say, in his first stage-Pflüger found that excitation took place by the make 
of both ascending and descending currents, but not at the break of either. Extending the clear inference of the previous case to cover this, it was supposed that here, too, the kathode-at one time near to, and at another far from, the responding muscle-excited at make. But this is not so conclusive, since the anode might equally well be regarded here as causing the excitation at make. Indeed this supposition that a very weak anode might cause excitation at make derives some support from Heidenhain's experiments. For, using a weak E.M.F., he found make-excitation to occur in the first stage only, when the current was ascending - that is to say, when the anode was near the responding muscle. This result would tend to show that there was a possibility of the reversal of normal polar effects when the acting E.M.F. was weak. With regard to this particular effect of minimal currents, there are considerable differences of opinion. The obtaining of such an effect is probably only possible when the nerve-muscle preparation is in an exceptionally favourable condition of excitability, a state of things not always possible to secure in the isolated specimen. It therefore occurred to me that the effect of a feeble anode in enhancing excitability might be demonstrated conclusively in the case of the vigorous intact animal. With this consideration in view, I carried out a number of experiments on certain of my students and myself.

If we make a slight wound, say one square $\mathrm{cm}$. in area, on the back of the hand, and apply a solution of salt, which is not too strong, a constant sensation is induced which cannot be called painful, but may best be described as smarting or irritating. If now we apply one non-polarisable electrode on this wound, and the other on a distant and indifferent point, then, on applying an E.M.F. to the circuit, characteristic variations of sensation will be induced, depending on whether the wound-spot is made anode or kathode (fig. 359). Employing an E.M.F. of 2 volts, it will be found that when the spot is made kathode, the sensation, which was previously one of mere general irritation, becomes 
intensely painful. This is because the kathode, at make and during its continuation, induces an enhancement of the excitability of the wound-spot. On the cessation of the current, the painful sensation disappears, and the normal smarting is restored. The wound-spot was next made anode, with

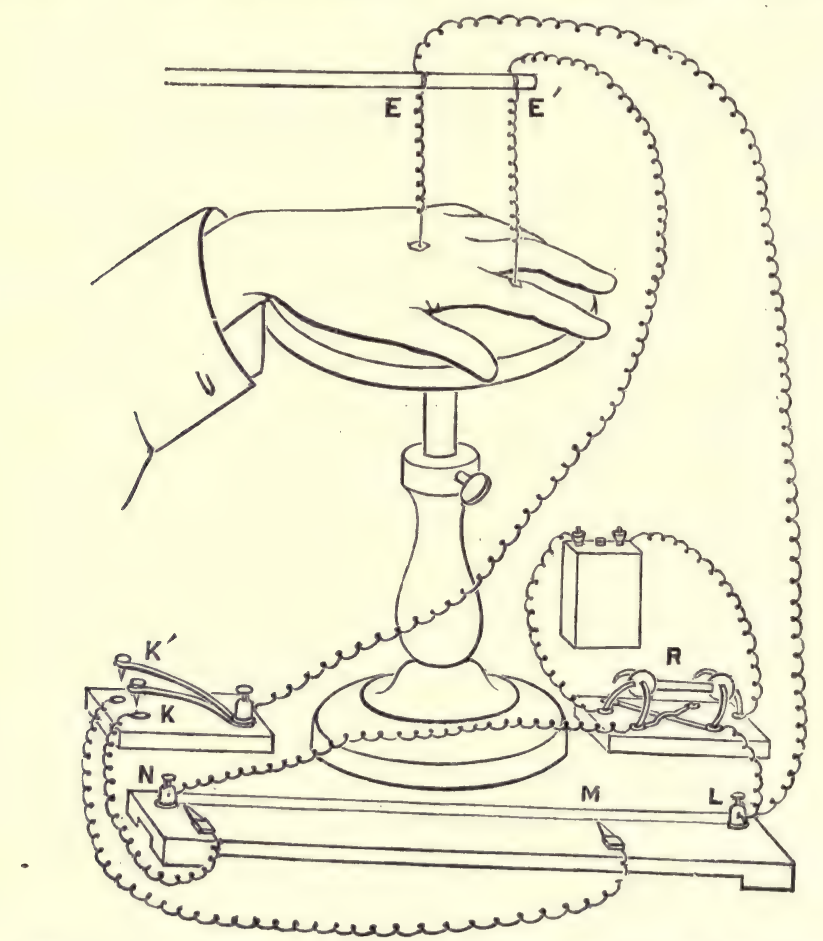

FIG. 359. Effect of Anode and Kathode on Responsive Sensation in Human Hand

By means of reversing key, R, E in connection with the wound-spot may be made anode, and $\mathrm{E}^{\prime}$ kathode, and vice versa. By alternately pressing the keys, $\mathrm{K}^{\prime}$ and $\mathrm{k}$, feeble or moderately strong E.M.F. may be employed.

the same E.M.F. as before. The sensation now experienced was one of soothing, the sense of smarting irritation having disappeared. On the stoppage of this current the original irritation was again restored.

In these experiments we have typical instances of the kathode inducing increase of excitability, and the anode 
depressing it, during the continuation of the current, a verification, by means of responsive sensations, of Pfliger's Law. Having thus, with moderate E.M.F. obtained the excitatory effect at kathode, and depressing effect at the anode, by means of the contrasted sensations of intense irritation and soothing, I was next desirous of seeing whether, with low E.M.F., these effects would be reversed. I therefore undertook investigations on a dozen different individuals, to determine the effect of anode and kathode, as the E.M.F. was gradually incrcased from $: 3$ to 2 volts. It should be mentioned here that the subjects of the experiments were totally ignorant of the object of the investigations, and were simply asked to describe their sensations at different points. Their ages varied from eighteen to twenty-five. As the critical point may undergo some variation with the season, it may be worth while also to mention that the experiments were carried out in summer, in the month of August.

The following case may be taken as typical :

Polar Effects of E.M.F. of Various Intensities on Responsive Sensation

\begin{tabular}{|c|l|l|}
\hline Acting E.M.F. & Effect on wound-spot when kathode & Effect on wound-spot when anode \\
\cline { 1 - 2 }$\cdot 3$ volt & Slightly soothing & Marked increase of irritation \\
$.6,3$ & Slightly soothing & Marked increase of irritation \\
I.0, 3 & Increase of irritation & Indifferent \\
I.5 ," & Increase of irritation & Slightly soothing \\
2.0 volts & Painful & Soothing \\
\hline
\end{tabular}

It will thus be seen that the kathode, which, at the moderately intense E.M.F. of 2 volts, induced a painful sensation, owing to the increase of excitability, induced the very opposite effect of depressing excitability at the low E.M.F. of 3 volt. Precisely the reverse, moreover, was the case with the anode. Here, with 3 volt, excitability was found to be enhanced, causing increase of irritation, while, with the moderately strong E.M.F. of 2 volts, it induced the opposite effect of soothing, by depression of excitability. 
The critical point of reversal would in this instance appear to be slightly below I volt, in the case of the kathode, while in that of the anode, it was at I volt, or slightly above. The effect observed at the extreme points were the same in all cases. Individual differences were concerned only with the exact point of reversal. Thus the point of reversal for the kathode varied in different cases between 6 and I volt; whereas with the anode it varied from $I$ to $I \cdot 5$ volt. In a subsequent chapter, this phenomenon of reversal of sensation under varying intensities of E.M.F., when other forms of stimulus are applied, will be studied in more detail. It may be stated here, however, that though the critical point of reversal varies to some extent with different individuals, and under different forms of stimulation, yet the law holds good that the excitatory effects induced by moderate E.M.F. are exactly reversed under feeble.

The main results regarding this opposition of the effects of feeble and strong E.M.F. may be still better demonstrated by the method of successive contrasts. In the last experiments, a long course of observations on the same individual, would be liable to fatigue the tissue. Moreover, the fine gradation of the changes induced is not calculated to exhibit the contrasts involved in their full intensity. Having, then, determined, from the previous experiments, that an E.M.F. of 5 volt and another of 2 volts were opposed in their excitatory effects, I now made special arrangements for applying these two intensities of E.M.F. alternately. For this purpose, I arranged a potentiometer which gave an E.M.F. of 2 volts between $\mathrm{L}$ and $\mathrm{N}$ (fig. 359), and of 5 volt between $\mathrm{L}$ and M. The end, L, was connected with the wound-spot by means of a non-polarisable electrode. A distant indifferent point, say on the surface of the finger, was connected with a double key $\mathrm{K} \mathrm{K}^{\prime}$. When $\mathrm{K}^{\prime}$ was pressed, $\cdot 5$ volt was applied, and when $\mathrm{K}, 2$ volts. Further, by means of a reversing-key, P, the wound-spot could be made either anode or kathode at will. In this way, first making the wound-spot anode, I applied alternately the 
E.M.F. of $\cdot 5$ and of 2 volts respectively. The lower voltage now gave rise to intense excitatory pain. On the cessation of the current the normal smarting sensation, due to salt, was restored, and on now applying 2 volts, this slight irritation was superseded by a sensation of soothing. This result was found to be repeated many different times.

The kathodic effect was next put to the test, and found to induce responsive sensations exactly the reverse. The application of 5 volt caused a soothing sensation, due to depression of excitability. An E.M.F. of 2 volts, on the other hand, induced an increase of excitability, with consequent pain.

These results are shown in the following table:

Method of Successive Contrasts to show Reversal of Sensation under Polar Currents

\begin{tabular}{|l|c|c|c|}
\hline \multicolumn{2}{|c|}{ Wound-spot anode } & \multicolumn{2}{c|}{ Wound-spot kathode } \\
\hline E.M.F. 5 5 volt & E.M.F. 2 volts & E.M.F. of '5 volt & E.M.F. of 2 volt \\
\hline Intense pain & Soothing & Soothing & Painful \\
\hline
\end{tabular}

From these experiments, then, it will be seen that during the passage of the current, and when the E.M.F. is low, it is the anode which increases the excitability, and the kathode which depresses. Pflüger's Law is thus seen not to be universally applicable, but to be true only within certain limits, the very reverse of this law holding good, in the case of excessively high, and in that of low E.M.F. The demonstration which has just been given of the latter of these two facts, is independently borne out by the results of electrotonic variations of excitability in nerves, described in the last chapter, where we saw that, with moderately feeble E.M.F., excitability was enhanced by the anode and depressed by the kathode.

It will thus be seen that polar variations of excitability are not always the same, but differ in character, according as the intensity of the acting E.M.F. is moderate or low. The great significance of this fact is apparent, with regard to the 
medical application of electricity, since the failure to recognise that reversal of effects which is to be expected under a feeble E.M.F. might here lead to a result the very opposite of that intended.

It has thus been shown that Pfliger's Law of the Polar Variation of Excitability is not universally applicable. It fails when the E.M.F. is either too high or too low, the effects observed under these circumstances being precisely the opposite of those enunciated by Pfliger. Under a low E.M.F. then, it is the anode which enhances the excitability, depression being induced by the kathode. This important fact, and the further fact that with low E.M F. conductivity is increased in the direction of the rising electrical potential, and depressed in that of the falling potential, will be found to explain all the varied electrotonic phenomena of nerves described in the previous chapter. 


\section{CHAPTER XLI}

\section{THE MOLECULAR THEORY OF EXCITATION AND ITS TRANSMISSION}

Two opposite responsive manifestations, negative and positive-Such opposite responses induced by polar effects of currents of different signs-Arbitrary nature of term 'excitatory'-Pro-excitatory and anti-excitatory agents-Molecular distortion under magnetisation in magnetic substances-Different forms of response under magnetic stimulation-Mechanical, magneto-metric, and electromotive responses-Uniform magnetic responses-Response exhibiting periodic groupings-Ineffective stimulus made effective by repetition-Response by resistivity-variation-Molecular model-Response of inorganic substance to electric radiation-Effect of rise of temperature in hastening period of recovery and diminishing amplitude of response-Sign of response reversed under feeble stimulation-Conduction of magnetic excitation-The Magnetic Conductivity Balance-Effect of A-tonus and $\mathbf{K}$-tonus, on excitability and conductivity - Conducting path fashioned by stimulus - Transmission of excitation temporarily blocked in iron wire, as in conducting nerve-Artificial nerveand-muscle preparation.

IT is admitted that the excitation of living tissues is brought about by some kind of molecular disturbance, and that the passage of this molecular disturbance from point to point is the transmission of excitation. As we do not possess the power of molecular vision we have perforce to be contented with the vagueness of the ideas which these terms connote, complicated as they are by the concomitance of other apparently mysterious properties of living tissues. If response and its variations, however, be in truth mainly dependent on the molecular condition of the tissue and its upset, then, from molecular considerations alone, it must be possible to explain why, under certain conditions, the responding substance is increased in excitability, and under others depressed. It has hitherto been found impossible to determine what is the nature of the antecedent molecular 
conditions to which these differences may be due: what it is that so determines the tone, that the excitability of a tissue is made to undergo a profound change during the action of a particular stimulus or on its cessation; and what finally causes the fact that one identical stimulus, say that of tetanising shocks, will sometimes act to exalt, and at others to depress, the excitability of the same tissue. It is the caprice which has seemed to preside over these phenomena that has forced observers upon the postulation of a hyperphysical 'vital force.' In the course of the present work, however, it has been shown that not only the simple phenomena of response, but all their complex variations also, are to be met with in the inorganic as in living matter, and that their explanation, therefore, must be sought for in the nature of antecedent molecular changes. As in the inorganic, the conditions of investigation are less complex than in living tissues, it follows that the study of molecular transformations and their after-effects there, is likely to throw much light upon that phenomenon of response which we have thus seen to be universal.

Taking first the response of living tissues, we find that the responsive change is of two kinds. This may be illustrated by the following experiment, carried out on the pulvinus of Erythrina indica during the season of its greatest sensitiveness. The stimulus employed was that of a constant electrical current. When the upper half of the pulvinus was made anode, response was found to take place by local expansion. This is seen in the up-record of figure 360 . On the break of the anode, we observe a movement of recovery in the opposite direction. The pulvinus was next subjected to kathode-make, and we observe a responsive contraction. At kathode-break, however, we have a recovery by expansion. We have thus observed two opposite responsive effects, according to the different polarities of the stimulating agent-namely, expansion at the make of anode, and contraction at that of kathode.

Since responsive effects must be due to molecular upset, 
or to new conditions of alignment, it is clear that contraction must be brought about by a one-directioned, and expansion by the opposite-directioned, change. This is evident in the present case, since the polar stimulating agents are opposite in their characters, and the opposition of their effects must correspond to this. Now it is necessary to distinguish these two responsive effects by opposite terms, which must needs be somewhat arbitrary. The contractile effect has thus been taken as the normal excitatory and negative. Having once adopted such a nomenclature, it is of course important that it should be strictly adhered to. Thus, if contraction be the normal response, then anything which tends to enhance it must be regarded as excitatory, and anything which opposes or retards it as depressing. This word 'depressing' is, however, unfortunate, since by it might be indicated a

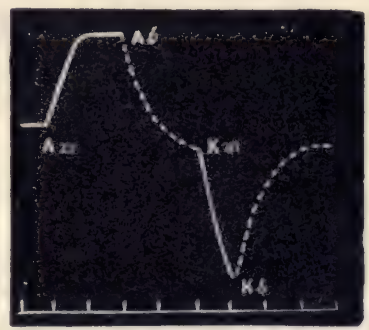

FIG. 360. Polar Effects of Currents due to Localised Application on Upper Half of Pulvinus of Erythrina indica

Up-curve represents expansion and convexity. Down-curve represents contraction and concavity. Continuous curve represents the action at make. The dotted curve shows the effect at break. $\quad \mathbf{A} m=$ convexity induced at anode-make. $\mathrm{A} b=$ responsive concavity at anode-break. $\mathrm{K} m=$ induced concavity at kathode-make. $\mathbf{k} b=$ expansion induced at kathode-break. The time-marks represent minutes.

permanent depreciation of the tissue, while diminution of the normal response is possible without such depreciation. Moderate rise of temperature, for example, with its expansive tendency, will lessen the contractile response without necessarily depreciating the tissue (p. I87). Reverting once more to the kathodic mode of stimulation, we know that a certain intensity of kathode is necessary, for the visible initiation of contractile response. ${ }^{1}$ Should the intensity employed be just short of this, there will be an

1 These kathodic and anodic effects refer to the normal moderate range of E.M.F, within which Pfliger's Law is applicable. 
incipient molecular distortion, in the same direction as that which precipitates the excitatory response, hence kat-electrotonus should prove to be excitatory. But a moderate anode, with its incipient molecular distortion in the opposite direction, will retard the normal response, and thus appear to be depressory. I must here point out that these terms excitatory and depressing have ordinarily speaking no absolute meaning, and can only acquire a definite significance when we have first fixed on that form of response which is to be regarded as normal. If, instead of contraction, we had regarded expansion as the normal response, then the effect of anode would have been regarded as excitatory, and that of kathode as depressing. We must therefore recognise that the very fact of contractile response being taken as excitatory, entails as a consequence the designation of all agencies, such as $\mathrm{K}$-tonus, which predispose the tissue to contraction, as excitatory, or better pro-excitatory, while those which, like an-electrotonus, oppose this, must be regarded as depressory, or better anti-excitatory.

From what has been said, it will be understood that it is the direction of the molecular derangement which determines the character of the response. That molecular upset, which expresses itself as excitatory contraction, we may call the $\mathrm{K}$-effect, and the reversed molecular movement, expressed as expansion, the A-effect. Thus, under anode, in fig. 360, the molecular distortion in one direction induces the expansive A-effect. On the cessation of this, the rebound of recovery causes a movement in the opposite direction, which may carry the molecules back, not merely as far as the equilibrium position, but beyond this. This movement, however, is in the same direction as that induced by $\mathrm{K}$-make. Hence we may understand how excitation is caused, not only by $\mathrm{K}$-make, but also by strong A-break. We may also understand how it is that the excitatory effect is much enhanced when A-break is immediately followed by $\mathrm{K}$-make. We also see, in a general way, that a particular-directioned molecular movement would have the most intense excitatory 
value when the molecular distortion was proceeding at a rapid rate, and not so much when a condition of permanent distortion had been attained. It is for this reason that; usually speaking, the excitatory effect is most pronounced at either kathode-made or anode-break,, and not so much during the continuation of kathodic action.

To recapitulate some of the principal facts enumerated above, the term 'excitatory' being applied to a particulardirectioned distortion or K-effect, then anything which induces an incipient molecular distortion in the same direction, tending to aid the $\mathrm{K}$-effect, and therefore to enhance that response, will be known as K-tonus. Anything, on the other hand, which induces an incipient distortion in the opposite direction, will oppose or retard the normal K-effect, and will, therefore, be known as inducing A-tonus.

In the examples given, the opposite $\mathrm{K}$ - or A-effects observed were the outward manifestations of the aggregate molecular effects induced. And from these we inferred the opposite-directioned changes which must have been their antecedent cause. In working with inorganic substances, however, and particularly in dealing with magnetic bodies, our power of molecular scrutiny is much keener. A rod of iron, for example, is known to consist of magnetic particles, each one of which is a true magnet, possessed of polar properties. Under ordinary circumstances these magnetic molecules are in close chains, but under the action of magnetising forces they become distorted in a directive manner. Under north-magnetising force they are distorted in one direction, and under south polar induction in the reverse. The intensity of the induced magnetisation is a measure of the degree of molecular distortion, and can be gauged by the deflection of the freely suspended needle of a magnetometer in the neighbourhood. Increasing magnetising force is thus seen to induce greater magnetometric deflections,

${ }^{1}$ It is conceivable that there should be occasions in which the final condition of distortion is not attained quickly, but slowly ; or where it is fluctuating instead of stable. Under such circumstances the excitation induced would be more or less persistent or tetanic. 
and on the cessation of the inducing force there is usually a molecular recovery, with a concomitant return of the magnetometric indicator to its original position.

Here, then, we have a means of recording the molecular distortions induced in a substance under a given external force. We are able also to study the relation between the acting force and the distortion induced, while it is increased or diminished in a known manner. And further, keeping the acting force the same, we are here able to study the effects of various modifying agents on the response, as recorded by the magnetometer,

In all these cases, then, we have a strict parallel to the excitatory molecular changes and their variations induced in a living tissue under stimulus. But besides this local action we have also, in the living tissue, nerves possessing the property of transmitting the state of excitation-that is to say, the molecular disturbance-to a distance ; and this transmission is modified appropriately by the various modifications which may be induced in the conducting nerve. Similarly I shall be able to show that, in an iron wire, excitatory magnetic disturbance is propagated to a distance; this conduction likewise being modifiable by the molecular changes induced in the conducting wire.

Thus, in those particular cases where molecular scrutiny is possible, we are enabled to visualise with considerable accuracy those molecular events on which excitation and its transmission depend. Afterwards, discarding this illustrative class of magnetic substances, I shall refer to other methods, by which the responsive manifestations of ordinary substances under stimulus, and the modifications of these responses under various conditions, will be recorded. From so comprehensive a study we shall find that whatever be the mode of record, and whatever the experimental substance employed, the fundamental reaction, and its variations under particular conditions, are curiously similar. It will then be realised that the response of living tissues is not alone of its own kind, but falls under a wide generalisation. 
But before proceeding further with the magnetic responses, we must call to mind two different responsive manifestations of living tissues. We have observed these under the polar action of electric currents, one being the $\mathrm{K}$ - and the other the A-effect. Similarly in magnetic substances also, under the action of the magnetising forces, we observe two different effects brought about by opposite polar changes. One of these is the result of north and the other of south polar induction; and of these, for the sake of convenience, we shall fix our attention on the effect induced by the north pole as the normal negative or $\mathrm{K}$-effect.

The fundamental molecular change induced may here, as in the case of living tissues, be recorded in various ways. In the present case, of the response of magnetic substances under magnetic stimulation, the methods of record may be classified as mechanical, magnetometric, and electro-motive. Joule discovered that a rod of soft iron undergoes a change of length on magnetisation. Though this variation is very small, I find it comparatively easy to demonstrate and record the responsive change concerned by means of the following device. One end of the iron rod is fixed, and the free end, carrying a wooden disc, rests on a tambour covered with stretched indiarubber. The tambour chamber is closed except at the point where a capillary tubing of glass enters it. This tube contains a short index. On now suddenly inducing magnetisation by a magnetising coil, the rod undergoes instantaneous elongation, and the resulting expulsion of air from the tambour causes a corresponding movement of the index outwards. Cessation of the magnetising current is attended by immediate recovery. It need only be mentioned here that by making the diameter of the tambour sufficiently large, and that of the capillary tube sufficiently small, and by optically magnifying the movement of the index, it is easy to obtain for this mode of experiment a very high degree of sensitiveness.

It is much easier, however, to record responsive molecular changes by the usual magnetometric, or by the induction or 
electro-motive method. According to the former of these, the magnetising coil, C, is placed broadside on, in reference

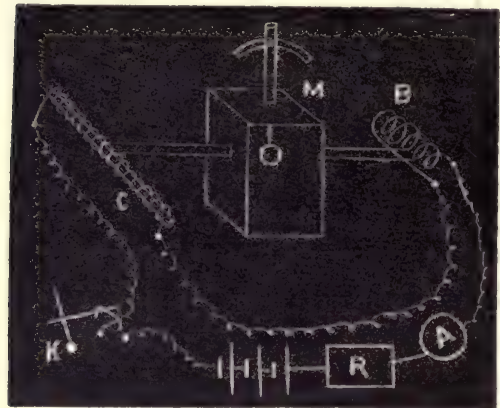

FIG. 36r. Experimental Arrangement for Magnetometric Method of Record

M, magnetometer ; C, magnetising coil ; B, balancing coil; A, ammeter; $\mathbf{R}$, rheostat; $\mathbf{k}$, key actuated by metronome.

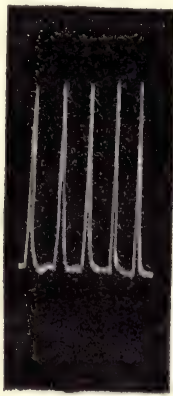

FIG. 362. Photographic Record of Uniform Magnetic Responses of Iron

to a freely suspended magnetic needle with its attached mirror (fig. 36I). A second balancing coil, B, is placed on

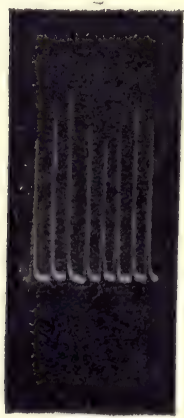

FIG. 363. Photographic Record of Periodic Groupings in Magnetic Responses the other side, and so adjusted as to nullify any disturbance of the needle by the magnetising coil. The experimental rod of iron is then introduced inside $\mathrm{C}$, and the responsive molecular action induced by the exciting current is recorded in the usual manner by the deflected spot of light from the magnetometer, $\mathrm{M}$, thrown on a revolving drum. The intensity of the exciting current, measured by the ammeter, A, is capable of adjustment by means of the rheostat, $\mathrm{R}$. The duration of application of the exciting current is determined by a metronome, and thus kept uniform in successive experiments. In fig. 362 is seen a series of records obtained in this manner, employing stimulation of moderate intensity. In fig. 363 is seen a curious instance of periodic groupings 
in magnetic responses, similar to those obtained in living tissues. In the next figure (fig. 364) is shown the effect of strong stimulation, which gives rise to responses, not only of greater amplitude, but also of prolonged recovery. Under the strong stimulation here employed, owing to persistent molecular strain, the recovery did not become complete. This is analogous to the contracture in strongly excited muscle. Such persistent strain may be removed by molecular vibration, the hastened recovery in the present record

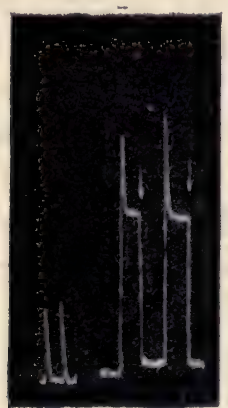

FIG. 364. Photographic Record or Response and Recovery of Steel under Moderate and Strong Magnetic Stimulus

First pair of records show response and recovery under moderate stimulus. In the next two, stronger stimulus induces response of greater amplitude and incomplete recovery. Molecular vibration by tap, at point marked by down-arrow $\downarrow$, hastens recovery. being the result of a tap. Magnetic stimuli, individually ineffective, become effective by repetition. In fig. 365 is

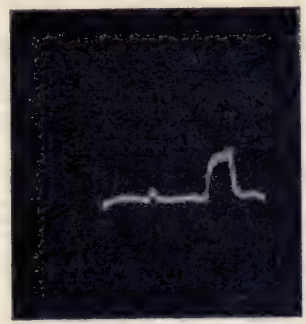

FIG. 365. Photographic Record showing Ineffective Stimulus made Effective by Repetition

A single brief magnetic stimulation induced little or no effect, but when rapidly repeated thirty times it became effective.

seen a record of this. Tetanisation also induces the maximum effect of fusion-as will be seen in the following chapter. Tetanisation, again, induces interesting after-effects in magnetic responses, precisely the same as those seen in living tissues. Under certain conditions, moreover, to be fully described later, tetanisation, as we shall see, enhances the subsequent responses, while under. other conditions, by inducing fatigue, it brings about their depression.

I have already mentioned the fact that in addition to 
the mechanical and magnetometric methods of studying response in magnetic substances, there is also a third means available, in the Induction or Electro-motive Method, to be fully described at the end of the present chapter.

I have now explained how the extent of molecular distortion induced in a magnetic substance by an external force can be gauged or measured by magnetometric or electric indications. For the detection of similar changes, however, in matter which is not pronouncedly magnetic, it is necessary to devise a method of record of more universal application. Such a method we have, as already said, in the record by resistivity-variation. It is here desirable, however, to give a more detailed account of this and the principle involved.

Our object being the deteçtion of the molecular changes induced by stimulus, let us briefly consider certain wellknown cases of molecular transformation induced by various stimulating agencies. Thus, when sulphur is subjected for a certain length of time to the action of light, there is no visible sign of any change. Its solubility in carbon disulphide, however, has been altered, and we can discriminate the portions acted upon from those unacted, by means of this 'developing' solution. But such discrimination is only possible when the molecular or allotropic modification has gone so far as to be somewhat stable-that is to say, when the after-effect of stimulus is persistent. The development of any after-effect would have been impossible had the substance in the meanwhile exhibited self-recovery. Between the original condition A, again, and the terminal modification $\mathrm{D}$, the substance must have passed through many gradations of condition, of a more or less impermanent stability. This case is analogous to that of a piece of iron under the action of magnetising forces, with their consequent molecular modifications. When the acting force is moderate, and the specimen has the power of selfrecovery, the induced molecular distortion-that is to say, the induced magnetisation-is fugitive, and there is no after- 
effect on the cessation of the force. But, under intense magnetisation, the molecular transformation is more or less persistent, and we observe an after-effect in the induced permanent magnetisation. To revert here to the illustration of sulphur, it is only because the persistent terminal change is the most easily distinguishable that we single it out for the name of 'allotropic change.' As a matter of fact we see that this is but the climax of a series of changes, and so incomplete a view has been made current by the fact that we had no means of recording the intermediate changes while they were in progress.

The next question is as to the possibility of making such records of molecular transformations, or of induced variations in the state of molecular aggregation, while they are taking place. This may be accomplished, as I shall show, by the concomitant variation of electrical conductivity. It is to be borne in mind that the state of molecular aggregation plays an important part in determining the conductivity of a substance, and as an example we may take the case of carbon, which exhibits wide differences of conductivity in its two allotropic conditions of graphite and diamond. Let us imagine a piece of carbon in an intermediate or neutral state between these two. We may suppose that an external force distorts it to a small extent towards the more conducting state of graphite. This distortion would be attended by an increase of conductivity, from which latter the extent of molecular distortion or upset involved might be inferred. Now, during the distortion from the equilibrium position, a force of restitution will tend to restore the carbon to its original neutral condition. If the distortion does not proceed beyond the elastic limit, then, on the cessation of the external stimulus, it will recover its original state, and this will be evidenced by the restoration of its original conductivity. But if the distortion be of a sub-permanent or permanent type, the recovery will be very much protracted, or will not take place at all. Such more or less permanent distortion, known as allotropic transformation due to stimulus 
of light, is seen in the production of red phosphorus from the yellow variety, and the insoluble from the soluble variety of sulphur.

It will thus be seen that the conductive aspect of a given substance is not definite, but variable, the conductivity being dependent on the particular molecular condition of the substance. This peculiarity may be represented in the accompanying model (fig. 366), if we give the cylinder representing the sensitive molecule three main-conducting aspects A B C. The non-conducting aspect is represented by $\mathrm{C}$. With the sensitive substance in this

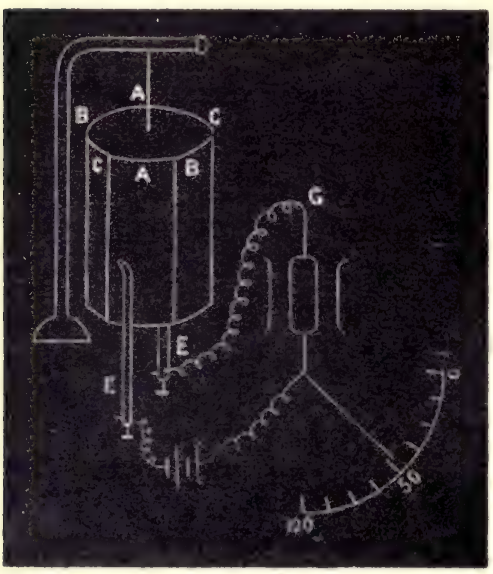

FIg. 366. Molecular Model particular condition, interposed in the electric circuit, the current in the galvanometer would be zero. A is the semi-conducting aspect of the substance, under which we may imagine the corresponding deflection of the galvanometer to be 50 . $\mathrm{B}$ is the highly conducting aspect, the corresponding galvanometer reading being IOO.

The model representing the sensitive substance has its surface divided into six parts, the opposite sextants being put in electric communication. The opposite sextants $\mathrm{C} C$ are coated with shellac to represent the non-conducting aspect; the sextants A A are coated with graphite to represent the semi-conducting aspect; and the highly conducting aspect is represented by the sextants B B coated with tinfoil. The three main aspects of the sensitive substance are thus represented in the model; it is to be understood that with sensitive substances, under the action of stimulus, the transition from one aspect to the next is gradual, and not abrupt, as represented. The sensitive substance is interposed between 
two electrodes. The torsion of the wire by which the cylinder is suspended represents the force of restitution. The galvanometer coil, by its deflections, exhibits indirectly the molecular strain produced in the substance by the action of stimulus.

Let us suppose that we start with the substance in its normal state A, with moderate conductivity, and let the corresponding galvanometer deflection be 50 . Let the substance belong to the negative class which exhibits an increase of conductivity, or diminution of resistance, under the action of stimulus. The stimulus will therefore distort the substance to a state of increased conductivity, the increased conductive aspect B B being brought opposite the electrodes. The enhanced current thus produced causes a deflection of, say, 100 in the galvanometer. If the strain has not been excessive, the substance will return, on the cessation of stimulus, to its original position of equilibrium, and the galvanometer deflection will fall from 100 to the original value 50. If the substance belong to the positive class, the distortion will be in the opposite direction, and the effect of stimulus will be to induce a responsive increase of resistance.

The coil of the indicating galvanometer thus moves in perfect response to the varying molecular strain induced in the sensitive substance by the action of stimulus. The invisible molecular distortions are thus revealed by the visible deflections of the galvanometric indicator-the effect on one is merely the reflection of the effect on the other. A curve of the molecular effect, induced by the action of stimulus, may thus be obtained with the galvanometer deflection as ordinate, and the time as abscissa. It is thus seen that these response-curves faithfully represent the invisible molecular strain-effect due to the stimulus, and the subsequent recovery.

I shall now describe how in practice, by this method of resistivity variation, we obtain responses of various substances to the stimulus of visible or invisible radiation. The 
sensitive substance may be made the fourth arm of the Wheatstone's bridge, and the responsive galvanometric deflection and subsequent recovery of the spot of light-by the upsetting of the balance, under the action of stimulus of radiation-is recorded in the usual manner, on a moving photographic plate. Or the sensitive substance may be placed in series with a galvanometer, a small E.M.F. giving a steady permanent deflection. Taking first selenium as the sensitive substance, the molecular change induced by the action of light, with its concomitant variation of resistance, causes a deflection of the galvanometer spot of light. On the cessation of the stimulus, molecular recovery takes place, and the deflected spot of light returns to its original position.

A series of such responses will be found on referring to page 3, fig. 3. The parallel method employed in recording

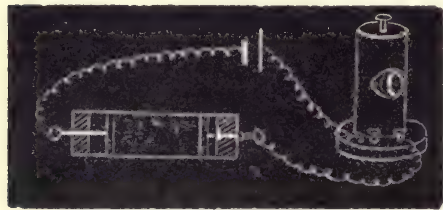

FIG. 367. Method of Resistivity Variation

Sensitive metallic particles placed in tube in series with galvanometer and E.M.F. This gives a steady permanent deflection. Stimulus of electric radiation induces a responsive variation of resistance with concomitant variation of galvanometric deflection. the responsive resistivity variation of masses of metallic particles of various kinds, under the stimulus of electric radiation, will be understood from fig. 367 . On obtaining records of the responses given under this method, I find, as I pointed out in the first chapter, that the responding substances are of two different types. The first, of which aluminium may be taken as the example, respond by diminution, or negative variation of resistance. The second, illustrated by potassium or arsenic, respond by an increase, or positive variation of resistance. In living tissues also, tested by various modes of response, we have seen two opposite types to occur-highly excitable nerve giving one, say, negative, while skin, on the other hand, gave the positive.

In the case of the inorganic substances referred to, we have extreme types, whose response is generally either positive or negative. There are, however, intermediate 
cases, where it is liable to change of sign according as the stimulus is feeble or strong. Certain substances, again, cannot quickly recover from the after-effect of stimulus; while, in others, recovery is fairly rapid. Recovery from intense stimulation is generally, other things being equal, more protracted than from feeble or moderate. Anything, however, which enhances molecular freedom or mobility will tend to hasten recovery.

I shall now give several typical records in illustration of the peculiarities of this form of response by resistivity variation, under various conditions. The first example

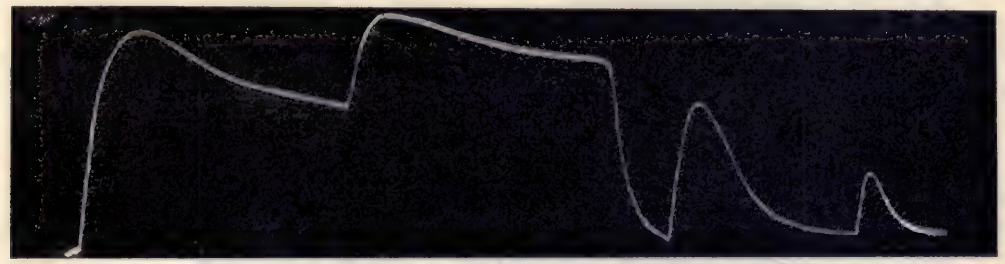

FIG. 368. Photographic Record of Response of Aluminium Powder in Sluggish Condition to Stimulus of Electric Radiation.

The first two responses exhibit incomplete recovery, which becomes complete on application of warmth. Note that warmth, increasing force of recovery, hastens recovery and also diminishes amplitude of response, as seen in the two succeeding records.

given, that of aluminium powder, will be of the negative type, the response being by diminution of resistance. When the substance tested happens to be in a sluggish condition, recovery is very protracted. There is then a responseremainder of persistent negative variation, corresponding to the contraction-remainder in muscle, or persistent electromotive negativity in other living tissues. But we know that a moderate rise of temperature is favourable to recovery; and on applying gentle heat, at the end of the second response, with its incomplete recovery, the persistent effect is seen to be removed, and there is an immediate completion of recovery (fig. 368).

This is the place to refer once more to an apparent 
anomaly, in the response of living tissues, where a slight rise of temperature increases conductivity, at the same time that it appears to diminish excitability, inasmuch as it brings about a lessened amplitude of response (Chapter XV.). This latter, however, may not really be due to diminution of excitability, since the same effect might equally well be brought about by an enhancement of the force of recovery. This view is supported by the further records given in fig. 368. We see here that incomplete recovery became complete, under the application of gentle heat. The next response given by this slightly warmed substance is seen to

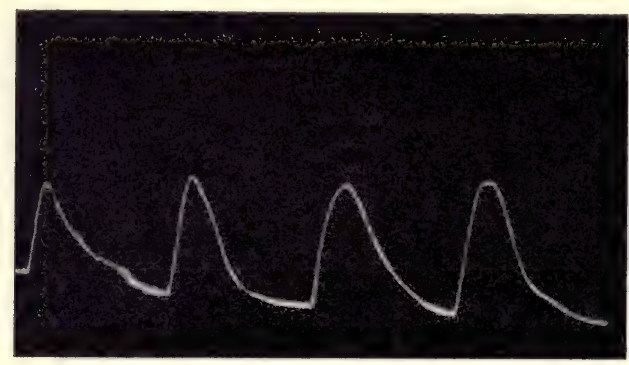

FIG. 369. Photographic Record Showing Uniform Response of Aluminiu n Powder to Uniform Stimulus of Electric Radiation.

show complete recovery within a relatively short time, this enhanced force of recovery bringing about at the same time a diminution in the height of response. The temperature of the substance was now again raised to a slightly higher degree, and the next response shows a still further diminished height and a considerably quickened recovery. When a substance is in a normal condition of excitability, its successive responses to uniform stimuli are found to exhibit complete recovery, and to be of equal amplitude. Figure 369 shows such a series obtained with powdered aluminium.

We have seen that the increased molecular mobility conferred by warming hastens recovery. A similar hastening of recovery may be brought about by a mechanical tap, as has already been shown (fig. 364) in the case of magnetic response. 
In fig. 370 is seen an example of the same thing in tungsten, where recovery from the effect of electric radiation is hastened by a tap.

It has been shown that the normal response by negativity in living tissues is liable to reversal under very feeble stimulation. This is better observed when the tissue is not highly excitable; because in this case it is easy to adjust the intensity of stimulation, so as to fall below the critical value for excitation. It is very interesting to observe similar opposed effects, under feeble and moderately strong stimulations, in the response of inorganic substances. For the reason just mentioned, it is desirable to select a substance for this purpose, which possesses a moderate degree of sensibility. Using a mass of tungsten particles, I found that under strong intensity of electric radiation--brought about by placing the radiator within a short distance of the substance-the response was by the normal negative variation, or diminution of resistance. But when the intensity

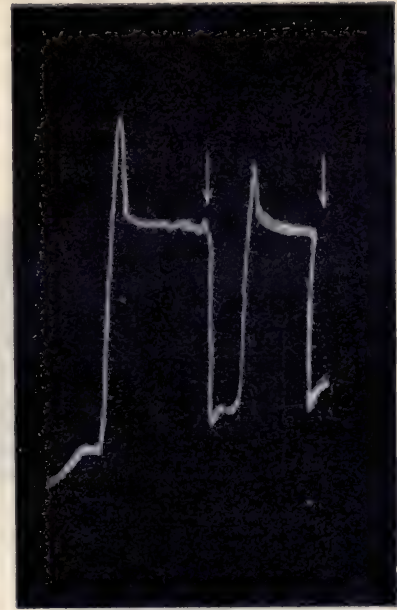

Fig. 370. Photographic Record of Response of Tungsten

The incomplete recovery is hastened by application of tap at points, marked with downward arrow. Cf. fig. 364 .

of stimulus was dimmished by placing the radiator at a greater distance, then the response was converted to positive. A record of this abnormal effect under feeble stimulation will be given later.

Thus, having observed molecular response and its variations by the Magnetic and Resistivity Methods of record, we now proceed to study the transmission of the state of excitation. We have seen that the essential condition of the transmission of excitation in living tissues lies in the 
propagation of molecular disturbance from point to point. The characteristics of such propagation must be-(I) that the transmitted molecular disturbance becomes enfeebled with distance, so that at a certain point the transmitted excitation would be reduced to zero; (2) that while a moderate stimulus is transmitted to a short distance, a stronger stimulus would be carried further; and (3) that the intensity of excitation transmitted would depend on the conducting power of the intervening tract, this conductivity being capable of enhancement by certain agencies, and depressible by others.

I shall now proceed to show that in an iron wire a transmission of molecular disturbance takes place which is

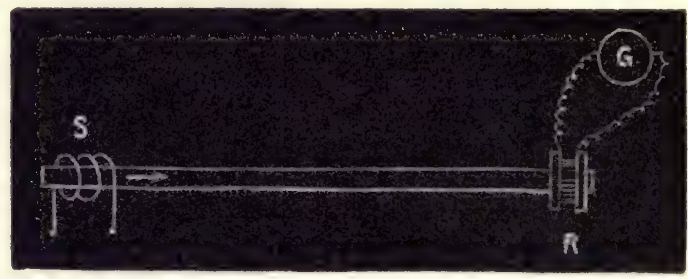

FIG. 371. Experimental Arrangement for obtaining Response in Iron by Induction Current

similar to that at the basis of the transmission of excitatory changes, both, as I shall show, being modifiable by similar circumstances in a similar manner. For these investigations I have employed the Induction or Electro-motive Method of observation. In the experimental arrangement-a diagrammatic representation of which is shown in fig. $37 \mathrm{I}-\mathrm{S}$ is the stimulating or exciting coil applied at the point to be excited. The conducting region intervenes between $\mathrm{S}$ and $\mathrm{R}$, which is the responding point, over which is wound the receiving coil, placed in series with either a telephone or a galvanometer. When the excitatory molecular disturbance reaches $\mathrm{R}$, it gives rise to an induction current in the coil, which in turn causes a sound in the telephone, or a responsive deflection in the galvanometer. For the purpose of simplicity, we shall take north polar or K-excitation as 
normal, and the resulting deflection of the galvanometer to the right as the normal response. The direct effect of the coil $\mathrm{S}$ on the coil $\mathrm{R}$ may be regarded as negligible, when they are separated from each other by a sufficient distance, and this would be even more true if the intervening iron wire were bent at an angle of 90 degrees.

Employing this mode of obtaining records of response to K- or.A-excitation, we meet with several curious analogies to the responsive effects seen in living tissues, under the electrical mode of stimulation. Electrical excitation of nerve and muscle, for example, is most effective when it is longitudinal, and ineffective when transverse. The same is true of magnetic excitation of iron, where longitudinal excitation is effectively transmitted to a great distance, whereas transverse excitation is relatively ineffective.

Again, in the case of the electrical excitation of living tissues, it is at the instant of kathode-make, as we have seen, that excitation is induced. Continued action exhibits in general no effect. The same normal excitatory effect seen at kathode-make, is induced again, but at anode-break. Similarly, in the case of an iron wire, the normal galvanometric response is seen at the moment of $\mathrm{K}$-magnetic excitation, but not during its continuance. The same excitation is also obtained, at break of $\mathrm{A}$, or south polar magnetisation.

We are led from such close analogies, not only to visualise, but also to obtain some insight into the sequence of molecular events which is the concomitant of excitation. I have already pointed out that excitation and its opposite, depression, being phenomena of molecular distortion, it is to be expected that a particular-directioned distortional movement should be associated with one of these, and the opposite with the other. We also know the further suggestive fact that it is the sudden change of the environment, inducing a sudden responsive molecular disturbance, that is most effective in bringing about excitation. The latent period, and a slowly-rising excitation, correspond to the 
slow initiation of the molecular upset. After this the rate of molecular distortion will be rapid, and in this second period we find that the excitatory reaction also is at its maximum. In any case, it is rather during the period of increasing molecular distortion that we should expect to see the most intense excitation, than when a static condition of derangement has been attained. Thus it is at the moment of $\mathrm{K}$-make that we obtain the excitatory indication, and not afterwards, when the molecules are being maintained in the distorted position.

Returning once more to the iron wire, we find that when the distorted molecules have been set free by the break of $\mathrm{K}$, there is a sudden movement of recovery in the opposite direction. If now the K-effect, with its particular-directioned molecular movement, be termed the excitatory, then the opposite movement must be regarded as one of depression, and it is interesting to note that in a living tissue there is an aftereffect of depression at kathode-break. The anode-make, on the other hand, with its opposite molecular distortion, is, as one would expect, depressory. But at the break, the direction of the rebound of the released molecules being the same as that brought about by K-make, must be excitatory. The close parallelism which we have thus traced out, forces upon us the conclusion that the molecular actions which underlie the excitation of living tissues may be polar in their character.

The fact that magnetic excitation undergoes diminution during transmission, can be shown by moving the receiving coil $\mathrm{R}$ further and further away from $\mathrm{S}$, when the responsive sound in the telephone, or deflection in the galvanometer, will be found to undergo a graduated diminution, till, with a given stimulus, the effect, from being considerable, is reduced at a certain distance to wil. Keeping this distance the same, however, a stronger stimulus will be found efficient to evoke response, and the responding coil will now have to be moved further, in order again to reduce the response to zero.

We shall next study the variation of conductivity induced 
by an external agent, as modifying the intensity of the transmitted effect. In order to study the phenomenon of conduction and its modification, as will be remembered, a delicate form of Conductivity Balance, fully described in Chapter XXXIII. was used. Excitation was here caused by $\mathrm{S}$ at a middle point, the transmitted excitatory effects at $\mathrm{E}^{\prime}$ and $\mathrm{E}$ being made to balance. This condition of balance was obtained when one arm, say the left $\mathrm{E}^{\prime}$, was kept at a fixed distance from $\mathrm{S}$, and the other, or right, was moved towards S, or away from it, as required. When $\mathrm{E}$ was too far from $\mathrm{S}$ the excitatory effect would be smaller than at $\mathrm{E}^{\prime}$, and this under balance would be indicated by a response, say downwards. When, again, $\mathrm{E}$ was too near to $\mathrm{S}$, there would be an over-balance, the resultant response being upwards. Between these could be found a point of exact balance where the record was horizontal ( $c f$. figs. 289, 290).

A high degree of delicacy in the study of similar phenomena in the case of iron wires may be obtained by

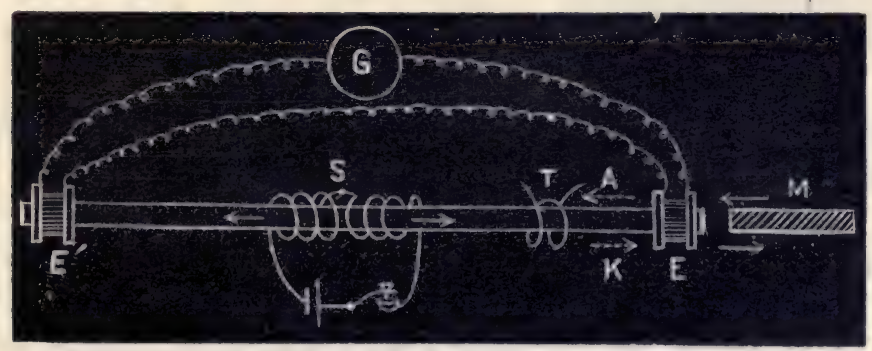

FIG. 372. Magnetic Conductivity Balance

$\mathrm{s}$, magnetising coil, by which north-polar or K-impulses are sent out in two directions as shown by arrows. $\mathbf{E} \mathbf{E}^{\prime}$, receiving coils, adjusted at balance. M, permanent magnet, by which either A- or K-tonus is induced at the responding ends of the iron rod. T, tonic coil, by which A- or $\mathrm{K}$-tonic molecular dispositions may be induced in one arm of the balance.

the employment of the Magnetic Conductivity Balance (fig. 372), which I shall now describe. The magnetic stimulator, S, consists of a pair of similar coils wound in opposite directions. When a magnetising current is suddenly 
sent through these two coils, in a proper direction, two equal north-polar impulses will be generated simultaneously, and travel, one to the right, towards $\mathrm{E}$, and the other to the left, towards $\mathrm{E}^{\prime}$. In order to obtain a balance of the excitatory effects at $\mathrm{E}$ and $\mathrm{E}^{\prime}$, we keep $\mathrm{E}^{\prime}$ at a fixed distance, and move $\mathrm{E}$ backwards and forwards till the balance is found. This process of balancing will be found graphically illustrated in the records given in fig. 373. E was placed at first too near to $\mathrm{S}$, and the over-balance is seen as up-responses. The coil was then moved away very gradually, and the response of over-balance is seen at each step to undergo a diminution or approach towards balance. We next note the attainment of exact balance, where the record is seen to be horizontal. The coil is now moved still further to the right, and the con-

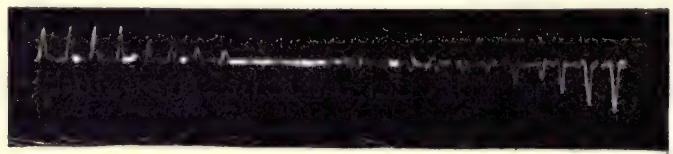

FIG. 373. Process of Balancing illustrated by Photographic Record of Responses

sequent increasing under-balance.is exhibited by the gradually increasing reversed down-responses.

Having thus obtained balance, we are able to record the variations induced in conductivity by a given agent. This is applied on the right arm of the balance, the subsequent upset of which, in one direction or the other, indicates the enhancement or depression of conductivity. Resulting upresponses will indicate enhancement, and down-responses depression. A well-known agent for the enhancement or depression of the conductivity of the nerve is the polar action of the kathode and anode. Moderate kat-electrotonus enhances conductivity, whereas the anode depresses or inhibits it. The explanation which I have already offered, regarding anodic and kathodic effects on excitability, will also be found applicable in the case of conductivity. An excitatory or kathodic effect will be facilitated in trans- 
mission, if the molecules in its path are already incipiently orientated, so that the incident stimulus finds them predisposed to respond in that direction and to transmit the excitation. Hence the kathodic effect is more easily transmitted through a tract which is in a state of $\mathrm{K}$-tonus, whereas it is retarded or inhibited under A-tonus. We shall now study the corresponding effect in magnetic conduction. Normal excitation in these experiments, it should be remembered, is taken as that which is brought about by the northpolar or $\mathrm{K}$-effect. If there is a tonic coil, $\mathrm{T}$, surrounding one arm of the balance, then, by sending a permanent current of moderate intensity round the coil, in one direction or the other, we may induce at will, in that arm, either K-tonus or A-tonus. The molecular disposition induced by the stimulus, and by $\mathrm{K}$ - and A-tonus respectively, will be understood from the diagrammatic representation given in fig. 372 . Local variation of excitability at $\mathrm{E}$ may be induced by bringing near to it either the north or south pole of a permanent magnet $\mathrm{M}$.

I shall now exhibit the enhancement or depression of magnetic conductivity by $\mathrm{K}$ - or A-tonus. A balanced record is first obtained, and $\mathrm{K}$-tonus then induced in the right arm. Successive K-make excitations are now applied, starting from the centre of the balance at $\mathrm{S}$, and proceeding onwards to left and right simultaneously. The resulting responses are recorded, records of the break-effect being

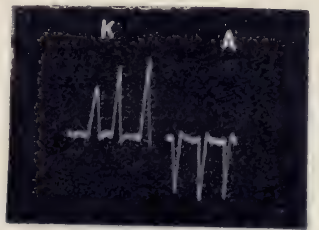

FIG. 374. Effect of $\mathrm{K}$ - and A-Tonus on Magnetic Conduction

The first series exhibit by resultant over-balance upwards the effect of $\mathrm{K}$-tonus in enhancing ' conductivity of right arm. The next series, with resultant down-responses, show depression by A-tonus.

avoided by timely interruptions of the galvanometer-circuit. It will be seen (fig. 374) from the upsetting of the balance in an upward direction, that K-tonus has induced enhancement of conductivity in the right arm. On now causing A-tonus, by reversing the current in the enclosing tonic coil, $\mathrm{T}$, a de- 
pression of conduction is induced, as shown by the upsetting of the balance in a downward direction.

We next turn to the question of the variation of conductivity induced by $\mathrm{K}$-tonus, when moderate or excessively strong; and it is here important to forecast from theoretical considerations what is to be expected under varying intensities of the polarising force. It is easy to understand that moderate $\mathrm{K}$-tonus, inducing an incipient orientation of the molecules, will predispose them to easy upset in a particular direction, thus greatly facilitating the transmission of excitation from point to point. Thus a moderate $\mathrm{K}$-tonus will enhance conductivity. But if the $\mathrm{K}$-tonus in question be excessive, so that the molecules are already distorted to their maximum position, incident stimulus can then induce no further change, and under such circumstances there can be little transmission. Hence, under increasing intensity of $\mathrm{K}$-tonus, we may expect to obtain increasing conductivity up to a certain point. But, beyond this, the conductivity will be decreased, or even actually inhibited.

These anticipations are seen fully verified in the accompanying record (fig. 375), which shows the opposite effects on conductivity of moderate

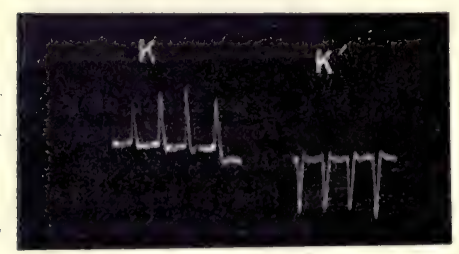

FIG. 375. Opposite Effects of $\mathrm{K}$-Tonus when moderate and strong and strong $\mathrm{K}$-tonus. The upsetting of the balance in an upward direction, $\mathrm{K}$, shows the effect of moderate K-tonus. Strong $\mathrm{K}$-tonus was next applied, with the effect of upsetting the balance in the opposite direction, $\mathrm{K}^{\prime}$. Thus we see that, while under moderate $\mathrm{K}$-tonus the conductivity is enhanced, under a much greater intensity it becomes depressed. This will, I think, be found to explain a somewhat anomalous occurrence, which has been observed in regard to the conduction of excitation through a kathodic region in nerve-and-muscle preparation. A stimulus applied on the extra-polar region in nerve is 
found to be transmitted through the kathodic area, inducing enhanced response of the indicating muscle, if the polarising current be weak. But when the intensity of the kathode is made stronger, even the strongest stimulus will fail to induce response. This is evidently due to the fact that a strong kathode induces a depression or abolition of conductivity.

Moderate $\mathrm{K}$-tonus, then; we have seen to induce enhanced conductivity, because of the favourable molecular disposition which it brings about. Even on the cessation of $\mathrm{K}$-tonus this disposition remains, owing to molecular 'retentiveness,' with its concomitant enhanced conductivity as an aftereffect. This induction of a favourable molecular disposition or habit is an interesting phenomenon, which we shall meet with again.

We shall next study the enhancement or depression of local excitability by $\mathrm{K}$ - or A-tonus. We saw, in Chapter XXXIII., that by means of the Conductivity Balance we might determine the variations, not only of conductivity, but also of local excitability. In magnetic experiments the responsive area at the right-hand end of the balance may be made either $\mathrm{K}$-tonic or A-tonic, by bringing near it one or other pole of a permanent magnet. Under induced A-tonus, the molecular excitability is depressed, and the balance upset in a downward direction; while under $\mathrm{K}$-tonus excitability is enhanced, the resulting response being upwards (fig. 376 ).

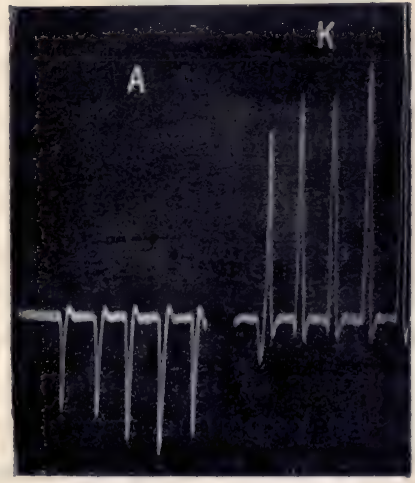

FIG. 376. Effects of $\mathrm{K}$ - and A-Tonus on Magnetic Excitability

From the resulting upset of the balance, $\mathbf{A}$ is seen to induce depression, and $\mathrm{K}$ enhancement, of excitability.

A still more interesting case is that in which the stimulus itself fashions, as it were, the path for its own conduction. The receiving coil is placed at such a distance from $\mathrm{S}$ 
(fig. 37I) that, owing to imperfect conductivity of the intervening tract, but little excitation reaches it. Excitation at S, however, distorts the molecules in its immediate neighbourhood, in a certain direction, incipiently distorting others at a little greater distance in the same favourable way. A second stimulus is therefore transmitted a little further, bringing about the same predisposition still further on. Thus an improved conducting-path is made, in a substance formerly but an indifferent conductor, by the action of the stimulus itself. In this way transmitted excitation, at first relatively ineffective, becomes increasingly effective (fig. 377). It is very interesting to note that I have obtained an effect exactly parallel in the case of nervous tissues.

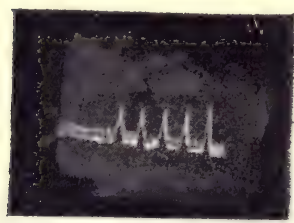

FIG. 377. Gradual Enhancement of Conductivity by the Action of Stimulus For example, when attempting to obtain the transmitted effect of excitation by mechanical response, in a vegetable or animal nerve in depressed tonic condition, the first series of tetanising shocks would induce no response. It would sometimes be only after long repetition that conductivity would be gradually restored, as seen in the initiation and subsequent enhancement of responses given at the distant responding-point:

It may be that few phenomena connected with the response of living tissues, bring home to us, so effectively as an experiment on a nerve-and-muscle preparation, the sense of the specific and mysterious character of the responsive manifestations of the living. The nerve, at its central termination, is locally excited by electric shocks, and some obscure impulse then passes through the long conducting tract to the muscle at the other end. Arriving there, this invisible nervous impulse initiates a new series of events, which find expression in visible motile indications The work performed at the responding end may be out of all proportion to the strength of the stimulus imparted at the centre. It is as if the nervous impulse tapped a relay, and 
set free a local store of latent energy. The conductor, moreover, is seemingly unlike the conductor in an electrical circuit, where the line wire and return wire must be periodically connected with terminals of an electro-motive source, for any message to be transmitted. In the nerve we have only a single conductor, without a return, an arrangement by which it would appear as difficult to send a message, as it would be to apply the two poles of a battery at the end of a single wire, in the expectation of a signal from the recorder at the far end. Inorganic matter again, is popularly regarded as susceptible only of impulses from the grosser physical forces, while the nerve-the vehicle of psychic impulses-is conceived of as played upon by forces of a finer order, and as itself modifiable, by subtler influences, notably that of its own previous history, or memory. There are, as we know, some conditions which induce such changes in the nervous channel itself, that messages from outside, previously scarcely perceptible, are accentuated. Under opposite influences, again, the conduction of impulse is interrupted. Similar results are brought about by certain agents of a polar character, like the action of anode and kathode. Under electrotonic action the transmission of impulses through the nerve may be blocked, conduction being renewed as soon as the electric block is removed. Or electrotonic action, again, may be used for the opposite purpose, of accelerating the transmission of impulses. Nothing more convincing than such facts could have been urged in support of the hyper-physical character of the phenomena in question.

But the experiments which I have described, relating to the conduction of excitatory molecular changes in a piece of iron wire, show that parallel phenomena occur in the physical domain also; and in order to demonstrate this in a striking manner, I cannot do better than describe an arrangement which I have devised, and which may be taken as an artificial nerve-and-muscle preparation. This consists of a thin iron rod for the transmission of magnetic 
excitation applied at one end, with a responding arrangement, to give motile indications, at the other. This latter consists of a secondary coil, which may be slipped over the responding point, being in series with some sensitive metallic powder in circuit with a galvanic recorder, and a voltaic cell as source of energy. The excitatory molecular disturbance transmitted through the conducting iron rod gives rise, on reaching the responding-point, to an electrical disturbance in the secondary coil connected with the motile indicator. This electrical disturbance causes secondary excitation of the sensitive substance, in consequence of which the electric conductivity of the particles becomes suddenly enhanced. By this 'relay' action the stored-up energy of the cell is suddenly released, with a consequent induction of motile response in the galvanic recorder. It is thus seen that this motile response, initiated by the transmitted stimulus, need not be proportionate to its primary exciting cause, since it may possibly be much enhanced by the amount of energy set free in the responding circuit itself. This transmission of excitation is liable, moreover, as in nerve, to be modified by subtle molecular changes induced in the conducting tract through which it takes place. Excitation may be arrested in the one case by an electrical block; and in the other, similarly, we are able to stop the transmission of a message, by means of a magnetic block. It is by no gross physical restraint that the impulse is so arrested, but by invisible molecular distortion within the rod. Molecular freedom is next restored by the removal of the magnetic block, and we find that the message, which, though constantly reiterated, was hitherto inhibited, is suddenly allowed to rush onwards and bring about the signal. 


\section{CHAPTER : XLII \\ MODIFICATION OF RESPONSE UNDER CYCLIC MOLECULAR VARIATION}

Anomalies of response-Explicable only from consideration of antecedent molecular changes-Continuous transformation from sub-tonic to hyper-tonic conditions-Two methods of inquiry, first by means of characteristic curves, second by progressive change of response-Abnormal response characteristic generally of A or sub-tonic state-Abnormal transformed into normal, after transitional B state-B state characterised by staircase response-Responses at C stage normal and uniform-At stages D and E responses undergo diminution and reversal-Responsive peculiarities seen during ascent of curve, repeated in reverse order during descent-All these peculiarities seen not only in living but also in inorganic substances, under different methods of observationElucidation of effect of drugs-Response modified by tonic condition and past history.

WE have seen that, normally, the phenomenon of response in living tissues is very definite. There are other conditions, however, under which it is found to be modified or even reversed. These abnormal effects may be brought about, either by feeble stimulation, or by changes in the responding tissue itself. Thus, though moderate stimulus evokes normal negative response, a feeble stimulus will often be seen to induce the abnormal positive, and this is most easily observed in certain particular modifications of the tissue associated with sub-tonicity. The fatigue-changes due to excess of stimulation are also, curiously enough, effective in bringing about the same abnormalities of response.

It is open to us to regard these anomalies as the result of obscure vital actions, and therefore incapable of further analysis. Or, since the phenomenon of response itself is admitted to be due to the molecular upset caused by stimulus, their origin may be looked for in the antecedent 
molecular condition of the responding substance. There is a school of investigators, again, who, appearing to discard the theory of vital action, in accounting for these changes, have substituted for it the hypothetical anabolic, or up-building, and catabolic, or down-breaking, chemical changes. And such assumptions have certainly the advantage of meeting every emergency, whether it be an expected effect or its direct opposite which occurs, for by their means it is always possible to make a reference to the one process or the other, whatever be the inconsistency involved.

As regards the interminable controversy on the physical versus chemical nature of response-phenomena, I have already drawn attention to the fact that on the border-line between Physics and Chemistry it is impossible to make any sharp demarcation. Changes, in themselves undoubtedly molecular or physical, may be attended by concomitant changes of chemical activity. An example will perhaps make this clear. We may take, for instance, the photographic action of light on a sensitive plate. This is regarded as due to chemical dissociation or break-down. If this were so, however, the effect would be permanent. But, instead of this, the latent image is liable to disappear, and in a Daguerreotype plate the after-effect of light-that is to say, the persistence of the image-has a duration of a few hours only. Such images, moreover, due to the action of light, have been found to form themselves even on elementary and inert chemical substances like gold. Here, any chemical break-down, in the ordinary sense of the word, is out of the question.

Stimulus in general we have seen to induce molecular distortion, the persistence of which is dependent on the strength of the stimulus, and also on the power of selfrecovery characteristic of the given substance. We have further seen a difference of electrical potential to be induced, as between molecularly strained and unstrained areas. When the substance, therefore, thus differentially acted upon, is placed in a suitable electrolyte, volta-chemical actions are 
necessarily set up, by which material in one part may be accreted, and in another dissolved. In this way a positive or negative image may be developed.

We have also seen, in the responses of living tissues, that while moderate stimulation induces one effect, the same stimulation, long continued, may cause the so-called fatiguereversal, such reversals sometimes, in fact, becoming recurrent. It is interesting to note that in a similar fashion a photographic plate, subjected to various durations of exposure, will give either negative or reversed positive images, or recurrences of these. ${ }^{1}$

From such facts it is clear that for the elucidation of response and its variations, we must look to its molecular antecedents, and not to its secondary chemical or other consequences. If response phenomena in general, then, are determined by molecular conditions, as such, it follows that in order to unravel the anomalies which occur in the response of living tissues, we must attempt to ascertain those conditions which induce any given variation of response in matter in general. That these phenomena are not peculiar to the response of living tissues, but take place in all matter under similar circumstances, is a fact which has been often reiterated in the course of previous chapters, and which I first pointed out in the course of my investigations on 'Response in the Living and Non-Living.' 2

In the work in question, referring to the occurrence of abnormalities in response, I said :

'Calling all normal response negative, for the sake of convenience, we observe its gradual modification, corresponding to changes in the molecular condition of the substance. Beginning with that case in which molecular modification is extreme, we find a maximum variation of response from the normal, that is to say, to positive. Continued stimulation, however, brings the molecular condition to normal, as 1902.

' Bose, ' On Strain Theory of Photographic Action,', Proc. Roy. Soc.

See Response in the Living and Non-Living (1902), pp. 129, 130. 
evidenced by the progressive lessening of the positive response, culminating in reversion to the normal negative. This is equally true of nerve and metal. In the next class of phenomena the modification of molecular condition is not so great. It now exhibits itself merely as relative inertness, and the responses, though positive, are feeble. 'Under continued stimulation, they increase in the same direction as in the last case-that is to say, from less negative to more negative, this being the reverse of fatigue. This is evidenced alike by the staircase effect and by the increase of response after tetanisation, seen, not only in nerve, but also in platinum and tin. The substance may next be in what we call the normal condition. Successive uniform stimuli now evoke uniform and equal negative responses - that is to say, there is no fatigue. But after intense or long-continued stimulation, the substance is overstrained. The responses now undergo a change from negative to less negative: fatigue, that is to say, appears. Again, under very much prolonged stimulation, the response may decline to zero, or even undergo a reversal to positive, a phenomenon which we shall find instanced in the reversed response of retina, under the long continued stimulus of light.

'We must, then, recognise that a substance may exist in various molecular conditions, whether due to internal changes or to the action of stimulus. The responses give us indications of these conditions. A complete cycle of molecular modifications can be traced, from the abnormal positive to the normal negative, and then again to positive, seen in reversal under continuous stimulation.' ${ }^{1}$

It is the molecular cycle here referred to, with the concomitant cyclic variation of response, that forms the subject of the present chapter. I shall attempt to show that the various anomalies in the response of living tissues, which were referred to in an earlier passage, may be elucidated

' In the above quotation I have, in accordance with the convention which I now uniformly follow, referred to normal response as negative, and abnormal as positive. - J. C. B. 
by this consideration. I explained, in the first chapter of the present work, the fact that the molecular derangement of matter under stimulus might be studied by recording any one of several concomitant physical changes. These are: $(a)$ the change of form-contraction or expansion; (b) the electro-motive change; and $(c)$ the variation of electric resistivity. By means of the first of these we investigated the responsive effects induced by stimulus in animal and vegetable tissues, and in the inorganic substance indiarubber. By the second, that of electro-motive variation, the excitatory change and its variations were studied in living tissues, animal and vegetable, and in inorganic bodies like metal wires. And, lastly; by variation of resistivity, we have obtained records of excitatory changes in living tissues, as also in masses consisting of metallic particles. In the last chapter, moreover, I have shown that the molecular responses of a magnetic substance may be recorded by means of appropriate magnetometric or galvanometric methods.

I shall now take up the question of the nature of those obscure molecular modifications which the response of a substance is found to undergo, and in consequence of which it exhibits variations either of intensity or of sign. The only conceivable reason for such changes would lie in some unknown transformation of the antecedent molecular condition. This being so, the next question is, whether we could possibly discover what these transformations are.

The properties of a substance at any given moment, we must remember, are not determined solely by the nature of that substance, but also by the energy which it possesses. It is obvious, for instance, that the responsive properties of matter, when its energy is depleted or its condition is a-tonic, will be different from those of matter in a higher tonic condition, and that there will be many gradations intermediate between the two. Thus, as a substance is gradually transformed, from a state of depletion to one of excessive energy, we -can see that, theoretically, there might be two possible ways of obtaining an insight into the progressive molecular 
changes occurring in it. First of these would be the continuous observation of the character of the replies marle by the changing substance to the shock of stimulus, with the progressive modification of those replies. And the second method would lie in taking a continuous record of some property of the substance, as a whole, which was undergoing a concomitant change. In the first of these modes of scrutiny the information would be obtained by an inspection of the varying responses. In the second, it would be arrived at by the examination of certain characteristic curves ;

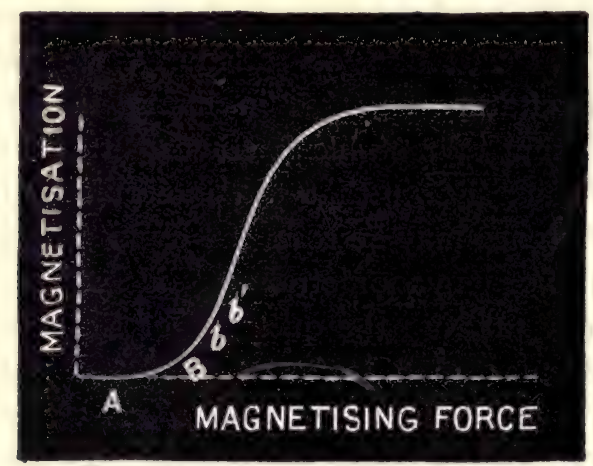

FIG. 378. Characteristic Curve of Iron under increasing Force of Magnetisation

and finally, if both these methods gave correct indications of the molecular state at the time being, then each particular response of the first method would be found to have its own place in the characteristic curve of the second. This characteristic curve will be best understood from a continuous record of the induced molecular change occurring under the action of an increasing external force. The simplest example of this is afforded by the curve which shows the relation between induced magnetisation and inducing magnetic force (fig. 378). This induced magnetisation, as will be understood, measures the amount of molecular distortion. A characteristic curve, essentially 
similar, is obtained from filings of a substance belonging to the negative class under increasing electro-motive force (fig. 379). Taking first the substance in a low or indifferent condition, we find the curve in its earliest stage, A, to be almost horizontal. That is to say, the molecular distortion induced is here very slight. We next arrive, however, at a stage, B, which I shall call transitional, where increasing force induces change at a rapid rate. In the third stage, subsequent to this, there is a decline in the rate of change, the molecules now approaching their maximum distortion.

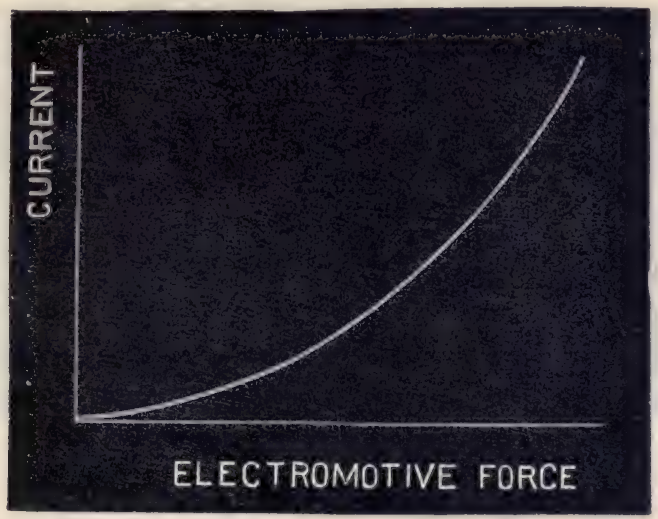

FIG. 379. Characteristic Conductivity Curve of Sensitive Metallic Particles belonging to Negative Class, under increasing Electromotive Force

These principal features are common to characteristic curves in general, slight deviations from the type being met with occasionally. In the cases given, for example, the substance starts from an indifferent condition. But it might have been in a still lower, or a-tonic, condition at starting. Under such circumstances I find that the tendency of the first part of the curve is to fall below the zero-line, crossing it, however, in an upward direction, at the transitional point $B$. When the curve, again, has reached the highest point, C, it may remain horizontal for a considerable time, or there may be a decline, owing, as we shall see, to fatigue. 
If we take a cyclic curve, recording the effects under increasing, followed by those under diminishing, force, it will be found that the forward and return portions of the curve do not in general coincide (fig. $380 \mathrm{a}$ ). Thus, when an increasing magnetising force starting from zero acts on an iron rod, and is brought back to zero, the condition of the rod at the end is not exactly the same as at the beginning. A certain amount of molecular work, which is not reversible, has been done during the cycle. A certain molecular distortion persists as an after-effect in residual magnetisation. Similarly, when

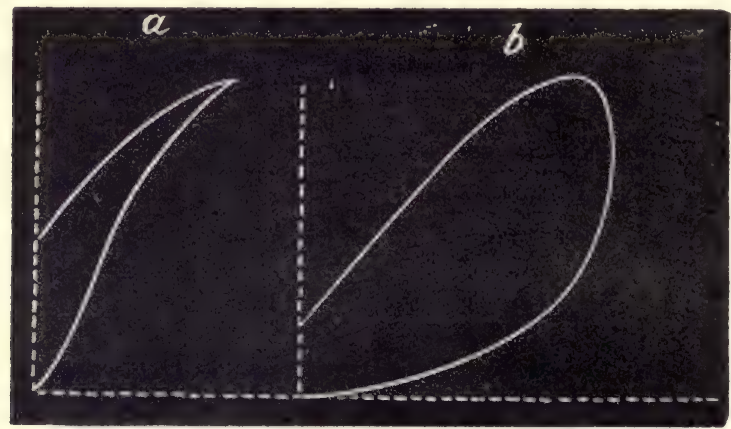

FIG. 380. Cyclic Curves of Magnetisation (a) and of Conductivity (b)

metallic particles are subjected to cyclic electro-motive variation, an after-effect is found to persist in a change of conductivity. In substances belonging to the negative class the after-effect is one of enhanced conductivity ${ }^{1}$ (fig. $380 \mathrm{~b}$ ).

Referring again to that molecular condition of the substance which is represented by $b$ in fig. 378 , we find that a new increment or accession of force will raise its condition to $b^{\prime}$. In this case the acting force has been continuously operative and continuously increasing. On the cessation of the acting force, a substance possessing marked self-recovery will fall back from $b^{\prime}$ to $b$. But if there be a certain persistence of after-effect, then a

1 Bose, 'On the Change of Conductivity of Metallic Particles under Cyclic Electro-motive Variation.'--The Electrician, September Igor. 
stimulating force which had raised the substance to $b^{\prime}$ would, when again applied, after a very brief interval, raise it to $b^{\prime \prime}$, and so on. That is to say, the molecular effect would in this case be additive. Tetanisation will thus give a curve bearing a great resemblance to the characteristic curve. This will be seen from the following record, obtained by magnetic tetanisation of steel (fig. 38I), which bears so close a resemblance to the record of electrical tetanisation of nerve ( $c f$. fig. 313). Both these curves, again, resemble the typical characteristic curve seen in fig. 378 . In

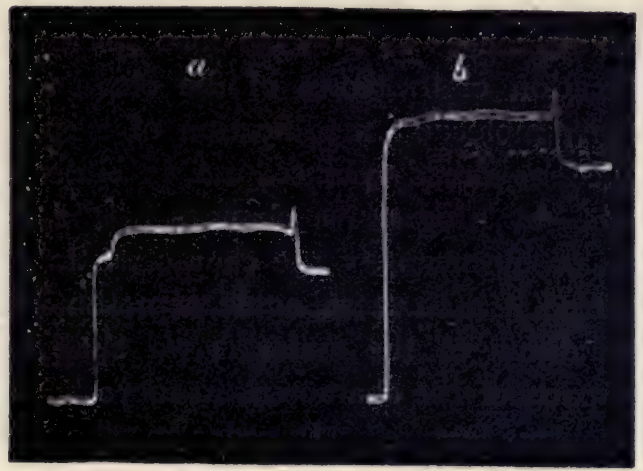

FIG. 381. Photographic Record of Magnetic Tetanisation of Steel, exhibiting Transient Enhancement of Response on Cessation

In $a$ tetanising shocks were moderate, in $b$ strong.

all these we find that the curve rises, after a longer or shorter horizontality, in an abrupt manner; that its rate of rise then undergoes a decline, the curve tending again to become horizontal ; after which fatigue-decline may be initiated. In the photographic record of the magnetic tetanisation of steel (fig. 38I), a remarkably suggestive phenomenon is observed. In that part of the tetanic curve which is horizontal the one-directioned molecular distortion, due to stimulus, is exactly balanced by the force of restitution. On the sudden cessation of tetanisation the state of balance is disturbed, and we obtain here the remarkable occurrence of a brief overshooting, or positive variation, in the curve, followed 
by recovery. This is exactly parallel to the sudden enhancement of response in the retina on the cessation of tetanising light, or to the enhancement of response in the nerve when the tetanising electrical shock is suddenly withdrawn (pp. 428, 536). From the present experiment it will be seen that the suggested explanation of the phenomenon, as due to anabolic or katabolic changes, is gratuitous.

In responding substances, where the persistence of aftereffect is relatively great, the successive shocks for the obtaining of the tetanic curve need not be repeated so quickly as where recovery is rapid. The shifting of the base-line of a series of even such responses as indicate incomplete tetanus, will give an indication of the form of the characteristic curve. The progressive molecular modification of a substance may thus be gauged, as already pointed out, in either of the two different ways-by progressive changes in the character of the response, or by means of the characteristic curve of the substance. And if both these, again, represent correctly the molecular condition of the material, we shall further find that definite parts of the characteristic curve have each their peculiar responsive features. In order to take these records of the characteristic curve and corresponding responses of a substance, moreover, we may adopt any method that is convenient-mechanical, electro-motive, magnetic, or that of the resistivity variation. The feasibility of such records is obviously a matter of the extent of the change induced and the sensitiveness of the recording apparatus. Of the various methods here mentioned, it may be said that there are no particular sources of uncertainty to be guarded against in regard to variations of resistance, of magnetisation, or of length. But in the method of electro-motive variation, as the change to be recorded is relative, being measured against a neutral or indifferent point, some difficulty occurs in securing a point which is invariable. This may be done more or less perfectly, however, by choosing an injured or killed point on the tissue for the second contact, in order that it may be subject to as little variation as possible from environmental changes. 
We shall now proceed to the description of the distinctive characteristics of certain molecular states. We may take first the case of nerve, which gives different characteristic responses under different conditions; and here, employing the simplest mode of record-namely, the mechanical-we find, as already said, that, when it is cut off from all sources of energy, the specimen is apt to fall into a condition of growing sub-tonicity. This is indicated in the mechanical

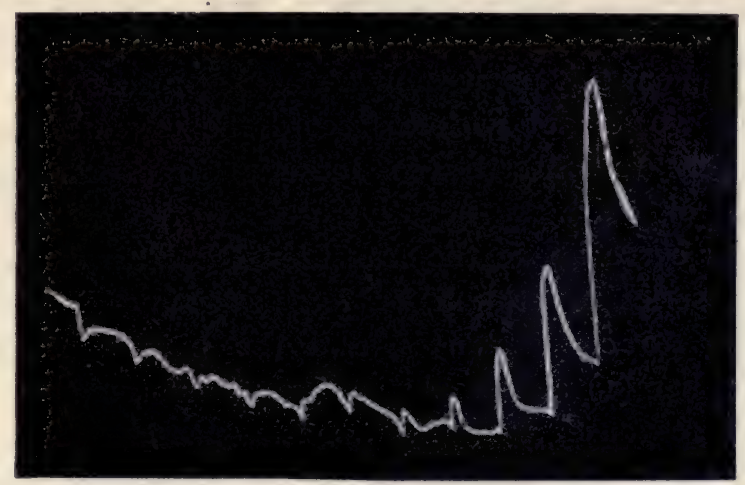

Fig. 382. Mechanical Response of Frog's Nerve to successive equal Stimuli, applied at Intervals of One Minute

The sloping line at the beginning shows growing elongation due to subtonicity. Stimulus here causes positive response. Fourth, fifth, and sixth responses are distinctly diphasic. Responses become normal and increasingly negative after the seventh, with marked staircase increases. Molecular transformation is seen to be very rapid, above the B point of transition. Record is a photographic reduction, half original size, of tracings obtained on smoked glass.

record by an increasing abnormal elongation, as in fig. 382 , given above. When the nerve is now subjected to the action of stimulus, its tonic condition is gradually restored, progressing towards a normal excitatory condition. The molecular transformation involved here is at first expressed by growing retardation of the abnormal elongation, and afterwards by gradual contraction. At the point of transition from positive to negative, or from elongation to 
contraction, that is to say, in stage B, we shall find that the rate of transformation becomes very rapid.

The second test, by which we may judge of the progress of molecular transformation in the experimental specimen, consists, as we have seen, in the nature of its reply to stimulus. Thus, in the sub-tonic condition, with its tendency to elongation, the responses are abnormal positive. From this they pass gradually, with the progress of molecular transformation, into the normal negative, the intermediate responses being either diphasic or zero. As the process is very rapid after passing the point of transition, the succeeding responses near this point show a staircase increase.

Or if we do not wish to record the intermediate series, but merely to observe the terminal transformation into negative, or enhanced negative, due to the ascent of the molecular curve above the transitional point, we may apply a rapid series of stimuli, or tetanisation. We may here, according to circumstances, and the point started from, obtain either (I) abnormal positive transformed to normal negative responses; or (2) diphasic, passing into normal negative; or (3) feeble, becoming enhanced, negative response. The idea has been put forward, as already said, that tetanisation enhances the responsiveness of the nerve, by some supposed evolution of carbonic acid. That this, however, is erroneous, has been shown by numerous experiments already related, and by the fact that even in inorganic substances, under given circumstances, tetanisation enhances response. Nor is it invariably true, in any case, that its effect is always to enhance response. Under certain conditions, it may actually cause depression. The decisive selement in the question of its effect lies in that part of the characteristic curve at which it is applied. If this be immediately after the point of transition its result will be an enhancement. Should tetanisation, however, be applied above the maximum or highest point in the curve, its effect will be the diminution of response by fatigue.

In order clearly to exhibit the fact that continuous 
molecular transformation shows itself in two different waysby a progressive physical change of the substance itself as exhibited by the characteristic curve, and also by a progressive variation in the character of the responses-I shall here give a pair of records of the mechanical response of frog's nerve. In fig. 382 the continuous molecular transformation caused by impinging stimulus is shown by the growing contraction, or responsive mechanical negativity of the nerve, as seen in the shifting of the base-line upwards. It is also interesting to notice here the continuous transformation of the individual responses from the abnormal positive through diphasic to the normal negative. There is also the noticeable additional fact that after the point of transition is passed the response undergoes a marked staircase increase. In fig. 383 is given another record, obtained with frog's nerve, where, after

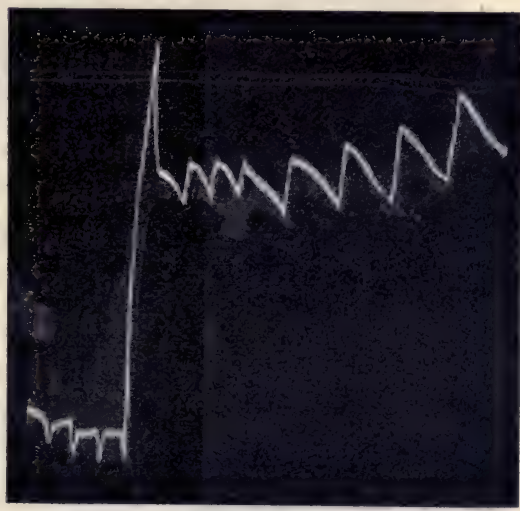

FIG. 383. Mechanical Response of Frog's Nerve, showing Conversion of Abnormal Positive into Normal Negative Response after Tetanisation

Note also the shifting of the base-line upwards, and that the individual period of positive is shorter than that of negative responses. an intervening period of tetanisation, the abnormal positive response is converted into normal negative with staircase increase. The shifting of the base-line upwards is also very noticeable here. Effects precisely similar are observed in the mechanical response of vegetal nerve.

If we now turn to a different mode of observation-say that by the electro-motive variation-the records will be found to bear a remarkable resemblance, in every particular, to those which have just been given. We find here the same 
continuous transition, from abnormal positive to normal negative, as before, through intermediate diphasic, with a shifting of the base-line upwards, exhibiting an increasing negativity. The gradual transformation of the character of the response may be seen when a long series of successive responses to successive stimuli is taken ( $c f$. fig. 277). Or the abnormal positive may be seen transformed into normal negative, after an intervening period of tetanisation ( $c f$. fig. 276). Or when the point of molecular transition is

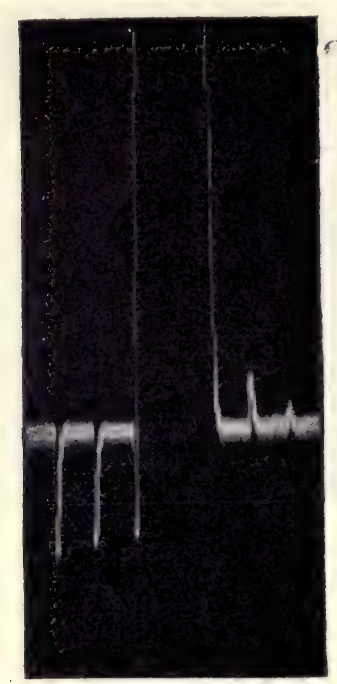

FIG. 384. Photographic Record showing Conversion of Abnormal 'Down' Response in Tin to Normal 'Up,' after Tetanisation passed, the effect of intervening tetanisation is to enhance the amplitude of response (cf. fig. 275). It will thus be seen that the characteristic response in the sub-tonic condition $\mathbf{A}$ is abnormal positive ; and that, when the substance is transformed by stimulation, to a point above the transitional $\mathbf{B}$, the response is converted into normal negative, and lastly, since the rate of transformation is very rapid above the point $\mathbf{B}$, that successive responses in that region exhibit a staircase increase, or moderate negative becomes the enhanced negative, after an intervening period of tetanisation. The underlying transformation is thus indicated by changes in the response, and also by the shifting of the baseline upwards, in exhibition of the characteristic curve. These changes, which have now been described in the case of nerve, will be found to apply in all other instances of molecular transformation equally.

Results in every way parallel are obtained with inorganic substances. In fig. 384 is seen the abnormal electro-motive response, represented as 'down,' converted into normal 'up' after an intervening period of tetanisation. In the 
next figure (fig. 385) is shown how this abnormal response in platinum, in consequence of successive stimulation, is

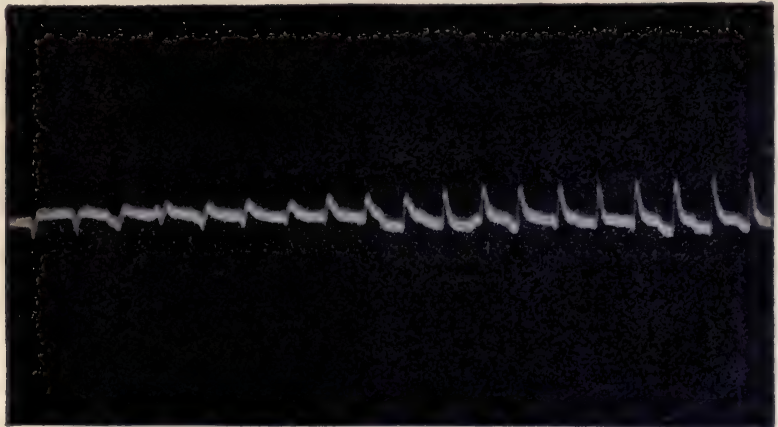

FIG. 385. Gradual Transformation from Abnormal to Normal Response in Platinum

The transition will be seen to have commenced at the third and ended at the seventh, counting from the left.

gradually transformed into a growing normal, through the intermediate diphasic. In fig. 386 is seen how the normal

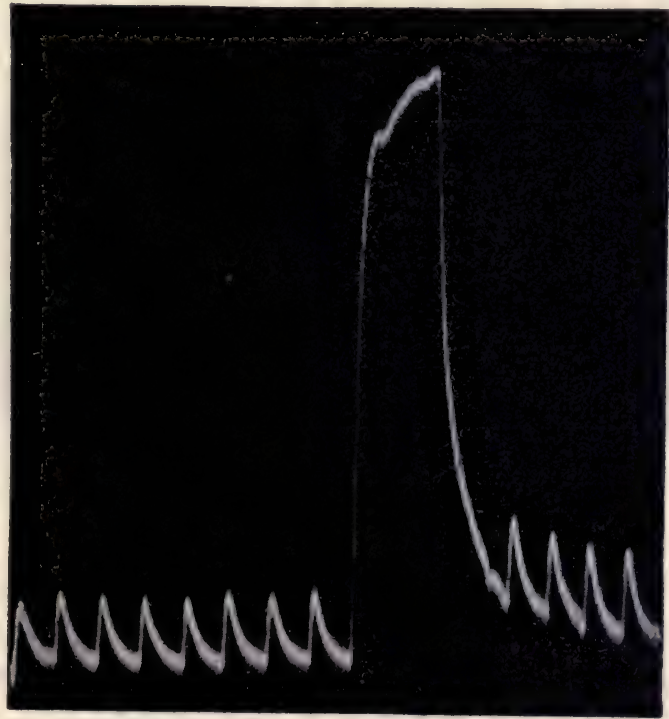

FIG. 386. Normal Electro-motive Response in Tin, enhanced after Tetanisation 
response is enhanced, after an intervening tetanisation, in a specimen of tin wire.

We pass next to the third mode of record, that, namely, by the Conductivity or Resistivity Variation. A selenium

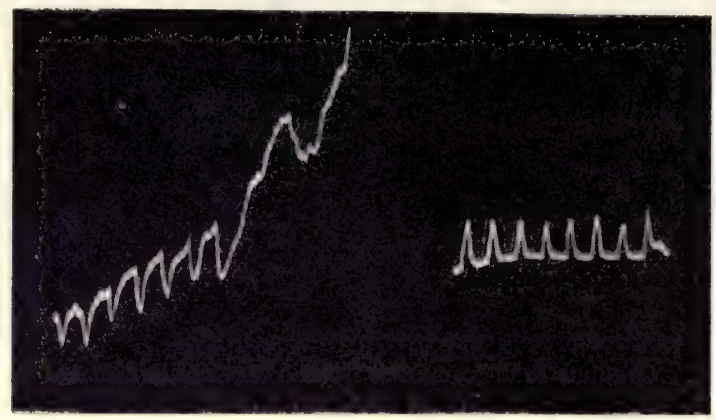

FIG. 387. Photographic Record of Abnormal Response of Selenium Cell converted into Normal after Tetanisation

Stimulus applied was high frequency electric shocks.

cell I find to be sometimes in a certain molecular condition in which it will respond to high frequency equi-alternating

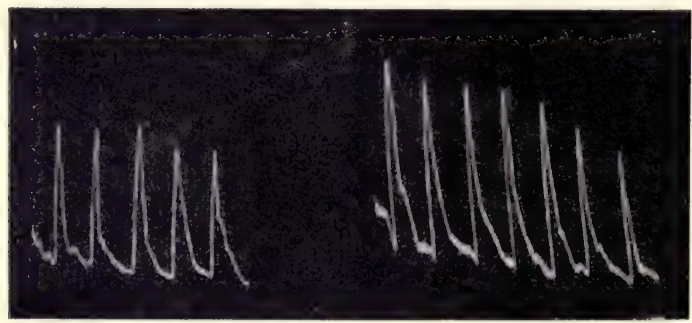

FIG. 388. Photographic Record showing Moderate Normal Response of Selenium enhanced after Tetanisation

Stimulus applied was light.

electrical shocks, of the order of a million times per second, by an increase of resistance. Tetanisation is found to induce a transformation, attended by a diminution, or negative variation, of resistance. After this, the responses are found 
to be converted into normal: that is to say, they now take place by the diminution of resistance. Fig. 387 gives a photographic record of these effects. Selenium cells, again, under normal conditions, respond to light by a diminution of resistance. After tetanisation, or continued application of light, the normal responses, under certain circumstances, undergo an enhancement. The transformation induced by tetanisation, it is interesting to note, also shows itself by the shifting of the base upwards (fig. 388).

I have found similar effects, again, to be exhibited by various metallic powders, under the stimulus of electric radiation. The record given in fig. 389 exhibits the abnormal positive response, of increase of resistance, as given by tungsten. After a short period of tetanisation the base-line is seen to be shifted upwards, the molecular condition being transformed, in a negative direction, and thus exhibiting a permanent diminution of resistivity. The response in this particular transformed condition is seen to be diphasic-positive followed by negative. A further period of tetanisation carries this transformation still further in the negative direction, and the individual responses now seen are augmented normal negative. I give also a second pair of records in which the normal response of moderate amplitude in aluminium is enhanced, after an intervening period of tetanisation (fig. 390).

We have seen, lastly, that molecular response may be recorded by means of the magnetic variation. And it is interesting to see, by employing this mode of record, that under certain conditions tetanisation will enhance magnetic response (fig. 39I).

In order to make a striking demonstration of the fact that the various phenomena described are not the result of some specific property of living tissues, with their hypothetical assimilation and dissimilation, but are determined by molecular conditions common to màtter both living and inorganic, I shall now give in verticàl columns, several series of records of responses, obtained, under parallel conditions, 
from living tissues; animal and vegetal, and from inorganic bodies. As the methods also, by which these records were

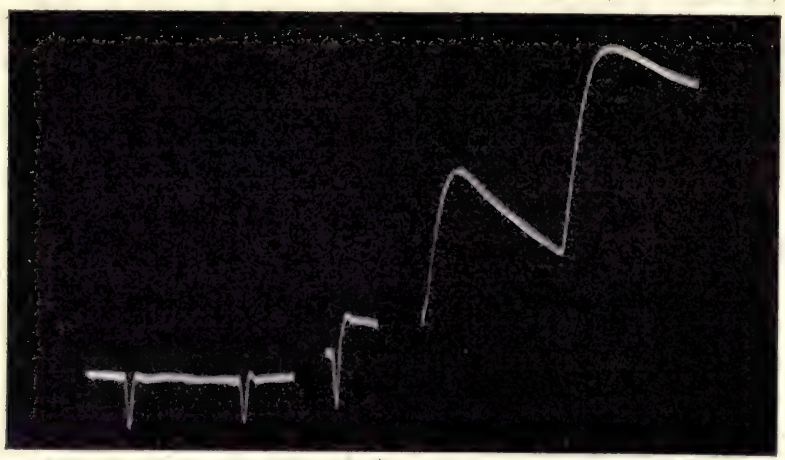

Fig. 389. Photographic Record of Abnormal Response of Tungsten to Electric Radiation, converted after Tetanisation into Diphasic and Normal

obtained, were so different as those of the mechanical, the electro-motive, the resistivity and the magnetic variations, it

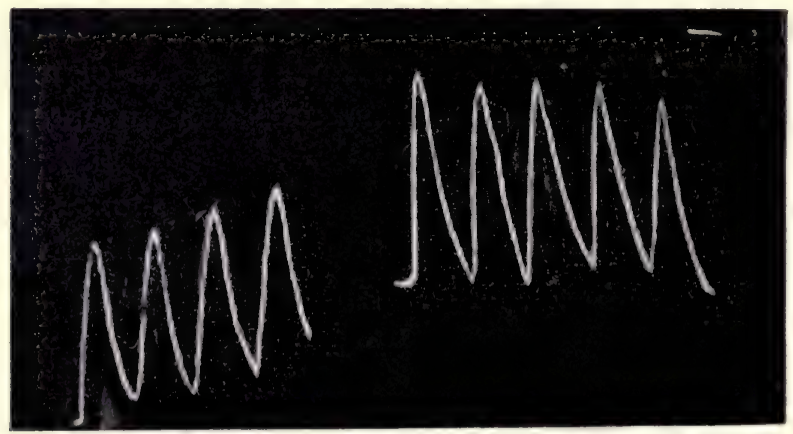

FIG. 390. Moderate Normal Response of Aluminium, enhanced after Tetanisation

follows that their similarities under parallel circumstances can only be due to certain fundamental molecular reactions, which are common to all alike. Further, since some of th 
responding substances were elementary, and the experimental arrangements offered no possibility of chemical reaction, it follows that similar responses, in other cases likewise, are determined, not 'by some antecedent chemical, but by molecular action, though chemical action may take place as a consequence of their responsive molecular derangement.

In the first of the series of records in vertical columns (fig. 392) we have: (a) a mechanical record of abnormal response by expansion,

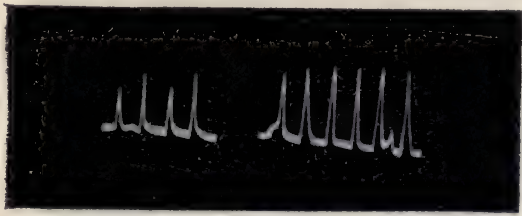

FIG. 39r. Photographic Record of Enhancement of Magnetic Response after Tetanisation

passing into normal response by contraction, after intervening tetanisation, in frog's nerve. In (b) we observe a similar transformation, as seen in the electro-motive response of frog's nerve. The abnormal response by galvanometric positivity is here converted by tetanisation into the normal negative. Turning next to inorganic substances, and taking the method of Resistivity Variation, we find in (c) the abnormal positive response of tungsten converted by tetanisation into normal negative. Finally (d) where the specimen is tin wire, and the record made by electro-

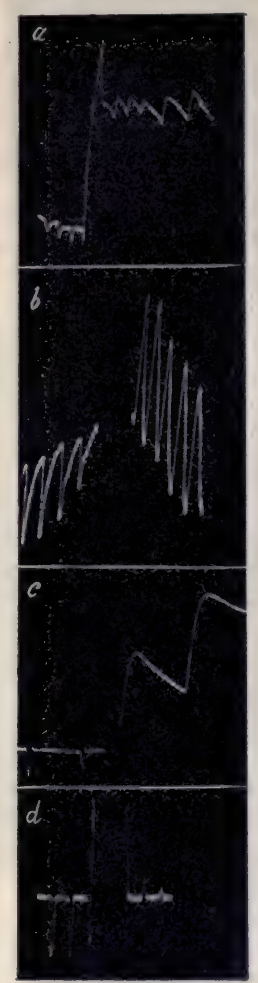

FIG. 392. Vertical Series of Records showing Transformation of Abnormal into Normal Response after Tetanisation in Living and Inorganic alike in the A phase

$a$, Mechanical response of frog's nerve to electric stimulation ; b, Electromotive response of frog's nerve to thermal stimulation; $c$, Response by resistivity variation in tungsten to electric radiation; $d$, Electro-motive response of tin wire to mechanical stimulation. 


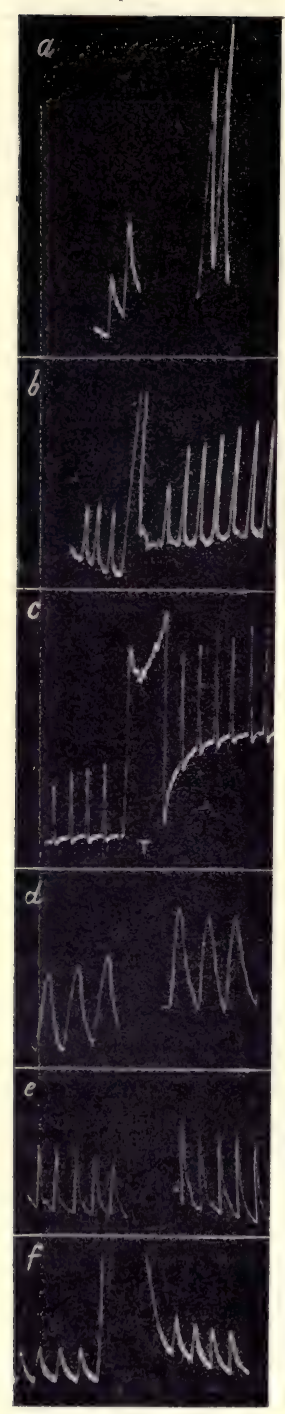

motive variation, the abnormal response is seen to be converted into normal after tetanisation. It will be noticed, in all these cases, that the antecedent molecular transformation, on which the conversion from abnormal to normal response depends, is also shown independently, by the shifting of the base-line of the record in the direction of the normal response-that is to say, upwards.

In the next series, again, in fig. 393, is shown the effect of tetanisation in enhancing feeble normal response. This moderate normal response, it will be remembered, is characteristic of the molecular condition, just above the point of transition from abnormal to normal. In $(a)$ is seen the enhancement of mechanical response in nerve of fern. In (b) we have the enhanced electromotive response of frog's nerve. In (c) a similar enhancement of electro-motive response is shown in plant nerve. In $(d)$ we see the enhancement of response after tetanisation in aluminium powder by the method of resistivity variation, the stimulus employed being Hertzian radiation. In (e) the method of record is also by resistivity variation, in a selenium cell, under the stimulus of light. And finally, in $(f)$ are given the responses of platinum wire, under the

FIG. 393. Series showing how Tetanisation enhances Normal Response in the B Phase

$a$, Mechanical response of frog's nerve; $b$, Electro-motive response of frog's nerve; $c$, Electro-motive response of plant-nerve; $d$, Response by resistivity variation in aluminium powder; $e$, Response of selenium ; $f$, Electro-motive response of tin. 
method of electro-motive variation, before and after mechanical tetanisation. The antecedent molecular transformation to which this enhancement is due may also be gauged, in all these cases, by the shifting of the base-line upwards.

We have up to this time dealt with the first part only of the characteristic curve, up to a point slightly above that of transition. The responding substance, however, in consequence of the after-effect of stimulation, now passes into

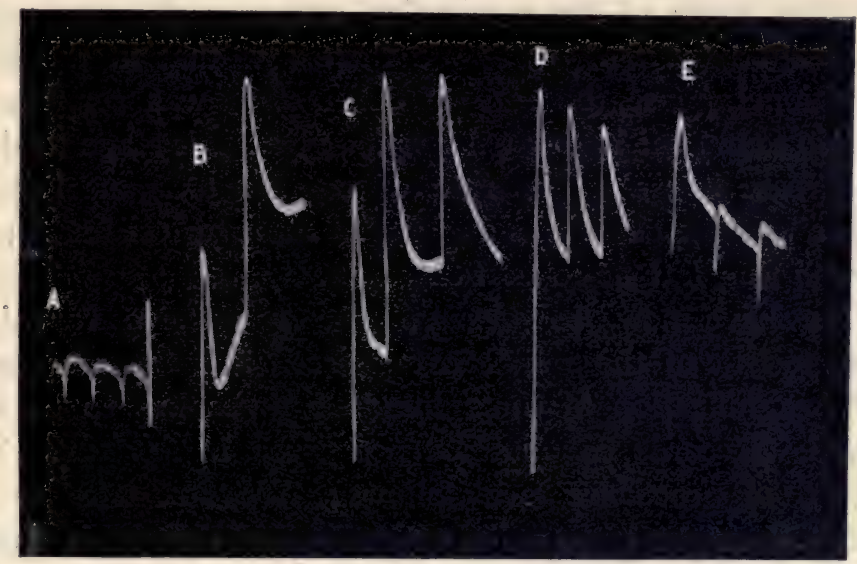

FIG. 394. Photographic Record showing Responses corresponding with different parts of characteristic curve in frog's nerve

$a$, Abnormal subtonic ; $b$, Staircase ; $c$, Uniform ; $d$, Fatigue decline ; $\ell$, Fatigue reversal.

various different phases of molecular condition. These may be short-lived, or more or less persistent.

We shall next study all the responsive modifications due to these induced molecular conditions, from the subtonic $\mathbf{A}$ to the post-maximum $\mathbf{E}$ conditions, in order. Selecting as our specimen for this purpose the nerve of frog, the different phases through which this is capable of passing may, for convenience, be divided into five classes (fig. 394). In the first of these - the abnormal A phase-the nerve is sub-tonic. It is here undergoing a relaxation, and its characteristic 
response to individual stimulus is abnormal positive. "In consequence of stimulation, however; we have seen this relaxation to be arrested, and to pass into growing contraction. The characteristic of response at this transitional stage is to be diphasic, passing gradually into the normal negative. On reaching this, the $\mathbf{B}$ phase, the responses, as we have seen, commence with feeble normal, and undergo a staircase increase. We may arrive at an idea of the rate of molecular transformation, in this and succeeding phases, from the curve of the mechanical response of nerve under tetanisation ( $c f$. fig. 3I3). We there saw that in the $\mathbf{B}$, or transitional, phase, the rate of contraction was very rapid; we also found the individual responses at this stage to show a staircase effect. The rate of contraction next became slower, and the curve was afterwards more or less horizontal. Beyond this, fatigue-relaxation set in.

We have now to observe the responsive variations characteristic of these different phases. For this purpose, a high magnification of three hundred times has to be employed. Records so obtained are given in fig. 394. The method of procedure is as follows: We first take two or three test-responses to individual stimuli of definite intensity, at the $\mathbf{A}$ phase. This test-stimulus is subsequently maintained at the same intensity. When the record of the $\mathbf{A}$ stage has thus been taken, continuous stimulation is applied for a time, till we arrive at the B stage, when the record of responses to individual stimuli is taken once more. The contraction due to the previous tetanising stimulus employed for the conversion of phase, is now so great that the recording spot of light is carried out of the field. At the commencement of each phasic record, therefore, the spot has to be brought back to the plate by suitable adjustment of the reflecting mirror. Thus the first record of each series really shows the effect of the termination of tetanisation, the subsequent records showing response to individual stimuli. We may, however, obtain some idea of the characteristic changes occurring in the nerve as a whole, by joining the tops of the 
response records. From the inclination of the line thus produced it is possible to see whether the nerve at each different phase was contracting; had assumed a stable length, or was relaxing. In the $\mathbf{B}$ phase, as here shown, for instance, it will be seen that the nerve, when undergoing an increasing contraction, shows a staircase enhancement of response ; at $\mathbf{C}$ we observe this change to arrive at a climax, with consequent stability of condition and uniformity of response.

The characteristic curve, after this, undergoes a reversal: that is to say, responsive contraction is now diminished, and eventually gives place to relaxation; and it is curious to find that all the responsive phenomena observed during the ascent are now repeated, but in the reverse order. That is to say, during the ascent of the curve we obtained the sequence of abnormal positive, diphasic, and increasing normal responses. And during the reversed process we obtain diminishing normal, diphasic, and the culminating abnormal positive response. The cycle of molecular phases, with their attendant variations, is thus complete.

An inspection of $\mathbf{D}$ shows the change in the condition of the nerve from the contracted to the relaxing state. The onset of fatigue is also seen in the diminishing amplitude of the responses. This process is seen accentuated, to the actual reversal of response, in the last phase E. I shall later give a special record exhibiting the diphasic responses intermediate between $\mathbf{D}$ and $\mathbf{E}$.

The response of nerve has hitherto been supposed, as already mentioned, to be specifically different from that of ordinary tissues. One characteristic particularly insisted upon was its indefatigability, or incapacity for fatigue, the nerve in this respect differing essentially from the muscle. On taking a general review, however, of nerve and muscleresponse, we find that there is no essential difference between the two. During the first phase of contraction, both alike show staircase increase. This is followed, in both, by a series of uniform responses. And in the stage of fatigue, in both, the process of contracture gives place to one of 
relaxation. The only difference lies in the fact that fatigue makes its appearance in the one case earlier than in the other.

When dealing with the subject of the enhancement of response by tetanisation, I stated that here it was not tetanisation, as such, which formed the determining factor in bringing about the increase of response; this was rather due to a phasic molecular transformation, induced by tetanisation. If the substance happen to be in the transitional $\mathbf{B}$ phase, then and then only will tetanisation enhance its response. If, however, it should happen to be in the optimum $\mathbf{C}$ phase, then the same tetanisation will have the effect of carrying it into $\mathbf{D}$ and $\mathbf{E}$, the phases of fatigue. The response here,

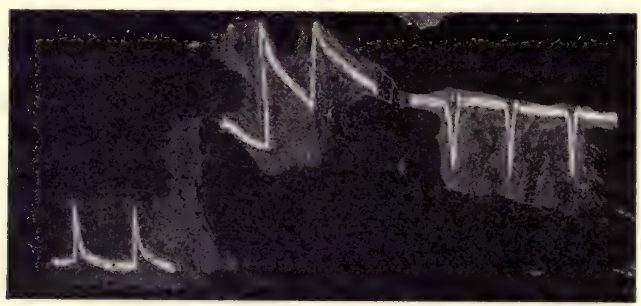

FIG. 395. Photographic Record of Response of Tungsten showing

Enhancement of Response after moderate Tetanisation, and Reversal of Response, due to Fatigue under stronger Tetanisation

instead of being enhanced, will be decreased or reversed. This is seen in the following record (fig. 395) obtained with tungsten when moderate tetanisation enhances response, whereas strong tetanisation, by bringing on fatigue, reverses the normal response.

How universal are these phenomena will be seen from the accompanying series of records, obtained not only with various living tissues, but also with inorganic substances under parallel conditions, the normal responses being in all these cases reversed by tetanisation, in consequence of the transformation from the $\mathbf{C}$ to the $\mathbf{E}$ phase. In fig. 396 (a) is seen the normal contractile response of a frog's nerve reversed to the positive or expansional, after tetanisation. 
In $(b)$ is seen the reversal of the normal electro-motive response in the digesting leaf of Drosera, after tetanisation, the stimulus here also being electrical. In $(c)$ we have reversal after tetanisation in the electro-motive response of the pulvinus of Mimosa. And in (d) is given a similar reversal after tetanisation, in the response of tungsten powder, the record being here of the resistivity variation, under the stimulus of Hertzian radiation. These, and other results already given, have been obtained by the employment of different forms of stimulation. We may, therefore, regard these characteristic transformations as brought about by all forms of stimulus alike.

We thus see that one identical stimulus may give rise to opposite effects, according to the molecular condition of the responding tissue. This molecular transformation, moreover, may be brought about by the previous action of the stimulus itself. These considerations will, I think, be found to elucidate the very obscure question of the effect of drugs, with special reference to the opposite actions of large and small doses. Since a chemical substance acts in a manner not unlike that of other stimulating agents, a moderate dose of a given reagent might be expected to induce effects similar to that of the action of moderate stimulation.

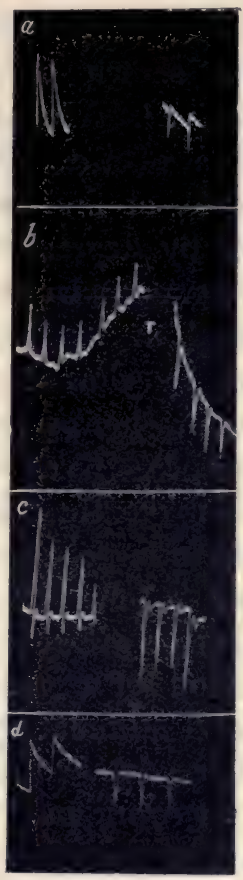

FIG. 396. Series showing reversal of Normal Response by fatigue due to strong Tetanisation inducing the $\mathbf{E}$ phase

$a$, Mechanical response of frog's nerve ; $b$, Electromotive response of Dro. sera ; $c$, electro-motive response of pulvinus of Mimosa ; $d$, Response by resistivity variation of tungsten powder.

Hence its effect in inducing molecular transformation will generally be to enhance excitability, as from $\mathbf{B}$ to $\mathbf{C}$. Too long-continued action, 
however, carrying the substance acted upon to the phase of $\mathbf{D}$ or $\mathbf{E}$, will cause depression. Or it is conceivable that the same depression might be more rapidly induced by more intense stimulation-that is to say, by a larger dose.

Now that this is what actually takes place has already been shown in several experiments which have been described. We saw, for example (fig. 95) that the continued action of the moderately stimulating agent, sodium carbonate, at first induced an exaltation of response, followed later by depression. In the case of vegetal nerve, again (cf. fig. 297), we found that the same agent, in smaller doses, caused at first an enhancement of conductivity, followed later by slow depression. A stronger dose of the same reagent, however, was found to cause rapid depression (fig. 298). Even in the case of poisons, so-called, the same facts make their appearance. Here an agent which proves toxic in large, appears as a stimulant when given in minute, doses. Thus in studying the effect of various chemical agents on growth-response, I found that while a one per cent. solution of copper sulphate was toxic, the same reagent proved stimulatory, if given in a solution of $\cdot 2$ per cent. A more detailed account of these experiments will be found in my work on 'Plant Response,' from which I quote the following summing up :

'A survey of the effects of drugs, both stimulating and poisonous, reveals the striking fact that the difference between them is [often] a question of quantity. Sugar, for instance, which is stimulating when given in solutions of, say, I to 5 per cent., becomes depressing when the solution is very strong. Copper sulphate, again, which is regarded as a poison, is only so at I per cent. and upwards, a solution of $\cdot 2$ per cent. being actually a stimulant. The difference between sugar and copper sulphate is here seen to lie in the fact that in the latter case the range of safety is very narrow. Another fact which must be borne in mind in this connection is that a substance like sugar is used by the plant for general metabolic processes, and thus removed from the 
sphere of action. Thus, continuous absorption of sugar could not for a long time bring about sufficient accumulation to cause depression. With copper sulphate, however, the case is different. Here, the constant absorption of the sub-tonic stimulatory dose would cause accumulation in the system, and thus ultimately bring about the death of the plant.' ${ }^{1}$

From all this it is clear that the progress of medicine may be greatly facilitated when the attention of investigators is drawn to the importance of the molecular aspects of the phenomena with which they have to deal. Thus, in examining the action of drugs, a threefold question is seen to arise. It must first be determined what is the nature of the responsive molecular change induced by the given reagent under normal conditions. The second matter of inquiry is, What is the critical dose, above and below which opposite effects may be expected? And, finally, as the nature of the response has been seen to be influenced by the part of the molecular curve, at which the responding tissue has arrived, when the chemical reagent is applied, it rollows that an important element in the problem lies in the determination of the tonic condition of the tissue. How important is this last factor will be seen from an experiment to be described at the end of the present chapter, where an identical course of treatment "in one condition of the tissue revives it from inanition, and in another hastens its death.

I have shown that when the condition of the substance is transformed from $\mathbf{C}$ to $\mathbf{E}$, the response also is reversed from normal negative to abnormal positive. I shall now, therefore, proceed to show that in the course of this transition there is an intermediate stage of diphasic response. Before exhibiting this in the case of nerve, I shall give an interesting record in which the same thing is seen to take place in the mechanical response of fatigued indiarubber. Before the onset of fatigue, the normal'contractile responses were large, but at that stage-that is to say before the record

\footnotetext{
${ }^{1}$ Bose, Plant Response, p. 488.
} 
commences - they had begun to decline. In the series then recorded (fig. 397) we see how the depressed contractile responses are gradually transformed into abnormally expansive, through an intermediate diphasic.

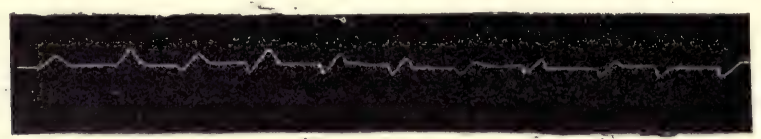

FIG. 397. Fatigue in Indiarubber giving rise to Diphasic and Reversed Responses

In the next series of mechanical records, obtained from nerve of frog (fig. 398), we have results exactly similar. The depressed contractile negative here passes through diphasic to abnormal positive. Thus, during the descent of the characteristic curve we obtain, as has been said before, a

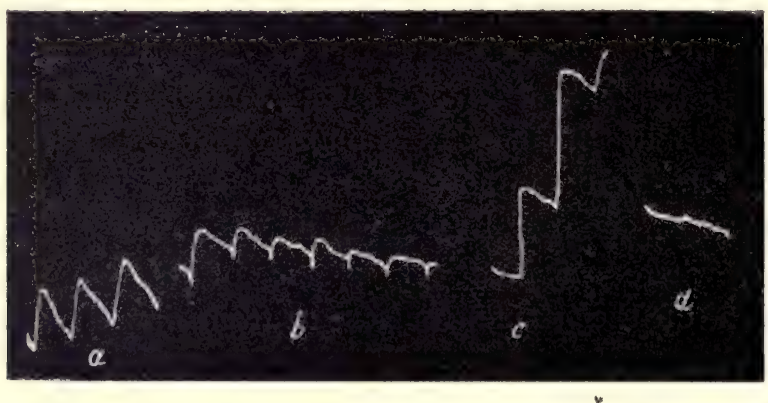

FIG. 398. Fatigue inducing Diphasic Variation and Reversal of Normal Response in Frog's Nerve

$a$, Diminished normal response; after tetanisation, enhanced fatigue induces diphasic passing into reversed positive response, $b$; a period of rest after this revived the normal response in i ; after long-continued tetanisation, response is seen to be abolished in $d$, by the death of nerve.

repetition, but in reverse order, of all the phenomena seen during the molecular ascent; the sequence of responsive variation was from abnormal positive, through diphasic, to increasing negative. During the descent the sequence is diminishing normal response, diphasic, and abnormal positive. 
The two halves of the cycle are thus strangely alike, one being, as it were, a reflection of the other. The cycle begins with sub-tonicity, due to a deficit of absorbed stimulus, and ends with the abnormality caused by excess of stimulation. The starting-point of the one may be supposed to meet the end of the other in a common fatality. The tissue comes to the same death by inanition on the one hand, through lack of stimulation, and by fatigue, on the other, through overstimulation. But though the one half thus mimicks the other, there is, as it were, a polar difference between the two, by reason of the difference in their past histories. To revive the dying tissue, in the beginning of the cycle, stimulation is necessary ; to revive it afresh, at the termination of the cycle, a period of rest is essential. 


\section{CHAPTER XLIII}

\section{CERTAIN PSYCHO-PHYSIOLOGICAL PHENOMENA-THF, PHYSICAL BASIS OF SENSATION}

Indications of stimulatory changes in nerve: I, Electrical; 2, MechanicalTransmission in both directions-Stimulatory changes in motor and sensory nerves similar-Responsive molecular changes and the correlated tones of sensation-Two kinds of nervous impulse, and their characteristics-Different manifestations of the same nervous impulse determined by nature of indicator -Electrical, motile, and sensory responses, and their mutual relations-The brain as a perceiving apparatus-Weber-Fechner's Law-Elimination of psychic assumption from explanation of particular relation between stimulus and resultant sensation-Explanation of the factor of quality in sensationExplanation of conversion from positive to negative tone of sensation after tetanisation-Various effects of progressive molecular change in nerve-Effects of attention and inhibition-Polar variations of tonus, inducing acceleration and retardation.

IT is admitted that the molecular changes induced in the nerve by stimulus, are followed by sensations perceived in the brain. The question as to the nature of these antecedent changes induced in the nerve, and the quality of the sensation that succeeds them, falls properly, then, within the scope of a physiological inquiry; and it is certain aspects of this which will be treated in the present chapter. I may here point out that the results which I have to describe consist of deductions drawn from direct experiment. They will in some cases lend support to the psychological hypotheses already advanced; while in others they will be found to be opposed. In such cases, therefore, it is perhaps not too much to hope, from their strictly experimental character, that they will prove of use in deciding between rival theories; while in others they will be found to introduce facts and considerations which are entirely new. 
Referring to the excitatory changes on which sensation depends, there has been much discussion as to whether the effects of stimulus in efferent and afferent nerves are of the same or of different natures. The difficulty in deciding this point lay in the fact that the indications of the state of excitation are different in the two cases, one exhibiting it objectively by the motile effect, and the other subjectively by sensation. It has been supposed, as we have seen, that the excitatory changes transmitted by the nerves were unaccompanied during their progress by any direct visible indications. It has been shown, however, in the course of previous chapters, that a change of form does in fact accompany the transmission of the wave of excitation along the nerve. It was also shown that this mechanical indication could be rendered extremely delicate, ranking, in degree of sensitiveness, between the galvanometer and the brain. Employing this mode of investigation, then, we found not only that the wave of excitation might be transmitted in either direction in any given nerve, but also that the changes induced by stimulus were similar in afferent and efferent nerves (p. 529).

Regarding the nature of this molecular change, again, it has been supposed that the nerve under excitation exhibited a specific variation, known as the neural, totally unlike those changes which take place, for instance, in muscle. We have seen, however, that this is not the case, the mechanical and electrical expressions of the molecular changes in excited nerve being of a nature essentially similar to those observed in muscle also. Even in the matter of conduction, we have seen that non-neural tissues transmit the state of excitation to a certain distance beyond the point of stimulation. The difference in this respect is one of degree, and not of kind.

We have next to deal with the question of sensation as induced by molecular changes in the nerve. It is widely admitted that the changes induced in the nerve by stimulus will cause responsive sensations. But the relation between 
the responsive sensation and the character of the molecular change that induces it has been regarded as unascertainable. ' That many of our feelings depend immediately upon the condition of the nervous elements is beyond doubt.... What is the peculiar nature of the excitation upon which the different feelings depend for their differences of quality? What is the characteristic change in the excitation that gives rise to two kinds of tone which the feelings possess, to pleasure and to pain? Physiological psychology can answer none of these questions with much confidence.' '

The fundamental contrast of tone in question raises the inquiry, therefore, whether it may be possible to discover any antecedent nervous changes of opposed character. Taking an instance of response by some simple form of sensation, it is well known that while moderate stimulus produces a feeling which may be described in general as not unpleasurable, or even distinctly pleasurable, an intense stimulus of the same nature will cause a displeasurable or even painful, sensatior. These fundamental differences of quality are classified as 'positive and negative tones' of sensation, the term 'positive' being here associated with perceptions which are not unpleasant, or even actually pleasant, while 'negative' refers to the reverse. While the sensations ensuing under moderate stimulus, then, such as moderate pressure or moderate light, are of 'positive' tone, those brought about by more intense stimulus are apt to become converted into negative. The positive sensation grows to a maximum, according to the rise of stimulus-intensity within a certain limit. Beyond this point, sensation becomes, first, less and less positive, and then increasingly negative, as the intensity of stimulus continues to be augmented. Or a simple stimulus, such as a light blow, which evokes a positive sensation, will, when often repeatedthat is to say, when employed tetanically induce a negative or painful sensation. It is thus seen that the tone of sensation 1s in some way associated with the intensity or duration of

- Ladd, Outlines of Physiological Psychology (1891), p. 387. 
stimulus. The question, however, remains, whether or not these opposite sensation-tones could be demonstrated to be dependent upon characteristic nervous changes of opposed characters. If we should succeed in making such a demon. stration a physico-physiological basis of psychical effects would have been established which would unquestionably prove to be of great value.

Now we have seen, referring to previous investigations on nerves, (I) that a feeble stimulus applied to the nerve is transmitted as a pulse of expansion. This we have designated the positive wave. The propagation of this wave being more or less of the nature of a hydrostatic disturbance, we have seen that its transmission is not affected to any great extent, even when the conductivity of the tissue is diminished. (2) A more intense stimulus we have found competent to give rise to a disturbance of opposite or negative sign - that is to say, to a pulse of contraction. The velocity with which this second, or, as we have called it, the true excitatory wave, was conducted, we found to increase with the intensity of the stimulus. While with feeble stimulus the positive wave alone was transmitted, with stronger, both negative and positive were propagated, but the more intense negative was liable to mask the feeble positive. As the negative wave was dependent on the conductivity of the tissue for its propagation, we have seen that it was possible to separate the two by any means which would diminish the conductivity of the tissue. By such means the negative could be made to lag behind the positive; or, by its complete suppression, it was even possible to exhibit the positive alone. Thus a tissue which normally gave only negative response, owing to the masking of the positive, might, by the depression of its conductivity, be made to give diphasic, or positive response alone (p. 530).

So far then, as regards the detection of two nervous impulses of opposite sign by means of the delicate mechanical method. The same facts may also be demonstrated by the less sensitive method of electrical response, according to 
which we saw that the two nervous impulses were exhibited by two opposite electro-motive variations - those of galvanometric positivity and negativity respectively. This reaction of expansion and galvanometric positivity, however, may also occur as the expression of the increase of internal energy, in whatever way produced. Indeed, the positive form of response under moderate stimulation may be regarded as a case falling within this definition. Thus we see that, beginning with very moderate stimulus, we obtain in the tissue a purely positive effect; and that, as the stimulus is augmented, the true negative excitatory effect also begins to make its appearance in increasing degree, the positive component of the response being now more or less masked. The energy that afterwards remains latent in the tissue goes to enhance the tonic condition. The amount thus held latent depends on the difference between income and expenditure. As a general rule, it will be under intense stimulation that the expenditure of energy will be likely to exceed the income. Thus we have two extreme cases, first, that in which moderate stimulus brings about increase of energy ; secondly, that in which excessive stimulus brings about run-down of energy; and between the two a large range of variation, within which either one condition or the other may predominate. It must, of course, be understood that anything which increases the tonic condition is for the well-being or health of the organism, and is associated with positivity. Similarly, any fall of the tonic condition below par makes for exhaustion and against healthy tone.

We have next to take a rapid survey of the changes induced by stimulus in the conducting nerve itself, or any of its attached indicators. Such variations may, for purposes of convenience, be classified as motile, electrical, and sensory. In the nerve itself we have found, as has already been pointed out, by means of the Kunchangraph, that the motile change induced by feeble stimulus was one of expansion, the same change being shown electrically by galvanometric 
positivity. The change induced by strong stimulus, on the other hand, was of contraction and galvanometric negativity.

In the terminal motile indicator also, there are two different modes of response of opposite signs-namely, expansion and contraction. In the highly excitable muscle, the occurrence of the former of these, brought about, as it is, by very feeble stimulus, is not easy to demonstrate. Bearing in mind, however, the fact that in nerve positive response is more easily obtained when the excitability is depressed, I succeeded in obtaining positive expansional response of the muscle, in a nerve-and-muscle preparation of frog, which had been depressed by the anæsthetic action of chloroform. At a certain stage of anæsthetisation, the response of the muscle under stimulation of the nerve was found to take place by expansion, followed by recovery. Just as in a nerve in a somewhat depressed condition, successive stimuli evoke positive response converted later into normal negative, so, in the muscle-pre-

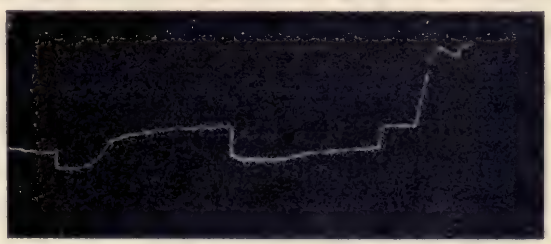

Fig. 399. Abnormal Response of Muscle by Relaxation, followed by Normal Response of Contraction

The first two responses by relaxation are followed by two contractile responses. paration described, the abnormal positive was followed by the normal negative response. In fig. 399 I give a photographic reproduction of the myographic record obtained on a smoked-glass surface.

In all these different effects we obtain, by means of the mechanical response of the terminal organ, what is merely a parallel expression of changes occurring in the nerve itself. As in the nerve, so also in the muscle, there are two different kinds of responsive expression-namely, expansion and contraction. Thus we see that the various manifestations registered by different modes of indication are only so many diverse expressions of the same fundamental molecular changes. 
We turn next to the sensory mode of indication, that is to say, to the psychic effects registered in the central perceiving organ by the positive and negative waves conveyed to it along the afferent nerves. We have already seen that the stimulatory changes induced in these sensory nerves are precisely the same as those which occur in the efferent. What, then, are the effects in the central apparatus induced (I) by that positive impulse which is associated with expansion, and (2) by the negative impulse associated with contraction?

It is a matter of universal experience, as already mentioned, that feeble stimulus gives rise to sensations of positive or pleasurable tone, while an intense stimulus of the same kind will induce a responsive sensation which is negative or painful. We have also seen, in the course of the present work, that a feeble stimulus will give rise to a wave of expansion and galvanometric positivity, while the same stimulus, when intense, will give rise to a negative wave. We are therefore justified in regarding the positive impulse, associated with expansion and galvanometric positivity, as pleasure-bearing, and its opposite as pain-bearing or doloriferous. Numerous experiments-some being of a crucial character-will be given, in the course of the present and succeeding chapters, which will be found to lend full support to this conclusion.

This fact, that the same stimulus may induce positive sensation in the central and expansion in the motile organ, or the negative painful sensation with muscular contraction, according only to the nature of the indicator, will furnish grounds of reconciliation to those who hold on the one hand that the motor reaction is secondary to the mental, and on the other, that sensation is merely an accompaniment of movements reflexly induced. ${ }^{1}$

1 'Many hold the motor reaction to be secondary to the mental. Of the coarser emotions it has been argued by James that the feeling does not cause, but is caused by, the bodily expression. The bodily changes, according to him, follow directly the perception of the exciting fact, and our feeling of the same 
If the sensation be in fact due to definite and ascertainable physico-physiological changes in the nerve, then the various modifications of sensation must, in like manner, be traceable to corresponding modifications in the physico-physiological process. In that case, the particular relation which is known to exist as between stimulus and sensation-expressed as Weber-Fechner's Law - must be demonstrable as directly dependent upon molecular changes induced, and not on the existence of some assumed psychic factor. This molecular theory, further, if it expresses a truth of universal application, ought to be capable of explaining not only the quantitative relation between stimulus and sensation, but also that qualitative variation of which Weber-Fechner's Law is unable to take account. Should the Molecular Theory prove adequate to this, its truth may be regarded as demonstrated.

We shall, however, subject this theory to further and still more crucial tests. If it be true that our sensations, painful and pleasurable, are due to nervous impulses of opposite character, then any modification of either of these impulses by any given agent should appropriately modify the resultant sensation. We have seen, for instance, that the negative wave is complex, and contains within it the masked positive. We have also seen that by appropriate means these two waves may be made to exhibit themselves separately; or the positive, by the total suppression of the negative, may be displayed alone. I shall therefore show that, by the employment of the same means, the subjective sensation of painful or negative tone may also be analysed into its component parts, which may thus be made to exhibit themselves in succession; and, on the other hand, that by

changes as they occur is the emotion. Certain experiments furnish evidencenot highly satisfactory-that all pleasurable states of consciousness are accompanied by bodily movements of extension, and all painful by movements of flexion. These movements may be very slight. Münsterberg concludes that the feeling of agreeableness is the mental accompaniment and outcome of reflexly-produced movements of extension, and disagreeableness of the movements of flexion.'--Schäfer, Text-Book of Physiology, vol. ii. (1900), p. 975. 
the complete obliteration of the negative element an already painful sensation may be converted into pleasurable.

Of the scheme thus laid down, the first part will be carried out in the present, and the second in the succeeding, chapters.

Turning now to our sensations themselves, it may be well to consider some of the characteristics of the central perceiving apparatus. As a detector of nervous changes the brain is undoubtedly the most delicate of instruments, surpassing in this respect not only the galvanometer, but also the Kunchangraph. It fails, however, strictly speaking, as an accurate metrical apparatus. It is not able to discriminate quantitatively, for instance, by means of sensation, through any wide range, between the finer differences of intensity in the nervous impulses it receives. In the painand-pleasure series, again, the distinctions which it is able to make are, to a certain extent, of a merely qualitative character, unmistakable only as between the two extremes of the series, the intervening region tending to be somewhat indefinite. The sensitiveness of the physical instruments, Kunchangraph and galvanometer, is always constant and reliable. For example, in the galvanometer, by adjusting the controlling magnet, we can obtain varying degrees of scnsibility, which at any particular adjustment will remain constant. But in the perceiving apparatus, not only is the sensitiveness of different individuals widely different, but even in a single individual it undergoes great variation under different conditions.

By deliberate attention or inhibition, as by raising or lowering of the controlling magnet in the galvanometer, the sensitiveness of the perceiving field can be almost indefinitely varied. Pursuing this analogy of the galvanometer further, we find that in the brain, instead of a single coil, with its one pair of terminals, there are many coils with many pairs of terminals, receiving impulses from every part of the organism. Confining our attention, moreover, to any single circuit among these, we find again that the impulses it 
conveys are varied in their character. There are, for instance, the immediate effects of stimulus, whether positive or negative, and also the persistent after-effects of stimuli previously absorbed. The central apparatus, however, is not acted on by these impulses from any single circuit alone, but from many circuits at the same time, the whole resulting in a vague tremor of generalised consciousness. The individual sensation evoked by any particular stimulus bears to the rippling surface of this consciousness the relation of a larger or smaller wave.

Thus we see that there are two conditions which will contribute to the intensity of the sensation evoked by an individual stimulus. There will be, first--to revert to the simile of the galvanometer-the enhancement of the conductivity of the particular circuit involved; and, secondly, the suppression of all interference caused by the semiconscious activity of other circuits. By the action of the will, producing the condition of attention or expectation, the excitability of the receptive or responsive points, and the conductivity of particular channels, may be exalted, while they may be depressed in others by the reverse process of inhibition. The extent to which it is claimed that this power of inhibition may, with practice, be carried, would appear almost unimaginable. I have myself known of an authenticated instance in which the pulsation of the heart was arrested and renewed at will. In India, indeed, it has been held, from very remote times, that such practices are capable of reduction to a science. It is thus believed to be possible that all nervous impulses due to external causes may one by one be inhibited, until the attention is concentrated on a given point, in complete isolation from any interference whatever by the physical organism. Regarding the physical aspects of these processes of inhibition and concentration, more will be said at the end of this chapter.

There are again other elements calculated to bring about further variations in the sensitiveness of the instrument which lie more or less beyond the control of the observer. 
His previous habits and prepossessions all contribute, as is well known, to modify it more or less permanently. The principal reason for the constancy of the records made by the physical indicators lies in the greater or less constancy of the properties of those elements of which they are composed. Even here there is a fluctuation of sensitiveness, owing to changes in the properties of the material. But these changes are neither so rapid nor so considerable as in the neural apparatus, whose excitability is extremely susceptible of modification under the influence of fatigue, and of such varying factors as health and tonicity, as well as by the action of the stimulus itself.

Looking now at the whole range of impulses generated in the nerves under increasing stimulus, we shall see that the positive effect is gradually augmented till it reaches a maximum. It then undergoes a decline, passing into a resultant negative. This resultant negative response contains, as we know, a masked positive component, which can be separated and exclusively demonstrated by appropriate methods. With increasing stimulus the negative response undergoes an enhancement till a limit is reached. Expressed in terms of sensation, then, the effect perceived is at first of positive tone, and this, growing in intensity, is pleasurable. This positive tone, however, afterwards undergoes a diminution, and finally passes over the zero-line; this constitutes the commencement of a somewhat extended range, in which the resultant negative, with its masked positive, undergoes increase. Referring to the curve at the crossing of the zero-line, it must be said that we have here a neutral point. This is not, however, the same as that absolute zero at which the curve of sensation was initiated, for here the neutrality is not due to absence of effect, but to the fluctuating equilibrium of two opposite effects, one positive and the other negative. It is thus to be understood that, while in the positive region the tone is simple, in the region of the resultant negative it is complex, the sensation here being compounded of positive and nega- 
tive (pleasure-pain). In this region, then, it is theoretically possible to bring out the positive element alone, by suppressing the negative. It is the predominance of either one of the two components which at any given moment determines the pleasure-pain character of the complex sensation. At a certain critical point it is the element of pain which will begin to appear as relatively conspicuous, this proceeding towards a climax with increasing stimulation.

It must be understood that we are dealing in general with nervous response under normal conditions of excitability. It will be sufficient here to make a cursory reference to certain exceptional cases which may occur. We have found that the character of the response given by a tissue is determined by the two factors of (I) the effective intensity of stimulus, and (2) the excitability of the responding tissue. If the nature of a given stimulus be such as to produce but a moderate effect, there will be a greater likelihood of its evoking only positive response. Or the responding tissue, in another case, may possess exceptional excitability; hence the responsive indication here will tend from the beginning to be negative. The different effects depending on the varying excitatory characteristics of the tissue, we have already seen illustrated in several cases. The slightly excitable epidermis was seen to give a predominantly positive response, whereas nerve which had been rendered highly excitable tended, on the other hand, to give negative response.

We shall now proceed further to consider the correspondence between the responsive sensation and the degree of nervous change induced by stimutus. And here the first question that arises is that of the relation which sensation bears to the intensity of the stimulus that provokes it. The difficulty of this investigation lies in the generally unsatisfactory character of the subjective standard, and in the variations induced by stimulus itself in the sensitiveness of the neurile elements.

According to what is known as Weber-Fechner's Law, the strength of stimulus must increase in geometrical ratio 
in order that the intensity of the sensation may increase arithmetically. The method of experiment on which this result was based is not, however, altogether unexceptionable. Against the generalisation itself, many objections have been urged, and even its supporters claim for it only a very limited range of application. It has been pointed out that Fechner starts with the assumption that the change of sensation under varying intensities of stimulus is merely quantitative; he does not take into account that the quality or sign of sensation is also liable to change.

Confining our attention to that range of sensation which does not involve any change of quality, it is urged that, starting from the minimally effective intensity of stimulus, excitation at first increases very rapidly, and subsequently more slowly, under successive equal increments of intensity. This particular complexity led Fechner to suppose that such complex phenomena as the quantitative relation between stimulus and sensation were not determined by mere physical or physiological factors. He therefore maintained that his generalisation expressed an ultimate law concerning the relation between physical stimuli and psychical reactions, or, in other words, between body and soul.

It is now possible, however, turning away from speculative hypotheses, to subject this question to direct and simple experiment. We have seen how delicate and free from complication is the record of the mechanical response of nerve. In these tracings we have the record of the direct effect of stimulatory action on the nerve itself. In order, then, to study the effect of stimuli of varying intensities, I first chose a specimen of the sciatic nerve of gecko. A sliding electric inductorium was used for the purpose of stimulation. From a previous experiment with a ballistic galvanometer the absolute intensity of the electric shocks at different distances of the secondary from the primary was calibrated. Marks were then made on the sliding base, which gave various intensities of stimulation-1, 2, 3, 4, 5-and so on. Thus, keeping the duration of the exciting 
primary current the same, and bringing the secondary nearer and nearer the primary, the intensity of the stimulus could be gradually increased quantitatively. The effective intensity of stimulation might also be increased in a graduated manner by fixing the primary inside the secondary, and gradually increasing the duration of stimulation.

The following record shows the effect of stimuli increasing from one to ten, by increments of one at a time (fig. 400). It will be seen that at the beginning, owing to growing sub-tonicity, the nerve was undergoing a gradual relaxation, the downward slope of the beginning of the record. On application of stimulus of intensity as shown by $\mathbf{I}$, there is a sudden responsive relaxation, followed by a partial recovery. As the intensity of stimulus is successively increased, the positive response undergoes an increase, the maximum-positive response being evoked when the stimulus-intensity is 3 .

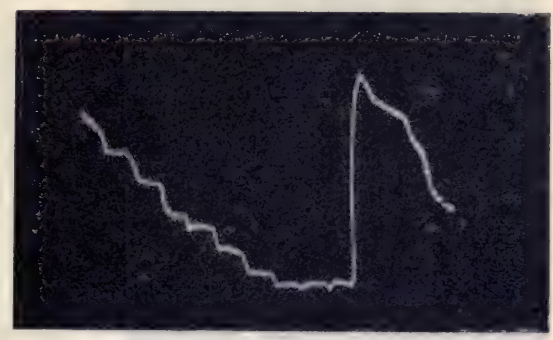

FIG. 400. Record of Response in Nerve of Gecko showing the Effect of Arithmetically increasing Stimulus After this the amplitude of response undergoes a progressive decline, the response to stimulus 8 being practically zero. This neutral point, as was shown earlier, is not to be regarded as the true zero, being in fact the balancing point of positive and negative. This will be seen when we inspect the record of intensity 9, where the increasing negative actually induces a minute diphasic response-positive followed by negative. The next response to stimulus Io gives us a sudden large negative response. Above this point of transition I find, as will be seen in the following records, that there is a rapid enhancement of normal negative response. Still later, this increase of rate would decline, and later again, by the setting-in of fatigue, the responses might undergo an actual diminution. Thus, in that 
part of the record which lies beyond the point of transition, we can see that the excitatory change at first increases very rapidly, and afterwards more slowly, under successive equal increments of stimulus-intensity.

In order to test the universality of these characteristics of nerve-responses, I obtained records with many different specimens. The record just shown was taken, as already said, with a sciatic nerve of gecko, which was in somewhat sluggish condition. The next (fig. 4OI) was obtained from the sciatic nerve of a vigorous bull-frog. On subjecting this nerve to increasing stimuli, I, 2, 3, 4, . . it was found that

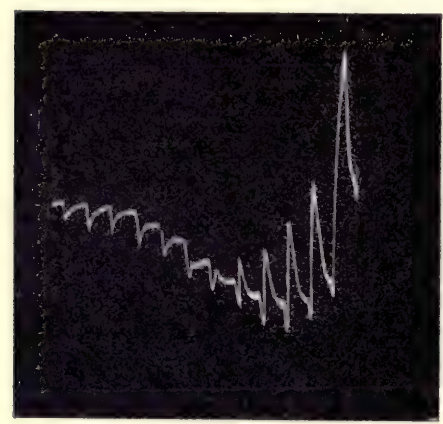

FIG. 401,-Response of Nerve of Bull-frog to Stimuli I, 2, 3, ... I2, increasing in Arithmetical Progression the positive response reached a maximum, after which it declined, and the response became diphasic. With gradually increasing stimulus, the positive element in the response now became smaller and smaller, while the negative grew larger. Above this the negative responses underwent a very rapid increase.

Up to this point, I have been describing the peculiarities of responsc, as seen in motor nerves. In order to show, however, that the same characteristics hold good of the responses of sensory nerves, I next took a specimen of the optic nerve of Ophiocephalus. As I here wished to demonstrate the possibility of the response after continuous increase, reaching a limit, I employed the very moderate magnification of only thirty times, in the recording Kunchangraph. With larger magnifications, the record exceeds the recording-plate, and such a demonstration is impossible. The object being thus to record the peculiarities of response in the negative region only, that stimulus which was taken as the unit was sufficiently strong to induce a contractile response which, under the given magnification, 
appeared moderate. Records were then made under increasing stimulus $I, 2,3,4,5 \ldots$ It will be seen from these (fig. 402) that increasing stimulus induces at first a rapid augmentation of the negative response, after which a limit is reached.

The same characteristics I find to hold good of the response of plant-nerve (fig. 403). Here the response-records were commenced at a point just above that of transition. Now, if these particular relations between stimulus and

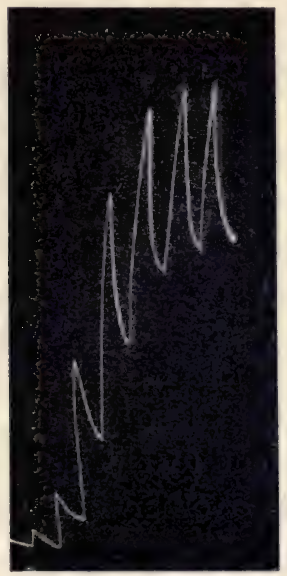

FIG. 402.-Response of Optic Nerve of Ophiocephalus to Arithmetically increasing Stimuli $\mathrm{I}, 2,3,4,5,6,7$ response be due, in the case of man, to some specific psychic reaction, then the same must also be true not

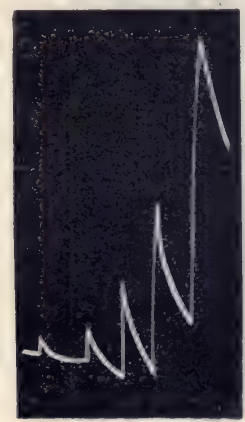

FIG. 403. Mechanical Response of Nerve of Fern to Arithmetically increasing Stimulus

only of the animal but also of the plant. And further, the records themselves, being mechanical; were direct records of undeniably physical changes. Even in the case of the inorganic, again, the same characteristic relations obtain, as we shall find in the next figure. Hence it is not true that the relation between stimulus and the responsive reaction is different in the inorganic from what it is in those living nervous tissues whose changes we perceive as sensation. That is to say, it is determined, in all cases alike, by the same underlying physical factors, 
In fig. 404 we have a series of magnetic responses to arithmetically increasing magnetic stimulation. The magnetising current by which this was accomplished increased from $\cdot 2$ ampère to 2 ampères by steps of 2 ampère at a time. The responses at first, as will be seen, increased at a very rapid rate, and afterwards the rate declined. The remarkable similarity between this record and that obtained with the optic nerve of Ophiocephalus is at once apparent.

In the chapter on the modification of Response under Cyclic Molecular Variation, I have already shown how the responsive

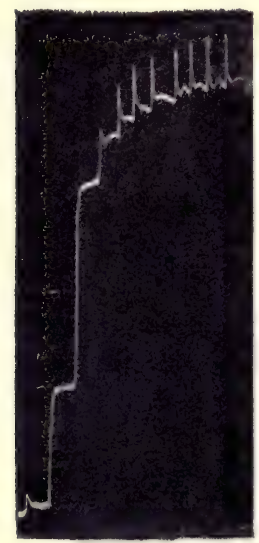

FIG. 404. Photographic Record of Magnetic Responses in Steel to Arithmetically increasing Stimulus change differs in intensity at different parts of the characteristic molecular curve. We there saw that just beyond the point of transition, in the B state, the rate of change was very rapid, and that it became slower in the higher part of the curve. The induced transformations in the condition of the nerve, by which it passes from the phase $\mathbf{B}$ to $\mathbf{C}$, and so on, are of themselves sufficient to elucidate not only these, but also other obscure characteristics of the phenomenon of sensation. They will, for example, explain the curious case in which an identical stimulus given at regular intervals proves at first not unpleasant, and at the end of the series actually painful. Here sensation has become intensified, though the exciting stimulus remains the same. I may quote here an account of an experiment by Professor Sherrington, which exhibits this fact in a very interesting manner:

'I found that by focussing the heat rays from a lamp upon a skin area of about 25 sq. $\mathrm{mm}$. (on the back of the hand), and allowing a perforated screen to intermittently intercept the radiation, the heat-pain begins to be perceptibly intermittent when, the times of play and of interception 
being equal, the intermittence falls below the rate of thirteen per minute. An intensity of this intermittent radiation stimuli, at first not painful, and yielding sensations strikingly discrete, soon becomes dolorific, and then the sensations remain discrete no longer, but are fused more or less together.' 1

This anomaly of gradual heightening of sensation, and later, fusion of effects, appears at first sight inexplicable. I shall give a satisfactory explanation of the latter point in the next chapter ; but as regards the former of its two elementsnamely, the heightening of sensation under uniform intensity of stimulus-we have seen that, owing to the after-effect of stimulus, the condition of responding substances in general, and nerve in particular, is gradually transformed, from a point below the transition $\mathbf{B}$ to one above it. But, owing to this transformation, the character of the response is changed (cf. fig. 382). If the original response be positive, it will be converted later into negative. If it be moderately negative, it will be converted into more intense negative.

It will also be seen that the intensity of response is not solely determined by the intensity of stimulus, but is modified by the existing condition of the nerve. And this latter undergoes a change by the action of stimulus itself. Thus there are certain times of the day when, owing to sluggishness of the tissues, our power of perception is dull. But acuity of perception becomes enhanced with each successive stimulus responded to. This is also true, more or less, of each individual undertaking. These facts are easily understood from a consideration of the different responses which are characteristic of the ascent of the molecular curve above the point of transition.

We have also to consider the fact that this progressive molecular modification, under certain circumstances, imposes the anticipation of the responsive maximum, with increasing stimulus, or even, in other cases, the induction of an actual decline. The molecular variation, in consequence of the

1 Texl-book of Physiology, edited by Schäfer (1900), vol. ii. p. 998. 
after-effect of previous stimulation, is seen by the shifting upwards of the base-line. We may here refer back to fig. 3 I where the responses of two different tissues to increasing stimulus, show, in the one case complete recovery, and in the other, a persistent after-effect. The base-line in the former coincides with the line of absolute equilibrium; and increasing stimulus consistently shows an increasing response, till, owing to molecular distortion reaching a maximum value, a limit in the response was reached. In the second of these records the persistent after-effect shifts the baseline, which now becomes the line of modified equilibrium. Owing to this fact, the relative maximum of variation from the condition of modified equilibrium is reached much earlier than would otherwise have happened. The extent of individual response, after this, appears in fact to undergo an actual diminution, though, measured from the line of absolute equilibrium, this is not the case. In our sensation we are unable to take cognisance of the absolute zero, the changed condition of the nerve itself at any given moment providing us with a relative zero, which is our only standard of comparison, and our whole perception of intensity depends upon induced variations from this changing zero.

The record which I have given in figs. 400 and 401 , showing the mechanical responses in the nerves of gecko and frog, under increasing stimulus, not only explains the quantitative change in the sensation, but also that change of quality or sign, under appropriate conditions, which had not hitherto found any explanation. We see in the curve of response, as has been said, that with feeble stimulus response is positive, and that this positive response attains a climax, after which it diminishes in amplitude, and then changes sign and passes over into negative under increasing stimulation. In the corresponding response by sensation, we find similarly, not only a difference of quality or sign, but also a transformation from one to the other, under increasing stimulation, from a climax where the sensation attains a maximum of the pleasurable tone. After 
this the positive declines, and passes over the line of transition into the region of the negative or painful.

The fact, again, that a moderate stimulus, such as is efficient to induce the positive sensation, will, if tetanically applied, become negative or painful, finds satisfactory explanation from the peculiar characteristics of the molecular curve. For we have seen, under tetanisation, that the curve is raised from the positive region, below the point of transition, into the negative, above it ( $c f$. fig. 383 ).

We have thus seen that by molecular transformation the excitability of the nervous tissue may be enhanced or depressed. There are other conditions also under which the conducting power of the nerve, as well as its excitability, appear to be modified by the exercise of will. Thus, by attention, a stimulus which was previously scarcely perceptible may be raised to sensory prominence. The reactiontime, again, may be diminished by attention. The very opposite of these effects, again, are induced by inhibition. As an example of the latter may be mentioned the inhibition by the will of the muscular movements natural under a given stimulus. It was at one time thought that this inhibition of movement was brought about by the in-nervation of antagonistic muscles. This theory, however, is held to be disproved by the fact that such inhibitory effects are seen, even where antagonistic muscles are not present. It would thus appear that the nerve is susceptible of being thrown into certain conditions, in response to internal action, of an unknown character, by which the transmission of excitation through it is either accelerated or inhibited. This power has been said to be ' unique and mysterious.'

That such opposite dispositions of the nerve may actually result from opposite incipient distortions of the molecules is well shown, however, by the action of electrotonus. Here we have seen that by the influence of one pole an incipient molecular distortion is brought about, which facilitates the transmission of the true excitatory wave, while by the opposite this transmission is hindered or blocked. Thus an 
identical nerve will be rendered acceleratory or inhibitory by the opposite effects of the inducing tonus.

If an external force be thus capable, according as it is positive or negative, of inducing opposite molecular dispositions, by which the conducting power of nerve is so profoundly modified as to render it for the time being either an accelerator or an inhibitor, then it is not difficult to understand that these molecular dispositions may also be yaried in a similar manner by impulses from an internal source. The molecular dispositions themselves, by which these effects are brought about, are no doubt curious, but they are neither mysterious nor unique; for we have seen that by molecular distortions of one sign or another, artificially induced in magnetic substances, a given magnetic impulse may be either accelerated or retarded.

We have seen that the transmission of excitatory changes is facilitated, if the nerve be subjected to incipient molecular distortion in a favourable direction. Now in considering the attitude of attention, we can see at once, if only from the muscular indications which it induces, that the nervous channel is probably thrown by it into a state of moderate contraction. Inattention, on the other hand, must generally be attended by an attitude of relaxation. We have again found that while incipient contraction or $\mathrm{K}$-tonus enhances conduction, an intense contraction, or very strong $\mathrm{K}$-tonus, will inhibit, because the molecular distortion thus induced is already maximum, and external stimulus can produce little further effect (p. 6ro). It is here interesting to note that the expression 'steeling the nerves to pain' is not altogether fanciful or metaphorical. The attitude of preparedness thus denoted is one of rigid contraction.

In the course of the present chapter, then, it has been shown that there are two distinct nervous impulses, positive and negative. The former, induced by feeble stimulus, gives rise to a positive tone of sensation. The negative, on the other hand, due to stronger stimulus, gives rise to a sensation 
of painful or negative tone. The particular relation which exists between stimulus and sensation has been shown to be an expression of the peculiar characteristics of the molecular curve. The ascent in the curve, being rapid immediately above the point of transition, and slow later, equal increments of stimulation cause in this region an increase of sensation which is at first rapid and then slow. Below the point of transition responses are positive. But tetanisation, inducing a molecular transformation, carries the curve above the point of transition. Hence moderate stimulus, inducing positive sensation, is converted, when tetanically applied, into negative or painful. The positive response is simple, but the negative is complex, containing a masked positive; and we shall see in the next chapter how this complex sensation can be analysed into its component parts.

We have also seen that, in addition to moderate stimulation, there are other agencies by which the tonus of nerves may be altered. In a magnetic substance, the incipient molecular distortions of one sign induced by K-tonus, enhance the excitability and conductivity, while those due to A-tonus depress them. Strong K-tonus, again, inhibits conduction. By such polar actions, then, an impulse is either accelerated or inhibited. Similar polar changes are seen in the nerve, moreover, under the action of an- and kat-electrotonus. In like manner, by the exercise of the internal stimulus of will, the tonus of the nerve may be so varied that, under different circumstances, the transmission of excitatory impulses is accelerated or inhibited. 


\section{CHAPTER XLIV}

\section{DISSOCIATION OF COMPLEX SENSATION}

Conversion of pleasurable into painful sensation, and vice versa, by electrotonusThe Sensimeter-Mechanical stimulation--Stimulation by thermal shocks-Chemical stimulation-Opposite effects of anode and kathode--Normal effects reversed under feeble E.M.F.-Negative tone of sensation blocked by alcohol and anæsthetics-Separation of positive and negative sensations, by lag of one wave behind the other-Dissociation of sensation by depression of conductivity-Abolition of the negative or painful element by block of conduction.

I SHALL next proceed to demonstrate, by means of decisive experiments, the fact that sensation and its variations are associated with certain physiological changes and their appropriate modifications. In the mechanical and electrical response of the nerve, we found that while moderate stimulus gave positive response, a stronger stimulus gave negative ; that while the positive was unmixed or elementary, the negative contained a masked positive; and further, that the velocity of the transmission of the positive was greater than that of the negative wave.

We shall now study the correspondence of the responsive sensation with these various nervous changes induced by stimulus, and their modifications under different agencies. I shall next, therefore, show, in accordance with the chief aim of this chapter, that the same conditions which determine the exhibition of positive, mechanical, or electrical response, will also, pari passu, determine positive tone in the responsive sensation. Those conditions, on the other hand, which bring about a negative mechanical or electrical change in the nerve, will also induce a negative tone in the sensation. The resultant transmitted effect recorded in the responding apparatus, was, as we saw, determined by the receptive ex- 
citability of the stimulated point, and the conductivity of the transmitting tissue. We shall, therefore, investigate separately the effects of various agents in the modification of receptive excitability and of conductivity respectively.

We saw, again, in the last chapter, that as the intensity of stimulation is continuously increased, the response gradually passes from the positive, through the point of transition, into the negative. This happens, as we have seen, immediately above the point B. This region, therefore, may be referred to as critical and indifferent, and must be regarded as having the peculiarity that if, by any means, the molecular curve be raised above it, the corresponding sensation tends to become actually painful, or if carried below, becomes pleasurable.

It follows that, in this critical indifferent region, any condition which tends to exalt the excitability of the tissue, and thus the negativity of the response, will also accentuate the negative tone of sensation, its mixed character thus passing into the distinctly painful. Those conditions, on the other hand, which lower excitability, and therefore responsive negativity, will also act by detracting from the painful element in the indifferent sensation in the critical region, and rendering it to a greater or less extent positive, soothing, or pleasurable. In this way, by depressing or raising the excitability at will, a stimulus which had already caused a painful sensation might be made not unpleasant, and an indifferent or positive sensation could be made negative or painful. We shall presently see how, by variations due to the presence of internal or external modifying factors, the positive tone of sensation is actually rendered negative, and vice versa, under such transpositions.

Now we have at our disposal various well-known agencies by which the excitability of the tissue can be raised or lowered. In order to lower excitability, certain anæsthetic agents may be employed, and their effect in the modification of sensation-tones will presently be described. The ideally perfect means, however, not only for the exaltation or depression of excitability at will, but also for the rapid 
interchange of the one effect with the other, is that of electrotonus.

Under the normal condition of a medium intensity of E.M.F., we know that it is the kathode which enhances excitability, and the anode which depresses it. Hence an indifferent sensation, however caused, will be rendered painful when the excited point is made kathode, or pleasurable when anode. If, again, the stimulus be of sufficient intensity to render the resulting sensation moderately painful, kat-electrotonus will make it intensely painful, and an-electronus convert it into soothing, by taking away from it the negative element.

The an- and kat-electrotonic effects referred to here are those which fall under the generalisation made by Pfliger. I have shown, however, that the application of this law is but limited. Under the action of a feeble E.M.F. these anand kat-electrotonic effects are exactly reversed, and it is then the anode which renders the tissue excitable, the kathode inducing relative depression. If, then, sensationchanges are dependent, both qualitatively and quantitatively, on antecedent physiological changes, we may expect corresponding reversals to take place in serisation, according as the anodic or kathodic applications are feeble or moderate.

And, lastly, we might expect to meet with other characteristic changes in responsive sensation, due to the peculiar differences shown in figs. 320 and $40 \mathrm{O}$, between the positive and negative effects. We there saw that the positive response was short-lived, whereas the negative was more persistent. Even the negative response itself further was, under moderate stimulation, of briefer duration than under strong. In accordance with these facts there ought to be a difference in the fusion of sensation. Let us suppose that the frequency of stimulus, for the induction of the indifferent sensation, be adjusted in such a way as to cause a sensation which is so fused as to be almost continuous. Under normal kathode, this response, being converted into more persistent negative, will now become completely fused and painful Under normal anode, on the other hand, the individua 
responses being converted into short-lived positive, the resultant sensation will become discrete and pleasurable Before proceeding to describe these experiments in detail, it will be well to present these inferences in the form of a tabulated summary :

EfFect of Electrotonus on Indifferent Sensation IMPERFECTLY FUSED

\begin{tabular}{|c|c|c|}
\hline Intensity of E.M.F. & Anode & Kathode \\
\hline Moderate. & $\begin{array}{l}\text { Soothing and conspicuously } \\
\text { discrete. }\end{array}$ & Painful and continuous. \\
\hline Feeble. & Painful and continuous. & $\begin{array}{l}\text { Soothing and conspicuously } \\
\text { discrete. }\end{array}$ \\
\hline
\end{tabular}

These generalisations I shall now demonstrate by means of experiments carried out under, not one, but various forms of stimulation. For experimental adjustment two conditions have to be fulfilled. The first is so to regulate the intensity of stimulus as to cause the indifferent sensation. The second is so to adjust its frequency as to induce a fusion of effects all but complete. It may here be pointed out that in general the degree of frequency necessary to bring about this semifusion will be a question of the effective intensity of stimulation employed, and the excitability of the subject. Having first decided on the intensity of stimulus to be employed, it is easy to adjust its frequency by means of an adjustable interrupter.

I shall first describe the Sensimeter, by means of which these experiments were carried out. Mechanical stimulation, when required, was caused by a tapper, actuated by an electro-magnetic arrangement (fig. 405). When an electrical current is sent round the electro-magnet, the soft iron armature is pulled down against an antagonistic spring. When the current is stopped the armature is released, and the tapper delivers a blow. The height from which the tapper falls determines the intensity of the blow, the former being dependent on the strength of the electro-magnetic pull, which is determined in its turn by the intensity of the 
current. This latter is adjusted by means of a carbon rheostat. In order to adjust the frequency of stimulation, a toothed-wheel is fixed on clock-work, the normal period of rotation of the axle being once in four seconds. This speed, however, may be continuously adjusted within a certain range by means of a regulating governor. When the toothed wheel has sixteen teeth, the frequency of stimulation is four times in a second. By inserting wheels with different numbers of teeth, and by means of the regulating

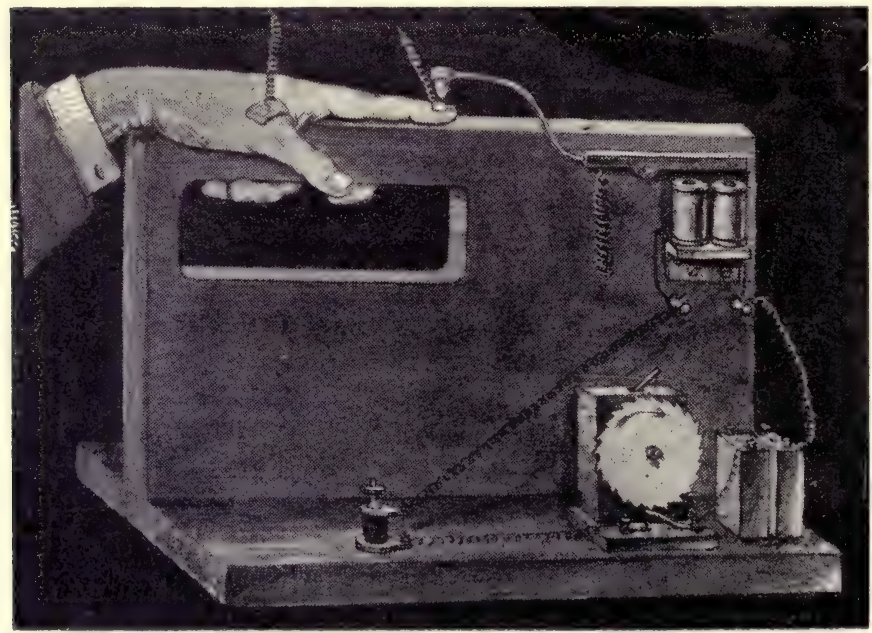

FIG. 405. The Sensimeter

governor, it is possible to obtain any desired graduation of frequency.

The same experimental arrangement, with slight modifications, may be employed to give a series of thermal shocks, For this purpose the electro-magnetic tapping arrangement is removed from the apparatus, and the electro-thermic stimulator attached to the circuit, instead of the tapper. The frequency of the thermal shocks may be regulated in the usual manner, their intensity being dependent on that of the heating current, which is adjusted by the rheostat. 
In experimenting on the effect of mechanical stimulation, the intensity and frequency were so adjusted as to bring about a neutral and imperfect fusion. In a particular experiment, for example, the frequency of stimulus was four times in a second. The receptive point for this experiment was the very sensitive back of the end joint of the human fore-finger-that is to say, the space immediately adjacent to the quick of the nail. In order to study the electrotonic effect, one electrode was applied by means of a piece of cotton, moistened in normal saline, and placed on the receptive area, the second being on a different finger. After so adjusting the intensity and frequency of stimulus as to give the required semi-continuous and indifferent sensation, the receptive point was made kathode, the E.M.F. employed being moderate - that is to say, of $I^{\prime} 5$ volt. The resulting scnsation was distinctly painful and continuous. By now reversing the electrotonic current, the receptive point was made anode, and the resulting sensation was not only positive or soothing, but also strikingly discontinuous. In order to exhibit the reversal of these effects under feeble E.M.F., I employed an E.M.F. of 2 volt. The initial indifferent sensation was now found to be converted under anode, to negative and continuous, the kathode inducing a soothing and discontinuous sensation. The effect under a feeble E.M.F. is thus found to be in every way the opposite of that under strong. When frequent experiments are carried out on the same finger, the result is apt to be blurred in consequence of fatigue. It is therefore advisable to carry out the preliminary adjustment with one, and to repeat the experiment on another finger. The normal opposite effects of anode and kathode may in general be easily demonstrated by an E.M.F. of $1 \cdot 5$ volt. But the value of the feeble E.M.F. which induces the reversal of these, varies with different individuals and with different modes of stimulation. It is, therefore, advisable to start with the lowest possible E.M.F. of the order of about or volt, and increase it gradually, till the reversal of effects is most pronounced. On 
increasing the E.M.F. still more, the reversed effect passes first into neutral and then back to the normal, characteristic of a moderate E.M.F.

Similar effects are also obtained on employing the stimulus of thermal shocks. The electro-thermic stimulator is now inserted in the Sensimeter, in place of the mechanical tapper. The indifferent and incompletely fused sensation becomes continuous and painful, on making the receptive point kathode, under a moderate E.M.F. of 2 volts. On now making the receptive point anode, the sensation becomes converted into a markedly discrete positive. The reversal of these normal effects under a feeble E.M.F. was obtained in a given experiment by thermal shocks, when the polarising E.M.F. was ${ }^{\circ} \mathrm{O}$ volt.

Turning next to the chemical mode of stimulation, it will be remembered that an experiment has already been described ( $p .582$ ) where a continuously irritating sensation was caused by the application of salt on a wounded spot, this moderate sensation of pain being rendered soothing when the spot was made normal anode, and intensely painful when normal kathode. Effects precisely opposite resulted from the application of a feeble E.M.F. It will thus be seen that those same conditions which depressed the normal negative response, also acted to obliterate the negative tone from the resulting sensation. Those conditions, on the other hand, which exalted the negativity of the response, would convert the positive tone of sensation into negative or painful. These general facts have been demonstrated by the employment of different forms of stimulation, the necessary depression or exaltation of excitability having been brought about by electrotonus. So far we have dealt with the modifications induced in the responsive sensation by the variation of the receptivity of the excited point. We shall next briefly discuss the effects which ensue on the variation of the excitability and conductivity of the nerve by different agents. In order to induce depression, there are various anæsthetic agents which might be used. 
In investigating the influence of alcohol (p. 493) we saw that three distinct effects were induced by it on the receptivity, responsivity, and conductivity of a tissue respectively, these effects themselves being further modifiable by the duration and intensity of the application. It was shown that, in the first stage of its application, alcohol exalted the power of receptivity. But its effect on conductivity and responsivity, especially after a certain duration of application, was one of great depression. The total effect of alcohol is thus somewhat complex. At an early stage of its application there is an effect of exaltation. After a while, however, the conducting power becomes increasingly depressed by its action. This means that the passage of the true excitatory or negative wave is progressively impeded or even blocked, the positive alone continuing to be transmitted for a time. At a certain stage of alcoholisation, therefore, the negative tone of an existing sensation, normally painful, will, as it were, be erased. And this withdrawal of the painful element, by causing sudden relief, together with the actual transmission of the positive wave, may induce a tone of sensation which might even perhaps be regarded as pleasurable. In any case the abolition of conductivity must eliminate the element of pain, and it was undoubtedly this which, in pre-anæsthetic medicine, caused its employment for certain minor operations. Long and intense alcoholisation will, of course, obliterate all sensation. On the after-effects of so depressing a reagent it is unnecessary to dilate.

Effects, in some respects parallel, may be observed under etherisation, where, at a certain stage in the action of the narcotic, there is a cessation, not of all sensation, but of its painful element alone. Thus, by depression of conductivity, and consequent suppression of the negative wave, the positive may be 'dissociated' from the negative sensation. Sustained pressure on a nerve is also known to depress conductivity, and it is interesting to note here that pressure on the ulnar nerve-trunk will abolish painful sensation, the positive, or mere contact-sensibility remaining practically undiminished. 
We shall next study the various effects induced by the variation "of conductivity. We have already seen that a moderately intense stimulus gives rise to two waves, one positive and the other negative, the former having the greater velocity of the two. Thus these two waves, starting from the receptive and reaching the distant responding point, will give rise to two different responsive effects, separated from one another by an appreciable interval. The separation depending on the lag of one wave behind the other, will be greater the greater the length of the conducting tract. The first to arrive at the perceiving centre being the positive impulse, we shall have there a positive sensation of mere touch or contact. The later-arriving negative wave gives rise, according to the nature of the indicator, to mechanical contraction, galvanometric negativity, or a painful sensation, as the case may be. If the conducting tract be not sufficiently long, the two waves will be superposed, or indistinguishable, one masking the other. But they may be analysed, or separated, by anything which diminishes the conductivity of the intervening tract, and this is rendered possible by the fact that the positive wave is not much affected by changes of conductivity. On the other hand, the velocity and intensity of the negative wave are both diminished by anything that diminishes the conductivity. Hence a diphasic response-the sensation of touch followed by pain-may be expected, wherever the intervening conducting tract between receptive and responsive points is sufficiently long. In other cases, where the tract is shorter, a sufficiently strong stimulation will give rise to a single sensation of negative or painful tone. This negative sensation, however, is complex in its character, masking as it does, a contained positive element. If then the conductivity of this transmitting nerve be in any way diminished to a moderate extent, the complex sensation will be found to be analysed, the negative being made to lag behind the positive. There will thus be a dissociation of the dual elements of the complex sensation, and the result will be a diphasic response 
-the positive or sensation of contact, followed by that of pain. With still greater depression of conductivity, the normally painful sensation, by the blocking of its negative element, will be turned into one of positive tone.

We shall now proceed to verify these theoretical inferences. The fact that there are actually two distinct nervous impulses, and that of these the positive travels faster than the negative, is demonstrated by the well-known experiment in which a smart tap is applied on the ball of the foot, with the result that we perceive, first, the sensation of touch, and then, at an appreciable interval afterwards, that of pain. The possibility of this demonstration in a normal nerve is due to the length of the conducting nerve here concerned.

In other cases the same dissociation is seen to be effected under varying degrees of loss of conductivity, in different forms of nervous paralysis. The considerations which I have advanced, will, however, I believe, offer a satisfactory explanation of those curious instances of 'dissociation' and 'delayed pain' which are known to pathology. In such cases, a prick with a pin, for instance, is first perceived as mere contact, and then, after an appreciable interval, as the sense of pain. Other cases are known in which, the loss of conductivity being very great; the patient could handle burning coal without pain, the resultant perception being entirely positive.

From the diverse phenomena described in this and the previous chapters, it is clear that a sensation of positive tone is associated with that particular responsive change in the nerve, which is expressed as expansion and galvanometric positivity. We have also seen that such effects are brought about by a feeble intensity of stimulus, which acts to increase the internal energy without inducing the true excitatory negative effect. Of anything which thus increases the internal energy, it may be said in general that it will induce positive mechanical and electrical expressions with concomitant positive sensation. With stronger stimulus, as we have seen, the negative mechanical and electrical responses are induced. But such negative responses contain, as we have 
also seen, the masked positive component, and from the point of view of energy, they represent the algebraical summation of income and expenditure, positive and negative, increase and diminution. Considered as sensation, then, this particular effect will be composed of dual elements, a mixture of positive and negative tones. With very strong stimulation, finally, the negative effect will be very great; expenditure will be greater than income; and the sensation will assume a predominantly and persistently negative tone.

The complex negative, moreover, containing a masked positive, is capable of dissociation into its component elements. By the partial or complete block of conductivity, it is exhibited as dual response of 'dissociation,' or by the total suppression of the negative, as positive alone. In this way, a sensation, originally painful, may be rendered not unpleasurable, or even pleasurable. It was further shown, employing electrotonus and the sensimeter, that an indifferent and semi-fused tone of sensation can, by merely exalting the excitability of the tissue, be converted at will into one which is continuous and painful. By depressing the excitability, on the other hand, this indifferent sensation may be made pleasurable and strikingly discrete. 


\title{
CHAPTER XLV
}

\author{
MEMORY
}

Memory an after-effect of stimulus-Persistence dependent on strength of stimulus - Rate of forgetting-Multiple after-effects in retina-Spontaneous revival of after-images-Theories of memory-Latent images and their revival-Aftereffect of stimulus on excitability and conductivity-Differential effect of diffuse stimulus-Revival of latent image in metal-Revival of latent image on phosphorescent surface-Negative or reversed memory-image-Psycho-physiological version of this experiment-Differential excitation under diffuse stimulus, internal or external-Continuity of this seen in mechanical response of plagiotropic stem and pulvinus of Mimosa, in the electrical discharge of certain fishes, and in psychic response of memory.

Up to the present we have considered only the immediate effects of stimulus. We know, however, that excitation entails, not only an immediate, but also an after-effect. We are thus led to the question of the physical aspects of the phenomenon known as memory, which is admittedly a matter of after-effects. Even with the somewhat insensitive apparatus for the detection of nervous changes which is at our disposal-the galvanometer or the Kunchangraph-we find that the after-effect of strong is more persistent than that of feeble stimulus. In the very sensitive neurile apparatus, nervous changes and their after-effects are more distinctly perceived. The psychological retention of an impression follows, in general, the curve of response and recovery. The fact that physiological recovery from the effect of strong stimulus is less rapid than from feeble, has its correspondence in the period required for the fading of sensory impressions, of which also it may be said that the stronger persist longer than the weak.

This fact that the after-effect of strong stimulus persists 
for a longer time than that of feeble, may be exhibited in an interesting manner as follows. A simple design is made with magnesium powder and fired in a dark room. A second observer, unacquainted with the design, observes the flash and closes his eyes. The instantaneous flash, obscured as it is by dense smoke, does not at once produce any definite impression. In the retina, however, the obscuring image of the smoke, being of little luminosity, quickly passes off, and the after-effect of the brilliant flash, thus separated from the obscuring smoke, grows into perfect distinctness. In this manner I have often been able, by subsequent closure of the eyes, distinctly to observe luminous phenomena of brief duration, which, while the eyes were open, had been indistinguishable.

We have thus touched upon the question of the time required for the obliteration of a mental impression. Another interesting aspect of this subject lies in the rate at which fading takes place, or, in other words, the rate of molecular recovery. In the curve of recovery we find that this rate is at first very rapid, and becomes increasingly slow with the descent of the curve. This is also the characteristic of the process of forgetting, Ebbinghaus, for instance, found that the forgetting of a series of 'nonsense syllables,' at first quick, became increasingly slower with time.

Certain excitable tissues, such as nerve and cardiac muscle, give responses to strong stimulus, which, as we have seen, are multiple in character. The retina, again, under intense stimulus of light, exhibits multiple after-excitations, which may be detected by a galvanometer (p. 426). This fact explains the multiple after-image often seen on closing the eyes after strong light. Another proof that these multiple after-images are physiological lies in the fact that their periodicity is modified by a previous condition of rest or activity. Thus, early in the morning, when fresh from rest, this period I find to be at its shortest, and later in the day to become gradually longer, owing to growing fatigue. In a given instance, the period at 8 A.M. was 3 seconds, 
which had lengthened to 5 seconds by 3 P.M.; and was further prolonged to 6.5 seconds by I I P.M.

One way of exhibiting the after-images in the retina is, as we have already seen, by means of a stereoscope containing two incised slits inclined to each other, instead of photographs. On looking through this at the bright sky for ten seconds, or longer, a composite image is formed of an inclined cross. The eyes are now closed, and the first effect noticed is one of darkness, due to the molecular rebound. By reason of the Binocular Alternation of Vision, already referred to, one luminous arm of the inclined cross now projects itself aslant the dark field, and then slowly disappears, after which the second, perceived by the other eye, shoots out suddenly in a direction athwart the first. This multiple alternation proceeds for a long time, and produces the curious effect of two luminous blades crossing and recrossing each other. At first the after-images of the cross, seen with the eyes closed, are very distinct, so distinct that any unevenness in the design at the edges of the slanting cuts can be made out clearly. There is here no doubt of the 'objective' nature of the strain impressed on the retina, which, on the cessation of direct stimulus of light, gives rise to after-oscillations with concomitant visual recurrences. This recurrence may be taken as a proof of the existence of physical strain in the retina. The recurrent after-image is very distinct at the beginning, but becomes fainter with each repetition. A time comes when it is difficult to tell whether the image is the objective after-effect due to previous strain, or merely an effect of 'memory.' There is, in fact, no hardand-fast line of demarcation between the two-one merges simply into the other. In connection with this, it is interesting to note that some of the phenomena of memory also are admitted to be recurrent.

Visual impressions and their recurrence often persist for a very long time. It usually happens that, owing to weariness, the recurrent images disappear, but in some instances, long after this disappearance, they will spontaneously 
appear at most unexpected moments. Thus in a given case of the present experiment, performed in the afternoon, the subject perceived this recurrence for some time, after which the alternating impressions seemed to disappear, and were completely forgotten. On retiring at night, however, these recurrent images suddenly reappeared. Thinking the matter to be an effect of light, the observer hastily extinguished his lamp. But the recurrent images now became only the more intense.

In another case the recurrence was observed in a dream, about three weeks after the original impression was made, and in this case it was seen as the crossing and recrossing of bright swords. 'These instances of the revival at night of impressions made in the daytime, when the interference o distracting influences is withdrawn, is significant. Since an intense stimulation of nerve is liable to recur spontaneously, without the action of the will, or even in spite of it, it follows that any single impression, when very intense, may become dominant, and persist in recurring automatically. Examples of this are only too familiar.

We have hitherto dealt with that aspect of memory in which it is a more or less immediate after-effect of sensory stimulation. But we encounter a much more difficult problem when we come to the question of the revival of an image long after it has apparently faded. It has been suggested that this process of revival depends upon the existence of some 'scar,' or fixed impression, in the brain, or on a certain persistent disposition or tendency to movement created there. It is perhaps worth while to point out here, however, that though when a blow is recent, the smarting effect will persist for some time, yet; when once healed, no scar could of itself reproduce the origirial excitation. It is of course recognised that such expressions are merely figurative, and that the entire process is not clearly understood. We are more likely, however, to arrive at a true explanation of the phenomenon if we recognise in it two distinct factors, first, that of molecular change, with concomitant change of 
properties; and, second, the effect of an internal stimulus, delivered as a blow from within, by an impulse of the will, upon the sensitive surface in which the image is latent.

We shall now first observe in some detail those changes which remain as an after-effect of previous stimulation. The differential effect caused by primary stimulus fixes the latent image, and it is only by the reproduction of the same differential excitation, that the memory-image can subsequently be revived.

We must here recall briefly the results which were established in Chapter XLII., on the modification of response under cyclic molecular variation. It was there shown that, under the action of stimulus, the molecular condition of a substance undergoes a progressive variation, exhibited in its characteristic curve; that the forward and return curves do not exactly coincide, because the history behind the two half-cycles has not been the same; and, finally, that on the cessation of stimulus, the original molecular condition is not exactly restored, a certain effect being residual. Owing to this residual effect, the properties of the responding substance are changed. We also saw that, in consequence of this impressed change, the conductivity and excitability of the substance might be enhanced. A frequent repetition of a stimulus was thus shown to create a habit or disposition by which the mass of a substance, formerly almost non-conducting, might be made a conductor of excitation.

These impressed molecular changes may not leave any visible impression behind. But let us look at the responding properties of a given substance at different points on the characteristic curve. In a sluggish $\mathbf{A}$ condition, that is to say, before it has even been excited, the power of response of the substance to a given stimulus will be slight or negligible. Let us suppose next that by the action of stimulus the substance is raised above B. On the cessation of stimulus a slow recovery will then take place, whose completion may be indefinitely prolonged. The substance will thus approach very near the point $\mathbf{B}$ in the curve, without actually reaching 
it. This difference between $\mathrm{B}$ and the point actually reached may be so small as to be undetectable by any ordinary mode of inspection. We therefore term the impression latent. But the properties of this B area, formerly acted upon, have been profoundly changed, being rendered more excitable by the impressed effect of previous stimulus. In this sensitive impression-surface will be certain areas in the $\mathbf{A}$ and certain others in the $\mathbf{B}$ condition, the former sluggish and the latter characterised by enhanced excitability. By the shock of an internal diffuse stimulus, a differential excitation may now be induced, exactly similar to that caused by primary stimulus. This is the revival of the memory-image.

We may carry out a physical experiment exemplifying this process of the rise of a latent impression into vividness under the action of diffuse stimulus. We may take a sensitive surface, in which different areas, in consequence of previous excitation, have impressed on them latent variations of excitability. Thus indifferent portions of the surface A, A may have their excitability represented by zero, another portion $\mathrm{B}$, whose excitability has been exalted as the aftereffect of stimulatory agents previously applied, will have its normal excitability enhanced. In still a third portion, $\mathrm{C}$, the excitability is artificially depressed or abolished. The responding substance was a tin wire; dilute solution of sodium carbonate, which is an exciting agent, was applied on the area B. The depressing or poisonous reagent, oxalic acid, was applied at C. After a short period of this application the wire was washed, and there was no outward indication of any difference between the areas A, B and C. Electrically also there was little or no permanent difference between them. One non-polarisable electrode connected with a galvanometer was kept permanently applied on the indifferent surface $A_{\|}$. The second exploring electrode was now moved along the wire, and while it rested on any point the wire was excited as a whole by vibration. The galvanometer, under this arrangement, would detect differential excitability. As long as the exploring wire moved over 
indifferent areas there was no effect detected in the galvanometer. But as soon as the exploring electrode rested on the area $\mathrm{B}$, the latent enhancement of excitability there showed itself by a sudden responsive up-movement of the galvanometer. When the electrode again passed over $B$ and reached an indifferent area, $A_{\diamond}$, response disappeared. But when it reached $\mathrm{C}$, with its depressed excitability, there was another responsive movement, this time in the reversed or down direction. It is thus seen that the impress made by the

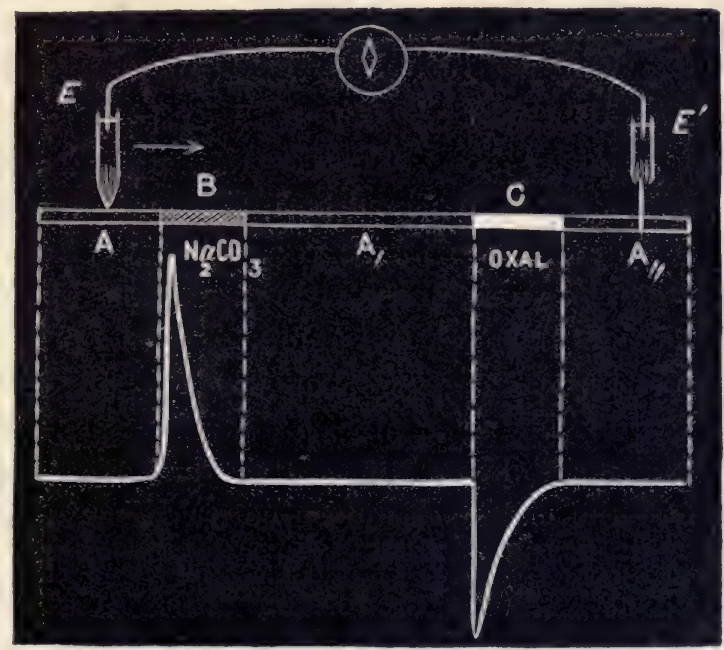

FIG. 406. Revival of latent Image in Metal

action of stimulus, though it remain latent and invisible, can be revived by the impact of a fresh excitatory impulse (fig. 406).

Again, this revival of the latent image by a subsequent stimulation may be exemplified in a simpler and more striking way. We take a card and coat it with some so-called phosphorescent material, such as luminous paint. This is kept a long time in the dark, till the whole is reduced to a uniform A condition. From a previous experiment we have determined what is that duration of exposure, $T$, to a given 
intensity of light, which will evoke a luminous or phosphorescent response. A stencilled pattern is now placed on the prepared card, and the whole is exposed to light for the time $T$. On now cutting off the light and removing the stencil a luminous pattern is seen, which is the primary response. This impression slowly fades out. But the cardboard now contains a latent image, whose revival will be analogous to that of memory. The stimulated areas which have now ceased to respond are still, in virtue of previous stimulation, in the $\mathbf{B}$ condition, which is more excitable than the indifferent A. A feeble diffuse stimulus should now, by its differential action, prove efficient to revive the latent image. We now expose the whole card to diffuse stimulation of light, of a duration shorter than $\mathrm{T}$. The excitation of the indifferent background will by this means be ineffective, whereas it will be effective wherever the image proper has been impressed. We shall, therefore, obtain a revival of the positive imagethat is to say, an image of the same kind as the original.

An interesting case occurs here, showing the theoretical possibility of obtaining a negative or reversed 'memory image.' The possibility of this will be understood, from an inspection of the characteristic curve. We saw that in the region $\mathbf{B}$ the substance rises in excitability. But in the region of $\mathbf{D}$ and $\mathbf{E}$, where the maximum molecular distortion has already been reached in consequence of over-stimulation, fatigue changes are induced, by which the excitability is depressed below the normal. It follows from what has already been said that an impressed image of this character will be revivable, under subsequent diffuse stimulation, but as a negative, or reversed memory-image.

I shall now describe a psycho-physiological version of this experiment. Let the observer stare at the incandescent filament of an electric lamp, preferably with one eye, say the right, the left being kept closed all the time. The right eye is next closed, and is further covered by the hand. Multiple after-images will now be seen for some time, till the impression seems to have completely disappeared. No trace of the 
latent image is now perceivable in the field of dark vision. When this point has been reached, the hand is suddenly withdrawn from its position over the closed right eye. The light in the room now percolates through the semi-translucent eye-lid, and suddenly gives a moderate diffuse stimulus to the retina. Under these circumstances, the latent image is revived, as a negative - that is to say, as a very dark filament against a brighter background. Thus the essential condition for reviving the latent impression of stimulus would seem to be the subjecting of the unequally impressed tissue to diffuse stimulation. The revival of the image as positive or negative will then be a question of whether the stimulus have been moderate or intense.

I have already shown, by actual experiment on nervous tissues themselves, that the differential excitability induced as an after-effect of moderate stimulus (memory-impression) will give rise, on diffuse stimulation, to one kind of response, and the after-effect induced by strong stimulus to the reverse (cf. figs. 3II, 3I2). In the former case, the moderately stimulated area, on diffuse re-stimulation exhibits induced galvanometric negativity, as compared with the indifferent contact, this being the sign of its relatively greater excitation. In the second case, the sign of response is reversed, the over-stimulated area, on re-stimulation, becoming galvanometrically positive.

The revival of memory-images is thus seen to be due to differential response, evoked by diffuse stimulus, in an organ rendered anisotropic, by the unequal impressions which it contains of previous stimulation. A similar differential effect under diffuse stimulation has been seen in plagiotropic stems. Here the upper surface has a deep impression or memory of over-stimulating sunlight, and on diffuse stimulation this upper surface becomes galvanometrically positive, a responsive current flowing from below to above. It will thus be seen that there is a continuity between the impressions made on the sensitive neurile elements, and the physiological anisotropy induced by the differential action of past stimulus. 
Diffuse stimulus, moreover, whether internal or external, acting on the differentially excitable tissue, gives rise to a marked indication, which may be either motile, electrical, or psychic. A stimulus is applied to the stem of Mimosa. This is transmitted as an excitatory impulse, and reaches the differentially excitable organ, the pulvinus. As far as this organ is concerned, the transmitted stimulus may be regarded as internal. This internal stimulus, then, gives rise to a conspicuous differential effect, shown in the fall of the leaf. In electrical fishes, similarly, the internal stimulus, delivered by the will of the animal upon the differentially excitable organ, becomes evident as an excitatory discharge. In man, again, the revival of memory constitutes a psychic response, due to the play of the diffuse internal stimulus of will upon a sensitive surface rendered differentially excitable by the presence of a latent image.

It will thus be seen that various after-effects of stimulus find expression as the phenomena of memory. The effect of primary stimulus does not disappear at once, but fades gradually, with a concomitant fading of the sensory impression. From the fact that the after-effect of feeble, is less persistent than that of strong, stimulus, we understand that the sensory or memory impression also lasts longer in the latter case than in the former. Very intense stimulation, again, is apt to give multiple responses as its after-effect, and the corresponding psycho-physiological phenomenon is seen in the recurrent after-images in the retina.

When a considerable interval has elapsed after the primary stimulus, there is apparently no trace left of the latent image. But the properties of the impressed portions of the sensitive surface have undergone a more or less permanent change in consequence of stimulation. Certain channels have been rendered more conducting, and certain areas more excitable. By an internal diffuse impulse it is now possible to cause differential excitation, and thus to revivify the latent image. 


\section{CHAPTER XLVI}

REVIEW OF RESPONSE OF ISOTROPIC ORGANS

Laws of response-Opposite responsive expressions of true excitation and increase of internal energy--Separation of the positive and negative wavesPosition in molecular cycle determines character of response-Abnormal sub-tonic positive and reversed fatigue positive-Effect of tetanisationSimilar effects in the inorganic-Phasic alternations-Multiple and autonomous response-Unmasking of antagonistic element by overshooting Different expressions of a single fundamental molecular change-Response by change of form, by secretion or absorption, by variation of electric resistivity, or by electro-motive change-For the last, induction of anisotropy necessary-Perfect modes of stimulation : (a) Torsional vibration ; (b) Rotary mechanical stimulation; (c) Thermal shocks; (d) Equi-alternating electric shocks-Accurate determination of the death-point by mechanical and electrical spasms-Current of injury due to after-effect of stimulusExplanation of characteristic electric distribution in plant and muscle cylinders-Relative positivity of dead tissue-Reversal of current of injury so-called-Unreliability of response by negative variation.

IT has been shown, in the foregoing chapters, that all the diverse phenomena of response may be summarised in the two following formulæ :

1. Excitatory response takes place by contraction and galvanometric negativity.

2. Increase of internal energy induces the opposite effect, of expansion and galvanometric positivity.

The first of these effects is simply demonstrated by direct excitation of an excitable tissue. In order to demonstrate the second, stimulus is applied at a distance from the responding point. In consequence of sudden local contraction at the receptive area, a wave of increased hydrostatic tension is transmitted with great rapidity. Energy is thus conveyed hydraulically, and at the distant responsive point the transmitted effect induces expansion and galvanometric 
positivity. This is followed by the more slowly transmitted wave of true excitation, which on its arrival gives rise to the normal response of contraction and galvanometric negativity. The two responsive effects can thus be exhibited separately, when one lags behind the other. When the intervening tract is short, or the conductivity great, the excitatory negativity masks the hydro-positive effect. But this hydropositive may again be unmasked, by various forms of selective physiological block, which depress the conduction of the true excitatory, without interfering to any appreciable extent with the passage of the hydraulic wave. In this way, the positive may be separated from the contained negative, the response being thus rendered diphasic-positive followed by negative. Or, by the complete suppression of the excitatory negative wave, a response originally negative may be converted into purely positive (figs. 45, 47, and 49).

As response is an expression of molecular derangement, it is the extent of this which determines its amplitude. The character of response is also modified by the molecular condition of the responding substance, and the different mólecular conditions through which a substance may pass are indicated by the characteristic curve. From the study of such a characteristic curve we find that these molecular transformations are not specific, but of general occurrencealike in inorganic and living tissues. When the energy of the responding substance is for any reason below par, that is to say, when it is in the extremely sub-tonic A condition, external stimulus will be absorbed without evoking the normal excitatory expression. Response will then be abnormal, or of opposite sign to the true excitatory effect. By the absorption of impinging stimulus the substance now passes into the next stage $\mathbf{B}$, where molecular transformation proceeds at a rapid rate. At this stage, the previous abnormal response is not only reversed to normal, but successive responses exhibit a staircase increase. At the next or $\mathbf{C}$ stage, the responses are uniform. Following this, we arrive at the maximally distorted position D. Stimulus 
at this stage induces little further excitatory distortion, while the tendency to recovery is great. In this fatigue-state the amplitude of response undergoes a decline, and in the succeeding stage $\mathbf{E}$ an actual reversal.

The various corresponding types of response-sub-tonic abnormal, staircase, uniform, fatigue-decline, and fatiguereversal-are not exhibited by any one particular kind, but by all forms of tissues. Thus muscle may exhibit a shortlived staircase effect, and nerve, supposed to be indefatigable, not only shows decline, but even reversal of normal response, under extreme fatigue.

There are two definite conditions under which the normal negative response is converted into abnormal positive, with an intermediate diphasic. These are (I) the condition of extreme sub-tonicity, and (2) that of fatigue brought on by over-stimulation. As regards the first, it is to be remembered that the normal excitability of a tissue is maintained by the supply of energy from the rest of the organism of which it forms a part. Under isolation, the latent energy or tonic condition of the tissue is liable to fall below par, under which circumstances the response becomes abnormal positive. By the absorption of the energy of stimulus, the substance is transformed from the $\mathbf{A}$ to the $\mathbf{B}$ condition, with restoration of its normal response. The process of gradual transformation may be seen in a series of records to successive stimuli, when the abnormal gradually passes into the normal, through an intermediate diphasic. Or, an intervening tetanisation will serve to convert response from the abnormal to the normal. Abnormal or reversed response is also seen to occur under fatigue, but its genesis in the molecular curve is here in reversed order to that of the abnormal response of sub-tonicity. In the latter, during the continuous transformation from the $\mathbf{A}$ to the $\mathbf{C}$ phase, stimulation converted the abnormal response into normal, through diphasic. But now, during transformation induced by stimulus from $\mathbf{C}$ to $\mathbf{E}$, the normal negative passes into abnormal positive, through intermediate diphasic. To transform the abnormal positive 
into normal negative in the first case, stimulation is necessary. To do the samein the second case, rest is necessary. In the records obtained from different animal tissues, various anomalies are met with, of which there has not hitherto been any satisfactory explanation. Thus the same tissue at different times will be found to give either the normal negative, or di-phasic, or abnormal positive response. Thus it has been shown that in the two extreme cases alike, of subtonicity and fatigue, the response of nerve is abnormal positive (p. 636). Numerous other examples of this fact have been met with in the course of this work, in, for example, the response of skin (p. 31 1), that of the glandular and digestive organs (pp. 342, 346), and that of retina (p. 423). It will thus be seen how important is the molecular condition of the tissue in determining the nature of response. This is strikingly shown in the fact that the same tetanisation which in the $\mathbf{A}$ condition converts the abnormal to normal, in the $\mathbf{D}$ will convert the normal into abnormal. Again, if tetanisation be applied at the beginning of the $\mathbf{B}$ stage, the subsequent responses are enhanced, whereas the same tetanisation at the end of $\mathbf{C}$ induces a fatigue reversal (figs. 394, 395, and 398).

That the explanation of these various results is to be sought for in molecular considerations, and not in that hypothetical assimilation and dissimilation which really explain nothing, is fully demonstrated by the fact that precisely similar responsive variations are obtained, in the same circumstances, in the case of inorganic matter, under different forms of stimulus and different methods of record. As an example, may be cited the transformation of abnormal response into normal, in tungsten, after tetanisation (fig. 39I), the stimulus employed being electric radiation, and the mode of record, resistivity-variation. Parallel effects have been shown in the case of tin, the response being recorded by the electro-motive variation, and the stimulus employed mechanical (fig. 386). The enhancement of normal response also under tetanisation, when at the B stage, has been shown in tin (fig. 388); and finally, the reversal of normal response 
by fatigue was shown in tungsten under electric radiation, while in the contractile response of indiarubber under thermal stimulation it took place with intermediate diphasic (fig. 397). The characteristic curve has been shown to exhibit the history of molecular transformation under continuous stimulation. In the first part of this curve a progressive change is shown to be manifested outwardly by increasing contraction or galvanometric negativity. In the second part a reversal of this process is seen to occur. This is illustrated in records of response under continuous stimulation. Thus muscle shows increasing contraction, to be followed by fatigue-relaxation (fig. 64). The same thing is observed in Mimosa, as a fall of the leaf, followed by its re-erection (fig. 65). Electrically, this is observed as increasing galvanometric negativity, followed by reversal to positivity. These phasic alternations may in some cases be exhibited only once, and in others repeatedly. Thus, in a certain style of Datura such phasic alternation is seen to occur twice (fig. 76); and again, in leaflets of Desmodium gyrans, at first quiescent, continuous stimulus of light gives rise to those repeated alternations of negative and positive which constitute multiple response (fig. I4I). The distinction between the tissue which gives only one such alternation, and others which display it in repeated succession, is not, it should be borne in mind, rigid. Even skeletal muscle, under certain circumstances, is found to give rise to rhythmic excitations. The fact that multiple response is a phenomenon of widespread occurrence, and not specifically characteristic of any particular kind of tissue, has been fully demonstrated in the course of the present work.

It would appear that there is a tendency of the incident stimulus, when applied continuously, to find an expression whose predominant characteristics are alternating. We may first have the exhibition of excitatory molecular distortion. But when this has reached a maximum, no further excitatory expression being possible, the incident energy becomes relatively effective in increasing the internal factor, with 
attendant expansion, galvanometric positivity, and enhanced power of recovery. At the maximum point-that is to say, at the top of the tetanic curve-the two forces are balanced; and at this point, if the stimulus be suddenly withdrawn, the particular state of unstable balance is often manifested by a brief overshooting in one or other direction. This effect is often noticed in the retina and in certain vegetable structures under the action of light (figs. 244, 247,254, and 260), and in nerve under electric tetanisation (p. 536). That it is not primarily dependent on assimilation and dissimilation, but on the molecular factor, is seen in the fact that similar effects are also to be observed, under corresponding circumstances, in the response of inorganic substances (figs. 258 and 383).

The phasic molecular alternation so conspicuously exhibited under continuous stimulation may also be seen in the record of responses to successive stimuli. The phenomenon is then regarded as an after-effect and shown by the shifting of the base-line of the record (figs. 208 and 396).

Since the effect of stimulus is to induce a molecular upset, the change in question must be attended by various concomitant physical changes. It will therefore be possible to record the excitatory effect by recording the attendant variations of any one of these. The effect of stimulus may thus be recorded by (I) the accompanying change of form, in contraction or expansion; (2) an attendant secretion or absorption; (3) a variation of electric resistivity by diminution or increase of resistance; and (4) electro-motive changes of galvanometric negativity or positivity. The changes in the responding substance as a whole, may be recorded by any one of the first three methods. But in the last," or that by the electro-motive variation, the method depends on the relative variations of the electric potential at two different points. For if the substance be isotropic and subjected to diffuse stimulation, the electro-motive change at the two contacts being similar, there will be no resultant effect to record. For the recording of electro-motive response, then, it is necessary to obtain an effect which is differential. 
The first way of doing this is to localise the stimulus at one of the two contacts. This may be done by interposing a physiological block between the two, so that the excitation of one does not reach the other. The second method is to select an experimental specimen which is anisotropic, whether naturally or artificially. Artificial anisotropy is induced by injuring one of the two contacts, and so bringing about a relative depression of excitability at that point.

The method of resistivity variation which I had previously employed, in observing the response of inorganic substances, proved capable of sufficient perfectibility for the study of similar phenomena in living tissues also. The main difficulty in applying this method had hitherto lain in the disturbing electro-motive variation, consequent on a-symmetrical excitation, or the differential excitability of the structure. A detailed account of the means by which this method was rendered reliable will be found in Chapter XXXVII., where it will be seen that records of the excitatory variation obtained by it are in every way similar to those made by other methods. All the different modes of taking records which have been enumerated are, it must be remembered, independent expressions of a common fundamental molecular change. Thus, on physically restraining that mechanical movement in a motile organ which is due to excitatory change, the electromotive response of galvanometric negativity continues to be given. Similarly; in a tissue in which, under the experimental arrangements, there can be no resultant electro-motive change and no contractile movement, the excitatory change may, nevertheless, be observed by means of the resistivity variation (p. 548).

For the obtaining of the electromotive response, the electrical mode of stimulation, unless special precautions are taken, is subject to various disturbing influences, such as current-escape and the occurrence of polarisation. For this reason it was desirable to devise sorne non-electrical form of stimulation which should be capable of quantitative application; and this I have been able to secure by no less than 
three distinct methods. I found that torsional to-and-fro vibration constituted an effective form of stimulus, the amplitude of which could be increased by increasing the angle of vibration. The intensity of stimulus was found to remain constant so long as the period and amplitude vibration were kept constant. The tissue, moreover, was not subject to injury by the use, within limits, of this method (p. 3I). My second method was that of Rotary Mechanical Stimulation, in which friction of the terminal area of a pumicestone electrode constituted the stimulus, whose intensity was determined by the number of rotations (p. 29I). The third non-electrical mode of stimulation employed was that of thermal shocks. The area to be stimulated was, in this case, enclosed within a thermal loop of platinum or german-silver wire, the requisite thermal variation being produced by the passage of a heating electrical current round the loop. The intensity of the stimulus could in this case be varied by increasing the intensity or duration of the heating current (p. 38). And finally I have shown that the drawbacks incidental to the electrical mode of stimulation might be overcome by the use of equi-alternating shocks, the indefinite polarisation factor being thus neutralised (p. $25 \mathrm{I}$ ).

As the intensity of stimulus is gradually increased, it is found that the amplitude of response reaches a limit. Beyond this, increase of stimulus evokes no increase of response. On the application of a very strong stimulus, then, there is an amount of energy which is unable to find expression in the single response given by the tissue. Under such circumstances, the excess of energy is held latent, and often finds responsive expression later in a series of multiple responses. This phenomenon of multiple response to a single strong stimulus I find to be of very extensive occurrence. As examples of the different kinds of tissues in which this may be observed, may be mentioned the stems and petioles of various plants (fig. I38), the digesting leaves of Drosera (fig. 209), the pitcher of Nepenthe 
(fig. 206), the animal stomach (fig. 213), nerves of animals, and the retina (fig. 252).

I have also shown that there is no strict line of demarcation between the phenomena of such multiple response and autonomous response so-called. Biophytum, for example, which, usually speaking, exhibits a single response to a single moderate stimulus, and multiple response to a strong stimulus, will, under exceptionally favourable tonic conditions-that is to say, when it has absorbed from its surroundings an excess of energy-exhibit responses which are apparently autonomous. A typically autonomous plant like Desmodium gyrans, again, when deprived by unfavourable circumstances of that excess of energy which it requires, will be reduced to the condition of a multiply responding plant merely. It then responds by a single response to moderate, and by multiple responses to strong, stimulus. When the energy imparted by strong stimulus is exhausted, these multiple responses come to a stop, to be once more renewed, on a fresh accession of strong stimulus. Or a lateral leaflet of Desmodium, originally quiescent, may be put into, and maintained in, a state of pulsation by the action of sunlight.

It is from the stored-up energy derived from its surroundings that the tonic condition of the plant is so raised as to maintain its so-called autonomous activity. From this it will be seen that, strictly speaking, there is no such thing as automatism. Movement can only be produced by the immediate action of stimulus, or by energy previously absorbed:

In recording the autonomous pulsation of the lateral leaflets of Desmodium gyrans, it is found that while the down-movement brought about by the contractile action of the lower half of the pulvinule is very rapid, the up-movement due to recovery, and to contraction of the upper half of the organ, is relatively slow. The two alternating excitatory impulses, in the lowerr and upper halves respectively, are in the ratio approximately of $I^{\circ} 5$ to $I$. This 
explains the peculiar electrical responses of Desmodium gyrans, which are concomitant with the autonomous mechanical pulsations of the leaflet. I find that, corresponding with one complete mechanical pulsation, there are two electrical pulses. Of these the principal electrical wave coincides with the down-movement of the leaflet, and the smaller with the up. The electro-motive intensity of the principal wave is nearly $I^{\prime} 5$ times that of the subsidiary. In a particular experiment, for example, while the value of the former was 0024 volt, that of the latter was. OoI 6 volt. These electro-motive variations are expressions of fundamental excitatory effects, and not dependent on the mechanical movement of the leaflets. For when the responding leaflet is physically restrained, the electro-motive responses exhibit even greater intensity than before. This will be seen in the simultaneous records of mechanical and electrical pulsations given in fig. 145 .

An important subject of inquiry lay in the accurate determination of the death-point. This investigation afforded striking demonstration of the fact that it is a single excitatory reaction which is expressed in different ways under different modes of record. It has been shown that when the experimental tissue is subjected to a gradual rise of temperature, there is a definite point at which an excitatory spasm occurs, marking the initiation of death. If a continuous record be taken of the concomitant variation of length, increasing expansion is found to be converted at this point into a sudden contraction. In an anisotropic organ like the pulvinus of Mimosa the erectile movement of the leaf is abruptly transformed into one of fall. A curled tendril exhibits at this point a sudden uncurling. Taking, again, the electro-motive method of record for the detection of the death-point, the increasing positivity of the specimen is spasmodically reversed to negativity. Finally, on employing for the record the method of resistivity variation the increasing is seen to become suddenly changed into a diminishing resistance. It is found, how- 
ever, employing numerous specimens, that these mechanical and electrical spasms take place, under normal conditions, at the same point. In the case of phanerogamous plants, this is found to be at or very near $60^{\circ} \mathrm{C}$. (figs. 328, 329, 330). That these mechanical and electrical spasms, further, constitute a true case of excitatory response, is proved by the fact that induced physiological depression also induces depression of the death-point. Fatigue may thus lower the death-point by as much as $19^{\circ} \mathrm{C}$.

The response of contraction, initiated at the death-point, is later converted into post-mortem relaxation, and galvanometrically the negativity initiated at the same moment becomes subsequently a post-mortem positivity.

With regard to the so-called Current of Injury it.was shown that this arises as the after-effect of strong stimulus. It should be remembered that a cut, or the application of a heated wire, constituting mechanical and thermal sections respectively, will act as a strong stimulus, and, further, that the after-effect of excitatory galvanometric negativity is persistent when the stimulus is strong. The excitatory effect, moreover, is transmitted from the point of application to greater or less distances, according to the strength of stimulus and the conductivity of the tissue. As this transmitted effect undergoes diminution with distance, it is obvious that the most intense negativity will be.induced at the point of section, undergoing a gradual diminution as we move further away from it. If, taking a given length of isotropic tissue, we make two opposite terminal sections, we shall clearly have a symmetrical distribution of electrical potential as regards the middle or equatorial zone, the two ends being most negative, while the equator is relatively most positive (fig. I IO). Two points symmetrically situated as regards this equator would thus be equi-potential, while a-symmetrical points would show appropriate differences of potential, a zone near the equator being relatively positive to one which is further away from it, or nearer to the terminal section. These considerations, supported as they 
are by experimental results, account satisfactorily for the particular electrical distribution in a muscle-cylinder.

It is often supposed that dead tissue is negative to living. But I have shown that this is not the case, the dead being actually positive to the living. It has already been mentioned, in connection with experiments described, on the mechanical and electrical spasms of death, that at the initiation of death, a tissue exhibits excitatory contraction and negativity, while the post-mortem effect is one of relaxation and positivity. This explains the peculiar electrical distribution which I have observed, in the exploration of tissues, of which some parts were dead, others dying, and still others, again, fully alive. It was there shown (figs. I I3, I I 5) that the greatest negativity occurred on the death-frontier. Proceeding in either direction from this point, whether towards the living or towards the dead, it is found that these points are increasingly positive, or decreasingly negative. But the maximum positivity of the dead portion is greater than that of the living. From this it is clear that the dead is positive to the living.

From these facts, that the dying is negativè, and the dead relatively positive to the living, it is clear that the so-called current of injury is liable to reversal. In the case of the former, the current of injury will be from the dying to the living; in the latter, from the living to the dead. This demonstration of the occurrence of a hitherto unsuspected reversal, demonstrates to us the possibility of many complications, and wrong theoretical inferences. For response by the negative variation of the current of injury is usually taken as the concomitant of the chemical process of dissimilation, while the positive variation is held to be associated with assimilation. Now, by the reversal of the so-called current of injury, one identical excitatory reaction may be made to appear, now as a negative, and again as a positive variation. This is sufficient to indicate the unreliability of the so-called Method of Negative Variation, 
an unreliability of which we shall further meet with many glaring instances.

An assumption more or less current is, that in order to obtain response, there must be an antecedent current; by whose negative variation it can be detected. Hence the supposed necessity of a current of injury prior to response. The real reason, however, for thus injuring one of the contacts is so to depress its excitability that, on diffuse stimulation, the excitatory response of the uninjured may remain unbalanced, and therefore unannulled. That it is this depression of excitability, and not the current of injury as such, which is the essential condition for obtaining resultant response, is seen from the fact that excitatory response may still be obtained, even when the so-called current of injury is zero, or reversed positive (fig. I I6). 


\section{CHAPTER XLVII}

\section{REVIEW OF RESPONSE OF ANISOTROPIC ORGANS}

Anisotropic organs-Laws of response in anisotropic organs-Natural current of rest and current of response-Reversal of natural current of rest--Unreliahility of positive and negative variations of current of rest--Determination of the differential excitability of a tissue-Resultant response of skin due to induced stronger negativity of inner surface and feebler negativity (tomato skin) or positivity (skins of grape and frog) of outer-Response of intact human skin-Response of intact human lip-High excitability of secretory and glandular surfaces-Response of glandular foot of snail-Response of intact human tongue-Response of digestive organs-Phasic alternations of secretion and absorption-Multiple response of digestive organs-Phasic changes induced by previous activity-Response of digestive organs of Nepenthe and Drosera - Electro-motive peculiarities of skin and mucous coat of stomach not similar - Normal response by galvanometric negativity in mucous coat of stomach of frog, gecko, and tortoise-The root as a digestive organ-Excitatory secretion and galvanometric negativity of young roots-Phasic alternations of secretion and absorption - Cognate subject of ascent of sap-Sap-wood not really deadProofs of physiological character of suctional response-Water-movement a mode of excitatory response-Response of electrical organs-Two types, Torpedo and Malepterurus - Vegetal analogues to electric plates of two types, Torpedo and Malepterurus, in Pterospermum and pitcher of Nepenthe-Multiple character of response of electric organs-Response of electrical organs constitutes an extreme case of differential excitability of anisotropic structures---Similar effects with inorganic structures--Excitatory effect of light on plant tissues-Phasic alternations-Initiation of multiple and autonomous response by light-Three types of direct and after-effects-Response of retina like, and not different from, that of other tissues-Error introduced by method of negative variation-Multiple responses in retina and their visual correspondences - Binocular Alternation of Vision-Three types of direct and after-effects in retina under light-Geo-electric response.

I SHALL next pass in review another class of phenomena, the want of a clear understanding of which is at the root of many supposed anomalies in the response of animal tissues. I allude to the natural anisotropy, with consequent differential excitability, of various organs. 
As an example of a differentially excitable organ we may take the pulvinus of Mimosu, in which the lower half is more excitable than the upper. In this case, strictly localised stimulation of either the upper or the lower evokes contraction and galvanometric negativity of that particular half, the effect in the lower half being the greater. But if the stimulus be diffused, whether internally or externally, the response will be differential, by the greater contraction or galvanometric negativity of the more excitable. From this we arrive at the general law of the electrical response of anisotropic organs.

1. On simultaneous excitation of two points $\mathbf{A}$ and $\mathbf{B}$, the responsive current flows in the tissue from the more to the less excited.

2. Conversely, if under simultaneous excitation, the responsive current be from B to A, B is the more excitable of these two points.

The second of these two laws enables us to determine the relative excitabilities of any two points. As a simple example of the anisotropy induced in a tissue by the unequal action of the natural stimuli of the enviroment, we may take a tubular organ, such as the hollow peduncle of Uriclis lily. Here the exposed outer surface, constantly subjected to external stimuli such as light, becomes as it were fatigued, and reduced in excitability. Other histological modifications follow on this, the external cells becoming thus cuticularised and protoplasmically defective. Owing to the depression of excitability on this epidermal surface, the intensity of its normal excitatory change by galvanometric negativity is decreased, a change which, in the case of certain skins, culminates in responsive positivity. The inner surface of the hollow peduncle, which may be regarded as epithelial, being, on the other hand, protected, remains normally excitable and is thus more so than the outer surface. The outer surface, however, probably by reason of the action of the external stimuli to which it is constantly exposed, is naturally negative, relatively to the protected and more excitable inner 
surface. And it will generally be found true that while this natural cnrrent of rest is from the less excitable $A$ to the more excitable $B$, the current of response, on the other hand, which occurs on excitation, is from the potentially more excitable, and therefore now more excited $B$, to the less excitable and therefore less excited $A$.

Such is the course of events in the normal or primary condition. But under the excitation due to preparation, or accidental disturbance, the more excitable surface becomes the more excited, and relatively to the other, galvanometrically negative. In consequence of this, the natural current is reversed, and we have a resting-current due to the aftereffect of injury or accidental excitation, flowing from the more to the less excitable. Thus, while the natural current, in the primary condition, was from the less excitable $A$ to the more excitable $B$, that is to say, $A \rightarrow B$, this reversed current of rest, due to accidental excitation or injury, is from $B \rightarrow A$. Even now, however, $B$ may be more excitable than $A$, hence fresh stimulation will induce a responsive current from $B$ to $A$. In the primary condition, such a responsive current would have appeared as a negative variation of the natural current $A \rightarrow B$. But when the primary condition has been so modified that the natural current is reversed, and has become $B \rightarrow A$, the normal responsive current $B \rightarrow A$ will appear as a positive variation. Still another variation is possible, when the normal response itself undergoes reversal owing to fatigue, under which condition this abnormal response, relatively to the reversed current of rest, appears as if it were the normal negative variation (fig. I I9). It has, however, been shown that if we discard this unreliable test of response, by the variation induced in an antecedent current of rest-the so-called negative variation-it will be found that the responsive current always flows from the more to the less excited.

In order to determine which of two points in an anisotropic tissue is the more excitable, it is necessary, as now understood, to determine the direction of resultant response, under 
stimulation which is equal and simultaneous. In order to do this, we may employ such a non-electrical form of stimulation as the mechanical or the thermal. For this it is possible to employ (I) the Vibratory Stimulator; (2) the Rotary Mechanical Stimulator ; or (3) stimulation by thermal shocks. When results are obtained according to these methods, there can be no uncertainty as to those complications of effects which might conceivably arise when the electrical form of stimulus is employed. The last-named may, however, be used without misgiving, when stimulation is effected by equi-alternating shocks. The ordinary Ruhmkorff's make- and break-shocks are not suitable for this purpose, inasmuch as the effective intensity is unequal for make and break, besides which the polarisation-effect may not be exactly neutralised. The equi-alternating shocks, from which these defects have been eliminated, are obtained by means of (I) a rotary reverser in the primary coil (fig. I 70), or (2) a motor-dynamo (fig. I72). The responses again, under these electrical forms of stimulation, may be photographically recorded as either the direct or the after-effect of stimulus. It was shown, by the employment of all these various methods of stimulus, mechanical, thermal, and electrical, that the responsive current to be obtained with an anisotropic organ was definite in direction, being always, under normal conditions, from the more excitable $B$ to the less excitable $A$. I shall now proceed to recapitulate briefly the results obtained by these methods in various cases of anisotropic tissues, such as skin, epithelium, glands, animal, and vegetal digestive organs, and electric organs generally.

Taking first the skin of tomato it has been shown that the separate responses of the outer and inner surfaces are unequal. The outer, owing to cellular modification under the stimuli of the environment, gives only a feeble negative response, whereas the internal surface gives a much stronger normal response by galvanometric negativity. On simultaneous excitation of both inner and outer surfaces, the responsive current is found to flow from the inner to the 
outer. Here the resultant current is brought about by the difference between the stronger responsive negativity of the inner, and the feebler responsive negativity of the outer surface, which may be represented as $\uparrow^{\downarrow}$. With certain specimens of tomato skin, however, the modification of the outer surface is so great that its individual response is reversed to positive, that of the inner being the normal strong negative. The resultant response, then, is still from inner to outer, but equals the summated effect of the two $\uparrow$. From this we pass to the response of grape-skin, which resembles the latter of these two cases. The response of the skin of frog is also of this type, and it may be said of skins in general that their response is from the more excitable inner surface to the less excitable outer. This conclusion has been verified by experiments on various skins, both vegetable and animal. Among the latter of these may be mentioned the skin of the neck of tortoise, and that from various parts of the body of gecko.

When the skin is isolated with very great care, so as to reduce to a minimum the excitatory effect of preparation, it is found that the natural current of rest is from the less excitable outer to the more excitable inner surface; the excitatory current being in the opposite direction. Owing to the excitatory effect of preparation, the current of rest of the skin of tortoise was found reversed. The responsive current, however, was found to flow from inner to outer, thus proving that the inner surface was the more excitable. In illustration of the great practicability of the methods employed, I may refer to the photographic records obtained of the response of the skin of the intact human forefinger (fig. I 80).

In describing the differential excitability of the hollow peduncle of Uriclis lily, it was shown that protected surfaces are, as a rule, more excitable than those which are exposed, and have thus undergone a greater degree of modification. On taking the plagiotropic stem of Cucurbita, the lower surface of which is protected from light, it is found that, 
while the current of rest flows from the exposed upper to the protected lower surface, the direction of the responsive current is opposite, namely from the lower to the upper, proving that the protected lower is the more excitable of the two. Similarly, in the case of the intact human lip, I found that the resting current was from the epidermal to the epithelial, the responsive current being in the opposite direction (fig. 196). Again, on testing the differential response of armpit and shoulder, I found that the responsive current was from armpit to shoulder, the former being thus the more excitable of the two (fig. 194).

We have seen that the lining membrane of the inner surface of the peduncle of Uriclis lily is very thin, and that, in distinction to the outer or epidermal membrane, it may be regarded as epithelial. As we approach the bulb-end of the peduncle, this inner layer of cells is found to be highly turgid, and secretion is found to take place into the hollow tube. The inner surface of the carpellary leaf of Dillenia indica, again, secretes a mucilaginous substance. In these two cases there are no definite glands. But definite glands are found to occur on the inside of the pitcher of Nepenthe. In all these cases the secreting layer, whether provided with glands or not, is found to be very highly excitable, and to respond by strong galvanometric negativity. Taking a carpel of Dillenia indica, it is found that the natural current is from the outer epidermal to the inner secreting surface, the responsive current being in the opposite direction. On making very careful connections, with the skin of the protruded body of the snail, and the glandular under-surface of its foot, it is found that the natural current is from the non-glandular to the glandular, but the responsive current from glandular to non-glandular. As an example of the way in which the true natural current of rest may be reversed by the excitatory effect of preparation, I showed that, while in the intact snail the natural current was from non-glandular to glandularthe gland being in this case relatively positive, to the extent of $\mathrm{OOI} 3$ volt-after the sectioning of the foot, the original 
natural current was reversed, owing to the greater relative excitation induced at the glandular surface, which now became relatively negative, to the extent of -.0020 volt. With the intact human tongue, further, I found that a very strong responsive current was induced on excitation, from the lower to the upper surface, thus showing that the lower was the more excitable of the two.

The response of digestive organs may now be passed in review. In these, as in glandular organs, excitatory response is supposed to take place by secretion. In connection with this, it must be borne in mind that in the tissue of the pulvinus of Mimosa, on the removal of the impervious skin, excitation induces secretion of the contained fluid, which, again, is re-absorbed on the cessation of excitation. We know the pulvinus to be contractile, and may therefore regard this secretion as an effect of contraction, causing expulsion of water. Apart from the differential action of the upper and lower halves of the organ, and the magnifying petiolar index, the fundamental contractile action would, in the case of Mimosa, as in others, have passed unnoticed. This goes to show that it is not impossible that the phenomenon of secretion through a permeable membrane may be associated with excitatory contraction. In favour of such continuity, it may be urged that tissues, hitherto regarded as non-motile, have been shown to exhibit excitatory contraction. In digestion, as a whole, we have to recognise two different processes, those, namely, of secretion, and of subsequent absorption. Parallel to these, we find that the electrical response of digestive organs exhibits phasic alternations of negativity and positivity.

It was shown that the pitcher of Nepenthe-which may be regarded as an open stomach-affords us unique facilities for the observation of the normal responses of digestive organs. In experimenting with the animal stomach, the specimen has to be cut open, in order to make the necessary connections; and, owing to the highly excitable character of the organ, this gives rise to intense excitatory action, the 
after-effect of which is necessarily to reverse the normal current of rest. With a pitcher of Nepenthe in a fresh condition, the natural current of rest is from the outer to the inner, the responsive current being in the opposite direction, and the glandular surface, on simultaneous excitation of the two, becoming galvanometrically negative (fig. 203). Digestive organs, moreover, tend to exhibit multiple responses, the response to a single strong stimulus, say thermal, or of mechanical section, consisting, whether in animal or vegetable organs, of a series that may persist for nearly an hour (figs. 206, 209, and 213). When the pitcher of Nepenthe contains a large number of captured flies, that is to say, when it has been subjected to long-continued stimulation, it exhibits a phasic change, the responses now becoming reversed to positive (fig. 205). This, as pointed out above, is probably significant of absorption. In Drosera, the normal response of the glandular surface is by induced negativity, but on long-continued stimulation, this is reversed to positivity (fig. 208).

In the animal stomach, the observed current of rest is generally from the glandular to the non-glandular surface. From the fact that the skin of the toad, which is also possessed of imbedded glands, gives a current of rest from the outer surface to the inner, it has been supposed that the mucous coat of the stomach of the frog had the same electromotive reaction as its outer skin. That this, however, is not the case is seen from the fact that on excitation the skin becomes galvanometrically positive, while the mucous membrane of the stomach becomes galvanometrically negative. The observed current of rest in the stomach would appear, from Nepenthe, to be, not the natural current of rest, but the reversed current, due to the excitatory effect of preparation. The normal effect of excitation in the stomach, I uniformly find, in such different instances as frog, gecko, and tortoise, to be by galvanometric negativity of the mucous surface (figs. 2IO, 2II, and 2I2). On applying a strong thermal stimulus to the stomach of frog, I obtained an interesting series of 
responses, of which the first were negative, the second part diphasic, and the last portion reversed positive (fig. 213 ).

Looking at the phenomenon of digestion, we see that it consists first of a secretory process, by which certain solid substances are dissolved, and secondly of the absorption of these dissolved substances. Similar functions are subserved in vegetable life by the root, by which solid inorganic foodmaterials are first dissolved by secreted acids, and then absorbed. The proof of the former is seen in the wellknown corrosion-figures produced by growing rootlets on a marble surface. I have also been able to demonstrate the phenomenon of excitatory secretion in young roots by allowing them to absorb dilute salt solution, and then under excitation to secrete it into highly dilute silver nitrate solution. This last was attended by the visible formation of a white precipitate. The electrical response of young roots of Colocasia, moreover, I found to be by induced galvanometric negativity (fig. 214), which, under long-continued stimulation, was apt to show reversal to positivity. The older roots, on the other hand, under the same intensity of stimulation, gave response by galvanometric positivity (fig. 21 5). The former of these responses, there is every reason to believe, is associated with secretion, and the latter with absorption.

This question of the absorption of inorganic food materials by the plant is naturally connected with the subject of the Ascent of Sap, which is regarded as one of the most difficult problems in plant physiology. The non-physiological theories advanced are admittedly inadequate to the explanation of this phenomenon. That the ascent, nevertheless, could not be due to physiological action was held to have been proved by the facts (I) that water-conduction takes place preferentially through sap-wood, assumed to be dead; and (2) that poisonous solutions, such as would kill a living tissue, have been found to be transported through the roots, or the cut ends of their trunks, to the tops of trees.

I have, however, been able to show that these objections are not valid. For in the first place, the supposed dead 
wood, concerned in the transport of sap, through the trunks of trees, can be proved, by electrical tests, to be fully alive. This living wood responds to stimulation by induced galvanometric negativity, such response disappearing on the death of the tissue, as, say by drying, after which it cannot be restored. The response of living wood is also depressed by anæsthetics, and abolished by poisons (figs. 216, 217). As regards the argument based on the transport of poison, it has been shown that as the active elements concerned in the transport of sap are diffused throughout the length of the trunk, the death of one individual zone, to which the poison has ascended, does not abolish the suctional activity of the zone above. It is only when the plant has been killed throughout, by the arrival of the solution at its top, that the complete arrest of suction could be expected to take place. And this is found to be the case. Various agents, on the other hand, which are known to induce changes, whether of exaltation or depression, in the physiological activity, are found to induce corresponding modifications in the rate of suction. A very delicate means of investigating this question has been shown to be that afforded by the records obtained with the Shoshungraph (fig. 218). Here, under the ordinary method of record, the slope of the curve indicates the normal rate of suction, and the effect of various agencies is immediately shown by the resulting flexure of the curve. This method of record, again, becomes extremely sensitive, when it is carried out under balanced conditions. By means of these records, it has been shown that depressing agents, such as cold or anæsthetics, depress or arrest suction, whereas warmth exalts it. It has been shown, further, that just as the multiple activity of the Desmodium leaflet is arrested, when the latent energy of the plant falls below par, so also, under similar circumstances, the suctional activity falls into abeyance, and that, as in the one case, so also in the other, the activity is renewed, by the application of an external stimulus. It has also been shown that the latent 
period which elapses, before the initiation of this responsive variation to external stimulus, is longer when the plant is in a sub-tonic condition than in the same plant when its tonic condition has been slightly raised by previous stimulation. Crucial experiments, finally, have been described, showing that water-movement is a mode of excitatory response.

A difficult problem in connection with electrical response is that of the discharge from the electrical organs of certain fishes. In a large number of cases, of which Torpedo may be taken as the type, the discharge takes place in a direction from the anterior or nervous to the posterior and nonnervous surface. Pacini's generalisation that the responsive discharge is always from the anterior to the posterior surface is negatived by the instance of Malepterurus, in which it is from the modified glandular posterior to the anterior surface. Another peculiarity of the response of electric organs in general is that the responsive current is always in the same direction - that, namely, of the organ-discharge-whether the exciting shock be homo- or hetero-dromous. No theory has yet been found which will fully explain all these peculiarities.

I have shown, however, that this phenomenon is not alone of its kind; nor is it dependent on any specific characteristic of the animal nerve-and-muscle, or gland, of which different electric organs are modifications. The response of the electric organ simply constitutes an extreme case of differential excitability, and follows the general law of response in anisotropic organs-namely, that on diffuse stimulation the responsive current flows from the more to the less excitable. The peculiarity of the organ simply depends upon the fact that owing to the serial arrangement of its elementary anisotropic plates the terminal electro-motive effect becomes very large by summation. We find vegetal analogues to the two types of electrical plates of Torpedo and Malepterurus, in the leaves of Pterospermum, and the pitcher.of Nepenthe. In the first of these, Pterospermum, as in Torpedo, the anterior nervous surface is relatively morc excitable than the mass 
of indifferent tissue on the posterior surface. Hence the current of response is from the more excitable anterior to the less excitable posterior. In the second type-the pitcher of Nepenthe and the electrical plates of Malepterurus-the posterior surface being glandular and therefore exceptionally excitable, the responsive current is from posterior to anterior.

In taking rheotomic observations on the response to electrical stimulation in various anisotropic leaves-virtually acting, as has been shown, like electrical plates - it was found that in sluggish specimens the maximum electro-motive value was attained 2 second after the exciting shock. This was also the value of the period which elapsed after the application of moderate mechanical stimulation. With vigorous specimens, however, such as the leaves of Nymphrea alba, the maximum effect was attained in a much shorter time, that is to say, in about ${ }^{\circ} \mathrm{O} 3$ second. In the electrical o:gan of Torpedo the corresponding period has been found to be oI second. The response of electrical organs is found to be repeated or multiple. In the rheotomic records obtained with leaves, further, the multiple apices of the curve show that the response of vegetable organs also has this multiple character Multiple response, however, is not the peculiar characteristic of the electrical organ, but has been shown to take place in various kinds of animal and vegetable tissues.

Again, that this peculiarity-of definitely uni-directioned response, whether the excitation be homodromous or heterodromous-is not distinctive of life, with its specific powers of assimilation and dissimilation, but of anisotropy in general, with its consequent differential excitability, was shown by the fact that similar uni-directioned responses to homo- or hetero-dromous shocks were given by an inorganic structure, consisting of prepared lead (fig. 167).

We have next to pass in review the question of the response of plant and animal tissue to stimulus of light The various motile responses, induced by light in plants, are so diverse and so apparently incapable of being explained by any single reaction of fundamental excitation, that it 
was thought that the effect of this stimulus was different in different cases, the specific reaction in each organ being determined by the ultimate advantage of the plant. But I have been able to show that the excitatory effect of light is normal and like that of any other form of stimulus. The various results induced by it depend, first, on the question whether stimulus has remained localised at the point of application, or been transmitted to distant areas. The effect is thus modified by the intensity of the stimulus and the conductivity of the tissue. These results, however, may be further modified by the differential excitability of the organ. Here, as in other cases of stimulation, the general rule holds good that response is by greater contraction and galvanometric negativity of the more excited. As a concrete example may be mentioned the case of the pulvinus of Mimosa, when the upper surface alone is subjected to the stimulus of light. Here, owing to local excitatory contraction of the upper, the expelled water reaches the lower half of the pulvinus and induces there the hydro-positive effect of expansion, both of these effects conspiring, in this first stage of response, to erect the leaf. The electrical variation at the lower half is here, then, found to be positive. But as the excitatory effect itself is gradually conducted to the lower half, it induces there an increasing contraction. The mechanical response is now therefore reversed, from one of erection to one of depression, the electrical variation of the lower half of the pulvinus undergoing at the same time a corresponding change from positivity to negativity (fig. 237). From this experiment it is clear that the electrical response under light exhibits the same stimulatory changes which are also visibly demonstrated by mechanical response. We see, moreover, from this experiment that light in general acts as a moderate stimulus. For while mechanical or thermal stimulus induces a sudden collapse of the leaf of Mimosa, the application of light brings about only a gradual fall.

Owing to this moderateness of the stimulus of light, and to the fact that its application is strictly local, it is easy to 
understand the possibility of certain modifications occurring in the response. Thus, in highly excitable and conducting tissues, the responses will be by galvanometric negativity, and the state of excitation will be conducted to a certain distance. But we have seen that in tissues which are not highly excitable, stimulus, falling below the excitatory value, gives rise to positive response. Thus, under the action of light, we obtain in plants two types of response, negative and positive. Moreover, under continuous stimulation of light, these may undergo phasic alternations $(-+-+)$ or $(+-+-)$. As an example of negative response to direct or transmitted stimulation of light may be seen the response of Bryophyllum (fig. 238), the positive response being exemplified in the record obtained with a petiole of cauliflower (fig. 240).

It has been explained how these alternating phasic responses lead us to the phenomenon of multiple and autonomous response.

A leaflet of Biophytum, or a Desmodium leaflet in a state of standstill, under the continuous action of strong light, will exhibit multiple mechanical responses. The corresponding multiple electrical responses are seen in the response of the lamina of Bryophyllum under the action of continuous light (fig. 242). It has also been shown that these phasic alternations are brought about by the fact that the antagonistic elements in the response become effectively predominant by turns. Either of these antagonistic factors may be unmasked more effectively by the arrest of external stimulus at a particular phasic maximum. Thus, in the case where the normal alternation is $(-+-+)$, if the stimulus be suddenly withdrawn at the end of the second phase, or positive maximum, the response overshoots in the positive direction (figs. 243, 244). The characteristic direct and after-effects in this Type I., then, during the application of light and its removal are $(-++)$. In specimens whose characteristic response under continuous stimulation is $(+-+-)$, if stimulus be again withdrawn at the end of the second 
phase-here negative maximum-the response overshoots in the negative direction (figs. 245, 247). The direct and after-effects in this Type III., therefore, may be represented by the formula $(+-\ldots)$. Between these two extremes lie instances of an intermediate Type II., which has cases $(a)$ and $(b)$, according as the stimulus is removed at maximum of the first or negative phase, or at maximum of the second or positive phase. The formula of Type II. $(a)$ is thus $(-+\ldots)$, while that of Type II. $(b)$ is $(-\ldots)$.

The response of the retina furnishes us with the most striking examples of the action of stimulus of light. The true character of this response has been supposed hitherto to be unlike that of other tissues, for while excited nerve and muscle were said to show response by 'negative variation,' the response of the retina was referred to as by ' positive variation.' This furnishes us with an instance of the confusion which is apt to result from making the so-called resting-current the standard of reference. On testing for the natural current, by making connections with the longitudinal surface of the optic nerve, and with the cornea, in an undetached eyeball of frog, I found that it flowed from the cornea to the nerve. But when the eye is detached, by section of the optic nerve, the after-effect of excitation on the more excitable nerve reverses this current, the nerve becoming relatively galvanometrically negative. The normal effect of transmitted excitation from the retina would now make the nerve still more galvanometrically negative, and this would appear as a positive variation of the reversed natural current. Hence, the responsive positive variation, met with in the eye under light, is in reality the same normal excitatory response, by galvanometric negativity, with which we are already familiar.

I have also shown, by means of equi-alternating electric shocks, that under normal conditions the optic nerve is more excitable than the cornea, and that the retina is more excitable than the optic nerve. The eyeball and retina have often been found by different observers to exhibit 
abnormal or reversed response. Now, with regard to reversed response in general I have shown it to be due to either of two different conditions which hold good for all responding tissues. These are in the first place sub-tonicity, and secondly, fatigue. The abnormal response caused by the first has been shown to be converted into normal, in the case of the retina, by the action of an agent which enhanced the excitability.

Another phenomenon which I discovered in the response of retina was that of multiple response, induced by the application of strong or of continuous stimulation. These multiple responses have visual correspondences in the multiple after-images seen in the retina, and in the visual fluctuations which occur under the constant stimulus of light. The latter of these facts was demonstrated by a specially devised stereoscope (p. 432). In this connection may be mentioned the interesting phenomenon of Binocular Alternation of Vision.

The various types of direct and after-effects observed in vegetable tissues under light I find to have their close correspondences in the responses of the retina. Just as in the highly excitable lamina of Bryophyllum, constituting Type I., we have the formula of $(-++)$, so also, in the highly excitable retina of Ophiocephalus, the same sequences of direct and after-effects is observed. In less highly excitable vegetable tissues, such as the petiole of cauliflower, affording us Type III., the sequence was shown to be $(+-\ldots)$. In correspondence with this may be mentioned the response of the isolated retina of fish, observed by Kühne and Steiner. In this case, as the effect of isolation, the retina must have become sub-tonic, which supposition is borne out by the fact that its response to the immediate action of light was abnormal positive instead of negative. I found a similar sequence to occur in an isolated sub-tonic retina of Ophiocephalus (figs. 260, 261).

Finally, in somewhat fatigued specimens, an intermediate Type II. was found, in which the sequence was $(-+\ldots)$ 
or $(-\ldots)$. Examples of these are afforded by the eye of the frog. These correspondences, between the effects of light in vegetable tissues and in the retina, will be clearly understood from the series of figs. 253 to $26 \mathrm{I}$.

The next subject to be summarised is that of the electrical response of plants to gravitational stimulus. In an apogeotropic organ like the stem, when laid horizontally, the mechanical response is such as to make the shoot once more vertical. The active factor in this curvature might obviously be, either the responsive contraction of the upper side, or the responsive expansion of the lower. The question to be decided here was whether the response of the plant, to geotropic stimulus, was or was not of the same nature as its response to other effective forms of stimulation-that is to say, by excitatory contraction. An experiment has been described ( $p .436$ ) in which this question was subjected to tests. The local application of cold is known to bring about the temporary abolition of the excitatory effect, and in the present case, its application on the lower side of a horizontally laid shoot was not seen to induce any effect on the response, while, when applied on the upper, it retarded and arrested response to gravitation. This shows that in this response it is the contraction of the upper side which is the active factor. This is independently verified by the test of electrical response, where I find that the upper side, when subjected to gravitational stimulus, exhibits the sign of true excitation-namely, by induced galvanometric negativity.

The important Theory of Statoliths offers us a suggestive explanation of the manner in which gravity exercises stimulation upon the responding tissue, by the weight of solid particles. When the stem is vertical, in consequence of the symmetry of distribution of the particles on all sides, there is no resultant action; and as soon as this symmetry is disturbed by laying the stem horizontally, response might be expected to be initiated. This, however, is not the case. The shoot first bends down, and it is not until after the 
expiration of nearly three-quarters of an hour that the first sign of apogeotropic action appears. This anomaly is probably due to the induced mechanical curvature caused by weight which has first to be overcome. We may, however, regard ourselves as independent of the mechanical indications, when recording the effect of gravitational stimulus by geo-electric response. The excitatory electric effect, as we have seen in other cases, takes place as before, when all responsive mechanical indications are restrained. Proceeding on this principle, therefore, I found that the geo-electric response was initiated within so short a time of subjecting the specimen to gravitational stimulus as one minute (fig. 27I). This experiment shows of what widespread application is the electrical mode of detecting the excitatory response of tissues, to many different forms of stimulus. 


\title{
CHAPTER XLVIII
}

\author{
REVIEW OF RESPONSE OF NERVE AND RELATED \\ PSYCHOLOGICAL PHENOMENA
}

Transmission of excitation in plants-Vegetal nerve-Similar variations of receptivity, conductivity, and responsivity, under parallel conditions in plant and animal nerves-Conductivity balance-After-effect of section on conductivity and excitability-Function of vegetal nerve in plant-economyLaminæ of plant form a catchment-basin for stimulus-Motile response of nerve-Molecular cycle and characteristic changes in response of nerveEffect of fatigue on transmitted excitation-Similarity of excitatory molecular changes in both afferent and efferent nerves-Multiple response induced by strong stimulus in nerve-Multiple excitations in nerve during drying Individual contractile responses to constituent tetanising shocks-Negative after-effect on abrupt cessation of tetanisation-Extra-polar effects similar in plant and animal nerve-Inadequacy of Pfligger's Law-Under feeble E.M.F. excitability enhanced by anode and depressed by kathode-Demonstration by subjective response-Under feeble current excitation travels better against than with it-Response by variation of electrotonic current due to algebraical superposition of excitatory effect-Physico-physiological basis of sensationIdentification of positive tone of sensation with hydro-positive wave and negative tone of sensation with negative wave-Natural and artificial induction of dissociation of sensation-Physical explanation of Weber-Fechner's Law-Quality of sensation also a factor-Conversion from painful to pleasurable and vice versa at will by electrotonus-Memory as an after-effect of stimulus-Persistent after-sensation-Revival of latent memory-image through differential excitation induced by diffuse stimulation--Same effects demonstrated in the inorganic.

THE next subject to be reviewed is that of the conduction of stimulus. It has been supposed that plants do not conduct excitation by the transmission of protoplasmic changes, as certain animal tissues are known to do. Even in the wellknown case of Mimosa, where stimulus is seen to induce movement at a distance, this was supposed to be the result of hydro-mechanical disturbance. This conclusion has been shown, however, to be erroneous, for pure hydrostatic disturbance 
has been proved to occasion an erectile movement of the leaf with galvanometric positivity (figs. 44,45 , and 46 ). The transmission of true excitation, on the other hand, gives rise to a fall of the leaf and the electrical response of galvanometric negativity. Again, the transmission of excitation in the plant is modified similarly by those varying physiological conditions which influence it in the case of the animal. Thus, a strong stimulus is transmitted more quickly, other things being equal, than a feeble. Fatigue, on the other hand, is found to depress the velocity. The application of cold reduces or temporarily abolishes the transmission, while warmth enhances its velocity. Anæsthetics, again, are found to depress conductivity. And lastly, the polar effect of currents, in the plant as in the animal, is to induce opposite changes, according as anode or kathode is applied. I have, moreover, been able to isolate certain tissues specially fitted for the conduction of excitation. These are found in the soft parts of the fibro-vascular bundles, and are particularly easy to isolate in the case of fern. They here possess the relatively high velocity of about $50 \mathrm{~mm}$. per second. It may be said, in view of their peculiar responsive characteristics, and the modifications of their response under given conditions, that these structures are indistinguishable from animal nerves, and may therefore be rightly designated vegetal nerves. On isolation, for example, these highly excitable vegetal nerves, like the animal nerve, when isolated, are liable to fall into a state of sub-tonicity, on account of which their conducting power is temporarily impaired. The transmitted effect of stimulus, then, as in the corresponding case of animal nerve, becomes one of abnormal galvanometric positivity. Continuous stimulation when in this state, however, by carrying the tissue out of the $\mathbf{A}$ into the B condition, converts the abnormal positive response into normal negative, through an intermediate diphasic, in the plant as in the animal nerve. When in the B stage, again, tetanisation has the effect of enhancing response in both. The effects of ether, carbonic acid, alcohol vapour, and 
ammonia are the same in the one case as in the other. The effects of various drugs on the receptivity, conductivity, and responsivity of the vegetal nerve are the same as on those of the animal, and finally, in the action of different salts, the acid and basic moieties exhibit the same characteristic effects in plant and animal nerves alike (Chapters XXXII. and XXXIII.).

In order to study the variation of excitatory effects in nerves I was able to devise a very delicate instrument, the Conductivity Balance (fig. 29r). This apparatus not only enables us to study the modifications of conductivity, excitability, and responsivity, induced by a given agent separately, but also to compare relative variations as between any two of these, say, for instance, conductivity against excitability, or receptive excitability against responsivity. It also enables us to compare and contrast the action of two different reagents applied simultaneously in different parts of the same nerve. In this way the factor of uncertainty introduced by the unknown individual differences between two nerves is eliminated.

The principle on which the Method of Conductivity Balance depends is that of applying stimulus at a point which, in the excitatory sense, is exactly midway between the electrodes $\mathrm{E}$ and $\mathrm{E}^{\prime}$; the excitatory effects at $\mathrm{E}$ and $\mathrm{E}^{\prime}$ exactly balance each other, and the galvanometric deflection is then zero. When the excitability of the right hand, $\mathrm{E}$, or the conductivity of the right arm, $\mathrm{C}$, of the balance is enhanced, the balance is upset and the resultant response is, say, up ; depression, on the other hand, upsets it in the opposite direction. Not only may the effects of various chemical agents be determined by this method, but it is easy also to study by its means the effects of temperature on conductivity. Cold is thus found to depress, and warmth to enhance it (figs. 307, 309). Another important investigation carried out by this means was on the curious phenomenon presented by the effect of section in enhancing the excitability of adjacent points. It was shown that this 
was due to the molecular transformation caused by the stimulus of the mechanical or thermal section. The effect of such stimulus on neighbouring points is to induce moderate excitation, raising them to the higher excitability of the condition B (fig. 31 I). . At or very near the section point itself, on account of over-stimulation, the transformation is to condition $\mathbf{D}$ or $\mathbf{E}$, and the result should be one of loss of excitability. In accordance with this, it is found that at such points there is depression of excitability (fig. 3I2).

As regards the place of the vegetal nerve in the plant economy, it may be said that the normal excitability of a tissue, by which its proper functions are discharged, can only be maintained fully by a supply of energy, which must be received from the environment. Both animal and vegetal nerves have been shown, when isolated, to lose their normal conductivity and excitability, their response becoming abnormal or being abolished. It is only by the accession of fresh energy of stimulus that the normal conductivity and excitability are restored. It is known, further, that when the nerve loses its excitability, undergoing consequent degeneration, the attached muscle also exhibits rapid decay. It will thus be seen that the various tissues of the organism are maintained in their normal functional activities by means of energy conveyed to them through the nerves.

One of the principal forms of energy in maintaining the tonic condition of a green plant is sunlight; when deprived of this, its various normal activities come gradually to a stop, and the plant ultimately dies. But if any portion of the plant be exposed to light even its shaded parts will be found to continue in natural vigour. This is exemplified by the experiment of Sachs, in which an undetached branch of Cucurbita was kept in a dark box, and was found to grow, and produce flowers and fruits, as if under normal conditions. The fact that a plant, when totally deprived of sunlight, dies, shows how essential to its tonic condition is energy of light. The fact that so long as a portion of it is kept in light the whole flourishes, proves the transmission of energy from one 
part to another, a transmission which is now made comprehensible, being effected through the intervention of the plant-nerves, whose existence I have demonstrated. In the case of trees, again, the interior tissues whose functions are of great importance in various ways, are inaccessible to such external energy as that of light. But no part of them is far removed from the vegetal nerves, whose outer endings are found in the ramified venation of the leaves. The laminæ of the plant thus in their aggregation form an extensive catchment-basin for the reception of energy from outside and its ultimate transmission within the plant. An experiment has been described which shows the enhancement of the excitability of the plant-nerve by energy of light (fig. 334).

I have next to summarise a new method for the study of excitatory reactions in nerves. It has been supposed that in certain respects the reaction of the nerve is specifically different from that of the muscle. It has been regarded as typically non-motile, the highest power of the microscope being incapable, it was said, of detecting any effect in responsive change of form. I have shown, however, that this conclusion was erroneous, there being in this respect a continuity between the responses of muscle and nerve. In a particular case of frog's nerve the responsive contraction under strong stimulation was as much as I4 per cent. of the original length, and in others, it was as much as 20 per cent. or more. With a magnification of about 200 times, which is afforded by my moderately sensitive Kunchangraph, the observer is able to study all the excitatory phenomena in nerve with as great ease, and much greater accuracy, as by the employment of a very highly sensitive galvanometer. Records of the electrical responses of nerve are obtained by the differential effects of excitation at the two contacts, when one of these has been subjected to injury. It has been shown that such injury does not always completely abolish the excitability of the second contact, for which reason there may be induced a local reaction of feeble negative or reversed 
positive response. The interference of this with the normal response at the uninjured contact is thus apt to give rise to various complications. In contrast with this we have the reliability of the mechanical response of the nerve, in which the effect recorded is direct, and not differential. Again, the electrical form of stimulus, which is almost universally employed for the excitation of nerve, is liable by leakage, unless very great precautions are taken, to vitiate the results obtained by the electrical mode of response. When the response observed, however, is not electrical but mechanical, this source of error is obviously eliminated.

By means of mechanical response, the molecular transformations through which the nerve passes, under the action of stimulus itself, may be observed with the greatest clearness. An isolated nerve, cut off from its natural supply of energy, generally falls into a sub-tonicity indicated in the mechanical record, as an increasing abnormal relaxation; and the application of stimulus induces at this point an abnormal positive response, of sudden expansion. Successive or continuous stimulations, however, transform the nerve from condition A to condition B, the abnormal expansion being arrested and converted into increasing contraction. During this stage, then, the responses to individual stimuli are transformed from the abnormal expansive positive to the normal contractile negative, through an intermediate diphasic. Molecular transformation is here very rapid and the responses show a staircase increase (fig. 382). An intervening period of tetanisation will now have the effect of enhancing the response (fig. 383 ). In the clear demonstration thus obtainable of a progressive molecular transformation, with corresponding variations of response at its different stages, we arrive at the true explanation of the change from the abnormal positive to the normal negative, in electrical response, and also of the enhancement of the normal negative after an intervening period of tetanisation (figs, $275-278$ ).

The next stage to be reached is $\mathbf{C}$, where the responses are uniform. After this, we arrive at $\mathbf{D}$, where fatigue-decline 
begins to make its appearance. $U p$ to this point, the nerve as a whole has been undergoing increasing contraction, the base-line of the series of records being thus tilted upwards. But after $\mathbf{D}$, it begins to show relaxation, and at the stage $\mathbf{E}$, the responses to individual stimuli are actually reversed, the region of transformation from diminishing to reversed response being often marked by the appearance of diphasic (figs. 396 and 400). The entire responsive cycle may thus be viewed as consisting of two halves of which one is the reverse of the other. From the state of extreme sub-tonicity at A with its abnormal positivity, the responses are transformed through diphasic to feeble normal negative at $\mathbf{B}$. They here increase in a staircase manner, till they become uniform at $\mathbf{C}$. After this begins the reversing process, due to fatigue, brought on by overstrain, with its diminishing normal responses at $\mathbf{D}$, through diphasic, to abnormal positive once more, at E. Excessive sub-tonicity and excessive stimulation alike find their extreme case in the abolition of all response at death. The difference between the abnormal positive response of sub-tonicity and the abnormal positive response of fatigue lies in their previous history. The one is due to lack of stimulation and the other to its excess. For the restoration of normal response, the treatment in the two cases must be opposite. In the first, the application of stimulus is necessary ; in the second, it is its cessation, or rest, which is required.

Similar effects are also met with, in the case of transmitted excitation. In the sub-tonic condition, conductivity is depressed, and the transmitted effect is abnormal positive. By the action of stimulus, however, the conductivity is gradually restored, and the response to transmitted stimulation is converted from abnormal positive to normal negative through the intermediate diphasic. After this, under increasing fatigue the diminishing responses are converted to abnormal positive through an intermediate diphasic (fig. 325). Another important demonstration was that of the perfect similarity of the molecular changes induced by stimulus in the afferent and efferent nerves respectively. 
The experiments which have just been described were carried out on the efferent gastrocnemius of frog and gecko. With the afferent optical nerves of certain fishes I obtained mechanical responses which were exactly the same as these (figs. 324, 402). That is to say, in a sub-tonic condition the optic nerve gave the abnormal positive or expansive response, and this was subsequently converted into the normal contractile responses, through an intermediate diphasic.

The mechanical response of nerve just described, may be recorded either photographically, by a reflected spot of light, or directly on a smoked-glass surface by means of a writingpoint. The difficulties due to friction in the latter case are obviated by the use of the Oscillating Recorder. By means, however, of a battery of levers, and using the optical method of record, it is possible to have a magnification by the Kunchangraph of one hundred thousand times or more, the sensitiveness of the record being correspondingly enhanced. By this means many new phenomena may be brought under observation, one of these being the multiple response induced by strong stimulus in nerve. It is known, again, that nerve becomes highly excitable during the setting-in of 'drying,' and under these conditions, in a nerve-and-muscle preparation, repeated mechanical spasms are exhibited by the attached muscle. In taking the mechanical record of nerve, it is found that the substitution of dry for moist air at once induces a visible contraction. Now this state of partial contraction, bringing the nerve, as it does, into condition $\mathbf{B}$, we know to be significant of enhanced excitability. If the drying of the nerve be now allowed to continue, it is found that there is induced a series of multiple responses (fig. 326). And the multiple spasms seen during drying in the muscle of a muscle-and-nerve preparation undoubtedly have, as one of their factors, these multiple excitations thus demonstrated to take place in the nerve.

With the highly magnifying Kunchangraph, again, the individual effect of a single shock is demonstrated with the greatest clearness. Under rapidly succeeding tetanising 
shocks, the response shows a serration of the apical line, proving that the individual responses are not completely fused. On the abrupt cessation of tetanising shocks a sudden enhancement of the contractile effect occurs, followed by the usual recovery. This is analogous to the sudden enhancement of response on the cessation of tetanisation, seen in the retina, in magnetic response, and in the response of certain sensitive inorganic preparations under similar circumstances (pp. 536, 428, 383).

The next subject to be surveyed is that of electrotonus, and the variations in excitatory effects induced by it. It has been shown that the polarising currents induce extra-polar currents in the plant nerve, exactly as in that of the animal (fig. 338). As regards the effect of electrotonic currents generally on excitability, the results obtained by Bernstein are described as polarisation-decrement, whereas those of Hermann are known as polarisation-increment. That is to say, with one experimental arrangement the induced electrotonic current is seen to undergo a diminution under excitation, and with a different arrangement an increase. These and other electrotonic variations appear to be very anomalous, and incapable of mutual reconciliation.

I have, however, been able to show that all these effects may be regarded as combining the variations of two distinct factors, namely conductivity and excitability, under the influence of an electrical current. One of the principal difficulties in the correct explanation of these phenomena has hitherto lain in the assumption that Pfliger's Law, relating to the polar effects of currents, was of universal application. I have shown, however, to the contrary, that it applies only to a certain middle range of electro-motive intensity, the excitatory effect at anode and kathode being, at a very high E.M.F., exactly reversed. Going, again, to the other extreme, of a low electro-motive force, I have shown that, in opposition to Pfliger's generalisation, it is the anode that enhances the excitability of a nerve, while the kathode depresses it. This $\mathrm{I}$ have been able to demonstrate by 
numerous experiments (figs. $35^{\circ}$ and 353 ). The fact that under feeble E.M.F. the variation of excitability is opposite to that under moderate E.M.F. can be demonstrated with great simplicity by means of the subjective response of sensation. A wound was made on the back of the hand, and the application of a dilute solution of salt caused a moderate irritation. The application of kathode to this wound now rendered the irritation intolerably painful, while that of the anode at once made it soothing, removing even the normal discomfort due to the salt. These effects-coming under Pfliger's generalisation that kathode enhances excitability, while anode depresses-held good so long as the acting E.M.F. was about $\mathrm{I}_{5} 5$ volt. But when the acting E.M.F. was reduced to 5 volt, the kathode was found to induce a soothing sensation, whereas the anode became painful.

I have also found that the passage of a current profoundly modifies the conduction of excitation in a directive manner, according as the excitation has to travel with or against it. In the simplest cases, where the polarising electrodes are so far apart as to eliminate the direct excitatory effect of the poles, and using a feeble current, I have shown that excitation travels better electrically uphill, that is to say, against the current, than down, or with it. Thus the normal responses to transmitted stimulation are found to be enhanced when the polarising current is against the direction of transmission. A polarising current in the same direction as that of excitation, has, on the other hand, the effect of retarding it. The normal responses are then diminished, or even reversed to positive, by the diminution or abolition of the power of true conduction (figs. $345,346,347$ ).

The various effects described as polarisation-increment and decrement have been shown further to be due to the increased galvanometric negativity of the more excited of two points, the responsive current being algebraically summated with the existing electrotonic current. The greater excitation of one of these two points was also shown to be due to the greater intensity of excitation conducted to it, or 
to the greater excitability induced by the action of the anode, or to both.

This summary of results will conclude with a brief account of the demonstration of the physico-physiological nature of the basis of sensation. The effect of a single stimulus has been shown to consist of two different waves sent out from the point stimulated, of which the hydropositive travels with a greater velocity than the true excitatory or negative. If the stimulus applied, moreover, be feeble, the positive wave alone will be transmitted. If the stimulus, again, be very strong and the path of conduction short, one wave will be superposed over the other, the negative masking the positive. The two waves, however, may be separated from each other by inducing a depression of the conductivity of the nerve, when the negative will be made to lag behind the positive. By the suppression of the negative, owing to sufficient reduction of conductivity, the positive may be made to arrive at the responding point alone.

Nervous impulses have thus been shown to be of two different kinds, positive and negative, and contrary to the universal assumption that the nerve gives no visible indication of its state of excitation, it has been shown that these are accompanied by waves of expansion and contraction respectively. In addition to these visible mechanical expressions, we have also the concomitant electrical expressions of galvanometric positivity and negativity. I have been able, moreover, to identify the wave of expansion as the vehicle of that change which gives rise to the positive tone of sensation, which may be described as pleasurable or at least not unpleasurable. The negative or contractile wave, similarly, has been shown to be doloriferous. These two waves we saw to be separable from each other, whenever the conducting nerve was sufficiently long. Thus, when the sole of the foot receives a smart stroke from a rod, two different impulses are sent out, first the positive or sensation of contact, which is not unpleasurable, followed by the negative, with its different and painful tone of sensation. In various 
well-known cases of nerve-disease, bringing on diminished conductivity, this dissociation of sensation is met with pathologically. In paralysis, again, burning coals may be held in the hand and induce only the feeling of contact, without any sensation of pain.

One very difficult problem in connection with psychological response is that of the peculiar relation between the intensity of stimulus and that of response. The generalisation known as Weber-Fechner's law, asserts that stimulus must increase in geometrical, for sensation to increase in arithmetical, progression. Fechner, moreover, regarded this relation, not as due to any physical or physiological factor, but as a particular case of some specific psychological law. On an inspection of the mechanical responses of animal nerve, given in figs. 400,401 , and 402 , however, we see that the peculiar relation between stimulus and sensation follows inevitably on the physiological character of those responses. We there see that under feeble stimulus the response is positive, connoting, as we know, a positive tone of sensation. After this, as stimulus increases, the sign of response undergoes a reversal into normal negative. From this point onwards, for some time, the response to increasing stimulus shows a rapid rate of increase; but this increase tends to reach a limit as the maximum molecular distortion is approached. These facts follow naturally from the molecular theory of response which has been described, and in such considerations we obtain an explanation of those changes in the tone or quality of sensation of which WeberFechner's law was unable to take account. That these responsive characteristics, again, are not peculiar to the animal nerve, has been seen in the fact that vegetal nerves also show a similar relation between stimulus and response (fig. 403). That this relation indeed is universal, will be understood from the response of an inorganic substance to increasing stimuli, as given in fig. 404 .

Another interesting proof of the dependence of the psychological upon physico-physiological changes_is afforded 
by the polar action of currents. It has been shown that the positive response is short-lived, whereas the negative is relatively more persistent, its persistence increasing with the intensity of the response. Now, by means of the Sensimeter (fig. 405), we can apply a series of stimuli of measured intensity in such a way as to induce the neutral sensation which is neither pleasurable nor painful. The frequency of this stimulation is so adjusted as to appear all but continuous. If we now render the excited point moderately anode, and thus reduce its excitability, the neutral will be converted to the positive tone, and the sensation, moreover, will be rendered strikingly discrete. If, next, the excitability of the stimulated point be enhanced by the application of moderate kathode, the neutral sensation will become converted into painful, becoming, further, fused and continuous.

It will thus be seen that in the determination of sensation the internal plays as important a part as the external. By the peculiar molecular disposition of the nerve, it is indeed possible, as we have seen, to convert one quality of sensation into another, and such dispositions are to a greater or less extent under the control of the will. It is not external circumstances, then, which are the dominant factor psychologically, for the impression created by these is capable of indefinite modification in any direction, by the action of habitual induced dispositions. The reader will see for himself what illimitable possibilities are opened up by the line of thought here suggested.

The last subject to be reviewed is the phenomenon of memory, which is an after-effect of stimulus. The aftereffect of strong stimulus is in general more persistent than that of feeble. Similarly, the memory of a strong sensation is moregenduring than of a weak one. Very strong stimulus, again, gives rise, as we have seen, to multiple responses. In the retina these are perceived as multiple after-images, which sometimes appear to be renewed spontaneously. This fact will often be found a sufficient explanation of visual phantoms and hallucinations. This, however, is not the usual method 
of reviving memory-images. Long after every trace of the primary stimulation has disappeared we can revive it by an impulse of the will. Memory-impressions are often likened to scars. Of this metaphor, however, it may be said, that though, no doubt, when the blow is recent the smarting effect will persist for some time, causing an ever-diminishing aftersensation, yet, when the scar has healed, how could it, of itself, reproduce the original sensation? To do this, the original excitation would require to be reproduced, in the absence of the primary exciting cause. If, then, instead of regarding it as a scar, we translate the original impression into shades of light and darkness, we see that such a picture was produced by different intensities of the primary stimulus acting on the sensitive surface-in other words, by means of induced differential excitation. To bring back the picture we have to reproduce, in the absence of prirnary stimulus, the same state of differential excitation as was at first induced by it.

Such a revival is possible, as already shown, under the combined action of two different factors. It has been shown that when an isotropic tissue is locally acted upon by stimulus, the excitatory manifestation thus induced disappears after a time. There is now nothing visible by which to distinguish the stimulated from the unstimulated areas. In consequence of this stimulation, however, there has been a transformation of the molecular condition of the portions acted upon. The tissue, which was originally isotropic, has now become anisotropic, by the impression of this latent image. On diffuse stimulation, the differentially excitable structure will now exhibit the latent image, by various forms of differential excitation, of which some one particular manifestation will, in the case of any given organ, be the most conspicuous.

Thus, in a metallic plate containing latent positive and negative chemical impressions, we shall obtain, on the application of diffuse stimulus, corresponding positive and negative galvanometric responses. In a phosphorescent plate, again, a small area may be subjected to the action of light. On the 
cessation of stimulus this will give luminous response, which may be taken as the immediate effect of primary stimulus. On the fading of this image, if the whole plate be subjected to feeble diffuse illumination for a short time, the latent image will once more appear as a bright patch against a dark background. This is because, as the after-effect of stimulus, the area $\mathbf{B}$ has been rendered more excitable. Hence, diffuse stimulation evokes more intense response from it than from its more inert background. Similarly, the memory-image is capable of revival by the internal impulse of the will, acting as a diffuse stimulus to evoke a differential sensation, which reproduces the light and shade of the primary picture.

The responsive phenomena seen in living matter are, undoubtedly, wonderful and mysterious; but those shown by the inorganic are no less wonderful. By ascribing all physiological occurrences to specific reactions, and by constantly postulating the intrusion of forces of a new order, the road to the further advancement of knowledge is closed. By the conception of matter itself, on the other hand, as possessed of sensibility - that is to say, of molecular responsiveness-we attain an immediate accession of insight into those physical interactions which must furnish the terms of any ultimate analysis. We are led by it to the discovery of the impressive fact of continuity as existent between the responses of the most complex living, and the simplest inorganic matter. Limiting ourselves, again, to the realm of living matter, we are impelled to recognise parallelisms, in the response of plant and animal, whose extent could never otherwise have been suspected. All the responsive phenomena of the animal are thus found to be foreshadowed in the plant, and this to such a degree that in the common script of the response-record the one is indistinguishable from the other. In both we observe a similar series of excitatory effects, whether these be exhibited mechanically or electrically. Both alike are responsive, and similarly responsive, to all the diverse forms of stimulus that impinge 
upon them. We ascend, in the one case as in the other, from the simplicities of the isotropic to the complexities of the anisotropic; and the laws of these isotropic and anisotropic responses are the same in both. The responsive peculiarities of epidermis, epithelium, and gland; the response of the digestive organ, with its phasic alternations; and the excitatory electrical discharge of an anisotropic plate, are the same in the plant as in the animal. The plant, like the animal, is a single organic whole, all its different parts being connected, and their activities co-ordinated, by the agency of those conducting strands which are known as nerves. As in the plant-nerve, moreover, so also in the animal, stimulation gives rise to two distinct impulses, exhibiting themselves by twofold mechanical and electrical indications of opposite signs. It is the nature of the indicator, again, which determines in any given instance the form of the responsive expression. A single molecular derangement may thus find manifestation as change of form, alteration of electrical condition, and subjective sensory variation. The dual qualities or tones known to us in sensation, further, are correspondent with those two different nervous impulses, of opposite signs, which are occasioned by stimulation. These two sensory responses-positive and negative, pleasure and pain-are found to be subject to the same modifications, under parallel conditions, as the positive and negative mechanical and electrical indications with which they are associated. And finally, perhaps, the most significant example of the effect of induced anisotropy lies in that differential impression made by stimulus on the sensory surfaces, which remains latent, and capable of revival, as the memory-image.

In this demonstration of continuity, then, it has been found that the dividing frontiers between Physics, Physiology, and Psychology have disappeared. 



\title{
CLASSIFIED LIST OF EXPERIMENTS
}

\author{
Molecular Responsiveness of Matter
}

\section{Mechanical response :}

1. Contractile response in indiarubber

Electromotive response :

2. Response of tin .

3. Fatigue in inorganic response

4. Action of stimulant on response of platinum . . . . 8

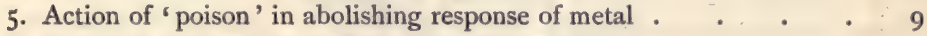

6. Response in metal by method of negative variation . . . 9

\section{Response by resistivity variation :}

7. Response of selenium to light . . . . . . . 3

8. Response of galena to electric radiation . . . . . . . . 3

9. Response of allotropic silver $\mathrm{Ag}^{\prime}$ to electric radiation . . . 4

\section{Fundamental Phenomenon of Response in plants}

ro. Simultaneous record of mechanical and electrical response . . .

11. Electrical response of pulvinus of Mimosa when physically restrained

I2. Response to sudden variation of tension . . . . . 25

13. Response to sudden compression . . . . . . . . 25

14. Response to tension and compression . . . . . . . 26

15. Response to mechanical blow . . . . . . . . 26

16. Response to vibrational stimulus $\quad . \quad+\quad . \quad$. $\quad . \quad 27$

17. Response to chemical stimulus _. . . . . . . 27

18. Response to thermal shock . . . . . . . 25, 38

19. Influence of sudden variation on efficiency of stimulation . . $\quad 32$

20. Additive effect . • • . . . . . . . . . 34

21. Genesis of tetanus in mechanical response of plants . . . 43

22. Genesis of tetanus in electrical response of plants . . . . 43

23. Rheotomic observation of time relation . . . . . . . . 48

24. Response to increasing intensity of stimulus of mechanical blows . $\quad 39$

25. Response to increasing intensity of vibrational stimulus . . . 40

26. Response to increasing stimulus, with or without complete recovery - 4 I 


\section{Positive and Negative Response}

27. Hydraulic response in Mimosa

28. Positive mechanical response followed by negative in Biophytum . . $\quad 58$

29. Positive mechanical response followed by negative in Mimosa . $\quad 59$

30. Simultaneous record of positive and negative mechanical and electrical response in Biophytum . . . . . . . . . . 6 6 I

31. Positive, diphasic and negative response in petiole of cauliflower $\quad .62$

32. Positive and negative responses in tuber of potato _ . . . . 64

33. Unmasking of positive element in response by selective block . . 66

34. Effect of stimulus of light on growth . . . . . . . 73

35. Effects of steady and sudden variation of temperature on growth response .

\section{VARIous Types of Response}

36. Abnormal positive response in sub-tonic tissue . . . . . 83

37. Staircase response in tissue originally sluggish $\quad . \quad . \quad$. 9 I

38. Staircase response in vegetal nerve . . . . . . . . 9 I

39. Staircase response in galena . . . . . . . . . 103

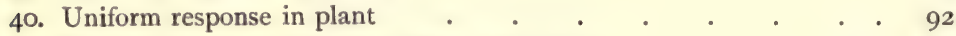

4I. Fatigue due to rapidly succeeding stimulation . . . . . 93

42. Fatigue due to overstrain . . . . . . . . . 96

43. Fatigue-decline under continuous stimulation, in mechanical response of Mimosa .

44. Fatigue-decline under continuous stimulation, in electrical response of celery

45. Oscillatory response in arsenic

46. Phasic alternation in mechanical response of style of Datura . . 100

47. Periodic fatigue in electric response of plants . . . . . $\quad$. 98

48. Periodic fatigue in autonomous response of Desmodium gyrans . . 99

49. Reversal of normal response in fatigued nerve . . . . . $\quad$ IO2

50. Bifurcated expression of response as growth and mechanical response 104

\section{Detection of Physiological Anisotropy}

51. Differential response of compound strip . . . . . . 108

52. Isolated responses of upper and lower halves of pulvinus of Mimosa, with resultant differential response . . . . . . . . I09

53. Transverse differential electrical response of pulvinus of Mimosa . I Io

54. Transverse differential electrical response of plagiotropic stem of Cucurbita . . . . . . . . . . . III

55. Transverse differential electrical response of petiole of Musa . . II4

\section{Natural Current of Rest and its Variations}

56. Effect of rise of temperature on current of rest . . . . . 119

57. Effect of falling temperature on current of rest _ . . . . 119

58. Effect of $\mathrm{Na}_{2} \mathrm{CO}_{3}$ solution on current of rest . . . . . 122

59. Effect of $\mathrm{CO}_{2}$ on current of rest . . . . . . . $\quad$. 122

60. Reversal of natural current of rest as after-effect of stimulation . . 127 


\section{Effect of Chemical Agents on Excitability}

61. Effect of chloroform

PAGE

62. Effect of chloral hydrate

63. Effect of formalin .

130

64. Effect of $\mathrm{NaOH}$

65. Effect of $\mathrm{KOH}$

I3I

132

66. Effect of sugar solution . . . . . . . . . . . 136

67. Effect of $\mathrm{Na}_{22} \mathrm{CO}_{3}$ solution . . . . . . . ${ }_{1} 36$

68. Effect of $\mathrm{HCl}$. $\quad . \quad$. . . . . . . . . 138

69. Effect of moderate and strong dose of $\mathrm{KOH}$. . . . $\quad$ I39

\section{Method OF INTERFERENCE}

70. Responsive effect of variation of phase . . . . . . I44

71. Diphasic response and its variation . . . . . . 147

72. Diametric method of recording response . . . . . . I47

\section{Current of Injury and Negative Variation}

73. Electrical after-effert in inorganic substances under strong stimulation . . . . . . . . . .

74. Resistant galvanometric negativity in vegetable tissue under strong stimulation. . . . . . . . . . 152

75. After-effect of persistent negativity due to section . . . . I 54

76. Electrical distribution in plant cylinder . . . . . . I55

77. Response by negative variation . . . . . . . 158

\section{Current of Death and Response by Positive Variation}

78. Response by abnormal positive variation . . . . . . . . 65

79. Electric exploration of dying and dead tissue . . . . . . 169

8o. Electric exploration of tissue one end of which is killed . . I7 I

81. Response by negative and positive variations of current of injury . 174

\section{EFFect OF TEMPERATURE}

82. Effect of cold in arresting autonomous pulsation in Desmodium . . I8I

83. Effect of warmth on autonomous pulsation of Desmodium . $\quad$ i 82

84. Effect of cold in permanent abolition of response . . . . . 183

85. Effect of cold in temporary abolition of response . . . . 184

86. Effect of cyclic variation of temperature on electric response . . 185

87. Effect of rising temperature on amplitude of response . . . 186

88. Effect of rising temperature on conductivity . . . . . I87

89. Abolition of response by high temperature . . . . . I90

90. Effect of cooling on frequency and amplitude of pulsation of Desmodium 


\section{DETERMINATION OF DEATH-POINT}

9I. Determination of death-point by abolition of electrical response . I95

92. Determination of death-point by means of thermo-mechanical curve 198

93. Determination of death-point by means of inversion of electromotive curve . . . . . . . . . 202

94. Simultaneous reversal of electrical curve and response . . . 204

\section{Multiple and Autonomous Response}

95. Multiple mechanical response in Biophytum . . . . . 207

96. Multiple electrotactile response in Mimosa . . . . . 208

97. Multiple electromotive response in Biophytum . . . . . 209

98. Multiple electric response in various tissues . . . . . 2 I0

99. Multiple electric response in stomach of frog . . . . . 2 I0

IOO. Autonomous response in Biophytum . . . . . . . 211

IOI. Initiation of multiple response in Desmodium under light . . . 212

102. Spark record of autonomous pulsation in Desmodium . . . 2 I4

103. Simultaneous record of mechanical and electrical response of pulsating Desmodium leaflet . . . . . . . . $2 \mathrm{I} 7$

104. Record of electrical responses in Desmodium leaflet when movement is restrained . . . . . . . . 220

105. Multiple response of growth . . . . . . . . 22 I

\section{REsponse OF LEAves}

I06. Effect of section of petiole of Ficus on the current of rest $\quad . \quad 227$

107. Effect of section of petiole of Citrus on the current of rest . . 226

108. Effect of stimulation of lamina . . . . . . $\quad 225$

I09. Parallelism between response of Musa and that of Dioncea . . 237

\section{Electric Discharge in Anisotropic Organ}

I 10. Response of leaf of Nymphrea to transmitted stimulation . . 245

II I. Response of leaf of Coleus to thermal shocks . . . . . 248

I12. Rheotomic observations . . . . . . . . 252

II3. 'Blaze current,' so-called, in inorganic substance . . . . 266

\section{Response to equi-alternating shocks in :}

I14. Carpellary leaf of Dillenia . . . . . . . 256

II 5. Leaf of Pterospermum . . . . . . . . . . 255

I16. Pitcher of Nepenthe . . . . . . . . . 256

117. Pulvinus of Mimosa . . . . . . . . . . 268

I18. Bulb of Uriclis lily . . . . . . . . . 257

I19. Prepared strip of lead . . . . . . . . . 267

120. Petiole of Musa . . . . . . . . . 283

'12 i. Plagiotropic stem of Cucurbita . . . . . . . 285

122. Eel . . . . . . . . . . 285

123. Variegated leaf of Pothos . . . . . , , , 286 


\section{RESPONSE OF SKIN}

124. Current of rest in vegetal skin

PAGE

125. Isolated responses to mechanical stimulus of upper and lower surfaces of grape skin

126. Isolated responses of frog's skin

127. Isolated responses of skin of tomato

300

128. Response of grape-skin to thermal shocks . . . . . . 30I

129. Response of grape-skin to equi-alternating shocks . . . . 302

130. Response of frog's skin to equi-alternating shocks . . . . 303

131. Response of tomato-skin to equi-alternating shocks . . . . 308

132. Exhibition of positive after-effect . . . . . . 309

133. Response of skin of tortoise . . . . . . . . 307

134. Response of intact human skin . . . . . . . 300

135. Illustrative response of pulvinus of Mimosa showing cyclic variation 303

136. Illustrative autonomous response of Desmodium exhibiting cyclic variation . . . $\quad . \quad$. . . . . 304

I37. Normal response of skin of gecko. , . . . . . 3 I0

138. Abnormal diphasic response of skin of gecko, converted to normal after tetanisation

3I I

\section{Response of Epithelium and Glands}

139. Resting current in foot of snail . . . . . . 318

I40. Reversal of true resting current by injury . . . . . . 319

14I. Response of water-melon . . . . . . . . . 315

142. Response of glandular foot of snail . . . . . . . 316

143. Response of human arm-pit . . . . . . . . 319

144. Response of human lip . . . . . . . . . 320

145. Response of human tongue . . . . . . . $\quad$. 322

146. Normal response of pulvinus of Mimosa, reversed after tetanisation

147. Response of Dillenia and its reversal after tetanisation . . 326

148. Reversed response of Dillenia under feeble stimulation . . . 328

\section{Response of Digestive ORgan}

149. Determination of natural current of rest in digestive organ of Nepenthe . . . . . . . . . . 335

150. Excitatory electric response of fresh pitcher of Nepenthe . $\quad 338$

151. Response of Nepenthe at intermediate stage . . . . . . 339

152. Response of pitcher of Nepenthe with entrapped insects . $\quad 339$

153. Normal response of digestive leaf of Drosera and its reversal after tetanisation . . . . . . . . 342

I54. Response of frog's stomach to mechanical stimulation . . . 345

155. Response of stomach of tortoise to electric stimulation . . , 345

156. Normal response of stomach of gecko and its reversal after tetanisation . . . . 346

157. Multiple response to strong stimulus in pitcher of Nepenthe when fresh. 
I58. Multiple response in pitcher of $N$ PAGE

158. Multiple response in pitcher of Nepenthe with entrapped insects . 34I

159. Multiple response in Drosera . . . . . . . . . . 343

160. Multiple response in stomach of frog . . . . . 347

\section{Ascent of Sap and Suctional Response}

161. Electrical response of young root . . . . . . . 353

162. Electrical response of older root . . . . . . . . 354

163. Responsive secretion by root . . . . . . . . 352

164. Response of root to chemical stimulation . . . . . 355

165. Electrical response of living wood and its depression under anæs-

thetic . . . . . . . . . . . 362

166. Abolition of electric response of wood by poison . . . . 363

167. Hydraulic response to stimulus . . . . . . . . . 366

168. Effect of cold on suctional response . . . . . . . 372

169. Effect of rise of temperature on suctional response . . . 372

170. Effect of anæsthetic on suctional response . . . . 375

171. Excitatory versus osmotic action . . . . . . . . . 376

172. Initiation of suctional response and enhancement under stimulus . 383

173. Diminution of latent period as after-effect of stimulus . . . 384

174. Responsive variation of suction under overbalance. . . . $\quad 386$

I75. Response to sub-terminal stimulation . . . . . . . $3^{88}$

\section{Response to Stimulus of Light}

I76. Transverse transmission of effect of moderate stimulus . . . 397

177. Transverse transmission of effect of strong stimulus . . . 399

I78. Mechanical response of Mimosa to unilateral light . . . . 400

I79. Electrical response of Mimosa to unilateral light . . . . $40 \mathrm{I}$

I80. Transmitted effect of stimulus of light . . . . . . 402

181. Electrical response of Bryophyllum to light . . . . . . 402

182. Electrical response of petiole of cauliflower . . . . . 406

183. Multiple growth response under light . . . . . . 405

184. Multiple mechanical response of Biophytum under light. . . 407

185. Multiple electrical response in Bryophyllum under continuous light 408

186. Normal negative and positive after-effect under light . . . 409

187. Influence of fatigue on after-effect. . . . . . . 4II

188. Third type of direct and after-effects in plants under light . . . 412

\section{REsponse of Retina}

189. Determination of differential excitability as between optic nerve and cornea . . . . . . . . .

190. Determination of differential excitability as between retina and optic nerve . . . . . . . . . . . . 419

191. Determination of true current of rest in frog's eye . . . . 4I8

192. Conversion of abnormal retinal response to normal by excitatory agents . . . . . . . . . . . 423

193. Normal retinal response of Ophiocephalus . . . . $\quad 427$ 
194. Reversed retinal response and after-effect in Ophiocephalus

PAGE

195. Three parallel types of direct and after-effect of light in plant and animal

I96. Multiple response in frog's retina

197. Multiple response in retina of Wallago . . . . . . . . 433

198. Multiple response in human retina . . . . . . . 430

199. Pulsating response in human retina under continuous light . $\quad .432$

200. Binocular alternation of vision . . . . . . . . 43I

20I. Analysis of composite image by after-effect . . . . . 432

\section{Geo-electric Response}

202. Response to unilateral pressure of particles . . . . . . 437

203. Determination of excited area under geotropic stimulus . . . 436

204. Geo-electric response - . . . . . . . . 440

205. Geo-electric response of an organ physically restrained . . . 442

\section{VELOCITY OF TRANSMISSION}

206. Determination of velocity of transmission by mechanical response - 447

207. Determination of centripetal versus centrifugal velocity . . . 448

208. Effect of fatigue on velocity of transmission . . . . . . . . 449

209. Effect of intensity of stimulus on velocity _. . . . . . . 449

210. Effect of temperature on velocity . . . . . . . 450

2 I I. Determination of velocity of transmission by electromotive method $45^{2}$

212. Longitudinal versus transverse conduction . . . . . 454

\section{Electric Response of Nerve to Thermal Stimulus}

213. Electrical response of frog's nerve to equi-alternating shocks . . 457

214. Normal response of animal nerve to thermal stimulation . $\quad 460$

215. Enhancement of normal response after thermal tetanisation . $\quad 462$

2I6. Abnormal positive converted to normal negative after thermal tetanisation. . . . . . . . . . 463

217. Gradual transition from abnormal positive to normal negative through diphasic $\quad . \quad . \quad . \quad . \quad . \quad .465$

218. Conversion of abnormal positive to normal negative through diphasic by increasing intensity of stimulus . . . . . . 466

\section{Electric Response of Plant Nerve}

219. Effect of ether on electric response of plant nerve . . . . 472

220. Effect of carbonic acid . . . . . . . . 472 .

221. Effect of alcohol . . . . . . . . . . . . . 473

222. Effect of ammonia . . . . . . . . . . . 474

223. Effect of ammonia on response of indifferent tissue . . . . 474

224. Three parallel types of response in plant and animal nerve $\quad$. 475

225. Effect of tetanisation on enhancement of normal negative response - 475 
226. Abnormal diphasic converted into normal negative after tetanisation

227. Conversion of abnormal positive to normal negative through intermediate diphasic under increasing intensity of stimulation

\section{Conductivity Balance}

228. Effect of $\mathrm{Na}_{2} \mathrm{CO}_{3}$ solution on responsivity of frog's nerve . . . 485

229. Effect of $\mathrm{CuSO}_{4}$ solution on responsivity of frog's nerve . . . 485

230. Effect of $\mathrm{CaCl}_{2}$ on responsivity of plant nerve . . . . . 486

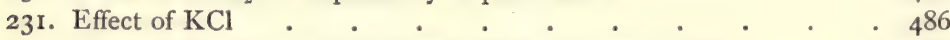

232. Comparative effects of $\mathrm{NaCl}$ and $\mathrm{NaBr}$ on responsivity . . . 487

233. Effect of dilute solution of $\mathrm{Na}_{2} \mathrm{CO}_{3}$. . . . . . . . 488

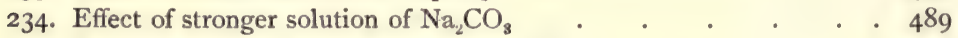

235. Excitability versus conductivity under $\mathrm{KI}$. . . . . . $49 \mathrm{I}$

236. Excitability versus conductivity under NaI : . . . . 492

237. Effect of alcohol on responsivity of frog's nerve . . . . 492

238. Effect of alcohol on receptivity of plant nerve . . . . . 493

239. Effect of alcohol on conductivity of nerve . . . . . . 493

240. Effect of alcohol on responsivity of nerve . . . . . . . 494

241. Receptivity versus responsivity under alcohol . . . . 495

242. Effect of cold on conductivity . . . . . . . . 499

243. Effect of warmth on conductivity . . . . . . 50 I

244. After-effect of moderate stimulation on conductivity and excitability 504

245. After-effect of strong stimulation on conductivity and excitability . 505

\section{Mechanical Response of Animal Nerve}

246. Mechanical response of frog's nerve under electric tetanisation . 509

247. Effect of ammonia on mechanical response . . . . . 516

248. Effect of morphia . . . . . . . . . . 516

249. Effect of aconite . . . . . . . . . 516

250. Effect of alcohol . . . . . . . . . . 517

25I. Effect of chloroform . . . . . . . . 518

252. Conversion of abnormal positive to normal negative through diphasic, by tetanisation . . . . . . . . 521

253. Occurrence of staircase effect _ . . . . . . $\quad$. 524

254. Comparison of mechanical responses in animal and plant nerve . $\quad \mathbf{5 2 8}$

255. Mechanical response of optic (sensory) nerve . . . . $\quad 529$

256. Fatigue of conductivity in nerve of gecko. . . . . . 530

257. Transient enhancement of contraction on cessation of tetanisation . 536

258. Multiple mechanical response in nerve as effect of drying . . 539

\section{Mechanical Response of Plant Nerve}

259. Enhancement of excitability after tetanisation . . . . . 554

260. Effect of light in enhancing excitability of plant nerve . . . 556

261. Determination of velocity by mechanical response of plant nerve $\quad 525$ 


\section{Response of Living Tissue by Resistivity Variation}

262. Determination of death-point by inversion of curve of resistivity . 546 263. Excitatory response by resistivity variation . . . . . 548 264. Action of anæsthetics on response by resistivity variation in frog's nerve

\section{Electrotonus}

265. Extra-polar effects in plant nerve . . . . . . . 56 I

266. Variation of conductivity in plant nerve by passage of current $\quad . \quad 566$

267. Conduction of excitation with the current . . . . . 567

268. Conduction of excitation against the current . . . . 568

269. Modification of excitability by anode . . . . . 569

270. Modification of excitability by kathode . . . . . 570

27I. Variation of excitability and conductivity with polarising electrodes in shunt . . . . . . . . . 572

272. Variation of excitability and conductivity with polarising electrodes in series.

\section{INADequacy OF Pflüger's LAW}

273. Normal effects of anode and kathode on plant . . . . 579

274. Reversal of normal effect under high E.M.F. . . . 579

275. Demonstration by subjective response of opposite effects induced by moderate and feeble E.M.F.

\section{Molecular Theory of Excitation}

\section{Mechanical Response :}

276. Mechanical response of pulvinus of Erythrina indica, by make of kathode and anode . . . . . . . . . 583

\section{Magnetic Response:}

277. Uniform magnetic responses . . . . . . . . 594

278. Periodic groupings in magnetic response . . . . . 594

279. Incomplete magnetic recovery after strong stimulation . . 595

280. Additive effect of magnetic stimuli, individually ineffective . $\quad 595$

281. Magnetic tetanisation, with transient enhancement of effect on cessation . . . . . . . . . . . 623

282. Demonstration of exact magnetic balance . . . . . 608

283. Effects of $\mathrm{A}$-tonus and $\mathrm{K}$-tonus on magnetic conduction . . . 609

284. Opposite effects of moderate and strong $\mathrm{K}$-tonus on magnetic conduction. . . . . . . 6ro

285. Effects of $\mathrm{A}$-tonus and $\mathrm{K}$-tonus on magnetic excitability . . . 6II

286. Enhancement of magnetic conductivity by successive magnetic stimuli . . . . . . . . . 6r2

287. Blocking of transmission of magnetic excitation . . . . 613 


\section{Response by resistivity variation :}

288. Response of aluminium powder to electric radiation . . . . 601

289. Persistent after-effect on strong stimulation . . . . . 603

290. Effect of warmth in hastening recovery . . , . . . . 60I

291. Uniform responses to electric radiation . . . . . . 602

292. Cyclic molecular variation and concomitant modification of response - characteristic curves . . . . . . . . . 620

Effect of tetanisation at A stage, conversion of abnormal positive to normal negative seen in :

293. Mechanical response of frog's nerve . . . . . . 627

294. Electromotive response of tin . . . . . . . 628

295. Conversion of abnormal to normal through diphasic in platinum . 629

296. Response by resistivity variation in selenium . . . . . . 63I

297. Response by resistivity variation in tungsten . . . . . 632

Effect of tetanisation at B stage, enhancement of normal response seen in :

298. Mechanical response of frog's nerve . . . . . . 634

299. Responsive resistivity variation in selenium . . . . . $63 \mathrm{I}$

300. Responsive resistivity variation in aluminium powder . . . 632

30 r. Electromotive response of tin . . . . . . . 630

302. Magnetic response of iron . . . . . . . . 633

Effect of tetanisation in inducing $\mathrm{E}$ stage, diminution or reversal of normal response seen in :

303. Mechanical response of frog's nerve . . . . . . . . 635

304. Mechanical response of nerve of gecko . . . . . . 642

305. Mechanical response of india-rubber . . . . . . . . 642

306. Responsive resistivity variation in tungsten . . . . . 638

\section{Correlation of Psychic and Physiological Response}

307. Expansive and contractile response in muscle . . . . . 649

308. Relation between stimulus and response in sciatic nerve of gecko . 657

309. Relation between stimulus and response in sciatic nerve of bull-frog $65^{8}$

310. Relation between stimulus and response in sensory optic nerve of Ophiocephalus. . . . . . . . . 659

311. Relation between stimulus and response in plant nerve . . . 659

312. Relation between stimulus and response in magnetic substance . 660

\section{Experiments with Sensimeter on Electrotonic Variation of SEnsation}

313. Conversion of pleasurable sensation to painful under kathode (mechanical stimulation) . . . . . . . 670

314. Conversion of painful to pleasurable sensation under anode (mechanical stimulation) . . . . . . . . . . 67I 
3I5. Positive tone of sensation due to thermal stimulus converted to PAGE negative under kathode . $\quad . \quad$. $\quad . \quad . \quad .672$

316. Negative tone of sensation due to thermal stimulus converted to positive under anode . . . . . . . . . . 672

317. Reversal of normal effects under feeble E.M.F. . . . 671, 672

318. Differences of fusion in sensation according as it is modified to positive or negative . $\quad . \quad . \quad . \quad . \quad 671,672$

\section{MEMORY}

319. Revival of latent impression in metal under diffuse stimulation . 683

320. Revival of latent or 'memory image' in phosphorescent screen under diffuse stimulation . . . . . . . . . 684

321. Reversed or negative image . . . . . . . 685 



\section{N D E X}

ADDiTIVE effect, 34, 595

After-effect, persistence of, under strong stimulation, I 5 I-I 54

Anæsthetics, effect of, on excitability, 130

$\begin{array}{lll}, & , & \text { on response of nerve, } 472,518,673 \\ , & , & \text { on response of wood, } 362 \\ , & \quad, & \text { on responsive resistivity variation, } 549 \\ \text { on suctional response, } 375\end{array}$

Anisotropy induced by stimulus, in memory image, 686

$\begin{array}{llll}, & , & , & \text { in metallic plate, } 683 \\ , & \text { ", } & , & \text { in phosphorescent screen, } 684 \\ \text {, } & \text { ", } & \text { in plagiotropic stem, III }\end{array}$

Arm-pit, response of human, 319

Ascent of sap, various theories of, 356

Assimilation and dissimilation, 68, 87, 308

Autonomous response, continuity with multiple, 2 I I

$\begin{array}{ll}, & \text { in Biophytum, } 2 \text { I I } \\ ,, & \text { in Desmodium, } 212\end{array}$

Avalanche theory, 502

BERNSTEIN on polarisation decrement, 562

Biedermann on response of glands, 289

Binocular alternation of vision, 43I

Biophytum, mechanical response of, 58

, multiple response of, 209

, positive and negative response in, 59

Blaze current, so-called, in lead, 266

Block, advantages of method of, 133

, method of, 5

Burdon Sanderson, on response of Dioncea, 12

Characteristic curves of conductivity, 62I

, , , of differentially excitable surfaces, 324

,, ", of magnetisation, 620

Complex sensation, dissociation of, by lag, 674, 675

, , obliteration of negative element in, by selective block, 673, 675

Composite image, analysis of, by after-effect, 432

Conduction of true excitation in plants, 446 
Conductivity balance, experiments with :

\begin{tabular}{|c|c|c|c|}
\hline ", & " & , & $\begin{array}{l}\text { on conductivity versus receptivity under alcohol, } \\
495\end{array}$ \\
\hline " & ," & $"$ & $\begin{array}{l}\text { on conductivity versus receptivity under } \mathrm{KI} \text { and } \\
\mathrm{NaI}, 49 \mathrm{I}-492\end{array}$ \\
\hline " & ", & ," & on receptivity under alcohol, 493 \\
\hline , & ", & ", & " versus responsivity under alcohol, \\
\hline & "' & & $\begin{array}{l}\text { on responsive variation under alcohol, } 494 \\
\text { on variation of conductivity under alcohol, } 494\end{array}$ \\
\hline
\end{tabular}

Conductivity balance, experiments with :

\begin{tabular}{|c|c|c|c|c|}
\hline & ," & variati & responsi & y by alcohol on frog's nerve, 492 \\
\hline & :, & ", & , & by $\mathrm{CaCl}_{2}$ on plant nerve, 485 \\
\hline & ", & ," & ," & by $\mathrm{CuSO}_{4}$ on frog's nerve, 485 \\
\hline & ," & ," & , & by $\mathrm{KCl}$ on plant nerve, 487 \\
\hline & , & $\therefore$ & ," & by $\mathrm{Na}_{2} \mathrm{CO}_{3}$ on frog's nerve, 485 \\
\hline & , & , & " & $\begin{array}{l}\text { contrasted effects of } \mathrm{NaCl} \text { and } \\
\mathrm{NaBr}, 487\end{array}$ \\
\hline & ," & ", & conduc & $\begin{array}{l}\text { ty by } \mathrm{Na}_{2} \mathrm{CO}_{3} \text { on plant ne ve, } 488- \\
489\end{array}$ \\
\hline
\end{tabular}

Conductivity balance, experiments with :

" $\quad$ effect of cold on conductivity, 499

, $\quad$ " of electric current, 565-568

$" \quad, \quad$ of excessive stimulus, 505

", " $\quad$ of moderate stimulus, 504

Correlation, 553

" of moderate stintus, 504

Corrosion figures, 349

Crescograph, 22I

Cucurbita, electric response of plagiotropic stem of, II I , 285

Current of death, 166

Current of injury : anomalous variation in, 165

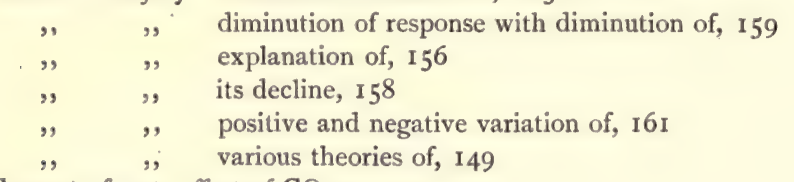

Current of rest, effect of $\mathrm{CO}_{2}$ on, 122

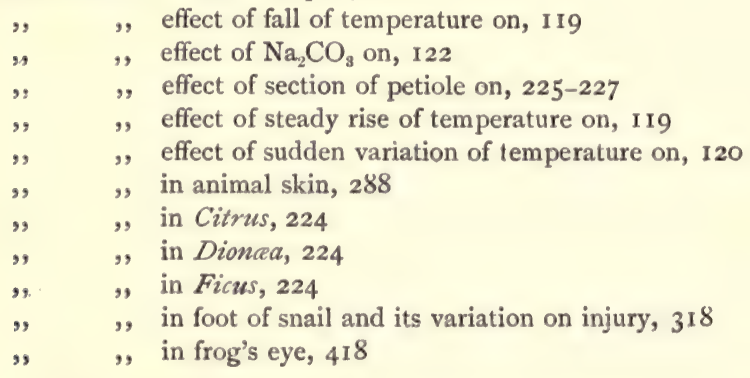


Current of rest, in Nepenthe, 335

\begin{tabular}{|c|c|c|}
\hline & ," & in vegetal skin, 298 \\
\hline & , & natural, and its variations, 317 \\
\hline , & , & phasic changes in, 302,303 \\
\hline & , & physiological condition determining, II 7 \\
\hline & , & positive and negative variations of, 126 \\
\hline & , & reversal of, as after-effect of stimulus, I 24 \\
\hline & , & variation of, $175^{-177}$ \\
\hline
\end{tabular}

DARWIN on excitatory reaction in Drosera, 33I

Dead tissue, positivity of, I70

Death, different post-mortem symptoms of, 192

Death-point, accurate methods of determining, 194

$\begin{array}{ccc}, & \text { determination of, by abolition or reversal of response, I95 } \\ , & , & \text { by electric spasm, } 202 \\ ,, & , & \text { by inversion of curve of electric resistivity, } 546\end{array}$

Depression, response by method of relative, 9

Desmodium pulsation, electro-motive response of free leaflet, 2 I 8

$\begin{array}{cccc}, & , & , & \text { leaflet physically restrained, } 220 \\ \text { " } & , & \text { principal and subsidiary waves in, }\end{array}$
" $\quad$, initiation of, under stimulus, 2 I 2
, $\quad$, $\quad$ rate of, 215
, $\quad$, spark record of, 2 I4

Dewar and McKendrick on retinal current, 4I5

Differential excitability, determination of : in eel, 285

\begin{tabular}{|c|c|c|c|}
\hline ", & ", & ", & in Musa, 114,284 \\
\hline ", & ", & "' & in plagiotropic stem, I I I , 285 \\
\hline ", & , & , & in pulvinus, $3 \circ 3$ \\
\hline "3 & " & , & in retina, 419 \\
\hline & , & , & in variegated leaves, 286 \\
\hline
\end{tabular}

Differential excitation, in memory revival, 680

Differential response, of compound strip, Io8

$$
\begin{array}{lll}
, & \text {, } & \text { of Mimosa, 108, } 303 \\
, & \text { laws of, I09 }
\end{array}
$$

\begin{tabular}{|c|c|c|}
\hline ", & ", & current of rest in, 335,344 \\
\hline , & , & multiple response in, $333,341,343,347$ \\
\hline "' & , & response of, in Drosera, 342,343 \\
\hline$"$ & , &,$\quad \quad$ in frog, 345,347 \\
\hline ," & , & in gecko, 346 \\
\hline$"$ & ", & in Nepenthe, 339-34I \\
\hline , & ", & in tortoise, 345 \\
\hline
\end{tabular}

Digestive organ, alternating phasic reactions in, 329

Digestive organ, response of,

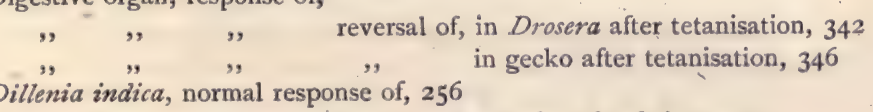

Dillenia indica, normal response of, 256 
Dillenia indica, reversal of normal response, under feeble stimulation, 328

Direct and after-effect, methods of, 275

\section{Dissociation and delayed pain, 675}

$$
\begin{gathered}
\text { of light, in plant, } 409-414 \\
, " \text { in retina, } 427-430
\end{gathered}
$$

, of complex sensation, by depression of conductivity, 674

Dose, effect of, on "excitability, r 36

$$
\text { by lag, } 675
$$

Drosera, response in digestive leaf of, 342,343

Drugs, modification of effect by tonic condition, 64 I

, significance of effect of dose, 639,640

Drying, effect of, on nerve, 539

Du Bois-Reymond on current of rest in frog's skin, 288

$$
\begin{array}{lll}
, & \quad, & \text { on organ current, } 260 \\
, & \text { on positive and negative polarisation, } 246
\end{array}
$$

Dying tissue, negativity of, 169,173

EBbinghaus, on rate of forgetting, 678

Eel, electric response of, 285

Electric discharge under excitation in leaf organ :

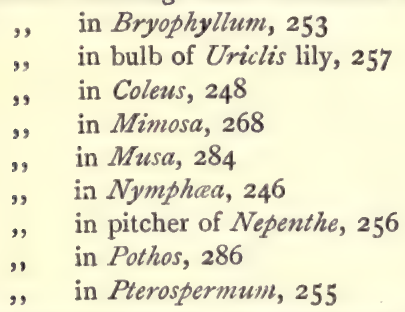

Electric distribution, explanation of, in dying and dead tissue, 173

, , $\quad$ in plant and muscle cylinder, I 50,156

Electric organ, anterior and posterior surfaces, 242

, . , laws of response in, 248

,, $\quad$, theories of, 260

Electrical response, in absence of mechanical, 20, 220

, , laws of, in anisotropic organs, 109

, , , , in isotropic organs, 75

Electrotonus, Bernstein's polarisation decrement, 562

, conversion of qualities of sensation by, 670-672

, extrapolar effects in plant nerve, 56I

"Hermann's polarisation increment, $56_{3}$

" law of variation of conductivity under, 568

", , , , excitability under, 57 I

,, modification of conductivity by, 566-568

, variation of excitability by, 569

Energy, hydraulic transmission of, 69

Engelmann on current of rest in skin, 288

Equi-alternating shocks : their advantage, 274 
Excitability, variation of, by chloral, I3I

\begin{tabular}{|c|c|c|c|}
\hline ," & ", & ," & chloroform, I 30 \\
\hline , & ", & ", & formalin, I32 \\
\hline , & ", & ", & $\mathrm{HCl}, \mathbf{I}_{3}^{8}$ \\
\hline ", & , & , & $\mathrm{KHO}, \mathbf{1}_{3} 8$ \\
\hline , & ," & ," & $\mathrm{NaHO}, \mathrm{I} 34$ \\
\hline & , & ," & $\mathrm{Na}_{2} \mathrm{CO}_{3}, \mathbf{1}_{3} 6$ \\
\hline & & ," & sugar solution, 136 \\
\hline
\end{tabular}

Excitation, true meaning of, I6

Fatigue, alternating, 98

,$\quad$ in response of metal, 7

., reversal of normal response in Dillenia under, 327

, , , in Drosera under, 342

," , , , in Mimosa under, 326

, $\quad, \quad$, $\quad$ in nerve under, ro2

, , , , $\quad$, $\quad$ in stomach of gecko under, 346

, $\quad$, transmitted effect in nerve under, 530

,, under continuous stimulation, 95

,, under overstrain, 96

Fibro-vascular bundles, distribution of, in stem, $55^{8}$

Forgetting, rate of, 678

Frog, response in retina of, 418,426

$, \quad, \quad$ in skin of, 300,302

, , $\quad$ in stomach of, 345,347

Functions of vegetal nerve, 559

GalenA, response of, 3

Gecko, fatigue of conductivity in nerve of, 530 ,

,$\quad$ response in nerve of, 657

, $\quad, \quad$ in skin of, 310,3 II

, , , in stomach of, 346

Geo-electric response, in apogeotropic organ, 440

, $\quad, \quad$ in organ physically restrained, 442

Geotropic action, hydrostatic theory of, 435

, $\quad$ statolithic theory of, 435

Geotropic stimulus, determination of area excited by, 436

Gotch on oscillatory character of electric discharge, 270

Growth, effect of stimulus on, 73

Growth pulsation, $22 \mathrm{I}$

temperature on, 73

Gymnotus, electrical discharge in, 242

HAAKE on electromotive difference in plants, 13

Hartig on ascent of poison, 363

Heidenhain on enhancement of excitability by section, 502

Hermann on current of rest in skin, 288

, on polarisation increment, 563

Holmgren on retinal current, 415 
Human lip, response of, $32 \mathrm{I}$

, skin, response of, 300

, tongue, response of, 322

Hydraulic response, 55

INJURY, degree determining sign of action current, 175 see Current of Injury

Interference, induced difference of phase causing, 142

, method of : effect of chemical agents determined by, 147

, , , effect of cold determined by, 147

Inversion of thermo-mechanical curve, 198

, of electro-motive curve, 202

, of curve of resistivity, 546

KÜHNE and Steiner on retinal current, 415

Kühne on polar effects in Protozoa, 579

Kunchangraph, 5I I

Kunkel on electro-motive variation due to water movement, 13

, on electric reaction in plants, $\mathrm{r} 3$

LATENT image, revival in phosphorescent screen, 684

Latent impression, revival of, in metal, 683

Laws of differential response, 109

, , electrical response of isotropic tissue, 75

, , , response in electric organs, 248

,, ,, variation of conductivity under electrotonus, 568

, , , , , excitability under electrotonus, 57 I

Leaves, electric response of Bryophyllum, 253

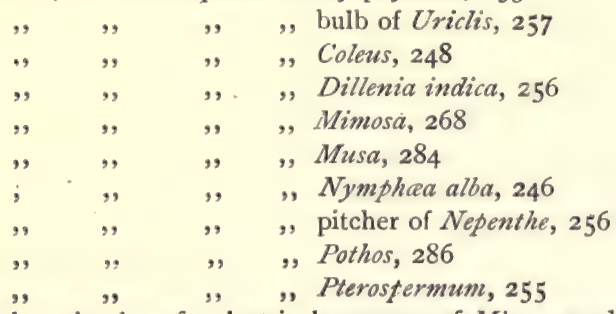

Light, stimulus of : electrical response of Mimosa under, 401

mechanical response of Mimosa under, 400

mechanical response under, in pulvinated and growing organs, 394

multiple electrical response induced by, in plant, 406,408

, $\quad$," multiple mechanical response induced by, 407

, , , , , , , , , , in retina, 426

, , $\quad$ multiple visual impulse induced by, 430

,, , transmitted effect due to, 402

Light, influence of fatigue on after-effect of, 4 I I

") negative and positive responses to, 402, 406

, phasic responsive alternations under, 408 
Light, positive and negative after-effects under, 409, 4I 2

,, three types of direct and after-effect in plant under, 409-4I4

, three types of direct and after-effect in retina under, 427-429

Lip, response of human, 32 I

MAGnetic balance, 607

Magnetic conduction, blocking of, 61 4

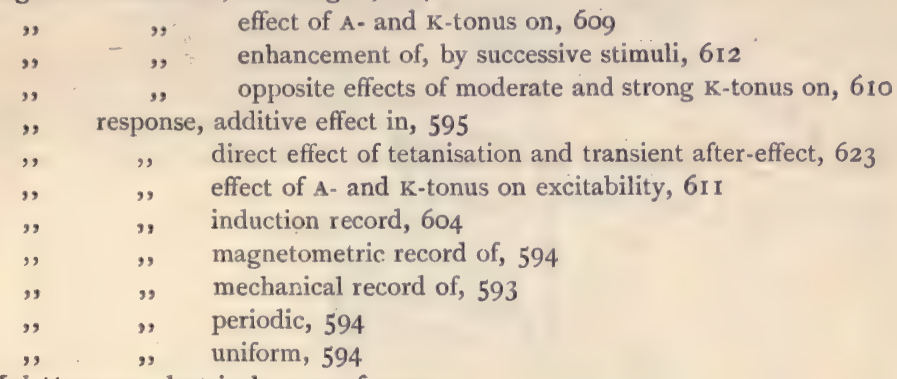

Malepterurus, electrical organ of, 242

Mechanical and electrical response, simultaneous record of, I 7, 19

Mechanical stimulator, rotary, 29I

Melon, electrical response of, 3 I 5

Memory, an after-effect of stimulus, 677

" explanation of revival of, 685

, persistence, dependent on strength of stimulus, 677

, spontaneous revival of, 680

Memory image, negative, 684

Metal, abolition of response by poison, 9

, fatigue in response of, 7

, response of, 6

Mimosa, electrical response of, 20, I10, 127, 268, 326

, electrical response under light, $40 \mathrm{I}$

, hydraulic response in, 55

, hydro-positive and negative response of, 56, 59

, mechanical response under light, 400

, phasic changes in response of, 303

, reversal of response by fatigue, 326

, variation of motile sensibility of, 2 I

," differential response of, 108

Molecular model, 598

Molecular modification, reversal of normal response due to, 7

Molecular response, persistent after-effect in, 597, 60I, 603

Molecular theory of excitation, 590

Molecular transformation, external tests of, $620^{\circ}$

Morograph, 197

Morographic record by electro-motive response, 202

", $\quad$ ", by mechanical response, t98

Motor transformer, 28i 
Munk on response in Dionca, 12

Musa, electrical response of, parallel to Dionce, 237

Muscle cylinder, electrical distribution in, 150

Multiple response, electrical in Biophytum, 209

\begin{tabular}{|c|c|c|}
\hline$"$ & ", & in Desmodium, 218, 220 \\
\hline ", & $"$ & ,, in Drosera, 343 \\
\hline ", & , & electro-tactile in Mimosa, 209 \\
\hline ", & ", & electrical in pitcher of Nepenthe, 34 I \\
\hline ", & , &,$\quad$ in stomach of frog, 347 \\
\hline " & $"$ & mechanical, in Biophytum, 208 \\
\hline ", & $"$ & in Desmodium, 212 \\
\hline " & $"$ & , $\quad$ in nerve, 539 \\
\hline "' & ', & rheotomic record showing, 52 \\
\hline ", & ", & under light in Biophytum, 407 \\
\hline ", & ", & in Bryophyllum, 408 \\
\hline "' & ", & in cauliflower, 406 \\
\hline$"$ & ", & in frog's retina, 426 \\
\hline "’ & ", &,$\quad$ in human retina, $43^{\circ}$ \\
\hline "' & ", & of growth, 22I \\
\hline
\end{tabular}

Nepenthe, current of rest in, 335

, multiple response in, $34 \mathrm{I}$

, three types of response in, 338-340

Nerve, excitatory electrical changes in, 508

, , mechanical changes in, 509

, failure to conduct, 530

Nerve of animal, electrical response of,

",
",


Nerve of animal, mechanical response of, effect of morphia on, 516

\begin{tabular}{|c|c|c|c|}
\hline , & צ' & , & , \\
\hline פ' & , & , & , \\
\hline , & ", & , & , \\
\hline , & , & ," & ", \\
\hline ," & ", & , & , \\
\hline , & , & ", & , \\
\hline , & , &, & , \\
\hline "9 & , & , &, \\
\hline
\end{tabular}

Nerve of plant, discovery of, 470
, of tetanising electric shocks, 510

five stages in, 89,635

multiple response in, 538

relation between stimulus and, $657-659$

similarities of, in plant and animal, 528

in motor and sensory, 529

three types of, 519

transient enhancement of, on cessation of tetanisation, 536

, , response of, conversion of abnormal to normal after tetanisation, 475

conversion of abnormal to normal by increasing in.

tensity of stimulus, 476

effect of alcohol on, 473

effect of ammonia on, 474

effect of $\mathrm{CO}_{2}$ on, 472

effect of ether on, 472

effect of tetanisation in enhancing, 476

" , ,,$\quad$ three types in, 475

$, \quad, \quad$ similarities of response in, and in animal, $47 \mathrm{I}-478$

Nerve of plant, mechanical response of, 528

\begin{tabular}{|c|c|c|c|c|}
\hline ", & ", & ", & ", & determination of velocity by, 525 \\
\hline & , & ", & " & effect of light on, 557 \\
\hline & , & , & ", & aiter tetanisation, \\
\hline
\end{tabular}

Nervous impulse, two kinds of, 647

,, , various direct and indirect manifestations of, 648

Noll, Haberlandt, and Nemec, statolithic theory of, 435

Nymphea alba, response of leaf of, 246

Ophiocephalus, mechanical response of optical nerve of, 529

Oscillating recorder, 527

response in retina of, $427-429$

Optic nerve and cornea, differential excitability between, 4I 7

\section{PACINI, law of, 242}

Pfeffer and Czapek on theory of geotropism, 435

Pfliger, avalanche theory of, 502

Pfliger's law : its failure with high E.M.F., 579

,, , , , with low E.M.F., 58I

$, \quad, \quad$, of polar effects of currents, 578

Phasic reactions, alternating, Ioo

,, variations of current of rest, 302, 303

, , , , , turgidity, 305

Phenolpthaline, detection of transport channels by, 360

Polar effects of currents, demonstration of :

\begin{tabular}{|c|c|c|c|c|}
\hline ", & " & ", & "' & by motile response in plants, 579 \\
\hline ", & ", & ", & , & by subjective sensation, $5^{82}$ \\
\hline
\end{tabular}


Poison, action of, modification of, by dose and tonic condition, 640, 64I , , , on inorganic response, 9

Positive response of sub-tonic tissue, 83

, $\quad, \quad$, unmasking of, from resultant negative, 66

Plerospermum, analogy with Torpedo, 255

Pulvinated and growing organs, similarities of response in, 394

REID, on response of skin of eel, 289

Resistivity variation, determination of death-point by, 546

$\begin{array}{ccc}\text { ", } & \text {, } & \text { response by, and its correspondence in the other modes of } \\ \text { response, } 549\end{array}$

Response, bifurcated expression of, 104

, considered as molecular derangement, 590

, law of differential, ro9

, law of isotropic, 75

," positive, diphasic, and negative in cauliflower, 62

, ., , , , in potato, 64

, simultaneous record of mechanical and electrical, I7, 19

, various forms of, 2

Response recorder, 34

Retina, determination of differential excitability of, and optic nerve, 418

, excitatory after-effect in, $427-430$

, multiple response in human, 430

, , , , in frog, 426

", ", ", Wallago, 425

, response of, in Ophiocephalus, 427-429

, , $\quad$ in frog, $4 \mathrm{I} 8$

,, so-called positive response of, 4I 9

Retino-motor effect, $42 \mathrm{I}$

Retinal response, conversion of abnormal to normal, 423

, " " explanation of abnormal, 423

, , $\quad$ three types of after-effect in, 427-429

Reverser, oscillating, 276

, rotating, 280

Rheotome, observations with, 47, 254

Root as digestive organ, 349

,, response of young and old, 353,354

,2 responsive secretion of, $35^{2}$

Rosenthal on current of rest in stomach, 288

Rotary Mechanical Stimulator, 29I

SACHS on growth of Cucurbita in darkness, 557

Schönlein on oscillatory character of electric discharge, 270

Season, influence of, on recovery from stimulus, 45

,,$\quad$ on suctional response, 390 
Selenium, response of, 3

Sensation, conversion from positive to negative by tetanisation, 665

, different tones of, 646

,, dual elements in, 674

, effect on, of attention and inhibition, 652

, effect on, of molecular disposition, 663,664

, effect on, of progressive molecular change in nerve, 66I

, identification with opposite nervous changes, 650

, $\quad$ interchange of pleasurable and painful under electrotonus, 670-672

, $\quad$ negative element in, blocked by anæsthetics, 673

, positive and negative, 664

, positive simple, negative complex, 665

Sensimeter, 670

Shoshungraph, 368

Skin, abnormal response converted to normal after tetanisation, 3 I I

, response of, in frog, 300,302

$\begin{array}{lll}, & , & \text { in gecko, 3I0, 3I I } \\ , & , & \text { in grape, 299, 30I, 302 } \\ , & , & \text { in intact human, 300 } \\ , & , & \text { in tomato, 307, 308, 309 } \\ ,, & , & \text { in tortoise, 307 }\end{array}$

Snail, foot of, response in, 316

, , $\quad$ resting current and its variation in, 318

Staircase response, 91, I03, 522,524, 625, 635

Stimulants, action of on inorganic response, 8

Stimulation, by chemical agents, 27

, by equi-alternating electric shocks, 277

,, by taps, 26

,, by tension and compression, 25

, by thermal shocks, 24

" effect of moderate, on conductivity, 504

, $\quad$, of strong, on conductivity, 505

, instantaneous mechanical, 47

, response to various forms of, 24-27

, ", to increasing intensity of, 40

, rotary mechanical, 29r

, vibratory, 27

Stimulator, electro-thermic, 38

, vibratory, 30

Stimulus, effect of, on growth, 73

" importance of, seen in ascent of sap, 382, 555

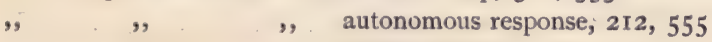

", , ", growth, 556

", , ", motile excitability, 554

, opposite effects of, on highly tonic and sub-tonic tissues, 77

, relation between, and response :

,,,$\quad$ in plant-tissue, 40, $4 \mathrm{I}$

", $\quad$, $\quad$ in nerve of bull-frog, 658

, $\quad$ in nerve of fern, 659 
Stimulus, relation betwe $\epsilon \mathrm{n}$, in nerve of gecko, 657

$\begin{array}{lll}, & , & \text { in optic nerve, } 659 \\ , & , & \text { magnetic, in iron, } 660\end{array}$

Strasburger on ascent of poisons, 363

Suctional response, diminution of latent period of, 384

\begin{tabular}{|c|c|c|}
\hline & , & effect of anæsthetics on, 375 \\
\hline ," & , & effect of poison on, 374 \\
\hline , & , & effect of season on, 390 \\
\hline , & , & effect of variation of temperature on, 372 \\
\hline & ,, & initiation of, by stimulus, 382 \\
\hline & , & osmotic versus excitatory action, 376,377 \\
\hline & , & persistence of, particular zone being killed, 373 \\
\hline & , & under method of balance, $3_{3} 8_{3}$. \\
\hline & ," & ," , • overbalance, $3^{86}$ \\
\hline & , & ,, unbalanced method, 388 \\
\hline
\end{tabular}

TABULAR statement :

,$\quad$ " $\quad$ electro-motive variation in Desmodium leaflet, 2 I9
,$\quad$ heliotropic effects, 394
,$\quad$ determination of death-point, 205
,$\quad$ velocity of transmission in living tissues, $45^{2}$

Tapper, the mechanical, 26

Temperature, different effects of, on conductivity and excitability, I 87

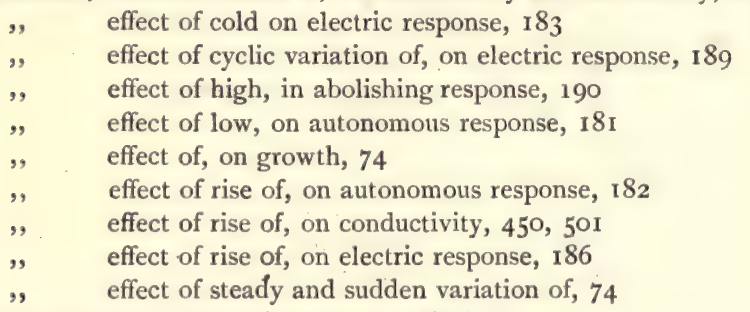

Tetanus, genesis of mechanical and electrical, 43

Tetanisation, transient enhancement of response on cessation of :

Tetanisation, effect of :

$\begin{array}{llll}, & , & , & \text { in brominated silver, } 429 \\ , & , & , & \text { in frog's nerve, } 536 \\ , & , & , & \text { in frog's retina, } 428 \\ ,, & \text { ", } & \text { in magnetic response, } 623\end{array}$

A stage-conversion of abnormal to normal response,

Electromotive response,

$\begin{array}{ll}\text {, } & \text { continuous transformation from abnormal to normal } \\ \text { in platinum, 629 }\end{array}$

Mechanical response,

" conversion from abnormal expansive to normal
contractile response in frog's nerve, 627 
Tetanisation, effect of :

Response by resistivity variation,

B stage-enhancement of normal response,

Electro-motive response,

$, \quad, \quad$ in nerve of fern, 475

",$\quad$ in nerve of frog, 462

, , $\quad$ in response of tin, 630

Mechanical response,

,, $\quad$, in frog's nerve, 634

, $\quad$, in nerve of fern, 554

Response by resistivity variation,

, $\quad$ in aluminium powder, 632

Magnetic response,

in selenium, 630

$, \quad, \quad$ in response of iron, 632

D stage- fatigue reversal of normal response,

Electro-motive response,

$\begin{array}{ccc}\text { Mechanical response, } & \text { reversal of normal response in Drosera, } 342 \\ \text { in stomach of gecko, } 346\end{array}$

Response by resistivity variation,

, , , fatigue reversal in tungsten, 638

Thermal chamber, 200

" cork chamber, 500

, shocks, quantitatiye stimulation of nerve by, 460

, , , stimulation by, 38

,, variator, II 3

Time-relations, difference of, in different tissues, 45

, rheotomic observations on, 50

Tongue, response of human, 322

Torpedo, electrical organ of, $24 \mathrm{I}$

Tortoise, response of skin of, 307

, , , of stomach of, 345

Transportation channels, detection of, by phenolpthaline, 36

VELOCITY of transmission :

determination of, by electro-motive method, $45^{2}$

, by mechanical method, 447

, centrifugal versus centripetal, $44^{8}$

, $\quad$ effect of fatigue on, 449 
Velocity of transmission :

determination of, effect of intensity of stimulus on, 449

, $\quad$ effect of temperature on, $45^{\circ}$

, longitudinal versus transverse, 454

Verworn on polar effects of currents on Protozoa, 579

Vibratory stimulator, 30

Vision, binocular alternation of, $43 \mathrm{I}$

Visual impulse, pulsatory character, 432

Von Fleischl on response of nerve, 278

WALLER on 'blaze current,' 260

, on response of green leaves to light, 395

", on response of skin, 294

, on three types of response in nerve, $46 \mathrm{I}$

Waves, positive and negative : their characteristics, 67

Weber-Fechner's law : its inadequacy, 656

Wood, demonstration of living characteristics of, $36 \mathrm{I}$

, effect of anæsthetics and poison on response of, 362, 36

, effect on, of drying, 36r

, normal response of, $36 \mathrm{I}$

ZANTEDESCI on organ current, 269 

- 


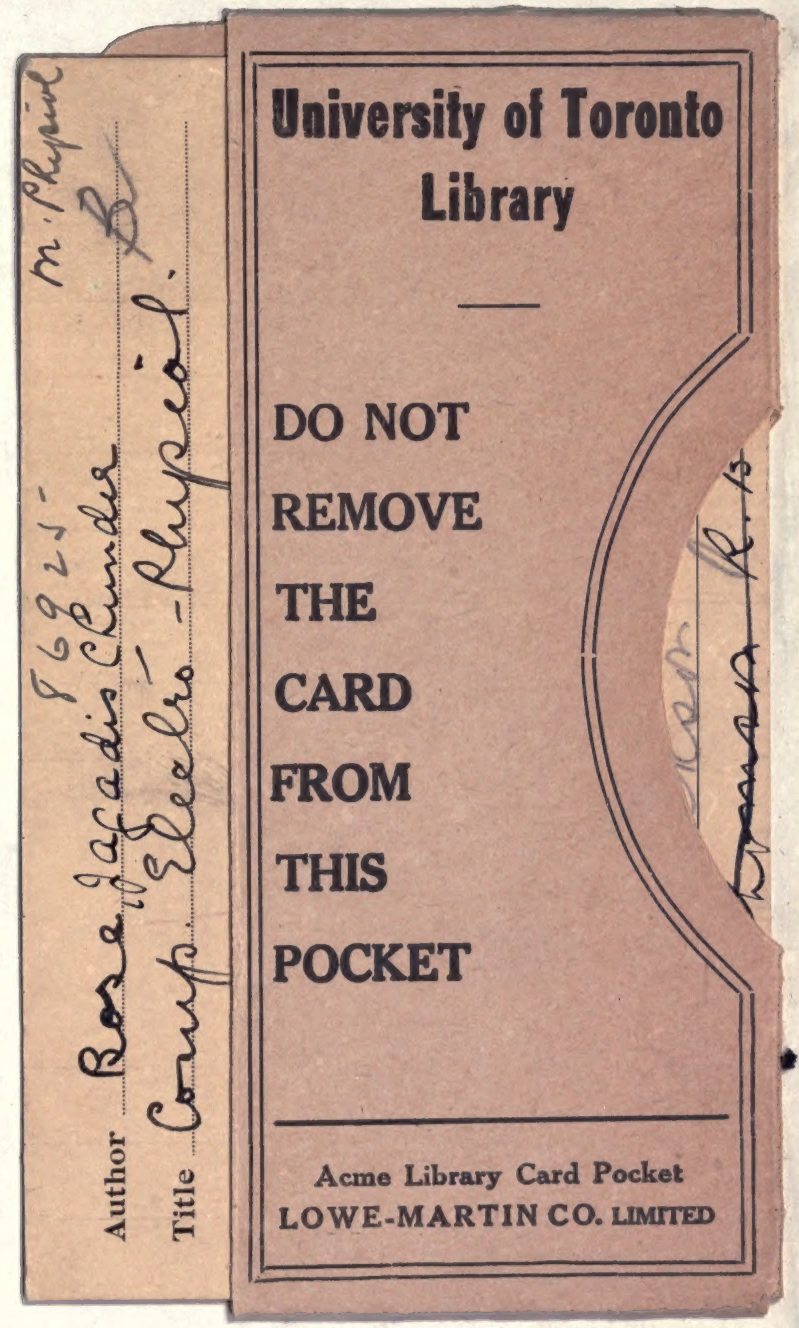


